
$\begin{array}{ll}518216 & \text { HIST } \\ \text { COL }\end{array}$ 
anl. 


$$
281101492 x
$$




\section{PATHOLOGY \\ AND \\ BACTERIOLOGY OF THE EYE \\ COLLINS AND MAYOU}


Digitized by the Internet Archive in 2014

https://archive.org/details/b21287442 


\section{PATHOLOGY}

\section{AND \\ BACTERIOLOGY OF THE EYE}

BY

E. TREACHER COLLINS, F. R. C. S.

CONSULTING SURGEON TO THE ROYAL LONDON OPHTHALMIC HOSPITAL AND CONSULTING OPHTHALMIC SURGEON TO THE CHARING CROSS HOSPITAL, ETC.

AND

M. STEPHEN MAYOU, F. R. C. S.

SURGEON TO THE CENTRAL LONDON OPHTHALMIC HOSPITAL, ETC.

SECOND EDITION

WITH 4 COLOURED PLATES

AND 306 FIGURES IN THE TEXT

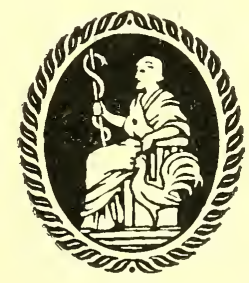

LONDON

WILLIAM HEINEMANN

(Medical Books) Ltd. 



\section{PREFACE TO SECOND EDITION}

It is thirteen years since the first edition of this book was published, during which time our knowledge of the pathology of eye has grown apace. In preparing a second edition we have, therefore, had to undertake a thorough revision of our former work and add much new matter.

The first edition appeared as one of a series of volumes constituting an International System of Ophthalmic Practice. The present one is produced as a separate and independent work.

Pathology is really disordered physiology, and the classification of diseases of the eye on a physiological rather than an anatomical basis, adopted as a novel procedure in the first edition, has been retained in the present one. The grouping of diseases in a new classification may aptly be compared to the working out of a jig-saw puzzle. The construction of the frame is usually easy, the chief difficulty arises in fitting the central pieces in to make a complete picture. Whilst in this edition we have retained the main divisions of our subject, we have, with the advance of knowledge, thought fit to alter in several instances the grouping of the individual diseases.

Slit lamp microscopy of the living eye is as yet in its infancy and we have not attempted to include all its findings, which still require much correlation with the histological characters of diseased condition. We have, however, quoted some of them where they seem to support our argument.

Since the appearance of the first edition, we have each of us contributed several articles dealing with the pathol- 
ogy of the eye to various periodical publications, many of the drawings by which these were illustrated are included in the present volume, and we have to express our indebtedness to the Council of the Ophthalmological Society of the United Kingdom for its permission to use them.

Though each chapter has had additions made to it, the most extensive alterations will be found in Chapters III, V and VII. The clinical importance of Glaucoma, or Hypertony, naturally led to a comprehensive study of the conditions giving rise to it, and its sequelæ. The opposite condition, Hypotony, though of just as much importance from a pathological standpoint, has received comparatively little attention. In Chapter III "On the disturbances in the circulation and constitution of the nutrient fluids of the eye," not only Hypotony, but the alterations in the circulation of lymph, with which it is so frequently accompanied, are more fully dealt with than in the previous edition.

The increase in our knowledge of the nature of immunity and of the influence of antibodies, has necessitated the rewriting of much of the first part of Chapter V "On inflammation."

The recognition of several degenerative conditions of the tissues of the eye as hereditary symmetrical affections, occurring apart.from any inflammation or toxic influences, has led to the regrouping of many of the diseases dealt with in Chapter VII entitled "Degenerations." We have much pleasure in acknowledging our indebtedness to Mr. Williamson Noble F.R.C.S., for his valuable assistance in correcting the proofs and constructing the Index.

E. Treacher Collins.

M. S. MAYou. 


\section{PREFACE TO FIRST EDITION}

In text-books dealing with diseases of the eye the method usually employed is to treat, separately, of the affections met with in each of its different anatomical divisions. As diseases are frequently not limited by anatomical landmarks such a classification necessarily results in overlapping.

It is possible to give an inclusive description of the various diseases which occur in an organism, or in one part of an organism such as the eye, by dividing them into the several processes which occur in its life history; this is the method which we have adopted.

The life of all organisms consists of a process of evolution or development to a state of maturity, which is reached and maintained by the absorption of nutrient material together with a capacity for resisting pernicious influences in the environment. Finally there comes a process of involution or degeneration.

The first chapter of this book deals with various aberrations which occur in the eye in its process of evolution.

Tissues after having arrived at a state of maturity may reassume some of their embryonic characteristics, such a condition is spoken of as an atavism of tissue or teleplasia, and the resulting new formations as teleplasms. These teleplasms are described in the second chapter on new growths.

The nutrition of the eye is maintained by various fluids, the changes which result from derangements in their circulation forms the subject of Chapter III.

Chapters IV, V and VI are devoted to disturbances resulting from pernicious influences in the environment. 
Chapter IV treats of injuries mechanical, chemical, thermal, electrical and radial. Chapter $\mathrm{V}$ of the reaction of the tissues to irritation, or inflammation; and Chapter VI of various parasites which invade the eye and the specific reactions to which they give rise. There are some affections that are presumably parasitic in origin but in connection with which up to the present no specific organism has been definitely demonstrated, these also are included in this chapter.

The process of involution or degeneration of the various tissues of the organ is described in the last chapter.

An appendix at the end of the book treats of the different laboratory methods employed in the pathological examination of the tissues of the eye and of the bacteria found in association with it.

The facts and theories of which our knowledge of the pathology of the eye is made up are the outcome of the work of an immense number of different observers. Anyone who writes a treatise on the subject must necessarily be indebted for much of his material to the writers who have preceded him.

About some matters there is much difference of opinion but we have not thought it necessary, in a book of this character, to discuss at length the rival merits of all the different theories which have been advanced, or even to mention some that have been found definitely untenable. We have preferred to dwell chiefly on those which accord best with our own observations, adapting or modifying them in such ways as we have found desirable.

We have not thought it advisable to insert any names of authors in the text of this book or to give anything like a complete list of references in the foot-notes. First, because we think the insertion of names in the text tends to distract the attention of the reader from the subject which is being dealt with. Second, because to have done so with anything like adequate justice would have invested the book 
with a bibliographical character which it is not desired that it should possess.

We have both enjoyed exceptional opportunities for the practical study of pathology of the eye, and it is the outcome of the experience we have thus gained which we have endeavoured to record. We have already written numerous separate articles bearing of the subject and references have been given to these in footnotes in appropriate places to enable the reader, who so desires, to find further evidence for the views expressed than it has been possible to include here. A few references to the articles by other writers which it has been thought would prove useful for a similar reason have also been given.

E. Treacher Collins.

M. S. Mayou. 



\section{CONTENTS}

\section{CHAPTER I}

\section{Aberrations in Development}

I. Aberrations Connected with the Formation of the Primary and Secondary Optic Vesicles ....... Cyclopia or synophthalmia; anophthalmia; congenitally cystic eyeball; shot-silk or watered-silk retina; coloboma of the retina and optic nerve; microphthalmia with cysts; congenital pigmentation of the retina; congenital ectropion of the uveal pigment of the iris; absence of nerve fibres; opaque nerve fibres in the retina; crater-like hole of the optic disc; physiological cup of the optic disc; pseudo-neuritis; congenital day-blindness with colour-blindness, and congenital night-blindness (Mooren's retinitis punctata albescens); congenital amblyopia; congenital colour-blindness; miosis congenita.

\section{Aberrations Connected with the Invaginating Surface} EPIBLAST.

Congenital aphakia; congenital nuclear cataract; defective development of the posterior capsule; opaque membrane behind the lens; shrunken fibrous tissue cataract; posterior lenticonus; disc-shaped cataract; congenital Morganian cataract; axial fusiform cataract; backward or forward displacement of the nucleus of the lens; triradiate opacities; coralliform axial cataract; congenital punctate cataract.

III. Aberrations Connected with the Invaginating MesoBLAST

Persistence of the central hyaloid artery or its sheath; abnormalities in the arteria centralis retinæ; cilio-retinal vessels; connective tissue formation in the vitreous chamber; muscæ volitantes; coloboma of the vitreous humour; congenital defects in the suspensory ligament of the lens; ectopia lentis; coloboma lentis; lenticonus anterior; cellular adhesion of the ciliary processes to the lens capsule. 
IV. Aberrations Connected with the Encircling Meso-

Congenital opacities and vascularity of the cornea; congenital anterior synechiæ of iris, pupillary membrane or lens; congenital anterior staphyloma; congenital glaucoma or buphthalmia; megalocornea and microcornea; keratoconus; astigmatism; persistent pupillary membrane; fibrous tissue formation on the anterior surface of the iris; aniridia; coloboma of the iris; polycoria; corectopia, discoria' and microcoria; coloboma of the choroid, typical and atypical; congenital crescent; muscular tissue external to the clioroid; blue sclerotics; axial myopia; hypermetropia; albinism; melanosis of the eye.

V. Aberrations Connected with Structures in the Line of the Orbito-nasal Fissure, the Formation of the Eyelids and the Orbital Muscles.

Coloboma of the eyelids; absence of the lacrymal canai; lateral probosis or demicyclops; epicanthus; congenital shallowness of the orbits and exophthalmos; orbital meningo-encephalocele; dermoid cysts along the line of the orbito-nasal fissure; congenital hernia of orbital fat; congenital notches in the lower lid at the outer canthus; abnormalities in the lacrymal passages; fistula of the lacrymal sac; cryptophthalmia; dermoid growths; abnormally large plica semilunaris; microblepharon and lagophthalmos; ankyloblepharon; trichiasis; distichiasis; congenital ptosis; associated movements of eyelid and jaw; congenital abnormalities in the extrinsic muscles of the eye; congenital ocular paresis; congenital enophthalmia; heterophoria or latent squint; concomitant strabismus.

\section{CHAPTER II}

\section{Neoplasms}

\section{Teleplasms Arising in Parts Derived from Cuticular} Epiblast . . . . . . . . . . . . . I60 Squamous carcinoma or epithelioma; papilloma; horns; epithelial plaques; glandular cancer of the skin or rodent ulcer; carcinoma of the Meibomian glands; adenoma; carcinoma and adenoma of the lacrymal gland; moles or nevi of the conjunctiva and the malignant growths arising from them.

II. Teleplasms Arising in Parts Derived from Neural EPIBLAST .

Glioma or neuro-epithelioma of the retina; gliomata of the 
optic nerve, gliomatosis; innocent tumours of the pars ciliaris retinæ (adenomata); innocent pigmented growths in the uveal tract (melanomata); malignant melanotic growths of the pigment epithelium of the uveal tract (melanotic carcinomata).

III. Teleplasms Arising in Parts Derived from Mesoblast . i 86 Intraocular sarcoma; epibulbar sarcoma; sarcoma of the orbit; endothelioma of the optic nerve; lymphoma; neuro-fibroma and neuro-fibromatosis; fibroma of the optic nerve; osteoma; myoma; hemangioma; lymphangioma.

IV. Metastatic Growths

Intraocular metastatic carcinoma; metastatic thyroid tumours in the orbit; orbital metastatic lympho-sarcoma.

V. Cysts .

i. Cuticular epiblastic. Dermoid cysts; implantation cysts; cysts of the lacrymal gland; cysts of Zeiss's glands; cysts of Moll's glands; cysts of Meibomian glands; cysts of Krause's glands; cysts of Henle's glands.

ii. Neural epiblastic. Cysts at the back of the iris; cysts on the inner surface of the cilary body; simple detachment of the retina; cysts in the retina.

iii. Mesoblastic. Extraocular lymphatic cysts; intraocular endothelial lined cysts.

\section{CHAPTER III}

\section{Disturbances in the Circulation and Constitution of the Nutrient Fluids of the Eye}

I. Disturbance in the Circulation and Constitution of the BloOD. . . . . . . . . . . . . . . 24 I

i. The blood supply to the interior of the eye.

ii. Complete obstruction to the central artery of the retina.

iii. Complete obstruction to the central vein of the retina.

iv. Obstruction of a ciliary artery.

v. Obstruction of a vortex vein.

vi. Impeded retinal circulation.

Albuminuric retinitis; diabetic retinitis.

vii. Impeded choroidal circulation.

viii. Extravasation of blood.

Hemorrhage from iris; hemorrhage into vitreous; hemorrhage from the choroid; hemorrhage from the retina; hemorrhage into the optic nerve. 
ix. Dilatation of orbital vessels.

Localised dilatation of the vessels connected with the orbit; general dilatation of the orbital vessels; exophthalmic goitre; cavernous sinus thrombosis.

$\mathrm{x}$. Diseases due to changes in the constituents of the blood.

(a) Ischemia from loss of blood.

(b) Deficiency of red blood corpuscles. Pernicious anæmia; secondary anæmia.

(c) Excess of red blood corpuscles. Erythræmia (Vaquez's disease).

(d) Deficiency of hemoglobin. Chlorosis.

(e) Increased white blood corpuscles. Leukemia; chloroma.

(f) Increased coagulability. Thrombosis; embolism; retinitis septica.

(g) Decreased coagulability. Hemophilia.

(h) Deficiency of vitamins. Vitamin A, xerosis of conjunctiva and night blindness;

Vitamin B, beriberi neuritis; Vitamin C, scurvy.

(i) Poisons in the blood.

Endogenous; exogenous.

II. Disturbances in the Circulation of Lymph

i. Serous detachment of choroid and ciliary body.

ii. Serous detachment of the retina.

iii. Serous exudation from the iris.

iv. Serous exudation from the ciliary body.

v. Serous effusion into the head of the optic nerve.

vi. Serous exudation from the retinal vessels.

vii. Edema of the cornea.

viii. Serous effusion into the conjunctiva.

ix. Serous effusion into the orbit.

$\mathrm{x}$. Serous effusion into the eyelids.

xi. Serous effusion into Tenon's capsule.

xii. Serous effusion secondary to general diseases.

III. Disturbances in the Circulation of the Intraocular

FluId. . . . . . . . . . . . . . . . . . 293

i. Mechanism for the maintenance of the normal intraocular tension.

ii. The causes of hypertony or increased intraocular tension. 
iii. The effects of hypertony on the various structures of the eye and their respective functions.

iv. The means employed for the reduction of tension in primary glaucoma.

(a) The opening up of the normal channels of escape of fluid from the eye.

Iridectomy; iridectomydialysis; cyclodialysis.

(b) The formation of new channels for the escape of fluid from the eye.

Filtration scars and cystoid cicatrices; detachment of the choroid and intraocular hemorrhage after glaucoma operations.

v. The causes of hypotony or decreased intraocular tension, and its effects.

(a) Diminished secretion.

Nerve inhibition; vascular disturbance; damage to epithelium.

(b) Increased excretion.

Through normal channels; through newly-formed channels.

(c) The sequelæ of hypotony.

\section{CHAPTER IV}

\section{Injuries}

I. To the Eyeball. . . . . . . . . . . . . . . . . . 339

i. Ruptures.

ii. Concussions.

Iridodialysis; rupture of the pigment epithelium of the iris; rupture of the sphincter pupillæ, mydriasis and cycloplegia; rupture of the ligamentum pectinatum and ciliary muscle; concussion injuries of the pars plana of the ciliary body; dislocation of the lens; retroflexion of the iris; concussion cataract; rupture of the choroid; rupture of the retina; Commotio retinæ; pigmentation of the retina; hole in the retina at the macula; rupture of the lamina cribrosa and evulsion of the optic nerve; concussion hypertony and hypotony; birth injuries.

iii. Wounds.

Wounds of the cornea; simple abrasion of the epithelium; simple wound of the anterior surface of the cornea; simple perforating wound of the cornea; filtering 
cicatrices; complicated wounds of the cornea; anterior synechia; cystoid cicatrix; an adhesion of lens capsule; an entanglement of vitreous in a corneal wound; operation wounds and perforating wounds which pass into the periphery of the anterior chamber; wounds of the conjunctiva; perforating wounds of the globe behind the limbus; wounds of the iris; wounds of the lens.

iv. Foreign bodies.

Foreign bodies in the cornea, conjunctiva or sclerotic; ophthalmia nodosa; foreign bodies in the interior of the eye; siderosis bulbi.

v. Effects of heat, cold and chemicals.

False pterygium; symblepharon; ultra-red ray cataract.

vi. Excessive or deficient light, electricity and x-rays.

Eclipse-blindness; night-blindness; erythropsia; snowblindness and ophthalmia electrica; nystagmus associated with head-nodding in infancy; miners' nystagmus; injuries from lightning; injuries from x-rays.

II. To the Eyelids. . . . . . . . . . . . . .

i. Contusions.

ii. Wounds.

Traumatic ptosis; symblepharon; cicatricial ectropion; cicatricial entropion; epiphora.

iii. Burns.

III. To the Orbit . . . . . . . . . . . . . . . .

i. Concussions.

Fractures of the roof of the orbit; fractures extending through the optic foramen; fractures involving the inner wall; depressed fracture of the floor of the orbit; fracture of the margin of the orbit; lesions of the contents of the orbit.

ii. Wounds.

Gunshot injuries; through and through wounds; arteriovenous aneurisms; traumatic enophthalmia.

iii. Foreign bodies.

\section{CHAPTER V}

\section{Inflammation}

I. The Changes Common to all Inflammatory Processes. 4 io Inflammation; definition; cause; the changes in the tissue; the part played by the blood-serum; immunity; antibodies 
and their derivatives; phagocytosis; virulence; hypersusceptibility; the part played by the cells.

II. The Modifications of the General Processes of InflamMation in the Various Structures of the Eye . . 438 Conjunctiva: new gland formation; cysts; xerosis; phlyctenulæ; follicular formation.

Cornea: ulceration; leukoma; keratocele; anterior staphyloma; edema; vesicular keratitis; herpes cornea; bullous keratitis; superficial punctate keratitis; dendritic keratitis; filamentary keratitis; annular infiltration; ring abscess; keratectasia.

Sclerotic: episcleritis; scleritis; sclerosing keratitis.

Uveal tract. Choroiditis; suppurative; non-suppurative; supra-choroiditis. Irido-cyclitis; serous; plastic; suppurative; sequelæ.

Retina: edema; retinitis-non-suppurative and suppurative. Optic nerve: retrobulbar; interstitial; perineuritis; papillitis. Lacrymal gland: dacroadenitis-acute, chronic.

Lacrymal canals: dacrocystitis.

Eyelids: blepharitis; trichiasis; madarosis; tylosis.

Tenon's capsule: inflammation-acute; chronic.

Orbit: cellulitis.

III. The Method of Infection of the Ocular Tissues. . 480 Infection-definition; Koch's postulates; dissemination of infection in eye diseases; stages of the infective process; ectogenous and endogenous infection. Pyemia, acute and chronic. Wound infection.

\section{CHAPTER VI}

\section{Parasitic Diseases Affecting the Eye}

I. BACTERIA . . . . . . . . . . . . 490

i. Cocci. Staphylococcus; streptococcus.

Diplococci (Gram positive).

Pneumococcus.

Diplococci (Gram negative).

Gonococcus.

Diplococcus intracellularis meningitidis.

ii. Bacilli.

(a) Influenza group.

B. of Koch-Weeks. 
B. of Müller.

B. of influenza.

(b) Diphtheroid group.

B. of Klebs-Loeffler.

B. Xerosis.

(c) Diplobacillary group.

B. of Morax-Axenfeld.

B. of Petit.

(d) Capsulate bacilli group.

B. of Friedlander (pneumobacillus).

B. mucocus capsulatus.

B. of ozena.

(e) Coli group.

B. coli communis.

B. of typhoid.

(f) The acid fast bacilli.

B. of tubercle.

B. of leprosy.

(g) Unclassified bacilli.

B. Mallei.

B. of Ducrey (soft sore).

B. of Zur Nedden.

B. Aerogenes.

B. subtilis.

B. pyocyaneus.

II. Fungi. . . . . . . . . . . . . . . . . . . . . 549

Streptothricæ; aspergillus; penicillium; botryomycosis; hefæ; favus; ringworm.

III. Animal Parasites . . . . . . . . . . . . . 553

i. Unicellular organisms.

Protozoon. Spirocheta pallida.

Malarial parasites. Trypanosomiasis.

ii. Multicellular organism.

Entozoon. Cysticercus; hydatid; filaria.

Insectivora.

IV. The Diseases Which are Probably Duf to MicroorganISMS. . . . . . . . . . . . . . . . 570 Trachoma; vernal catarrh; sympathetic ophthalmitis. 


\section{CHAPTER VII}

\section{Degenerations}

I. Cuticular Epiblastic . . . . . . . . . . . . 588

(a) Atrophy of epithelium.

(b) Edema, vesiculisation and desquamation.

(c) Keratinisation and hyperkeratosis.

(d) Mucoid, fatty and hyaline changes.

II. Lenticular Epiblastic

(a) Capsular cataract.

Anterior polar cataract.

(b) Subcapsular cataract.

Diabetic cataract; cataracts secondary to disturbances in the circulation of the ciliary body; napthalin cataract; senile degeneration of capsular epithelium.

(c) Equatorial cataract.

Senile cataract.

(d) Nuclear or perinuclear cataract.

Lenticular myopic degeneration; black cataract; zonular or perinuclear cataract.

III. Neural Epiblastic.

(a) Degeneration of the ganglion cells of the retina.

Amaurotic family idiocy.

(b) Degeneration of the rods and cones of the retina.

Retinitis pigmentosa; symmetrical macular pigmentary degeneration.

(c) Atrophy of the optic nerve.

Secondary atrophy; primary atrophy; tabetic atrophy; hereditary optic atrophy.

(d) Degeneration of retinal pigment epithelium.

Hereditary hyaline infiltration of the retina.

(e) Degeneration of the neuroglia of the retina and optic nerve.

Hyaline nodules in the optic disc; amyloid bodies in the optic nerve and retina.

(f) Degeneration of the vitreous humour.

Synchisis scintillans; detachment of the vitreous humour.

(g) Degeneration of the pars ciliaris retinæ and pars iridis retinæ. 
IV. MESOBLASTIC

(a) Degeneration in the fibrous tissues.

Transverse calcareous film of the cornea; arcus senilis; marginal atrophy of the cornea and marginal keratectasia; hyaline thickenings in connection with Descemet's membrane and the ligamentum pectinatum; nodular and reticular opacity of the cornea; staphylomata; pinguecula; pterygium.

(b) Degeneration of the blood vessels.

Vascular sclerosis; sclerosis in the retinal arteries; choroidal vascular sclerosis.

(c) Degenerations in the ocular muscles.

(d) Degenerations in inflammatory exudates.

Hyaline degeneration; amyloid degeneration of the conjunctiva; fatty degeneration; cholesterine crystals; calcareous degeneration and bone formation; ossification of the choroid.

\section{APPENDIX ON LABORATORY METHODS}

Methods of Obtaining Material for Examination. . . . 679 To make a bacteriological examination; to examine cytology of cells; the examination of the aqueous for organisms; the removal of a piece of conjunctiva for microscopical examination; the examination of excised eyes.

Postmortem Methods and Injection of the Blood Vessels of the Eye. Methods of Fixation and Hardening . . . 683 Smear preparations; heat; ethyl alcohol; methyl alcohol; formaline; Müllers fluid; saturated solution of perchloride of mercury; decalcifying fluid.

External Examination of an Eyeball after Removal . . . 687 Side to which the eye belongs; diameters of the eyeball; palpation of the eyeball; transillumination; condition of the cornea; condition of the sclerotic; condition of the anterior chamber and its contents; condition of the iris; condition of the lens.

Freezing, Division of Eyeball and Macroscopical Examination After Division . . . . . . . . . . . .690 Preparation of freezing mixture; cornea; anterior chamber; iris; lens and its capsule; vitreous; retina; choroid; optic disc; optic nerve; method of mounting macroscopical specimens. 
Embedding and Cutting Microscopical Sections ...... Page

Celloidin; paraffin; freezing in gum; cutting sections.

Staining And Mounting . . . . . . . . . . . . . . 697

Logwood and eosin; carbo-thionin; borax methylene blue; carbol-fuschin; carbol-gentian violet; Gram's iodine solution; safranine; Gram's method of staining hair, scales, etc.; Weigert's fibrin stain; Weigert's iodine; Weigert's elastic tissue stain; sudan III; acid methylene blue; methylene blue; bismark brown; Leishmann's stain; Leishmann's method of staining the spirocheta pallida; Giemsa's method of staining the spirocheta pallida and so-called trachoma bodies; method of demonstrating the spirocheta pallida on a dark background; silver method of staining the spirochæta pallida in the tissues; to demonstrate capsules of bacteria; to demonstrate flagella of bacteria; to make a hanging drop preparation; staining flagellæ; Pappenheim's stain; eosin aqueous; Van Gieson; iron hematoxylin; method of staining the medullary nerve sheaths; method of staining early fatty degeneration in the medullary sheaths of nerve tissue; Nissl's method of staining nerve cells; Mallory's axis-cylinder stain; Ehrlich's "intravitam" methylene blue method of staining axiscylinders of nerves; Golgi's method of staining nerve fibres, ganglion cells and neuroglia; method of staining karyokinetic figures in the nucleus; methods of demonstrating hyaline changes; methyl violet for demonstrating amyloid changes; method of removing pigment from cells; method of demonstrating the cement material between epithelial and endothelial cells; method of demonstrating cholesterin crystals; method of demonstrating calcareous and bony changes; method of demonstrating the staining of the eye with iron and copper.

Demonstration of the Microscopical Structure of Various Parts of the Eye.

Cornea; uveal tract; lens; vitreous; retina; optic nerve.

Examination of Blood and Various Fluids of the Eye . . 7 I4

Cleaning slides and cover-glasses for making smear preparations; pipettes; to make a sterile glass needle; Wright's blood capsules; method of obtaining blood for Wassermann reaction, opsonic index, etc.; to make blood films; method of preparation of vaccines. 



\section{LIST OF ILLUSTRATIONS}

I. The cells found in the conjunctiva ..................... 433

II. Follicular formation in the conjunctiva ............. 446

III. Trachoma............................. 575

IV. Trachoma and tubercle..................... 576

Fig.

I. Human cyclops with extreme degree of microphthalmia..... I 2

2. Human cyclops, showing absence of proboscis and notching of the upper lids......................... I2

3. Human cyclops (twin) ....................... I

4. Human cyclops, showing two secondary optic vesicles and two lenses in one globe.................... I 3

5. Human cyclops, showing optic disc with two retinal and two central hyaloid arteries...................... I4

6. Human cyclops showing base of skull and cranial nerves.... I4

7. Human cyclops showing absence of convolutions and fissures in the brain.............................. I5

8. Case of anophthalmia...................... I6

9. Contents of the orbit of a chick the subject of congenital anophthalmia......................... I7

Io. Diagram showing normal involution of secondary optic vesicle............................. I8

II. Diagram showing normal upgrowth of mesoblastic tissue into the cavity of the secondary optic vesicle, etc....... I9

I2. Diagram of a coloboma of retina and choroid........... 2I

I3. Microscopical appearances of posterior half of a microphthalmic eye......................... 22

I4. Diagram of a congenitally microphthalmic eye with a cyst attached to it.......................... 23

I5. Diagram of a congenitally microphthalmic eye with a cyst attached to it.......................... 23

I6. Diagram of a congenitally microphthalmic eye with a cyst attached to it........................... 24

I7. Diagram of a congenitally microphthalmic eye with a cyst attached to it.............................. 
S. Shows por

I9. The iris in a case of congenital partial aniridia......... 28

20. Section of a horse's iris showing the corpus nigrum....... 29

2I. Lateral view of head of anencephalic fetus........... 29

22. View of skull from above of the fetus of Fig. $21 \ldots \ldots \ldots \ldots 30$

23. Transverse section of optic nerve of anencephalic fetus..... 30

24. Horizontal section through posterior part of the eye anencephalic fetus.......................... 3 I

25. Section through head of the optic nerve in a case of opaque

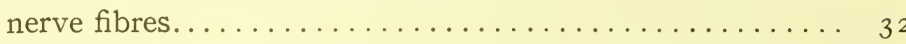

26. Section through a lens with a congenital gap in the posterior capsule.......................... 46

27. Section through a lens from a microphthalmic eye with a

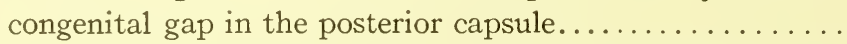

28. Section through the front part of a microphthalmic eye in

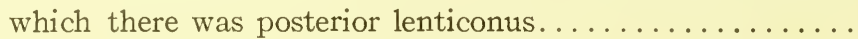

29. Antero-posterior section through a congenital disc-shaped

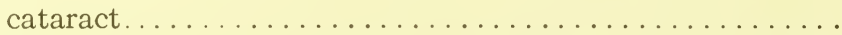

30. Diagrammatic section through a microphthalmic eye in which the whole fetal vascular system of the lens persisted...... 5 I

3I. Section through a lens with congenital punctate cataract.... 55

32. Transverse section of the central artery of the vitreous in a human fetal eye.......................... 58

33. Transverse section of the same artery shown in Fig. 32 further forward in the vitreous............. 58

34. Transverse section still further forward where the artery has divided up.................... 58

35. Diagram of section of an eye with persistent and patent

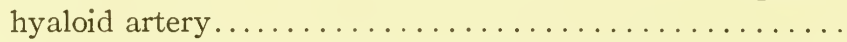

36. The same condition as Fig. 35 with the ciliary processes

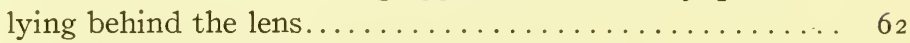

37. An eyeball with sight destroyed by ulceration of the cornea showing persistent remains of a hyaloid artery........ 63

38. Semi-diagrammatic section of microphthalmic eye with coloboma of iris and choroid ............... 66

39. Portion of retina and atypically developed vitreous from

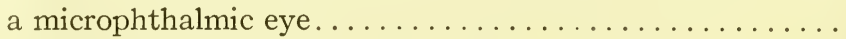

40. Branching fibres with granules on them from the vitreous humour of a normal fetal eye............... 68

4I and 42. Show suspensory ligament of the lens in sections of a normal human eye....................... 69

43. Congenital coloboma and displacement of the lens........ 7 I

44. Section through front half of an eye lost from glaucoma, with congenital coloboma of iris and lens........... 
45. Section of anterior half of eye of an infant with congenital anterior staphyloma and anterior lenticonus.......... 74

46. Section through the ciliary body in an eye which had atypical development of the vitreous and suspensory ligament

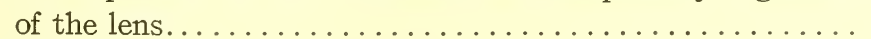

47. Section through another part of ciliary body from same

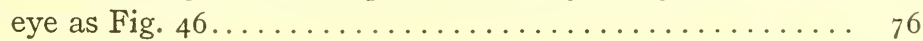

48. Congenital anterior synechia of a persistent pupillary mem-

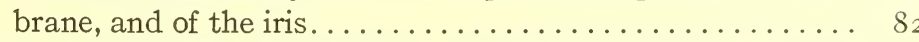

49. Lateral half of a cat's eye with a congenital anterior synechia

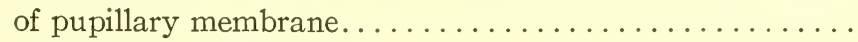

50. Microscopical appearances of the pupillary membrane in the cat's eye depicted in Fig. $49 \ldots \ldots \ldots \ldots \ldots \ldots$.

5I. Pigmented pupillary membrane adherent to the substantia propria of the cornea from the cat's eye depicted in Fig. 49

52. Microphthalmic eye of a chick with lens adherent to the

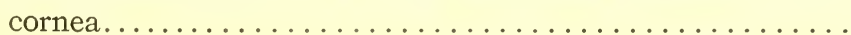

53. Section through an eye the subject of congenital glaucoma

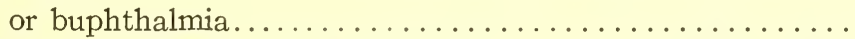

54. The angle of the anterior chamber in an eye affected with

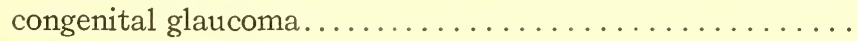

55. Persistent vessels in the lamina irido-pupillaris carrying

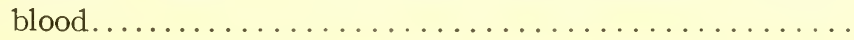

56. Congenital microphthalmia and persistent pupillary mem-

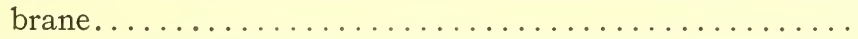

57. Anterior half of right eye of a patient with apparent aniridia. .

58. Thickening on the surface of the iris due to persistence of a portion of the iridic part of the fetal lamina irido-pupillaris.

59. The iris from the same eye as shown in Fig. $5^{8}$ where no thick-

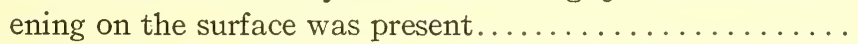

6o. Section of eye showing a rudimentary iris in which clinically

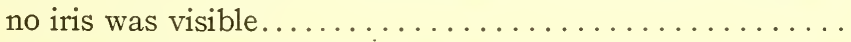

6r. A congenital abnormality at the angle of anterior chamber

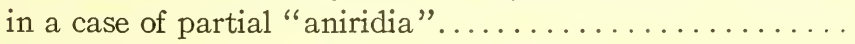

62. Section of the eye of a woman who had congenital aniridia and, after a perforating ulcer of the cornea, developed glaucoma............................ 100

63. Section showing mode of termination of ciliary body at the seat of a congenital coloboma of the iris............ I00

64. Microphthalmic eye with coloboma of the iris........... IOI

65. Congenital coloboma of iris with a bridge........... I03

66. Section through head of optic nerve in case with a congenital crescent at the lower border of the optic disc......... I09 
67. Section of cornea and sclerotic from a case with blue sclerotics I I I

68. Antero-posterior section through a highly myopic eye...... I I 2

69. Section through head of optic nerve in an eye with a myopic crescent....................... II 4

70. The ciliary muscle in a myopic eye ............ I 6

7 I. The ciliary muscle in a hypermetropic eye ......... I 7

72. Hyperplasia of endothelium on surface of iris ........ I 26

73. Outer wall of the nose in a new-born child........... I 27

74. Congenital coloboma of upper lid.............. I 3 I

75. Small congenital indentation in border of upper eyelid...... I3 I

76. Case of lateral proboscis or "demicyclops".......... I 32

77. Symetrical congenital notches in lower lids........... I 37

78. Case of unilateral cryptophthalmia............... I 40

79. Eyelids of a cat three days after birth, showing commencing

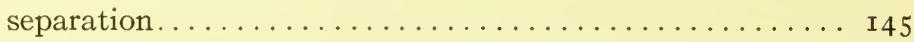

8o. Line of junction of eyelids in human fetus of the fourth month I 45

8I. An epibulbar epithelioma which completely disappeared after one application of radium bromide.............. I $6_{3}$

82. Section of commencing epithelioma of ocular conjunctiva.... I64

83. Section through a hard papilloma from the margin of an

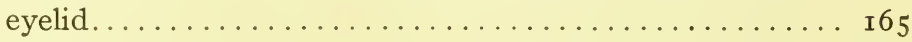

84. Glioma of the retina................ I 73

85. Section through an eye containing glioma of retina....... I 75

86. Section through a glioma of the retina showing rosette-like formation of cells.................... I 6

87. Gliomatous growth in two eyes, one much shrunken..... I 78

88. Intradural tumour of optic nerve............. I 79

89. Longitudinal section through tumour of optic nerve....... I 80

90. Transverse section through a glioma of the optic nerve..... I8 I

9I. Innocent pigmented growth of the iris........... I 83

92. Congenital pigmented tumour of the ciliary body........ I 84

93. Melanotic carcinoma of the ciliary body........... I 85

94. Bleached section of the melanotic carcinoma depicted in

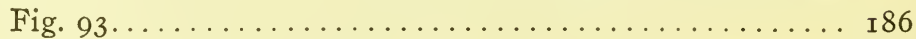

95. Melanotic sarcoma of ciliary body............. I 88

96. Lateral half of an eyeball containing a melanotic sarcoma of the ciliary body ................... I 89

97. Sarcoma growing from the choroid.............. I90

98. Section of an eyeball with a flat sarcoma of the choroid..... I9I

99. Section of flat sarcoma of choroid shown in Fig. 98 under higher magnification. ............... I92

I00. Melanotic sarcoma growing from the choroid at the margin of

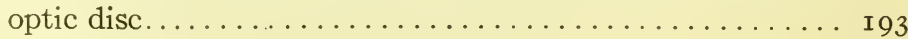


Iог. Lateral half of an eyeball containing an angiosarcoma of the

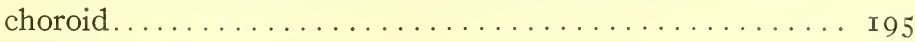

I02. Section through an angiosarcoma of the choroid........ I96

I03. Epibulbar melanotic sarcoma of the conjunctiva......... I 99

ro4. Sarcoma which started at the limbus and involved the cornea 200

105. Endothelioma arising from the blood-vessels of the orbit, a scirrhus-like growth..................... 203

Io6. Endothelioma arising probably from the lymphatics of the orbit, a soft friable growth................. 204

I07. Microscopical appearances of a tumour of the lacrymal gland. 207

I08. Microscopical appearances of the skin of the eyelid in a case of neuro-fibromatosis..................... 208

I09. Microscopical appearances of a buphthalmic eye in a case of neuro-fibromatosis.

IIO. Section through the choroid of an eye affected by neuro. fibromatosis........................... 2 Io

III. The hypertrophied nerve end-organs in the choroid under a higher power than in Fig. IIo................

I I2. Angioma of conjunctiva................... I 4

I 3. Half an eyeball containing an angioma of the choroid..... 2 I6

I 4 . Section of a cavernous angioma of the choroid ......... 2I 7

I 5. Cavernous angioma of the choroid shown in Fig. I I4 under higher magnification................. 2 I 8

I 6. Ophthalmoscopic appearances of an angioma of the retina... 2 I9

I I 7. Microscopical appearances of a vascular growth of the retina. 220

II 8. Section of the posterior part of an eyeball with a metastatic

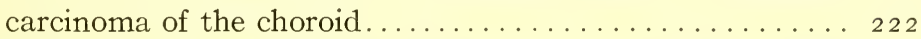

II9. Section of a metastatic carcinoma of the choroid under higher magnification than in Fig. I $8 \ldots \ldots \ldots \ldots \ldots \ldots 223$

I 20. Epithelial lined cyst in the anterior chamber.......... 225

I 2 I. Lining epithelium of cyst shown in Fig. I $20 \ldots \ldots \ldots \ldots 225$

I 22. Cyst in the iris following the implantation of an eyelash... . 226

I 23. Part of the wall of the cyst shown in Fig. I $22 \ldots \ldots \ldots \ldots 227$

I 24. Epithelial implantation cysts in the cornea........... 227

I 25. Epithelial implantation cyst in the cornea........... 228

I 26. Laminated epithelium lining cyst in the cornea depicted in

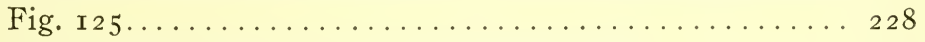

I 27. Large epithelial lined cyst in a shrunken globe.... . . . . 229

I28. Plastic iritis after a perforating wound producing cyst between the two pigment-epithelial layers on the back of the iris... 232

I 29. Cyst formed by the separation of the two layers of the pars

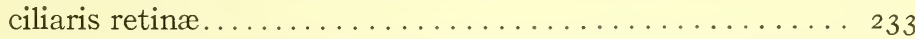

I30. Section through the iris with a cyst on its posterior surface. 234 
PAGE

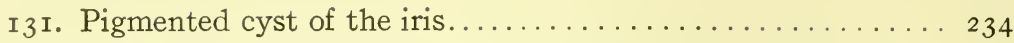

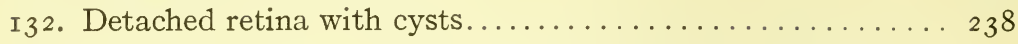

I33. Transverse section of the optic nerve showing embolism in

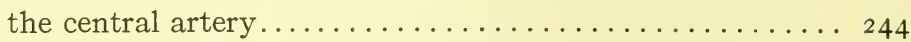

I34. Section through the retina with a thrombosed retinal vein cut transversely............................ 245

I35. Section of iris in a case of glaucoma following thrombosis of the central vein, with thickened endothelial membrane on its anterior surface.................. 247

136. Piece of iris from same case as Fig. I35 under higher magnification........................... 247

I37. Section through the coats of the eye in case of albuminuric

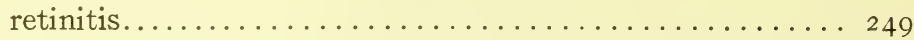

I38. Section through the macula lutea in case of albuminuric retinitis............................ 250

I39. Mass of retained and degenerate blood clot at the angle of the

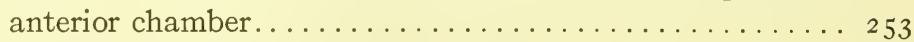

I40. Blood staining of the cornea................. 254

I4I. Microscopical appearances of stained area in cornea depicted

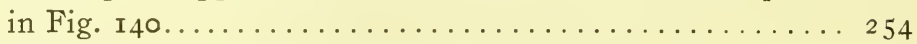

I42. Angeoid streaks in the fundus oculi............ 257

I43. Section through the coats of the eye in a case of subhyaloid

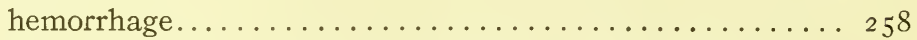

I44. Section through the coats of the eye in a case of albuminuric

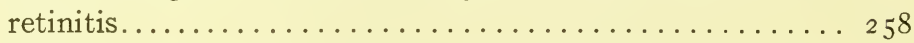

I45. Section through coats of the eye showing mass of organising

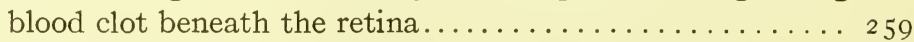

I46. Serous effusion between the ciliary body and sclerotic..... 28 I

I47. Subretinal serous effusion the result of thrombosis of a

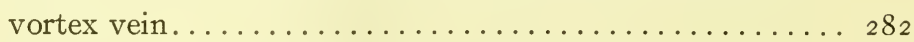

I48. Microscopical section of the coats of the eye shown in Fig. I47 283

I49. Serous effusion from iris into the anterior chamber....... 284

I 5 O. The same as Fig. I 49 under higher magnification. . . . . . 285

I 5 . Vesicles in the epithelium on the anțerior surface of a sta-

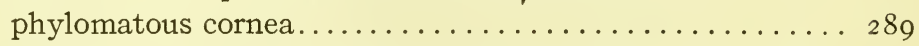

I52. Organising edematous exudate on the surface of the cornea in case of hereditary syphilitic kerato-iritis.......... 290

I53. Anterior part of the cornea in an eye with "filamentary

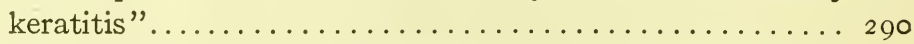

I54. Bleached section of the ciliary body of a normal eye showing processes of cells dipping down from the pigment-

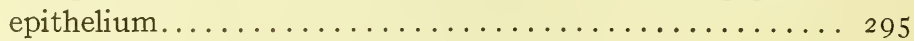

I 55. Processes of cells depicted in Fig. I 54 more highly magnified. 296 
I 56. Bleached section of ciliary body with some of the downgrowths from the pigment epithelial layer cut transversely....... 297

I 57. The normal angle of the anterior chamber........... . 298

I 58. Angle of anterior chamber in recent glaucoma.......... 30 I

I59. A condition of iris bombé resulting in secondary glaucoma... 302

I60. Lateral half of an eye with wound of cornea through which lens and iris escaped. It subsequently developed glaucoma.................................. 303

I6I. Section through angle of anterior chamber of the eye depicted

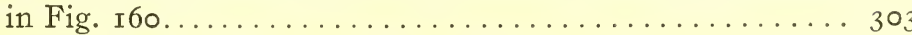

I62. Lateral half of an eye from which a cataract was extracted and which afterwards developed glaucoma.......... 304

I63. Glaucoma secondary to dislocation of the lens........ . . . 305

I64. Section through the cornea in case of congenital glaucoma showing rupture in Descemet's membrane............ 3 I I

I65. Ectropion of the uveal pigment at pupillary margin of the

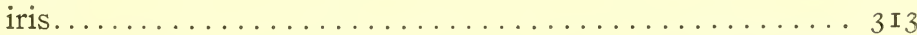

I66. Angle of anterior chamber in case of glaucoma of long standing 3 I 3 I67. Section through the optic nerve in case of glaucoma...... 3 37

I68. Diagram showing the distribution of the nerve fibres in the

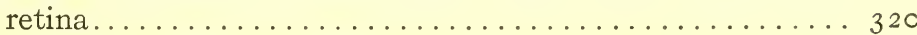

I69. Section through angle of anterior chamber of an eye upon which iridectomy had been performed............ 324

I70. Section of an eye with a cystoid cicatrix........... 325

I 7 I. Section of an eye in which iridectomy failed to relieve tension

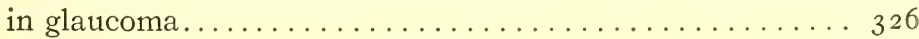

I72. Iridectomy performed for absolute glaucoma; lens adherent

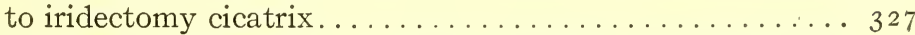

I73. Section of cornea from an eye with hypotony showing wrinkling of Bowman's and Descemet's membranes......... 335

I 74. Wrinkling of Bowman's membrane............ 336

I75. Puckering of sclerotic in a shrinking eye ........ 336

I76. Wrinkling of the membrane of Bruch in an eye with hypotony 337

I77. Wrinkling of the membrane of Bruch and heaping up of pigment epithelium in an eye with hypotony........ 337

I 78. Lateral half of an eye ruptured by blow from fist. . . . . . 340

I79. Rupture of globe showing a prolapse of the iris......... 34 I

I80. Rupture in the sclerotic and subconjunctival dislocation of

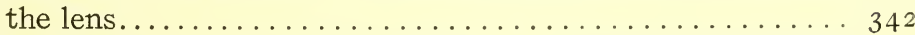

I8I. Eye injured by blow from stick of a catapult, producing iridodialysis and rupture of the ligamentum pectinatum... 344

I82. Rupture of the pigment epithelium on the back of the iris . . 346 I83. Partial rupture of the ligamentum pectinatum........ 347 
I 84. Complete rupture of the ligamentum pectinatum. . . . . . 348

I85. Concussion injury to eye and rupture of ligamentum pecti-

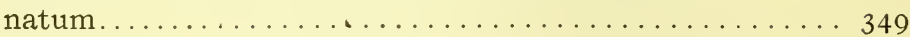

I86. Wide separation of ciliary body from sclerotic due to concussion............................ 350

I87. Rupture of the pigmented and unpigmented layers of pars plana of the ciliary body................. 35 I

I88. Detachment of the unpigmented layer of the pars plana of the ciliary body......................... $35^{2}$

I 89. Eye injured by concussion showing rupture of retina at the

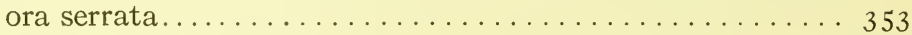

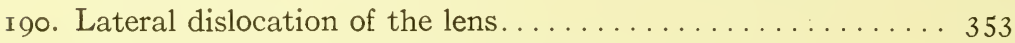

I9I. Lens dislocated into anterior chamber. . . . . . . . . 354

I92. Rupture of the posterior capsule of lens . . . . . . . 356

I93. Rupture of the choroid (late stage) . . . . . . . . . . . 357

I94. Subchoroidal hemorrhage compressing the choroid ....... 360

I95. Rupture of the lamina cribrosa .............. . . 363

I96. Recent perforating wound of the cornea.......... . 368

I97. Healing perforating wound of the cornea . . . . . . . . 368

198. New formation of hyaline membrane on posterior surface of a scar in the cornea........................ 370

199. Extension down of the surface epithelium along the lips of an

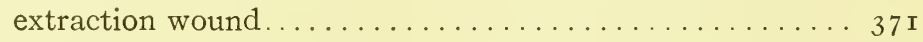

200. Anterior synechia of the iris ............... 372

20I. Prolapse of a knuckle of iris into a wound at the sclero-corneal

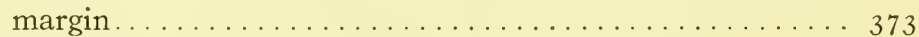

202. Cystoid cicatrix in an eye lost from glaucoma......... 374

203. Entanglement of lens capsule in wound, after extraction of

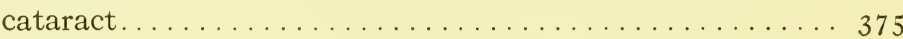

204. Adhesion of lens capsule to posterior surface of an extraction

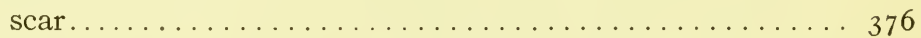

205. Wound of the cornea and suspensory ligament of lens with prolapse of vitreous humour.............. 377

206. Cicatrix left after incision through the cornea with a keratome for iridectomy for glaucoma............... 378

207. Gaping wound at the sclero-corneal margin with prolapse

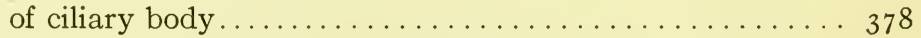

208. Perforating wound at the limbus with a conjunctival flap... 379

209. Limbal cicatrix with a conjunctival flap nine months after extraction of cataract........................... 380

210. Perforating wound of cornea and lens............ 384

2 II. Two layers of the lens capsule after extraction with laminated tissue lying between them................. 386 
2 I2. Two layers of lens capsule after extraction of cataract with bladder-like cells between them................ 386

2I3. Section of iris with two eyelashes lying on its anterior surface. 389

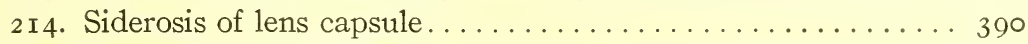

2I5. Section of a polypus in the plica semilunaris showing the process of new gland formation.............. 439

2 I6. Section similar to Fig. 2 I5 showing more advanced gland formation.

2 I7. Epithelium of conjunctiva from a case of ectropion showing changes due to exposure................... 440

2 I8. New gland formation in conjunctiva from a case of ectropion. 44I

2 19. New gland formation in conjunctiva from a polypus of the plica semilunaris.

220. Section through a phlyctenule of the conjunctiva........ 444

221. Same as Fig. 220 in a later stage . . . . . . . . . . . 445

222. Extensive suppurating ulcer of the cornea . . . . . . . . . 447

223. Section through cornea showing inflammatory cells infiltrating the anterior layers of the substantia propria......... 448

224. Anterior synechia of the iris following perforating corneal

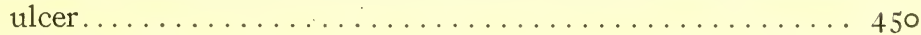

225. Perforating ulcer of the cornea and incarceration of the iris.

226. Cornea destroyed by ulceration and replaced by mass of fibrous tissue...................... $45^{2}$

227. Lens capsule adherent to posterior surface of a staphy-

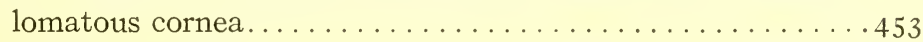

228. Ring abscess of the cornea................... 455

229. Mooren's ulcer involving whole cornea........... 456

230. New formation of connective tissue between the sclerotic and choroid in a shrunken eyeball.............. 460

23I. Ciliary body and angle of anterior chamber in a case of cyclitis........................ 462

232. Posterior surface of cornea in an eye with keratitis punctata. 463

233. Plastic iritis as a result of a perforating wound of the cornea

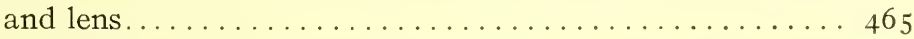

234. The iris drawn back into the angle of anterior chamber the result of iritis following a perforating wound........ . 469

235. Section of an eye with plastic cyclitis............ 467

236. Tubular processes of epithelial cells in cyclitis . . . . . . . 4 468

237. "Pseudo-glioma". ... . . . . . . . . . . . . . . . . . 469

238. Degeneration of macular fibres in the optic nerve after

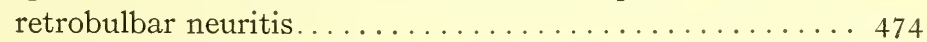

239. Optic neuritis secondary to a tuberculous mass at margin

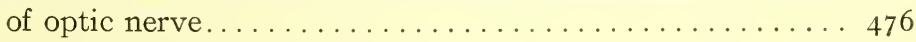


240. Microörganisms found in smear preparations of the conjunctival secretion in different forms of ophthalmia...... 500

24I. A smear preparation from discharge from the conjunctiva containing Klebs-Loeffler bacilli................ 5 I 6

242. Tubercular nodules involving root of iris............. 535

243. Iris with tubercular nodules at pupillary margin......... 536

244. Tubercle of the iris........................ 536

245. Iris thickened with tubercular nodules.............. 537

246. Tuberculous mass in choroid causing intense neuritis...... 540

247. Tuberculous mass in choroid................... 54 I

248. Conglomerate mass of tubercle starting in choroid....... 542

249. Streptothrix Fosterii removed from the canaliculus....... 550

250. Aspergillus growing in the cornea................ 55 I

251. Penicillium glaucum......................... $55^{2}$

252. Gumma in the root of the iris................ 557

253. Changes left by a patch of syphilitic chorio-retinitis....... 558

254. Cornea affected with interstitial keratitis............ 559

255. Cornea which had become vascularised as the result of interstitial keratitis............................ 560

256. Gumma of the ciliary body.................... 56 I

257. Papillary formation in early trachoma............ $57 \mathrm{I}$

258. Cornea affected with trachomatous pannus............ 579

259. Discharge from a case of vernal catarrh.............. 580

260. Section of one of the papillæ from a case of vernal catarrh... $58 \mathrm{r}$

26r. Vernal catarrh showing necrosis in the plasma cells........ 582

262. Section through choroid in a case which excited sympathetic ophthalmitis............................ $58_{3}$

263. Conjunctiva in a case of xerosis............... $59 \mathrm{I}$

264. Shrunken dry conjunctiva and keratinisation.......... 592

265. Anterior polar cataract of less than six weeks' formation.... 597

266. Anterior polar cataract of seven months' formation....... 598

267. Anterior polar cataract of eleven years' formation......... 599

268. Anterior polar cataract of twenty-one years' formation..... 600

269. Anterior polar cataract of eleven years' formation......... 60I

270. Melanotic sarcoma of ciliary body giving rise to capsular cataract............................ 602

27 I. Section through a cataractous lens from an eye with a melanotic sarcoma of the choroid..................... . 604

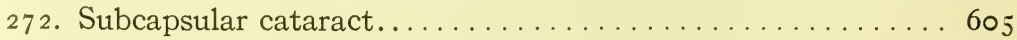

273. Shrunken eyeball with Morgagnian cataract............ 6 I I

274. Early changes in senile cataract............... 6 12

275. Spreading changes in senile cataract............. $6 \mathbf{I}_{3}$

276. Zonular cataract......................... 6 8

277. Section through macula in amaurotic family idiocy....... 627 
PAGE 278. Section through macula in a case of symmetrical pigmentary degeneration........................ 633

279. Sections of retina from the same case as Fig. 279 showing degeneration in a ganglion cell................. 634

280. Section through head of optic nerve in case of simple optic

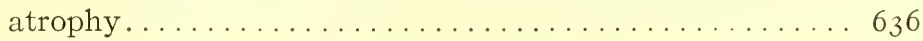

28 $\mathrm{I}$. Hyaline formations on inner surface of the elastic lamina

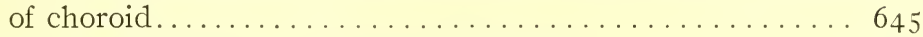

282. Hyaline formations in head of optic nerve........... . 645

283. Hereditary hyaline infiltration of the retina.......... . 646

284. Amyloid body in the retina. . . . . . . . . . . . . . . . 649

285. Section through a transverse film of the cornea......... 655

286. Hyaline excrescences on inner surface of Descemet's membrane.............................. 659

287. Nodular opacity of the cornea................. 66 I

288. Reticular opacity of the cornea.............. 66 I

289. Intercalary staphyloma................... 663

290. Ciliary staphyloma....................... 664

291. Equatorial staphyloma....................... 664

292. Section through a sclerosed artery in the retina.........6670

293. Section showing sclerosed vessels in choroid and retina.... 672

294. Section through band of bone formed in a mass of inflammatory exudate from the choroid...............6 677

295. Method of making a smear preparation with a platinum

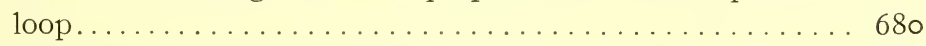

296. Glass tube for centrifugalisation. . . . . . . . . . . . . 68 I

297. Museum jar for mounting macroscopic eye specimens . . . . . 692

298. Opal glass plate to fit in above jar to hold eye in position... 692

299. Globe-Wernicke book case for macroscopic specimens....... 693

300. Leitz "Base sledge" microtome... . . . . . . . . . . . . . . . . 695

30г. Leitz "Base sledge" microtome for cutting paraffin sections. . 696

302. Method of drawing up fluid into pipettes........... 7 I 4

303. Wright's blood capsule.................... 7 5

304. Ehrlich eye-piece dividing the field into squares......... 7 I 6

305. Sealed glass capsules for vaccine, serum, etc.......... 7 I 7 



\section{ERRATA}

Page 321. Last line, the word "temporal" to read "nasal."

Page 322. Line 6, the word "temporal" to read "nasal."

Page 633. Line 3 of the legend Fig. 278, the word "inner" should read "outer." 



\title{
PATHOLOGY AND BACTERIOLOGY OF THE EYE
}

\author{
CHAPTER I
}

\section{ABERRATIONS IN DEVELOPMENT}

Aberrations occurring in the development of the eye are necessarily most of them congenital defects, but not all, e.g., the medullary substance around the fibres of the optic nerve is not formed until after birth, and the abnormality due to formation of medullary substance around the nerve fibres in the retina, though an aberration in development, is not a congenital defect.

On the other hand it is necessary to remember that all congenital defects are not aberrations in development; some of them may be changes due to intrauterine inflammation and others due to injuries at birth. These will be described under inflammations and injuries, respectively.

The cycle of changes which ensues after fertilization of an ovum is due to inherent capacities transmitted by the germ cells, and influences brought to bear on it through its environment. Aberrations in development may, therefore, be attributed, either to inherent defect in the germ cells, of one or other parent, or to chemical or mechanical influences affecting the nutrition of the embryo during the time of its growth.

The eye commences to develop early after fertilization, and soon becomes a prominent feature; it is therefore exposed to disturbing influences occurring over a considerable period of fetal life. The earlier the dystrophic influence arises the greater the deformity which is pro- 
duced. There is evidence to show, that some relation may be traced between the time at which a disturbing influence occurs, and the character of the aberration.

Experimental embryologists have of recent years demonstrated that arrests of development in the eyes may be brought about by several different chemical and physical means, applied either directly to the embryo or through the mother. A variety of different congenital malformations of the eyes have been produced, in the off-spring of rabbits, and guinea pigs, by feeding the pregnant mother with napthaline; none, however, have transmitted the defects thus produced in them to their descendents. ${ }^{1}$

Arrests of development of the optic vesicle in various stages have been produced in fish by subjecting their developing eggs to magnesium salts, to ether or alcohol, or to certain mechanical operations. ${ }^{2}$

It has also been found that the normal course of development of an embryo, or of some part of it, may be interfered with by lowering the temperature, and thereby reducing the rate of oxidation, or by directly cutting off the supply of oxygen. ${ }^{3}$

The young of rats exposed to the emanations of radium during late pregnancy have developed eye defects.

Experiments have been performed with cytolysins, which produced ante-natal deformities of the eyes capable of transmission to subsequent generations by inheritance. ${ }^{4}$

An emulsion of pulped lens matter from newly killed young rabbits, diluted with normal saline, was injected at intervals, intra-venously and intra-peritoneally, into fowls. The immunized serum, thus produced, was subsequently injected at intervals, intra-venously, into pregnant rabbits, at a time when the lens was undergoing its most rapid development. Many of the young of the rab-

${ }^{1}$ H. E. Pagenstecher. Arch. f. Vergleichende Ophth. II, IV, 424, I9I2.

${ }^{2}$ C. R. Stockard. J. Exp. Zool., V, 6, I909.

${ }^{3}$ C. R. Stockard. Am. J. Anat., V, I92 I, 28.

${ }^{4}$ M. F. Guyer. Internal. Cong. of Ophth. Washington, I922, 669. 
bits so treated died in utero, but amongst those which survived a certain number had at birth very conspicuous eye defects; the commonest being complete or partial opacity of the lens. Other abnormalities were also met with, but most of them were attributable to arrests of development following on the early defect in the lens.

The arrests in development of the eyes of rabbits started in this way by cytolysins, occurred not only in the animals treated, but were transmitted to their descendents through nine generations, and there is no apparent reason why they should not go on indefinitely.

The general characteristics of the inheritance was that of a Mendelian "recessive," and transmission was effected through the males as well as the females, so that there seems no doubt that the germ cells must have become affected.

A similar series of inheritable defects was produced by the use of pulped rabbits lens intravenously injected directly into rabbits, just before or during their pregnancy. In this case the rabbit herself must have developed antibodies against the invading lens material, which were capable of directly inducing germinal changes.

These experiments show that in trying to determine the cause of arrests of development in human beings, the ante-natal condition of the parents calls for the most careful consideration.

Congenital malformations of the eyes are sometimes sporadic in origin, and sometimes hereditary. In the sporadic cases the mother frequently gives a history of having received some shock or fright during the course of her pregnancy. Very frequently the time of the shock corresponds fairly accurately with that at which the part of the eye affected was likely to be undergoing formation. During fetal life the human embryo receives its nourishment from the maternal blood, through the placental circulation. Any temporary arrest of this circulation, 
such as that produced by cardiac inhibition in the mother from a shock, might deprive the embryo of the requisite amount of oxygen at a critical moment in the development of a certain part of the eye, and so lead to its arrest in formation.

Cases have been recorded in which a parent, who has lost an eye from destructive inflammation, has subsequently had a child born suffering from microphthalmia. In such cases it is conceivable, that as the outcome of the destructive inflammation, the parent might have developed antibodies against the disturbed tissues of his own eye capable of producing changes in the germ cells.

At present we know but little as to what influence the secretion of the maternal endocrine glands have on the developing fetus. That they do exert some influence seems highly probable, for both the thyroid, parathyroids and pituitary body are in a state of heightened activity during pregnancy. There is experimental evidence to show that in dogs excision of the parathyroids produces cataract; it is likely, therefore, that a maternal deficiency of these glands during pregnancy, might result in failure of some of the essential constituents of the fetal lens in its development.

The pressure caused by abnormal bands in the amnion plays a conspicuous part in the production of malformations connected with the facial fissures, doubtless it may also sometimes mechanically interfere with the formation of the eyelids, or even cause arrests in the development of the eye itself.

Many eye affections are hereditary. An infectious disease such as syphilis may be transmitted in utero from the mother to the child. Certain degenerations of the tissues of the eye, coming on after they have reached their full development "abiotrophies," are found in members of the same childship, and also in different generations in the same family. Such conditions will be described respectively under the head of inflammation and degenera- 
tion, here heredity with relation to aberrations in development will be discussed.

Congenital malformations, such as microphthalmia and albinism, are often met with in members of the same childship, and not in successive generations, this collateral inheritance is known as "familial," it is probably due to intrauterine toxemia or malnutrition. Where an abnormality is transmitted from one generation to another, as is sometimes the case in congenital aniridia, correctopia and some forms of congenital cataract, if the transmission is through the father there must be a germinal cause, if the transmission is through the mother the cause is likely to be germinal but cannot with certainty be regarded as such.

Some abnormalities of the eye, like congenital colour blindness, are as a general rule limited to the male sex, but are transmitted from one generation to another through apparently normal females. The rule, however, is not absolute, an affected male has been known to transmit colour blindness to his son, and it is sometimes found in females, but an unaffected male never transmits the disease. ${ }^{1}$ As the cause in the exceptional cases transmitted by the male must be germinal, it is probably so in those transmitted through the females.

Many of the congenital malformations of the eyes which are transmitted from one generation to another behave, as defined in Mendelism, as "unit-characters." In other words the condition is transmitted in its entiety or not at all. Allowing for numerical discrepancies, which must occur in dealing with the offspring of human beings, many pedigrees which have been constructed of congenital malformations are in their broad feature consistent with the Mendelian theory of inheritance. ${ }^{2}$ In some cases the abnormality behaves as a "dominant" over the normal condition, and in others as a "recessive."

${ }^{1}$ Nettleship. Trans. Ophth. Soc. of the U. K., XXIX, I909, 1xxv.

${ }^{2}$ Nettleship. Ibid., lxviii. 
This chapter is headed aberrations in development because it is intended to include in it other conditions besides mere arrests of development. The evolution of the fetus is a modified recapitulation of the evolution of the race; or in the words of Hæckel "ontogeny is a recapitulation of phylogeny, " hence in arrests of development we have reproduced conditions suggesting the normal characteristics of lower animals. An arrest of development represents, therefore, a backward tendency from an evolutionary stand point, and if these were the only departures from the normal in the course of development no progressive evolution could take place. Besides arrests of development we meet with aberrations which are termed "Mutations" or "Sports." Such aberrations form inheritable units, and when advantageous to the individual in connection with its environment, tend, through natural selection, to become incorporated as a normal characteristic of the species. Many mutations which arise are of no value, these always exist as abnormalities, and tend after existing for a few generations to die out and disappear. As to the cause of mutations we have even less evidence than as to the cause of arrests of development.

The development of the eye is divided for purposes of description into several separate stages: (I) The formation of the primary and secondary optic vesicles. (2) The ingrowth of invaginating surface epiblast. (3) The upgrowth of invaginating mesoblast. (4) The elaboration of surrounding mesoblast. (5) The formation of the eyelids and structures in the line of the orbito-nasal fissure and the orbital muscles. After a brief description of each of these stages the various conditions which arise from aberrations in connection with them will receive detailed consideration. 


\section{ABERRATIONS CONNECTED WITH THE FORMATION OF THE PRIMARY AND SECONDARY OPTIC VESICLES}

The first visible antecedent in the development of the eyes consists in the lateral outgrowth of two hollow processes from the anterior cerebral vesicle; these are termed the primary optic vesicles. It is, however, probable that the original tissue from which these outgrowths proceed is medial and single, and that it later separates into two areas of growth which sprout out laterally to form the primary optic vesicles.

After the formation of a primary optic vesicle the next stage consists in its invagination anteriorly, and inferiorly, which results in the formation of a cup composed of two layers, the secondary optic vesicle. From the inner of these two layers is formed the retina, and from the outer the retinal pigment epithelium.

Into the anterior part of the cup of the secondary optic vesicle there passes a downgrowth of the surface epiblast which forms the lens vesicle; into its lower part proceeds an upgrowth of mesoblast which develops into the bloodvessels of the retina and vitreous humour. The space through which the upgrowing mesoblast passes to enter the cup of the secondary optic vesicle is termed the fetal ocular cleft; this cleft ultimately becomes closed by union taking place between the layers of the secondary optic vesicle at its margins.

The stalk which connects the brain with the secondary optic vesicle takes no part in the formation of nerve fibres, but develops into the supporting neuroglia of the optic nerve. The distal part of this stalk becomes invaginated below like the primary optic vesicle, and the invaginating mesoblast develops into the central artery and vein of the optic nerve.

The axis-cylinders composing the optic nerve and the nerve-fibre layer of the retina, are in part formed from outgrowths of the ganglion cells of the retina, which extend 
up into the brain; and in part from outgrowths of the ganglion cells in the brain which extend down into the retina. The axis-cylinders in the optic nerve are at first devoid of medullary sheaths, the formation of these sheaths begins at the cerebral end and extends downwards, they do not reach the posterior surface of the lamina cribrosa, where they end, until after birth.

The development of the ganglion cell layer of the retina, which is the first layer to become differentiated, commences centrally and extends peripherally. Though development starts in the area centralis it is the last to become completed. The macula, together with the papillo-macular nerve fibres is, both phylogenetically and ontogenetically, of late formation. At birth, the external nuclear layer at the macula consists of only a single layer of nuclei, and the cones are short, thick and few in number. It is not until some months after birth that the fovea centralis attains its full development.

The inner layer of the secondary optic vesicle from the ora serrata to the root of the iris forms a single row of columnar cells. From it are also probably formed, the matrix of the vitreous humour, and the fibres of the suspensory ligament of the lens (see page 56 ).

At the root of the iris the inner layer of the secondary optic vesicle commences to be deeply pigmented, and the arrangement of its cells can only be seen in bleached sections. They are at first wedge shaped with their bases directed backwards, giving the posterior surface of the iris near its base a crenated appearance, they then become more regularly columnar, and terminate at the pupillary border where they unite with the outer layer of the secondary optic vesicle.

The outer layer of the secondary optic vesicle develops different characteristics in the three different divisions of the uveal tract, choroid, ciliary body and iris.

On the inner surface of the choroid it forms a single row of cells which manifest positive phototactic amæboid move- 
ments, and which are concerned in the secretion of the visual purple. From them also is developed the cuticular part of the lamina vitrea (membrane of Bruch), the outer elastic part of that membrane being composed of condensed yellow elastic fibres, which are mesoblastic in origin.

In the ciliary body the outer layer of the secondary optic vesicle consists of a single row of flattened pigmented cells, projecting outward from which are a number of processes, which when bleached are seen each to consist of a group of cells. These processes are most numerous where the nonplicated joins the plicated part of the ciliary body, they are there pear-shaped, and when cut transversely are seen as a ring of cells one layer thick with a central lumen. (Figs. I 55 and I 56.) From the ciliary body is secreted the aqueous humour and the nutrient fluid of the vitreous humour, presumably these gland-like projections play some part in the production of these fluids.

From the outer layer of the secondary optic vesicle in the iris is developed its muscular tissue, the dilator fibres and the sphincter. ${ }^{1}$ Bleached sections have shown that the cells in this layer are at first cylindrical in shape with a central nucleus, later the outer part of each cell is differentiated by the outgrowth of a contractile fibre, while the nucleus and pigment are displaced towards the posterior end. The contractile fibres blend to form what is termed the membrane of Bruch, the other parts of the cells with the pigment and nucleus, remain attached to them, and together they form the dilator muscle of the iris.

The cells forming the sphincter muscle originate from the outer epithelial layer of the secondary optic vesicle at the pupillary margin, where it turns back to join the inner layer. They commence to develop about the fourth month of fetal life, and about the end of the fifth month become

${ }^{1}$ Forsmark. "The Muscular Tissue of the Human Iris; its Structures and Development," Jena, I905. See Ophthalmic Review, I905, I34. 
separated off from the pigment epithelium by the growth in between of iris stroma.

The aberrations which may arise in connection with the formation of the primary and secondary optic vesicles are as follows:

Failure in the original cells, from which the primary optic vesicles sprout out, to become differentiated into two distinct growing areas-cyclopia or synophthalmia.

Failure of the primary optic vesicles to bud outanophthalmia.

Failure in the invagination of the primary optic vesicle - congenitally cystic eyeball.

Imperfect coaptation of the two layers of the secondary optic vesicle - shot-silk or watered-silk retina.

Failure in the closure of the cleft in the secondary optic vesicle - coloboma of retina and optic nerve; microphthalmia with cyst.

Union of the two layers of the secondary optic vesiclecongenital pigmentation of retina.

Unusual extension forward of the secondary optic vesicles - ectropion of the uveal pigment of iris.

Defective downgrowth or upgrowth of axis-cylinders from ganglion cells-absence of nerve fibres in optic nerve and retina.

Abnormal medullation around axis-cylinders in retinaopaque nerve fibres.

Atypical development of neural portion of secondary optic vesicle-crater-like hole in optic disc.

Excessive space for passage of nerve fibres into the eye - physiological cupping of optic disc.

Restricted space for passage of nerve fibres into the eye-pseudo-neuritis.

Defective formation of light-percipient elements in retina-congenital day-blindness with colour-blindness; congenital night-blindness (Mooren's retinitis punctata albescens).

Failure in differentiation of macula-- congenital amblyopia. 
Defective development of the cones-congenital colour blindness.

Defective deposition of pigment in the outer layer of the secondary optic vesicle-albinism (see page i 25 ).

Failure in the development of muscle fibres from the outer layer the secondary optic vesicle in the iris-miosis congenita.

Cyclopia or Synophthalmia.-Cyclopia or Synophthalmia is a condition in which the eyes or orbits of the two sides are to some extent united. It is always associated with other extensive malformations, especially of the face and brain, so that those affected do not long survive birth.

Many gradations in the degree of approximation, or blending, of the two orbits and eyes are met with. ${ }^{1}$ In the least marked cases there are two orbits, each with a normally formed eyeball, only separated from one another by a single very small nasal aperture. In the most marked cases there is a central orbit with a single eyeball, none of the parts of which are duplicated, and with only one optic nerve.

The failure in separation of the two orbits results in the fronto-nasal process, from which the nose should have developed, being pushed forwards, and hanging down from above the orbit in the form of a proboscis (Fig. I).

When one central orbit is formed it is surrounded by four eyelids, the palpebral aperture between which is roughly diamond shaped (Fig. 2). The upper canaliculi are suppressed, and the lower terminate in a blind canal.

The degree of union of the two eyeballs varies considerably, often besides the changes due to mere union there are other defects of development. The united eyes though usually abnormally large may be microphthalmic.

When one eye is present, without any duplication of its structures, the cornea is generally of an oval shape with its long axis horizontal.

${ }^{1}$ M. S. Mayou. Trans. Ophth. Soc. of the U. K., XXV, I906, 267. 


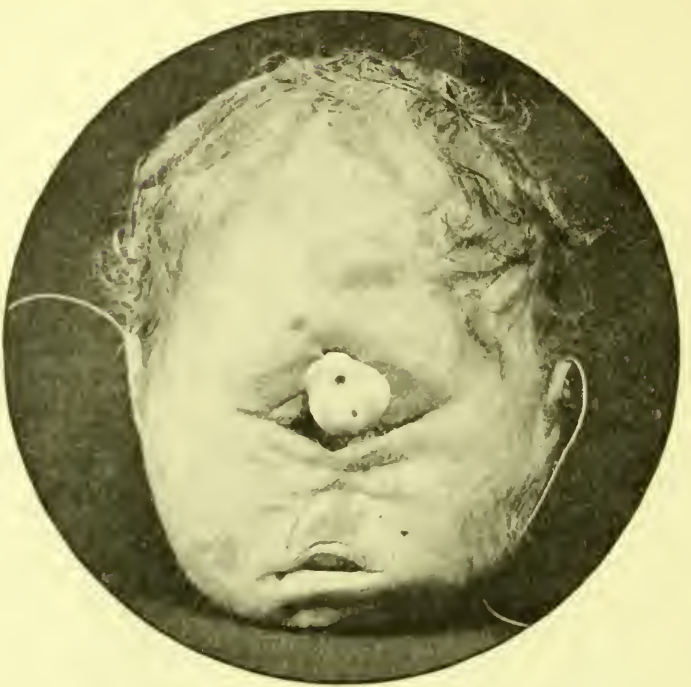

FIG. I.-Human cyclops with extreme degree of microphthalmia. Note proboscis with opening at the end (the upper hole is due to a pin mark).

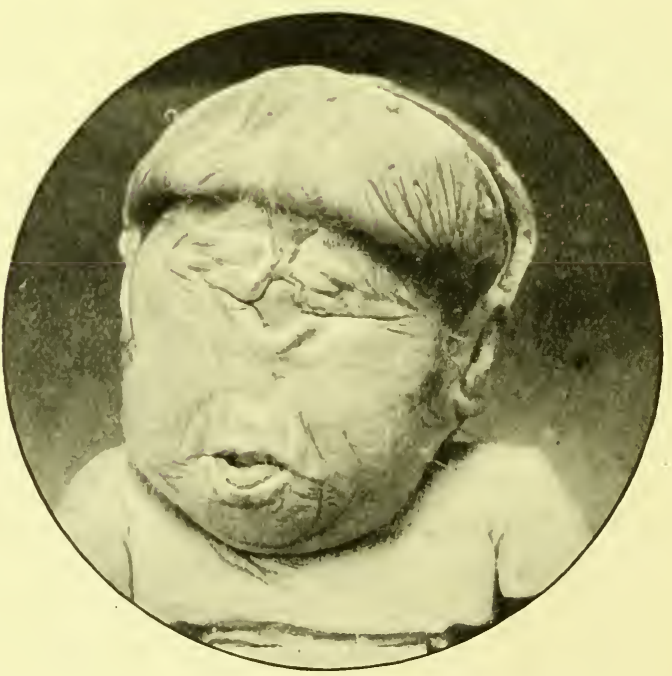

FIG. 2.-A human cyclops, showing the absence of a proboscis and notching of the upper lids. 


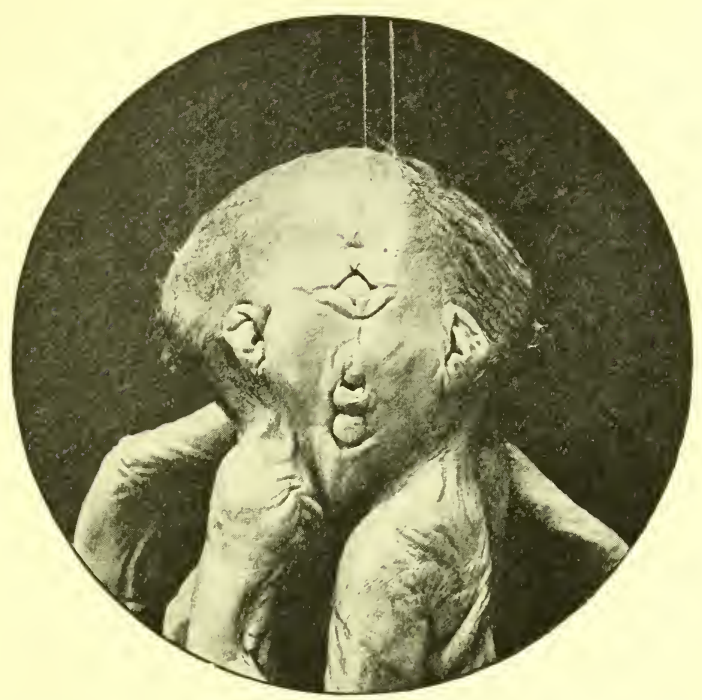

FIG. 3.-Human cyclops (twin). There is an exactly similar face on the other side of the specimen. The eye, although practically fully formed except for a coloboma of the optic nerve, is derived half from each fetus.

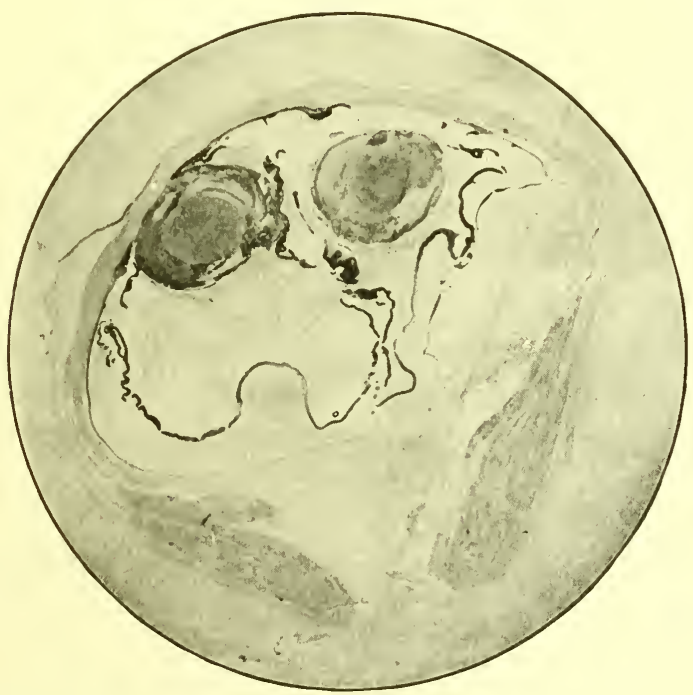

FIG. 4.-A human cyclops, showing two secondary optic vesicles and two lenses in one globe. 


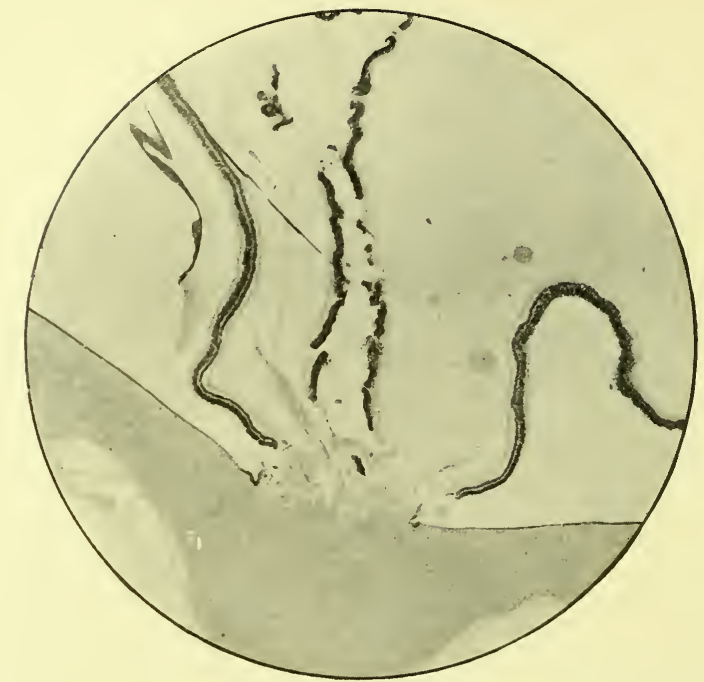

FIG 5.-A human cyclops, showing the optic disc with two retinal and two central hyaloid arteries.

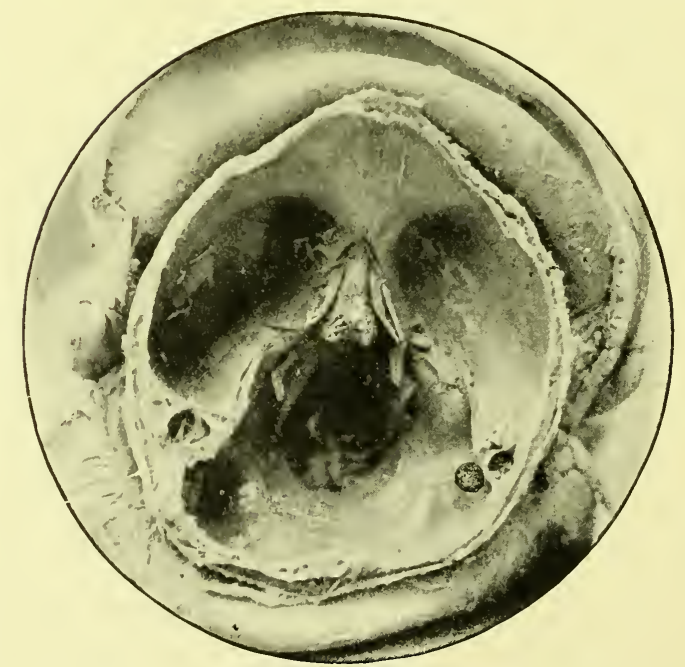

FIG. 6.-Human cyclops showing the base of the skull and cranial nerves (note the single optic nerve). 
All the structures in the eye may be single and the lenses alone show signs of union. The cornea and sclerotic may be single, but the iris, choroid, retina, lens and vitreous contained within them double (Figs. 4 and 5 ). The sclerotics on the inner side of each eyeball may be the only parts united. Two entirely separate eyes may lie side by side in the same orbit.

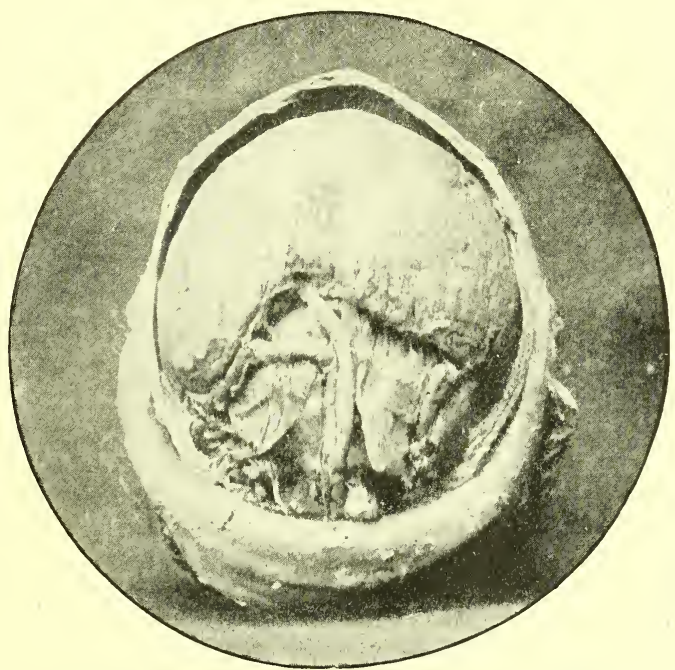

FiG. 7.-Human cyclops, showing the absence of convolutions and fissures in the brain, the posterior part of the cerebrum is occupied by a large cyst (local hydrocephalus).

The condition is due to an arrest in development of the anterior part of the neural tube. It has been shown that in certain species of fish cyclopia can be induced, almost at will, by subjecting their eggs to the action of various magnesium salts dissolved in sea water. ${ }^{1}$ From these and other embryological experiments it seems probable that the original tissue from which the primary optic vesicles are formed is median and single, and that cyclopia is due to an arrest in the formation of the eyes whilst the elements from which they start are still in the median axis of the developing nervous system. This differs from the

${ }^{1}$ C. R. Stockard. J. Exp. Zool., V, I909, 6. 
older view which attributed the defect to a fusion, more or less complete, of the incipient optic vesicles in the median plane.

Anophthalmia.-One or both eyes may be congenitally absent. The abnormality is met with in healthy well formed children (Fig. 8) but not uncommonly other malformations, such as hare-lip and supernumerary digits, occur in association with it.

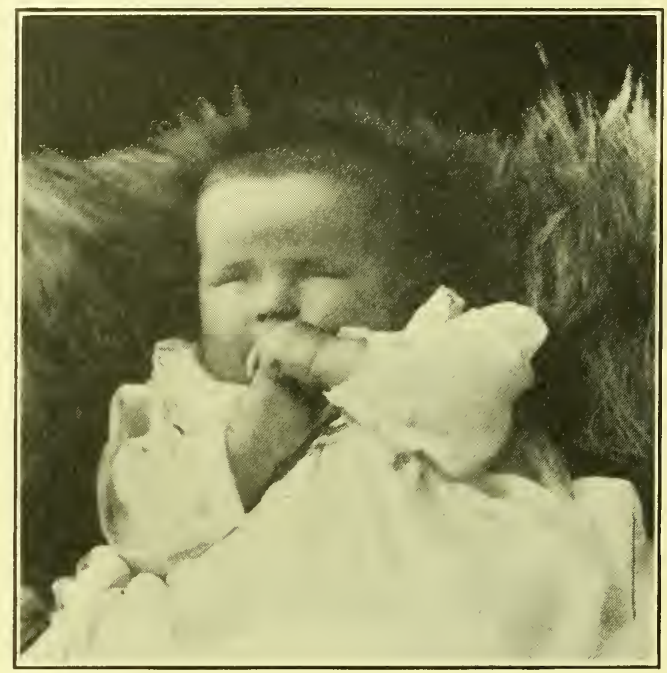

FIG. 8.-Case of bilateral anophthalmia, child aged 4 months.

The eyelids though small are usually well formed; they may be adherent at their margins. The orbit, smaller than normal, is lined throughout by conjunctiva. Digital examination of it frequently reveals a small hard, mobile, nodule near the extreme apex. The lacrymal puncta may be absent in one or both lids but the lacrymal gland is usually present.

In these cases the optic nerve has never been found to enter the orbit; it either ends as a cone or fibrous filament at the optic foramen, or it is entirely absent together with the chiasma. The olfactory lobes and cerebral hemispheres 
have also been found deficient, which is of interest in that they, like the primary optic vesicles, are outgrowths of the anterior cerebral vesicle. It is a failure of the primary optic vesicles to bud off from the anterior cerebral vesicle which seems to be the usual cause of anophthalmia.

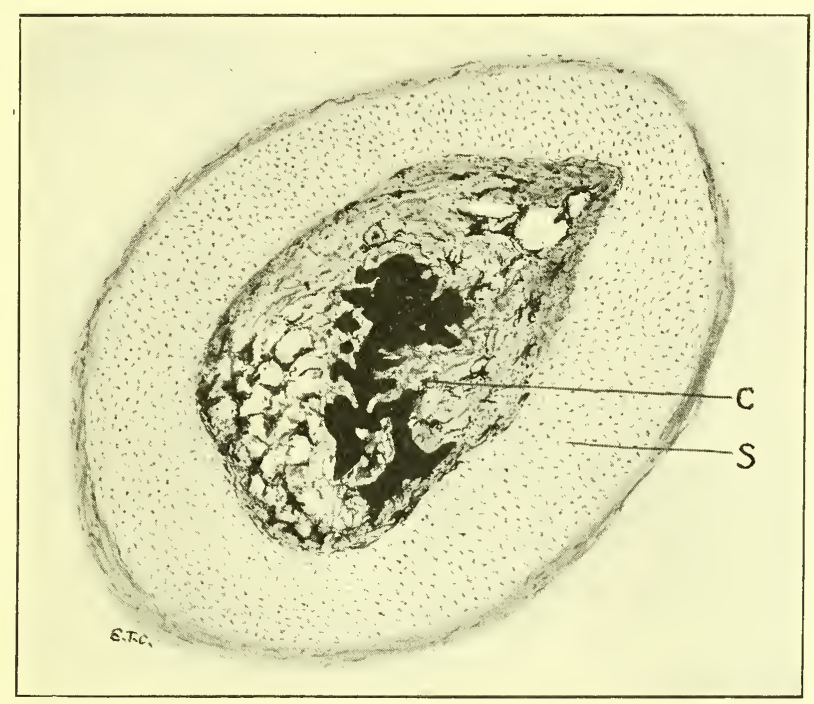

FIG. 9.-The mesoblastic constituents of an eyeball from the orbit of a chick the subject of congenital anophthalmia. $S$ points to a ring of hyaline cartilage representing that normally found in the sclerotic; $C$ points to tissue like that of the uveal tract contained within this ring. There was no optic nerve or any tissue representing that formed from the secondary optic vesicle.

Microscopical examination of the nodules met with in the orbits has shown ${ }^{1}$ them to be composed of subsidiary parts of the eye, of mesoblastic origin. A capsule of fibrous tissue like the sclerotic containing pigmented choroidal tissue but not any retina (Fig. 5), i.e., there is complete absence of the essential nervous elements constituting an eye. It is to these cases that the term anophthalmia should,. strictly speaking, be limited. Unfortunately, the presence or absence of the essential nervous elements in a nodule in

${ }^{1}$ Treacher Collins and J. H. Parsons. Trans. Ophth. Soc. of the U. K., XXIII, 1903, 244. 
the orbit, can be determined with certainty only by microscopical examination. So, that clinically it becomes very difficult to distinguish between true anophthalmia and very high degrees of microphthalmia.

Congenitally Cystic Eyeball.-A few cases have been recorded in which an infant at birth has been found to have a cyst in the orbit replacing the eyeball, of which clinically none of the normal structures can be detected.

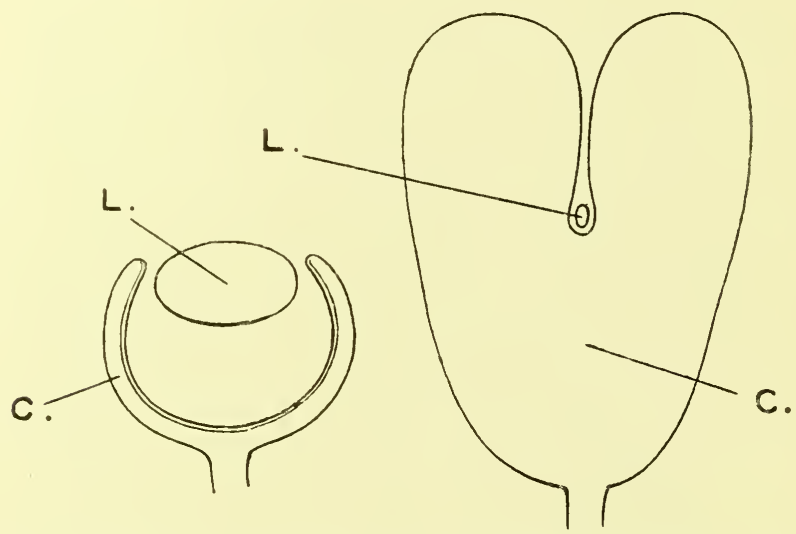

FIG. I0.-Diagram showing the normal involution of the secondary optic vesicle, and how a failure in its involution may result in the formation of a cystic eye. $C$, Cavity of cyst; $L$, lens.

Pathological examination of two such cysts $^{1}$ has shown them to have an outer wall of fibrous tissue, into which the tendons of the orbital muscles were inserted, and a lining membrane of retinal tissue. Posteriorly this lining membrane consisted of a single row of pigmented cells, representing the outer layer of the secondary optic vesicle, and anteriorly of ill-formed retinal tissue, representing the inner layer of the secondary optic vesicle. Apparently, the normal involution of the primary optic vesicle had

${ }^{1}$ M. S. Mayou. Trans. Ophth. Soc. of the U. K., XXIV, I904, 340.

Treacher Collins and Johnson Taylor. Trans. Ophth. Soc. of the U. K.,

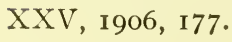


failed to take place, the two layers of secondary optic vesicle never having come into apposition; the cavity of the cyst corresponding to the cavity of the primary optic vesicle (Figs. Io and I I).
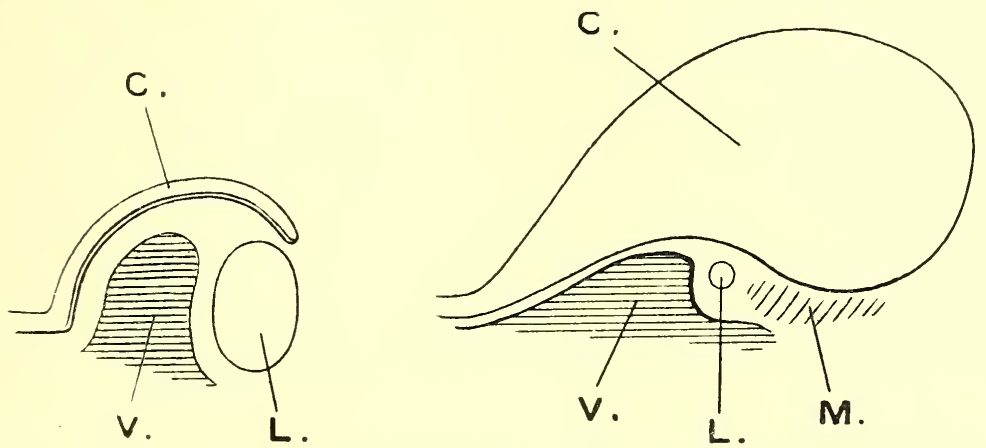

FIG. II.-Diagram showing the normal upgrowth of mesoblastic tissue into the cavity of the secondary optic vesicle, and the upgrowth of this tissue when involution of the primary optic vesicle has failed to take place and a cystic eye is developed. $V$, Vitreous; $L$, lens; $M$, mesoblastic tissue anterior to lens; $C$, cavity of cyst.

Shot-silk or Watered-silk Retina.-The condition which is described by one or other of the above names is very common in the eyes of young people, and tends to disappear on the growth of the eye to its full dimensions. It often remains permanent in hypermetropic eyes and is not met with in myopia. A dark background to the retina from a deeply pigmented choroid renders it most conspicuous.

With the ophthalmoscope a number of light reflexes are seen which shimmer and shift with every movement of the mirror. They are most marked along the line of the retinal vessels and around the macula.

The inner layer of the secondary optic vesicle during fetal life grows more quickly than the outer layer, or the surrounding mesoblast. It becomes, therefore, thrown into folds which project inward toward the vitreous chamber. As the eyeball approaches its full dimensions these folds gradually become flattened out, so that the two layers of the secondary optic vesicle lie everywhere in close contact. 
In the eyes of young people, and in small hypermetropic eyes, the inner surface of the retina is imperfectly flattened out; slight inequalities in the level of its inner surface are present, especially in the position of the retinal vessels, and it is these inequalities which catch the light and reflect it so as to give rise to the watered-silk appearance.

Coloboma of the Retina and Optic Nerve. Microphthalmia with Cysts.-The fetal ocular cleft normally closes early in fetal life, cutting off the mesoblastic structures external to the secondary optic vesicle from those internal to it.

In some animals a permanent union between the mesoblastic structures inside and outside the eyeball exists, the ocular cleft never becoming entirely closed. In fish the falciform process projects upward through the cleft, and in birds the pecten.

Occasionally in man, a union is found to have persisted between the tissues derived from mesoblast inside the secondary vesicle and those external to it, in the region of the ocular cleft. Such a union must necessarily prevent the edges of the secondary optic vesicle coming together, and a gap or coloboma in the retina results. In these eyes there is an abnormal development of fibrous tissue in the vitreous chamber, which extends through the cleft, they do not expand to the full extent, and are microphthalmic.

A delay in the separation of the mesoblast outside, from that inside the eye, without permanent union, may also result in failure in the closure of the cleft in the secondary optic vesicle, and the formation of a coloboma. When this occurs the changes are not limited to the structures derived from the secondary optic vesicle, but involve also those immediately external to it. If the defective closure is in the extreme posterior part of the cleft, there is a coloboma of the optic nerve sheath; if somewhat further forwards, a coloboma of the choroid; if near the anterior lip of the cup, a coloboma of the ciliary body and of the iris, which normally grows from it. Colobomata of these 
structures will be described more fully under the aberrations connected with the formation of the enveloping mesoblast (see page 105).

The region in which a coloboma occurs is frequently a weak spot in the walls of the globe, there being a defective closure of the cleft in the retina or optic nerve, and a faulty formation immediately external to it of the structures derived from the mesoblast. On the expansion of the

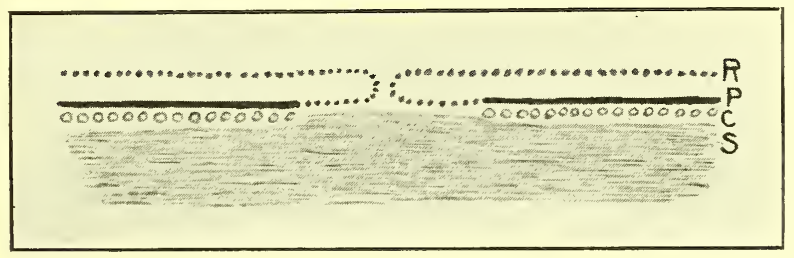

FIG. I2.-Diagram of a coloboma of the retina and choroid showing how folds of the retina may extend across the area in which the choroid is deficient. $R$, Retina; $P$, pigment epithelium; $C$, choroid; $S$, sclerotic.

globe, as the result of the intraocular pressure, the weak colobomatous area is very liable to become ectatic, sometimes to such an extent as to produce a considerable cystic protrusion.

Microscopical examination of eyes with colobomata of the choroid have shown varying conditions of the retina in the region of the defect. In most specimens vertical antroposterior sections have alone been made, and no gap in the retina has been demonstrated. The absence of such a gap can only be definitely determined by equatorial sections.

The pigment epithelium representing the outer layer of the secondary optic vesicle has usually been found absent over a part of the defect. It frequently exists as an unpigmented row of cells before it finally ceases altogether. The retina proper, representing the inner layer of the secondary optic vesicle, has been found in various degrees 
of attenuation and maldevelopment. Two layers of fairly well formed retina have been met with in the colobomatous area, situated with their normally outer, rod and cone surfaces, in contact with one another. Such an arrangement suggests that across a gap in the region of the cleft between the outer layers of the secondary optic vesicle, folds of the inner layer of the secondary optic vesicle had extended. It has already been pointed out that the inner layer of the

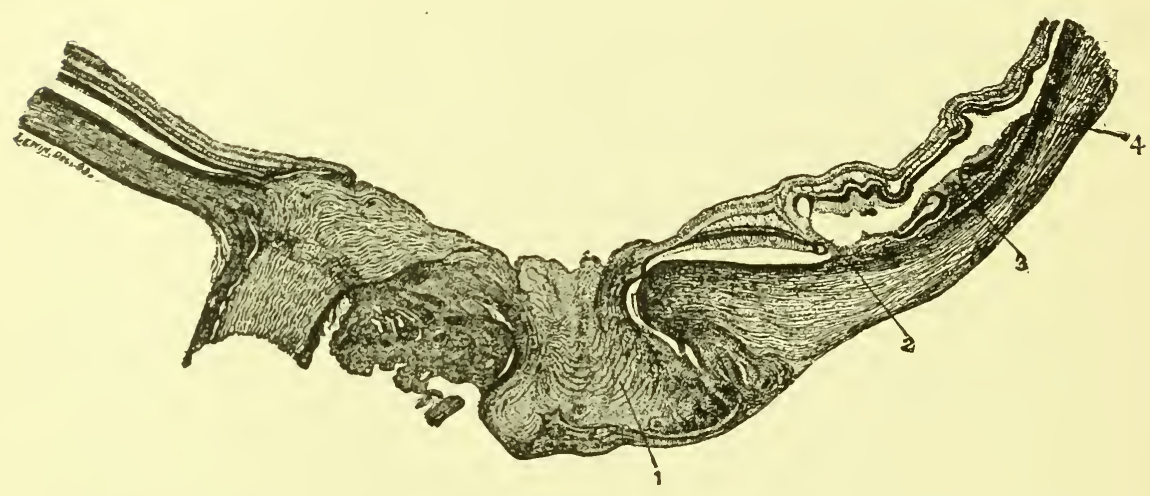

FIG. I3.-Microscopical appearances of the posterior half of a microphthalmic eye, showing a break in the continuity of the sclerotic and choroid through which knuckle of retina (I) tissue is protruding. A coloboma of the choroid commences at (2) in front of which the pigment epithelial layer is replaced by tissue like retina (3) but so placed that its nerve fibre layer faces the sclerotic. At (4) the pigment epithelium is again present and the coloboma of the choroid ceases.

secondary optic vesicle grows faster than the outer and in the fetal eye is thrown into numerous folds, an extension of them from each margin of the cleft, over the colobomatous area might therefore readily occur (Fig. I 2). When a coloboma becomes ectatic, and when from absence of the choroid the overlying retina is imperfectly nourished, it fails to develop or becomes atrophied.

In some eyes a portion of retinal tissue is found protruding into or through a gap in the choroid and sclerotic, in the region of the fetal cleft (Fig. I3). The size and extent of such a protrusion varies considerably. It may be only a small knuckle of retinal tissue, or consist of quite two- 


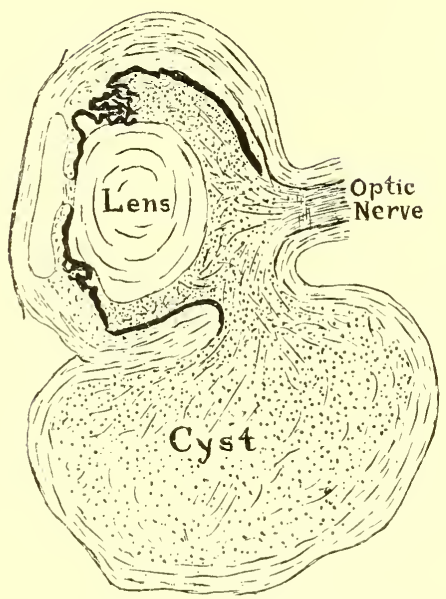

FIG. I 4.-Diagram of a congenitally microphthalmic eye with a cyst attached to it. Retinal tissue protrudes through a gap in the sclerotic and choroid at the lower and posterior part and fills the cavity of the cyst.

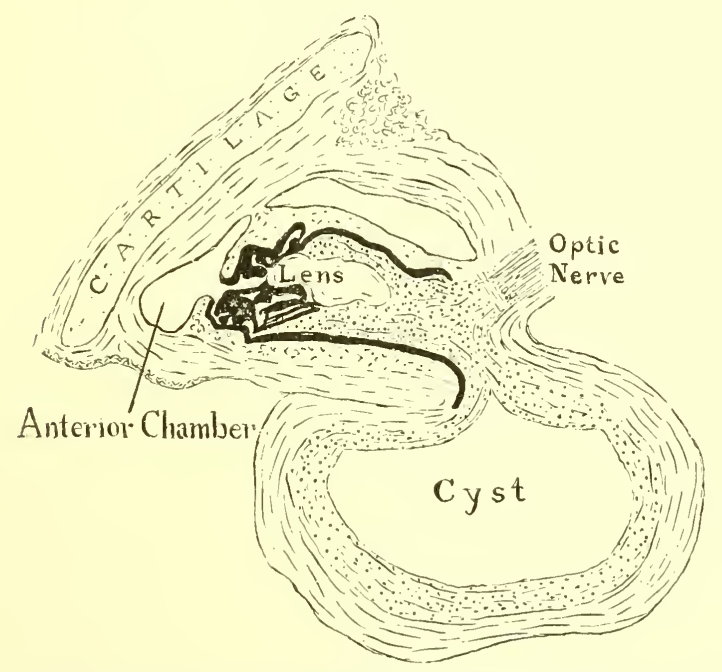

FIG. I5.-Diagram of a congenitally microphthalmic eye with a cyst attached. The cyst is connected with the eye at its lower and posterior part, and is lined by retinal tissue. There is an abnormal development of hyaline cartilage in the sclerotic. 
thirds of the membrane. ${ }^{1}$ In some cases it forms a solid mass with an external fibrous tissue covering, in others folds of retinal tissue become distended by fluid into cysts with an

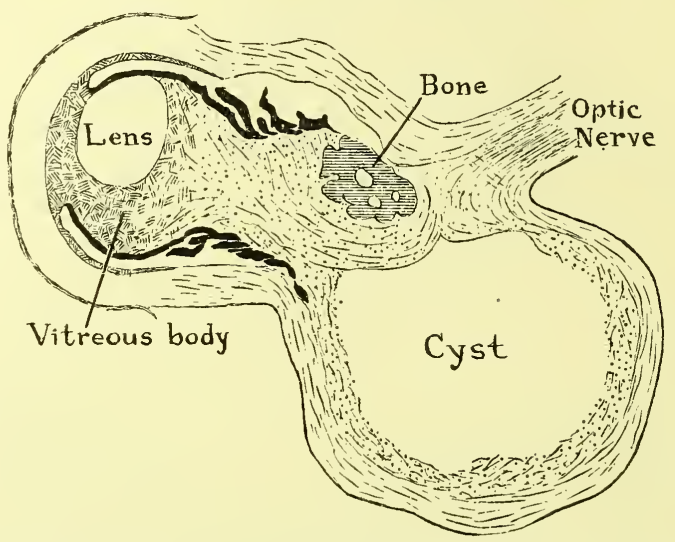

FIG. I6.-Diagram of a congenitally microphthalmic eye with a cyst attached. There is a wide opening of communication between the interior of the eye and the cavity of the cyst which protrudes from its lower and posterior part. The optic disc faces downwards towards the cavity of the cyst and the lining of the latter is composed of retinal tissue.

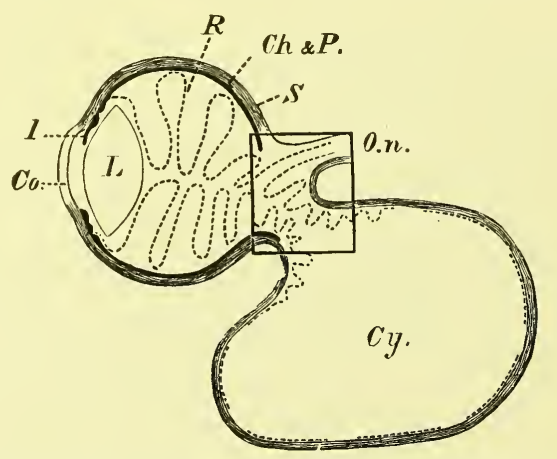

FIG. I 7.-Diagrammatic representation of a microphthalmic eye with a cyst attached. Co, Cornea; $L$, lens; $I$, iris; $R$, retina much folded; $C h$ and $P$, choroid and pigment epithelium; $S$, sclerotic; $O n$, optic nerve; $C y$, cyst lined by atrophied retina. The part marked off by straight lines is shown magnified in Fig. I7. Case recorded R. Lond. Ophth. Hosp. Reps., XII, I889, 289.

outer fibrous tissue coat (Figs. I5, I6, I 7). The protuberant retina may consist merely of neuroglial tissue with a few

${ }^{1}$ Treacher Collins. Trans. Ophth. Soc. of the U. K., XII, I892, 289 and XVII, I897, 254. 
scattered nuclear bodies, and sometimes hyaline nodules; or be so well developed that several of its layers can be easily differentiated. When well formed retina is present the rod

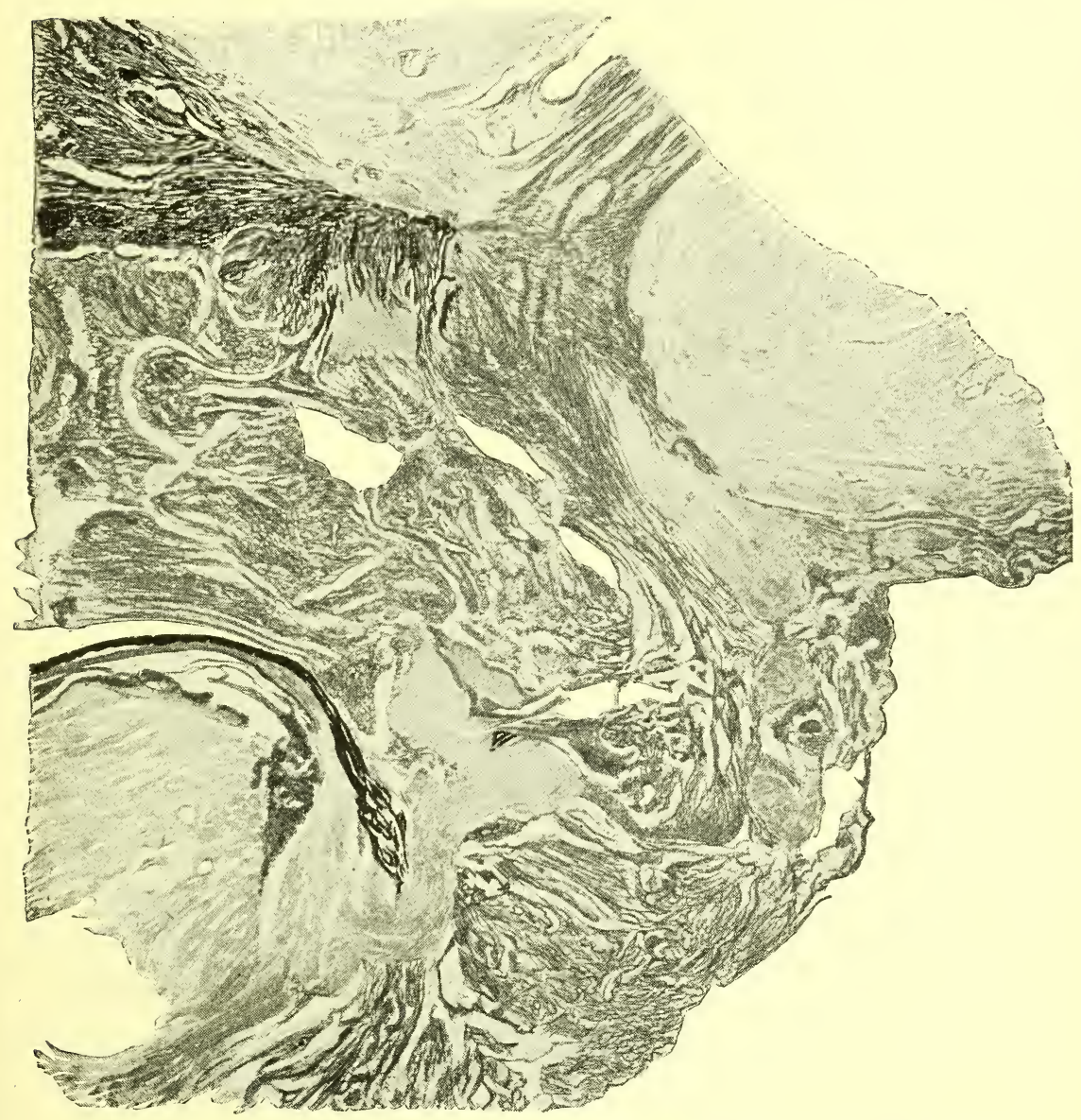

FIG. I8.- Shows the portion of the eye represented in Fig. I7, in the square, highly magnified. The neck of the cysts is seen with retinal tissue passing through it from the eyeball into the cyst.

and cone layer is directed inwards towards the cavity of the cyst. The eyeballs with which such cysts are connected are always microphthalmic, the degree of microphthalmia varying with the amount of protuberant retina. 
The cyst usually forms a marked translucent swelling, of a bluish colour, in the lower lid, and may be so extensive as to hide from view the small eyeball, to the lower part of which it is attached.

These protuberant nodules of retinal tissue and cysts, which are evidently only different degrees of the same defect, may be accounted for by non-expansion of the globe from faulty development of the vitreous humour; one or more of the folds of the secondary optic vesicle then grow out, through the cleft in the outer layer of the secondary optic vesicle, into the mesoblastic tissue beneath. If a fold of this sort became distended with fluid, the position of the retina would be such as has been found in these cysts.

When a coloboma involves the head of the optic nerve the expansion of the gap, from intraocular pressure, produces marked changes in the region of the optic disc. The disc itself becomes enlarged and depressed, sometimes only in its lower part, at other times over its whole extent, but to different degrees in different parts. The colour of the depressed area is white with greyish markings. The retinal vessels may not have attained their normal position in the centre of the disc, those going to the lower part of the fundus curving with a sharp bend round the lower border of the colobomatous opening, whilst those going to the upper part run a straight course from their point of entrance. In other cases the whole retinal blood supply seems to be derived from cilio-retinal vessels which curl round the margins of the area corresponding to the optic disc.

Conditions which have been termed colobomata of the optic nerve are often really colobomata of the choroid, which have become ectatic, and extend up to the margin of the optic disc. The lower border of the papilla becomes displaced upwards and backwards, so that its surface faces downwards. 
Small folds of retinal tissue are sometimes met with at the margin of the optic disc projecting into the underlying sclerotic, the sheath of the optic nerve, or even into the tissue of the nerve itself.

A coloboma of the nerve alone, without involvement of the adjacent choroid, is of rare occurrence. ${ }^{1}$ Pathologically it is recognised by the relation which the excavation bears to the intervaginal space in the sheath of the nerve, if internal to it, we have a true coloboma of the nerve, if external, a coloboma of the choroid.

In a coloboma confined to the optic nerve, the nerve entrance is of nearly normal dimensions, and the abnormalities met with are contained within its boundaries. A fold of retina is found passing through a gap in the lamina cribrosa into a hollow space in the nerve.

Congenital Pigmentation of the Retina. ${ }^{2}$ - Congenital pigment spots of a chocolate-brown colour are met with in the retina, they are angular in outline, and situated in groups suggesting the arrangement of collections of sarcinæ. They are usually restricted to a wedge-shaped area of the fundus and do not give rise to any symptoms.

Microscopically ${ }^{3}$ they have been found due to aggregations of deeply pigmented epithelial cells in the retina, some of them surrounding nodules of hyaline material. This hyaline material, which is similar to that composing the membrane of Bruch, is doubtless secreted by the abnormally placed pigment epithelial cells.

The condition is due to some localised failure in the development of the rods and cones, the absence of which permits migration of the pigment cells into the retina, and gives rise to an abnormal union of the two layers of the secondary optic vesicle.

Congenital Ectropion of the Uveal Pigment of the Iris.When the secondary optic vesicle is first formed, the point

${ }^{1}$ George Coats. R. Lond. Ophth. Hosp. Reps., XVII, I908, I 78.

${ }^{2}$ S. Stephenson. Trans. Ophth. Soc. of the U. K., XI, I 89I, 77.

${ }^{3} \mathrm{~J}$. H. Parsons. X Congress Internat. D'Ophth., I904, I 52. 
of junction of the two layers anteriorly, corresponds, to what in the fully developed eye is the termination of the retina proper, or pars optica retinæ at the ora serrata. The pigmented and unpigmented layers of cells lining the ciliary body, or pars ciliaris retinæ, and the two pigmented layers of cells on the back of the iris, or pars iridis retinæ, are extensions forward from the secondary optic

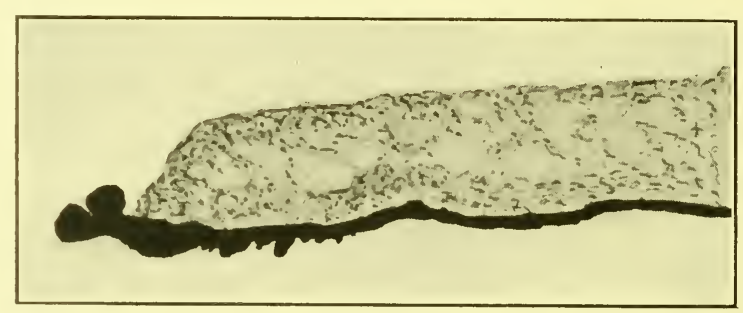

FIG. I9.-The iris in a case of congenital partial "aniridia" showing well marked ectropion of the uveal pigment. The sphincter pupillæ muscle is absent.

vesicle and develop later. In the fully formed eye, the anterior termination of the secondary optic vesicle, may be regarded as the point of junction of the two pigmented layers of the pars iridis retinæ at the pupillary margin.

Ectropion of the uvea is the abnormal extension forwards of these two layers to the anterior surface of the iris. It is occasionally met with as a congenital over-growth in the form of one or more small deeply pigmented nodules at the pupillary margin. When it occurs in connection with coloboma of the iris, or incomplete "aniridia," it is the outcome of defective development of the mesoblastic portion of the iris, and not overgrowth of the pigment epithelium (Fig. I9).

In many of the Ungulata, which have large oval pupils with the long axis horizontal, there is an extension forwards of the pigment epithelium of the iris round the pupillary margin; this at the upper part forms a black mass termed the corpus nigrum (Fig. 20). 


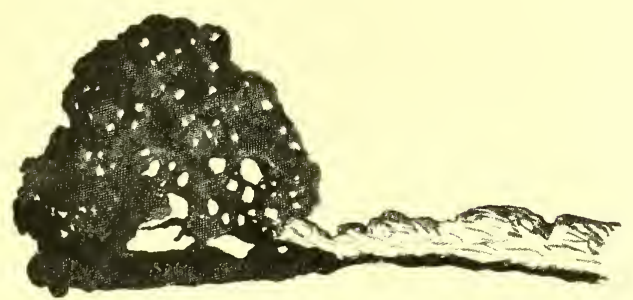

FIG. 20.- Section of a horse's iris, showing the prominent mass of pigmented epithelial tissue at its pupillary border, the corpus nigrum.

Absence of Nerve Fibres.-In cases of anencephaly, (Figs. 2I and 22) and in some cases of defective development

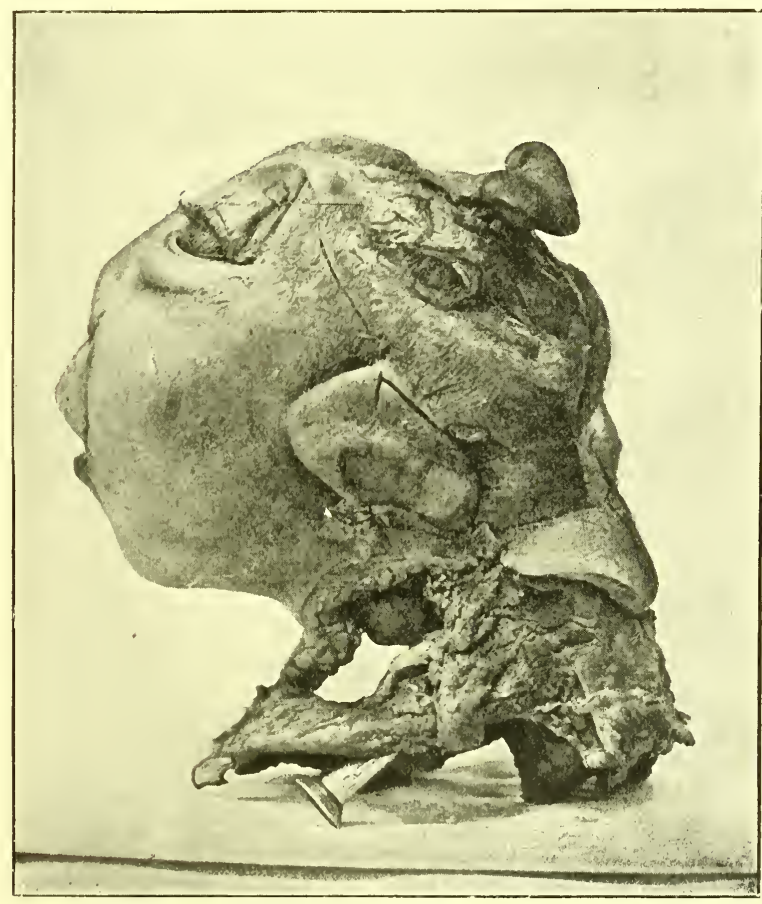

FIG. 2I.-Lateral view of the head of an anencephalic fetus, showing the deficient frontal eminences and proboscis.

of the occipital lobes resulting from cystic distension of the lateral ventricles, there is a complete absence of all 


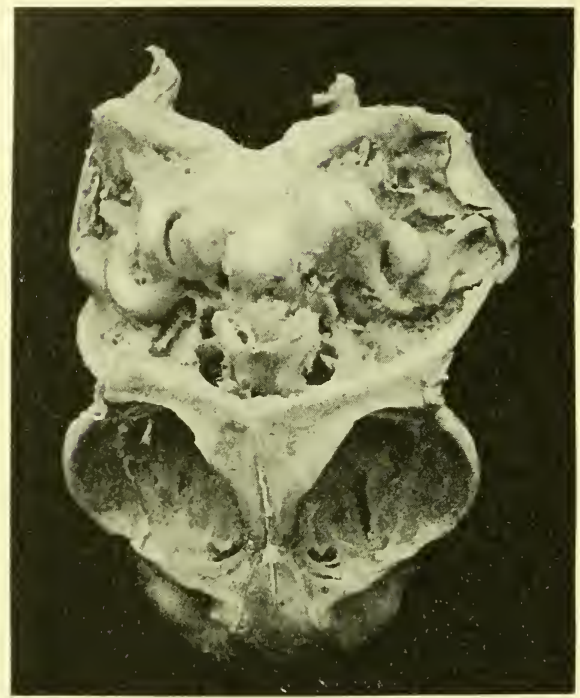

Fig. 22.-View from above of the skull of the anencephalic fetus depicted in Fig. 2I. The absence of the vault of the skull is shown, deficient foramen magnum, large semicircular canals, and the opening of the optic foramen.

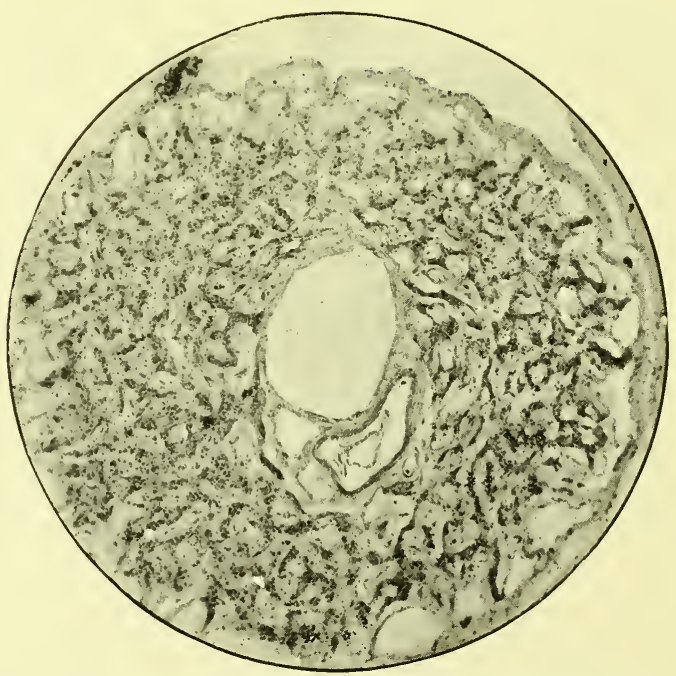

FIG. 23.-Transverse section of the optic nerve of an anencephalic fetus, showing empty trabectlæ and a large central artery and vein. 
the axis-cylinders in the optic nerve and retina. The fibrous tissue elements of the nerve, i.e., its sheath and trabeculæ are formed but the spaces in the trabeculæ contain no nerve fibres (Fig. 23). The optic disc is deeply cupped, due in part to the absence of the nerve fibres and in part to the yielding of the weak lamina cribrosa before the normal intraocular pressure (Fig. 24). The layers of the retina show much their usual arrangement; its ganglion cells may be present but its nerve-fibre layer is absent, so that its blood-vessels lie in direct contact with the hyaloid membrane of the vitreous.

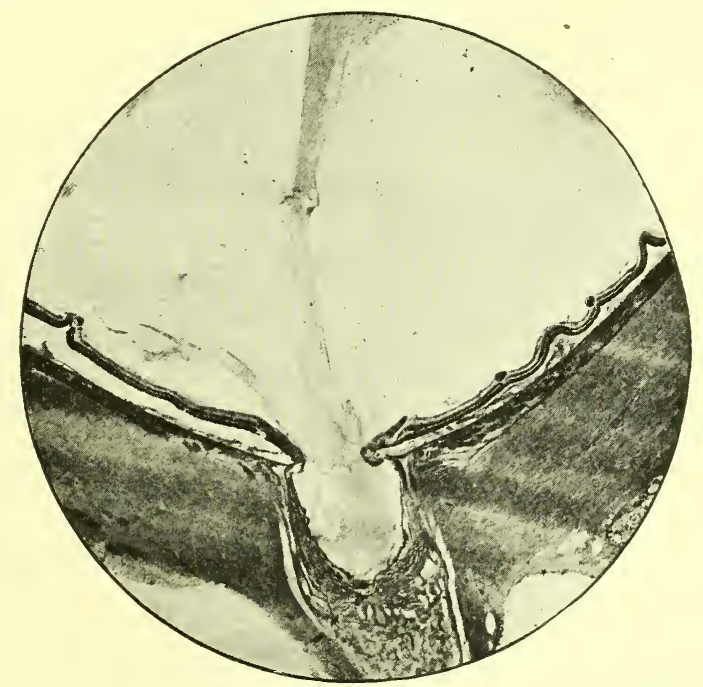

Fig. 24.- Horizontal section through the posterior part of an eye of an anencephalic fetus, showing deep cupping of the optic disc.

These cases ${ }^{1}$ suggest that the axis-cylinders of the optic nerve and retina are largely cerebral in origin and that the integrity of the cerebral centres is necessary for their formation.

Opaque Nerve Fibres in the Retina.-In some animals nerve fibres in the retina are normally surrounded by medul-

${ }^{1}$ M. S. Mayou. Trans. Ophth. Soc. of the U. K., XXIV, I904, 150. 
lary sheaths, and when viewed ophthalmoscopically, appear as white striated areas obscuring the red reflex of the choroid. In the rabbit, bundles of such opaque fibres spread out horizontally from each side of the optic disc, all the retinal blood-vessels being confined to those areas.

In the normal retina of man the nerve fibre layer consists only of axis-cylinders and it is a transparent membrane. Occasionally, as an abnormality, areas are found in the retina in which medullary sheaths have developed around

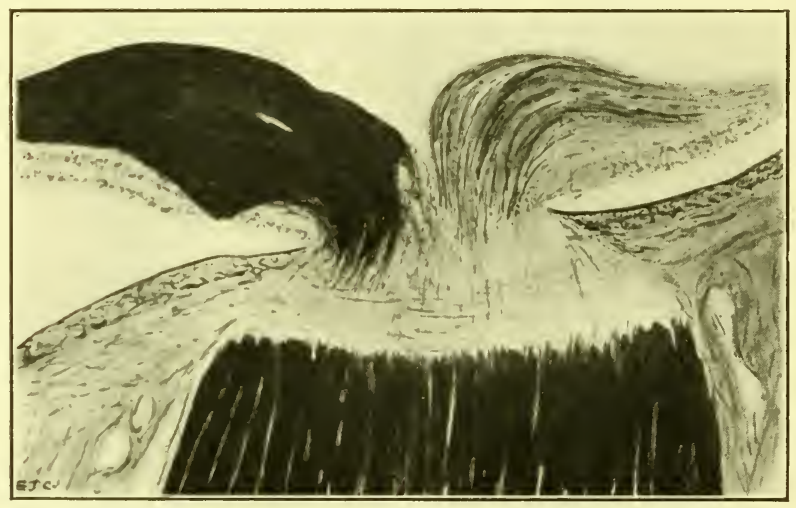

FIG. 25.-Section through the head of the optic nerve in a case of opaque nerve

fibres. The medullary substance is stained black by Weigert's method.

the axis cylinders, causing them to be white and opaque. In most such cases the patches of opaque nerve fibres are situated at the margin of the optic disc, but they may occur unconnected with it elsewhere in the fundus. Ophthalmoscopically, striation at the margin of these patches is a characteristic diagnostic feature of the affection. As the retinal blood-vessels lie in the nerve fibre layer of the retina, where they pass through an opaque patch they become entirely, or in part, obscured from view.

Microscopical sections of patches of opaque nerve fibres at the margins of the disc, stained either by Weigert's or Pal's method, show that the medullary sheaths around 
the fibres in the optic nerve end in the usual way, at the posterior surface of the lamina cribrosa. Axis cylinders unsheathed pass through the lamina cribrosa, and it is only on emergence from that structure into the retina that they again become invested with the darkly staining medullary substance (Fig. 25).

This abnormality of medullation of the nerve fibres in the retina is developed after birth, for, as already pointed out, the nerve fibres in the optic nerve itself are not medullated at birth.

Crater-like Hole of the Optic Disc.-The condition which has been described under the above name differs from a coloboma of the optic disc; it is rarely situated in the line of the ocular cleft, and two holes may be present in different parts of the same disc. It consists of a pit situated in the papilla, near its margin, varing in size from $1 / 6$ to $1 / 3$ of the diameter of the disc. In depth it may be an amount which is just appreciable by the observation of a parallax ophthalmoscopically, or as much as 8 to 9 $\mathrm{mm}$. The walls and floor of the pit are usually pigmented, but to a different degree in different cases.

Microscopical examination of specimens $^{1}$ in which this condition is present shows a deep pocket in the nerve at its border, which passes backwards separating the lamina cribrosa from the sclera promontory. The pit is entirely in the nerve, it is lined by pigment epithelium, and filled with altered retinal tissue. Ophthalmoscopically the pigmented floor of the depression is seen through the retinal tissue, which is transparent.

It has been suggested ${ }^{2}$ that in these cases the neural portion of the secondary optic vesicle instead of forming neuroglia for the support of the nerve fibres, develops atypically into tissue like that normally met. with in the eyeball. A layer of pigment epithelium, corresponding to the outer layer of the secondary optic vesicle, forming

${ }^{1}$ G. Coats. R. Lond. Ophth. Hosp. Reps., XVII, I908, 2 I 5.

${ }^{2}$ Monthus. and Opin. Archiv. D'Ophtal., XXIII, 32. 
on the inner surface of the pial sheath and aberrent retinal tissue, corresponding to the inner layer of the secondary optic vesicle, forming deeper in the nerve.

Physiological Cup of the Optic Disc.-A much commoner form of depression in the optic disc than that last described is the "physiological cup." It varies in size, does not extend to the margin of the disc, and is usually situated a little external to the middle line. The floor of the cup is of a lighter colour than the rest of the disc, and on it some grey markings are often seen, due to the fibres of the lamina cribrosa which are exposed to view. The retinal vessels on emerging from the nerve curl round the inner margin of the cup.

The fibres of the optic nerve in passing into the eye to reach the retina traverse an opening in the sclerotic and choroid. They are always grouped about the sides of the opening, and if its size is excessive they do not entirely fill it, a vacant space which appears ophthalmoscopically as a depression, being left in the centre of the disc.

Pseudoneuritis.-In eyes which are abnormally small, i.e., in cases of high hypermetropia, an appearance of the optic disc is met with which closely resembles that brought about by inflammation, for which it is very liable to be mistaken. Ophthalmoscopically, the disc is seen to have no central depression, to be unusually protuberant, of a greyer colour than normal with a striated and somewhat ill-defined margin. ${ }^{1}$

This condition may be regarded as the reverse of a physiological cup. The small eye has a small opening in the choroid and sclerotic for the passage of the fibres of the optic nerve; they become therefore packed unusually close together, and no space is left at the point from which they radiate outwards to form a central depression.

${ }^{1} \mathrm{~W}$. T. Holmes Spicer. Trans. Ophth. Soc. of the U. K., XVI, I896, $13+$. 
Congenital Day-blindness with Colour-blindness, and Congenital Night-blindness (Mooren's Retinitis Punctata Albescens).-These two rare conditions will be described together, because both are probably due to some defective development of the percipient end organs in the retina, and because it is interesting to contrast the symptoms which they present.

The affection which has been termed ${ }^{1}$ congenital dayblindness with colour-blindness always affects both eyes, the patients have nystagmus, are amblyopic and colourblind. Usually they are totally colour-blind, but gradations in the severity of the affection occur, some having only a central scotoma for colours. The ophthalmoscopic changes are very slight, some pallor of the disc may be present and frequently some small white dots at the macula, or a little stippling, has been noted. The condition is hereditary, members of the same childship or of successive generations being affected. Some of the patients are mentally defective.

The name "retinitis punctata albescens" has been applied to several different conditions, but should ${ }^{2}$ be restricted to an affection with stationary night-blindness and numerous minute white spots in the fundus.

The night-blindness is doubtless congenital, dating from infancy and showing no tendency to increase. Both eyes are involved. The acuteness of vision in bright light is normal or very slightly defective; there may, however, be marked amblyopia. In dull lights it is always reduced. The field of vision is in some cases restricted in bright light, in others only in dim lights. Ophthalmoscopically, a multitude of tiny dull white dots are seen disseminated over the fundus. They are never present at the macula, and usually become smaller and less numerous near the periphery. The dots are round or oval in shape, some seem to be joined

${ }^{1}$ E. Nettleship. St. Thomas's Hosp. Rep., X, I880. Grunert. Arch. für Ophth., LVI, 1903, i, I32.

${ }^{2}$ E. Nettleship. Trans. Ophth. Soc. of the U. K., VII, I887, 301. 
together and look like dumb-bells. They are entirely free from any pigmentation and are situated at a deeper level than the retinal blood-vessels. Their average width is about half that of a medium-sized retinal artery. They remain indefinitely without undergoing any alteration in appearance. The condition has been met with several times in members of the same childship, but not so far in successive generations. Consanguinity in the parents of those affected is not uncommon. No pathological examination of the retina in these cases has yet been made, and the exact nature and position of the white dots can only be conjectured.

It has been pointed out ${ }^{1}$ that the retina has a dual function, one associated chiefly with the cones and the other with the rods. The one form of vision has the power of distinguishing colours, requires high intensity of light, and gives great definition and clearness. It is most marked in the centre of the field and is associated with the cones. The other form is characterised by an inability to appreciate colours, has its greatest sensibility in weak illuminations, and a marked preference for rays of medium or short wave-lengths. It is wanting in definition, absent at the macula, and is associated with the rods.

Congenital day-blindness has been described as coneblindness, and it will be seen, that the visual functions just described as associated with the cones, are those which are found to be absent or weakened in that affection.

In congenital night-blindness, on the other hand, the symptoms which are usually present seem to indicate defective rod vision. It is true that in some cases there has been defective form sense, which is not what would have been expected, but this may have been due to some other cause superadded. The absence of the white dots at the macula in all the cases shows, that the region in which cones only exist was unaffected.

\footnotetext{
${ }^{1}$ Von Kries. Arch. für Ophth., XLIII, I 896, iii, 95
} 
Congenital Amblyopia.-That congenital amblyopia, or congenital defect of form-sense, is due to some imperfect development of the macula seems highly probable. Mammals as a class are characterised by the absence of a fovea, the Primates being the only ones in which it is found. It is developed in association with the higher forms of binocular vision, when the production of one highly sensitive spot in the retina for form-sense becomes essential.

The anatomical characteristics at the fovea to which the hightened acuity of form-sense may be attributed are: (a) The close congregation together of cones. (b) The isolation of each cone to one nerve-path. (c) The opening out of the ininer layers of the retina, allowing the unimpeded access of rays of light to the cones. Probably all three factors are essential for clearness of definition in vision, and an abnormally low acuity of central form-sense dating from birth, unaccompanied by any obvious ophthalmoscopic changes, may be due to the absence of any one of these characteristic features.

Some eyes with congenital amblyopia and nystagmus, in association with aniridia and albinism, have been examined microscopically. ${ }^{1}$ In these cases there was an absence of the fovea and of the usual distinctive characteristics of the retina at the macula.

When congenital amblyopia is present in both eyes, the capacity of fixing an object with one part of the retina in preference to another is not acquired, and the condition, characterised by involuntary rapid movements of the eyeballs, termed congenital nystagmus, is developed.

When congenital amblyopia is present in only one eye, the fusing of the images seen with the two eyes, for the purposes of central sterioscopic vision is of little use, and we have a condition which predisposes to the development of concomitant strabismus.

${ }^{1}$ Seefelder. Arch. für Ophth., LXX, 1909, i, 39 and C. H. Usher. Biometrika, XIII, I920, 50. 
Congenital Colour-blindness.-A person with normal sight can differentiate four simple unmixed colours in the spectrum, called primary colours, these are red, yellow, green and blue. The four primary colours can be further grouped into two pairs; red and green, and blue and yellow. The two colours in each of these pairs are complementary colours, i.e., when mixed together in suitable proportions they give rise to the sensation of white. They are also fatigue or contrast colours, the negative after image produced by looking at one colour in the pair corresponding to the other.

All parts of the retina are not equally sensitive to colours; for the perception of colours in the peripheral parts of the field a larger object, intenser light and greater saturation of colour, is required, than in the centre. If colour stimuli of moderate intensity are employed no sensation of colour is excited in the periphery of the field. On approaching the centre the first colours to be recognised are blue and yellow; it is not until we reach still nearer the centre that red and green become visible. If care be taken to make the coloured test objects of the same size, brightness and degree of saturation, the size of the fields for blue and green is practically the same, likewise those for red and green.

Though the final appreciation of colour is a psychological process, it seems to be an essential preliminary step that some change, either mechanical, electrical or chemical, should be produced in the end organs of the nerve fibres of the retina. Where colour sense is most acute, at the macula, cones only are present, so it would seem that the first change resulting in the appreciation of the different wave lengths of light must take place in them. Of what this change consists we have at present no definite knowledge. That the change in the cones which gives rise to the sensation of form, which is also most acute at the macula, is different from that which gives rise to the sensation of colour, is shown by the fact that the one may be deficient without the other being impaired. In cases of 
congenital amblyopia the form-sense may be defective whilst the colour sense is normal, and in cases of congenital colour-blindness the form-sense may be normal whilst the colour sense is defective.

A comparison of the cones at the periphery of the retina, where the colour sense is weak, with those at the macula, where the colour sense is acute, shows differences in arrangement and differences in shape. The cones at the macula are closely packed together, and on extension to the periphery they become widely spaced out. The cones at the macula, especially those at the centre of the fovea, are long and slender, those outside the macula gradually decrease in length as they extend towards the periphery; the decrease is most marked in the outer segment which at the same time increases in width. As yet we do not know to what extent the loss of power in distinguishing colours, on proceeding from the centre to the periphery of the retina, is to be associated with the wide spacing out of the cones or with their alteration in shape.

It has been already mentioned that those suffering from what is termed congenital day-blindness are also usually totally colour blind and amblyopic; some having an absolute central scotoma. These cases of achromatopsia confuse all colours, the spectrum being seen by them as a band of light, the different parts of which vary only in the degree of luminosity. Though as yet no pathological examination has been made of an eye affected with achromatopsia, there can be little doubt that it is due to some defect of development in the cones. A defect of so gross a character as to affect, not only the part they play in connection with colour sense, but also that in connection with form-sense.

Congenital Achromatopsia is a very rare affection, a much commoner congenital defect in colour vision is that in which only two colours are seen in the spectrum; those suffering from this affection are termed congenital dichromics. It has been estimated that from 3 to 4 per cent. of the population suffer from this form of colour-blindness, 
and a remarkably larger percentage of men than women; as many as 3 per cent. of the former and only 0.2 per cent. of the latter. It is frequently hereditary, being transmitted usually through unaffected females. Both eyes are as a rule affected, but well authenticated cases of monocular colourblindness have been recorded.

The commonest type of congenital dischromatopsia is that in which the individual affected is unable to distinguish between red and green. On looking at a spectrum he sees only two colours separated by a grey area called the neutral band. A much rarer form of congenital dischromatopsia is where the individual confuses yellow and blue. The first of the two forms, the red-green blindness, may again be divided into two classes; those to whom the spectrum appears shortened at the red end, and who receive no sensation from rays of long wave length; and those in whom the spectrum appears to be of its normal length and who can see only two colours in it.

With what structural defects in the retina these dichromic defects are associated has not yet been ascertained. The cases of congenital monocular colour-blindness, which have been met with, can only be explained by a defect situated in front of the commissure, and not in the brain. If there were defect of colour sense on one side of the brain, half of each eye would be affected not the whole of one eye. As the capacity for the appreciation of colour varies in the normal eye in different parts of the retina, being apparently associated with the distribution or construction of the cones, it seems probable that congenital defects of colour vision are due to some, at present unascertained, aberration in the cones.

Miosis Congenita.-A congenital smallness of the pupils may be met with apart from any evidence of fetal iritis. It is usually bi-lateral and may be met with in more than one member of a family. The pupils may be so small as to measure only $1 / 3 \mathrm{~mm}$. in diameter, they are inactive to light and convergence. Midriatic drugs produce but 
comparatively slight effect, the diameter of the pupil only expanding to $2.5 \mathrm{~mm}$., even after repeated instillations.

A want of response of the pupils to midriatics, apart from any obvious miosis, is also frequently met with in case of congenital cataract.

A microscopical examination ${ }^{1}$ of bleached sections of pieces of iris, which have been removed in cases of miosis congenita, has shown an absence of the dilator muscle fibres, they having failed to develop from the outer layer of the secondary optic vesicle on the back of the iris. There was also found an absence of the radial folds on the back of the iris, which it is presumed are formed to give a wider surface over which the dilator muscle elements may be spread out. The sphincter muscle, and the part of the iris in its neighbourhood, presented their normal appearance and dimensions; the more peripheral parts of the iris, where the dilator fibres are situated, was abnormally thin from before backwards.

A defective development of the sphincter muscle has so far only been met with in cases with deficient growth of the iris, cases of "partial aniridia," such as is shown in Fig. I 9. In it the pigment epithelium of the iris is seen to have extended further inwards than the mesoblastic tissue, and the sphincter muscle fibres, which should have formed at the point of junction of the two layers of the secondary optic vesicle, have failed to develop.

\section{ABERRATIONS CONNECTED WITH THE INVAGINATING SURFACE EPIBLAST}

The development of the lens from the surface epiblast may be divided into the three following stages:

I. The downgrowth of a fold of cuticular epiblast, which is separated in the form of a hollow vesicle from the surface epiblast, and then becomes surrounded by a hyaline capsule.

${ }^{1}$ S. Holth and O. Berner. “Miosis Congenita," Kristiania, 1922. 
2. The lengthening out of the cells composing the posterior layer of the vesicle so that they fill its entire cavity.

3. The proliferation of the cells composing the anterior layer of the vesicle, their migration to the sides of the vesicle, and transformation there into lens fibres.

The hyaline lens capsule was at one time regarded as the product of the fibro-vascular sheath which surrounds it during fetal life, and mesoblastic in origin. There is now considerable evidence to show that it is developed as a kind of secretion from the epithelial cells lining it, beginning to make its appearance before the fibro-vascular sheath is formed, and therefore epiblastic in origin.

The fibres formed from the posterior layer of the lens vesicle become encircled by those developed laterally, and form the most central part of the fully developed lens. The laterally developed fibres grow forwards and backwards, surrounding the nucleus, and meeting at the anterior and posterior poles, where they form lines of suture. The laying on of fresh lens fibres at the sides continues throughout life, but its rapidity is lessened as age advances, through the increased intracapsular tension tending to check the proliferative activity of the cells.

The lens during its most active period of development receives its nutrient supply from a special set of bloodvessels, which are arranged in a plexus around it, constituting what is called the fibro-vascular sheath. The bloodvessels in this sheath are derived from the central hyaloid artery posteriorly, and the anterior ciliary arteries anteriorly. Before birth, when the anterior chamber is formed, this sheath disappears, the lens for the rest of life receiving its nutrition by osmosis through its capsule.

The aberrations which may arise in connection with the invaginating surface epiblast are as follows:

A failure in the downgrowth of the surface epiblastcongenital aphakia. 
Delayed closure of the anterior wall of the lens vesicle - congenital nuclear cataract.

Defective development of the posterior capsule resulting in:

I. Opaque membrane behind the lens.

2. Shrunken fibrous tissue cataract.

3. Posterior lenticonus.

A failure in development of the nucleus from the cells of the posterior layer of the lens vesicle-disc-shaped cataract.

A failure in development of cortical fibres from the proliferating cells of the capsule-congenital Morganian cataract.

Defective extension of the laterally formed lens fibres around the front or back of the nucleus-axial fusiform cataract; backward or forward displacement of the nucleus.

Defects in the line of sutures between the lens fibrestriradiate opacities; coralliform axial cataract.

Abortive development of some of the lens fibres laid on at the sides-congenital punctate cataract.

Congenital Aphakia.- In cases of true anophthalmia, in which the primary optic vesicle fails to bud out, though a hard nodule is sometimes met with in the orbit, composed of the tissues of the eye of mesoblastic origin, no lens or remnant of lens matter has been found in association with them. Apparently congenital absence of the neural epiblastic elements of an eye also implies an absence of the cuticular epiblastic elements.

Many experiments have been performed of recent years on developing frogs, salamanders and minnows, the results of which, though in some respects contradictory, tend to show that the contact of the optic vesicle with the ectoderm stimulates the latter to produce a downgrowth, which results in the formation of the lens. ${ }^{1}$

Complete aphakia has been met with in a human embryo which measured $8.64 \mathrm{~mm}$., and was otherwise well

${ }^{1}$ M. F. Guyer. Internat. Cong. of Ophth., Washington, 1922, 676. 
developed. The optic cup was well formed and in its normal position; the choroidal fissure was still open but its margins were in close apposition. ${ }^{1}$ A case such as this shows that the conversion of the primary optic vesicle into the secondary optic vesicle is an innate capacity in the vesicle itself, and not in any way dependent upon a growth down into the primary vesicle of a portion of the ectoderm.

Microphthalmic eyes have also been described in which, after careful examination, the lens appeared to be completely absent. It would seem improbable, however, that a functional eye could be produced in which no downgrowth of cuticular epiblast took place to form a lens.

Congenital Nuclear Cataract.-Sections of the lens of an embryo fowl, I 50 hours old, have been described ${ }^{2}$ in which the lens vesicle had not separated from the surface epithelium, its anterior wall being still open. Through the gap in the anterior wall, lens fibres derived from the posterior wall protruded and were undergoing degeneration on the surface.

It is suggested that if in this case development had continued, the vesicle would ultimately have separated and closure of the anterior wall have taken place. A nuclear opacity would then have been formed around which clear cortical fibres would subsequently have been laid on.

Defective Development of the Posterior Capsule.The lens capsule is the product of the epithelial cells which line it, as the cells which compose the posterior layer of the lens vesicle soon lengthen out into lens fibres; the posterior capsule for the remainder of life is devoid of any cellular lining, there is therefore only a very short time in which it can be formed. Hence it is not surprising that defects in the development of the central portion of the posterior capsule should occasionally occur, causing it to be either abnormally thin or absent altogether.

${ }^{1}$ I. C. Mann. Brit. J. of Ophth., V, I92 I, 301.

${ }^{2}$ C. Hess. Handbuch der Gesamten Augenheilkunde, VI, 1905, 202. 
Congenital defects in the posterior capsule of the lens may be divided into three classes:

I. Where the gap in the capsule is filled by fibrous tissue, formed in the anterior part of the vitreous (atypically developed vitreous), which gives rise to an opacity at the back of the lens.

2. Where there is considerable extension forwards, through the gap in the capsule, of atypically developed vitreous, giving rise to a shrunken, tough, fibrous cataract.

3. Where there is a protrusion, backwards of the lens substance through the gap in the capsule into the vitreous; or if the capsule is only abnormally thin and not actually deficient, a bulging backwards of the lens substance at the posterior pole. Such protrusions give rise to what is known as posterior lenticonus.

I. Opaque Membrane behind the Lens.-Eyes which have been examined microscopically, in which the first of the above conditions was found, have usually been removed from infants on the suspicion that they contained a glioma of the retina.

A grey reflex was seen at the back of the lens, and sometimes in the tissue which gave rise to the grey reflex bloodvessels were detected, or a hemorrhage was present. At first the lens substance in front of the opaque membrane is clear, but in course of time it may become opaque.

Eyes thus affected are usually smaller than normal. The central hyaloid artery of the vitreous is found to have remained persistent, and sometimes to have continued to carry blood. Tags of pupillary membrane may also be present.

The gap in the capsule of the lens at its posterior pole is filled by tissue, composed of elongated cells and fibres, which is continuous with the vitreous humour (Figs. 26 and 27). It is into this tissue that the central hyaloid artery, when present, passes and breaks up. The lens capsule bounding the gap is usually found to be corrugated and thicker than normal. 
The lens substance, even in cases in which it appeared quite clear before removal of the eye, has shown changes microscopically which cannot be attributed wholly to hardening reagents. In some the nucleus has been displaced backward. Probably in many of the cases the lens would in time have become completely opaque if the eye had not been removed.

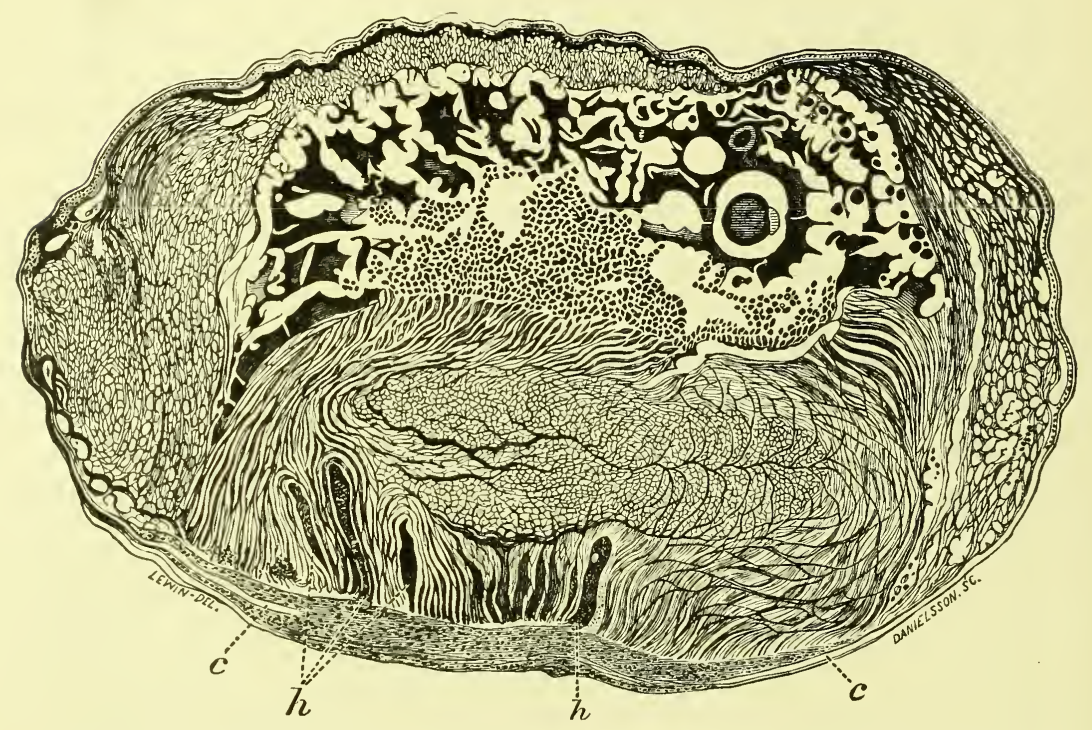

FiG. 26.- Section through the lens from the eye of a child aged 9 months. Clinically a gray reflex was seen from its posterior surface with a hemorrhage apparently in the lens itself. The lens fibres have become much broken up as the result of the hardening reagent. There is a mass of fibrous tissue shown at the posterior surface of the lens filling a gap in the posterior capsule $(c)$. In front of this fibrous tissue in the lens substance are hemorrhages $(h)$ which doubtless came from the central hyaloid artery. Case recorded in R. Lond. Ophth. Hosp. Reps., XIII, 363.

If in a case of this sort, in which the lens has become completely opaque, the operation of discission of the anterior capsule is performed, the lens matter in front of the fibrous membrane will become absorbed by the action of the aqueous humour, but the membrane itself will remain unchanged. Being exceedingly tough in consistency it cannot be torn with a needle, and any attempt to draw it 
out with forceps is almost certain to be followed by a loss of vitreous of which the membrane forms part, and to which it is united. Forcible displacement downwards of the membrane out of the visual axis seems to be attended by the best results.

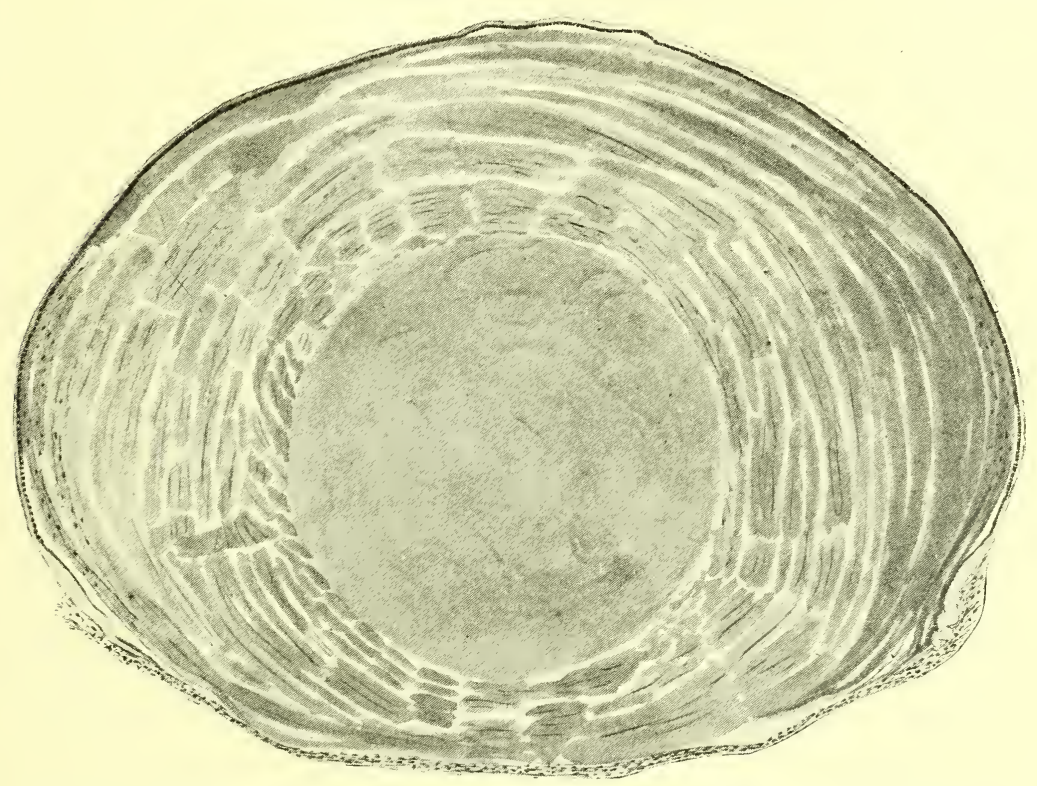

FIG. 27.-Section through the lens from the microphthalmic eye of a child aged Io weeks, which had atypical development of the anterior part of the vitreous, a persistent hyaloid artery, and a congenital gap in the posterior capsule of the lens. Fibro-cellular tissue fills the space where the capsule is absent. The nucleus of the lens is sharply differentiated off from the cortex. Case recorded by J. H. Parsons, Trans. Oph. Soc. of the U. K., XXII, I902, 258.

2. Shrunken Fibrous Tissue Cataract.-This condition resembles the former, but in it instead of the fibrous tissue, or atypically developed vitreous, merely bridging across the gap in the posterior capsule it extends through the gap into the lens. The invasion of the lens by vascular tissue causes it to absorb and shrink.

3. Posterior Lenticonus.-In the large majority of cases of posterior lenticonus in which an ana tomical examina- 
tion has been made ${ }^{1}$ a gap has been found in the posterior capsule. In the few in which its continuity was unbroken it was abnormally thin (Fig. 28). Where the gap existed the lens fibres were either in direct contact with the vitreous humour or separated from it by a connective-tissue membrane, similar to that mentioned in the first class of cases.

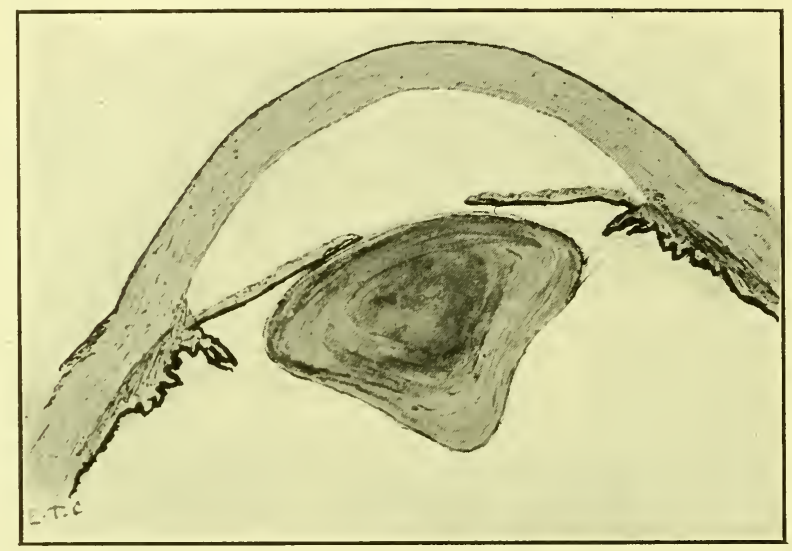

FIG. 28. - Section through the front part of a microphthalmic eye in which there was posterior lenticonus. The capsule is intact throughout, but thinner than normal posteriorly. Case recorded Trans. Ophth. Soc. of the U. K., XXVIII, I908, 107 .

In some of the cases, the only changes in the lens were in the fibres projecting backward into the vitreous, or in those in their immediate neighbourhood; in others there were anterior cortical changes, or the nucleus of the lens was displaced backward.

It has been suggested that the gap in the capsule in these cases is due to rupture, either from increase in size of the lens, or traction produced by a persistent hyaloid artery. There is, however, nothing to account for any increase in the size of the lens, and in a large proportion of them no

${ }^{1}$ A. Patry. Sur 1'histologie et l'etiologie du Lenticone Postèrieur. Genève, I906. 
trace of a hyaloid artery is present. The condition has, moreover, been met with in a chicken embryo I 50 hours old.

It seems more probable that the thinness of the capsule at the posterior pole, or the gap in it, are developmental defects. That on the growth of the lens, the thin capsule has bulged outward, or when deficient, the lens fibres have protruded through the opening into the vitreous.

Disc-shaped Cataract. ${ }^{2}$ - In disc-shaped cataract the lens is much flattened from before backwards due to absence of its nucleus. It is always associated with a dense white anterior polar opacity, which clinically is seen to lie at a deeper level than the surface of the iris at its pupillary margin. This central white opacity is usually surrounded by an irregular greyish area in which it appears to be set. The central opacity may fill the whole of the undilated pupil, and will then resemble a very shrunken opaque lens; when, however, sufficient dilatation of the pupil can be obtained, a perfectly clear marginal area will be discovered. If an iridectomy be performed, so that the edge of the lens becomes visible, it will be found not to present its normal rotundity, but to be abnormally thin from before backwards.

When a discission operation is performed on these cataracts, the central white opaque part can, with a little manœuvering, be separated from the rest of the lens, and made to drop down to the lower part of the anterior chamber. It will remain there lying in front of the iris for an indefinite time, giving rise to no irritation and showing no tendency to become absorbed. After the white central plaque has been picked out a central black opening is left through which the patient can see. Sections of these cataracts examined anatomically show flattening anterio-posteriorly, most marked in the centre, so that two rounded lateral masses are seen connected by a central band, an appearance suggesting

${ }^{1}$ Hess. "Handbuch der gesamten Augenheilk." VI, 201.

${ }^{2}$ Treacher Collins. Trans. Ophth. Soc. of the U. K., XVIII, I898, I24, and The Ophthalmoscope, VI, 1908, 669. 
that of a dumb-bell (Fig. 29). In the central flattest part of the lens, a laminated mass of tissue is situated similar to that met with in anterior polar cataracts. It extends backward from the anterior to the posterior capsule, no lens substance intervening. In the rounded parts on either side of the central laminated mass, there is lens substance, showing a variable amount of disturbance in the vicinity of the central part, and becoming more normal and regular in its arrangement toward the periphery.

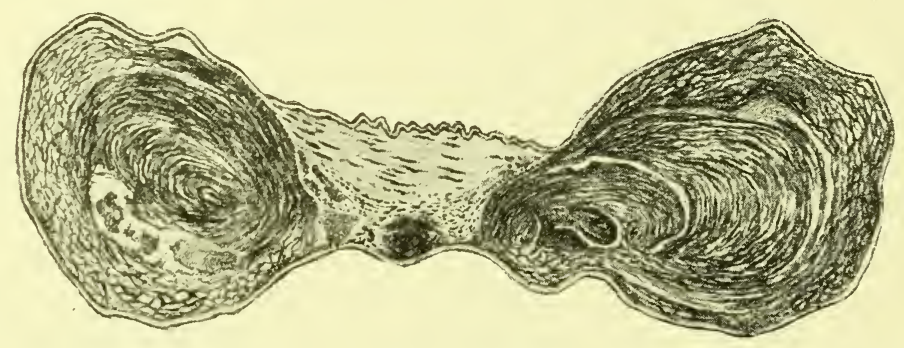

FIG. 29.-Antero-posterior section through a congenitally disc-shaped cataract. The nucleus of the lens has failed to develop and there is a dense laminated mass at the anterior pole.

The explanation of the condition seems to be a failure in the development of the nucleus; i.e., a failure of the cells which compose the posterior layer of the lens vesicle to lengthen out into lens fibres. This failure in the development of the nucleus does not seem to check the activity of the cells lining the anterior and lateral parts of the lens capsule. The laminated mass in the centre, which resembles in structure an anterior polar cataract, is probably produced in the same way as anterior polar cataracts in other conditions, i.e., by the proliferation of the cells lining the anterior capsule as the outcome of low intracapsular tension in their vicinity (see page 602). In these lenses, in which the nucleus fails to form, there would be abnormally low intracapsular pressure in the neighbourhood of the anterior pole of the lens, hence the formation of an anterior polar cataract. 
The activity of the cells lining the sides of the capsule not being interfered with, lateral lens fibres develop. These having no nucleus around which to group themselves form accumulations of fibres at the sides of the anterior polar mass, with irregularities and vacuolations of varying distribution.

Congenital Morganian Cataract.-This is a complete cataract which presents a characteristic uniform milky

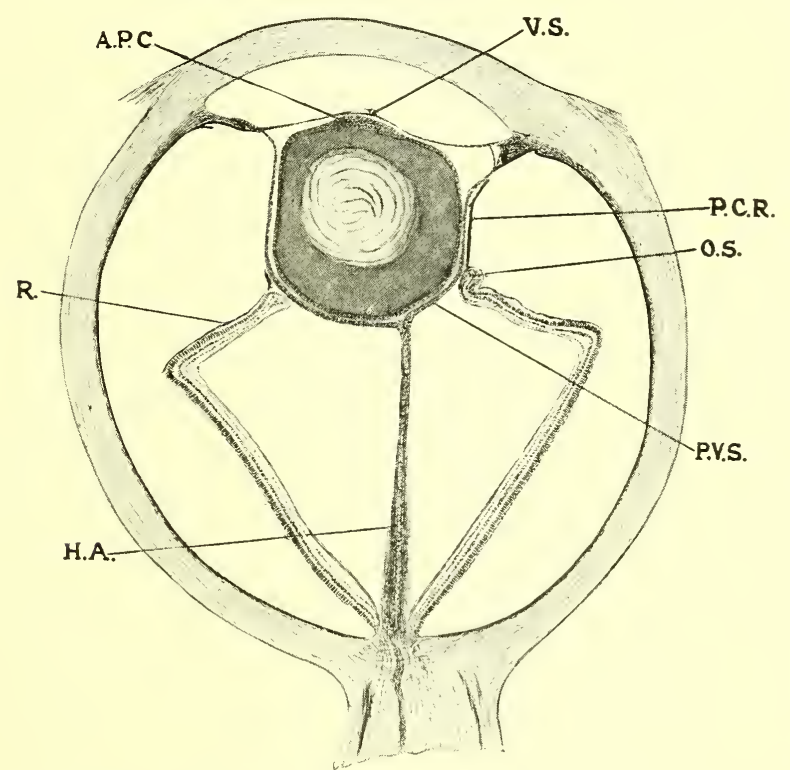

FIG. 30.-Diagrammatic section through a microphthalmic eye in which the whole fetal vascular system of the lens had persisted; the central hyaloid artery of the vitreous H.A.; and fibro-vascular sheath, P.V.S. and V.S. There was arrested development of the iris, "partial aniridia;" failure in separation of the pars ciliaris retinæ P.C.R., and retina $R$. at the ora serrata, O.S. from the sides of the lens; and a congenital Morganian cataract, with an anterior polar opacity A.P.O.

white colour, sometimes with a few dense white specks on the surface. When the capsule is pricked with a needle opaque fluid, containing flocculent material, escapes into the anterior chamber. If left there and not evacuated, increased tension often ensues. 
On anatomical examination of such cataracts the cortex is found almost entirely composed of a homogeneous material (Fig. 30), while the nucleus consists of lens fibres concentrically arranged, sometimes with vacuoles in them like those seen in the affected area in lamellar cataract. ${ }^{1}$

In the disc-shaped cataract some dystrophic influence, exceedingly early in fetal life, arrests the development of the lens fibres which should form from the cells of posterior layer of the lens vesicle, but does not interfere with the later development of the fibres from the cells at the sides of the capsule. In congenital Morganian cataract the lens fibres derived from the cells forming the posterior layer of the vesicle develop in a normal, or comparatively normal, manner; and the occurrence of some dystrophic influence later, interferes with the development of the laterally-formed lens fibres from the cells at the sides of the capsule. The one condition therefore appears to be the reverse of the other.

\section{Axial Fusiform Cataract. Backward or Forward Dis-} placement of the Nucleus of the Lens.-Under the name congenital axial fusiform cataract a condition has been described in which a spindle shaped opacity extends from the anterior to the posterior pole of the lens. It has been suggested that it is due to adhesion of the nucleus to the anterior and posterior capsule, so that on growth and expansion of the lens, it becomes drawn out into a fusiform shape.

A backward displacement of the nucleus of the lens is frequently met with where there has been a defect in development of the posterior capsule. It has been several times recorded in specimens with posterior lenticonus. It can be recognised clinically when the nucleus is opaque and the cortex clear. Adhesion of the nucleus to the anterior capsule has also been described.

The lens fibres which are developed laterally, in order to encircle those which are formed from the posterior layer

${ }^{1}$ Treacher Collins. The Ophthalmoscope, VI, 1908, 67I. 
of the lens vesicle, have to lengthen out anteriorly and posteriorly. Some extend more forward and others more backward. Adhesions of the nucleus to the anterior or posterior capsule would prevent the forward or backward growth of the fibres.

An adhesion to the anterior capsule may be due to defective closure of the anterior surface of the lens vesicle. An adhesion posteriorly to defect of development in the posterior capsule.

Triradiate Opacities. Coralliform Axial Cataract.-At the anterior and posterior poles of the lens, where its fibres meet, lines of suture are formed, varying in their arrangement at different stages of its development, and at different depths in the fully formed lens.

The lens when first formed is globular in shape, and the fibres composing it all meet at a point, anteriorly and posteriorly. As new fibres become laid on at the sides the lens alters in shape, its lateral diameters increasing more than its antero-posterior, then, instead of the lens fibres meeting at a point they meet along the two sides of a line; the direction of the line of suture at the anterior pole being at right angles to that at the posterior. This allows of some shortening of the lens fibres, those which extend furthest anteriorly not having to extend so far posteriorly, and vice versa. To effect the same purpose, as the lens becomes larger, the lines of suture between the lens fibres assume a triradiate arrangement, the three arms of the triradiate figure at one pole being situated in the reverse direction to those at the other. This is the arrangement met with on the surface of the lens at birth. After birth the lines of suture assume a still more complicated pattern, a star-shaped figure with primary and secondary radiations being produced; a fibre starting from the tip of a radiation on one surface, extending right into the centre of the star on the other.

With slit lamp-microscopy, the triradiate suture can be detected at the anterior surface of what corresponds to 
the embryonic lens in the form of a vertical $\mathrm{Y}$, and at its posterior surface in the form of an inverted $\mathrm{Y}$, even at an advanced age. By this method of examination the lens is also seen to present concentric areas of increased reflection, due to slightly changed physical characters in its different strata. These have been termed "optical laminated zones;" they afford assistance in determining the date at which pathological changes met with in the lens occurred. For instance if the changes are situated within the zones in which the triradiate figures are situated the inference is that they were congenital, whereas, if they are outside those zones they must necessarily have been produced since birth.

Congenital opacities are sometimes met with situated along the lines of sutures between the lens fibres. Triradiate opacities may form either in front or behind the nucleus; they are sometimes seen on the anterior surface of a zonular cataract. They are met with behind the nucleus in the hereditary disc shaped opacities, termed "Doyne's cataract;" a form of cataract which must not be confused with "discoid cataract," which has been already described, and in which the whole lens has the shape of a disc. In Doyne's cataract it is the area of opacity which is shaped like a disc the lens being of normal dimensions.

At birth these triradiate opacities appear to be situated close to the poles of the lens, as age advances, unaffected lens fibres are laid on in front of them, and the opacities then appear to be some distance away from the surface.

In the form of axial cataract which is termed coralliform cataract $^{1}$ opacities having a tube-like appearance are met with radiating forward and outward from the center. They terminate just short of the capsule in an ampulliform manner. So far no anatomical examination has been made of this form of cataract. The position occupied by the opacities is that of the lines of the sutures in the antro-posterior

${ }^{1}$ R. Marcus Gunn. Trans. Ophth. Soc. of the U. K., XV, I 895, II 9. 
plane and it is probable that they are due to some disturbance situated in them.

Congenital Punctate Cataract.-In congenital punctate cataract small, circumscribed, grey or white opacities are seen in the cortical layers of the lens. They vary in number in different cases and may be exceedingly numerous. They usually remain stationary, showing no tendency to increase

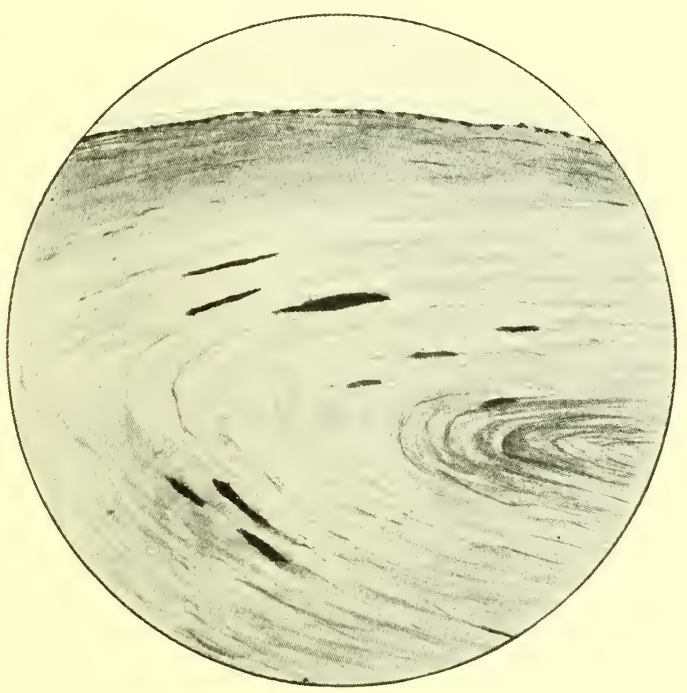

FIG. 3I.-Section through a lens with congenital punctate cataract showing spindle-shaped spaces between the lens fibres filled with deeply staining granular material.

either in number or size, and due to their peripheral distribution rarely cause any disturbance in vision. Both eyes are generally involved and frequently several members of the same childship.

Microscopically, spindle-shaped spaces are found between the lens fibres, filled with a granular material, which stains more deeply than the surrounding parts with nuclear stains (Fig. 3I).

The position and distribution of these opacities suggest, that they represent laterally formed lens fibres which have 
broken down into a granular substance, instead of developing in the normal way, and which have subsequently become surrounded by healthy lens fibres.

\section{ABERRATIONS CONNECTED WITH THE INVAGINATING MESOBLAST}

From the mesoblast which grows in to the primary optic vesicle from below are developed the central blood-vessels of the optic nerve, the blood-vessels of the retina and the central hyaloid artery.

There has been much difference of opinion as to the mode of development of the vitreous humour. Formerly it was thought that it came from the invaginating mesoblast, more recent observations have tended to show that it is mainly epiblastic in origin, and derived from cells in the region of the pars ciliaris retinæ. The firm adhesion of the vitreous to the pars ciliaris retinæ in the fully developed eye, and its freedom from attachment to the retina elsewhere, is explained by this view of its origin.

The tissue formed from the inner layer of the secondary optic vesicle, immediately in front of the ora serrata, is termed the primitive vitreous, its further growth is due to vascularisation by vessels developed from the invaginating mesoblast.

Blood-vessels have been detected in the invaginating mesoblast in sections of a human fetal eye of the sixth week. At first they lie behind the primitive vitreous, between it and the inner layer of the secondary optic vesicle. Many of them remain in this position becoming incorporated in the nerve fibre layer of the retina as it develops, and ultimately forming the vascular system of that structure. One of the arteries grows up from below into a depression in the primitive vitreous, which corresponds to the ocular cleft. On closure of the cleft it is encircled and becomes the central hyaloid artery. The hindermost part of the invaginating mesoblast grows up into what subsequently becomes the optic nerve, and forms its central blood-vessels. 
The central hyaloid artery in its fully formed condition arises from the central retinal artery on the optic disc, and as it passes forwards divides dichotomously. The branches thus formed spread out on the posterior surface of the lens anterior to the vitreous in what is termed the posterior fibro-vascular sheath. From there they extend round the sides of the lens, anastomosing, when they reach its anterior surface, with branches of the anterior ciliary vessels.

These branches of the anterior ciliary vessels extend inwards in the form of loops on the anterior surface of the lens, in what is termed the lamina irido-pupillaris which forms the anterior part of the fibro-vascular sheath of the lens.

All the blood from the plexus of vessels around the lens is carried into the anterior ciliary veins, there being no central hyaloid vein running with the artery.

The central hyaloid artery lies in a canal in the vitreous lined by a hyaline membrane, the canal of Cloquet. The posterior part of the artery is also encircled by a cellular sheath (Fig. 32), as it passes forwards the cellular sheath ceases, and the artery then lies in the hyaline canal devoid of any covering (Fig. 33). As the artery divides up, on approaching the back of the lens, the hyaline canal broadens out in a funnel-shaped fashion (Fig. 34).

The vascular supply for the vitreous and lens reaches its fullest development at the seventh month of fetal life, after that the vessels gradually become obliterated. With slit lamp-microscopy of the living eye, ${ }^{1}$ remnants of the central hyaloid artery can be detected in the eyes of most persons, varying much in form and extent. They are seen most distinctly when the micro arc-slit lamp is employed.

The point of attachment of the artery to the back of the lens is found not to be immediately at its posterior pole, but a little to its nasal side. This is due to its passing straight forward through the vitreous from the optic

1 A. Vogt. Atlas. 192 I, 128. 
nerve, which enters the eye to the nasal side of the mesial line. In some people the point of attachment of the central hyaloid artery to the back of the lens is seen as a button like thickening, and in others as a plexus of lines, remnants of the branches into which it divides up. Proceeding backwards from the point of attachment a floating corrugated filament can sometimes be detected.

The anterior termination of the hyaline canal, in which the branches of the central hyaloid artery lie when they reach the back of the lens, shows up in the eyes of some people as a curved line or a complete circle, which emits an asbestos like radiance.

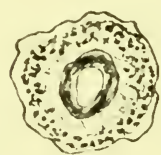

FIG. 32 .

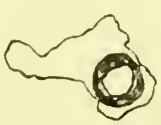

FIG. 33.

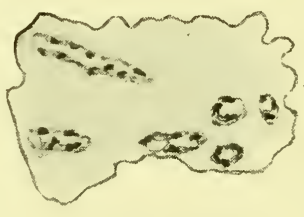

FIG. 34 .

FIG. 32.- Transverse section of the central artery of the vitreous, showing the cellular sheath and hyaloid membrane surrounding it. From a human fetal eye of between the 5 th and 6 th months.

FIG. 33. - Transverse section of the same artery shown in Fig. 3 I further forward in the vitreous. The cellular sheath has ceased, but the artery is still surrounded by a hyaloid membrane.

FIG. 34.- Transverse section of the same artery shown in Figs. 32 and 33 further forward in the vitreous, after it has divided up. The canal bounded by the hyaloid membrane has enlarged.

The supporting structure of the normal vitreous, viewed with the micro arc-slit lamp, presents many variations, and the distinctness with which it may be seen also varies in different individuals; generally there are layers of membrane presenting a fibrillar structure. This framework as a rule does not extend anteriorly to the lens surface, between it and the lens there is a relatively "optically empty" space, which probably represents that in which the vessels of the posterior vascular sheath lie in fetal life, and which after birth remains filled with tissue fluid. The vitreous is separated from this retro-lental space by what appears to be a characteristically folded membrane. 
The external hyaloid membrane of the vitreous can be detected as early as the tenth week in fetal life with nucleated cells in it widely spaced apart.

During part of fetal life the whole vitreous is permeated by a close network of delicate fibres with tiny granules lying on them, for the most part at the points where the fibres intersect one another. These granules, which are about one-eighth the size of the nucleus of a cell in the sheath of the hyaloid artery, stain deeply with hematoxylin. As the vitreous increases in size these granules and fibres stain more faintly, become more spaced out, and far less conspicuous.

The ciliary body when it commences to develop lies in contact with the side of the lens, while so situated cellular adhesions form between it and the lens capsule, which subsequently develop into the fibres of the suspensory ligament of the lens.

As the eyeball grows it does so at a greater rate than the lens; so that the ciliary body, at first in contact with the lens, gradually becomes separated further and further from it. The adhesions between the two structures, originally cellular, become stretched out into fibres, to which for some time the nuclei adhere and then gradually disappear.

As in the case of the vitreous humour, it was at one time thought that the fibres of the suspensory ligament were mesoblastic in origin, being derived from the cells of the fibro-vascular sheath. Now, however, it seems more probable that both the suspensory ligament and the vitreous humour are epiblastic, being derived from the inner layer of the secondary optic vesicle in front of the ora serrata. The fibres of the suspensory ligament are analogous with the radial fibres of Müller in the retina, and like them are developed from columnar epithelial cells, the cell substance of which becomes converted into neuro-keratin. The delicate network which permeates the vitreous during fetal life, may likewise be regarded as analogous with the neurokeratinal basketwork which forms the supporting frame- 
work for the nerve elements of the retina; and its hyaline membrane with the external and internal retinal limiting membranes.

Though the suspensory ligament of the lens and the vitreous humour are probably epiblastic in origin, during the whole period of their growth and development they are nourished by the vascular system formed from the invaginating mesoblast.

The aberrations which may arise in connection with the invaginating mesoblast are as follows:

Failure in the disappearance of the fetal vascular system of the vitreous-persistent central hyaloid artery-persistent sheath of central hyaloid artery.

Defective or irregular formation of the retinal bloodvessels-abnormalities in the arteria centralis retinæcilio-retinal arteries.

Atypical development around the fetal vascular systemcongenital connective tissue formation in the vitreous chamber.

Persistence of portions of the framework of the fetal vitreous-muscæ volitantes.

Imperfect closure of the cleft in the vitreous into which the mesoblast extends - coloboma of the vitreous humour.

Failure in the formation of adhesions between the ciliary processes and the lens capsule in a portion of its circumference-ectopia lentis, coloboma lentis, lenticonus anterior.

Persistence in the cellular character of a portion of the developing suspensory ligament-adhesion of ciliary processes to the back of the lens.

Persistence of the Central Hyaloid Artery or Its Sheath.

In all vertebrates except mammals a permanent vascular system is met with in the vitreous. It presents many variations in different species. There may be either a plexus of vessels in the centre of the vitreous such as the pecten in birds and the processus falciformis in fish and reptiles; or 
a network of vessels spread out on its surface lying in contact with the retina.

In many mammals a vestigial pecten or remains of a central hyaloid artery is present. It is met with in all ruminants and in a large number of rodents and marsupials. ${ }^{1}$

The different conditions which may arise from the abnormal persistence of the central hyaloid artery, or its sheath, in the human eye, may be summarized as follows:

I. The central artery persisting throughout its whole length and continuing to carry blood.

When such an abnormality is present there is a formation of fibrous tissue in the vitreous chamber (Figs. 35 and 36 ).

2. The persistence of the central artery throughout its whole length as a slender cord without any blood circulating through it.

Such a cord is usually thinnest in the centre, expanding where it is attached to the optic disc and to the back of the lens. Its anterior extremity is generally placed a little below the centre and to the nasal side of the posterior surface of the lens. In a few rare cases instead of starting from a vessel on the optic disc it has sprung from one in the lower part of the fundus. In one recorded case it was found to start in the centre of a coloboma of the choroid situated at the macula.

Microscopically these cords are composed of rather dense fibrous tissue lying in a persistent canal of Cloquet.

3. The persistence of a cord, representing the posterior part of the central artery, attached to the optic disc but ending in a free extremity in the vitreous (Fig. 37), with or without an opacity on the posterior surface of the lens.

The length of the cord varies considerably in different cases. It may be of a dark colour and easily seen, or light grey and semi-transparent when the recognition of its presence calls for careful observation. The free extremity of the cord is sometimes rounded and knob-like, at others

${ }^{1}$ Lindsay Johnson. Philosoph. Trans. of the Royal Society of London, I901, 53 . 
fine and tapering. It may bifurcate once or oftener. Ophthalmoscopically the cord, on movements of the eye, often oscillates with an undulating motion, and might easily be mistaken for a filaria in the vitreous. The opacity at the posterior surface of the lens, when present, may be flat and have a tag protruding back from it with a free extremity in the vitreous.

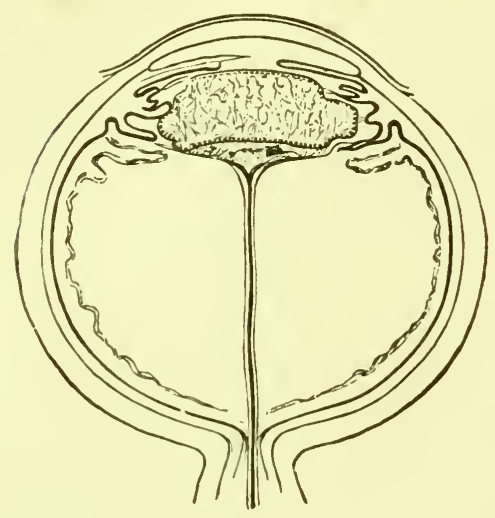

FIG. 35.

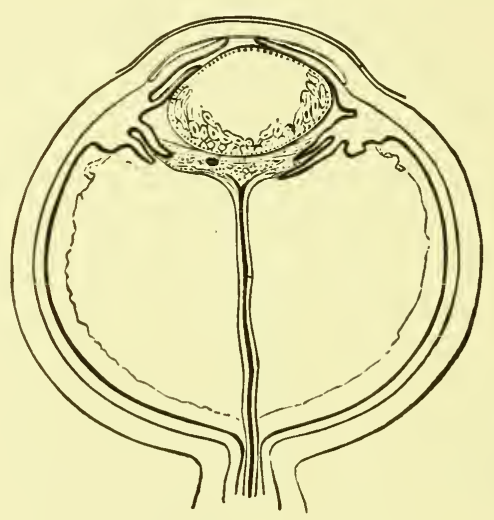

FIG. 36 .

FIG. 35.-Diagrammatic representation of a section of an eye with a persistent and patent hyaloid artery which terminated in a thick fibrous tissue membrane at the back of the lens.

FIG. 36.-Diagrammatic representation of a section of an eye presenting much the same type of malformation as in Fig. 35. The ciliary processes nearly all lie behind the lens, being attached to the mass of fibrous tissue in the anterior part of the vitreous in which the central hyaloid artery terminates.

4. The persistence only of the anterior extremity of the artery in the form of an opaque patch at or near the posterior pole of the lens. As in the last class the opaque patch may have a free and mobile tag attached to it.

5. The persistence of the cellular sheath which surrounds the posterior part of the artery inside the canal of Cloquet; the artery itself having entirely disappeared.

This persistent sheath is seen ophthalmoscopically as a grey membrane filling up the depression in the head of the nerve, or covering over one or more of the retinal vessels on the optic disc. 
Little rounded bodies of steel grey colour, presenting a cyst-like appearance, are sometimes seen ophthalmoscopically in front of and attached to the optic disc. They are probably cystic distentions of the sheath of the artery.

Abnormalities in the Arteria Centralis Retinæ. Cilioretinal Vessels. - It is only in mammals that blood-vessels are present in the retina and in the different species there are striking variations in their arrangement.

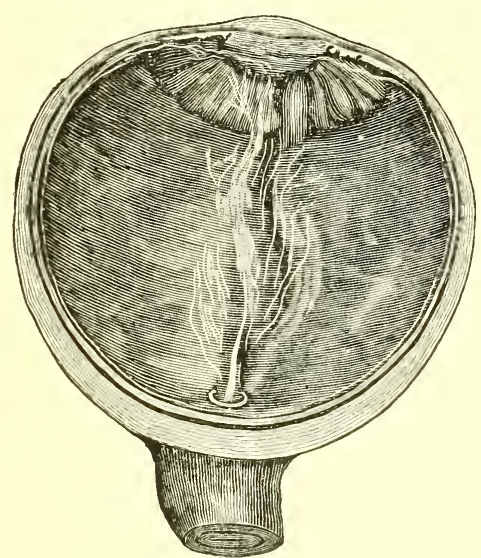

FIG. 37.- An eyeball the sight of which had been destroyed by ulceration of the cornea. Prolonged forward into the vitreous from the optic nerve is a tag of white membrane, which is due to a persistence of the central hyaloid artery.

In some mammals the retina is devoid of blood-vessels. In some they come entirely from the ciliary blood-vessels in the sclerotic, and gain access to the retina at the margin of the optic disc, around which they curl. In others there is a mixed supply of blood-vessels, some coming, as just mentioned, from the ciliary vessels, and some from the central retinal vessels.

In all primates the chief supply is the central retinal artery. In all the Leporidæ ${ }^{1}$ what are called the retinal vessels are internal to the membrana limitans interna.

${ }^{1}$ Lindsay Johnson. Philosoph. Trans. of the Royal Society of London, I 901,42 . 
The congenital abnormalities which are met with in the retinal blood-vessels in man are comparable, in many instances, with the different arrangements met with in the mammalia.

I. A case has been recorded ${ }^{1}$ of a boy who was born blind and had a complete absence of all retinal blood-vessels.

2. As already mentioned in some cases of coloboma involving the optic disc, the whole of the retinal blood supply has come from vessels which emerged from the margin of the optic disc at different portions of its circumference (cilio-retinal vessels). A similar abnormal blood supply to the retina has also been met with when no coloboma was present.

3. A mixed blood supply to the retina from the central vessels and one or more cilio-retinal vessels is by no means infrequent.

Cilio-retinal vessels have been estimated as present ${ }^{2}$ in 17 per cent. of a series of cases examined ophthalmoscopically. They are recognised by emerging from the margin of the optic disc with a sharp bend, away from the central vessels, in the direction of the sclerotic. Cilio-retinal veins are less common than cilio-retinal arteries. The connection of these vessels with the ciliary vessels in the sclerotic has been demonstrated ${ }^{3}$ anatomically.

Retinal vessels are sometimes found to arch inwards for a portion of their extent towards the vitreous. Whether or not they penetrate the hyaloid membrane, and pass actually into the vitreous humour, has not been determined. Their abnormal position is due to the external vessels of the vitreous humour in the fetus not becoming incorporated in the nerve-fibre layer of the retina.

Congenital Connective Tissue Formation in the Vitreous Chamber.-The vascular supply to the developing vitreous humour and lens normally ceases about six weeks before

\footnotetext{
1 V. Graefe. Arch. f. Ophth., I, 1854, I.

${ }^{2}$ Lang and Barrett. R. Lond. Ophth. Hosp. Reps., XIi, I 888, 59.

3 E. Nettleship. R. Lond. Ophth. Hosp. Reps., IX, I877, I6I.
} 
birth. Should its continuance be unduly prolonged a development of fibrous tissue, in the course of the bloodvessels, is liable to take place.

This abnormally developed fibrous tissue may be situated either in the vitreous chamber, about the capsule of the lens, or in the anterior chamber on the surface of the iris, and sometimes in more than one of these situations in the same eye. In the vitreous chamber it may form a thick membrane at the posterior surface of the lens, a band or cord along the track of the central hyaloid artery, or a spur-like prominence projecting upwards in the region of the fetal ocular cleft.

When there is a congenital development of fibrous tissue at the back of the lens, the central hyaloid artery passes into the mass and divides up in it (Figs. 35 and 36 ), this condition, as already mentioned, is frequently associated with a defect in development of the posterior capsule of the lens, and seems to be a sort of compensatory change to make good the defect. If the lens is clear, the symptoms to which this condition gives rise closely resemble those of a glioma of the retina; several eyes in which it was present have been excised under the supposition that they contained such a growth.

In a new-born child there is seen immediately behind the lens the glistening grey reflex to which the fibrous tissue gives rise, and the branches of the central hyaloid artery, which course through it, resemble very closely the branches of the retinal vessels seen on the surface of a gliomatous growth.

The way in which the blood in the central hyaloid artery escapes from the eye in these cases cannot always be definitely determined. There is no central vein running with the artery. The exit of blood by the anterior ciliary veins, which takes place in the fetal state, though present in some cases, cannot always be traced.

Congenital fibrous tissue formation in the region of the ocular cleft can sometimes be seen ophthalmoscopically 
as a grey mass projecting up into the vitreous chamber. Pathologically such fibrous tissue has been found continuous with the sclerotic through the ocular cleft, the adhesion of the two structures having prevented its closure. Often white cords stretch between the optic disc and the spur of fibrous tissue, each of which contains a persistent hyaloid artery.

Cases have been described in which a fibrous membrane behind the lens has extended forwards round its lower

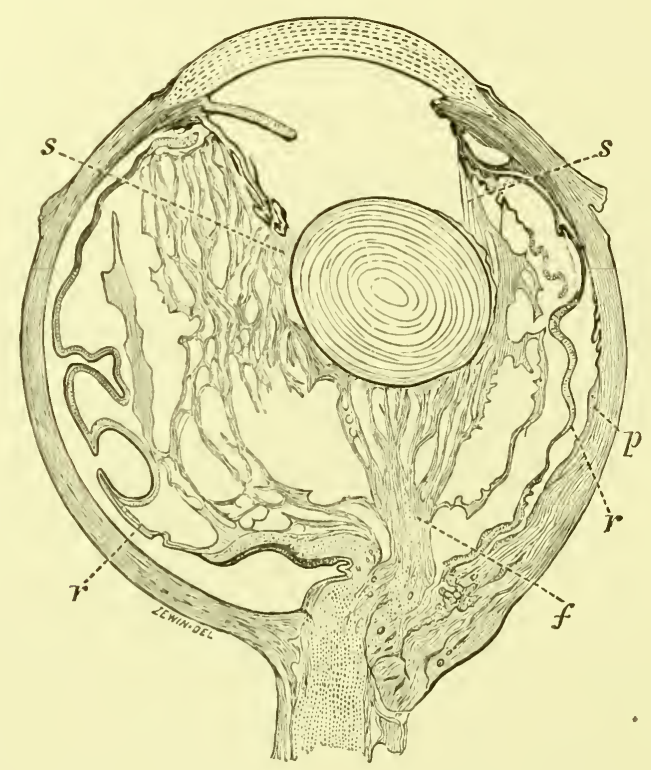

FIG. 38.-Semi-diagrammatic section of a microphthalmic eye with a coloboma of iris and choroid and a band of fibrous tissue in the vitreous, stretching from the posterior pole below the optic disc to back of the lens, which latter has been held back by it, the anterior parts of the eye having grown forwards, the ciliary processes being much elongated. $s$, Suspensory ligament; $r$, retina; $f$, fibrous tissue in vitreous; $p$, coloboma of choroid.

border and become adherent to the back of the cornea, just in front of the ciliary body. Its union to the cornea checks the forward growth of the iris, and the formation of the fibres of the suspensory ligament, so that colobomata of those structures are formed. 
Eyes which have this congenital formation of fibrous tissue in the vitreous chamber frequently do not expand to their normal dimensions, and are microphthalmic. The fibrous tissue is often tough and inelastic, so that on growth and expansion of the globe, the structures to which it is attached may become distorted or displaced. The ciliary processes, if attached to a membrane behind the lens, become stretched out and attenuated. A fold of the retina in the region of the ora serrata may become drawn

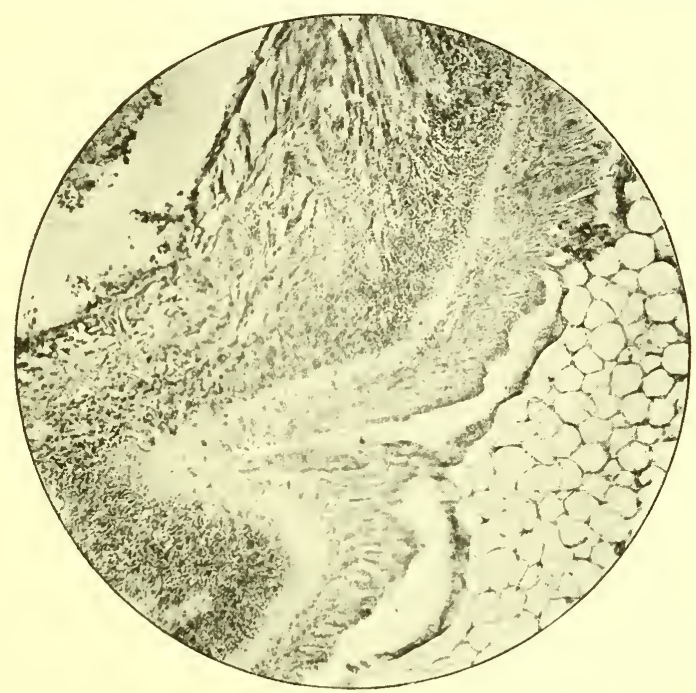

FIG. 39.-A portion of the retina and atypically developed vitreous from a microphthalmic eye. The atypically developed vitreous shown on the righthand side of the figure is transformed into tissue resembling adipose or myxomatous tissue.

forward by an adhesion to the membrane. A band adherent to the back of the lens may fix it to the walls of the globe, so that on expansion of the latter, it becomes held backward and altered in shape ${ }^{1}$ (Fig. 38).

Bands of this non-expansile fibrous tissue may, by the growth of the eyeball, lead to separation of the retina from the pigment epithelial layer, i.e., separation of the two layers of the secondary optic vesicle. Sometimes the

${ }^{1}$ Treacher Collins. Trans. Ophth. Soc. of the U. K., XIII, I893, II7. 
separation extends into the region of the ciliary body, the non-pigmented layer of the pars ciliaris retinæ being drawn away from the pigmented layer (Fig. 30).

Occasionally this abnormally developed fibrous tissue, becomes in part replaced by tissue resembling adipose or myxomatous tissue (Fig. 39). ${ }^{1}$

For a description of congenital connective tissue formation in the anterior chamber, in front of the iris, see page 95.

Muscæ Volitantes.-Most people can perceive entoptically when looking at a white surface, opacities which slowly

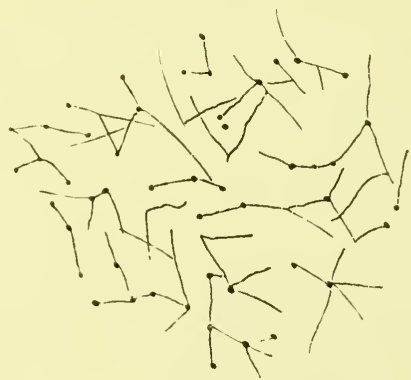

FIG. 40.-Branching fibres with granules on them from the vitrous humour of a normal human fetal eye. $\times 300$.

float in front of them. They present various shapes, usually resembling threads or portions of a net with beads on them.

These so-called "musca volitantes" are due to opacities in the vitreous humour and must be regarded as a physiological peculiarity. They consist of portions of the network of fibres and granules which, as already mentioned, extends throughout the developing vitreous in the fetal eye (Fig. 40).

Coloboma of the Vitreous Humour.-Failure in the closure of the cleft in the vitreous humour, into which the mesoblastic tissue grows which develops into central hyaloid artery, leads to a coloboma of the vitreous. It is a very rare form of abnormality, and when it occurs, is

${ }^{1}$ M. S. Mayou. Trans. Ophth. Soc. of the U. K., XXIV, r904, 355. 


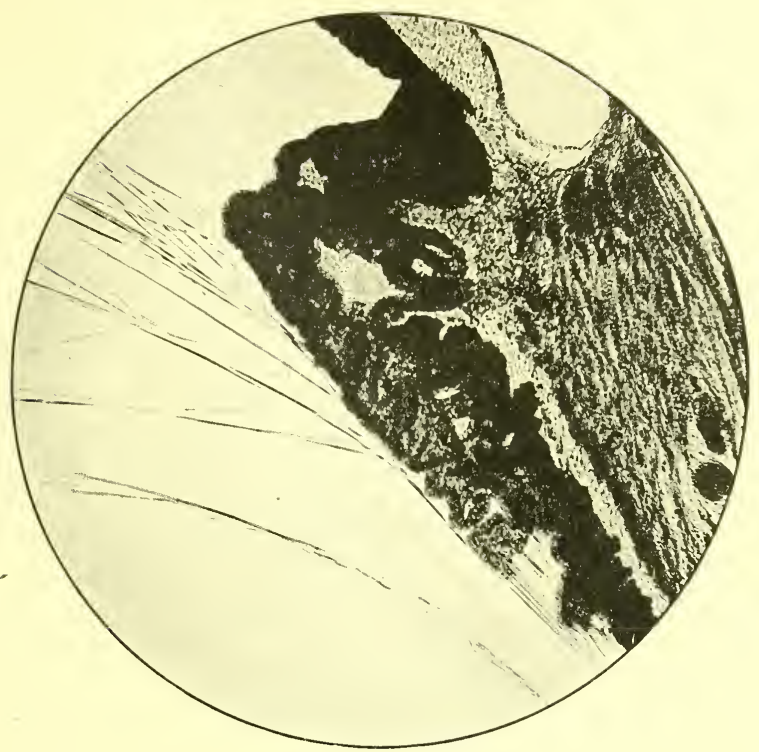

FIG .4I.

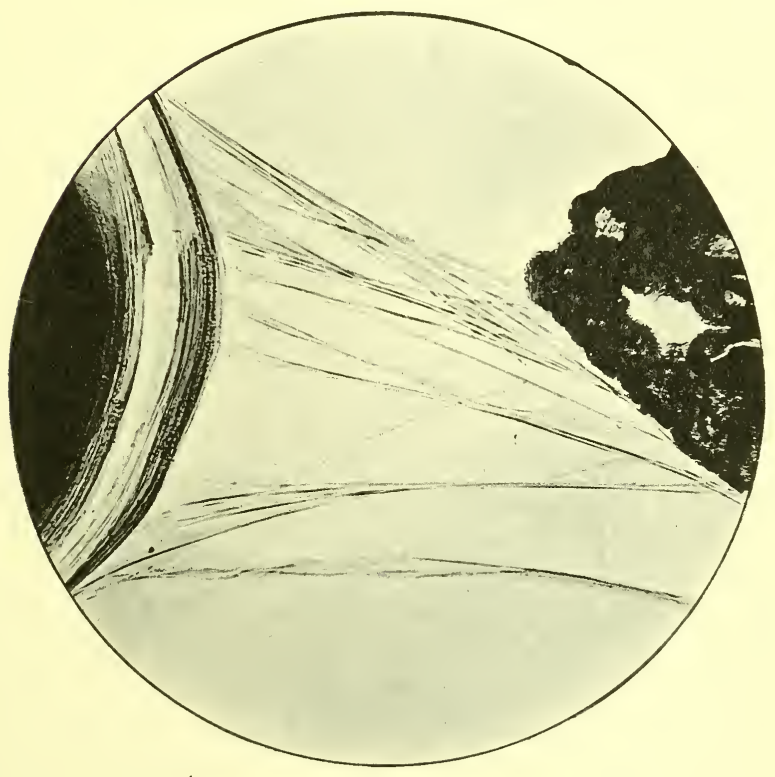

FIG. 42 .

FIgs. 4I, 42.- Show the suspensory ligament of the lens in sections of a normal human eye. The foremost fibres shown in Fig. 42 are the orbiculo-anterocapsular fibres, the prolongation of which backwards to the non-plicated part of the ciliary body is shown in Fig. 4I. The hindermost fibres shown in both figures are the orbiculo-posterio-capsular fibres. The cilio-equatorial fibres can be seen in Fig. 42 crossing the orbiculo-antero-capsular fibres, and passing direct from between the ciliary processes to the equator of the lens. The cilio-posteriocapsular fibres are shown in Fig. 42, and at their commencement in Fig. 4I. They cross the cilio-equatorial and orbiculo-antero-capsular fibres, and pass to the back of the lens. From photomicrographs by E. Collier Green. 
usually associated with some development of fibrous tissue around the hyaloid vessels. This, as already mentioned, may be adherent to the sclerotic, and project upwards into the interior of the eye in the form of a spur. Such a formation of fibrous tissue in the region of the ocular cleft prevents its closure, not only in the vitreous humour, but also in the secondary optic vesicle.

\section{Congenital Defects in the Suspensory Ligament of the} Lens.-Defect in the development of the suspensory ligament of the lens may result in absence of some of its fibres, or in a persistence in the cellular character of the adhesions between the ciliary body and the lens capsule from which its fibres are formed. ${ }^{1}$

The absence of a portion of the suspensory ligament is most frequently met with in the lower part of its circumference; i.e., in the position in which a coloboma of the iris and ciliary body usually occurs. The probable explanation of its failure in development in such cases is, that imperfect or delayed closure of the fetal ocular cleft prevented the ciliary body from coming in contact with the side of the lens at the time when the adhesions between those structures are usually formed. There must, however, be other causes for the defect, as it is occasionally met with in eyes in which there is no sign of any coloboma of the uveal tract.

Defects in development of the suspensory ligament may consist of either a complete gap, involving all its fibres over a certain area in its circumference, or a partial deficiency in which only a certain set of its fibres have failed to form. The orbiculo-antero-capsular fibres, which pass from the non-plicated part of the ciliary body to the anterior capsule of the lens, may be absent, whilst the cilio-posterio-capsular, passing from the ciliary processes to the posterior capsule, and the orbiculo-posterio-capsular, passing from the nonplicated part of the ciliary body to the posterior capsule, are present (Figs. $4 \mathrm{I}$ and 42 ).

${ }^{1}$ Treacher Collins. Royal London Ophth. Hosp. Reps., XIII, I890, 8I. 
Absence of a portion of the suspensory ligament produces changes in either the shape or position of the lens, malformations which are termed, congenital ectopia lentis, coloboma lentis and lenticonus anterior.

When the suspensory ligament retains its cellular character abnormal union of the ciliary processes to the lens capsule takes place, which causes them to become attenuated and displaced.

Ectopia Lentis.- Lateral displacement of the lens from a congenital defect in the suspensory ligament, must be differentiated from backward displacement due to fibrous tissue formation in the vitreous chamber, which has already been referred to.

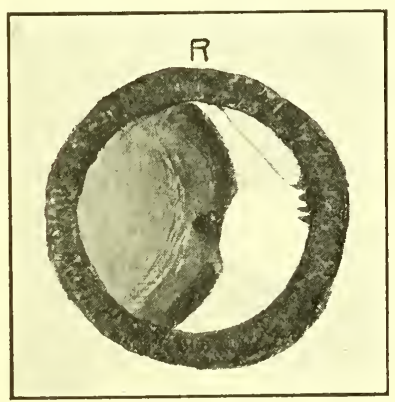

FIG. 43.-Congenital coloboma and displacement of the lens. Opposite the coloboma is a complete gap in the suspensory ligament. There are some persistent remnants of the pupillary membrane.

It is a rare malformation, but markedly hereditary, usually bilateral, the direction in which the lenses are displaced being symmetrical.

In the large majority of cases the lens is displaced upward with a slight inclination either inward or outward. It may be displaced horizontally (Fig. 43), or downward with an inclination outward or inward, but never directly downward.

Very frequently there is myopia, and monocular diplopia may be complained of. 
The iris is tremulous, and the anterior chamber generally deeper on the side the lens is absent than elsewhere. On oblique illumination with a dilated pupil, and sometimes without, the curved edge of the lens can be seen, the lens appearing grey and the aphakic area outside its margin black. By reflected light the curved edge of the lens is seen as a dark line in the area of red reflex. In some of the cases, due to the prismatic action of the edge of the displaced lens, a reduplicated picture of the optic disc can be seen ophthalmoscopically.

The lens often appears smaller and more globular than normal; though usually quite clear it may have opacities in it.

Varying conditions of the suspensory ligament in the aphakic area have been met with clinically. In some cases it was entirely absent and the lens mobile; in others there were only a few fibres present or gaps in it were visible, (Fig. 43), in a few cases no break in its continuity could be detected.

Congenital displacement of the lens is brought about by unequal traction on its sides as the ciliary body grows away from contact with it. This unequal traction may be due to:

I. Absence of the whole thickness of the ligament over a large area.

2. Weakness of the ligament over a large area due to an absence of some of its bundles of fibres.

3. Abnormal development of fibrous tissue in connection with the fibro-vascular sheath of the lens, in one sector of its circumference.

Coloboma lentis is also a rare form of abnormality; it consists in a congenital defect in the border of the lens presenting the appearance of a loss of substance. It varies in shape, extent, and position. It may be a triangular, crescentic (Fig. 44), or saddle-shaped notch; or the margin of the lens may, instead of presenting its usual curve, at the seat of the defect appear as a straight line, sometimes 
with little projecting bosses on it. The depth of the defect varies from the slightest indentation to one-fourth of the diameter of the lens. The commonest position for a coloboma of the lens is at its lower border, but it has been met with elsewhere, and two separate defects have been seen in the margin of the same lens.

The suspensory ligament is usually absent in the position of the coloboma, though cases have been observed clinically in which no break could be detected in its continuity.

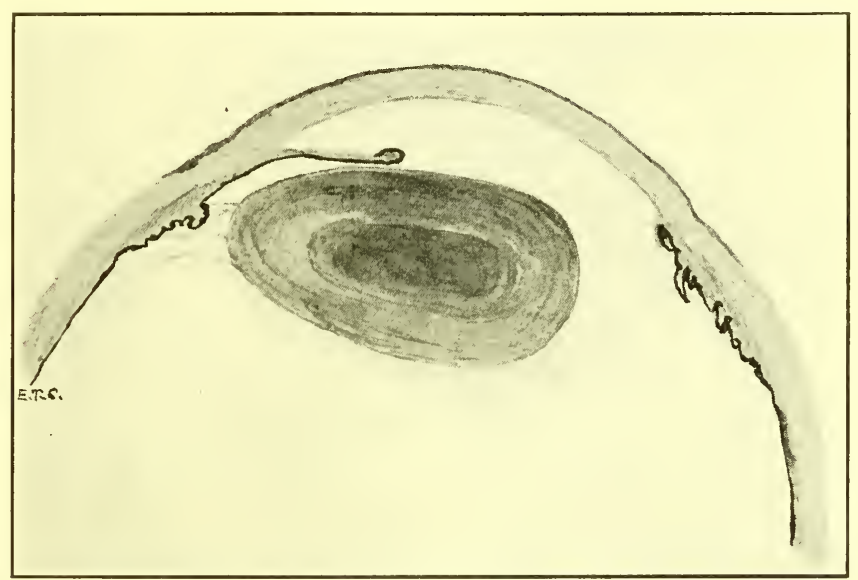

Fig. 44.- Section through the front half of an eye lost from glaucoma, which had a congenital coloboma of the iris and lens. The section passes through the seat of the coloboma in both structures. The suspensory ligament is absent opposite the coloboma of the lens and the latter is broader in that situation anterio-posterior than in the rest of its circumference. The angle of the anterior chamber is closed on the opposite side by the root of the iris and there is ectropion of the uveal pigment.

Myopia is frequent in these cases. In patients who have had the defect in one eye only it has been observed that the defective eye is myopic and the unaffected eye emmetropic.

In eyes which have been examined pathologically with coloboma of the lens an absence of the suspensory ligament at the site of the defect has been found, and the lens in that region was broader antero-posteriorly than in other parts of its circumference (Fig. 44). 
The size of the defect in the suspensory ligament which occasions a coloboma of the lens is a less extensive one than that which causes its congenital displacement.

On the growth of the ciliary body away from the sides of the lens the part of it opposite the gap in the suspensory ligament is not drawn upon in the same way as elsewhere. The absence of traction on the anterior and posterior surfaces of the lens capsule prevents the lens fibres from becoming compressed and bent in that position. The defect in the transverse diameter is compensated for by an increase in the anterio-posterior. The lens is there rounder than

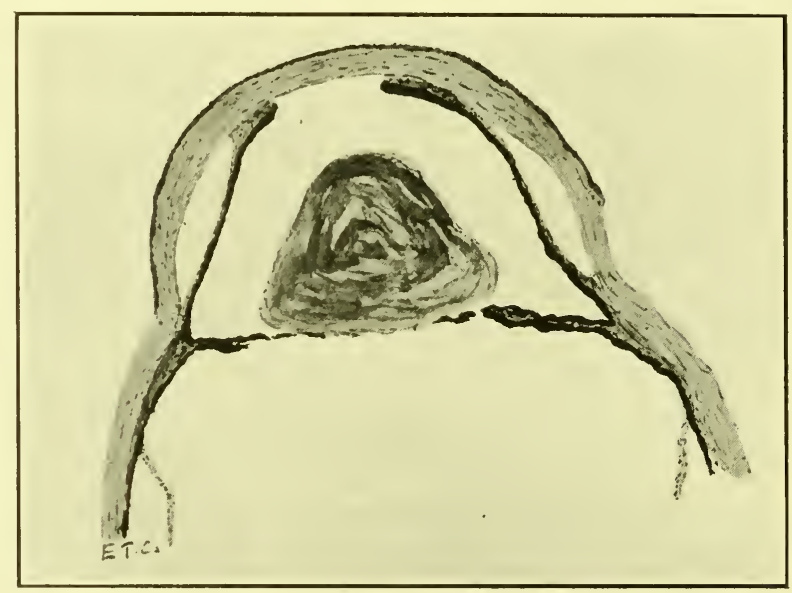

FIG. 45.-Section of the anterior half of the eye of an infant æt. 7 weeks. It shows a congenital anterior staphyloma with anterior synechiæ of the iris. Also lenticonus anterior. The ciliary processes are adherent to the posterior surface of the lens. The fibres of the suspensory ligament which should go to its anterior surface have failed to develop.

normal, as in accommodation when the suspensory ligament is relaxed; hence the frequency with which myopia is met with in these cases.

The variations in the shape of the defect in the lens margin in these cases is attributable to varying degrees of defect in the suspensory ligament. The bosses projecting from a straight margin are accounted for by the presence of 
a few strands in an area where it is otherwise defective. The presence of fibres in the colobomatous area, when viewed clinically, does not prove the absence of a defect in the suspensory ligament. One set of fibres may be present, such as those going to the front or sides of the lens, while those going to the back are absent. It is necessary for fibres of the suspensory ligament to go to both surfaces of the lens to produce the compression which keeps the lens fibres bent in a state of tension.

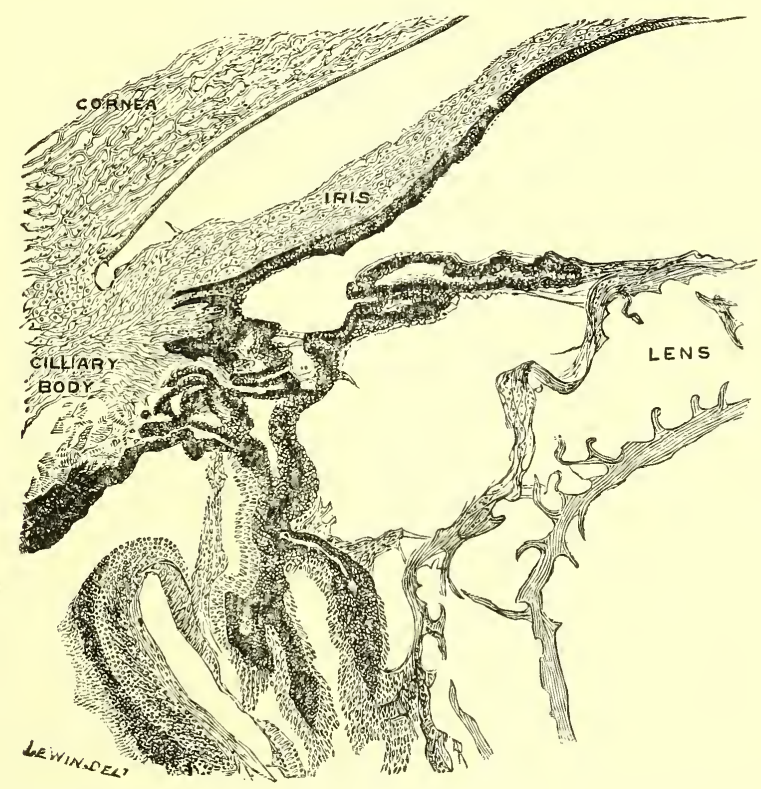

FIG. 46.- Section through the ciliary body in an eye which had fibrous tissue formation at the posterior surface of the lens with a persistent hyaloid artery. The fibres of the suspensory ligament are represented by cellular adhesions passing from the ciliary body to the lens capsule. The lens fibres have become much disorganised as the result of the hardening reagent. Case recorded in the $R$. Lond. Ophth. Hosp. Reps., XIII, r890, 8 r.

Lenticonus anterior has been seen clinically in only a few cases, it has been found pathologically in eyes with congenital buphthalmos and other gross malformations. ${ }^{1}$ A conical projection is seen from the surface of the lens at

${ }^{1}$ M. S. Mayou. Trans. Ophth. Soc. of the U. K., XXX, I9Io, I20. 
the anterior pole usually unassociated with any opacity (Fig. 45). Its occurrence may be attributed to a failure in development of the orbiculo-antero-capsular fibres of the suspensory ligament, which stretch from the anterior capsule to the hindermost part of the ciliary body. By the growth of the ora serrata and non-plicated part of the ciliary body outward and backward from the side of the lens, these fibres come to exert considerable traction on its anterior capsule and flatten out its anterior surface.

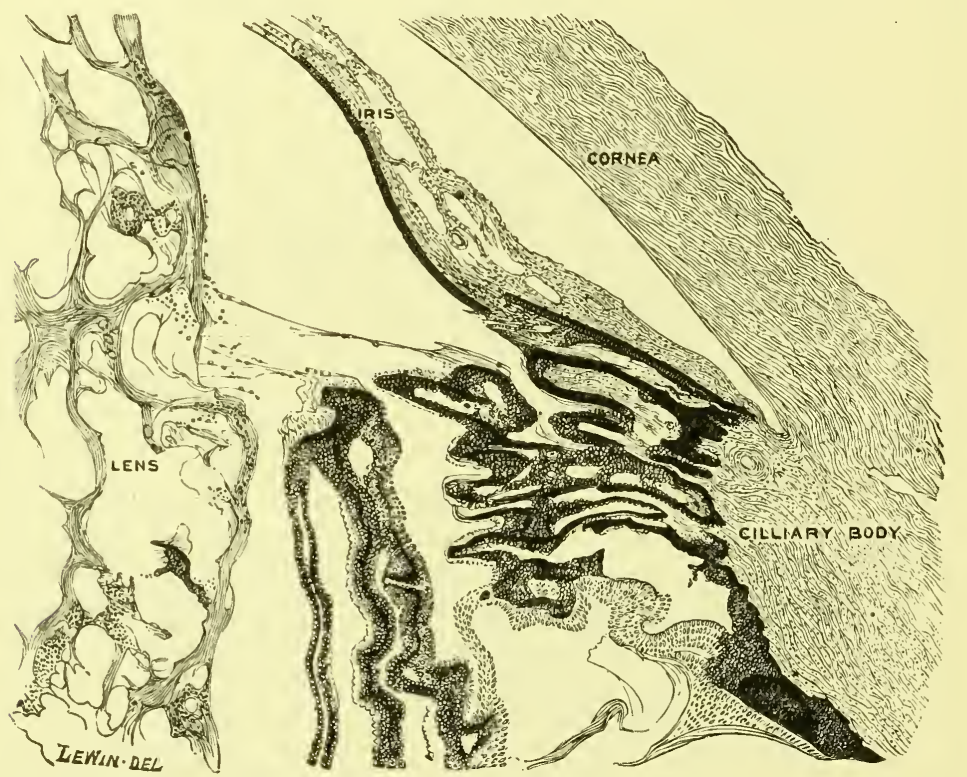

FIG. 47.-Section through another part of the ciliary body from the same eye as Fig. 46. The fibres of the suspensory ligament of the lens are shown with nuclei on them.

When they become relaxed in the act of accommodation the anterior surface of the lens becomes more convex, the most marked alteration taking place at the anterior pole; i.e., there is an approach to the condition of anterior lenticonus which defect is probably due to their complete absence. 
Cellular Adhesion of the Ciliary Processes to the Lens Capsule.-When there is an abnormal development of fibrous tissue at the back of the lens, with or without a persistence of the hyaloid artery, irregularities in the formation of the fibres of the suspensory ligament are frequently met with (Figs. 46 and 47 ).

The cilio-posterio-capsular fibres of the ligament, instead of becoming elongated into strands of kerato-hyaline, may retain their cellular character. These cellular adhesions then hold the ciliary processes in contact with the posterior capsule of the lens, so that on the expansion of the globe, the processes become stretched out and attenuated. If the lens remains clear these elongated ciliary processes can be seen clinically attached to its posterior surface. Pigmented patches on the back of the lens are sometimes seen as a congenital abnormality, they probably mark the site of adhesions of the ciliary processes which have subsequently given way on growth of the eyeball.

\section{ABERRATIONS CONNECTED WITH THE ENCIRCLING MESOBLAST}

The lens vesicle becomes separated from the surface epiblast by the growth in between them of mesoblastic cells from the sides. From this mesoblast are gradually differentiated the substantia propria of the cornea; Descemet's membrane and its lining endothelium; the ligamentum pectinatum; and the lamina irido-pupillaris.

The hyaline layer of Descemet's membrane can be seen as a thin delicate strand in the human fetus of the sixth week. It increases in thickness with the growth of the eye and is the product of the endothelial cells on its inner surface.

The cells of the mesoblastic tissue anterior to Descemet's membrane multiply rapidly, many of them becoming elongated into fibres to form the substantia propria of 
the cornea. The cells posterior to it become arranged into two rows, from the anterior of which is developed the endothelium of Descemet's membrane; whilst from the posterior is formed the lamina irido-pupillaris, which becomes vascularised by the extension into it of loops of vessels from the anterior ciliary arteries.

The site of the ligamentum pectinatum in the fetal eye is at first occupied by a collection of round cells, radiating from a single row lining the back of the cornea. The fibres of the ligamentum pectinatum stain and react in precisely the same way as the hyaline layer of Descemet's membrane and, like it, are probably the product of the cells which ultimately line them. Schlemm's canal can be distinguished as a ring of cells before the development of the ligamentum pectinatum is complete.

The blood-vessels which pass into the lamina irido-pupillaris, from the anterior ciliary arteries, anastomose with branches prolonged forward from the central hyaloid artery round the sides of the lens. In this way the whole lens becomes surrounded by a fibro-vascular sheath, the anterior part of which is composed of the central part of the lamina irido-pupillaris.

The vessels in the lamina irido-pupillaris are arranged in loops, from which fine anastomosing branches are given off. The main loops come close together at the anterior pole of the lens, but do not join.

The iris is formed by the prolongation forward of the two layers of the secondary optic vesicle beneath the lamina irido-pupillaris, and a deposition of mesoblastic tissue external to them, which constitutes its stroma. As the iris extends forward it insinuates itself between the lamina irido-pupillaris and the lens capsule, pushing in front of it the prolongation forward of the posterior fibro-vascular sheath. The central portion of the lamina irido-pupillaris which forms the anterior fibro-vascular sheath of the lens, on the growth of the iris, becomes raised up and forms the pupillary membrane. 
The mesoblastic tissue immediately surrounding the secondary optic vesicle consists at first of a mass of undifferentiated cells. The earliest indication of the formation of the choroid is the development of a larger number of bloodvessels in the inner, than in the outer part of this tissue. The cells composing the outer part become lengthened out and transformed into fibrous tissue to form the sclerotic. In the inner part are developed the branching cells of the choroid which, however, do not commence to show signs of pigmentation until the seventh month of fetal life. The stroma of the iris does not become pigmented until after birth.

The fibres of the ciliary muscle are mesoblastic in origin, being formed in spaces on the outer surface of the ciliary body, corresponding to the spaces in the lamina suprachoroidea on the outer surface of the choroid, and from cells analogous to the cells which line those spaces.

The growth of the eyeball is not complete at birth. Its anterior segment increases rapidly in size during the first year of life. The cornea reaches its full size at the end of the first year, but not its full radius of curvature until adolescence. The posterior segment of the globe continues growing up to the age of twenty. The cornea and sclera both increase in toughness during the early years of life, the number of fibres of yellow elastic tissue increasing in the latter.

The aberrations which may arise in connection with the formation of the encircling mesoblast are:

Atypical development of the mesoblast intruding between the surface epithelium and lens vesicle - congenital opacities and vascularity of the cornea - congenital anterior synechiæ of iris, pupillary membrane and lens-congenital anterior staphyloma.

Defective development of the ligamentum pectinatum and canal of Schlemm - congenital glaucoma or buphthalmos.

Overgrowth or undergrowth of the cornea-megalocornea-microcornea. 
Failure in the post-natal toughening of the corneal tissuekeratoconus.

Unequal development of the cornea in its different meridians - astigmatism.

Failure in the disappearance of the pupillary portion of the lamina irido-pupillaris-persistent pupillary membrane.

Thickening of the iritic portion of the lamina irido-pupillaris-fibrous tissue formation on the surface of the irishypoplasia of the surface endothelium of the iris.

Failure in the growth forward of the secondary optic vesicle and in the deposition of mesoblastic tissue external to it-aniridia-coloboma of the iris-pseudo-coloboma of the iris-polycoria.

Defective development in the stroma of the iriscorectpoia-discoria-microcoria.

Failure in differentiation of mesoblast immediately external to the hinder part of the secondary optic vesiclecoloboma of the choroid.

Atypical development of the cells lining the spaces of the lamina suprachoroidea-muscular tissue external to the choroid.

Defective development in thickness of the scleroticsblue sclerotics.

Post-natal overgrowth and undergrowth of the posterior segment of the eyeball-axial myopia-hypermetropia.

Failure in deposition of pigment in mesoblastic tissue encircling the secondary optic vesicle and in the outer layer of the secondary optic vesicle - albinism.

Excessive deposition of pigment in the above structures-melanosis.

Congenital Opacities and Vascularity of the Cornea.Congenital opacities of the cornea are often the outcome of injuries at birth (see page 365 ), some may be due to keratitis, but others are undoubtedly developmental defects.

Congenital opacity and vascularity are often associated but not always. In the form of congenital opacity termed 
arcus juvenilis, there is a complete ring of opacity at the margin of the cornea without abnormal vascularity. It resembles somewhat an arcus senilis, but differs from it in not having a diaphanous zone between the margin of the cornea and the opacity.

In the form of congenital opacity known as sclerophthalmia the margin of the cornea cannot be defined; it looks as though a portion of the sclerotic were prolonged on into the cornea, there is also abnormal vascularity. The opacity is of a dense white colour, and may occupy the whole, or a portion of the circumference of the cornea.

Congenital opacity of the cornea is frequently met with in cases of microphthalmia and congenital anterior staphyloma; microscopical examination in such cases shows an absence of the anterior limiting membrane, some irregularity of the fibrous-tissue lamellæ of the substantia propria, and an abnormal development of blood-vessels (Fig. 64).

At what period of fetal life the cornea becomes a transparent tissue is not known. It is never normally a vascular structure, and the extension of blood-vessels into it from the sclerotic is probably one of the causes of congenital opacity.

Congenital Anterior Synechiæ of Iris, Pupillary Membrane or Lens. - Imperfect differentiation of the mesoblast, which intrudes between the surface epithelium and the lens vesicle, into the several structures which are derived from it, may result in the formation of congenital synechiæ of the tissues bounding the anterior chamber.

An anterior synechia of the iris may result from failure in differentiation of the endothelium lining Descemet's membrane from the peripheral part of the lamina iridopupillaris, beneath which the iris grows (Fig. 48). A piece of the central part of the lamina irido-pupillaris (which becomes the pupillary membrane) may, if it fails to disappear, remain attached to the back of the cornea (Figs. 48 and 49 ). ${ }^{1}$

1 Treacher Collins. Trans. Ophth. Soc. of the U.K., XXVII, I907, 203. 
In some cases of congenital anterior synechia of these structures there is an absence of the hyaline layer of Descemet's membrane at the seat of the adhesion, the stroma of the iris or pupillary membrane being continuous with the substantia propria of the cornea, a differentiation of the mesoblast into the several layers having failed to take place. ${ }^{1}$

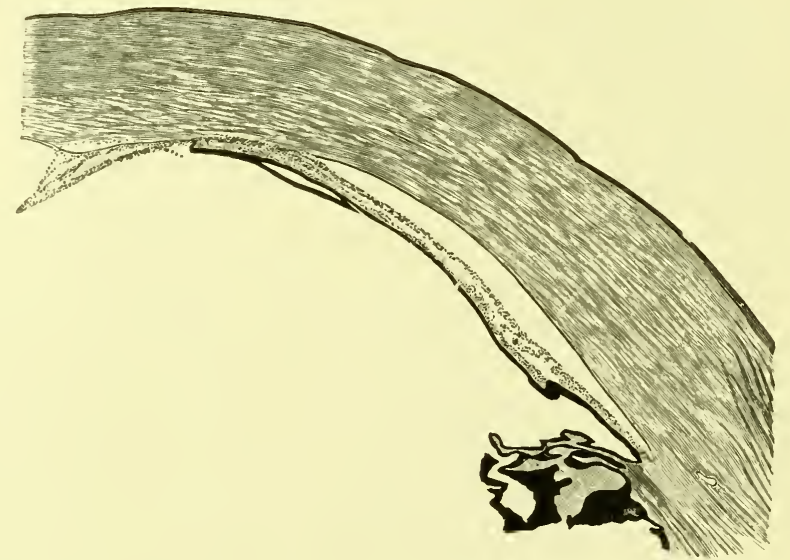

FIG. 48.-Congenital anterior synechia of a persistent pupillary membrane and of the iris.

When there is a very extensive congenital adhesion of the iris to the cornea the channels for exit of fluid from the eye at the angle of the anterior chamber are blocked, the tension of the eye becomes increased, and an anterior staphyloma is formed.

The case of a microphthalmic eye in a chick has been recorded $^{2}$ in which Descemet's membrane had failed to develop, and the anterior capsule of the lens was adherent to the substantia propria of the cornea. The adhesion of these structures obstructed the growth inward of the iris between them. In part of its circumference it was absent and in part had extended behind the lens (Fig. 52).

${ }^{1}$ M. S. Mayou. Trans. Ophth. Soc. of the U. K., XXX, I9Io, I20.

${ }^{2}$ Treacher Collins and J. H. Parsons. Trans. Ophth. Soc. of the U. K., XXIII, I903, 24I. 


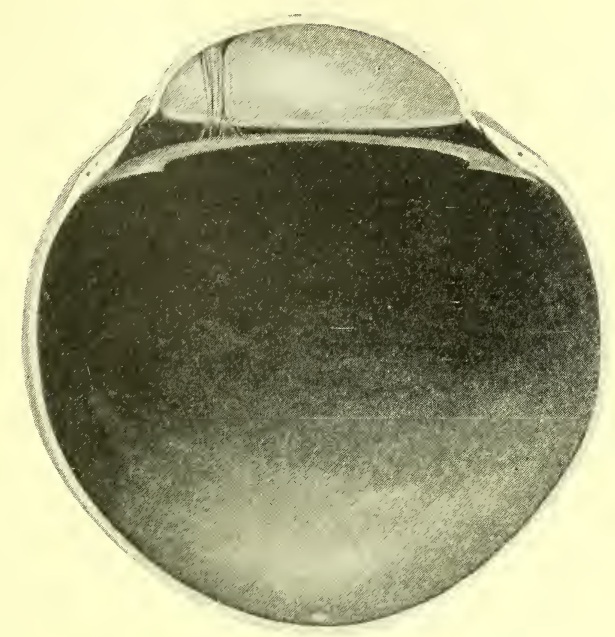

FIG. 49.- Shows the lateral half of a cat's eye from which the lens has been removed. Persistent tags of the pupillary membrane stretch from the anterior surface of the iris to the back of the cornea. Case recorded in the Trans. Ophth. Soc. of the U. K., XXVII, 1907, 203.

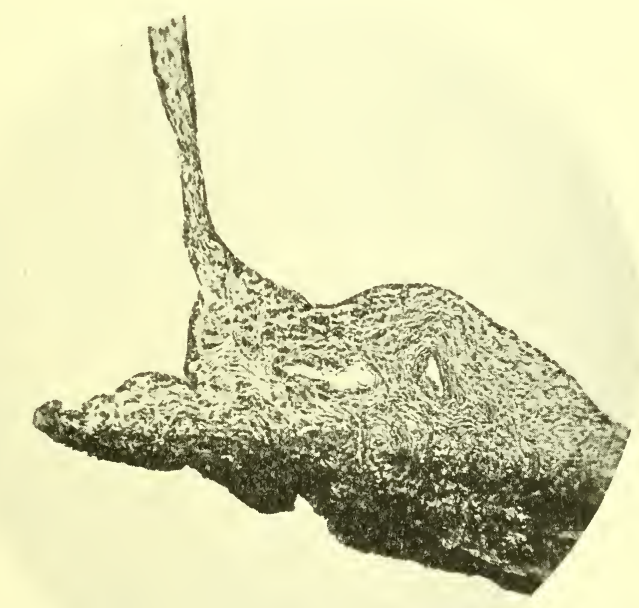

FIC. 50.- Shows the microscopical appearances of the pupillary membrane where it starts from the iris present in the cat's eye depicted in Fig. 49. From a photo-micrograph by E. Collier Green. 
Congenital Anterior Staphyloma.-Congenital anterior staphyloma of the cornea is a very rare condition. It presents, both clinically and pathologically, appearances very similar to those of an anterior staphyloma formed in infancy as the result of a perforating ulcer.

The cornea in these cases has a variable amount of opacity and vascularity. The iris lies in contact with, or

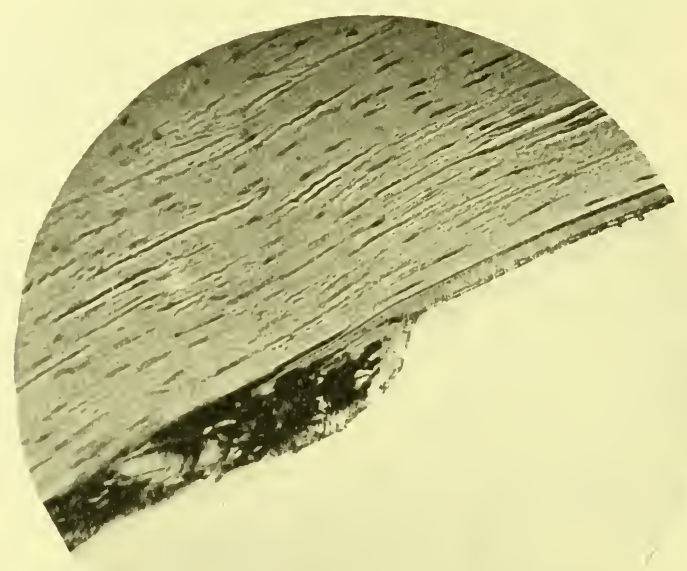

Fig. 5I.- Shows a piece of pigmented pupillary membrane adherent to the substantia propria of the cornea, Descemet's membrane being there absent: From the cat's eye depicted in Fig. 47. From a photo-micrograph by E. Collier Green.

is adherent to, the posterior surface of the cornea, so that there is no anterior chamber, and no means of escape for the aqueous humour from the eye. The increased tension thus produced is the cause of the expansion of the anterior part of the globe.

Several observers ${ }^{1}$ have been led to infer that the change in these cases must have resulted from intrauterine ulceration. It is, however, very difficult to believe that perforating ulcer of the cornea could occur in utero, heal, and result

${ }^{1} \mathrm{G}$. Coats. Ophthalmoscope, VIII, I910, 248. 
in a fully developed anterior staphyloma before birth. In one recorded case of congenital anterior staphyloma the whole of Descemet's membrane and the ligamentum pectinatum were absent; ${ }^{1}$ ulceration of the cornea never produces complete destruction of these structures.

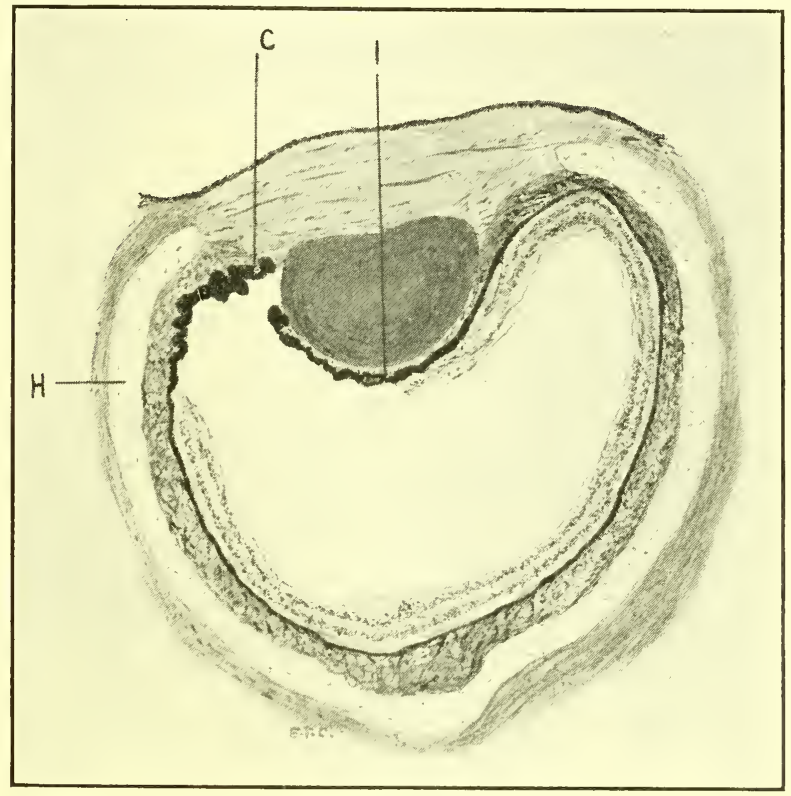

FIG. 52.-The microphtha1mic eye of a chick in which the lens has remained adherent to the cornea. The iris at $C$ has been arrested in its growth inward. At $I$ it has extended round the posterior surface of the lens instead of between it and the cornea. $H$ points to the hyaline cartilage in the sclerotic.

Apparently in these cases, the mesoblast which grows in between the surface epithelium and the lens had, in the whole, or a portion of its extent, failed to become in any way differentiated. The lamina irido-pupillaris and Descemet's membrane were not formed in the affected area, the mesoblast from which they should have developed being converted into a mass of vascularized fibrous tissue. ${ }^{2}$ Beneath this fibrous tissue spread the two layers of the secondary

1 Treacher Collins. Trans. Ophth. Soc. of the U. K., XXIX, I909, I69.

${ }^{2}$ M. S. Mayou. Trans. Ophth. Soc. of the U. K., XXX, I9Io, I20. 
optic vesicle, which form the pigment epithelium of the iris; but in the absence of any lamina irido-pupillaris no stroma of the iris was formed. The blood-vessels which should have supplied it passed instead into the mass of undifferentiated fibrous tissue.

Congenital Glaucoma or Buphthalmia.-Congenital glaucoma can usually be accounted for by some defect in the development of the ligamentum pectinatum or canal

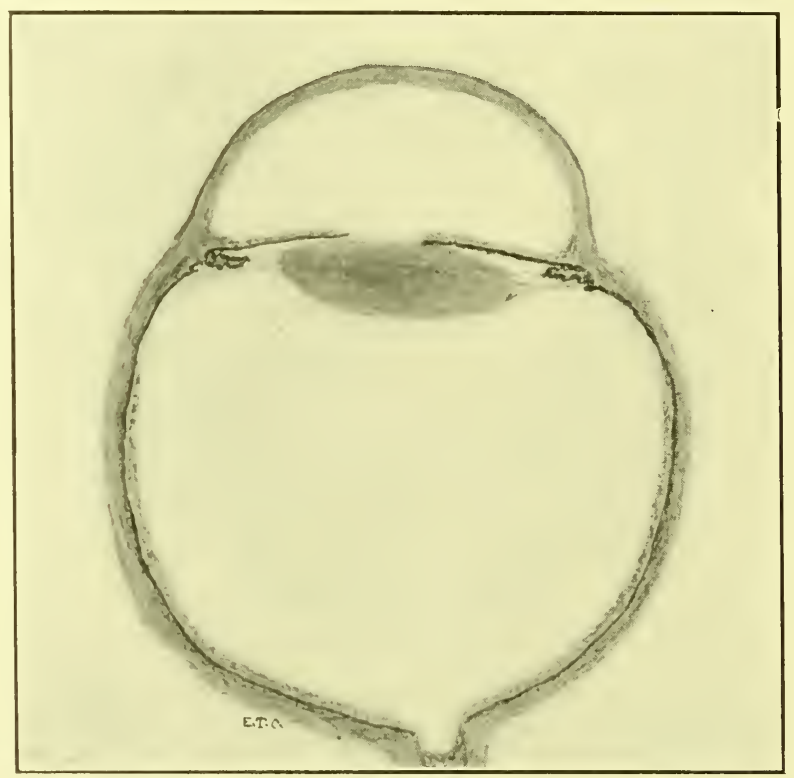

FIG. 53.- Section through an eye the subject of congenital glaucoma or buphthalmia. There is an enlargenent of the globe in all its diameters. Both cornea and sclerotic are stretched and thinned. The effect of the stretching on the lens has been to flatten it antero-posteriorly. The optic disc is cupped.

of Schlemm, the main channels through which the intraocular fluid escapes from the eye. The cornea and sclerotic in an infant's eye are very expansile structures, and increase of tension occurring at that stage of life causes them to stretch, the whole globe becoming enlarged. An enlargement of the cornea and deepening of the anterior chamber produces the appearance of a bullock's eye, hence the term "buphthalmia" (Fig. 53). 
Various defects of development have been met with in connection with the filtration area at the angle of the anterior chamber in cases of buphthalmia. ${ }^{1}$ The ligamentum pectinatum and canal of Schlemm have both been found absent, Descemet's membrane instead of splitting up into a number of fibres at the angle of the chamber being con-

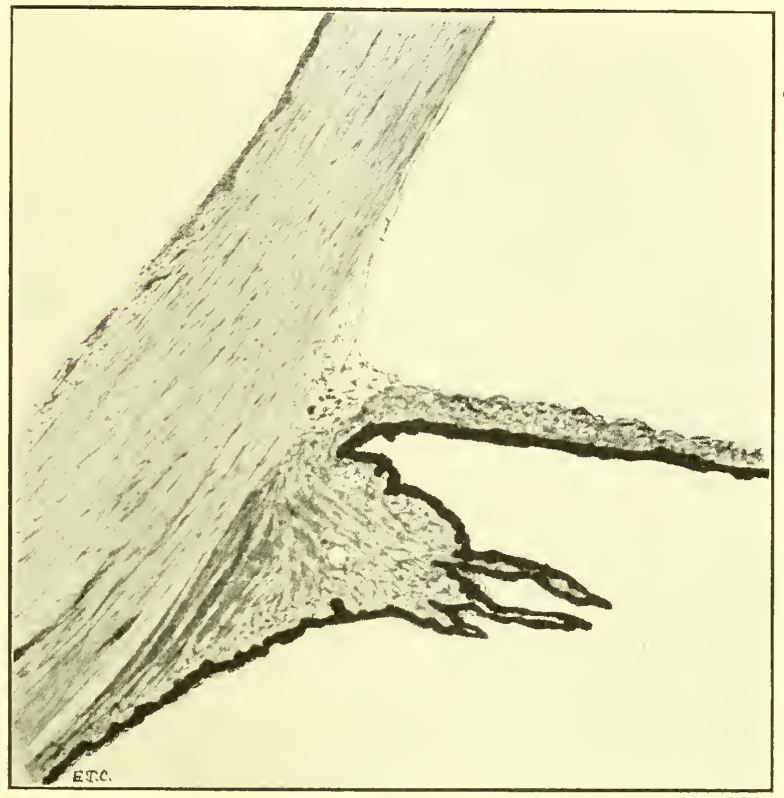

FIG. 54. - The angle of the anterior chamber in an eye affected with congenital glaucoma, or buphthalmia. It shows an imperfect separation of the root of the iris from the back of the cornea.

tinued round on to the anterior surface of the iris, the ciliary muscle taking origin from the sclerotic to which also the root of the iris has been found attached.

The ligamentum pectinatum has been found normal but the canal of Schlemm absent.

In nearly all mammals the fibres of the ligamentum pectinatum are more numerous, and fill up more of the angle of the anterior chamber, than in the human eye. The size

${ }^{1}$ F. Richardson Cross. Trans. Ophth. Soc. of the U. K., XVI, I896, 340. 
of the cornea compared with that of the globe is less in the human eye than in other mammals. Its diameter in all mammals, except the higher apes, is more than half the antero-posterior diameter of the globe, in man's eye it is considerably less than half.

The reduction in the size of the cornea, as compared with that of the globe, seems to be associated with a prolongation outward of the angle of the anterior chamber, and a simplification of the structure of the ligamentum pectinatum. In the human fetus the size of the cornea relative to the globe, and the condition of the angle of the anterior chamber and ligamentum pectinatum, resemble that met with in the lower animals.

In some cases of buphthalmia the prenatal condition of the ligamentum pectinatum is found; a network of fibres filling up a space at the angle of the anterior chamber, which in the normal fully developed eye is part of the anterior chamber (Fig. 52). It seems probable that the persistence of this prenatal state of the ligamentum pectinatum is responsible for obstructed exit of fluid from the eye, and the occurrence of increase of tension. ${ }^{1}$

The adhesions which are met with stretching across the angle of the chamber in these cases vary in density. They may be broad and compact, or only a few fibres or strands extending across a widely open angle. Doubtless these adhesions, with the increase in the size of the globe, and deepening of the anterior chamber, become considerably stretched and attenuated, some even giving way.

Cases of buphthalmia are occasionally met with in which the progress of the affection, after a certain time, seems to become spontaneously arrested, the tension becoming normal and the globe ceasing to enlarge. The breaking through and stretching of congenital adhesions, such as those above referred to, may be the explanation of this arrest.

${ }^{1}$ Treacher Collins. Compte.-Rendu, IX, Congrès Internat. D'Ophthal., I 899,88 . 
Megalocornea and Microcornea.-The average diameter of the normal cornea is Ir. $67 \mathrm{~mm}$., and it reaches its full size as early as the end of the second year. From the sixth month of fetal life, when the anterior chamber is first formed, up to the end of the second year, its growth is rapid, during that time it doubles its size.

As already mentioned, the cornea is at first a very expansile structure, and increase of intra-ocular tension in early life causes it to become abnormally enlarged.

Sometimes, apart from any increase of tension, and in eyes in other respects perfectly normal, it will grow beyond its usual dimensions; it is to this condition that the term megalocornea is applied. ${ }^{1}$ That the change in these cases is not due to expansion from increased tension, as in buphthalmia, is shown by the absence of any excavation of the optic nerve or contraction of the field of vision. The diameter of the cornea in cases of megalocornea may reach to $14.75 \mathrm{~mm}$.; unlike the buphthalmic cases, where the enlargement is due to stretching, it shows no ruptures in Descemet's membrane, no opacity, and no widening of the limbus with blurring of the sclero-corneal margin.

In cases of microphthalmia, due to defect in development of the vitreous humour, or imperfect closure of the fetal cleft, the cornea is usually abnormally small. There is also a well-recognised condition of microcornea associated with a perfectly formed but generally dwarfed eye, socalled nanophthalmia. Besides these conditions microcornea is met with as a form of undergrowth in eyes otherwise well formed, just as megalocornea is met with as a form of overgrowth. In otherwise normally developed eyes the diameter of the cornea may be only $8 \mathrm{~mm}$. This condition of microcornea is sometimes hereditary. As yet we know nothing as to the cause of this abnormal under or overgrowth of the cornea.

Keratoconus.-In the condition termed keratoconus there is a bulging of the central part of the cornea, occurring

1 Treacher Collins. Trans. Ophth. Soc. of the U. K., XI, I920, I32. 
apart from any inflammation or increase of intra-ocular tension. In some cases the ectasia is confined to the central zone, a shallow furrow being present at the junction of the cone with the more normal peripheral portion of the cornea. In others there is a hyperbolic curvature of the cornea as a whole, with a gradual diminution in the thickness of the cornea from its periphery towards the centre.

It is a rare affection but may occur in several members of the same family. As a rule both eyes are affected though often to an unequal extent. The ectasia for a time tends to increase and then becomes stationary; it never decreases in amount. The apex of the cone manifests no tendency to ulceration, but an opacity may form there which remains permanent; sometimes this opacity makes its appearance very rapidly. Microscopical examination of the cornea in advanced cases has shown thinning of Bowman's membrane and gaps in its continuity; the gaps being filled with adventitious connective tissue; a tendency to splitting apart and the formation of abnormally large lacunæ between the deep layers of the substantia propria; and the formation of ruptures in Descemet's membrane.

Examination of the apex of the cone by slit lampmicroscopy ${ }^{1}$ shows, besides the diffuse opacity of the substantia propria and the ruptures in Descemet's membrane: other parallel grey lines situated at varying depths, and usually running in a vertical direction, the real nature of which has not yet been determined. The nerve fibres in the affected area are also seen with unusual distinctness; they form a net-work of grey lines with fine white dots on them. This abnormal appearance of the nerve fibres has been found associated with a loss of sensitiveness at the apex of the cone.

The affection usually first attracts attention about adolescence by the failure in sight to which the distortion of the

${ }^{1}$ A. Vogt. Atlas, r92r, p. 44. 
cornea gives rise. It probably, however, starts much earlier, as slight degrees are difficult to detect and liable to be mistaken for cases of astigmatism.

The changes found histologically in advanced cases are evidently those which have resulted from stretching of the corneal tissue; the opacity which sometimes appears rapidly at the apex of the cone, may be accounted for by ruptures taking place in Descemet's membrane allowing the filtration of the aqueous humour into the substantia propria.

It seems most probable that the cause of the ectasia is some aberration in the development of the cornea. A1though the cornea reaches its full dimensions as early as the second year of life, it has not by that time completed its full development, it gradually goes on becoming tougher and less expansile, and its radius of curvature continues to increase up to the time of puberty. It has been estimated that the radius of curvature of the central third of the cornea is $7.36 \mathrm{~mm}$. from the 5 th to the 6 th year, $7.45 \mathrm{~mm}$. at the I 2 th year, and $7.84 \mathrm{~mm}$. in the adult. In the adult the peripheral parts of the cornea are markedly flatter than the centre, this is said to be the reverse of the condition at birth, when the cornea is more curved at the margins than in the centre.

It will be seen then, that the change in shape which the cornea undergoes in keratoconus, is only an exaggeration of the change which takes place in the post-natal development of the normal cornea, and that it may be regarded as an aberration in development due to some failure in the normal toughning process which takes place in its tissues.

Astigmatism.-Emmetropia is that ideal optical condition of the eye, in which parallel rays of light passing through its dioptric media are brought to a focus accurately on the retina, where they form a distinct inverted image of the point or object from which they proceed.

When parallel rays of light passing through the dioptric media of the eye are so refracted that they are not brought 
accurately to a focus on the retina we speak of the condition as Ametropia.

One of the commonest forms of Ametropia is due to an inequality in the curvature of the cornea in its different meridians, so that rays of light which pass through in one meridian are brought to a different focus from those which pass through it in some other meridian. This condition is spoken of as Corneal Astigmatism.

If the defect in the curvature of the cornea is such that the meridian having the greater curvature is at right angles to that having the smaller degree we have what is called regular astigmatism; and if the maximum and minimum degrees of curvature are not at right angles, irregular astigmatism.

Usually it is the vertical meridian of the cornea which has the greater amount of curvature, and the astigmatism which is then produced is said to be with the rule; should the opposite condition be present, and the horizontal meridian have the greater curvature, the astigmatism is against the rule.

We do not know whether corneal astigmatism is a congenital defect, or one which is produced in the first years of life, during post-natal development; but we do know that once established it usually remains stationary during the rest of life. It is such an exceedingly common defect that it is difficult to determine if heredity plays any part in its production. That slight ectasia in the region of the ocular cleft may sometimes be concerned in its formation seems probable, from its frequent occurrence in cases with congenital crescents at the margin of the optic disc. It is also of common occurrence in cases of asymmetrical facial development; this suggests that abnormalities in the conformation of the orbit, by means of the extra-ocular muscles, may in some way influence the curvature of the cornea. In the early plastic state of the cornea, the greater power of the lateral over the vertical recti muscles, may tend to flatten out the lateral curvature of the cornea 
more than the vertical, and so give rise to corneal astigmatism with the rule.

The lateral diameter of the normal cornea measures I I.6 mm., and the vertical Ix.o mm., there is, however, no evidence to associate this difference in the size of the two diameters with a difference in curvature. In eyes with the normal difference in the diameters of the cornea both astigmatism with, and against, the rule has been met with. In animals, in whom the difference in size of the two diameters of the cornea is more marked than in man, astigmatism is no more frequent than in animals in whom the cornea is nearly round.

Persistent Pupillary Membrane.-The pupillary membrane consists of the central part of the lamina irido-

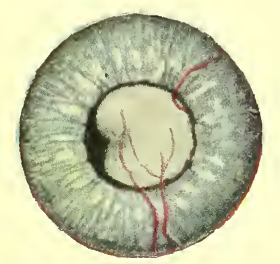

FIG. 55.- Eye of an infant aged 8 months with a congenitally cataractous lens, notched at its outer border and displaced slightly inwards. Fetal blood vessels carrying blood have persisted in the lamina irido-pupillaris. One curls round the margin of the pupil to pass behind the lens. Case recorded in Trans. Ophth. Soc. of the U. K. XXXIII, I9I3, I80.

pupillaris, and it forms the anterior part of the fibrovascular sheath of the lens. It exists until the 7 th or 8 th month of fetal life and then, its blood-vessels becoming obliterated, gradually disappears. Minute stars and threadlike remnants of the pupillary membrane can be found in all eyes by slit lamp-microscopy. Normally it is composed of delicate fibres and a plexus of small bloodvessels, whose walls consist of endothelial tubes with spindle cells external to them.

In rare instances the blood-vessels in this membrane may persist, remain patent, and continue to carry blood. They are seen clinically as red lines extending from the 
surface of the iris over the pupillary area. ${ }^{1}$ Sometimes one or more may be found to curl round the pupillary margin, and pass behind the lens, to join a persistent central hyaloid artery (Fig. 55).

More commonly portions of the pupillary membrane persist after its blood-vessels have disappeared. These persistent tags usually become of stouter consistency, and more cellular in structure, than the membrane in its fetal state. Like the stroma of the iris, before birth the pupillary membrane is unpigmented, but portions of it which persist may also, like the stroma of the iris, become pigmented later.

The frequency of the persistence of portions of the pupillary membrane has been estimated by two different observers as follows: Out of $3508^{2}$ people it was present in thirty-two, or 0.9 per cent.; in eighteen there were filaments only, in fourteen a membrane; three cases had remnants present in both eyes.

Out of 34 I 4 people $^{3}$ it was found in 68 or 1.7 per cent.; in thirteen, remnants were present in both eyes.

The appearances presented by the persistence of a portion of the pupillary membrane vary considerably; but whatever the arrangement it is always seen to start from the anterior surface of the iris. Not immediately from the pupillary margin, but a little distance external to it, usually from one or more of the teeth of the small circle of the iris.

The various forms of persistent pupillary membrane may be classified as follows:

I. Fibres arising from the small circle of the iris and uniting into a membrane in the centre of the pupil.

2. Fibres arising at different points in the circumference of the small circle of the iris and stretching across the pupil to form a delicate network in front of it.

1 Treacher Collins. Trans. Ophth. Soc. of the U. K., XXXIII, I913, I73.

${ }^{2}$ Franke. Archiv. f. Ophth., XXX, I884, 4.

${ }^{3}$ S. Stephenson. Trans. Ophth. Soc. of the U. K., XIII, I893, I39. 
3. Fibres running tangentially between two points in the small circle of the iris (Fig. 43).

4. All the toothed projections of the small circle prolonged inward, and projecting beyond the pupillary margin (Fig. 56).

5. One or more fibres attached to the small circle of the iris floating free at the other extremity.

6. A loop formed in front of the pupil (Fig. 57).

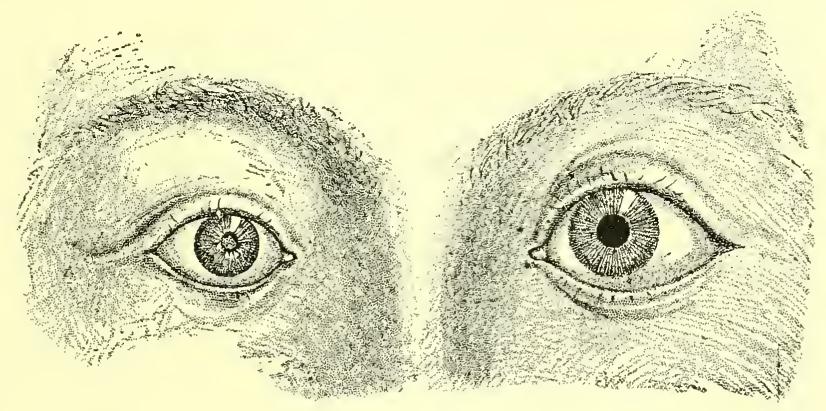

FIG. 56.-Congenital microphthalmia and cataract with persistence of numerous tags of pupillary membrane in the right eye of a girl aged 8 months.

7. One or more fibres arising from the iris attached to the capsule of the lens. Sometimes spoken of as capsulopupillary membrane.

8. One or more fibres arising from the iris attached to the posterior surface of the cornea. A condition already referred to on page $8 \mathrm{I}$.

9. A piece of membrane or a few dots, pigmented or unpigmented, adherent to the anterior capsule of the lens without any attachment to the iris.

A persistent pupillary membrane may be met with in association with other abnormalities of the iris such as coloboma and aniridia; also with anterior polar cataract or an atypical development of vitreous behind the lens.

Fibrous Tissue Formation on the Anterior Surface of the Iris.- It has already been mentioned how an abnormal development of fibrous tissue may take place in connection 
with the fetal vascular system, either in the vitreous chamber, or at the back and sides of the lens. A similar development of fibrous tissue has been met with, in rare instances, in connection with the anterior part of the fetal vascular system, on the anterior surface of the iris, and extending from it to the pupillary area. ${ }^{1}$ Fibrous tissue

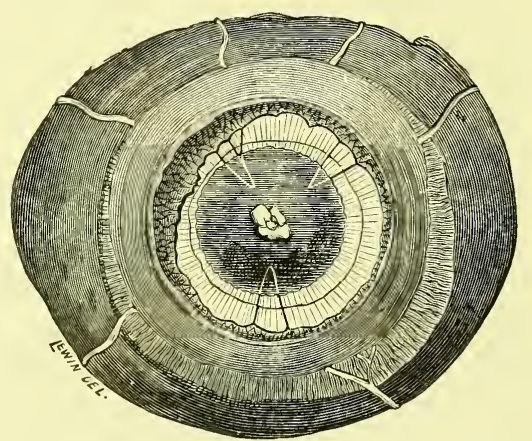

FIG. 57.-Anterior half of the right eye of a patient with apparent aniridia, from which the cornea and sclerotic have been removed, exposing a rudimentary iris, tags of pupillary membrane, lens with an anterior polar opacity, and fibres of the suspensory ligament.

thus formed shows clinically as a greyish membrane which hides from view the stroma of the iris, and can be seen to be situated at a slightly higher level (Figs. 58 and 59).

Aniridia.-Apparent complete congenital absence of the iris is nearly always a bilateral affection. The sight is defective, and the patient acquires the habit of screwing up the eyelids to shut off the excess of light which enters the eye. The condition is frequently hereditary being transmitted from one generation to another.

On looking into the eye the part where the iris should be is seen black like the pupil. In some cases no iris tissue whatever is visible, in others crescentic pieces or small nodules can be detected at the periphery of the anterior chamber, such cases strictly speaking should be termed partial aniridia. When examined by reflected light the margin of the lens, the fibres of the suspensory

${ }^{1}$ G. Coats. The Ophthalmoscope, VIII, I910, 706. 
ligament, and sometimes also the ciliary processes are clearly visible.

Opacity of the periphery of the cornea (arcus juvenilis), anterior polar cataract, persistent tags of pupillary mem-
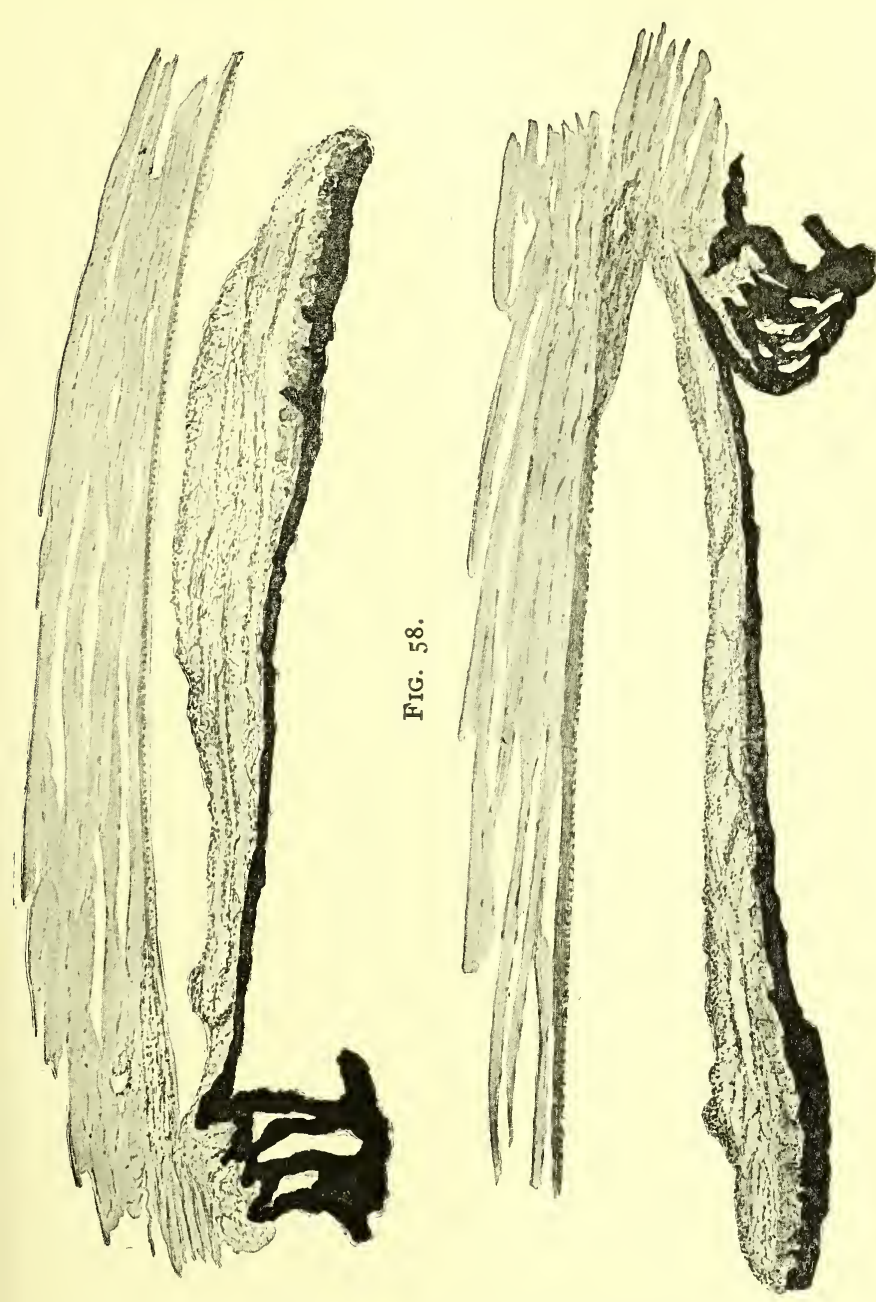

䒿总总

号焉.

0 검

के त्ष

당

w.

祉过

ज्ञ

언 0

吾岂志

넝 th. 당

표웛

论

ه 엉

प्र म

iे चै

(i)

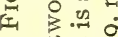

0 जo

吾起我

ت $\infty 0$

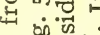

经㐘

0 0

ज的

○势。

대욤

造

ทें 웡

的芫㟧

$\infty$ ㄷํㅇ है

in

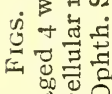

brane, and shallowness of the anterior chamber are occasional concomitants.

Though clinically no iris may be seen in these eyes, when they are examined anatomically a small rudimentary 
iris is always found to be present, the length of which varies in different cases and in different parts of its circumference in the same case. The way in which the sclerotic overlaps the cornea at its periphery serves to hide it from view clinically (Figs. 57,60). ${ }^{1}$

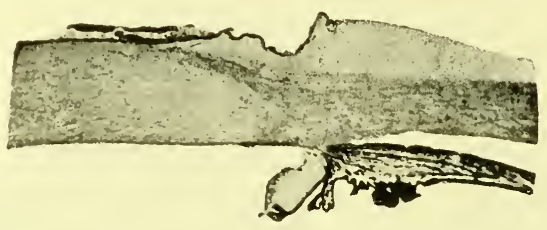

FIG. 6o.- Section of an eye, showing a rudimentary iris in which clinically no iris was visible. A persistent tag of pupillary membrane is attached to the tip of iris, and an adhesion stretches between its root and the ligamentum pectinatum.

In the rudimentary iris the sphincter muscle is undeveloped, and the pigment epithelial layers at its free border may be plicated, or extend further inward than the stroma, producing what is termed ectropion of the uvea (Fig. 19) (see page 27). Defect in development of the ligamentum pectinatum, such as has been described on page 87 , is a common accompaniment of aniridia. The angle of the anterior chamber appears to be imperfectly opened out, adhesions stretching across it between the dwarfed iris and the cornea (Fig. 6I). This probably accounts for a predisposition to the development of glaucoma which has been observed in these cases (Fig. 62).

When the iris commences to grow, the two layers of the secondary optic vesicle have to insinuate themselves beneath the lamina irido-pupillaris, between the cornea and the lens, which at that time lie in close apposition. They also have to push in front of them the prolongation forwards of the posterior fibro-vascular sheath of the lens, which passes round its sides to join the lamina iridopupillaris.

${ }_{1}^{1}$ Treacher Collins. Ophthalmic Review, X, I89I, Ior and Trans. of the Ophth. Soc. of the U. K., XIII, I 893, I 28. 
It is possible for the growth inwards of the iris to become obstructed in the following three different ways:

i. Prolonged, or abnormally firm adhesion of the cornea and lens.

ii. Adhesion of the pupillary portion of the lamina irido-pupillaris to the lens capsule.

iii. Adhesion of the lateral prolongation forwards of the posterior fibro-vascular sheath of the lens to the periphery of the cornea.

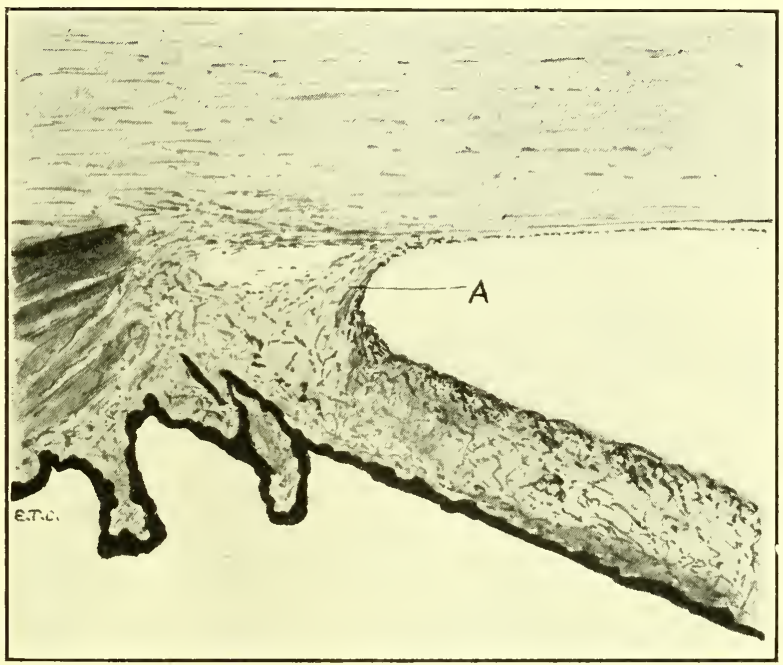

FIG. 6I.-A congenital abnormality at the angle of the anterior chamber in a case of "partial aniridia." An adhesion $(A)$ exists between the rudimentary iris near its root and the posterior surface of the cornea in the region of the ligamentum pectinatum, the two structures have failed to become completely separated from one another.

As already mentioned a permanent congenital adhesion of cornea and lens, arresting the growth of the iris, has been met with in the microphthalmic eye of a chick (Fig. 52 ). Adhesions of the pupillary membrane to the lens capsule frequently occur. Adhesion of a thickened prolongation forward of the posterior fibro-vascular sheath to the periphery of the cornea has been found associated with coloboma of the iris (Fig. 63). 


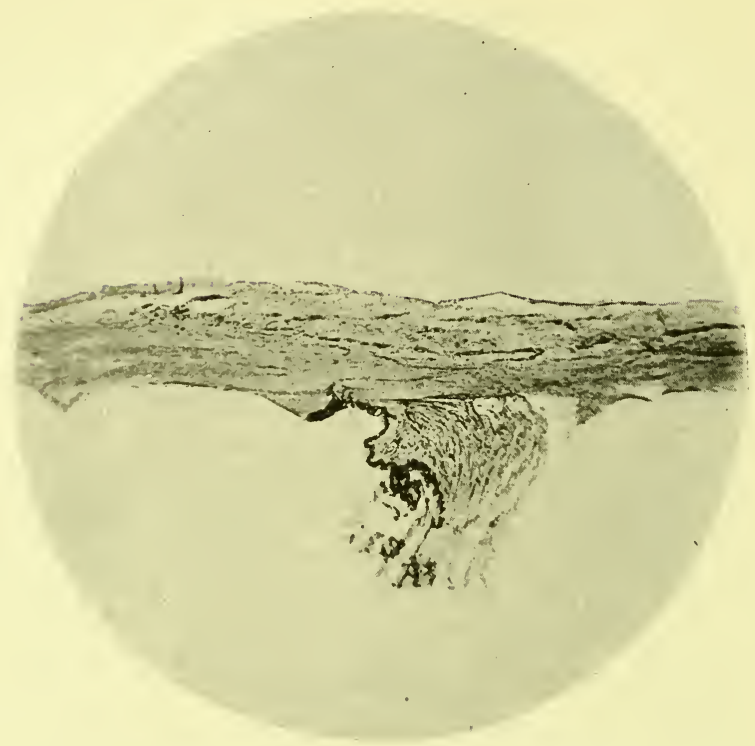

FIG. 62.- Section of the eye ot a woman who had congenital aniridia and after a perforating ulcer of the cornea developed glatucoma. A rudimentary stump of iris is shown in contact with the back of the cornea in the region of the ligamentum pectinatum. Case recorded in the Ophthalmic Review, X, I89 I, Ior.

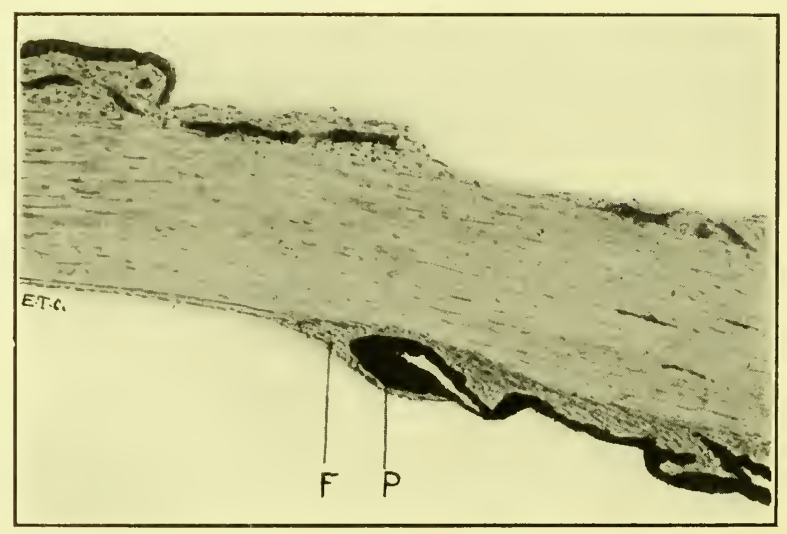

FIG. 63.-Section showing the mode of termination of the ciliary body at the seat of a congenital coloboma of the iris. $P$ points to the pigment epithelium on the posterior surface of the ciliary body where the uveal tract terminates; $F$ points to some tissue adherent to the ligamentum pectinatum in front of the pigment epithelium and continuous with a layer of cells posterior to it which probably represents a portion of the fibro-vascular sheath. 
That aniridia is due to some mechanical obstruction to the growth inwards of the secondary optic vesicle, which forms the posterior layers of the iris, seems probable, because, the pigment epithelium on the back of the small rudimentary iris in these cases is often found in excess and plicated. The frequent association of anterior polar cataract with aniridia also suggests prolonged contact of lens and cornea.

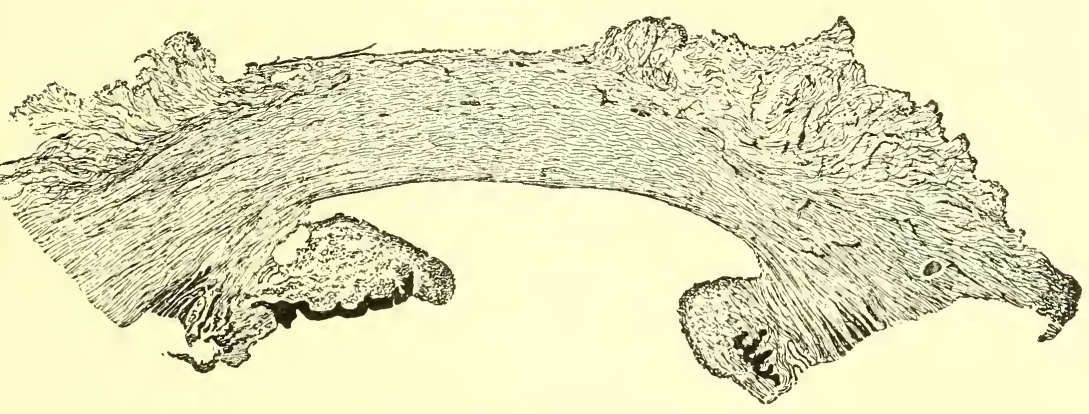

FIG. 64.-Shows the microscopical appearances of the front part of a microphthalmic eye with coloboma of the iris. Note the presence of blood-vessels in the anterior layers of the cornea. Case recorded R. Lond. Ophth. Hosp. Rep., XII, I $889,289$.

Coloboma of the Iris.-A coloboma of the iris is a deficiency in the tissue of the iris causing the pupil to be altered in shape (Fig. 64). It is one of the commonest malformations of the eye. The term pseudo-coloboma of the iris is applied to cases in which a portion only of the stroma of the iris is deficient, the deeper layers being present and left exposed.

In the large majority of cases of coloboma of the iris the defect is situated in its lower half, either directly downward, or downward with a slight inclination inward or outward; i.e., in the position of the fetal cleft. It has, however, been met with in exceptional cases upward, inward, and outward.

Two colobomata of the iris may in rare cases occur in the same eye, and in some cases more than half the iris has 
been absent, these latter merge into those of partial aniridia already referred to.

The extent and shape of the defect is even more variable than the direction. It may consist of only a slight notch in the pupillary border, or the whole thickness of a sector of the iris may be absent from the margin of the pupil up to the ciliary body. Usually the edges of the gap converge to a point at the ciliary margin, the coloboma then, together with the normal pupil, forming a pear-shaped opening. In other cases the margins of the gap run parallel to one another and occasionally, in rare instances, diverge as they proceed outward so that the widest part is at the ciliary border.

A coloboma of the iris is frequently associated with a coloboma of the other structures of the eye, viz., coloboma of the ciliary body, choroid, suspensory ligament of the lens; but may occur without any other malformation.

Its frequent association with abnormalities of the fetal ocular cleft, and its common occurrence downward, suggest that it is in some way connected with defective closure of the cleft.

The iris is formed by an extension forward of the two layers of the secondary optic vesicles beneath the lamina irido-pupillaris; if the cleft in the secondary optic vesicle failed to close, then necessarily in that region there would be a defect in development of the iris. If the closure of the cleft was delayed, until after the usual period at which the iris commenced to grow, then the time for its development would be shortened.

There are cases of coloboma of the iris which cannot be explained by defective closure of the fetal cleft; e.g., cases where it is situated upward or laterally, or where two gaps are present in the same iris. Such cases can be accounted for in the same way as aniridia (see page 98). Any cause which will arrest the growth of the whole iris, acting less extensively, will account for a partial absence of the iris. 
The iris is composed partly of mesoblast and partly of neural epiblast. In the cases of "pseudo-coloboma" the parts developed from the latter, viz., the pigment epithelial layers, have apparently developed in the normal way, but there has been a defective deposition external to them of mesoblast. This defective deposition of stroma is most likely due to an absence of some of the anterior ciliary blood-vessels.

Polycoria.-The term polycoria is applied to the rare condition in which there is more than one opening in the iris. The extra openings are not analogous to the normal pupil for they are never encircled by a sphincter muscle.

Polycoria may be divided up into three classes:

I. Cases in which a normally situated pupil is divided into two by a band passing across it, so that it presents the appearance of a figure 8 . The band in

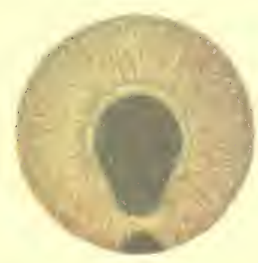

FIG. 65.-Congenital coloboma of the iris with a bridge. most cases would seem to be a thickened piece of the pupillary membrane which has remained persistent.

2. Cases in which a coloboma of the iris has a band of tissue passing across it similar in appearance to the stroma of the iris; these cases are sometimes spoken of as "coloboma with a bridge." The bridge is situated a little below the level of the pupillary border and is continuous with the tissue forming the toothed margin of the small circle of the iris. The opening above it is usually pear-shaped and that below it crescentic (Fig. 65).

In these cases the bridge stretching across the coloboma is composed entirely of mesoblastic tissue developed from the lamina irido-pupillaris.

3. Cases in which there are a number of openings variously situated, usually associated with the absence of a large area of the iris in one direction. In these cases there seems to have been in a large part of its circumference a failure in the growth forwards of the secondary optic vesicle, from 
which the pigment epithelial layers of the iris are formed. The mesoblastic stroma, derived from the lamina iridopupillaris, instead of forming a continuous layer being deposited as bands with gaps between them.

Corectopia, Discoria, Microcoria.-Eccentricity, undue smallness, and a want of rotundity in shape, of the pupil, are sometimes associated congenital malformations but may occur independently.

Normally the pupil is not exactly in the centre of the iris, being usually situated slightly downward and inward. In cases of corectopia it is generally displaced upward and outward. Very frequently displacement of the pupil in one direction is associated with displacement of the lens in the opposite. Corectopia is usually bilateral and symmetrical, and often hereditary.

In cases which have been examined microscopically ${ }^{1}$ it has been found that where the iris was short it was thick from before backwards, and where it was long it was thinner than normal. Where the corectopia was associated with ectopia of the lens, on the side from which the lens was displaced the fibres of the suspensory ligament were absent and the ciliary body in an imperfectly developed condition. It would appear, therefore, that an arrest in the development of the iris, ciliary body, and suspensory ligament in a portion of their circumference occurs in these cases. Such an arrest is probably due to some abnormal adhesion, thickening, or prolonged persistence of the lateral prolongation forwards of the fibro-vascular sheath of the lens.

A pupil may present congenital irregularities in its shape due to persistence of portions of the pupillary membrane. It may be congenitally pear-shaped from the presence of a small coloboma, or otherwise altered in shape from defective development of the sphincter muscle. For a description of Microcoria see page 40.

${ }^{1}$ R. A. Greeves. R. Lond. Opth. Reps., XIX, I9I9, p. 428. 
Coloboma of the Choroid.-Typical Coloboma of the choroid is situated downward, or downard and inward, in the position of the fetal ocular cleft; it is frequently bilateral.

The absence of the choroid in the affected area exposes to view the sclerotic, so that on looking into the eye with the ophthalmoscope, instead of the red reflex from the vascular choroid, a yellowish-white reflex from the sclerotic is seen.

A typical coloboma of the choroid is usually oval in shape with its long axis from before backwards. Posteriorly it may extend up to, or even encircle the optic disc. Anteriorly it may reach as far forwards as can be seen ophthalmoscopically, and is sometimes continuous with a coloboma of the ciliary body and iris. In some cases a band of healthy choroid extends across the coloboma from side to side dividing it into two. The margins of the colobomatous area are usually pigmented; its floor is always at a deeper level than the surrounding fundus, and sometimes, due to its having become ectatic, at a considerably deeper level, or deeper in some parts than in others.

The retinal blood-vessels are frequently seen crossing over a coloboma of the choroid, showing that some retinal tissue must be present where the choroid is absent. Occasionally a few large ciliary blood-vessels, situated at a deeper level than the retinal vessels, are met with in the defective area.

In an eye with a coloboma of the choroid there is generally a defect in the field of vision, though not so extensive as to correspond to the whole of the affected area. The retina which covers a coloboma of the choroid may be capable of perceiving light, and even of distinguishing colour.

The microscopical appearances of the retina overlying a coloboma of the choroid have already been referred to (page $2^{\mathrm{I}}$ ). The vascular layers of the choroid usually end abruptly at the edge of the coloboma, the retina, choroid and sclerotic being frequently adherent there. A thin, fibrous membrane may bridge across the gap in the choroid. 
In an ectatic area in a coloboma the sclerotic becomes abnormally thin.

Besides the typical form of coloboma of the choroid, arising in connection with the fetal ocular cleft, several atypical varieties are met with. In the condition termed choroideremia ${ }^{1}$ the whole choroid, except a small area in the region of the macula, appears to be absent. It is a bilateral affection, the patients are night-blind, have exceedingly small fields and a variable degree of central vision. Ophthalmoscopically a glistening white reflex is seen all over the fundus, except at the macula. Crossing this white area of exposed sclerotic large choroidal vessels are sometimes seen, as in cases of typical coloboma of the choroid; patches of pigmentation are also sometimes met with. At the macula there is a patch in which the red reflex obtained from the choroidal blood-vessels, as in the normal choroid, is seen. The retinal blood-vessels, somewhat smaller than usual, course over the fundus in the normal way.

The similarity of this condition in all the recorded cases, its bilateral character, the fact that the defect of vision in it remains stationary and seems to date from birth, all show that it is due to some defect in development and is not the result of inflammation.

It is interesting to compare and contrast these cases of choroideremia with another form of atypical coloboma of the choroid, which seems to be exactly the reverse. In what is termed macular coloboma there is a round, or oval, shining white patch, due to exposed sclerotic at the macula, the rest of the fundus presenting the normal red reflex. The size of the central patch varies in different cases, it may be that of the optic disc or as much as five times its dimensions. That retinal tissue must extend over the affected area is shown by the passage of retinal vessels across it, and also in some cases by the absence of any

${ }^{1}$ E. Nettleship. Royal London Ophth. Hosp. Reps., XVII, I908, 373. 
absolute central scotoma. The presence of retinal tissue has, moreover, been proved in several cases by microscopical examination, where the choroid was absent the retina was imperfectly developed and no pigment epithelial layer was to be seen.

Atypical coloboma of the choroid may occur in other parts of the fundus besides the macular region. It has been found situated upward, inward and outward. Indeed it seems that a localized congenital absence of the choroid may occur in any part of its extent.

A failure in the development of the choroid is due to a failure in the formation of blood-vessels in the inner part of the mesoblast which surrounds the secondary optic vesicle.

The region of the cleft in the secondary optic vesicle, through which mesoblast grows up to form the blood vessel of vitreous humour, and where for a time there is a connection between the encircling mesoblast and the intruding mesoblast, is the position in which this failure in vascularisation most frequently occurs, giving rise to a typical coloboma. A delayed separation of the intruding mesoblast from the encircling mesoblast is the probable cause of the defective vascular formation.

The choroid receives its chief blood supply from the short ciliary arteries. These are about twenty in number, and perforate the sclerotic in the vicinity of the optic nerve. They proceed from two main trunks which arise from the ophthalmic artery. A failure in the formation of all these vessels, except those which supply the region of the macula, would account for the condition described as choroideremia. The ciliary body and iris which receive their blood supply from the long posterior ciliary arteries and the anterior ciliary arteries are not affected.

The large ciliary vessels, which are sometimes seen in cases of coloboma of the choroid coursing over the affected area, are most likely branches of the long posterior ciliary arteries, which perforate the posterior part of the sclerotic further forward than the short ciliary arteries, and pass to 
the ciliary body before breaking up. Some of them may also be veins proceeding from the ciliary body to the vortex veins which perforate the sclerotic at the equator.

In a macular coloboma there seems to be a normal development of all the blood-vessels which are absent in choroideremia, but a failure in formation of those supplying the macular region which are present in choroideremia.

The other forms of atypical coloboma of the choroid may be attributed to the absence of the short ciliary arteries supplying the particular region affected.

Where coloboma of the choroid is associated with coloboma of the ciliary body there has been, not only a failure of development of short ciliary arteries in the defective area, but also of a long posterior ciliary artery.

The retina receives a considerable amount of nutrient supply from the capillary blood-vessels of the choroid; it is only natural, therefore, that where the choroid has failed to form, the outer layers of the retina should be defectively developed. It appears, however, that the vascular supply of the retinal vessels alone suffices for the formation of some light percipient elements.

Congenital Crescent.-In this condition there is at the lower margin of the optic disc a crescentic white area due to absence of the choroid and exposure of the sclerotic. The disc itself has an oval shape with the long axis horizontal, the physiological cup points downward, the retinal blood-vessels emerging in a downward direction.

The condition, except for its position, resembles a myopic crescent. It is frequently met with in hypermetropic eyes, often associated with astigmatism and sometimes amblyopia.

It has been described as a minimal form of congenital coloboma at the nerve entrance, and there can be no doubt that small colobomata are occasionally met with in this position.

The microscopical appearances of some of these congenital crescents, ${ }^{1}$ however, resemble closely those of a

${ }^{1}$ Elschnig. Arch. f. Ophth., LVI, I, 49. 
temporal myopic crescent. The scleral promontory in the region of the crescent is rounded off instead of projecting as a sharp spur. The choroid, its elastic lamina, and the nuclear layers of the retina end some distance from the nerve entrance (Fig. 66). It would seem likely that both forms of crescent are produced in the same way by stretch-

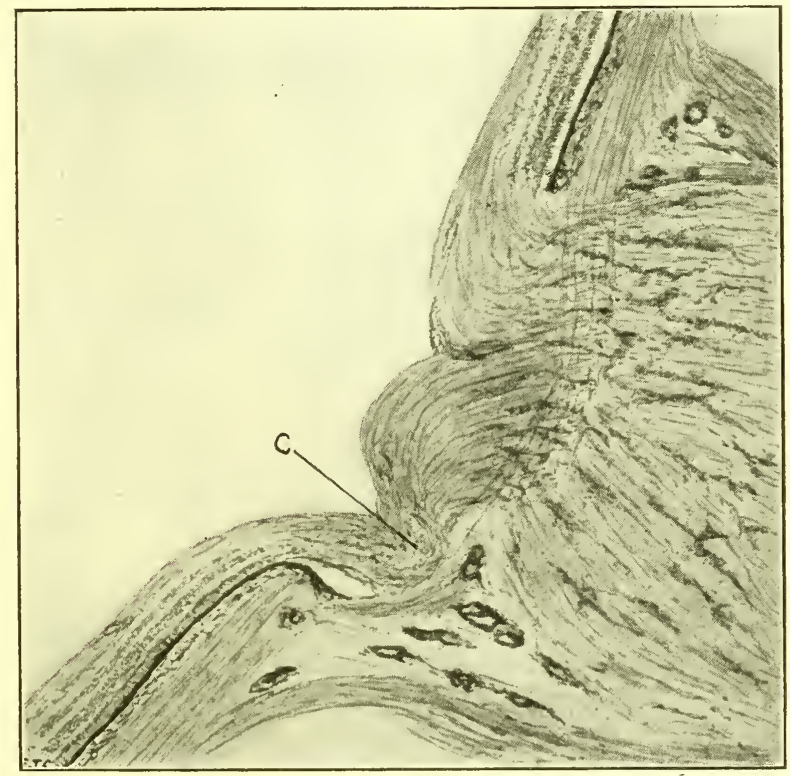

FIG. 66.- Section through the head of the optic nerve in a case in which there was a congenital crescent at the lower border of the optic disc. $C$ points to the site of crescent. The choroid and nuclear layers of the retina are seen to end at some distance from the margin of the disc and there is a depression backwards of the retina between these two points.

ing. In the myopic crescent the stretching is due to ectasia of the sclerotic on the outer side of the disc. In the congenital inferior crescent the ectasia is in the inferior part of the globe, due probably to some congenital weakness in the region of the fetal cleft. The shape and appearances of the disc in these cases are quite in keeping with this hypothesis. In myopia, where the stretching is on the outer side, the disc is oval vertically and the retinal vessels 
directed temporally. In the congenital crescent the stretching is below and the disc is oval horizontally, with a downward direction of the retinal vessels.

Muscular Tissue External to the Choroid.-The fibres of the ciliary muscle under normal conditions do not extend backwards beyond the region of the ora serrata. Cases $^{1}$ have recently been recorded in which microscopically, smooth muscle fibres have been found in the lamina suprachoroidea in the equatorial region, and even further back in the neighborhood of the optic papilla. No clinical symptoms have so far been associated with the presence of such abnormal muscular developments; they are, however, of interest in connection with the development of the ciliary muscle, in showing the analogy which exists between its fibres and the endothelial cells lining the lymph spaces of the choroid, for it is from such cells that these abnormal fibres must have been formed.

Blue Sclerotics.-When the sclerotic becomes thinned, either as the result of stretching or inflammation, the pigmented tissue of the uveal tract shows through and it assumes a slatey blue appearance. As a congenital malformation the sclerotic is sometimes abnormally thin, apart from any stretching or inflammation, and the whole of it then, from the corneal margin back as far as can be seen, presents a bluish hue, which has been described in different cases a "sky-blue," "azure blue," and "slate-blue." In one case ${ }^{2}$ in which the eyeball was examined microscopically the sclerotic was found to be so thin that the addition of the tendon of the internal rectus muscle fully doubled its thickness, the cornea was also thin having about half its usual number of layers (Fig. 67).

This condition of congenital thinness of the sclerotics is frequently accompanied by a deficiency or weakness of

\footnotetext{
'Salzmann. "The Anatomy and Histology of the Human Eyeball," I9I2, p. III. Fuchs. Archiv. f. Ophth., XCV, I9I8, 4.

${ }^{2}$ Leslie Buchanan. Trans. of the Ophth. Soc. of the U. K., XXIII, I 903,267 .
} 
other supporting fibrous tissue in the body. Those affected are sometimes of small stature, have a marked tendency to fracture of the long bones, and to dislocations and sprains of the joints. In one case ${ }^{1}$ skiagrams of the legs and arms showed the middle of the shafts of the long bones to contain the normal amount of compact bone but towards the extremities they appeared to be abnormally

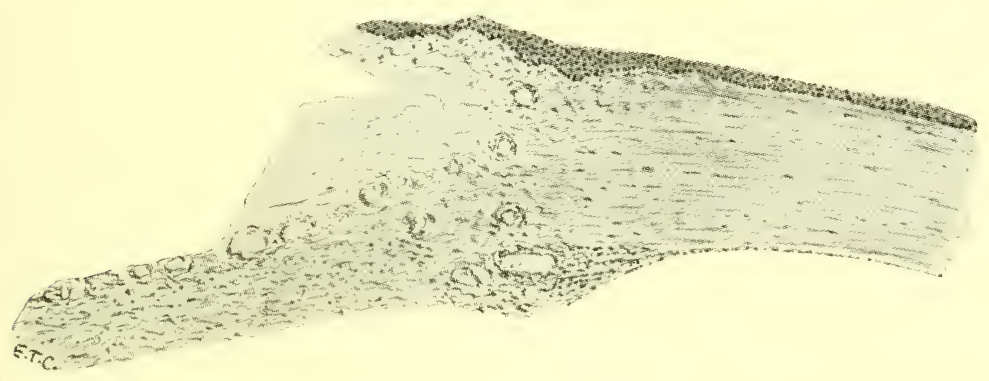

FIG. 67.-Case of maldevelopment of the cornea and sclerotic, "blue sclerotics." Recorded by Leslie Buchanan, Trans. Ophth. Soc. of the U. K. XXIII, $1903,267$.

transparent. In others there appear to have been enlarged medullary cavities. Some of the cases in later life develop progressive deafness.

This thinness of the sclerotics is a markedly hereditary affection, the blueness of the whites of the eyes to which it gives rise being an easily observed symptom, it has been possible to get histories of its occurrence in several successive generations. Out of a family of 55 , comprising four generations, 3 I members were found to be affected. It descends through affected members only, transmission to males through unaffected females does not appear to take place.

Axial Myopia.-Myopia is the term applied to the refractive condition of the eye in which parallel rays of light falling on the cornea are brought to a focus in front of the

${ }^{1}$ E. A. Cochayne. The Ophthalmoscope, XXII, I9I4, 27 I. 
retina. It may be due to an abnormal condition of the refractive media or a lengthening of the antero-posterior axis of the globe. The refractive media may have an increased refractive index or an abnormally strong curvature. The lens may be displaced forward or the ciliary

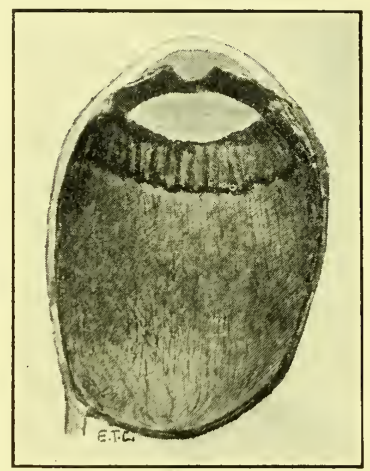

Fig. 68.- Shows an antero-posterior section through a highly myopic eye, actual size. There is considerable stretching and thinning of the whole posterior half of the globe. The specimen is preserved in the R. Lond. Ophth. Hosp. Museum.

muscle act spasmodically. By far the commonest cause of myopia, however, is an increase in the length of the eyeball, what is termed axial myopia. The increase is entirely due to overgrowth, or stretching, in the posterior half of the globe, the parts in front of the equator maintaining their normal dimensions. In some cases there is an ectasia, strictly localised to the region of the macula and optic disc, and having a sharply defined margin. In others the enlargement involves the whole of the hindermost part of the globe, there being a gradual merging of the affected and unaffected areas, and no sharply dividing line (Fig. 68). In the first named variety, which is described as staphyloma verum, the ectasia can be recognized ophthalmoscopically by the presence of a characteristic, dark, crescentic line on the nasal side of the disc, and about one and a half disc's breadth from 
it. This dark line corresponds to the edge of the ectasia, and the retinal vessels can be seen to curl round it in the same way as they do round the edge of a glaucomatous cup. That the floor of the ectasia, inside the crescentic edge, is at a deeper level than the surrounding parts of the fundus can be recognised ophthalmoscopically, by its higher refraction, and by the parallactic displacement which takes place on movement of the lens.

The sclerotic in the region of the ectasia may become so thin that after removal of the eyeball, it becomes dimpled inwards, failing to maintain its normal curvature. The choroid lining the ectasia is also much stretched and shows signs of atrophy.

The changes in the choroid in axial myopia occur at the margin of the optic disc and in the macular region, and can be viewed ophthalmoscopically. As a general rule the higher the degree of myopia the more extensive are these changes, but many exceptions occur.

The change at the margin of the optic disc consists of the so-called "Myopic crescent or conus." Small myopic cresents are limited to the temporal side of the optic disc, the larger ones to its upper and lower borders, whilst in very high degrees of myopia an area of choroidal atrophy may entirely encircle the disc.

A conus appears as a white area with a pigmented outer margin, varying in breadth from a narrow crescent to a patch the width of two or there optic discs in its largest diameter. The larger ones are frequently not of a uniform whiteness throughout, the external part being often pinkish in colour, or showing a network of choroidal vessels on its surface. The floor of a conus can be recognised as situated at a deeper level than the surrounding parts, the depth of the ectasia being greater there than elsewhere. Microscopically, the choroid, elastic lamina, and pigment epithelium are found to be absent over these crescents, terminating some little distance away from the position where they usually end at the border of the disc (Fig. 69). Thesclerotic 
which is thereby exposed to view, gives rise to the white reflex when seen ophthalmoscopically.

The changes at and around the macula in axial myopia consist of, light coloured patches where through atrophy of the choroid the sclerotic is exposed to view, and areas

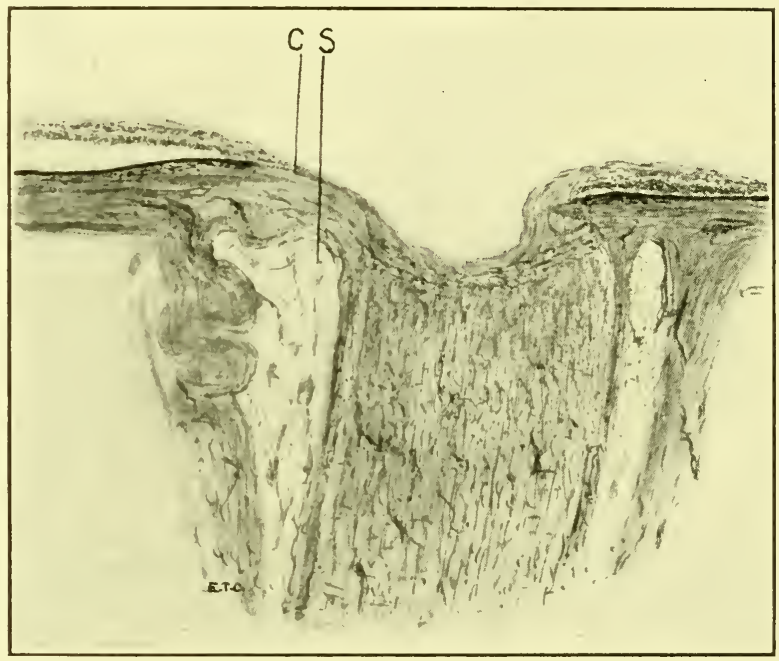

FIG. 69.- Section through the head of the optic nerve in an eye in which there was a myopic crescent. $C$ points to the position of the "distraction crescent," the choroid and pigment epithelial layer being there absent. Note the tendency to the formation of a "supertraction crescent" on the opposite side. Also note the outward extension of the intervaginal space $S$ on the side where the choroid is absent.

where the capillary layer of the choroid has disappeared and the network of its larger outer layer of vessels is rendered visible. Sometimes there are narrow irregular white lines to be seen, which microscopically are found to be due to ruptures in the elastic lamina, and red patches which look like hemorrhages, but do not alter in shape or size when kept under observation, and are probably dilated choroidal veins. Choroidal hemorrhage may occur at the macula in high degrees of myopia causing sudden loss of central vision; such hemorrhages gradually become converted into densely black circular patches. 
As a case of myopia progresses these atrophic changes, the result of stretching, are seen to increase in size and extent. Separate white patches become joined together to form larger ones. Patches of thinned choroid become converted into areas of complete atrophy. Patches of atrophy arising in the vicinity of the macula become joined to those extending from the temporal margin of the optic disc, and in the most extreme cases the whole posterior pole of the eye becomes converted into one large white area.

The changes. in the retina in axial myopia are chiefly secondary to the choroidal atrophy. As the result of the stretching, in which the retina participates with the other two coats, the fovea tends to become flattened out and the retinal vessels assume a markedly straight course. From the traction backward the choroid, as already stated, becomes drawn away from the margin of the optic disc on the temporal side, a distraction crescent being formed. Traction acting in a similar way on the nasal side of the disc tends to draw the retina, and to a less extent the choroid, over its inner margin, which gives rise ophthalmoscopically to a hazy yellowish crescentic area of the inner side of the papilla, a supertraction crescent.

Where the choroidal blood-vessels have atrophied the outer layers of the retina cease to receive their nutrient supply, and together with the pigment epithelium, undergo degeneration. When the elastic lamina becomes ruptured, or destroyed, adhesions form between the remains of the two inner coats. If detachment of the retina occurs in myopia, and a fused area of retina and choroid exists, the retina either tears away around it, leaving an isolated piece of the retina adherent to the choroid; or the adhesion persists and a tubular prolongation from the external surface of detached retina extends outward to the choroid.

In axial myopia the position of the optic disc is altered, the temporal border becoming displaced backward. Ophthalmoscopically this-gives rise to an apparent change in its shape, so that it appears to be oval with the long axis 
vertical. Partly due to this tilting, and partly to the dragging of the retina over from the nasal side, as already mentioned, the physiological cup has a markedly overhanging inner margin from beneath which the retinal vessels are seen to emerge.

In sections through the head of the optic nerve, where it enters the eye in axial myopia, the intervaginal space at

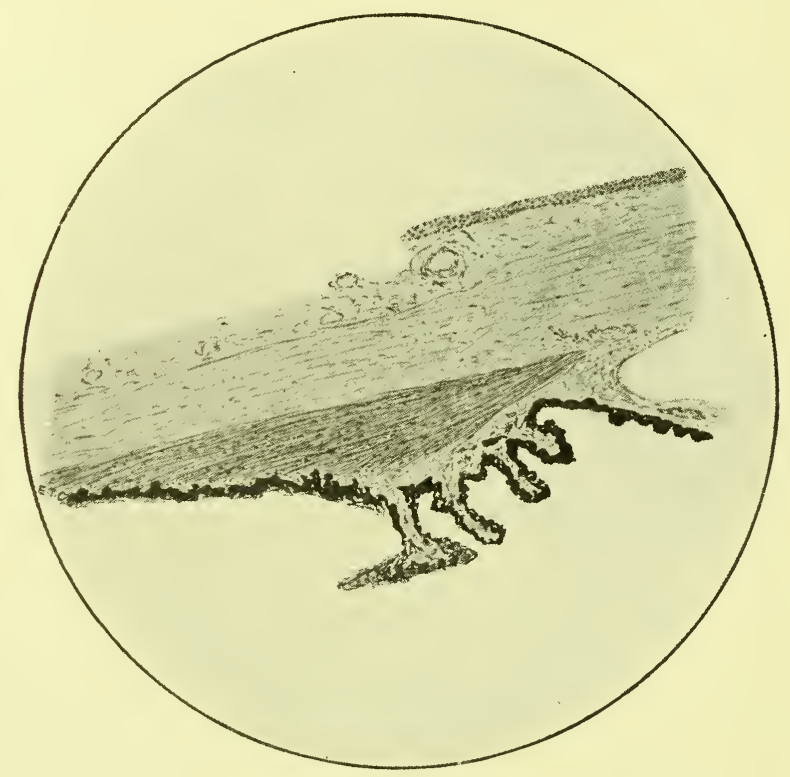

FIG. 70.-The ciliary muscle in a myopic eye.

its termination is found to extend outward laterally to a greater extent on the temporal than on the nasal side.

With the enlargement of the vitreous cavity, which takes place on the formation of a posterior staphyloma, there is no compensating new formation of vitreous humour. Either the vitreous becomes rarefied and of more fluid consistency, or the hyaloid membrane becomes detached from the retina, and a fluid of the consistency of the aqueous humour fills the intervening space. In high degrees of axial myopia both these conditions are frequently met with. 
A detachment of the vitreous can sometimes be recognised ophthalmoscopically by its giving rise to a bright reflex, which is evidently situated nearer the centre of the eye than the retina. When the vitreous humour becomes liquefied, the delicate fibres which form its matrix become broken $\mathrm{up}$ and move freely in the thin fluid media in which

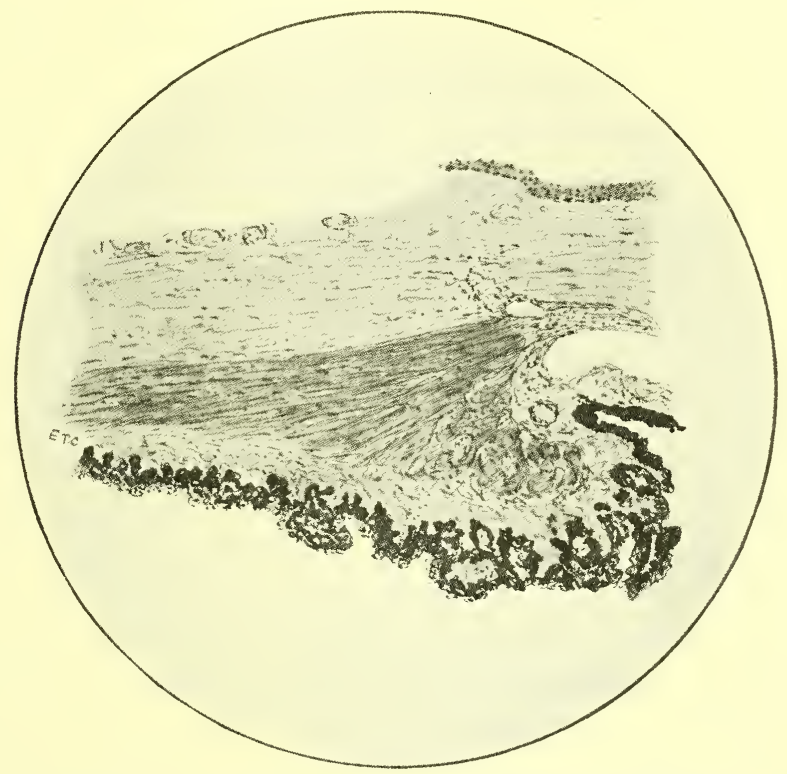

FIG. 7I.-The ciliary muscle in a hypermetropic eye.

they are situated. Hence most patients with axial myopia become conscious of floating opacities in front of their eyes. In time the strands of the matrix moving about in the fluid become entangled, and collect into web-like masses, which are not only seen by the patients but are visible ophthalmoscopically.

The ciliary muscle in eyes with axial myopia is flatter than normal and has scarcely any circular fibres (or muscle of Müller) (Fig. 70). In hypermetrophia, on the other 
hand, there is usually a marked development of these circular fibres (Fig. 7I).

A myope who reads at his far point does not use his accommodation, a hypermetrope, on the other hand, requires to use his accommodation to an excessive amount; we should expect therefore to find the ciliary muscle atrophied in the former and hypertrophied in the latter.

The direction in which the fibres of the ciliary muscle run would seem, however, to be largely influenced by the length of the anterio-posterior axis of the eyeball. When this is abnormally increased, as in axial myopia, the uveal tissue is considerably stretched, the ciliary processes are drawn backward, and the spaces in the tissue immediately external to them are also drawn backward and elongated. The fibres of the ciliary muscle, which are developed from cells lying in these spaces, likewise become altered in direction, and assume a longitudinal position anteroposteriorly instead of running circularly. In hypermetropia, where the eyeball is abnormally short from before backward, and not fully developed, the ciliary processes, as in the embryonic condition and as in the lower animals, project farther forward and inward, so that the spaces external to them, and the muscle fibres contained therein, have a circular direction.

In myopia the anterior chamber is usually deep and in hypermetropia shallow; the difference in the two conditions is in part attributable to the condition of the ciliary muscle, and in part to the consistency of the vitreous humour. An excess of circular fibres displaces the ciliary processes inward and these hold the base of the iris forward, which, in their absence tends to recede. A fluid vitreous does not form such a substantial support to the lens posteriorly as one of the normal jelly-like consistency, hence in myopia the lens tends to drop backward, and in high degrees both it and the iris may be slightly tremulous.

Axial myopia is often hereditary but rarely a congenital defect; it usually first attracts attention at the school age, 
and tends to increase with lessening rapidity up to the end of the second decade of life, after which it remains stationary. Very high degrees may go on increasing to later in life, such cases have been termed malignant myopia. In the rare congenital cases no crescentic atrophy of the choroid is found at the margin of the disc.

At birth the human eye is hypermetropic, the changes which take place in it to produce the emmetropic condition are the result of post-natal development. Growth is most rapid during the first years of life, and then decreases in rate, showing again increased activity from the I4th up to the 2 oth year. The rapid growth which takes place soon after birth affects mainly the anterior segment of the globe, the part in front of the insertions of the recti muscles. This expansion in the ciliary region, through the suspensory ligament, draws on the sides of the lens tending to flatten it in its then plastic state, and so reduces the amount of the hypermetropia. It is still further reduced, and ultimately obliterated altogether, by the increase in the anterioposterior axis of the eyeball.

The increase in growth of the eye which takes place after the I 4 th year is entirely due to enlargement of its posterior segment, that situated behind the insertion of the recti muscles. As the growth decreases there is a corresponding increase in the toughness of the fibrous tissue of the sclerotic, and an increase in the number of elastic fibres.

Axial myopia is due to some aberration in this late postnatal development of the posterior segment of the eyeball, corresponding with it in the time of onset, and usually ceasing to increase when the period of development is complete. In the malignant cases the sclerotic has probably become so thinned that the natural toughening process does not suffice to arrest its further expansion.

A tendency to undue expansion, or to some delay in the toughening process in the sclerotic are often hereditary conditions, but there are also other conditions which aid in the production of axial myopia. It is not met with in 
savage races, and is most prevalent in those who during the period of growth have been engaged in prolonged application to near work. In high degrees of myopia abstention from all near work will often arrest altogether its tendency to increase, and restricted near work in the lower degrees will suffice to produce the same result.

There has been much speculation as to how close application to near work produces an increase in axial myopia. Several theories have been put forward, and whilst some have been definitely disproved, of the others, no one can be said to be entirely satisfactory.

The bulging at the back of the globe is certainly not due to a sclero-choroiditis posterior, as was at one time thought to be the case, the changes met with in the choroid in myopia are the result of stretching and not inflammation. It can not be associated with the act of accommodation, for a myope of 3.5 dioptres will read at his far point of I 2 inches, and does not require to use his accomodation, moreover, there is much experimental evidence to show that the tension of the eye does not become increased during accommodation.

The theories which associate the bulging with the act of convergence would appear to have a more substantial basis. During convergence the posterior pole of the eyeball is displaced outward, and therefore becomes pulled upon by the optic nerve, which is attached a little to the inner side; the amount of traction would be increased where the nerve was comparatively short. Such traction it is suggested would account for the backward prolongation. It is difficult, however, to see why, under such circumstances, the greatest prominence should be to the outer side of the nerve and not at the nerve itself.

During convergence several of the extraocular muscles are brought into use, not only the internal recti. During the act of reading the eyes have to make excursions from one side of a line of print to the other and be kept steady all the time; this necessitates both the recti and oblique muscles 
being called into action. The two oblique muscles encircle the equator of the globe and the recti muscles, in spite of the check ligaments, may tend to compress its sides; the posterior part of the eyeball, the bulging of which produces axial myopia, is not touched by the muscles. Careful tonmetric measurements on a series of $\operatorname{cases}^{1}$ has shown that during convergence the intra-ocular pressure becomes increased in amount varying from $2 \mathrm{~mm}$. I0.5 mm. Hg., the mean being $4.9 \mathrm{~mm}$. Hg. It seems likely, therefore, that compression by the extraocular muscles may be an exciting cause.

A modification of the compression theory is that which accounts for the condition by congestion of the choroidal vessels, from obstruction to the vortex veins which emerge beneath the tendons of the oblique muscles. It is, however, doubtful if congestion of the choroid would result in softening of the sclerotic so as to cause an ectasia.

Hypermetropia.-Hypermetropia is the term applied to the refractive condition of the eye in which parallel rays of light falling on the cornea are brought to a focus behind the retina. Such a condition may be brought about by:

i. Flattening of the cornea decreasing its normal degree of curvature.

ii. Flattening of the lens anterio-posteriorly, decreasing its amount of refractive power.

iii. Absence of the lens, either by its removal from the eye, or by its displacement out of the line of vision.

iv. Displacement forwards of the back of the eyeball, either by an effusion or a new growth in the orbit.

$\mathrm{v}$. An abnormal shortness in the anterio-posterior diameter of the eyeball.

The first four conditions may be produced as the outcome of disease. By far the commonest cause of hypermetropia is the last named condition, which is due to an imperfect development of the eyeball.

${ }^{1}$ M. L. Hine. Trans. Ophth. Soc. of U. K., XXXV, I9I6, 230. 
The imperfect growth in hypermetropia is mainly in the sclerotic, the retina and choroid attaining their normal dimensions. This inequality of growth in the different tunics produces certain characteristic features in hypermetropic eyes. The retina does not lie quite smoothly on the inner surface of the globe, being imperfectly flattened out. Its inequalities catch the light and give rise to the condition of watered silk retina already described (see page I 9). The opening in the sclerotic through which the optic nerve passes being abnormally small, the nerve fibres become closely packed together, so that hardly any central depression or physiological cup is formed, and sometimes the condition described as "pseudoneuritis" is produced (see page 34 ). The choroid extends close up to the margin of the optic disc, and is not dragged away from it so as to produce the appearance of a crescent or a cone, as in myopia. Occasionally in addition to the defective development causing hypermetropia there is a small congenital coloboma of the choroid at the lower margin of the optic disc which must not be mistaken for a myopic crescent (see page ı 8 ).

In describing myopia it was mentioned that the ciliary muscle in hypermetropic eyes has a marked development of the circular fibres of Müller on its inner surface, this is largely due to the spaces in which they lie not having become lengthened out longitudinally, by stretching of the uveal tract on elongation of the eyeball. This marked development of the circular fibres causes the ciliary processes to project inwards behind the base of the iris, so narrowing the circumlental space.

The narrowing of the circumlental space in hypermetropic eyes, and the pressure which the ciliary processes are able to exert on the root of the iris if congested and swollen, predispose such eyes to glaucoma.

The incompletely developed eye of the newborn child is nearly always hypermetropic, the mean amount met with having been estimated by different observers as 2.3 diopters and 3 diopters. Under normal conditions 
during the first decade of life the hypermetropia curve rapidly falls. When hypermetropia exists as a permanent condition it is due to some failure in the post-natal development.

Albinism.-Albinism may be defined as a general deficiency in the development of pigment throughout the body, both in mesoblastic and epiblastic tissues. Thus in the eye there is defective deposition of pigment, both in the mesoblastic stroma of the uveal tract, and in the epiblastic outer epithelial layer of the retina.

Varying degrees of albinism occur, and in the same case different degrees of defect are met with in different parts. Sometimes the hair and skin are chiefly affected and sometimes the eyes. Within the eye itself different degrees of defect are met with in its different divisions. In albinotic rabbits' eyes the complete absence of pigment from all the tissues has been proved microscopically. In an albinotic human infant's eye no trace of pigment could be detected; but in all the adult human albinotic eyes, which have so-far been examined microscopically, some traces of pigmentation have been discovered. Pigment has been found present in the retinal epithelium when it was entirely absent from the stroma of the iris, ciliary body and choroid. Pigment may be entirely absent from the stroma of the uveal tract except in the choroid at the macular region. Infants who at birth appear not to have any pigment, in either eyes or hair, may develop some in both structures as they grow older.

The acuity of vision in albinotic eyes is defective, but there is no limitation of the field of vision, or any difficulty in the perception of colours. The defect of form sense has been found due to imperfect development of the fovea centralis (vide page 37); it causes the onset of nystagmic movements, and also, very frequently, of myopia. The patients are always very photophobic, and try to lessen the amount of light entering their eyes by keeping them screwed up. A red reflex is seen from the 
pupil and also through the iris. Ophthalmoscopically the network of the choroidal vessels can be seen most distinctly, especially toward the periphery of the fundus where the yellow reflex from the sclerotic shows between them. It is the presence of pigment in the branching cells of the choroid which gives rise to a uniform red reflex of the fundus, and also to the dark streaks between the vessels which are seen in people of dark complexion.

Melanosis of the Eye.-Melanosis of the eye consists of excess of pigment in those parts in which it is found normally situated in small quantities. It is therefore just the reverse of albinism.

A very deeply pigmented condition of the tissues of the eye, which is normal in negroes, is to be regarded as an aberration in development when it appears in the white races.

The patches of deeply pigmented embryonic tissue, termed "nevi" or "moles," which are met with in the conjunctiva and uveal tract, are different from the condition which is described here. So also are the congenital pigment patches in the retina due to adhesion of the two layers of the secondary optic vesicle (see page 27 ).

In the conjunctiva a little pigment is always present at the limbus, when this is increased in amount it gives rise to a black ring surrounding the cornea. In melanosis, patches of pigment are seen in the sclerotic, especially at the points where it is perforated by the anterior ciliary arteries. Microscopically the pigment is found in the episcleral tissue and the lymphatic sheaths around the blood-vessels.

The iris is of a dark brown colour. When the condition is unilateral, as it frequently is, the contrast between the colour of the two irides forms a striking feature-heterochromia iridis.

Microscopically the excess of pigment in the stroma of the uveal tract may be so great as to obscure the arrangement of its structure. The reflex from the fundus seen 
ophthalmoscopically is of a chocolate brown colour instead of a rose red, and the shimmering light reflexes of the retina are very numerous.

Some of the inner fibres of the lamina cribrosa represent the stroma of the choroid; it is asserted that pigment cells are normally met with amongst them. It is in keeping with this that in melanosis pigment patches may be present in the optic disc.

Out of 26 recorded cases of melanosis of the eye no less than 7 developed melanotic sarcoma, ${ }^{1}$ either extra-bulbar or of the choroid. In several of the recently recorded cases of this affection abnormal elevations have been observed scattered for the most part uniformly over the anterior surface of the iris, but showing no signs of taking on any malignant activity. In one such case in which sections of the iris were examined microscopically, the thickening was found to be due to hyperplasia of the surface endothelium (Fig. 72). It would seem, therefore, that melanosis of the eye may not infrequently be associated with melanotic new growths, innocent or malignant.

In the eyes of the white races of mankind, at birth the mesoblastic tissue of the irides is devoid of pigment, and they are of a slaty blue colour. In the dark races, ${ }^{2}$ however, pigment is found in the mesoblastic tissue of the irides considerably before birth, so that their infants are born with brown eyes. It has been noted, that in eyes affected with melanosis, the irides appear brown at birth like those of the darker races, so that the affection is a truly congenital one. In the white races pigment makes its appearance in the stroma of the iris after birth, but varies considerably in amount in different individuals according to their complexion. When examined microscopically it is rare, except in a case of albinism, to find an iris in which the stroma is entirely free from pigment granules, and all gradations in the degree of pigmentation may be met with. It is not,

${ }^{1}$ G. Coats. Trans. Ophth. Soc. of the U. K., XXXII, I912, I65.

${ }^{2}$ C. H. Usher. Biometrika, XIII, I920, I. 
therefore, possible to divide individuals up into two classes ${ }^{1}$ according to the colour of their eyes; those in which pigment is present only in the epiblastic layers on the back of the iris, which have been described as simplex; and those

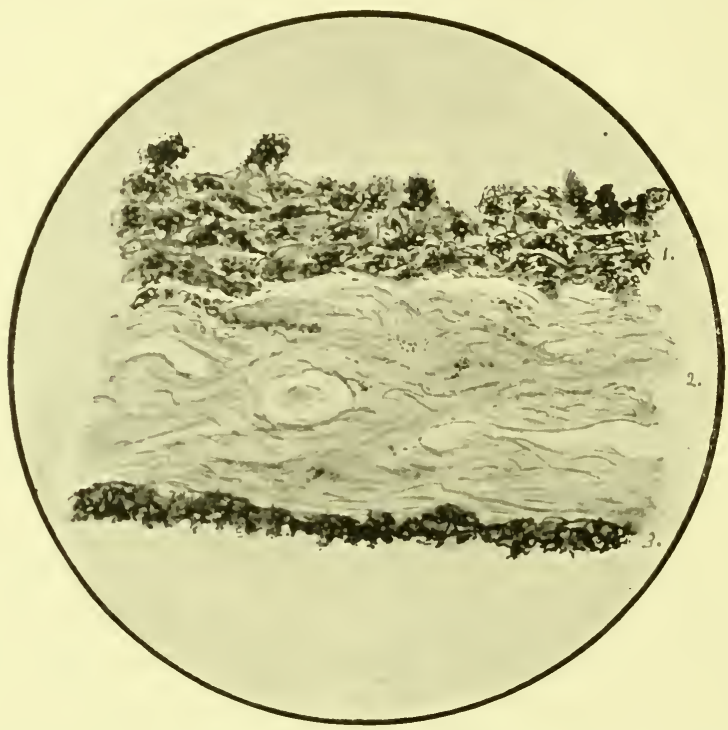

Fig. 72.-Thickened pigment endothelium on the surface of the iris in a case of melanoma. Case recorded by Alex. W. Stirling. Trans. Ophth. Soc. of the U. K. XL, I920, I43.

in which it is situated in both epiblastic and mesoblastic structures, which have been described as duplex. They are not clearly defined "unit characters," and to use them, as has been done, ${ }^{2}$ in an attempt to work out the laws of Mendelian inheritance must necessarily prove fallacious.

\section{ABERRATIONS CONNECTED WITH STRUCTURES IN THE LINE OF THE ORBITO-NASAL FISSURE, THE FORMATION OF THE EYELIDS AND THE ORBITAL MUSCLES}

In the centre of the face of the human embryo at the fourth week there is a cavity called the oral cavity out of which five fissures radiate. An upper pair, the orbitonasal,

${ }^{1}$ R. Galloway. Biometrika, VIII, I9II, 3 I4.

${ }^{2}$ Proc. of the Roy. Soc., LXXX, 1908, 85. 
a lower pair, the mandibular, and a vertical one, the intermandibular.

The upper pair radiate upward and outward between a central fold, which grows down from above, the frontonasal plate, and processes which extend in from either side, the maxillary processes. The outer parts of these fissures ultimately remain as the orbits, the inner parts become closed by fusion of the maxillary processes with the frontonasal plate, which takes place about the sixth or seventh week.

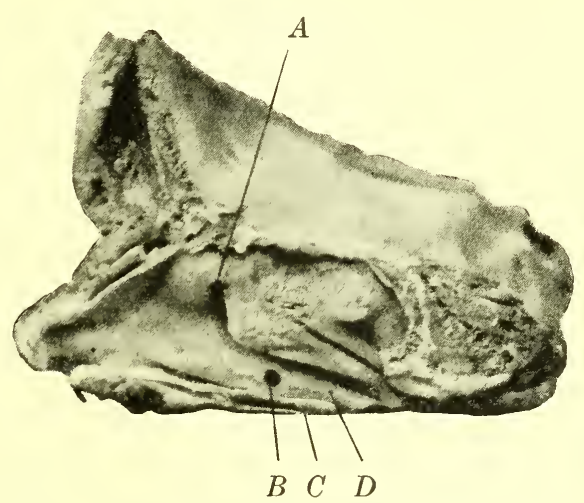

Fig. 73.- The outer wall of the nose in the new-born child. $A$ is placed over the situation of the upper end of the duct; $B$, over the lower end; $C$, the inferior turbinate bone which presses both on the floor of the nose and the lower end of the lacrymal duct; $D$, the floor of the nasal cavity. M. S. Mayou, Roy. Lon. Ophth. Hosp. Rep., XVII, I908, 246.

The lacrymal canals are developed from the epithelium lining the inner part of the frontal nasal fissure. A thickened strand forms which gradually becomes separated from the surface and surrounded by mesoblastic tissue. This strand, which ultimately becomes the nasal duct, is at first a solid cord of cells without any lumen. It is from the upper part of this cord that the canaliculi are developed, commencing as out-growing sprouts, which, by the end of the third month come into contact with the surface epithelium. ${ }^{1}$ From the upper extremity of the primary epithelial cord sprouts out, what ultimately becomes the cephalic

${ }^{1} \mathrm{~J}$. Parsons Schaeffer. The Am. Jour. of Anat., XIII, I9I2, I. 
end of the lacrymal sac. The lumen of the duct first makes its appearance in the upper part; the point of coalescence between the nasal duct and the mucous membrane of the inferior nasal meatus is the last to become patent, the lumen here is established approximately "at term" or even later Fig. 73 .

Owing to the lateral position of the eyes at the sixth month, the facial clefts, in which the lacrymal ducts and sacs are formed, are situated further forwards than the eyes, resembling at this stage of their development the condition found in lower animals. The last part of the facial cleft to become closed is in the region of the lacrymal sac. Bone is first deposited on the inner surface of the duct and around its lower end.

In the human fetus the formation of the eyelids commences about the eighth week, previous to that time the surface of the eye is directly continuous with the surface of the head. They appear first as ridges of mesoblastic tissue covered with epiblast which bud out above and below the cornea. Two weeks after their first appearance they have grown to such an extent that their margins meet over the front of the globe; the epithelium along their borders then becomes united, and the conjunctiva is for a time a closed sac, lined by a single layer of cells. At the inner surface of the eyeball a vertical fold forms inside the other two, which in man becomes the plica semilunaris.

While the epithelial bond of union at the margin of the lids exists, processes of cells grow down from it into the mesoblast beneath, from which are formed the hair follicles of the eyelashes and the Meibomian glands. In the human fetus the epithelial union breaks through and the lids become separate again before birth. This separation commences anteriorly on the growth of the eyelashes about the fifth month. The uniform curve which the lashes acquire outward is caused by their impinging, in their growth forward, on the edge of the opposing eyelid. 
The extrinsic muscles of the eyeball have been observed in man to arise from a common pre-muscle mass, first recognisable in an embryo of $7 \mathrm{~mm}$. in length. Cleavage takes place in this mass, and by the time the embryo has reached I4 mm., most of the muscles can be differentiated in the positions which they normally occupy in relation to the eyeball in the adult. The levator palpebræ superioris does not become separated off from the superior rectus until much later. The internal rectus attains maturity sooner than the external.

Many mammals, unlike man, have besides the four recti and the two obliques, another muscle, the retractor bulbi, which arises at the apex of the orbit inside the cone of the recti muscles, and is inserted into the sclerotic inside the insertion of the recti. From the cave-like fashion in which it encircles the optic nerve is often spoken of as "the Choanoid muscle." It draws the eyeball back in the orbit, and attains its greatest development in the Ungulata, in whom the movements of the third eyelid are more active than in other mammals.

The aberrations met with in connection with structures in the line of the orbito-nasal fissure, the formation of the eyelids, and the orbital muscles are:

Failure in union of the maxillary processes with the nasal portion of the fronto-nasal process-coloboma of the eyelid-absence of the lacrymal canal.

Failure in downgrowth of the frontal-nasal process on one side-lateral proboscis or demicyclops.

Imperfect bone formation in the frontal-nasal processepicanthus.

Imperfect development of the orbit-congenital exophthalmos.

Protrusion of contents of cranial cavity into the orbital portion of the fissure-orbito-meningo-encephalocele.

Sequestration of a portion of the surface epithelium along the line of the fissure-dermoid cyst. 
Defective closure at the outer angle of the fissurehernia of orbital fat-notches in lower lid at outer canthus.

Imperfect budding out, and canalisation, of the epithelial processes from which the lacrimal passages are developed. defective canaliculi-congenital lacrymal obstructionlacrymal fistula.

Failure of the eyelids to bud out in the whole or a portion of their extent-cryptopthalmia - coloboma of the eyeliddermoid growths of the eye.

Failure of the eyelids to unite over the front of the eye - microblepharon - congenital lagophthalmia-dermoid growths of the eye.

Undue development of the third eyelid-abnormally large plica semilunaris.

Formation of a persistent band uniting the two eyelidscongenital ankyloblepharon.

Perversion in the direction of the eyelashes-congenital trichiasis.

Formation of eyelashes in the place of Meibomian glands - congenital distichiasis.

Defective development of the levator palpebræ superioris muscle-congenital ptosis-associated movements of the eyelid and jaw.

Defects in development of the extrinsic muscles of the eye-congenital ocular paresis-congenital enophthalmos - heterophoria-concomitant strabismus.

Coloboma of the Eyelid.-A coloboma occurs more frequently in the upper than the lower eyelid. Whether in the upper or the lower lid, it is always situated to the inner side of the middle line. Both lids of the same eye may be affected. The inner third of one lid may be entirely absent; there may be a quadrate or triangular gap extending from the palpebral to the orbital margin (Fig. 74) or only a small indentation of the free border of the lid (Fig. 75). The part of the eyeball left uncovered by the defective eyelid is often the seat of a dermoid growth (see page I4I). 
The abnormality is frequently associated with other congenital defects about the face, attributable to defective development in the line of the facial fissures, such as hare-lip,

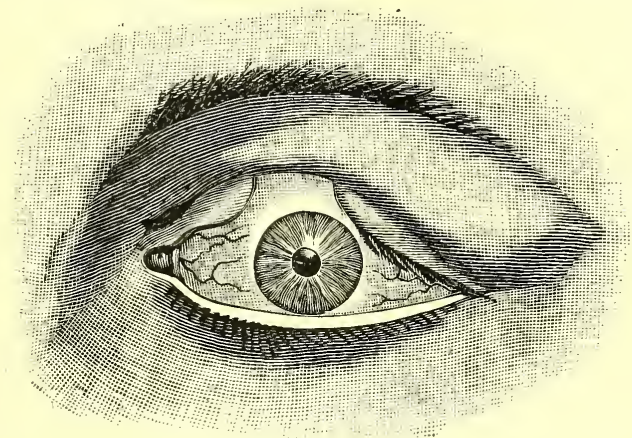

FIG. 74.-Congenital coloboma of the upper eyelid in a boy aged two years.

cleft-palate, macroglossia, supernumerary auricles, etc. Many of such defects are due to pressure caused by bands in the amnion. Some cases of coloboma of the eyelid may be

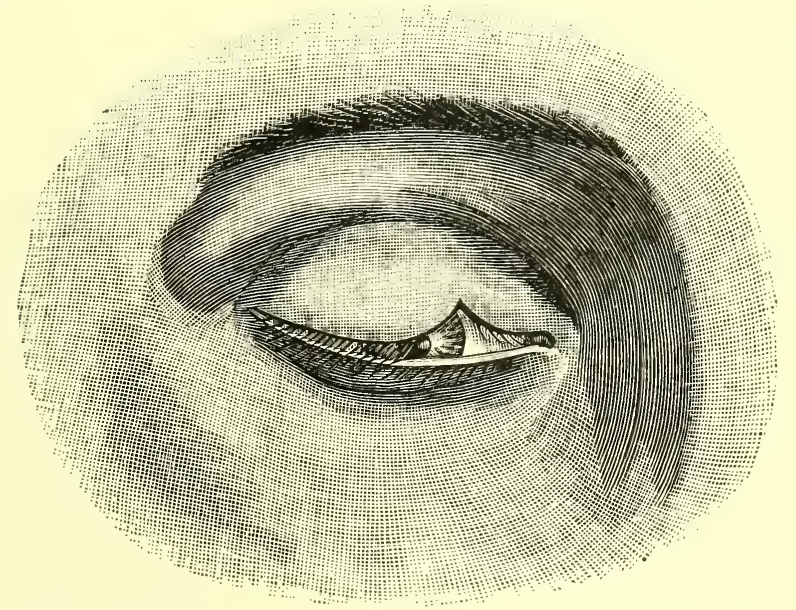

FIG. 75.-Small congenital indentation in the border of the upper eyelid.

best accounted for by pressure of such amniotic bands along the line of the orbitonasal fissure, interfering with its closure. There may, however, be other causes leading to 
defective or delayed closure of this fissure which will give rise to the same condition.

Absence of the Lacrymal Canal.-In sharks there is an open skin area between the nose and the eye. The nasal duct appears first amongst the amphibia.

In some very malformed fetuses the nasal duct has been found represented by a wide groove lined by mucous mem-

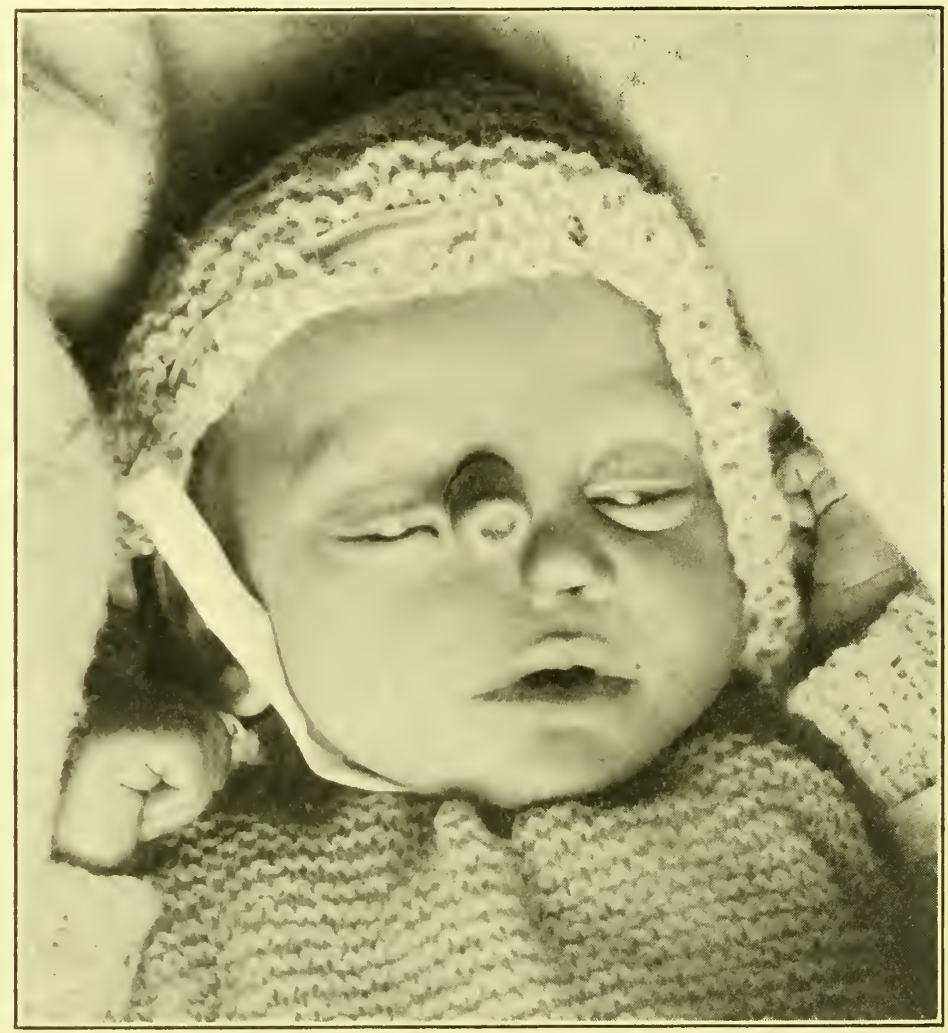

FIG. 76.-Case of lateral proboscis a "Demicyclops," recorded by Philip Franklin, Proc. Roy. Soc. of Med., Laryn. Sect. XIV, I92I, 48.

brane shelving off from the inner angle of the eye into the nasal cavity, a condition comparable with that met with in sharks, and attributable to failure in the union of a maxillary process with the nasal part of the frontal-nasal process. 
Lateral Proboscis or Demicyclops.-Children presenting this rare form of malformation seldom survive birth, but may occasionally do so. A tube or proboscis, resembling somewhat a penis, is found projecting forwards from the upper and inner angle of the orbit (Fig. 76). The central canal can be traced to the depths of the orbit, its secretion has been described in different cases as salty, mucous, or sebaceous material. The nasal bone on the affected side has been found absent, also the premaxilla and the lacrymal passages. The proboscis itself consists of the soft parts of the nose, the hard parts being undeveloped. The condition is due to the failure in downgrowth of the frontalnasal process on one side, the maxillary process uniting in beneath, and leaving it projecting above in the form of a proboscis.

Epicanthus.-In the condition termed epicanthus, which is nearly always bilateral, a crescentic fold of skin, with its concavity outwards, overhangs the inner canthus. It occurs in connection with marked defect in the development of the bridge of the nose, and is not uncommonly associated with congenital ptosis and defective movement of the eyes upward. The fold, when large, covers a portion of the inner side of the globe producing an appearance resembling convergent strabismus.

Congenital epicanthus is due to defective development of the bones in the fronto-nasal plate which form the bridge of the nose. If the skin covering the bridge of the nose is pinched up the epicanthus disappears. It is quite common to see it in infants, and for it to disappear as the bridge of their nose becomes formed. It may be met with in children the subjects of congenital syphilis who have had their nasal bones necrose from that disease. It is a characteristic of the mongolian races to have flat noses, and in them epicanthus is a normal feature. In the form of idiocy known as "mongolian idiocy," the characteristic appearance of the face is a flattened bridge to the nose with epicanthus. 


\section{Congenital Shallowness of the Orbits and Exophthalmos.} An imperfect development of the orbits causing them to be abnormally shallow is met with in oxycephaly or "tower skull," an affection in which the skull is unusually high; the average height of four such skulls in the Heidelberg Museum was found ${ }^{1}$ to be $143 \mathrm{~mm}$. while that of Ioo normal skulls was I 3 I. $6 \mathrm{~mm}$. The average depth of these orbits was $28.4 \mathrm{~mm}$. as against the normal depth of 39.05 $\mathrm{mm}$. The superciliary ridges are flattened, and in some cases there is a marked protrusion in the temporal regions with bowing outward of the zygomatic arches. Males are much oftener affected than females. The condition has been attributed to premature ossification of the coronal sutures. $^{2}$

As a result of the shallowness of the orbits exophthalmos is produced, which is sometimes so extreme that the eyelids fail to close over the eyes, and keratitis ensues. The roof of the orbit may form an angle of $45^{\circ}$ with the floor. From deformity about the optic foramen affections of the optic nerve are liable to occur, such as optic atrophy, and papilledema with ensuing atrophy.

Orbital Meningo-encephalocele.-A hernia of the cranial contents may be attributed to some arrested development of the bony walls of the orbit along the line of the orbitonasal fissure, or to some increased intracranial pressure during their formation.

The commonest site in the orbit for such a hernia is at the upper and inner angle, the cranial contents then protruding through the fronto-ethmoidal suture. An enlarged sphenoidal fissure or the optic foramen have also been found to be the channels of exit. The increase in the contents of the orbit, to which the hernia gives rise, presses on the eyeball, causing exophthalmos and sometimes defects in ocular development.

${ }^{1}$ Velhagan, Munich, medizin, Wochensch., I904.

${ }^{2}$ W. M. Beaumont. Trans. Ophth. Soc. of the U. K., XXX, I9Io, 44. 
In nearly all the cases which have been examined anatomically, besides a protrusion of the meninges a protrusion of brain tissue has been found; it is doubtful if a meningocele alone apart from an encephalocele ever occurs. The protrusion forms a fluctuating cyst in which pulsation synchronous with the respirations is sometimes observed. The fibrous tissue of the skin covering the swelling is continuous with the dura mater; the arachnoid and pia-mater are generally too much stretched and atrophied to be recognisable. The fluctuation of these cyst-like swellings is due to the presence of cerebro-spinal fluid. When, through some mistake in diagnosis, one of these protrusions has been opened, cerebro-spinal fluid has continued to drain away until convulsions and death ensued.

\section{Dermoid Cysts along the Line of the Orbito-nasal}

Fissure.-Dermoid cysts form smooth rounded tumours beneath the skin, which is freely movable over them. They may be of various sizes, usually about that of an acorn; they often grow rapidly at puberty causing the patient's attention to be first attracted to them then, though they are always congenital. There is often a depression and sometimes a complete absence of the bone beneath them, in the latter case the cyst lies in direct contact with the dura mater.

They may be situated in various parts of the orbitonasal fissure. The commonest position is near the upper and outer angle of the orbit, overlying the fronto-malar or fronto-temporal suture. They are also frequently found at the upper and inner angle of the orbit overlying the frontoethmoidal suture. They have been discovered lying at some depth in the orbit external to the cone of muscles, also in rare instances on the side of the nose in the line of the lacrymal canal.

The characteristic histological feature of these cysts is a lining of laminated epithelium with hair follicles, sebaceous glands, and sometimes sweat glands in connection with it. The surface of the epithelial lining, like that of the skin, is 
usually papillated, but when the cyst is much distended it may become flattened out and thinned.

The contents of these cysts is composed of sebaceous material, epithelial débris, and hairs. In rare cases a tooth has been found. In the outer fibrous tissue wall muscle fibres, and sometimes plates of cartilage, may be present.

Though dermoid cysts are often met with in the course of the fetal clefts about the face they are also found in other positions, for instance they occur on the vertex of the skull. A more comprehensive statement would be, to say that they are found opposite the lines of suture of the cranial bones during fetal life.

Early in embryonic life the skin covering the head and the dura mater are in contact, on the formation of the cranial bones the two become separated, but remain longest in contact along the lines of the sutures. Should a piece of the skin adhere to the dura mater it might become cut off, with some of its epithelium, and sequestered, so forming the starting point of a cystic growth. Where a cyst is situated the formation of bone may be interfered with, hence the occurrence of depressions in it beneath the cysts.

Congenital Hernia of Orbital Fat.-The orbital fat may be divided into, the central part situated within the cone of the muscles at the back of the eyeball, and the peripheral part situated outside the cone of the muscles, between them, and behind the lacrymal gland. It is this latter part which may extend forwards on the surface of the eyeball, as a hernia-like projection, through some congenital deficiency in Tenon's capsule and the expansion of the muscle sheaths, which normally holds back the fat between the globe and the walls of the orbit. The condition presents clinically the appearance of a smooth, yellow coloured, raised mass on the surface of the sclerotic. It is usually situated between the upper and external rectus muscles, sometimes also below the external rectus. When small it is hidden beneath the upper eyelid, but when large may 
protrude below; it becomes most exposed to view when the eye is rolled inwards.

These herniæ have generally been described as fibrofatty growths of the conjunctiva, there real nature becomes manifest on attempting to remove them. It is then found that they are not localized growths, but are continuous

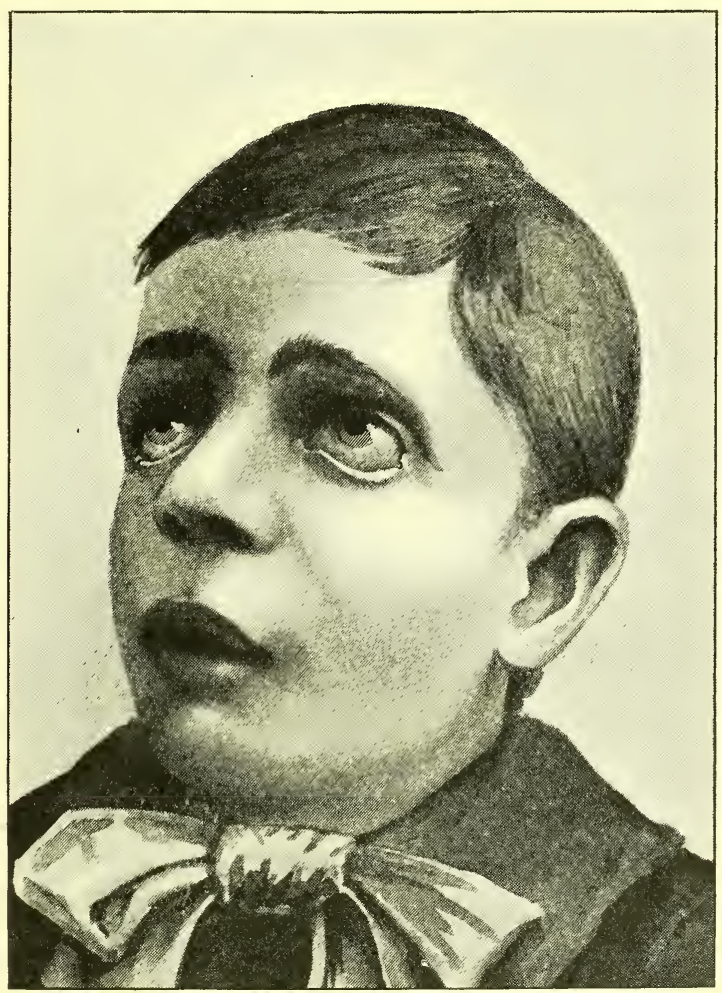

FIG. 77.-Symmetrical congenital notches in the lower lids near the outer canthus and flattening of both cheek bones.

with, and really prolongations forward of orbital fat. The peripheral fat of the orbit consists of large firm masses containing a quantity of connective tissue; it is the presence of a large quantity of connective tissue in these fat herniæ, which has led to them being described as fibro-fatty in consistency. They are often associated with congenital 
dermoid growths of the eyeball and colobomata of the eyelids, and are attributable to some congenital defect in the septum which holds back the fat at the outer extremity of the orbito-nasal fissure.

Congenital Notches in the Lower Lid at the Outer Canthus. ${ }^{1}$ - These cases, which are entirely different from the colobomata of the eyelids already referred to, are also manifestations of some defect in closure of the orbito-nasal fissure at its outer extremity.

The notch is always small though in some cases deeper than others. It often suggests from its appearance a duplication of the outer canthus (Fig. 77). The defect in the lid is associated with a flattening or want of prominence of the malar bone. This flattening of the cheek bones, when the condition is bilateral, as it usually is, gives the patient a very characteristic physiognomy, and causes all those affected to resemble one another so that they might readily be taken for members of the same family. This defect of the eyelid may be accompanied by dermoid growths of the cornea, congenital hernia of the orbital fat and trichiasis of the lower eyelid.

Abnormalities in the Lacrymal Passages.-Congenital abnormalities connected with the lacrymal passages may be met with in any part of their course. They may be divided into those connected with; (i) the canaliculi; (ii) the naso-lacrymal duct; (iii) the nasal ostium.

i. The puncta may be absent or multiple, two, three and even four have been noted in connection with one canaliculus. One or both canlaiculi may be absent; or a super-numerary one may be present, opening sometimes into one of the normal canaliculi and at others directly into the lacrymal sac.

These abnormalities may be accounted for by irregularities in the sprouts which bud out of the upper part of the column of epithelium. from which the nasal duct is formed,

${ }^{1}$ Treacher Collins. Trans. Ophth. Soc. of the U. K., XX, I900, 190, and $19 \mathrm{I}$. 
or in the establishment of their connection with downgrowths from the free bodies of the eyelids.

ii. Atresia of the lower part of the duct, where it joins the nose, is the commonest of all the malformations in connection with these passages. It manifests itself soon after birth by epiphora, or by the onset of secondary dacryocystitis, with the regurgitation of muco-purulent discharge through the puncta on pressure over the sac. The atresia in these cases is due, either to the failure of union between the column of epithelium, from which the duct is developed, with the up-growing epithelium of the nasal mucous membrane which forms the ostium, or, to imperfect canalisation of the column of epithelium which forms the duct. The nose together with the lacrymal ducts are before birth filled with epithelial debris, which normally become cleared away by aspiration, when the child commences to breath. When this plug remains impacted in the lower end of the duct congenital obstruction exists. ${ }^{1}$ One passage of a probe down the duct into the nose is generally sufficient to effect a cure in such cases. It is important to remember, however, that in rare cases the whole duct may be absent, due to some failure in the separation off of the column of epithelium from which it is formed, an atresia of which nature is much less amenable to treatment.

Diverticula and sinuses may form in the course of the duct, and in one case it was found to be double throughout.

iii. The ostium of the nasal duct, usually single, opens on the lateral wall of the inferior nasal meatus. A unique case is on record in which it opened into the middle meatus. Two or three orifices have been met with, and somtimes the ostium is found covered by a valve like fold.

Fistula of the Lacrymal Sac.-A congenital fistula of the lacrymal sac presents the appearance of a small dimple in the skin on the side of the nose a little below the level of the inner canthus. From the bottom of the dimple a pin-point

${ }^{1}$ M. S. Mayou. Royal Lond. Ophth. Hosp. Rep., XVII, I908, 446. 
aperture leads into the sac. ${ }^{1}$ The condition is frequently bilateral. It is due to incomplete closure of the facial cleft, the situation in which the fistula occurs, being the last part of the cleft to be shut off from the surface epithelium.

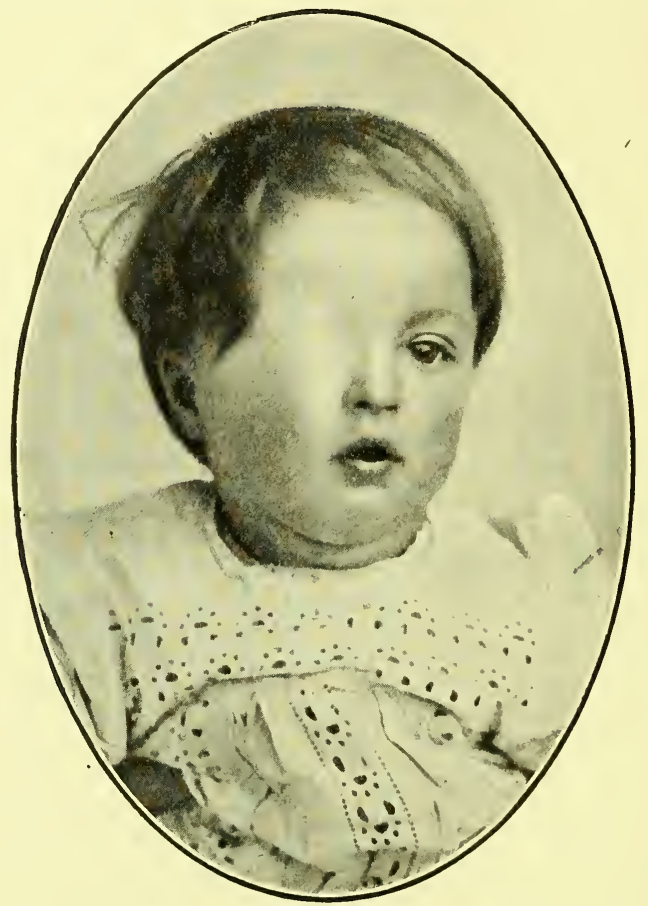

FIG. 78. - Boy aged 2 I months with unilateral cryptophthalmia. Case recorded by W. Sinclair, Trans. Ophth. Soc. of the U. K., XXXVIII, I9I8, I42.

Cryptophthalmia.-Cryptophthalmia is an exceedingly rare condition in which there is complete absence of the eyelids and their appendages, together with the lacrymal gland and lacrymal duct. The eyeball is entirely covered and hidden by skin (Fig. 78) beneath which it is seen to move about, and to which it is connected by a subcutaneous cellular membrane. On dissection the orbicularis muscle has been found present; a wrinkling of the skin about the eye due to its action may be noticed on exposure to

${ }^{1}$ N. Bishop Harman. Trans. Ophth. Soc. of the U. K., XXIII, I903, 256. 
bright light. The condition is generally bilateral, but unilateral cases have been met with. The eyeball itself is always malformed and other gross malformations are frequently present.

In these cases the eyelids have failed to bud out and no conjunctival sac has been formed. The conjunctiva is a portion of the surface-covering of the body, which becomes modified in structure by seclusion from external influences, through the union of the lids over the front of the eye. When the lids fail to grow out, the external covering of the eye is exposed to the same influences as other parts of the surface of the body, and assumes the same characteristics, viz., those of skin. There is an alteration in both the character of the epithelium and of the sub-epithelial mesoblastic tissues; the latter simulates the corium of the skin which is very different from the fibro-adenoid tissue of the conjunctiva.

An absence of the eyelids is the normal condition in some fish, but the surface of their eyes is kept moist by the fluid media in which they live.

Dermoid Growths.-In "cryptophthalmia," there is a dermoid or skin-like growth covering the whole of the surface of the eye. It is interesting to compare with this exceedingly rare condition the much commoner one in which the dermoid growth forms a small isolated patch, covering only a part of the surface of the globe.

It has already been mentioned, under coloboma of the eyelid, that the surface of the globe opposite the defect in the lid has frequently a skin-like covering. The dermoid growths which occur, unassociated with any malformation of the eyelids, consist of oval or circular isolated patches of skin on the surface of the globe situated at the margin of the cornea, a little below its horizontal meridian, more often on the outer than the inner side. There may be two growths on the same eye on opposite sides of the cornea. Usually only one eye is affected but sometimes symmetrical growths are found in the two eyes. In size, colour, and 
appearance, the growths often resemble a split pea, but may be much bigger and protrude through the palpebral fissure. Should they get nipped between the lids they become pedunculated. They often show a tendency to enlarge about puberty, at which time also hairs may start to grow from them.

Dermoid growths have been met with in the eyes of dogs, horses, sheep, oxen, and pigs as well as in man. In a sheep's eye, wool has been seen springing from the tumour.

Histologically, these dermoid growths present the characteristics of skin. Beneath a covering of laminated epithelium is dense fibrous tissue as in the corium, often mixed in the deeper parts with adipose tissue. In the corium are found yellow elastic fibres, hair-follicles, sebaceous glands, sweat glands and occasionally also glands of an acinous type like Krause's glands. Blood-vessels course through the growth and medullated nerve fibres have been demonstrated in them. The surface cells of the laminated epithelium are keratinised.

When the eyelids fail to bud out and the whole surface of the eye is left exposed, as in cryptophthalmia, it becomes covered with skin. When a portion of the surface of the eye is left exposed, as in coloboma of the upper lid, skin forms on the uncovered part. It seems probable, therefore, that when patches of skin form on the surface of the eye opposite the palpebral aperture, there has been some failure of union of the lids over the front of the eye in a portion of their extent which has left the affected part exposed.

A few rare cases of congenital staphyloma have been recorded in which the whole surface of the cornea was covered by a dermoid growth. In such cases the enlarged bulging cornea protruded through the palpebral aperture, this prevented the union of the lid margins in front of it, and skin was formed over the exposed area.

On the surface of the eyeball at its upper and outer quadrant, between the superior and external rectus muscles, congenital growths are sometimes met with containing 
plates of bone, hyaline cartilage, or acini-tubular gland tissue resembling the lacrymal gland. These growths are entirely in the conjunctiva being movable on the surface of the sclerotic, they sometimes have a cutaneous and at other times a mucous surface.

These congenital osteomata, enchondromata and adenomata, have been classed as teratomata, they are essentially aberrant developments of the corium, and it is better to regard them as an atavism of tissue. In support of this view is the fact that the corium of the skin in some animals is capable of producing cartilage and bone in the form of an exoskeleton.

Abnormally Large Plica Semilunaris.-The plica semilunaris of the human eye is a vestigial structure representing the membrana nictitans in other animals. The membrana nictitans consists of a plate of fibro-cartilage covered by conjunctiva, which when out of use lies tucked away at the nasal side of the orbit. It is best developed in birds who have two special muscles concerned in its movements over the front of the eye, known as the quadratus and the pryamidalis. The quadratus arises from the sclerotic above the optic nerve and forms a sling for the tendon of the pryamidalis to work in. The latter muscle arises from the nasal side of the sclerotic, and ends in a long tendon which passes through the sling, round the temporal side of the globe to the lower end of the membrane.

In crocodiles the nictitating membrane is transparent and has a powerful muscle for its movements. In the ophidia and some lacertili the third eyelid forms a permanent transparent covering to the eye, the conjunctiva being a closed sac, lined by single layer of epithelial cells, the animal seeing through the lid.

Among mammals, a nictitating membrane sufficient to sweep over the whole cornea is only present in the ungulata. In them its posterior border is continuous with the fat of the orbit behind the eyeball. The pushing forwards of the fat on the retraction of the globe, by the retractor bulbi 
muscle, causes the membrane with its thin, smooth, anterior border to pass in front of the cornea (see page $\mathrm{I}_{50}$ ).

An abnormally large plica semilunaris is sometimes found as a congenital defect in man. It may extend as far as the cornea and sometimes be capable of slight movement. Such cases would appear to be examples of atavism.

Microblepharon and Lagophthalmos.-The union of the lids at their margins for a time during fetal life seems essential for the eyelids to attain their normal length. When this union is not effected or separation takes place unusually early, either from the pressure of amniotic bands or some other cause, an undue shortness of the lids or microblepharon is liable to occur. In this condition there is an incomplete closure of the palpebral fissure when the eyes are shut, which is termed lagophthalmos. The lid margins can usually be brought together by a strong contraction of the orbicularis, but they remain open during sleep.

Ankyloblepharon.-The edges of the eyelids are united during several months of fetal life. In some animals (carnivora) the epithelial band of union persists until after birth, during which period the conjunctival sac is lined by a single layer of cells (Fig. 79) which does not become thickened until after separation of the eyelids. Ankyoblepharon, or union of the lid margins, is a rare form of congenital malformation, sometimes bands or strands of skin-like structure stretch between them. It has been met with in association with anophthalmia.

Trichiasis.-Congenital trichiasis is a simple inturning of the eyelashes without any bending in of the lid margin. That this is so is shown by the inter-marginal zone retaining its normal position. It is only met with in the lower lids. It is attributable to faulty union of the margins of the eyelids during fetal life, which prevents the lashes of the lower lid, as they grow, impinging against the border of the upper lid in such a way as to assume their normal curve outward (Fig. 80), and allowing them either to take a straight course or assume an inward tilt. 


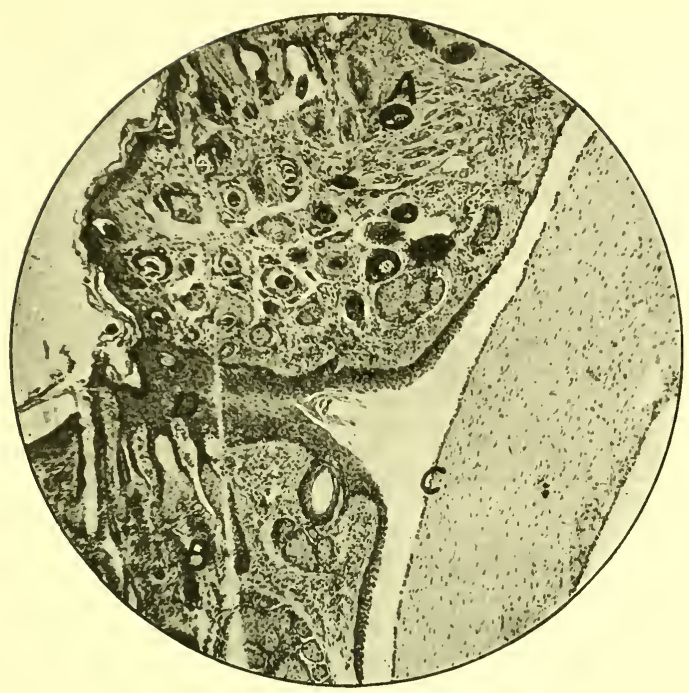

FIG. 79.-Eyelids of a cat three days after birth, showing separation commencing anteriorly and posteriorly by the degeneration of the cells, the middle portion being the last to yield.

$A$, Upper lid; $B$, lower lid; $C$, cornea covered by a single layer of epithelium; $D$, union of eyelids.

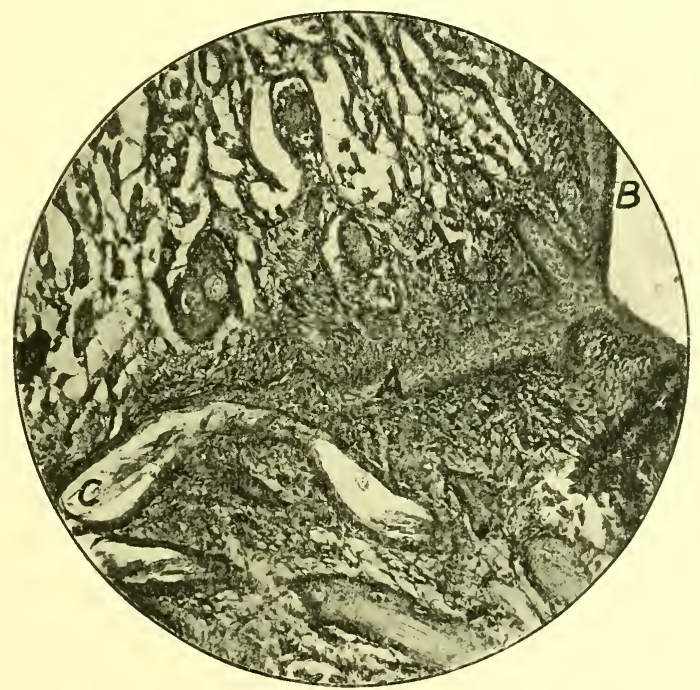

FIG. 80.-Line of junction of eyelids in human fetus of the fourth month II $\mathrm{cm}$. Shows curve produced by the turning forwards of the hair by the opposing lid.

$A$, Union of eyelids; $B$, conjunctival surface; $C$, hair follicles and Meibomian glands causing separation of the anterior part of the union. 
Distichiasis.--In congenital distichiasis there are two rows of eyelashes instead of one, the inner row being composed of fine hairs turning inwards toward the eye. Both upper and lower lids may be affected.

Histological examination ${ }^{1}$ of a lid in one case has shown complete absence of the Meibomian glands, their place being taken by the second row of hair follicles. There was also an over development of Moll's glands.

When the eyelids have their margins united, two rows of epithelial downgrowths form; the outer developing into the hair follicles of the cilia, and the inner into the Meibomian glands. In distichiasis both rows develop into hair follicles and no Meibomian glands are formed. It has been suggested that the hypertrophy of Moll's glands is due to an attempt on their part to supply the missing secretion.

Congenital Ptosis.-The levator palpebræ superioris is the last of the orbital muscles to become completely differentiated, and also the one which is most frequently ill developed. It may be absent altogether or have some of its muscle fibres replaced by fibrous tissue. If altogether absent, when care is taken to prevent the occipito-frontalis muscle coming into action, there is complete inability to raise the lid; if some muscle fibres are present, a slight upward movement may be produced. The condition may be either bilateral or unilateral. Patients suffering from the affection assume a very characteristic attitude. They throw their heads backward to see beneath their drooping lids, and by wrinkling their foreheads with their occipitofrontalis muscles so as to arch up the eyebrows, produce some elevation of the lids by traction on the skin.

Cases of congenital ptosis are very commonly associated with defective upward movement of the eyeball. As the levator muscle and the superior rectus muscle are, for part of fetal life, closely united, it is likely that any

${ }^{1}$ Kuhnt. Zeitsch. f. Augenheilk., I889, II, 46. A. R. Brailey. Trans. Ophth. Soc. of the U. K., I906, XXVI, I6. 
aberration affecting the development of one muscle would affect that of the other also. It may be, however, that the defective upward movement of the eyes in these cases is due to ill development of the superior rectus muscle from want of use; the inability to raise the lids doing away with any desire to elevate the eyes. In cases of unilateral ptosis, where the elevator muscles on the affected side are brought into play in association with those on the unaffected side, the upward movement of both eyes is generally unimpaired, this lends support to the latter view.

The levator palpebræ muscle besides raising the upper eyelid gives off fibres which are attached to the skin. It is this cutaneous insertion which gives rise to the superior palpebral fold which is such a great line of beauty in the face. When absent, as it is in cases of congenital ptosis, its value in this respect is realised for the patients then have a vacant stupid look. In several of the operations which have been devised for congenital ptosis attempts are made, not only to increase the power of raising the upper lid, but also to produce something which simulates the normal palpebral fold.

Associated Movements of Eyelid and Jaw.-In some cases of one sided congenital ptosis the drooping eyelid cannot be voluntarily raised, but it is vigorously raised when certain movements of the lower jaw occur. The movement is strictly limited to the levator palpebræ superioris muscle and cannot be controlled by the patient. In some cases the drooping eyelid is raised when the mouth is opened or when the jaw is directed to the opposite side, in other cases it is raised exclusively with only one of these movements of the jaw. It would seem, therefore, that this involuntary action of the levator is sometimes associated with contraction of the digastric muscles which depress the lower jaw, and sometimes with that of the external pterygoids which move the lower jaw from side to side.

In quite a large proportion of the cases of this abnormality the ptosis was noted to be on the left side, no case 
has been described in which the ptosis was bilateral, but cases have been met with of associated movement of the eyelid and jaw in which there was no ptosis present.

One hypothetical suggestion which has been made to account for these cases is, that there is an abnormal connection between the central nervous mechanism for the jaw muscles and the levator. ${ }^{1}$ The simplest explanation of this sort would be that some of the fibres of the levator palpebræ portion of third nerve arise, not from the nucleus of the third nerve, but from that of the fifth. No dissection has so far been made in any of these cases to prove or disprove this theory.

Another suggestion made is that the condition should be regarded as what is termed an "atavistic anomaly,"2 and to be in some way connected with the synchronous movements which take place between the jaw and the operculum during respiration in fish. Morphologically the orbicularis muscle is derived from the superficial musculature which forms the sphincter of the spiracle in fish, and the external pterygoids from the deep musculature connected with their maxillary cartilages. It is suggested that when the action of the levator palpebræ is weak the old-time associated action of these two musculatures becomes re-established. So that on movements of the jaw the orbicularis muscle becomes relaxed, allowing its weak antagonistic muscle, the levator palpebræ, to assume control and raise the eyelid. How it is that all cases of congenital ptosis do not show these associated movements is not accounted for by this theory.

Congenital Abnormalities in the Extrinsic Muscles of the Eye. Congenital Ocular Paresis.-The aberrations in development met with in the extrinsic muscles of the eye may be attributed to, imperfect or irregular cleavage of the pre-muscular mass, the formation of fibrous tissue in

\footnotetext{
${ }^{1}$ Marcus Gunn. Trans. Ophth. Soc. of the U. K., III, I88.3, 283.

${ }^{2}$ N. Bishop Harman. Trans. Ophth. Soc. of the U. K., XXIII, 1903, 356.
} 
place of muscle fibres, or to faulty insertion of the muscles on the surface of the sclerotic.

Some of the congenital abnormalities have been discovered in the course of anatomical dissections, having been unsuspected during life; others at post mortem examinations made on patients who have been known to have congenital defects in the movements of the eyes; whilst some have been detected during life in the course of operations performed for strabismus.

Except in cases in which there was gross defect of the nervous system, all recorded cases of congenital ocular paresis have been found to be due to developmental abnormalities in the ocular muscles.

Failure in separation has been met with of the superior and external recti, of the external and internal recti, and of the superior oblique and internal rectus. Muscular fasciculi have been found to pass from the superior to the inferior rectus, from the external to the internal rectus, and from external to the inferior rectus. In cases of congenital paralytic strabismus the rectus muscle on the affected side has been found either absent altogether or composed only of fibrous strands.

Congenital Enophthalmia.-In connection with congenital defects of the extrinsic ocular muscles displacement backwards of the eyeball in the orbit is sometimes met with, either as a permanent condition, or on attempted movements of the eye inwards. The symptoms met with in these cases may be enumerated a follows:

i. Inability to move the eye outwards.

ii. Impaired movement of the eye inwards.

iii. Either constant enophthalmos of the eye in its primary position, becoming increased on attempted movement of the eye inwards, or, if not present in the primary position, becoming manifest when such attempted movements are made.

iv. Narrowing of the palpebral fissure as the eye recedes, the amount of narrowing depending on the amount of recession. 
v. In some cases a forward movement of the eyeball, accompanied by widening of the palpebral fissure during attempts to move the eye outward from the primary position, i.e., just the converse of the two previous symptoms.

vi. In some cases, together with the retraction of the eye, an upward or downward turning of the cornea on attempted movement inwards.

A collection of the recorded cases of this abnormality shows that it is more often met with in females than males, and far more frequently on the left side than the right. In cases in which an anatomical examination has been made the external rectus consisted of a tendinous band, its muscle fibres being defective, and the internal rectus was inserted into the sclerotic abnormally far back; in one case it had a subsidiary insertion behind the normal one.

The capacity of translating the eyeball backwards and forwards in the anterio-posterior axis is a normal function in some mammals, for which a special musculature is provided. Burrowing mammals are able to retract their eyes below ground and protrude them when they come to the surface. Grazing mammals, whose safety depends on their swiftness of foot, are able to protrude their eyes on the approach of danger, so as to enlarge their field for circumferential vision; whilst they can retract them, and thereby move forwards their third eyelids, when feeding in long grass.

This translation of the eyeball is effected by two muscles, the retractor bulbi or choanoid muscle, and the protrusio bulbi or Gegenbauer's muscle. In those mammals in which these muscles are well developed the outer bony wall of the orbit is incomplete, the space between the bones being filled up by a fibrous membrane termed the "cornet," it is on the inner surface of this membrane where the unstriated muscle fibre constituting the protrusio bulbi muscle are found. The retractor bulbi arises from the bones at the apex of the orbit, and is inserted into the posterior part of the sclerotic behind the insertion of 
the recti muscles. When this muscle contracts it draws back the eyeball and compresses the fat of the orbit, so pressing forward the third eyelid which is attached to the fat. The contents of the orbit also becomes pressed against the fibro-muscular membrane composing its outer wall. It is partly the elastic recoil of this membrane, and partly the contraction of the protrusio bulbi muscle, which restores the eyeball to its usual position, and brings about the retraction of the third eyelid.

In man in whom the third eyelid only exists as a vestigial structure, the plica semilunaris, the muscles which translate the eyeball backwards and forwards are also vestigial.

The protrusio bulbi is only represented by an attenuated layer of unstriated muscle fibres, called Müller's muscle, on the inner surface of the orbital periosteum in the region of the spheno-maxillary fissure, supplied by the cervical sympathetic nerve. Paralysis of the cervical sympathetic nerve may be accompanied by slight enophthalmos due to paralysis of these muscle fibres. It has been suggested that the protrusion of the eyes in exophthalmic goitre is due to their prolonged stimulation and hypertrophy; certainly the retraction of the eyelids in this affection is due to stimulation of the cervical sympathetic, producing contraction of the other un-striated muscle fibres of Müller in the upper eyelids.

Vestiges of the retractor bulbi are met with in the form of connective tissue bands at the back of the orbit, and in rare instances strands of muscular tissue have been found. It has been suggested that the cases of congenital enophthalmos might be due to the abnormal development of such strands, but there is no anatomical evidence in support of this view.

As a general proposition it may be stated that the human eye is, under normal conditions, capable of rotation but not of translation, and that the steadiness of its position is due to the nicely balanced action of the muscles which act upon it from different directions. The recti muscles arising 
at the apex of the orbit and inserted in front of the equator of the globe tend to act as retractors, the oblique muscles passing backwards from the inner wall of the orbit to the posterior half of the globe act as protractors; as also do the check ligaments, containing the muscle fibres of Sappey, which pass from the sides of the orbit to the anterior surface of the external and internal recti muscles.

It is when, through faulty development of some of these muscles, this nicely balanced equilibrium is interfered with that the condition of congenital enophthalmos occurs.

The several different symptoms above mentioned as met with in these cases may be accounted for as follows: Inability to move the eye outward, to failure in the development of muscle fibres in the external rectus; impaired movement of the eye inwards to abnormal insertion of the internal rectus far back on the sclerotic; enophthalmos, increasing on movement of the eye inwards, to the abnormal insertion of the internal rectus, and to deficiency of the check ligament connected with it; narrowing of the palpebral fissure, to the eyelids falling backwards with the receding eye; forward movement of the eye and widening of the palpebral fissure on looking outwards, to the protracting influence of the oblique muscles uncompensated for by the defectively developed recti muscles; upward or downward turning of the cornea on retraction of the eye, to faulty insertion of the superior or inferior rectus.

Heterophoria or Latent Squint.-In heterophoria, or latent squint, there is a departure of the visual lines of the two eyes from parallelism when the stimulus to maintain binocular vision is removed. It is best made manifest by the use of a Maddox rod. If a spot of light is looked at, and the rod be held in front of one eye, the light appears to that eye as a streak, whilst the other eye sees it as a spot. There is then no desire to fuse the images seen with the two eyes, and they take up a position in which there is the least muscular effort, i.e., they assume a position of rest. 
If the balance between the ocular muscles is such that the visual lines remain parallel, and the image of the line of light is seen superimposed in front of the spot, the condition is termed orthophoria. If the visual lines diverge, and the images looked at appear crossed, the condition is termed exophoria. If the visual lines converge, and the images looked at are homonymous, the condition is termed esophoria. If the visual lines are displaced vertically, and the image of the line viewed horizontally is seen above or below the spot, the condition is termed hyperphoria. Similarly a want of balance in the oblique muscles has been termed cyclophoria.

The commonest cause of these latent squints is, either some congenital defect in the position of insertion of the ocular muscles, or some congenital weakness in certain muscles themselves.

It has been already mentioned, that in some of the rare cases of congenital defect of movement of the eyes, an insertion of the affected muscle abnormally far back on the sclerotic has been discovered. Much slighter variations of the insertions of the ocular muscles, such as would be likely to favour either the abductors or adductors, and the elevators or depressors, is of common occurrence.

The average distance of the centre of the tendon of each rectus from the corneal margin in the adult has been estimated as follows:

Internal rectus $5.5 \mathrm{~mm}$., External rectus $6.9 \mathrm{~mm}$., Superior rectus $7.7 \mathrm{~mm}$., Inferior rectus $6.5 \mathrm{~mm}$.

Similar measurements in the new born child were as follows:

Internal rectus $3.6 \mathrm{~mm}$., External rectus $4.9 \mathrm{~mm}$. Superior rectus $5.8 \mathrm{~mm}$., Inferior rectus $5 \mathrm{~mm}$.

From these measurements it will be seen that variations in the degree of heterophoria, due to the position of insertion of the recti muscles, are liable to occur until the eyeball has attained its full dimensions. 
In congenital ocular paralysis it has been mentioned that sometimes the place of a rectus muscle has been found occupied by a band of tendinous tissue, lesser degrees of a similar defect would account for congenital weakness of the ocular muscles. The morphological and pathological transformation of muscles into tendons, and of tendons into muscles, is well known to depend on blood supply and use, the degree of one often regulating the amount of the other. Some cases of heterophoria, with its accompanying disablements, are said to be materially benefited by gymnastic prism exercises or orthoptic training. In such cases the increased use and blood supply of the faultily developed muscles probably results in the formation of muscle fibres in place of tendinous tissue.

Concomitant Strabismus.-In concomitant strabismus there is no restriction in the movements of either eye, but a deviation of the visual axis of one eye from parallelism with the other, in whatever direction the eyes are turned.

The deviation may be inwards, convergent strabismus; outwards, divergent strabismus; upwards or downwards, vertical strabismus.

When both eyes are open, fixation may always be made with the same eye and the other eye always deviate, the strabismus is then said to be unilateral. If, when both eyes are open, fixation can be made with either eye, and either eye be allowed to deviate, the strabismus is said to be alternating.

Conjugate movements of the eyes in the vertical plane are innate in the human infant, but in the horizontal plane are only gradually acquired during the first six months of life. Vertical concomitant strabismus, unassociated with any horizontal strabismus, is of exceedingly rare occurrence.

The central acuity of vision, the power of accommodation and of convergence also develop very largely during the first year of life, and it is upon their due coördination that 
the capacity of maintaining the visual axes of the eyes in their normal relations depends.

Many concomitant squints, especially those of the alternating variety date from the first year of life. In some infants, from the very first, no effort is made to keep the eyes straight. In the majority of cases, the children acquire normal conjugate movements and keep the visual axes of the eyes parallel for a time, only beginning to squint when the powers of convergence and accommodation become more developed or actively employed. After the sixth year concomitant strabismus only develops if the sight of one eye becomes destroyed, then a previously latent squint may for the first time become manifest.

Morphologically the development of conjugate movements is found to be closely associated with the development of semi-decussation of the nerve fibres at the chiasma, so that the impressions received from the corresponding halves of the two retinæ are superimposed on the same half of the occipital cortex.

In Rodents the decussation of the optic nerve fibres is complete and they have no conjugate movements of the eyes. In the Carnivora semi-decussation of the optic nerve fibres, and conjugate movements, are both more marked than in the Ungulata. In Primates, who have the most extensive conjugate movements, and in whom the powers of accommodation and convergence become developed to a far higher degree than those of any other mammals, there is, besides semi-decussation of the optic nerve fibres, a very highly developed spot of central vision with a special bundle of nerve fibres leading from it. What happens to these papillo-macular bundles of nerve fibres at the chiasma has not been definitely proved anatomically, but there seems little doubt that there must be semi-decussation of them, so that impressions received from each macula are superimposed on each half of the occipital cortex, allowing of stereoscopic vision for detail. 
In the alternating concomitant strabismus cases that date from birth, there is a complete absence of any desire for such central stereoscopic vision; they often have perfect acuity of central vision in each eye, with little or no refractive error, but cannot be induced to make any attempt to use the two eyes together. A very simple explanation of such cases might be found in a congenital defect in the course of the macular nerve fibres, whereby complete decussation, instead of semi-decussation had taken place. With complete decussation the impression received by the visual cortex from each macula would be unilateral, instead of bilateral, as with semi-decussation. The acuteness of perception would not be affected, only the desire to see singly with the two eyes, and the stimulus which it produces to keep the visual axes parallel.

The close association which exists in the evolution and development of acute central form sense, accommodation and convergence is of considerable importance in considering the ætiology of concomitant strabismus.

A desire for binocular vision, or rather an intense dislike of double vision, is the stimulus which leads to the normally regulated conjugate and convergent movements of the eyes. Those who develop concomitant strabismus get over the difficulty of seeing double by mentally suppressing the image seen with one eye. The capacity for such involuntary suppression varies considerably in different individuals. When difficult much inconvenience and discomfort will be endured to avoid squinting. When easy the habit of squinting may be acquired on very slight provocation. A defect of sight in the squinting, eye is a common accompaniment of concomitant strabismus, such defect is usually due to congenital amblyopia, but may also be caused by nebulæ of the cornea, some refractive error or some intraocular affection. The presence of defect of sight in one eye facilitates the suppression of the indistinct mental image received from it, and so favours the development of a squint. 
Hypermetropia is frequently met with in association with convergent concomitant strabismus, it necessitates an excessive amount of accommodative effort in focussing near objects, the development of accommodation and convergence being so closely related, the excessive use of one evokes an excessive use of the other. A hypermetrope if he uses the requisite amount of accommodation to see near objects distinctly, uses so much convergence that he sees double, if he only uses the necessary amount of convergence to see singly the object looked at is out of focus, and is indistinct. Those who find it easy to suppress the image seen with one eye overcome the difficulty by squinting, so allowing all the excessive tendency to convergence to become manifest.

The presence of a latent squint, heterophoria, necessitates a constant effort of the ocular muscles to maintain parallelism of the visual axes and avoid diplopia. This effort is often well kept up when the health is good but fails when it becomes debilitated, if the image of one eye is easily suppressed when the eye assumes the position of rest, then a latent squint becomes manifest. The onset of squint in a child is often found to date from some attack of one of acute specific fevers, or some other general disturbing influence.

In any one case of concomitant strabismus there are often several factors which lead to its development. 


\section{CHAPTER II}

\section{NEOPLASMS}

Neoplasms of the eyeball and its appendages are divided into (i) teleplasms, (ii) metastatic growths, and (iii) cysts.

A teleplasm is a growth formed by a tissue which has reassumed some of its embryonic characteristics, i.e., an atavism of tissue. The power of rapid reproduction is one of the characteristics of cells during. embryonic life. As a tissue reaches maturity this proliferating power of its cells becomes reduced to that which is required for its repair and maintenance. In all teleplasms there is reassumption of the embryonic power of proliferation; sometimes resulting in a mass of cells simulating those from which the tissue is primarily developed; at others in the formation of tissue of a more highly organised character. Like the normal tissue the more highly specialised the structure of a neoplasm, the slower the power it has of reproducing itself; whilst the more embryonic its character the greater the rapidity of its growth, and of its liability to dissemination through the blood-vessels or lymphatics, i.e., the greater its malignancy.

In malignant tumours some of the cells when undergoing mitosis show a deficiency in the number of chromatophores in the nucleus, a condition simulating that which is found in the ovum before fertilisation.

Why certain cells in adult tissue should regain their embryonic powers of growth and form a tumour is not yet known. Some experimental work bearing on this subject may here suitably be referred to.

The characteristic features in the formation of an epithelioma are, the invasions of the mesoblastic tissue by 
extensions down into it of surface ectoderm, and the formation in the sequestered epithelium of compressed laminated masses which undergo keratinisation. This pathological process is a reproduction of what occurs physiologically in the formation of the crystalline lens. It has been shown, ${ }^{1}$ in frogs and salamanders, that the stimulus which gives rise to the down growth of ectoderm to form the lens is contact of the secondary optic vesicle with its inner surface. If by injury the development of the optic vesicle is arrested no lens is formed. If the optic cup is removed from its normal position, and engrafted under the epidermis in some other region of the head, the epidermis in this new region will be stimulated to form a lens. If a piece of ectoderm from some other region of the body be engrafted, so as to overlay the optic cup, from it a lens will be developed.

By the cultivation of tissues in vitro with suitable technique $^{2}$ a pure growth of cells can be obtained in the form of undifferentiated sheets of tissue. If connective tissue be added to such a culture the epithelial cells undergo a characteristic differentiation. In kidney cultures tubules are formed, and in cultures of alveolar mammary tissue acini develop. These experiments show the intimate dependence of the character of the growth in one tissue on the presence of another.

The growth of adult tissue in artificial media is found to be slow as compared to that of embryonic tissue. The rate of growth of the former may, however, be made equal to that of the latter by the addition of an extract of autolysed cells to the medium. This seems to show that the increased cell proliferation in wound repair is dependent on chemical excitation rather than variations in tissue tension.

Cells of malignant growths, cultivated in vitro, behave more like those of embryonic tissue than those of adult tissue in their growth, and do not require an extract of

${ }^{1} \mathrm{~W}$. H. Lewis. Am. Journ. of Anat., V, 1907.

${ }^{2}$ A. H. Drew. The Lancet, Apr. 28, I923. 
autolysed normal cells to call it into activity. Presumably the cells of malignant growths contain these growth-stimulating substances, which are only liberated from normal adult cells when they undergo autolysis.

In the eye and its appendages there are tissues developed from different divisions of the blastoderm, the cuticular epiblast, the neural epiblast, and the mesoblast. The teleplasms starting from each of these divisions may be classified as follows:

I. Teleplasms arising in parts derived from cuticular epiblast.

(a) Surface Epithelium.

Squamous Carcinoma or Epithelioma. Papilloma.

Horns. Epithelial Plaques.

(b) Glandular Epithelium.

Glandular Cancer of Skin or Rodent Ulcer.

Carcinoma of Meibomian Glands. Adenoma of the Eyelids.

Carcinoma and Adenoma of the Lacrymal Glands.

(c) Congenitally Sequestered Epidermic Masses.

Moles or Nevi and the Malignant Growths arising from them.

II. Teleplasms arising in parts derived from neural epiblast.

Glioma or Neuro-epithelioma of the Retina.

Glioma or Gliomatosis of the Optic Nerve.

Innocent Growth of the Pars Ciliaris Retinæ, "Adenoma."

Melanoma, Innocent Pigmented Growth in the Uveal Tract.

Melanotic Carcinoma, Malignant Growth starting in the Pigment Epithelium.

III. Teleplasms arising in parts derived from mesoblast.

Sarcoma, Intra-ocular, Epibular, and Orbital.

Lympho-sarcoma and Lymphoma.

Mixed Mesoblastic Growth. Neuro-fibroma and Neuro-fibromatosis. Osteoma. Myoma. Hemangioma. Lymphangioma.

\section{TELEPLASMS ARISING IN PARTS DERIVED FROM CUTICULAR EPIBLAST}

The parts of the eye and its appendages derived from cuticular epiblast are: the crystalline lens, the surface epithelium of the skin of the lids, conjunctiva, cornea, and the lining membrane of various glands. 
No new growths are met with in connection with the lens. The teleplasms arising from cuticular epiblastic structures may be divided into those starting in (a) surface epithelium, (b) glandular epithelium, (c) congenitally sequestered epidermic masses, moles or nevi.

(a) In the embryonic condition extensions downward of the surface epithelial cells occur into the subjacent mesoblast, preliminary to the formation of hair follicles, glands, etc. After embryonic life the surface epithelium ceases to send prolongations downward and only grows upward. When a reversion to the embryonic condition occurs, and prolongations downward of the surface epithelium into the tissues beneath again develop, a malignant teleplasm is formed which is termed squamous carcinoma or epithelioma.

In extending down through the basement membrane into the subepithelial tissue, cells of the growth gain access to lymphatic channels along which they spread, to the lymphatic glands, where fresh nodules of the new growth develop.

A thickening of the epithelium on the surface without any extension of it downward gives rise to innocent new formations termed papillomata, horns, and epithelial plaques.

(b) The cells which line glands lose their primitive tendency to rapid proliferation and become specialized to produce a secretion. If their secreting function ceases, and they regain their proliferative activity, a teleplasm is formed. If it remains located in the region in which it started it is innocent and is termed adenoma. If it tends to invade surrounding structures it is malignant and termed glandular carcinoma.

(c) Much difference of opinion exists as to the nature of moles or nevi. Some authorities hold that the cells composing them are mesoblastic in origin, and derived from the endothelium lining lymphatic channels; others regard them as epiblastic, the cells of the growth having become snared off from the surface epithelium, and sequestered in the sub- 
epithelial tissue. Neither view has yet been definitely substantiated. Those in favour ${ }^{1}$. of the latter, claim that in sections of moles in an early state the snaring-off process can be actually observed in its different stages. The fact that moles are only met with in close proximity to an epithelial lined surface is in favour of an epithelial origin. The cells composing them, however, differ from epithelial cells elsewhere, which either proliferate throughout life or break down to form a secretion. The majority of nevi or moles are pigmented, if the cells forming them resume their proliferative activity and invade surrounding tissues, a malignant melanotic growth is produced; the name applied to which depends upon the view taken as to the origin of the primary formations. If mesoblastic they are melanotic sarcomata, if epiblastic melanotic carcinomata.

Squamous Carcinoma or Epithelioma.-An epithelioma arising in the skin of the eyelids may extend to and involve the conjunctiva. Epithelioma arising primarily on the surface of the eye usually starts at the limbus. The epithelium of the cornea and of the conjunctiva presents a very regular base line, except just at the limbus where there are down-dipping processes of cells. It is in this locality that the pathological downgrowths, which are the characteristic feature of an epithelioma, make their appearance.

At first an epithelioma of the surface of the eye forms a small, opaque, whitish, raised patch; it then becomes a warty growth which after a time ulcerates (Fig. 8I), and may protrude through the palpebral fissure as a bleeding mass. Its growth forward is at first in excess of its invasion of the tissues beneath, more so than in growths of a similar character in other localities, due to the hard unyielding character of the sclerotic and the few lymphatic channels which it contains.

On extension inwards the growth meets with resistance from Bowman's membrane, and will often spread some distance external to it without invading the substantia

${ }^{1}$ A. Whitefield. The British Jour. of Dermatology, XII, 1900, 207. 
propria of the cornea. When the substantia propria does become invaded, columns of cancerous cells extend along the lymphatic spaces between its layers.

An epitheliomatous growth which has penetrated to the substantia propria at the limbus soon reaches the lymphatics connected with the canal of Schlemm, it will extend down along them to the canal itself, and from it reach the interior of the eye.

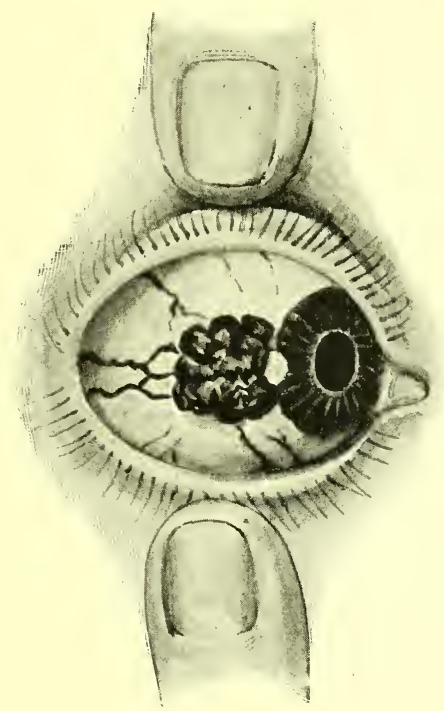

FIG. 8I.-An epibulbar epithelioma which completely disappeared after one application of radium bromide. Trans. Ophth. Soc. of the U. K., Vol. XXXV, I 9 I 5,2 I 5 .

If extension of an epitheliomatous growth occurs backwards from the limbus it reaches the large lymph space of Tenon's capsule, and in it will spread round the surface of the globe external to the sclerotic. If the whole cornea becomes encircled by growth, it is termed as a pericorneal epithelioma, if the whole globe becomes surrounded, it is termed a peribulbar epithelioma. From a growth in Tenon's capsule permeation takes place along the lymphatic sheaths of the blood vessels which penetrate and pass 
into the sclerotic. An extension to the eyelid, secondary to involvement of Tenon's capsule has also been met with. Involvement of the lymphatic glands is apparently of rare occurrence, but cases in which the preauricular and submaxillary glands were involved have been recorded.

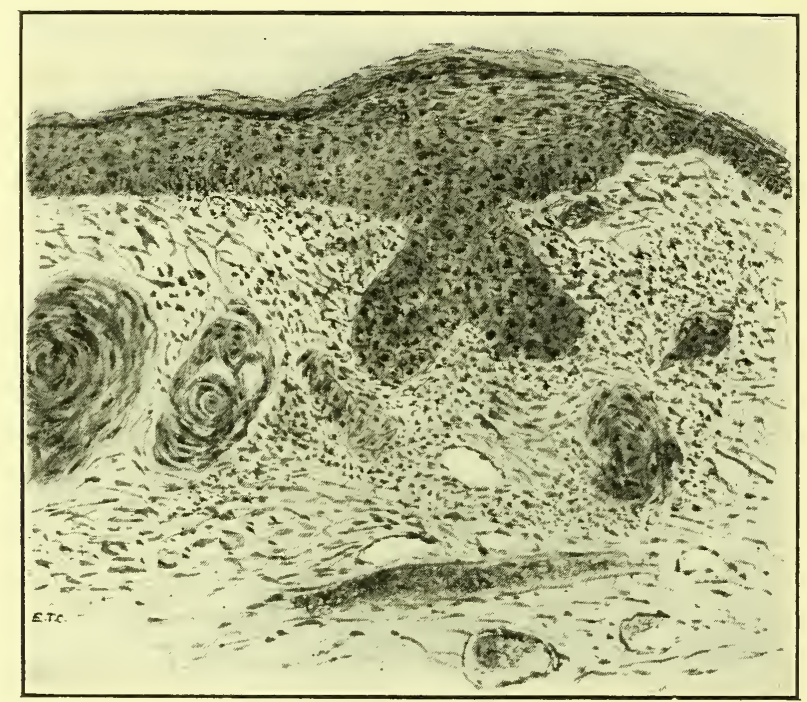

FIG. 82.- Section of commencing epithelioma of the ocular conjunctiva.

5 The microscopical characters of an epithelioma arising on the surface of the globe are similar to those starting in other situations. There are down-growths of branching columns of cells from the surface epithelium which break through the basement membrane (Fig. 82).

Proliferation goes on in these columns of cells, and as none of the cells can be thrown off, the central ones become compressed and flattened. In this way laminated nests of cells are formed, the central layers of which undergo keratinisation. Extensive keratinisation also occurs in the cells on the surface of the growth; other cells show vacuolation and signs of degeneration in their cytoplasm. There are always numerous polygonal prickle cells. In the peripheral parts active karyokinesis takes place. 
In an epithelioma, besides the cells of epiblastic origin, polymorphonuclear, mononuclear leucocytes, and endothelial cells are met with. The leucocytosis is doubtless excited by pyogenic organisms from the surface, and is most marked when ulceration has taken place. The proliferating endothelial cells around the growth resemble closely the cells of the growth itself, for which they are liable to be mistaken. When organisation takes place, bands of fibrous tissue with blood-vessels become formed, between the groups of epithelial cells.

Papilloma.-Papillomata arise either from the skin of the lids or conjunctiva. Those starting in the skin form small, hard, nodulated, sessile protuberances which are

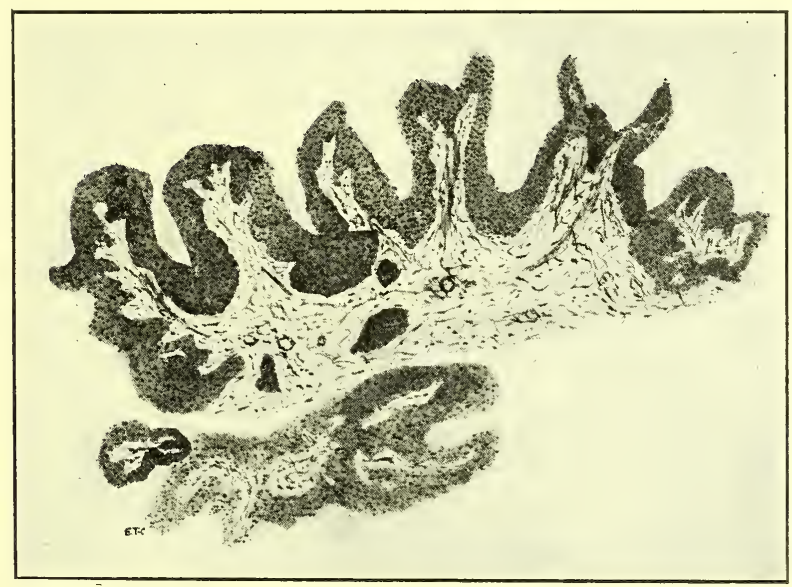

FIG. 83.-Section through a hard papilloma from the margin of an eyelid.

termed warts. They often occur at the margins of the lids and may be multiple.

Those starting in the conjunctiva are soft, red or pink, pedunculated growths with numerous delicate finger-like processes; springing most frequently from the fornix or plica semilunaris.

Though the preponderating tissue in these growths is the epithelium there is also hyperplasia of the sub-epithelial fibrous and vascular tissue; i.e., a new growth of tissue of 
mesoblastic origin. It is the thickening of the sub-epithelial tissue which gives rise to the papillated character of the tumour.

The relative amount of fibrous tissue, blood-vessels, and overlying epithelium varies in different growths, and for purposes of classification they are named fibromata, fibroangiomata or papillomata according to which element preponderates. Often growths are found presenting the appearance of a papilloma in one part, and of a fibroma or fibro-angioma in another.

In the soft papillomata of the conjunctiva, the fibrous tissue consists of only a few strands lying outside loops of thin-walled blood-vessels, and covered by stratified epithelium. In the harder papillomata of the skin the fibrous tissue forming the papillæ is dense and compact, and there are fewer blood-vessels (Fig. 83). The epithelium covering them is much thicker than the normal epithelium, the surface layers frequently undergoing hyperkeratosis.

Horns.-Horns composed of desiccated surface epithelial cells, which have failed to be cast off in the usual way, are met with on the surface of warts growing at the margins of the eyelids. They are of a brown or greyish color, hard in consistency, usually a few millimetres in diameter, and not longer than the eyelashes. They may, however, attain much larger dimensions. A specimen of one in the Moorfields Hospital Museum measures $28 \mathrm{~mm}$. in length with a diameter of $26 \mathrm{~mm}$. at its base.

If an anterior staphyloma protrudes between the eyelids so that it is not covered by them, the epithelium becomes dry and undergoes a process of keratinisation, these cells not being rubbed off sometimes become converted into horn-like incrustations.

Epithelial Plaques.-A purely epithelial innocent new growth of the surface of the eye is termed an epithelial plaque. $^{1}$ It is essentially a localised hyperplasia of the

${ }^{1}$ W. T. Lister and W. I. Hancock. R. Lond. Ophth. Hosp. Reps., XV, I903, 346 . 
surface epithelium; appearing clinically as a sharply defined white or yellowish patch, a few millimetres in diameter, with a dull granular surface. These plaques resemble closely the patches formed in xerosis of the conjunctiva, but occur in parts of the eye in which there has been no undue exposure, are not accompanied by night-blindness or other general symptoms, and do not disappear with general treatment.

The epithelium of the cornea and conjunctiva differs from the epithelium of the skin in being considerably thinner; the superficial keratinised layer, stratum corneum and prickle cells, are absent. In epithelial plaques the epithelium is found to have assumed the characters of the skin, and to possess surface layers containing keratin, keratohyalin, and prickle cells:

Glandular Cancer of the Skin or Rodent Ulcer.Rodent ulcer is a new growth in the skin, which as it spreads undergoes ulceration; it is only locally malignant.

It frequently starts on the side of the nose near the inner canthus, or on the eyelids, commencing as a small, hard, raised nodule, which may remain as such for several years before any ulceration takes place. Extension of the new growth is always accompanied by ulceration, the edges of the ulcer presenting a characteristic raised and thickened appearance. As a rodent ulcer spreads it may destroy the eyelids and invade the surface of the eyeball. By invasion of Tenon's capsule it may lead to compression and loss of mobility of the globe. A further extension into the sclerotic will ultimately result in perforation and gradual destruction of the eyeball. The destructive process of the growth is not checked when it reaches bone, which also becomes infiltrated and eroded.

Rodent ulcer commences usually between the ages of thirty and fifty, but has been known to begin as early as fourteen. Men are more frequently affected than women.

Microscopical examination of nodules of the growth in an early stage shows that it begins sub-epidermally, appar- 
ently in the sebaceous or sweat glands of the skin, presenting the characteristics of a glandular carcinoma. It is at first distinct from the covering epithelium, which only becomes involved later in the ulcerative stage.

The cells composing the growth, which are smaller and more pear-shaped than those of the surface epithelium, are grouped in columns or irregular masses, contained in alveoli bounded by newly formed connective tissue. Sometimes the alveolar arrangement is so marked that the appearance of the growth simulates the gland tissue from which it takes origin. The outermost cells of an alveoli are usually cylindrical, and the central ones round, oval, or polyhedral. They have a tendency to undergo vacuolation and degeneration so that spaces become formed in the growth. As the growth is derived from glandular epithelium the cells composing it are very different from those of a carcinoma derived from the surface epithelium, i.e., an epithelioma. The keratinisation, prickle cells, and nests of flattened cells, which are characteristic appearances in the latter are not found so frequently in the former.

The inflammatory changes around the growth are more extensive in rodent ulcer than in an epithelioma, and there is also a greater development of fibrous tissue.

Carcinoma of the Meibomian Glands.-A few cases of carcinoma which appeared to originate in the Meibomian glands have been recorded. A tumour forms in the eyelid which as it extends may result in ulceration, on either or both, the skin and conjunctival surfaces. Microscopically these growths have the characters of a typical alveolar carcinoma, they are different from those of a rodent ulcer, the cells being larger and more of the type of sebaceous epithelium.

Adenoma.-Adenomata of the conjunctiva or skin of the eyelids may be of a racemose or acino-tubular type. The former arise in either the Meibomian or sebaceous glands of the skin, the latter from either the modified sweat glands of Moll or the glands of Krause. 
An adenoma of a Meibomian gland is not of very rare occurrence; it is very likely to be mistaken for a chalazion. It sometimes develops most on the conjunctival surface of the tarsus and at others on the skin surface. Microscopically lobules are found resembling large normal alveoli distended with polygonal cells, the more central of which show a tendency to degenerative changes.

The distinctive features of an adenoma of a gland of Moll are its location at the margin of the lid, a tendency to cystic formation, and the presence of a double row of cells lining its tubules, an inner of cylindrical epithelium and an outer of endothelium - a similar arrangement to that which is found in the normal gland. ${ }^{1}$

In an adenoma of the gland of Krause the lining of the acini and ducts is composed of a single layer of epithelium.

Adenomata of an acino-tubular character have also been found starting in the plica semilunaris, probably from rudimentary glands.

Another vestigial structure at the inner canthus from which glandular growths sometimes arise is the caruncle. It is the vestige or remnant of the suborbital pit, which reaches its greatest development in some of the Ungulata. In them it contains numerous glands which secrete a highly odouriferous substance. In man two different varieties of glands are met with in the caruncle, modified sweat glands resembling the glands of Moll, and sebaceous glands, cases are recorded ${ }^{2}$ of new growths starting in each. Those from the sweat glands are mainly cystic, with papillomatous proliferations, covered by two layers of epithelium, springing from the inner walls of the cysts. In the growths from the sebaceous glands a reproduction of the normal gland tissue is very closely imitated.

Carcinoma and Adenoma of the Lacrymal Gland.Tumours arising in the lacrymal gland have been described as carcinomata or adenomata. A more careful study of

${ }^{1}$ M. Salzmann. Arch. of Ophth., I89I, XX, 380.

${ }^{2}$ G. Coats. Royal London Ophth. Hosp. Rep., XVIII, p. 269 and 280. 
new growths of this structure of recent years has, however, shown that the large majority of them originate in its mesoblastic constituents ${ }^{1}$ (see page 206).

Moles or Nevi of the Conjunctiva and the Malignant Growths Arising from Them.-Moles or nevi are met with in the ocular conjunctiva presenting the same characteristics as those occurring in the skin. They form flat or slightly raised spots, usually deeply pigmented, standing out very conspicuously in contrast with the surrounding white of the sclerotic. They may, however, be unpigmented and of a pinkish yellow colour.

The surface epithelium overlying them, instead of presenting its usual regular base line, frequently shows some down-dipping extensions of its deeper cells. The cells of the growth itself, though in close proximity to the surface epithelium, are separated from it by strands of fibrous tissue. They are of an epithelial or endothelial type, of various sizes, but always smaller than the surface epithelial cells. They are grouped in spaces bound by fibrous tissue, and are mostly polygonal in shape with a round nucleus. The pigment, when present, is located in the cells of the growth itself, in the connective-tissue cells, and in those of the overlying epithelium. Cystic spaces may form amongst the cells of the growth due probably to their disintegration; some of them swell up and resemble goblet cells.

In rare cases a mole may spread over the whole conjunctiva without showing any tendency to invade the deeper tissues.

A malignant growth starting in a mole of the conjunctiva presents symptoms similar to those described under epibulbar sarcoma (page I99). The history of the growth having commenced in a small pigmented patch, the existence of which had been recognised for many years previously, would suggest its real nature. Microscopically such a growth presents an alveolar structure, its cells

${ }^{1}$ R. A. Greeves. R. Lond. Ophth. Hosp. Reps., XIX, 237. 
resembling those met with in a mole, but showing a more active karyokinesis.

\section{TELEPLASMS ARISING IN PARTS DERIVED FROM NEURAL EPIBLAST}

The teleplasms arising in neural epiblast are either unpigmented or pigmented. The former are by far the more frequent; they are usually termed gliomata and start either in the pars optica, pars ciliaris retine, or the supporting tissue of the optic nerve.

The name glioma was given to these malignant growths of the retina on the assumption that they commence like the gliomata of the brain in cells of the neuroglia. More extended observations have not supported this view. The new growths of the retina present very marked differences, both clinically and histologically, from gliomata of the brain; e.g., they occur only in early life, are more highly malignant, and are composed of an altogether different type of cell. It seems, therefore, unfortunate that they should have received the same name.

The cells composing malignant growths of the retina are of the same character as the cells in the retina during fetal life. The name neuro-epithelioma has therefore been suggested as a substitute for that of glioma for these tumours, and regarding them as teleplasms it would seem more appropriate.

In the optic nerve some of the tissues are derived from neural epiblast and others from mesoblast. The nerve fibres themselves are developed as outgrowths from the ganglion cells of the retina or brain. From the tissue which forms the stalk of the secondary optic vesicle is produced the delicate supporting tissue of the nerve, or neuroglia. The dural and pial sheaths of the nerve, the endothelial cells in the intervaginal space, and the fibrous tissue trabeculæ containing blood vessels which extend into the nerve, are all mesoblastic in origin. No new 
growths occur in connection with the nerve fibres, but they may start either in the epiblastic neuroglia or in the mesoblastic fibrous tissue. Those originating in the neuroglia do not form well defined encapsuled tumours, but like the gliomata of the brain are diffuse growths in which the margins merge gradually into the surrounding unaffected tissue. For this reason the condition has been termed gliomatous degeneration or gliomatosis.

Innocent new growths which arise occasionally in the pars ciliaris retinæ, have been described by different observers as adenomata and epithelial hyperplasias.

Congenital pigmented patches comparable to moles or nevi of the skin and conjunctiva are met with in the uveal tract; they are termed melanomata. Their mesoblastic or epiblastic origin, as in the case of nevi of the skin, is a matter about which there is considerable difference of opinion. Sometimes they become malignant.

A few undoubted cases of melanotic carcinoma arising in the pigment epithelial layer have been recorded.

Glioma or Neuro-epithelioma of the Retina.-A glioma of the retina usually first attracts attention in the second year of life, and has never been known to manifest itself later than the age of eleven years. Sometimes it is undoubtedly congenital, but not always, since eyes which have been subjected to the most careful ophthalmoscopic examination, and in which no changes could be detected, have subsequently developed a gliomatous growth.

In about one-fourth of the cases the growth is bilateral, appearing either simultaneously in the two eyes or with an interval of only a few months. An interval of as much as three years has, however, been recorded. ${ }^{1}$ The growths in the two eyes arise independently; one is not metastatic to the other, and there is no extension of growth along the optic chiasma from one eye to the other. This is shown by patients' living for years after the removal of the two

${ }^{1}$ Treacher Collins. Trans. Ophth. Soc. of the U. K., XVI, I896, I42. 
affected eyes, without any fresh manifestations of the disease.

The right eye is affected with the same frequency as the left, and the two sexes equally. Members of the same childship have in rare instances been the subjects of glioma of the retina, but it has not been met with in more than one generation in a family.

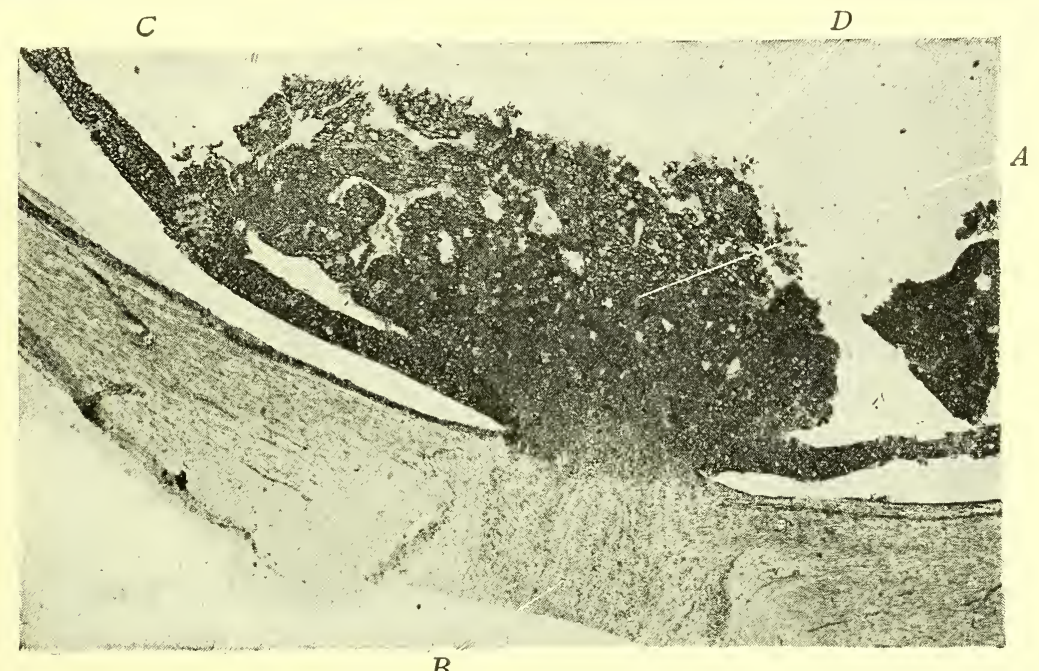

FIG. 84.-Glioma of the retina $A$ commencing to involve the optic nerve $B$. The glioma growing from the nuclear layers of the retina $C$ has converted it into a mass of new growth. The portion of the growth $A$ is fungating into the vitreous $D$.

The symptoms at the commencement of the disease vary according to the situation of the growth. If it protrudes from the outer surface of the retina it is termed glioma exophytum, if from the inner surface glioma endophytum. In the former, which is the more frequent, the retina is displaced inward and seen clinically behind the lens with the opaque white growth beneath it. In the latter the retina remains in position, the tumour extending inward to the vitreous chamber (Fig. 84), detached flocculent nodules often forming in the vitreous humour.

In the rare cases where it starts from the pars ciliaris retinæ it grows over the inner surface of the ciliary body, posterior surface of the iris, and anterior surface of the lens. 
In course of time glioma of the retina gives rise to increased tension, the effect of which in a child's eye is to cause expansion of the elastic cornea and sclerotic as in buphthalmos. It tends to spread along the lymphatic sheaths of the blood-vessels, and after invading the choroid, will extend out of the eye into the orbit along the perforating vessels. It may also, after having reached the anterior chamber, extend over the iris or perforate the globe in the region of the canal of Schlemm.

When a glioma escapes from the eye its rate of growth becomes accelerated, and it fungates outward, forming on the anterior surface of the eye a nodulated bleeding mass which was termed by older writers "fungus hematoides."

Glioma of the retina frequently extends backward along the optic nerve to the brain. Metastatic growths may form in the eye itself from cells of the primary growth which become detached and carried to some fresh situation, either in the vitreous chamber, anterior chamber, or subretinal space. Extraocular metastatic growth may form in the preauricular gland, in the periosteum of the cranial and facial bones, and occasionally in the liver.

Local recurrence after enucleation is exceedingly common and a frequent cause of death. If a patient lives three years after the removal of the eye for glioma, and during that time no fresh growth has occurred, the risk of a recurrence is most improbable. ${ }^{1}$

An early gliomatous growth is gelatinous in character and of a greyish colour; on division its blood-vessels show plainly as red points. When the growth has existed for some time degenerated areas form giving it a patchy appearance. They are of an opaque white or yellowish colour, and have sometimes particles of a dead white colour and gritty consistency in them, due to calcareous changes.

Microscopical sections of glioma of the retina stained with hematoxylin show clearly the difference between the

${ }^{1}$ J. B. Lawford and Treacher Collins. R. Lond. Ophth. Hosp. Reps., I890, XIII, 12. 
degenerate and undegenerate parts of the growth. The nuclei of the cells in the latter staining deeply and those of the former very slightly or not at all (Fig. 85).

The proliferation of the cells of the growth appears to proceed at a rate which is out of proportion to the rapidity of increase of its vascular channels; so that areas in it become badly nourished and undergo degeneration. The

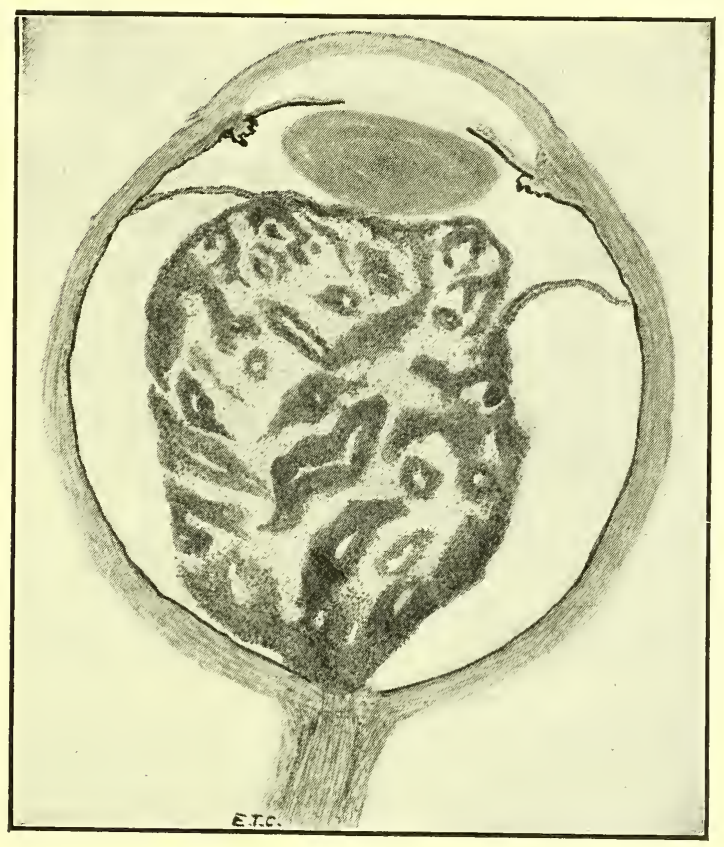

FIG. 85.-Section through an eye containing glioma of the retina. The growth is mainly from the outer surface of the membrane "glioma exophytum." The characteristic patchy way in which it stains, due to areas of degeneration, is shown.

best formed cells, and those where the most active karyokinesis is going on, are found in the proximity of the bloodvessels, the most degenerate being situated furthest from them.

A glioma does not usually start from one point only in the retina, there may be several separate foci. In sections of early foci the layer of the retina in which the growth com- 
menced can be detected. It is usually the outer or inner nuclear layer, but occasionally the ganglion-cell layer.

The cells of which a glioma of the retina is composed are peculiar to it, and are not met with in growths in other parts of the body, except as metastases. They have large round or oval nuclei with very little cytoplasm. In teased-out specimens little delicate processes can often be seen projecting from them. They closely resemble the

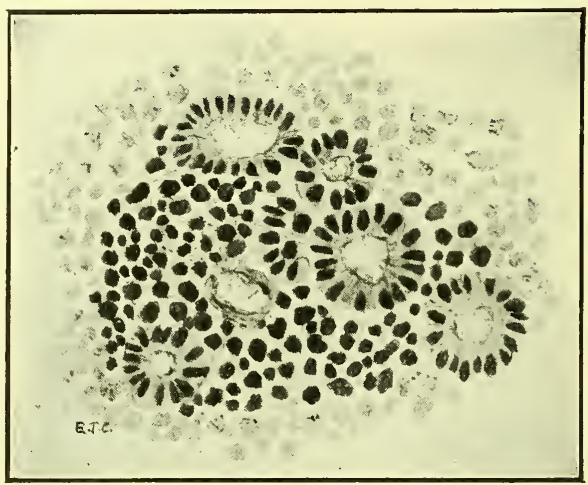

FIG. 86.- Section through a glioma of the retina showing the rosette-like formations of cells, which appear to be rudimentary rods and cones.

cells of which the whole of the retina is composed at the third month of fetal life, before any of its several layers have become differentiated. They are also very similar to the cells forming the nuclear layers of the retina. In some gliomata of the retina all the cells are of this type, in others, cells which may be regarded as rudimentary rod and cone cells, and rudimentary ganglion cells, are also found.

The rudimentary rod and cone cells occur grouped around a space which may be circular like the lumen of a tubular gland, or of an irregular shape. The circular groups of cells have been described as "rosettes" (Fig. 86).

The most highly developed cells in the rosettes are cylindrical in shape and have round or oval nuclei. The ends toward the open space taper somewhat, rod-like pro- 
jections protruding from them. The ends of the cells nearest the lumen rest on a basement membrane comparable to the membrana limitans externa. Similar rosette-like formations of cells are sometimes seen in the maldeveloped retina of congenitally deformed eyes.

Columnar-shaped cells bounding round or irregular spaces are met with, which may be regarded as a still more elementary stage of a rod or cone cell; and cells can be found in the various stages of transition from them, to those which form the chief mass of the growth.

The rudimentary ganglion cells are more angular than the ordinary glioma cells, have more cytoplasm, and a projection from them which may be regarded as a rudimentary axis cylinder. There is but little intercellular substance in gliomata, no true stroma.

In gliomata commencing in the pars ciliaris retinæ, single rows of cells, like those composing that structure, and cellular membranes like embryonic retina are found. Sudden transitions in the same growth from one of these conditions to the other occur, resembling the transition from the pars optica into the pars ciliaris retinæ. ${ }^{1}$

In a single section of a glioma of the retina various stages in the disintegration of its cells are often to be seen. At first the chromatin granules of the nucleus disappear, so that it no longer takes up the hematoxylin, but stains with eosin as a uniform pink circle. Fatty globules will next form in this circle, then the cell breaks down, becoming with others similarly affected a granular material, in which lime salts may become deposited and cholesterin crystals formed.

In rare cases an eye affected with glioma of the retina, instead of becoming glaucomatous and enlarged, shrinks and atrophies, the progress of the growth in it becoming checked or arrested. In the majority of such cases both eyes are affected but only one becomes atrophied (Fig. $8_{7}$ ). The cause of the atrophic process and cessation of

${ }^{1}$ F. H. Verhoeff. Trans. Am. Ophth. Soc., VIII, 1904, 35 I. 
growth cannot be said to be definitely determined. It may be simply a blockage of blood supply, or due to the occurrence of some intercurrent inflammation starting in the uveal tract, the toxin exciting which, acts on the cells of the growth besides causing the other destructive changes
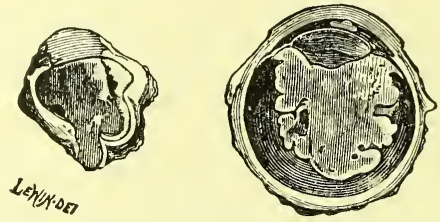

FIG. 87.- The lateral halves of the two eyes of a boy aged sixteen months. The left which is much shrunken is full of gliomatous growth, the lens is absent. The right is of normal size, a gliomatous growth involves the whole retina. Case recorded in the R. Lond. Ophth. Hosp. Reps., XIII, I892, 393.

in the eye. The fact that several shrunken eyes containing gliomatous growths, which have been examined histologically, showed inflammatory infiltration of the choroid lends support to this latter view.

The blood-vessels in glioma of the retina have thin walls composed of a lining endothelium with connective tissue external to it. This latter is liable to undergo hyaline and calcareous degeneration. Hemorrhages into the growth are of frequent occurrence and sometimes extensive.

Gliomata of the Optic Nerve or Gliomatosis.-New growths of the optic nerve starting in the neuroglia, as already stated, do not form well defined localised tumours, but diffuse intra-dural thickenings. They frequently involve not only the orbital but also the intracranial portion of the nerve, sometimes extending right up to the chiasma. Occasionally a new growth in the optic nerve is only an extension of a wide spread intracranial gliomatosis.

This form of new grow th of the optic nerve usually attracts attention in early life, the large majority manifesting themselves before the age of twenty.

A new growth situated in the cone-shaped area behind the globe, bounded laterally by the recti muscles, causes proptosis in the line of the orbital axis, without at first any 
lateral or vertical displacement. If, as the growth increases in size, it extends more to one side of the nerve than another, displacement of the globe in a direction away from the side of the tumour may occur. The movements of the globe often remain unrestricted, or only slightly limited, for a long time.

Glioma of the optic nerve does not invade the eyeball, but steadily and slowly enlarging behind it causes a gradu-

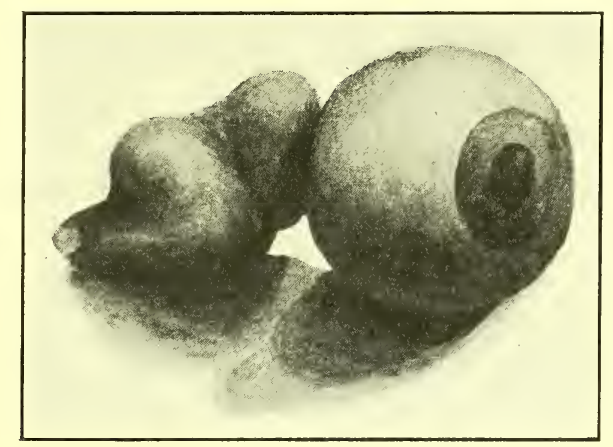

Fig. 88.- Intradural tumour of the optic nerve removed together with the eyeball from a boy aged twelve years. Case recorded by G. Lawson, R. Lond Ophth. Hosp. Reps. vol. XII, I888, I.

ally increasing amount of proptosis. A characteristic symptom, caused by the pressure of the growth on the back of the globe, is a slowly increasing amount of hypermetropia. In very advanced cases the tumour may distend the orbit, and even, through pressure, cause erosion of its bony walls.

In gliomatous growths of the optic nerve a defect of vision usually, but not always, precedes the onset of the proptosis. The defect of sight may be out of proportion to the extent of the ophthalmoscopic changes. In some cases the appearances of simple atrophy are early visible, in others, those of papilledema, or atrophy secondary to papilledema. The swelling of the disc is caused by pressure on the vein, due to a kink being formed in the nerve close to where it joins the globe (Figs. 88 and 89). 
Microscopically, in the affected areas of these cases, the nerve fibres are found replaced by a network of branching cells and fibrils, which latter are arranged at right angles to the connective tissue septa (Fig. 90). This characteristic of the fibrils, together with the way in which the new

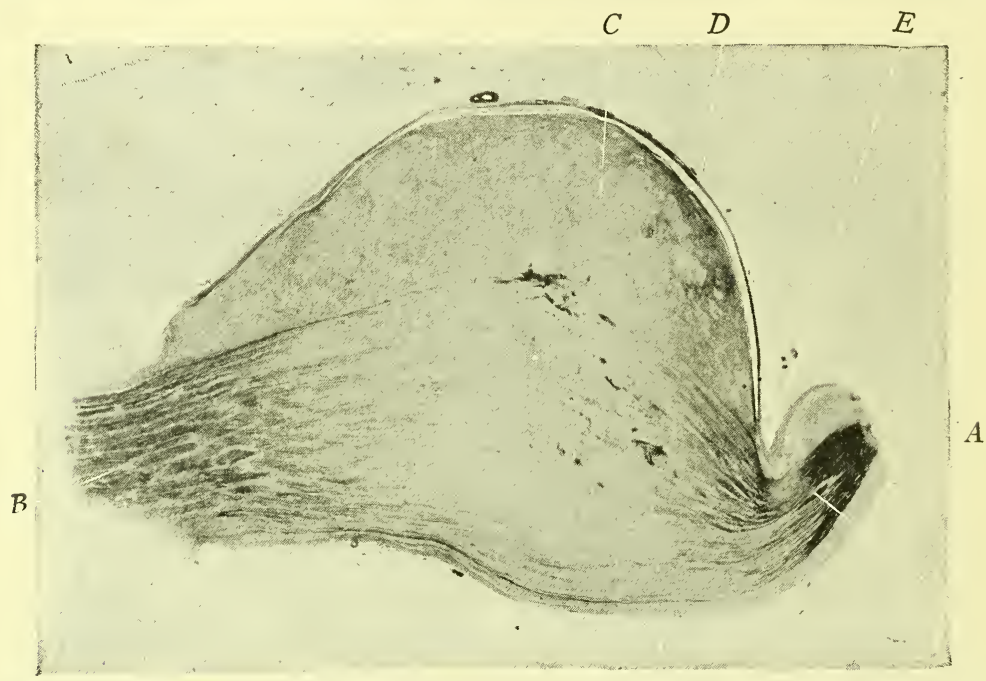

Fig. 89.- Shows a longitudinal section through a tumour of the optic nerve. $A$, The nerve divided close to the globe; $B$, the thickened nerve at the optic foramen; $C$, overgrowth of the supporting structure of the nerve; $D$, dural sheath of the nerve, $E$, kinking of the nerve which caused pressure on the vessels and thus produced edema of the optic papilla. Case recorded by M. S. Mayou, R. Lond. Ophth. Hosp. Reps. vol. XVI, I905, I55.

formed tissue reacts to specific glial tissue stains, serves to differentiate these epiblastic growths from those of mesoblastic origin, starting either in the nerve sheaths or in the connective tissue septa. ${ }^{1}$

Like the gliomata occurring in the brain the amount of cellular elements in these growths varies, some of them being more allied to embryonic tissue than others. The degree of development of the glial tissue may also vary in different parts of the same growth. Areas in it sometimes present a jelly like appearance, which has led to the mistake of describing the growth as myxomatous, but no mucin

${ }^{1}$ A. C. Hudson. R. Lond. Ophth. Hosp. Reps., XVIII, I9I 2, 5 I 7. 
is found in the fluid from these areas, which are in origin comparable to the vitreous humour. Microscopically they present the appearances seen in various stages of development of the vitreous humour, that is a felt work of fibrils more or less compactly grouped with small granules or thickenings on them.

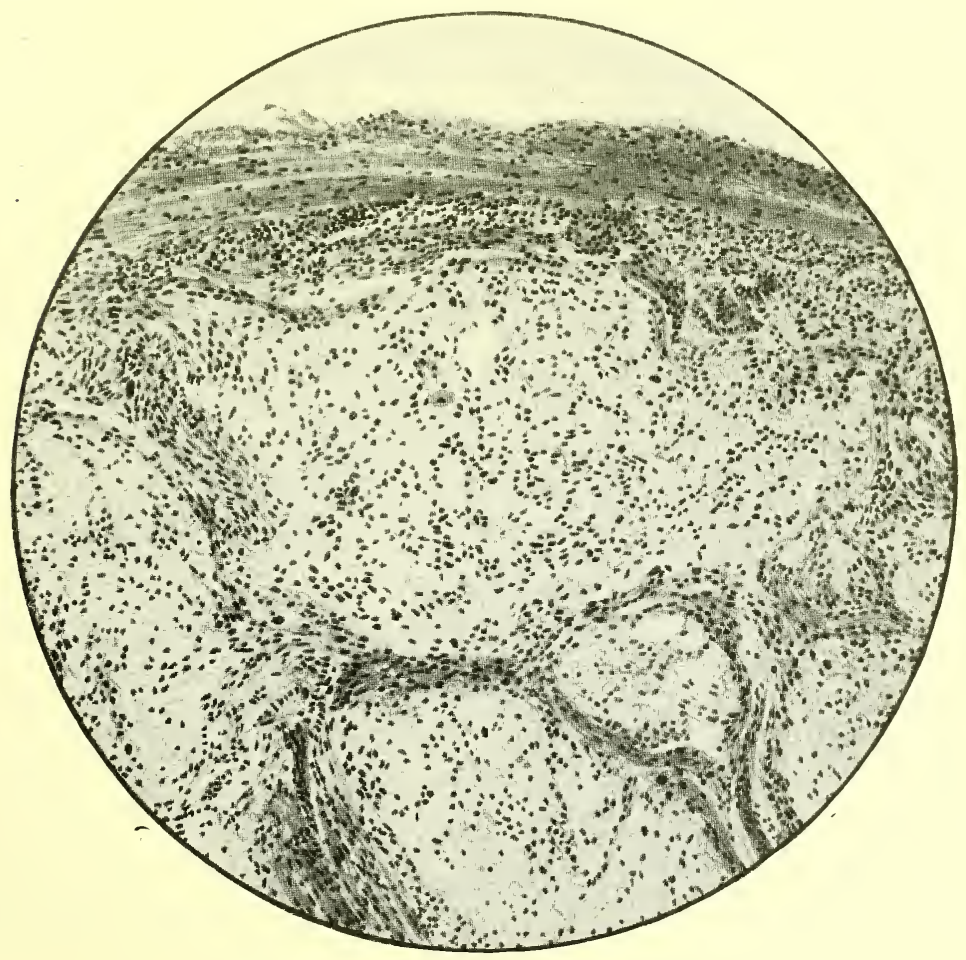

FIG. 90.-Transverse section through a glioma of the optic nerve. $\times$ Ioo. The pial sheath and fibrous trabeculæ in the substance of the nerve are considerably thickened; the spaces between the trabeculæ are occupied by a network of irregular branching cells, no healthy nerve-fibres being left. Case recorded by Treacher Collins and C. D. Marshall, Trans. Ophth. Soc. of the U. K., XX, I900, I59.

\section{Innocent Tumours of the Pars Ciliaris Retinæ ("Adeno-} mata").--Small innocent growths arising from the inner unpigmented layer of cells lining the ciliary process have been met with in the eyes of elderly people. As a rule they are not more than $\mathrm{I} \mathrm{mm}$. in diameter and do not give rise 
to any clinical symptoms, their presence being discovered only on pathological examination. In one case, ${ }^{1}$ however, the growth was unusually large from the formation of a cyst, and it pushed forward the periphery of the iris in its locality, so leading to the recognition of its presence clinically.

These growths start at the summit of a ciliary process and usually extend outward into it; sometimes they give rise to thickening on the inner surface of the ciliary body.

Springing from the unpigmented layer of cells the growth itself is entirely without pigment, but carries the pigmented layer in front of it as it extends outward. Both layers become invaginated, the lumen of the space so formed communicating with the interior of the eye. If the communication becomes closed and secretion from the lining cells continues, a cyst is developed.

The growth consists essentially of an increased proliferative activity of the epithelial lining of the ciliary body. This lining epithelium has been described as glandular in character; it is situated in that part of the eye in which the aqueous humour and nutrient fluid of the vitreous is poured out, and has been regarded as concerned in their elaboration. Tumours arising in it have therefore been termed adenomata. They $^{2}$ differ, however, from adenomata arising elsewhere in not possessing any fibrous-tissue stroma, so that sometimes they are called epithelial hyperplasia ${ }^{3}$ or innocent tumours of the ciliary epithelium.

Innocent Pigmented Growths in the Uveal Tract ("Melanomata").--Innocent pigmented growths comparable to the moles or nevi of the skin and conjunctiva are met with in the iris. They are usually situated near the pupillary border and appear clinically as small, dark, raised patches in the iris, which do not increase in size.

${ }^{1}$ G. Coats. R. Lond. Ophth. Hosp. Reps., XVII, I907, I43.

2 A. Alt. Am. J. of Ophth., XV, I898, 32 I.

${ }^{3}$ J. H. Parsons. R. Lond. Ophth. Hosp. Reps., XV, 1903, 375. 
Microscopically they have been found composed of polygonal, pigmented cells, arranged usually in an alveolar fashion in the deep layers of the iris (Fig. 9I).

As already pointed out, there is some evidence suggesting that the cells of which a nevus of the skin is composed are snared off from the surface epithelium. The most recent investigations into the embryology of the iris show that the sphincter muscle and the dilator muscle arederived

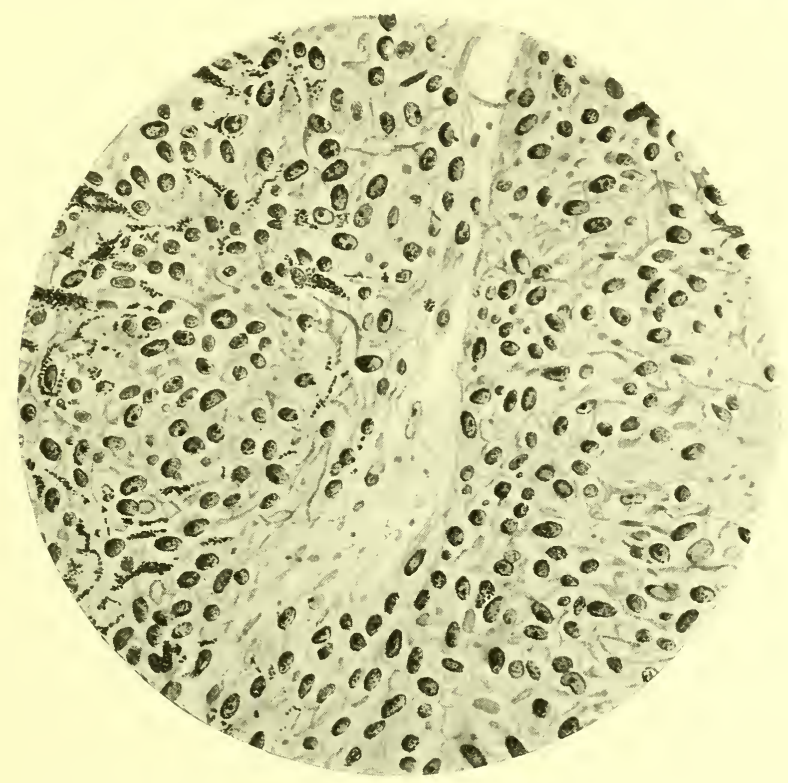

FIG. 9r.-Section showing the microscopical characters of an innocent pigmented growth of the iris. $\times 300$. Case recorded in the Trans. Ophth. Soc. of the U. K., XIX, I899, 53 .

from the anterior layers of the secondary optic vesicle, the former becoming snared off and entirely surrounded by mesoblast. It seems possible, therefore, that a melanoma of the iris may be composed of cells which have extended forward into its stroma from the pigment epithelium lining its posterior surface.

A pigmented new growth derived principally from the cells of the outer layer of the optic vesicle covering the ciliary body has been found in a congenitally malformed eye 
(Fig. 92); the cells for the most part were arranged in contact with each other, but toward its outer part there was an attempt at alveolar ${ }^{1}$ formation.

Oval dark patches with a sharply defined margin are seen ophthalmoscopically in the choroid. They do not increase in size or alter in appearance. It seems likely that such patches are innocent melanomata of the choroid like

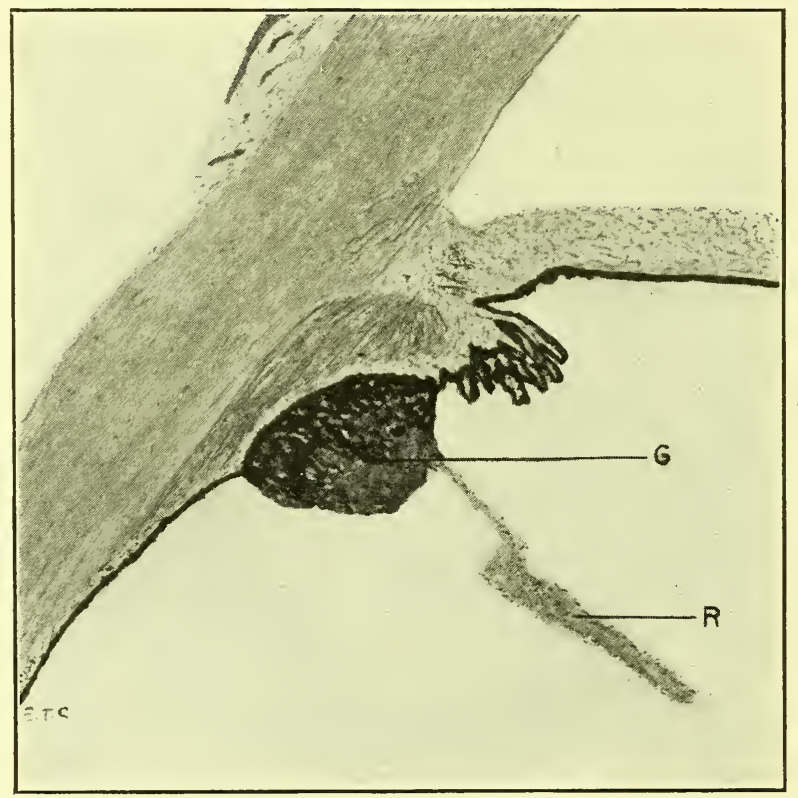

FiG. 92.-Congenital pigmented tumour of the ciliary body $G$, in a microphthalmic eye. Note the fetal condition of the angle of the anterior chamber and the separation of the two layers of the optic vesicle; the retina $R$ has never been in contact with the pigment cell layer.

those seen in the iris. In one ${ }^{2}$ of them, which was examined pathologically after having been seen ophthalmoscopically, the cells were found to be so deeply pigmented that its structure could only be studied in sections at the edge of the growth or after they had been bleached. It was composed of large spheroidal cells with circular nuclei

${ }_{1}^{1}$ M. S. Mayou. Trans. Ophth. Soc. of the U. K., XXVIII, I908, I07.

${ }^{2}$ Foster Moore. R. Lond. Ophth. Hosp. Reps., XIX, I9I4, 4 II. 
lying amongst a stroma consisting of normal choroidal chromatophores.

Malignant Melanotic Growths of the Pigment Epithelium of the Uveal Tract (Melanotic Carcinomata).Malignant growths arising from the pigment epithelium of the uveal tract are exceedingly rare. Cases have been described starting in its three different divisions, the iris,

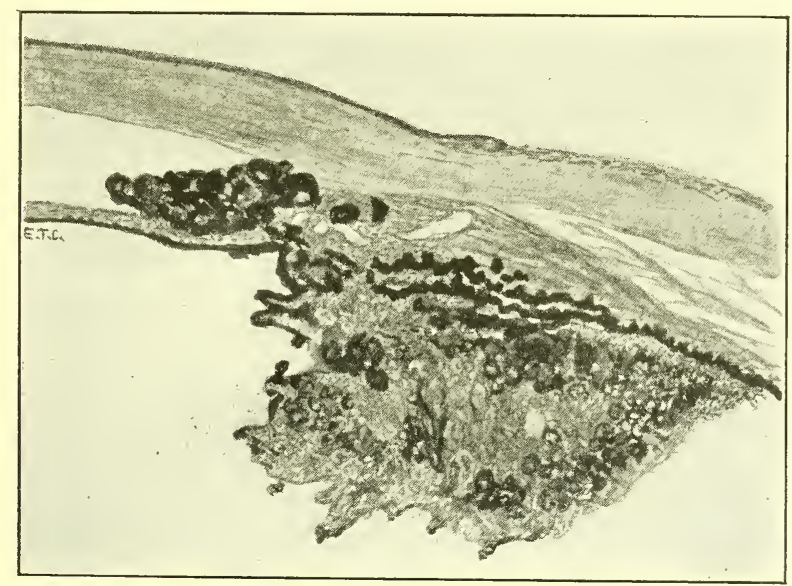

FIG. 93.-Melanotic carcinoma of the ciliary body starting as a primary growth from the pigment epithelium of the ciliary body in a woman aged 63 years. The root of the iris has become invaded.

the ciliary body, and the choroid. They may arise directly from the epithelial layer covering its inner surface or from a melanoma. In the iris the history of a melanotic nodule for many years before the onset of a melanotic growth in it has several times been obtained.

Those who consider nevi to be of mesoblastic origin, from the appearance of their cells, regard the malignant growths which originate in them as endotheliomata; whilst those who hold that the nevi are of epiblastic origin consider them carcinomata. It is possible that some of the cases reported as alveolar sarcoma of the uveal tract may have arisen in innocent melanomata. 
There seems to be no doubt about the epithelial origin of some of the melanotic malignant growths originating in the ciliary body. ${ }^{1}$ Melanotic carcinoma has been found growing from the surface and involving the substance of the ciliary body and iris (Figs. 93, 94), also forming a raised annular mass on the inner surface of the ciliary body with

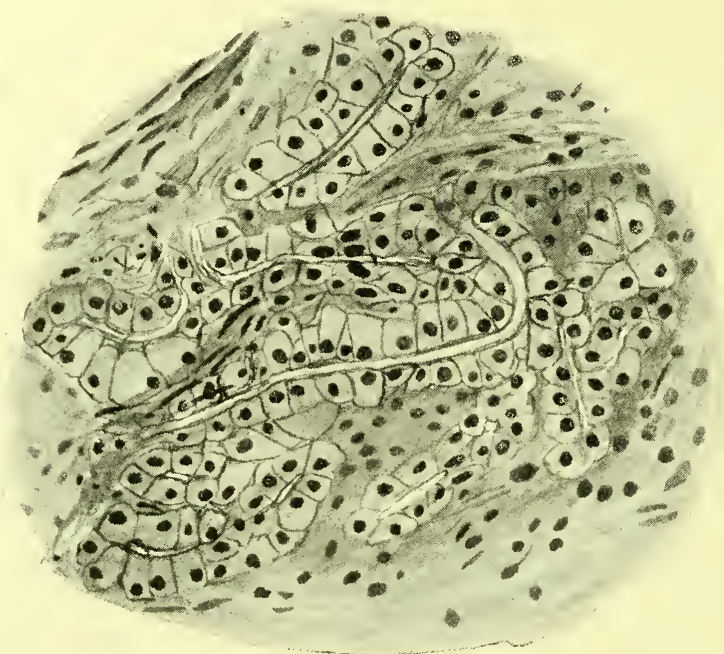

FIG. 94.--Bleached section of the melanotic carcinoma of the ciliary body depicted in Fig. 93. $\times 300$.

local metastases. In some cases this form of new growth was found in eyes which had previously been the seat of plastic endophthalmitis.

\section{TELEPLASMS ARISING IN TISSUES DERIVED FROM MESOBLAST}

A teleplasm arising in a tissue derived from mesoblast in which an early embryonic condition of that tissue is reproduced is termed a sarcoma. Such a growth is malignant. It is mainly composed of cells which are constantly pro-

${ }^{1}$ Treacher Collins. Trans. Ophth. Soc. of the U. K., XI, I89I, 6I. E. Fuchs. Arch. f. Ophth., LXVIII, I908, 3, 534. 
liferating, hence it rapidly increases in size. Its cells are liable to penetrate into the blood channels where, becoming detached, they form emboli; the transference of these cells to other parts of the body accounts for the occurrence of metastatic growths.

Sarcomata have usually been subdivided into classes according to the shape of the cells of which they are composed: hence the terms round, spindle, and mixed cell sarcoma. A more satisfactory classification has, however, been formed, based on the type of mesoblastic tissue which the growth simulates and hence the terms endothelioma, perithelioma, cylindroma, and lympho, fibro and myeloid sarcoma; the nomenclature describing the shape of the cells being reserved for the growths of a more rudimentary type.

The sarcomata described here are divided into intraocular, epibulbar and orbital.

A teleplasm composed of mesoblast more highly organised than a sarcoma is less cellular and less malignant. Any of the tissues into which the mesoblast becomes differentiated may form the predominant constituent of a growth; hence the terms, fibroma, osteoma, angioma, myoma, and lymphoma.

Intraocular Sarcoma.-Sarcoma of the uveal tract, although the most common form of new growth arising within the eye, is a comparatively rare disease. The anterior parts of the tract are much less frequently affected than the posterior. The ciliary body is more often the primary seat of sarcoma than the iris, the choroid than the ciliary body, and the posterior half of the choroid than the anterior.

Sarcoma of the uveal tract may occur at any age, it has been met with in a child two years old and a man of eightyfour; it is most frequent between the ages of forty and sixty. The two eyes and both sexes are affected almost equally. No hereditary tendency to it has been traced, except in one case where a mother and two daughters were similarly 
affected. ${ }^{1}$ Some general tendency to tumour formations in near relatives is, however, not infrequent.

The symptoms produced vary according to which of the three divisions of the uveal tract, iris, ciliary body, or choroid, is primarily involved. Whichever part is attacked, glaucoma, and the formation of extrabulbar and metastatic growths may ensue.

Sarcoma commencing in the iris manifests itself as a pigmented or unpigmented nodular swelling which steadily

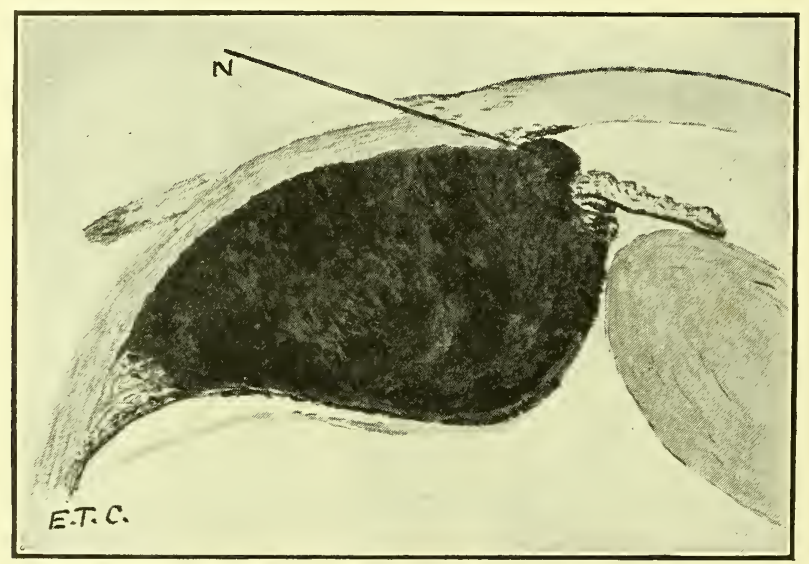

FIG. 95.-Melanotic sarcoma of the ciliary body extending forward through the root of the iris and appearing in the anterior chamber at $N$; also infiltrating the spaces of Fontana.

increases in size, is very vascular, and is frequently accompanied by hemorrhage into the anterior chamber. The ciliary body and ligamentum pectinatum become invaded early in the disease. The involvement of the latter causes increase of tension by preventing filtration from the eye. Extension of growth along the walls of the canal of Schlemm, or the anterior perforating vessels, gives rise to the formation of extraocular nodules.

Sarcoma commencing in the ciliary body soon invades the root of the iris, and makes its appearance at the extreme

${ }^{1}$ J. H. Parsons. Trans. Ophth. Soc. of the U. K., XXV, I905, 205. 
periphery of the anterior chamber, where it simulates clinically an iridodialysis (Fig. 95).

The interference which it causes in its early stages with the secreting function of the ciliary body may give rise to decrease in the intraocular tension. Later owing to the iris becoming pushed forward, lateral displacement of the lens, or invasion of the ligamentum pectinatum, the exit of the intraocular fluid becomes obstructed, and glaucoma ensues.

A new growth of the ciliary body which spreads forward through the base of the iris extends into the anterior chamber, and may eventually fill it. Enlargement of the growth directly inward raises and ruptures the elastic

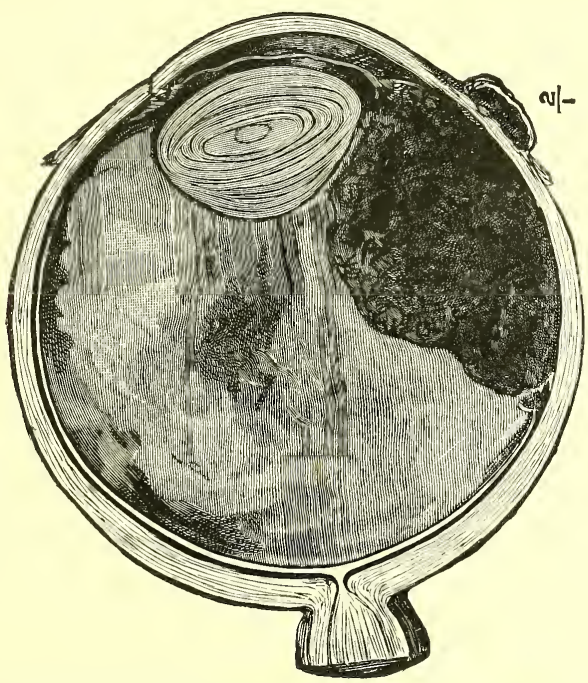

FIG. 96.-Lateral half of an eyeball containing a melanotic sarcoma of the ciliary body. There is a small extraocular nodule over the seat of one of the anterior perforating vessels, a short distance from the sclero-corneal margin, Specimen in the R. Lond. Ophth. Hosp. Museum.

lamina, later pressing on the side of the lens, distorting it in shape and displacing it laterally. An invasion of the lens substance by a sarcomatous growth through a rupture in its capsule has been described.

From extension backward of the growth the choroid becomes implicated, and in the later stages of the affection 
the retina may become detached. On its outer surface the growth has the tough sclerotic which resists invasion, the channels of the anterior perforating vessels passing through it are, however, early implicated. The formation of a nodule of pigmented growth on the surface of the globe at the site of one of these vessels being sometimes the first symptom to attract attention (Fig. 96).

In some rare cases of sarcoma of the ciliary body, the growth infiltrates the whole circle of that structure instead

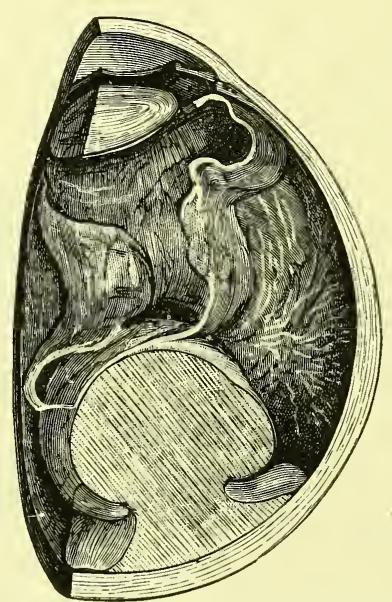

FIG. 97.- Shows an eye with a sarcoma growing from the choroid. The growth has extended inward through a rupture in the elastic lamina and then expanded into a rounded knob. Specimen in the R. Lond. Ophth. Hosp. Museum.

of forming a protuberant mass in one part of it. Such cases are known as ring or annular sarcomata and are comparable to the diffuse or flat sarcomata of the choroid. ${ }^{1}$

Sarcoma commencing in the choroid begins by spreading laterally, forming a disc-shaped thickening. The resistance to its extension is greater on the outer surface, where it is in contact with the firm sclerotic, than on the inner surface, where there is only the elastic lamina and retina. The elastic lamina, therefore, becomes raised up by the growth until the tension of it is so great that it

${ }^{1}$ J. H. Parsons. Arch. f. Ophth., I902, 2, LV, $35^{\circ}$. 
ruptures. The resistance to its extension inward being removed, the growth fungates through the rupture in the form of a rounded knob, the whole growth assuming a mushroom-like shape (Fig. 97).

In rare cases, a sarcoma of the choroid instead of growing inward spreads laterally, infiltrating and thickening the membrane instead of forming a protuberant mass on its

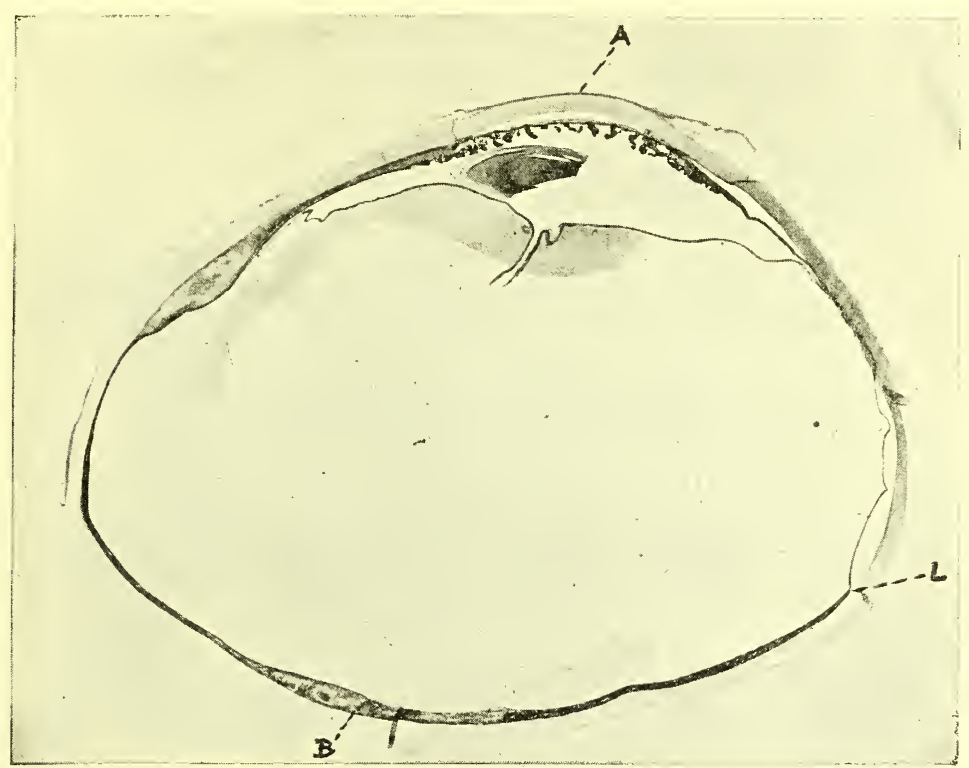

FIG. 98.- Section of eyeball with a flat sarcoma of the choroid. $A$, detached retina; $B$, main mass of the growth; $C$, spreading margin.

inner surface. Such sarcomata (Figs. 98 and 99) are termed diffuse or flat in contrast to the commoner variety which are circumscribed or nodular.

In sarcoma of the choroid the retina very soon becomes detached. The detachment extends over a wider area than that occupied by the growth; albuminous fluid which becomes coagulated by hardening reagents into a jelly-like material, filling the sub-retinal space. Besides the detachment in the vicinity of the growth there is often a second 
independent detachment in the lower part of the globe, ${ }^{1}$ due to gravitation of the fluid. The extent of the detachment tends to increase until it becomes complete, the retina assuming an umbrella shape. Frequently the retina retains an adhesion to the apex of the rounded knob of the growth, where there may be hemorrhages into it.

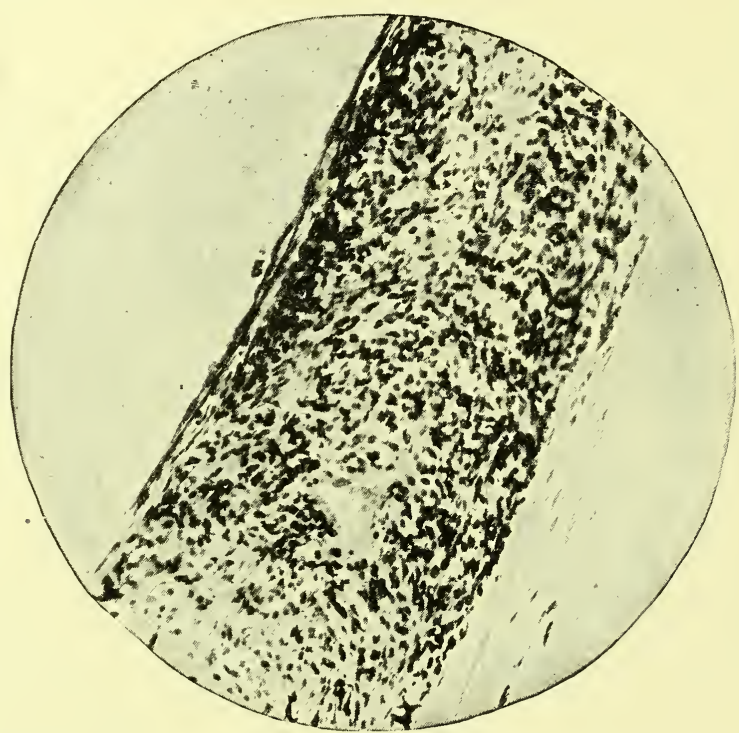

FIG. 99.-Section of the main mass of the growth shown in Fig. 98 under higher magnification. It is composed of spindle cells with a few large scattered pigment cells, and shows no tendency to alveolar arrangement. Case recorded by Cargill and Mayou, Trans. Ophth. Soc. of the U. K., XXVII, I907, I49.

The detachment of the retina is caused by an out-pouring of serous fluid from the choroidal vessels, due to obstruction to the venous circulation from pressure by the growth.

In the first stage of a sarcoma of the choroid there is no increase of tension. The space in the eye occupied by the growth and sub-retinal exudate is compensated for by fluid being squeezed out of the vitreous humour. U1timately the vitreous becomes so compressed and shrunken

${ }^{1} \mathrm{~J}$. H. Parsons. Ophthalmic Review, XXIV I905, I6r. 
that the lens and iris are forced forward, the angle of the anterior chamber becomes closed, and glaucoma ensues.

Extension of the growth outside the eye may occur along any of the channels in the sclerotic penetrated by vessels or nerves (Fig. I0०), commonly along the sheaths of the perforating vessels in the vicinity of the base of the growth. An extrabulbar growth behind the globe increases rapidly and gives rise to proptosis.

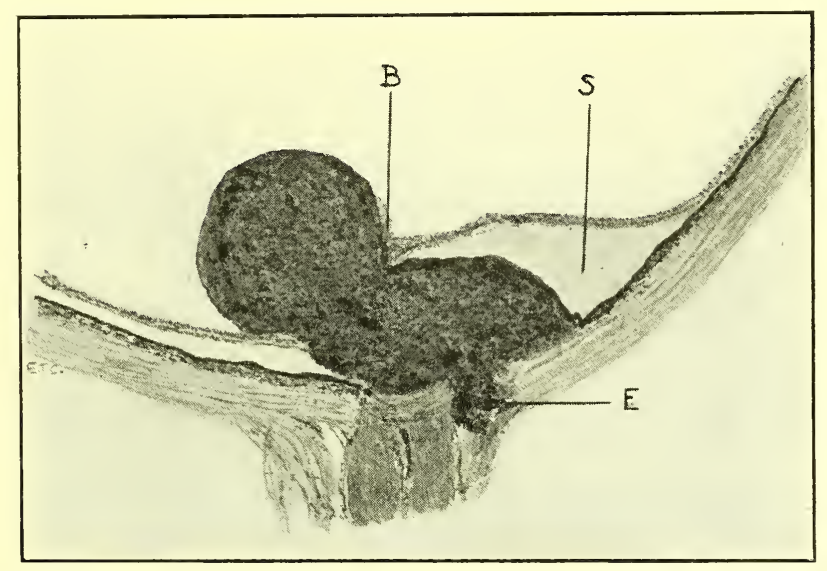

FIG. I0o.-Melanotic sarcoma growing from the choroid at the margin of the optic disc; breaking through the elastic lamina at $B$, and forming a round protuberance overlying the disc. Also extending through the sclerotic into the sheath of the optic nerve at $E$; the retina is partly detached and $S$ points to the subretinal albuminous exudate.

Metastatic growths occurring in connection with sarcoma of any part of the uveal tract are the result of transference of emboli of cells of the growth by the blood. That such emboli should occur is not surprising as the newly formed blood channels in the growth consist only of endothelial tubes, or even sometimes seem to be bounded by the cells of the growth itself. Metastases are mostly found in the viscera, the liver and lungs being the commonest sites. The lymphatic glands connected with the eye do not become affected.

Patients who have had an eye removed for sarcoma of the uveal tract more often die of metastatic growths than of 
a recurrence in the orbit. In the majority of cases, a patient with metastases, succumbs to their effects within three years from the date of excision of the eye. Much longer intervals may, however, elapse. ${ }^{1}$

Sarcomata of the uveal tract are sometimes divided into melanotic sarcomata and leuco-sarcomata, the former being far the more frequent. Such a classification cannot be very accurate or of much importance. Growths which appear to be quite white macroscopically are often found to contain pigment granules when examined microscopically. They may sometimes be densely pigmented in one part and devoid of pigment in another. A growth which is deeply pigmented in the eye is frequently unpigmented when it becomes extraocular. The primary growth in the eye may be pigmented and the metastatic growths unpigmented. The pigmentation of sarcomata in the uveal tract is generally due to melanin; in some, however, the pigment is derived directly from the blood usually as the outcome of hemorrhages into it; such pigmentation is termed hematogenous.

The character of the cells met with in sarcomata of the uveal tract varies considerably in different cases, and also sometimes in the same growth. The cells are derived either from connective-tissue cells or endothelial cells.

Connective-tissue cells of the uveal tract in their development are first round, then spindle-shaped and branching; melanin granules being deposited when they are fully formed. Endothelial cells are first small round cells, then increase in size and become polygonal.

A malignant new growth of the uveal tract may be composed of cells of any of the above types. When two different types of cells are met with in the same growth it probably represents, either a reversion to different stages of development in the cells, or to proliferative activity

${ }^{1} \mathrm{~J}$. B. Lawford and Treacher Collins. R. Lond. Ophth. Hosp. Reps. XIII, I892, I04. 
excited in cells of different types, such as the connective tissue and endothelial cells.

In spindle-celled sarcomata the cells have oval nuclei and are arranged in groups. In a microscopical section of the growth some of these groups may be cut transversely so that both the cells and their nuclei appear round. This might lead to the mistaken diagnosis of the growth as a mixed round and spindle-celled sarcoma, a mistake which can be prevented by the examination of a teased out specimen of the tumour.

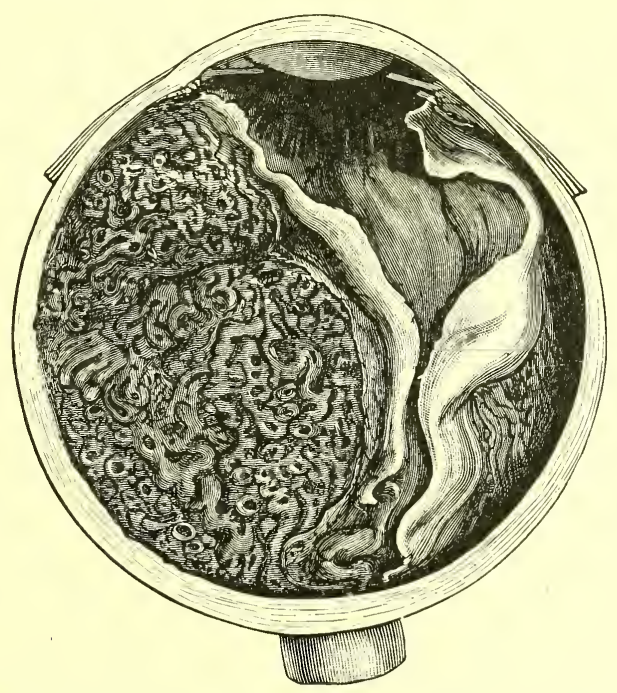

FIG. IOI.-The lateral half of an eyeball containing an angiosarcoma of the choroid. Note the foliated arrangement of the tumour at the seat of section. Specimen in the R. Lond. Ophth. Hosp. Museum.

In what are termed round-celled sarcomata it is the nucleus of the cell which is round, the cytoplasm in teased out specimens often showing projecting processes.

Endothelium in the normal uveal tract is found lining blood-vessels and lymph spaces; the latter are inter-fascicular and perivascular. Enhanced proliferative activity of any of these three different sets of cells is a possible source of a sarcomatous growth. The endothelium lining the blood channels rarely give rise to new growths. 
What are termed angiosarcomata or peritheliomata of the uveal tract are growths arising in the perivascular lymphatic sheaths. Some angiosarcomata present on section macroscopically a foliated appearance, simulating the convolutions of the brain (Fig. IOI). Microscopically blood-vessels with thin walls are seen surrounded by zones of cells which may be large and typically endothelial in origin (Fig. I02) or small with round nuclei and of irregular shape. The cells which are furthest from the blood-vessels

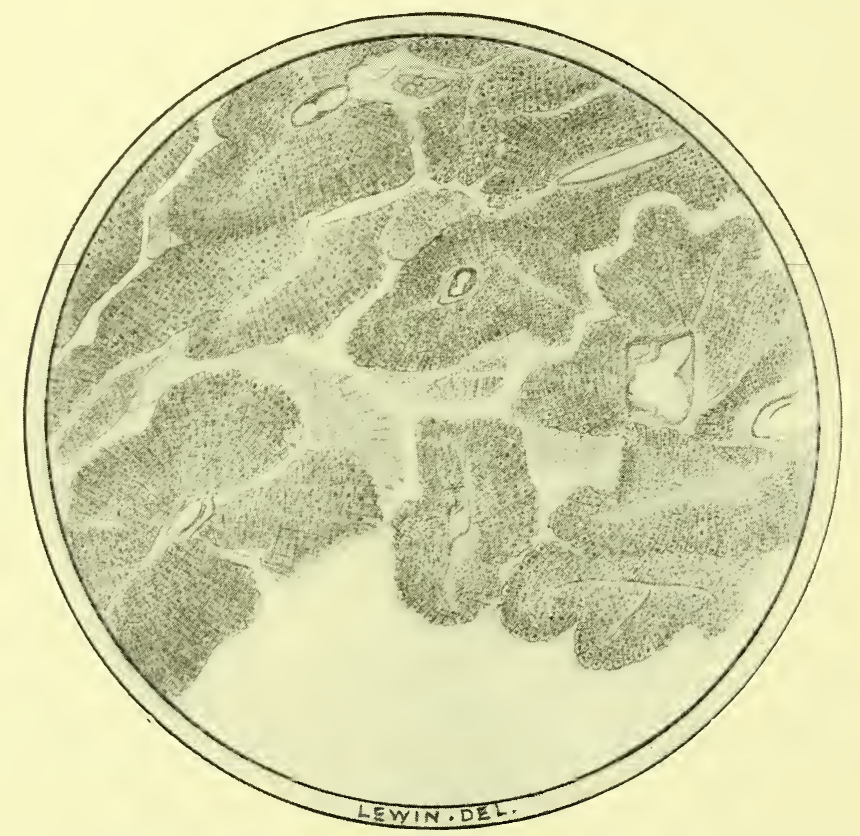

FIG. I02.- Section through an angiosarcoma of the choroid. Showing thinwalled blood-vessels surrounded by zones of cells of an endothelial type.

in this form of growth show a marked tendency to degeneration, their nuclei staining badly and the cytoplasm undergoing fatty changes. Hyalin and myxomatous degeneration may also be present. Angiosarcomata are frequently found without any melanin but are often the seat of hematogenous pigmentation, hemorrhages into them being exceedingly common. In some cases the hemorrhage is so 
extensive that on section of the globe the presence of a growth is easily overlooked, the interior of the globe appearing to be entirely filled with degenerate blood clot. ${ }^{1}$ In these cases degenerative changes are found not only in the growth itself, but also in the other tissues in the interior of the eye. There seems to be a general necrosis of most of the intraocular contents the cause of which has not yet been determined. It is suggested that it is the result of some toxin due to endogenous microbic infection.

There are rare cases of sarcoma of the uveal tract which, instead of going on to the glaucomatous stage, develop a plastic uveitis causing a shrinking of the globe; it may be that these cases are a later stage of degenerating angiosarcomata.

A malignant growth commencing in the endothelium of the lymph spaces of the uveal tract is termed interfascicular endothelioma. ${ }^{2}$ It presents an alveolar formation, and when its cells are large and polygonal simulates a carcinoma, from which it may be differentiated by the intimate connection of the tumour cells with the connective tissue. In the early stage of a carcinoma no such intimate connection exists, the cells of the growth being separated from the connective tissue by the layer of endothelial cells lining the lymphatic spaces along which it spreads.

Ring, diffuse, or flat sarcomata already referred to, are mostly interfascicular endotheliomata,$^{3}$ their tendency to extend laterally along the neighbouring lymph spaces, the line of least resistance, accounting for their mode of growth. In this particular they resemble "metastatic" carcinoma of the uveal tract (see page $22 \mathrm{I}$ ).

Interfascicular endotheliomata grow slowly and are liable to degenerative changes, both in the cells of the growth itself and in its connective tissue.

${ }^{1}$ J. H. Parsons. Trans. Ophth. Soc. of the U. K., XXV, I905, I93.

${ }^{2}$ H. Coppez. Arch. d'ophtalmol., XXI, I9oI, I4I.

${ }^{3}$ M. S. Mayou. Trans. Ophth. Soc. of the U. K., XXVII, I907, I49. 
In the oldest parts of the growth these degenerative changes may be so extensive as to destroy its characteristic features. The cells undergo hyalin or colloid degeneration which may result in cystic formation. The connective tissue undergoes hyalin or mucoid degeneration, and in some rare instances there has been a formation of cartilage. It is always necessary to examine the spreading edges, and more recently formed portions of the growth, to ascertain its real nature.

Tumours of pigmented endothelium arising primarily in the ligamentum pectinatum have been described. ${ }^{1}$ The endothelial cells lining the spaces of Fontana are usually pigmented, so that a new growth starting from them might be expected to be of a similar character. These growths are composed of polygonal cells arranged in an alveolar manner, after filling the angle of the anterior chamber they invade the cornea, iris, and ciliary body.

In the normal choroid, pigment is found in the large flattened cells of the lamina suprachoroidæ and in the branching cells of the outer vascular layers.

The pigment in melanotic sarcomata of the uveal tract is located in the cells of the growth, in the endothelium of the new vessels, and sometimes also in the intercellular fibrous tissue.

The chromatophores in sarcomata of the uveal tract are either of an embryonic type, or similar to the fully formed cells. Some are met with which appear swollen and distorted in shape from degenerative changes. The melanin in them, as in the normal cells of the choroid, is in the form of granules.

It has been suggested that some of the pigmented cells in a sarcoma of the choroid, which has ruptured the elastic lamina, are retinal pigment epithelial cells which have proliferated into the growth; their shape and the appearance of the pigment granules in the form of short rods favouring

${ }^{1}$ Hanke. Arch. f. Ophth., XLVII, I 899, 3, 463. 
such a view. It is difficult, however, to see how they could make their way through the capsule surrounding the growth.

Hematogenous pigmentation, following hemorrhage into a sarcomatous growth, may be differentiated from melanotic pigmentation by its lighter colour, being golden or brown, never black, and by giving an iron reaction.

Epibulbar Sarcoma.-Epibulbar sarcoma starts usually in the conjunctiva at the margin of the cornea (Fig. I03). The connective-tissue cells of the conjunctiva in this region

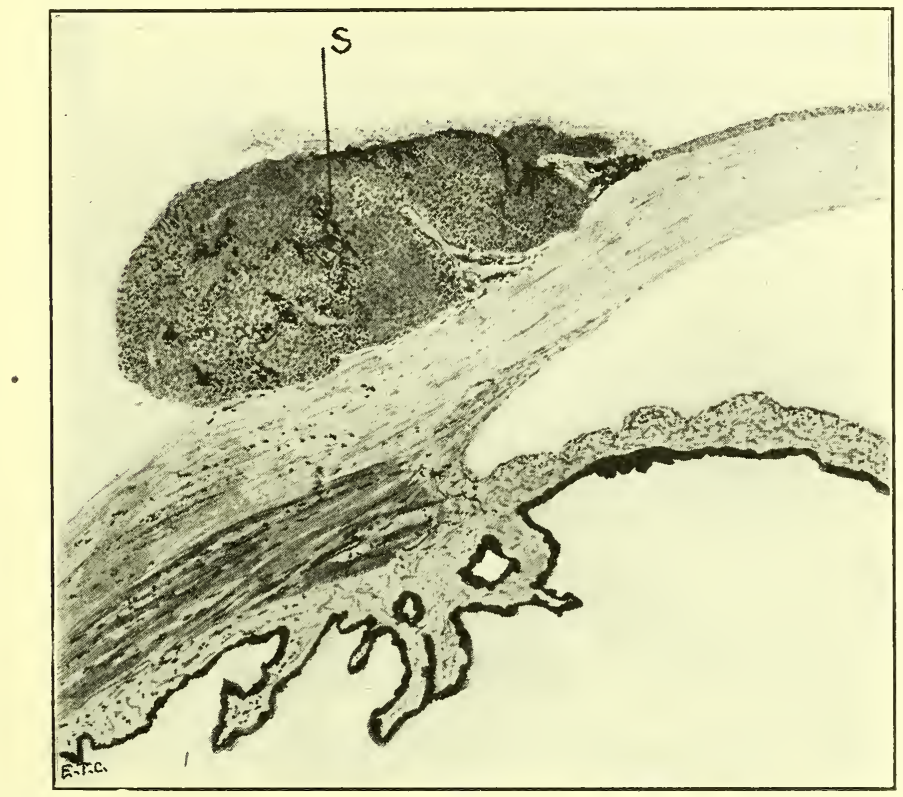

F1G. I03.-An epibulbar, melanotic, sarcoma of the conjunctiva, $S$, starting at the limbus.

contain pigment granules. In the dark races the amount of pigment is such as to produce a brown ring around the cornea. As epibulbar sarcomata are generally pigmented, the pigment in them being melanin and not hematogenous, it is probable that many of them originate in these cells.

Though the limbus is the commonest site for a sarcoma of the conjunctiva, it may occasionally begin in other parts, and has been seen to start from the plica semilunaris. 
The malignant epibulbar growths which arise from congenital nevi of the conjunctiva clinically resemble sarcomata. Some authorities regard such growths as endotheliomata, but for reasons already stated it has been thought best to group them with the carcinomata (see p. I6I).

True endotheliomata may arise in the conjunctiva from the lining cells of the blood or lymphatic vessels, and in rare cases extend into the cornea where they present an alveolar arrangement of cells of an endotheliomatous type.

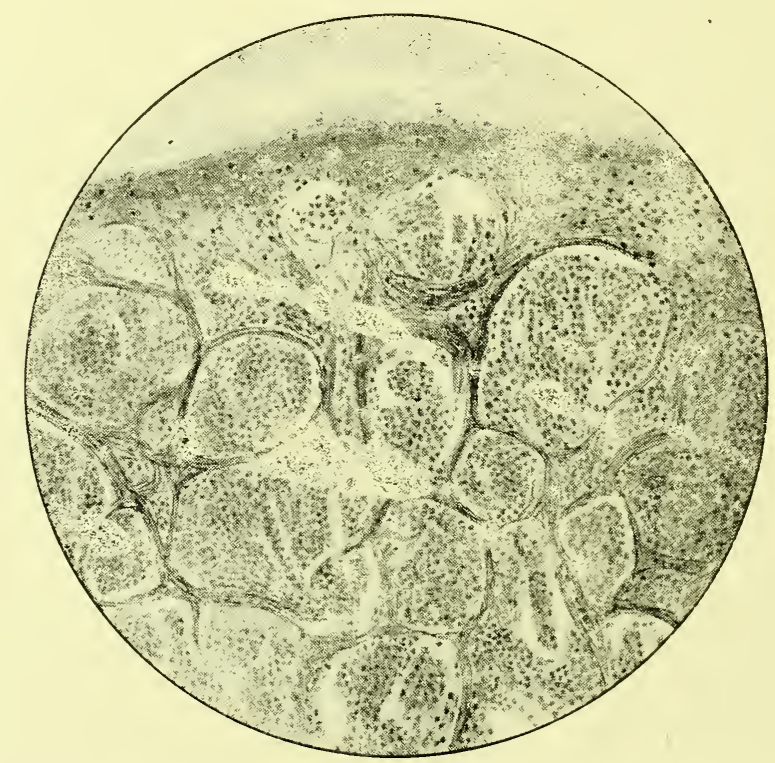

FIG. 104.- Shows the microscopical appearances of a growth which, starting near the limbus, involved the cornea. It is composed of collections of round cells grouped together in spaces bounded by bundles of fibrous tissue. Case recorded in Trans. Ophth. Soc. of the U. K., XV, I895, 90.

Epibulbar sarcoma may occur at almost any age; it has been met with at the age of eight, but usually appears between forty and fifty.

It commences as a small patch which generally develops into a nodular pedunculated mass but may form a flattened sessile growth. When pigmented, as it usually is, the pig- 
ment may not be confined to the new growth but become disseminated in the tissue around, especially along the margin of the cornea.

The tough fibrous tissue of the sclerotic and cornea does not readily become invaded (Fig. I04), and until it does the growth will be found to move with the conjunctiva on its surface. Intraocular invasion is rare but has been met with, the extension backward taking place along the track of the anterior perforating vessels or the canal of Schlemm.

The difficulty which the growth has in penetrating the sclerotic allows, when a case comes under observation early, of the entire growth being removed from the surface of the eye so that no local recurrence takes place. Metastatic growths from an epibulbar sarcoma occur with considerable frequency.

The shape of the cells in an epibulbar sarcoma may be round or spindle-shaped, but as in most sarcomata multinucleated cells are occasionally met with. The granules of melanin are found located both in the cells of the growth itself and in the intercellular tissue.

Sarcoma of the Orbit. - The orbit contains a variety of tissues from which a sarcoma may originate. The periosteum, the intermuscular septa, the endothelium of the blood and lymph channels, the sheath of the optic nerve and the lacrymal gland have all been located as the primary seat of this form of growth.

Sarcoma of the orbit may also arise from extensions into it, either of a growth originating in the eyeball, or in one of the neighbouring sinuses. The former have already been referred to in treating of intraocular sarcoma, the latter are outside the scope of this work.

Primary sarcoma of the orbit is a rare affection. It may arise before birth and at all ages, most frequently it occurs before the age of ten. Cases having growths in the two orbits have been recorded, it would seem unlikely that these were two primary independent tumours, but were probably, either lymphomata, (see Metastatic Growths) or growths 
originating in an ethmoidal or sphenoidal sinus which extended laterally into each orbit.

All sarcomata arising in the orbit give rise to proptosis, and usually to a sense of resistance on pressure of the eyeball backward. The direction of the proptosis, and the possibility of recognising the presence of a tumour on palpation, depend on its seat of origin.

The degree of malignancy varies with the character of the growth. Small, round-celled sarcomata extend rapidly and are very liable to cause metastases.

A tumour consisting of a large amount of fibrous tissue with round or spindle shaped cells is termed a fibro-sarcoma, and is the commonest form of sarcoma met with in the orbit. It represents a reversion to varying degrees of development in the tissue from which it starts, and has a low degree of malignancy.

The endotheliomata, though they extend rapidly locally do not give rise to metastasis with the same frequency as the small, round-celled growths.

Histologically it is often difficult to differentiate the small, round-celled sarcomata from gummata and lymphomata. The appearance of the blood-vessels may be of some assistance; in a sarcoma their walls are frequently composed almost entirely of the tumour cells. In a gumma, on the other hand, the thickened walls, well differentiated, from the small round cells by which they are surrounded can be made out.

A group of cases has been described under the name of inflammatory pseudotumour of the orbit $;^{1}$ a rare condition which cannot usually. be diagnosed without microscopical examination of the tissue involved.

Clinically it gives rise to the usual symptoms of an orbital neoplasm viz., proptosis and displacement of the globe with some loss of mobility. In the course of time the eye gradually becomes more firmly imbedded in the new tissue and

${ }^{1}$ W. L. Benedict and Mary S. Knight. Archiv. of Ophth., LII, I923, 582. 
cannot be pressed backwards. The progress of the affection is usually slow and is unaccompanied by any inflammatory symptoms, the recognition of its real inflammatory nature only being ascertained by the microscopical examination of a piece of the affected tissue after its removal.

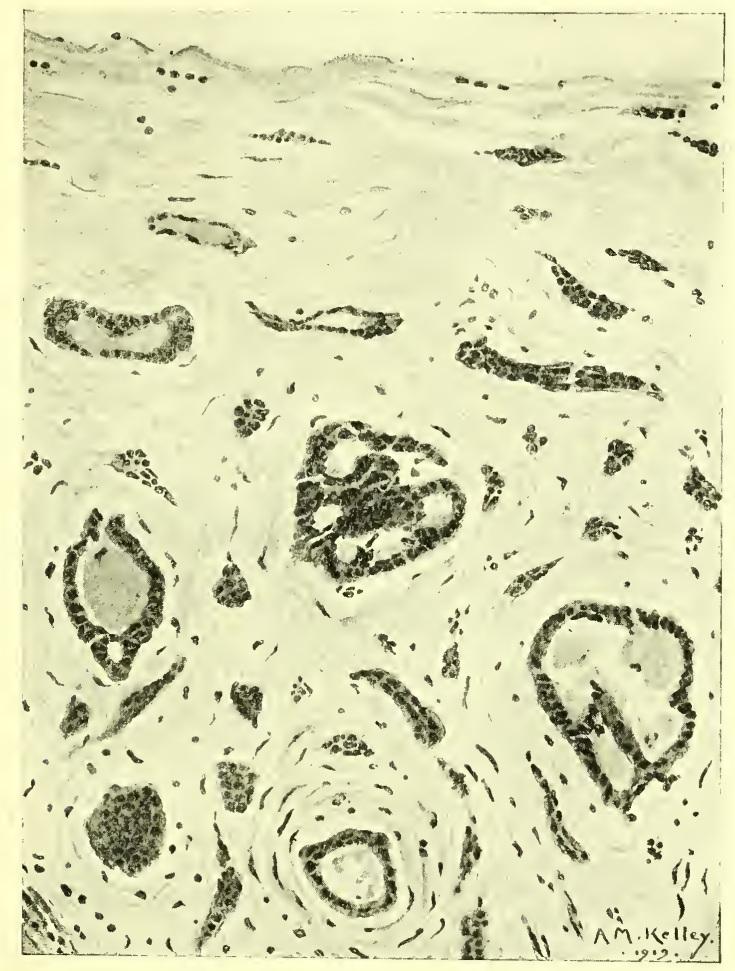

FIG. I05.-Endothelioma arising from the blood-vessels of the orbit, a scirrhuslike growth. Recorded in Trans. Ophth. Soc. of the U. K., XXXIX, I9I9, I35.

The condition has been ascribed to infection from some focal inflammation elsewhere which has escaped detection, and also to recurrent orbital hemorrhages which result in proliferation of the connective tissue and organisation. Certainly some of the recorded cases may best be accounted for as the result of hemorrhage.

The commonest seat of origin of a round-celled sarcoma is probably the periosteum. Some sarcomata, which are 
undoubtedly periosteal in origin, contain osteoblasts and have fragments of bone formed in them; these are termed osteosarcomata.

The endotheliomata which grow from the perivascular endothelium form columns of cells, and are termed cylindromata or angiosarcomata. The endotheliomatous character

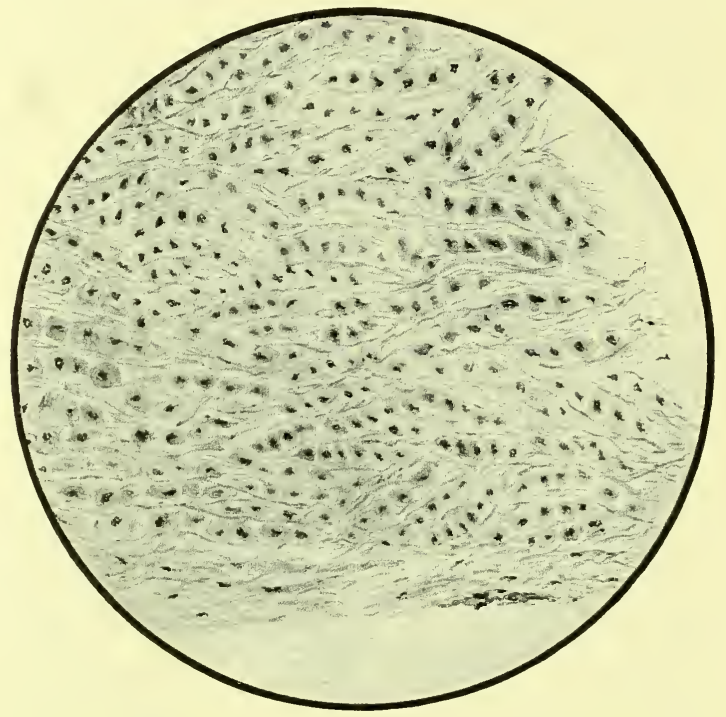

FIG. I06.-Endothelioma arising probably from the lymphatics of the orbit, a soft friable growth. Ibid.

of the cells can usually be made out in some parts of the growth, in others they may be less fully formed and of a small round or spindle-shape, while in others again degenerative changes may obscure their characteristics. The stroma of these tumours consists often of dense fibrous tissue, which gives them a scirrhous-like consistency (Fig. I05).

The endotheliomata originating in lymphatics show large well marked endothelial cells, grouped in spaces bounded by a small amount of fibrous tissue, alveolar sarcomata. Their microscopical appearance simulates a carcinoma, and they are of a soft consistency (Fig. I06).

Endothelioma of the Optic Nerve.-The endothelial cells found normally lining the arachnoid sheath of the optic 
nerve, and covering the trabeculæ of the intervaginal space, may be the starting point of endotheliomatous growths. Such tumours present a close histological resemblance to the endothelial tumours of the brain membranes, ${ }^{1}$ being composed of masses of typical endothelial cells, supported in a usually scanty connective tissue. The cells exhibit a strong tendency to whorled formations, in the centre of which laminated concretions are not infrequently met with. The tumour is either situated in the intervaginal space or intimately related to the dural sheath. It has been estimated that in 50 per cent. of these cases the onset of the symptoms dated from after the zoth year, and that a strikpreponderance of them were in females. Unlike the cases of gliomatosis of the optic nerve evidence of tumour formation usually precedes that of failure of vision. The tendency which these endotheliomatous growths have to infiltrate the neighbouring tissues is also in marked contrast to the gliomata. Local recurrence after operation is liable to occur.

Lymphoma.-The conjunctiva and the lacrymal gland are the only structures in connection with the eye which normally contain lymphoid tissue; they are, therefore, the only structures in which primary lymphomata originate. The lymphomata which are met with in the orbit and eyelids are described with the metastatic growths.

Lymphomata of the conjunctiva arises usually either in the fornix or the plica semilunaris. They have been met with starting at the limbus. Clinically they present the appearance of very large follicles and give rise to no inconvenience to the patient, their presence usually being discovered accidentally, or from the presence of some slight conjunctivitis. In rare instances a lymphoma has been know to assume malignant characters, lympho-sarcoma.

Histologically a lymphoma presents the structural characters of a lymphatic gland. There is a delicate stroma composed of fibres, endothelial cells and small blood-

${ }^{1}$ A. C. Hudson. R. Lond. Ophth. Hosp. Reps., XVIII, I9I2, 317. 
vessels, which is closely packed with mononuclear leukocytes and plasma cells.

The parenchymatous tissue of the lacrymal gland may be the primary seat of new growths presenting different characteristics. In the stroma of the normal lacrymal gland, between its gland tubules, there is some lymphoid tissue which may be the starting point of either innocent or malignant new-growths, Lymphomata or Lymphosarcomata.

Lymphomata may arise in one gland only or as multiple growths of the lacrymal and salivary glands. Mikulics considers that these multiple lymphomatous growths represent a definite morbid entity allied to but not identical with pseudo-leukæmia.

Though some of the unilateral cases which have been recorded as sarcomata were probably really lymphomata (Fig. I07), undoubted small round celled sarcomata of the gland do occur. Such tumours spreading in the stroma of the gland, separate widely its alveoli, and cause extensive degeneration of their lining epithelium. Though at first such tumours may be of slow growth, ultimately they break through the capsule of the gland and invade the orbit.

These lymphomatous growths are met with usually in persons under 40 years of age, and when first noticed form freely movable semifluctuating swellings at the upper and inner angle of the orbit.

In another form of growth starting in the parenchymatous tissue of the lacrymal gland, a variety of tissues of mesoblastic origin are found. Such growths occur in adult life, are never multiple, do not recur locally if completely removed, and do not give rise to metastasis in the lymphatic glands or elsewhere. A fatal issue has, however, been reported in some cases owing to the direct spread of the growth to the meninges. ${ }^{1}$

The structure of these growths is made up of the following tissues in various combinations, columns of endo-

${ }^{1}$ R. A. Greeves. R. Lond. Ophth. Hosp. Reps., XIX, 19I4, 237. 
thelial cells, spindle-shaped cells, fibrous tissue, myxomatous tissue, and hyalin cartilage, i.e., a mixture of the various tissues into which mesoblast may become differentiated. Growths of the parotid gland presenting a similar structure have been described as endotheliomata, and considered to arise from the flattened cells lining the

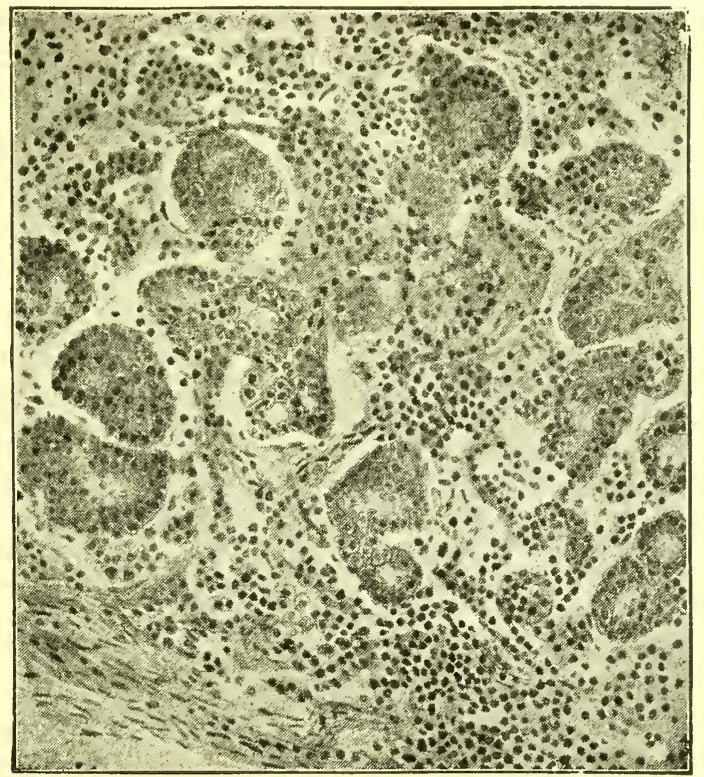

FIG. I07.- Shows the microscopical appearances of a tumour of the lacrymal gland composed of lymphoid tissue. The tubes of the gland are shown separated from one another by the new growth. Case recorded R. Lond. Ophth. Hosp. Reps., XIII, I893, 398.

lymph spaces. Endotheliomata starting in other parts of the body are, however, very different, they are not composed of such a heterogenous mixture of tissues, are more cellular and more malignant. It seems unsatisfactory to employ the same name for the two classes of growth and, therefore, those of the lacrymal gland are here termed tumors of mixed mesoblastic tissue.

Neuro-fibroma and Neuro-fibromatosis.-Neuro-fibromata occurring in connection with the eyeball or its appendages may be diffuse or localised. 
A diffuse neuro-fibroma, or neuro-fibromatosis, is a form of new growth of the fibrous tissue of the affected area, associated with a marked increase of the fibrous tissue elements of the nerves. When affecting the skin it was formerly known as the molluscum fibrosum of von Reckinghausen. The extreme hypertrophy of the part

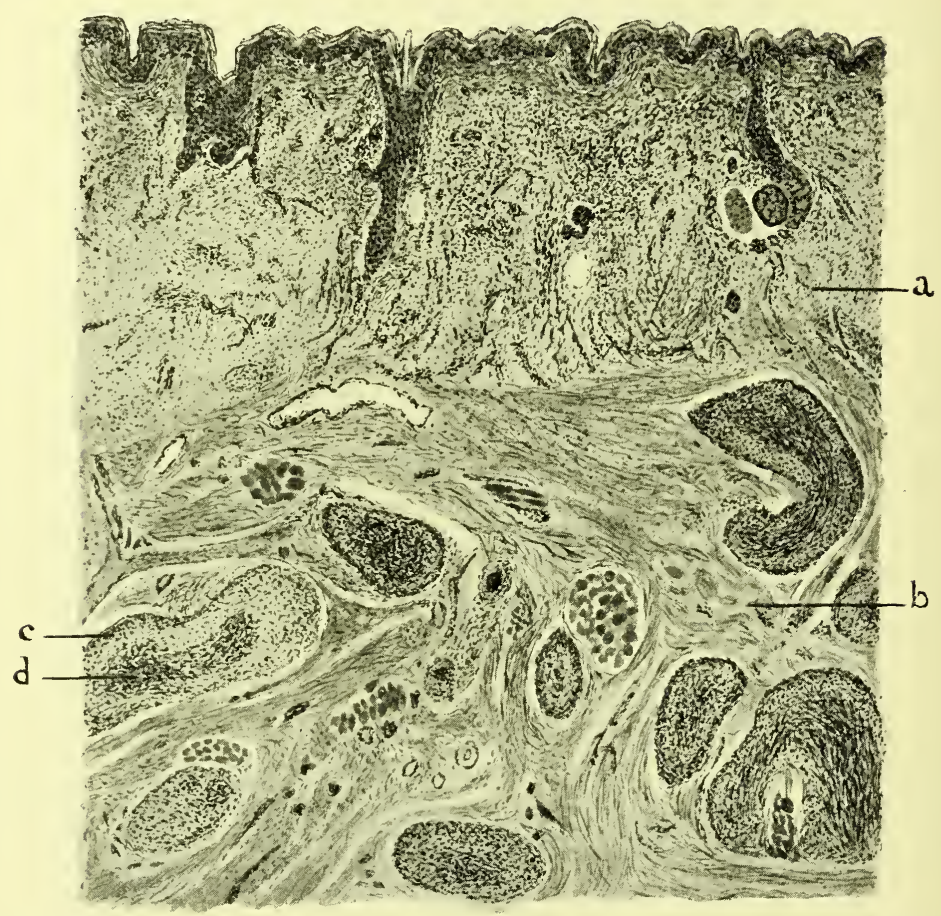

FIG. I08.--Shows the microscopical appearances of a section through the skin of the eyelid in a case of neuro-fibromatosis. $a$, Thickened corium; $b$, subcutaneous tissue with thickened nerves in it cut in various directions; $c$, the thickened perineurium of one of the nerves; $d$, nerve-fibres in the centre of the mass of thickened fibrous tissue.

involved led to the use of the clinical term congenital elephantiasis. The thickening of the nerves in some cases is so extensive that they become converted into a mass of convoluted cords with nodular and fusiform thickenings on them; hence the term plexiform neuroma. 
Though not a malignant form of growth it often shows a tendency to continuous, slow, local increase.

Microscopically the affected nerves show an increase of both the peri- and endoneurium, the nerve fibres themselves being unaffected. When the eyelids are involved they become considerably enlarged from a general hypertrophy of their connective tissue (Fig. I08). In some cases the upper lids form a large pendulous mass, which the patient is unable to raise sufficiently to see beneath. ${ }^{1}$ The palpebral conjunctiva becomes involved in the general hyperplasia. When the ocular conjunctiva is affected wormlike tortuous bodies, the thickened nerves, are seen on the surface of the globe. In the orbit the nerves may become converted into a convoluted

${ }^{1}$ S. Snell and Treacher Collins. Trans. Ophth. Soc. of the U. K., I903, XXIII, I 57. M. S. Mayou and G. Sutherland. Trans. Ophth. Soc. of the U. K., I907, XXVII, I 79 .

FIG. I09.-Shows the microscopical appearances of the ciliary region and sclerocorneal margin in a buphthalmic eye occurring in connection with neurofibromatosis of the eyelids. The root of an ill-developed iris is seen to be very thin and adherent to the cornea. In the small piece of iris left free, a wavy line is just indicated, showing the way in which Descemet's membrane terminated. (a), Thickened nerves in the cornea; $(b)$, thickened perineurium around the anterior ciliary nerves in the sclerotic cut longitudinally; $(n)$, nerves thickened in the sclerotic.

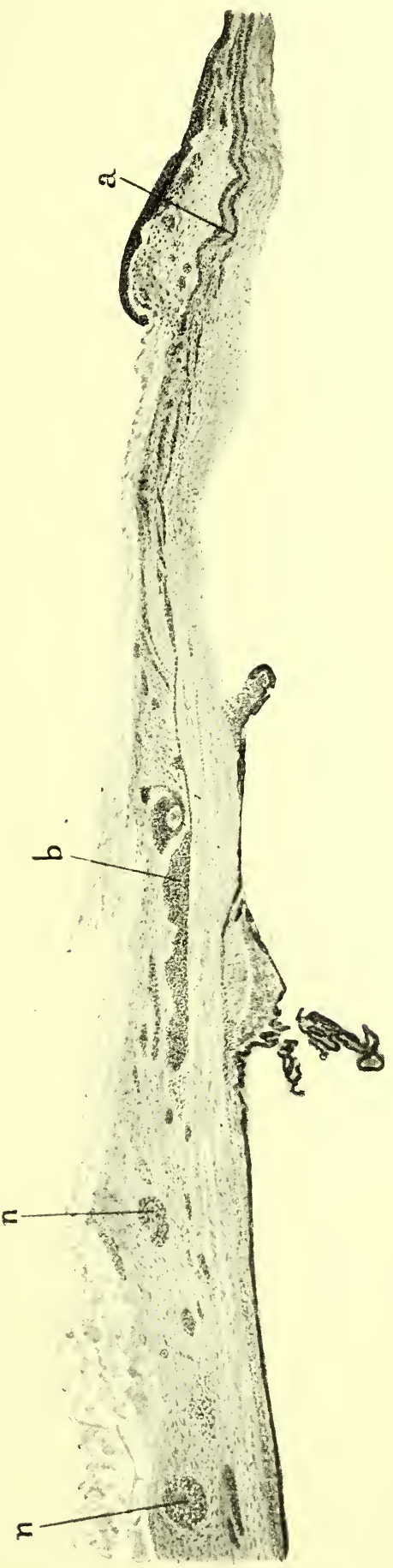


mass which gives rise to proptosis, and on palpation feels like a bag of worms.

In some of the cases in which the eyelids or orbit have been affected, the eyeball has been found enlarged as in buphthalmos. At first it might seem natural to assume that the enlargement of the eyeball was part of the elephan-

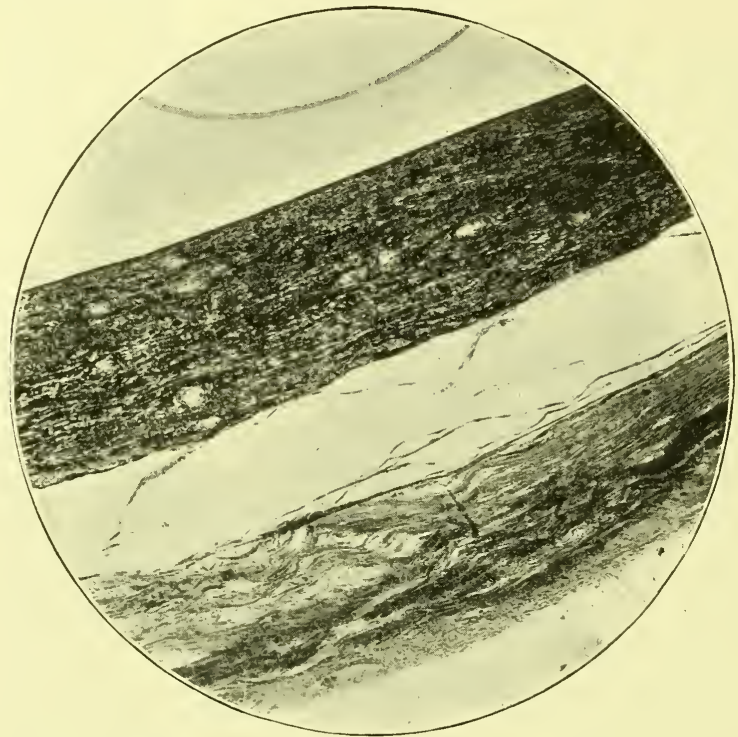

Fig. I I0.- Section through the choroid of an eye affected by neuro-fibromatosis. It is much thicker, denser in structure, and more highly pigmented than normal. Scattered throughout it are shown numerous small, oval, light-coloured bodies; they are the hypertrophied nerve-end organs. From a photomicrograph by E. Collier Green.

tiasis. Histological examination of such eyes has, however, shown a congenital malformation about the angle of the anterior chamber sufficient to account for the obstruction to exit of fluid from the eye (Fig. I09), the resulting increase of tension evidently being the cause of the expansion of the eyeball.

Intraocular changes of the nature of neuro-fibromatosis do, however, occur. ${ }^{1}$ The ciliary nerves external to the

${ }^{1}$ Treacher Collins and Rayner D. Batten. Trans. Ophth. Soc. of the U. K., I905, XXV, 248. 
sclerotic at the posterior pole of the globe may be thickened and plexiform. After entering the eye an increase of their peri- and endoneurium has also been found where they lie in the uveal tract, sclerotic, and cornea. The choroid may, moreover, become much thickened from hyperplasia of its fibrous tissue, the blood-vessels in it forming a much

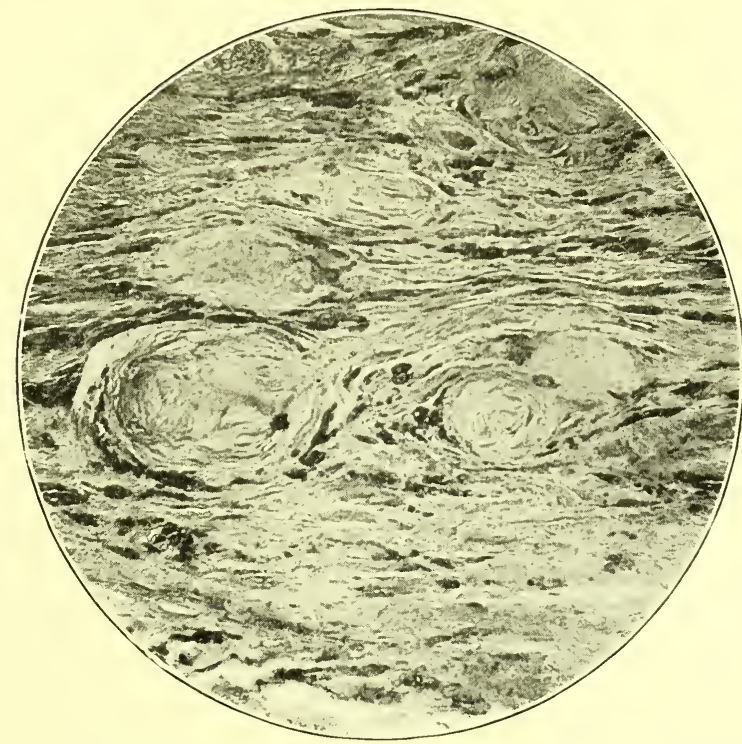

FIG. III.-Shows the hypertrophied nerve-end organs shown in the choroid in Fig. I I o under a higher power. A convoluted delicate fibre is faintly shown in the centre of each of them. An enlarged nerve is seen to be attached like a stalk to the largest of the bodies. From a photomicrograph by E. Collier Green.

less conspicuous element than usual. In a thickened choroid of this character enlarged nerves and end organs have been discovered; these latter were small oval bodies with nucleated cellular capsules and a central core composed of convoluted nerve fibres (Figs. IIo, III). Bodies of a similar character have been found in the orbital tissues in cases of neuro-fibromatosis.

Localised neuro-fibromata may occur in connection with the branches of the fifth nerve supplying the skin of the eyelids. They form small, hard, freely movable, and well defined nodules in the subcutaneous tissue. Microscopi- 
cally they are found to be due to thickening of the perineurium and endoneurium of the nerve fibre with which they are connected.

Fibromata of the optic nerve appear to be of less frequent occurrence than new growths starting in its neuroglia, ${ }^{1}$ the gliomatosis already described (see page 178 ). They form enormous thickenings of the dural sheath of the nerve, which by pressure cause atrophic changes in the nerve itself. They commence early in life, are of slow progress, and manifest no signs of malignancy. The exophthalmos which they produce always precedes the loss of vision.

Osteoma.-Though bone sometimes forms in the interior of the eye as a sequela of plastic inflammation of the uveal tract (see page 470) no new growth of bone occurs in the form of an intra-ocular osteoma. The orbit is, however, one of the favourite seats of such growths, though even in this position they are of rare occurrence. In the large majority of cases in which the orbit becomes involved the growth commences in one of the sinuses surrounding it, a few start primarily from the outer wall of the orbit. The frontal and ethmoidal sinuses are the commonest seats of origin of these growths, rarely the antrum of Highmore. It has been estimated that 40 per cent. appear first at the upper and inner angle, 30 per cent. at the inner wall, I per cent. at the floor and 0.5 per cent. at the outer wall. Bony growths are also met with in the orbit in connection with that form of hyperostosis affecting the facial bones which is known as leontiasis ossea.

Osteomata may have a rounded or nodular surface and start usually from a broad base, but vary much in shape according to their site of origin. Those starting in the wall of the orbit usually fungate out in all directions and become pedunculated. One starting in the frontal sinus extends, not only downwards into the orbit, but also upwards into the cranial cavity. An osteoma starting in the ethmoidal

${ }^{1}$ A. C. Hudson. R. Lond. Ophth. Hosp. Reps., XVIII, I9I2, 317. 
sinuses may extend laterally in both directions and involve the two orbits. Separate growths have also been met with in the two orbits.

In about two-thirds of the recorded cases of orbital osteoma the growth was of extreme hardness, like ivory. Some have had an external covering of ivory-like consistency, whilst the central part was composed of cancellous bone. A few have been described as of a spongy consistency, and others as semi-cartilaginous.

The age at which an osteoma of the orbit commences to form is difficult to determine, as they are of exceedingly slow growth. The first symptoms to attract attention are proptosis and failure of sight, these usually manifest themselves about the age of 25. The proptosis steadily increases, and may in time become so marked as to cause destruction of the eye from ulceration of the cornea. The failure of sight in the early stage is due to compression of the optic nerve, which may gradually become completely atrophied. Cases have been recorded in which the soft tissues overlying the growth have given way, and where the bony tumour thus exposed has become spontaneously detached, due to necrosis taking place in its pedicle. Osteomata starting in the frontal sinus, which break through into the orbit, sometimes extend so far up into the cranial cavity as to cause death from cerebral compression.

Myoma.-As has already been pointed out there is evidence to show that the muscle fibres in the iris are derived from neural epiblast, whilst those of the ciliary body are undoubtedly derived from mesoblast. Tumours, composed of what have been considered to be unstriated muscle fibres, are described as arising from each of these divisions of the uveal tract. The points relied on in diagnosing these growths as myomata rather than spindle celled sarcomata have been: the position in which they started, their slow rate of increase, the similarity in the shape and size of their cells to unstriated muscle fibres and the rodshaped appearance of their nuclei. 
In one recently recorded $\operatorname{case}^{1}$ a special selective stain was employed, Mallory's phosphotungstic hematoxylin. It picked out the myoglia fibrics coursing along the muscle cells and their terminal processes, so apparently leaving no doubt as to the myomatous nature of the growth.

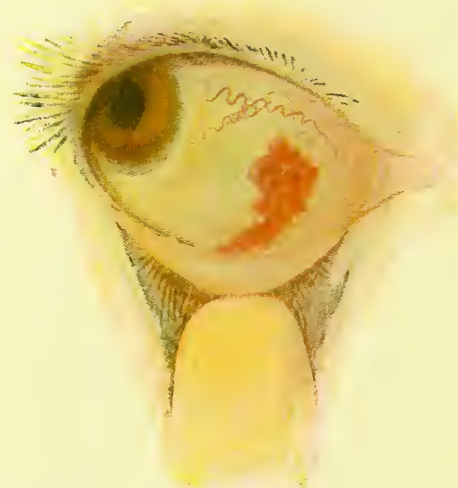

FIG. II2.-Angioma of the conjunctiva. Recorded in Trans. Ophth. Soc. of the U. K., XXXIII, I913, 55 .

Hemangioma.-The blood channels in hemangiomata may consist of $(a)$ a network of endothelial-lined spaces, cavernous angioma; $(b)$ a collection of thin-walled capillary tubes, capillary angioma; $(c)$ a plexus of moderately sized blood-vessels, arteries, or veins, or both, plexiform angioma.

The tissue between the blood channels varies in amount and character. It may consist of many of the different structures into which mesoblast becomes elaborated; e.g., fibrous tissue, adipose tissue, striated muscle fibre and bone. When the intervascular tissue is small in amount the growth is termed a simple angioma, when considerable a double name is employed. If the tissue between the vessels is very embryonic and cellular in character the growth is spoken of as an angiosarcoma, if it is mainly fibrous tissue angiofibroma, or if fatty angiolipoma.

${ }^{1}$ F. H. Verhoeff. Arch. of Ophth., LII, 1923, I 32. 
Most of the hemangiomata are met with in early life, a large number are certainly congenital; it is very probable that in nearly all there is some congenital rudiment which only becomes manifest as it increases in dimensions.

The hemangiomata dealt with here may be divided into the (I) extraocular and (2) intraocular.

I. The extraocular hemangiomata occur in the skin of the lids, the conjunctiva, and orbit.

Hemangiomata of the skin of the lids and conjunctiva are either cavernous or capillary (Fig. II2). In the latter situation they form either flat or polypoid growths, hemorrhages from them may occur causing the patient to shed "bloody tears." They may arise from any part of the conjunctiva but most commonly occur in connection with the plica semilunaris, the caruncle, and the fornices.

A hemangioma of the orbit, like any other new growth similarly situated, usually gives rise to proptosis. A distinctive feature of the proptosis due to vascular growths is the variability of its amount. Obstruction to the outflow of venous blood from the orbit, as by compression of the jugular vein, causes it to increase. Rest in the recumbent position and pressure backward of the globe will often cause it to decrease.

There are cases in which a patient is found to have, while in the erect position, one eyeball somewhat sunken in the orbit (enophthalmos), but where after hanging the head down it becomes abnormally prominent (exophthalmos). It has been suggested that in such cases there is a varicose condition of the orbital veins, or vascular growth, which has caused absorption of orbital fat, so accounting for the enophthalmos. On stooping, or compression of the jugular vein, the blood-vessels in the orbit become turgid and then exophthalmos results.

Hemangiomata of the orbit, unless of the very rare plexiform variety, do not pulsate or give rise to any bruit.

Capillary angiomata involving the eyelids may extend backward into the orbit. The hemangiomata confined to 
the orbit are usually cavernous and are surrounded by a fibrous capsule.

2. Intraocular hemangiomata are exceedingly rare but occur in connection with both the ciliary and retinal blood vessels.

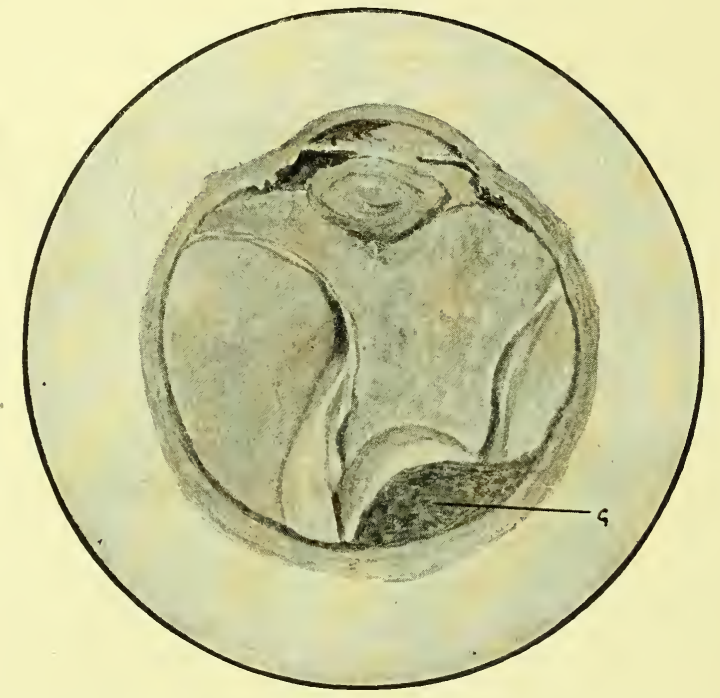

FIG. II3.-Lateral half of an eyeball contain a lens-shaped angiomatous growth $(G)$ of the choroid at the posterior part, above the optic disc. The retina is detached and a homogenous coagulum fills the subretinal space. The angle of the anterior chamber is closed by apposition of iris and cornea.

An angiomatous mass in the form of a rete mirabilis is a normal part of the choroid in some of the teleostean fish, and is termed the choroidal gland.

A few cases of vascular new-growths protruding from the anterior surface of the iris have been described. They form reddish nodules, from which hemorrhage into the anterior chamber is very liable to occur. There is some doubt as to whether they are simple angiomata or angio-sarcomata.

Several cases of cavernous angioma of the choroid have been met with, ${ }^{1}$ but only one case is on record in which a vascular growth in that membrane consisted of a plexus

${ }^{1} \mathrm{~L}$. Paton and Treacher Collins. Trans. Ophth. Soc. of the U. K., XXXIX, 1919, I57. 


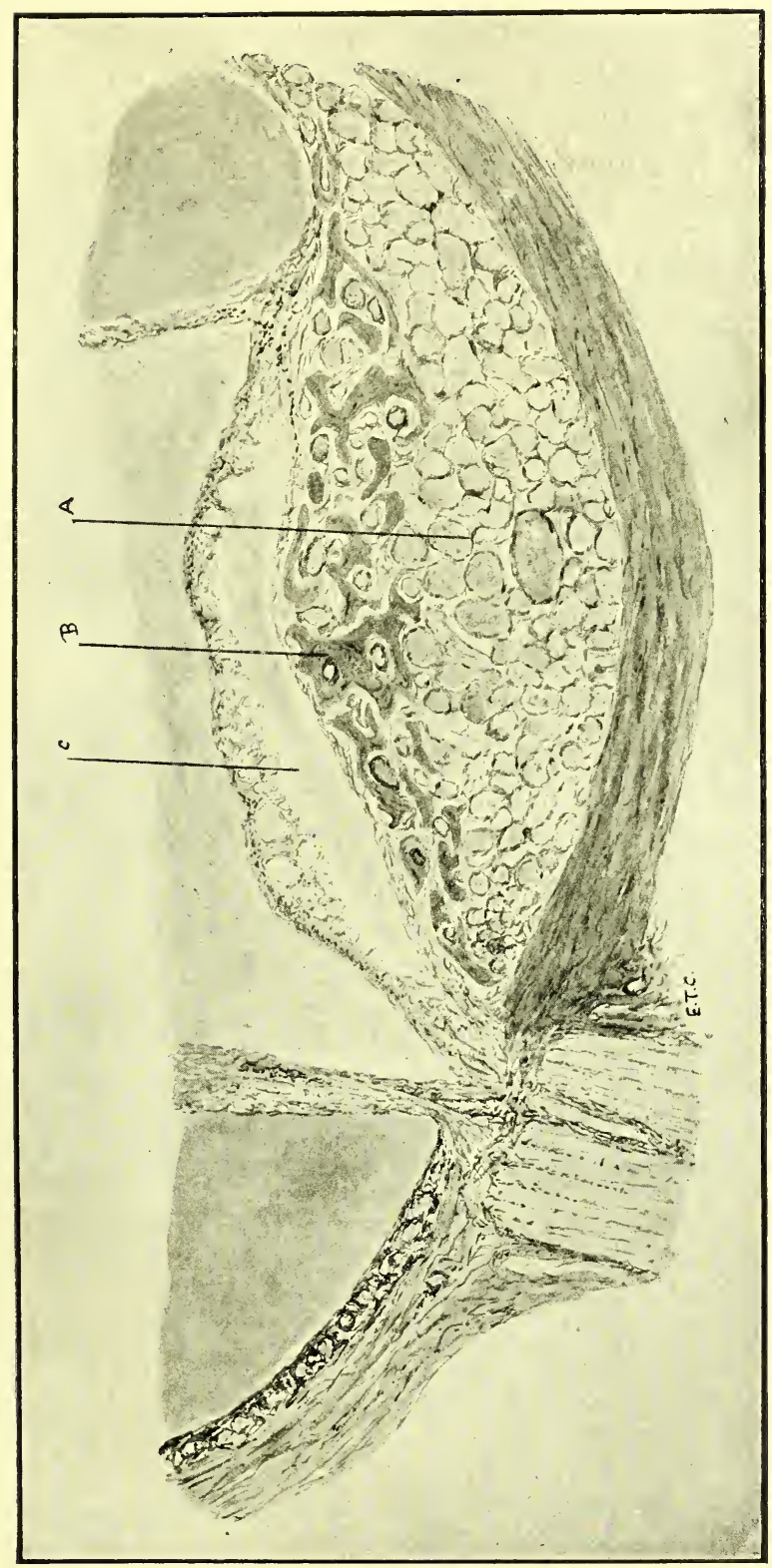

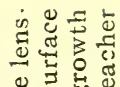

牙焉

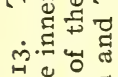

ํㅛㅇㅛ

造焉究

I क क

चृ

总 등

究

न ज्ञ

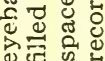

요

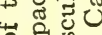

पै कि

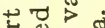

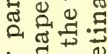

䨔

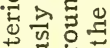

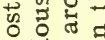

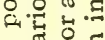

要求的

岁这昆

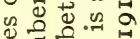

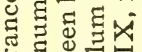

至

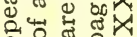

今.

สำ

ชึ

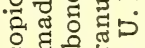

U.

包。胥

द

\& 8 .

†

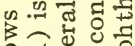

खी

v.

1.

$\exists$ 品它

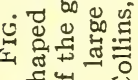


of thin walled vessels. Angiomata of the choroid are usually situated near the posterior pole (Fig. I I3), they give rise to a thickening of the membrane, and gradually merge into the surrounding choroid without any limiting capsule. A formation of fibrous tissue, or of bone, may arise on the inner surface of the growth (Figs. II4, II5). In some of the cases, a capillary nevus of the skin of the lids or an

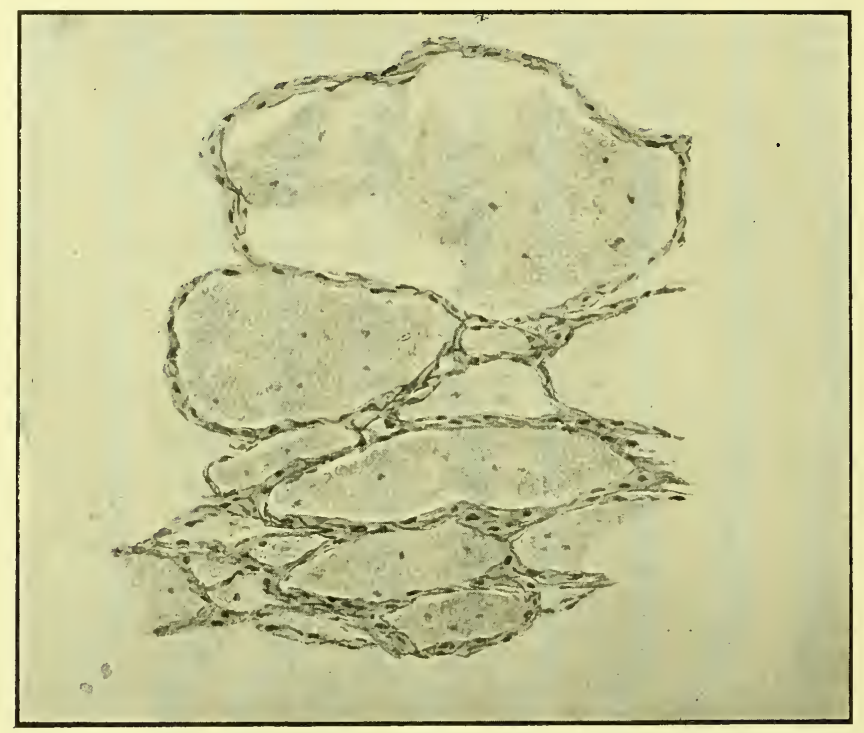

Fig. II5.-A small portion of the angiomatous growth shown in Figs. II 2 and II3 more highly magnified.

enlargement and tortuosity of the retinal vessels has been present. These growths certainly tend to increase in size, and in course of time give rise to detachment of retina and increase of tension.

Angioma of the retinal vessels is often bilateral, is usually met with in young people, and has twice been observed in two members of the same childship. Ophthalmoscopically an enormous enlargement of some of the retinal vessels, both arteries and veins, is seen. The enlargement so changes the appearance of the vessels that it is difficult or impossible to differentiate arteries from veins. The 
enlarged vessels terminate in a raised reddish or yellowish area (Fig. I 6). As the disease progresses vitreous hemorrhages occur and the retina becomes detached; in the course of years the surrounding parts may become invaded and

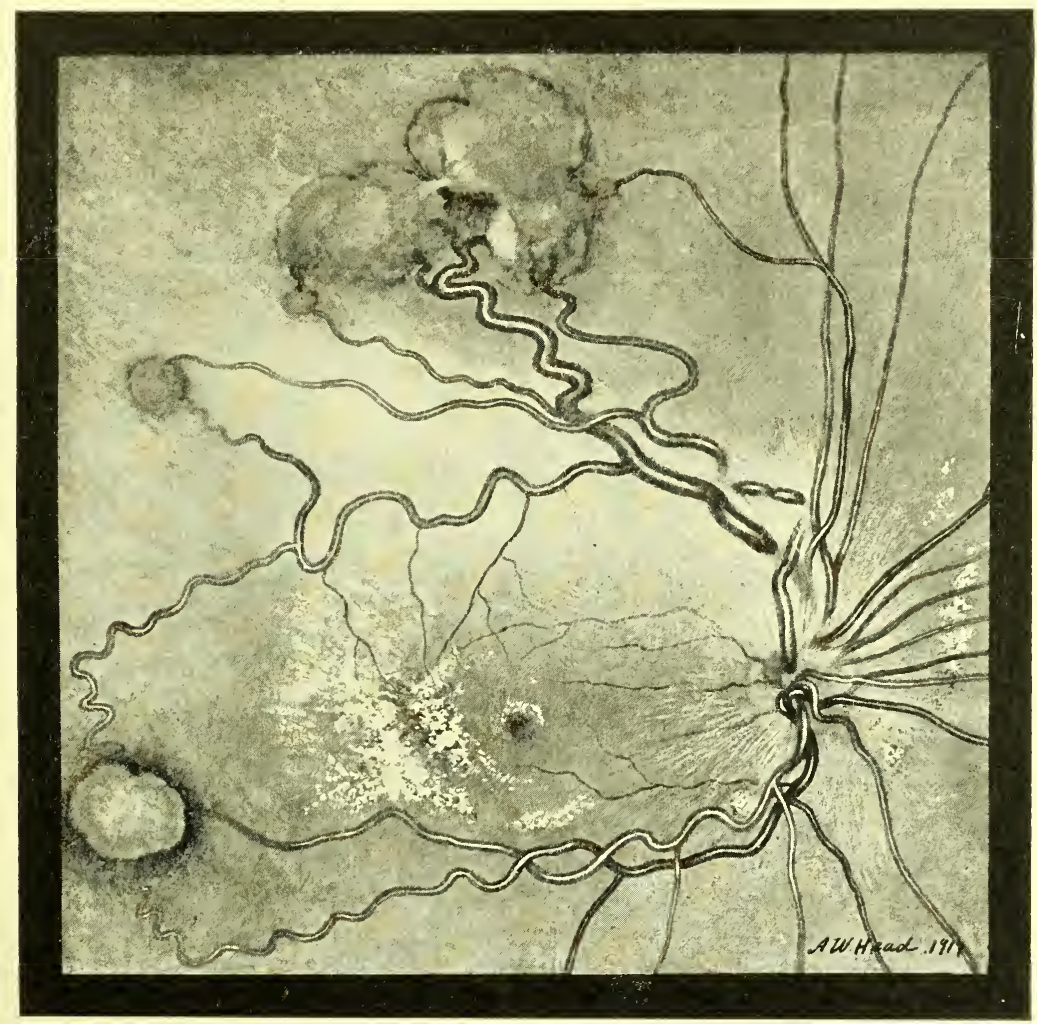

FIG. II6.-Case of multiple angiomata of the retina. Recorded in Trans. Ophth. Soc. of the U. K. XXXVIII, I9I8, I65.

secondary glaucoma followed by a staphyloma occurs. Pathological examination of eyes affected in this way has shown the presence of a capillary angioma in the tissue of the retina (Fig. II 7 ).

Lymphangioma.-Lymphangiomata like the hemangiomata may be cavernous and consist of a network of endothelial-lined spaces, or capillary and composed of thinwalled tubes. Cysts often arise in connection with them. 
The intra-ocular lymphatics consist of perivascular channels or endothelial-lined spaces, and no new growths which can be classified as lymphangiomata occur in connection with them.

Extraocular lymphangiomata arise in the skin of the lids, the conjunctiva, and orbit.

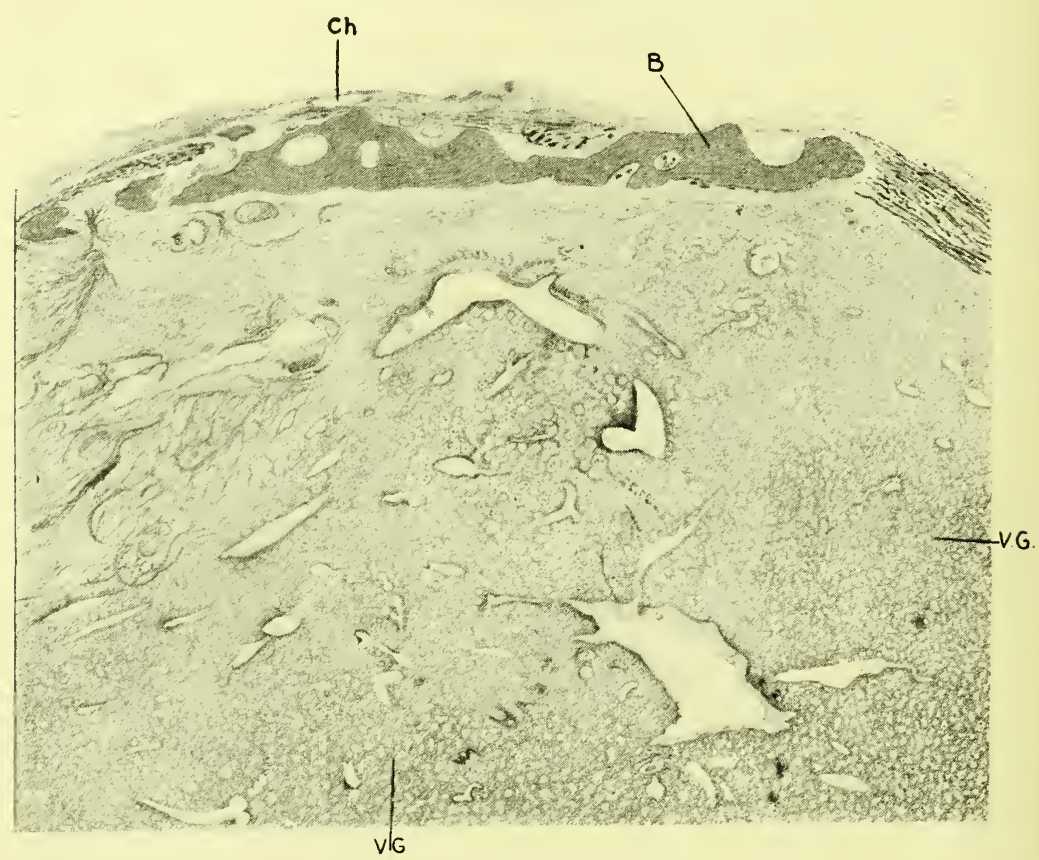

FIG. II7.-Shows the microscopical appearances of a vascular growth of the retina. Case described by Treacher Collins, Trans. Ophth. Soc. of the U. K., XIV, I894, I4I. V.G., Vascular growths; $C h$, choroid; $B$, bone in choroid.

A small dilatation of the lymphatics of the ocular conjunctiva, or lymphangiectasis, is frequently met with. True lvmphangiomata of the conjunctiva are rare, they are usually congenital and slowly increase in size. The growth has the appearance of a collection of small vesicles. If cut into, clear fluid escapes, the spaces afterwards becoming filled with blood; the growth then looks like a hemangioma. In course of time the blood absorbs and its original charac- 
teristics are restored. An angioid condition of the bloodvessels is sometimes associated with a lymphangioma.

Lymphangiomata of the orbit, unless they extend forward into the eyelids, are difficult to diagnose from other forms of new growth which produce proptosis. When cystic formation occurs in them a marked increase in the proptosis takes place.

\section{METASTATIC GROWTHS}

Metastatic growths, both carcinomatous or sarcomatous, were thought at one time to be always due to emboli of cells from the parent growth. This is undoubtedly the way in which sarcomatous growths become disseminated, but it has been shown that secondary carcinoma is very often due to a process of permeation. The cancer cells at the seat of the primary growth extend into the lymphatics, then infiltrate and grow along them to surrounding parts. Whether metastatic carcinoma of the uveal tract is due to a process of permeation or to emboli of cancer cells has not yet been definitely determined, but the balance of evidence is in favour of the former.

Intraocular Metastatic Carcinoma. ${ }^{1}$ - It has been suggested that sarcoma in the uveal tract may sometimes be metastatic, but the only metastatic growths occurring in the eye of which we have definite evidence are carcinomata. The condition is an exceedingly rare one and only arises in association with a widespread dissemination, so that patients when the eye is affected do not usually live longer than a few months.

In one-third of the cases both eyes are involved either simultaneously or within a very short time of one another. When only one eye is implicated, the right is affected as often as the left. More than two-thirds of the cases occur in females, which is accounted for by the primary growth

${ }^{1}$ C. D. Marshall. R. Lond. Ophth. Hosp. Rep., XIV, I897, 6I5. C. H. Usher. Brit. Jour. of Ophth., VII, Io, I923. 
in the large majority of cases being in the breast. It has also been found in the lungs, stomach, thyroid, liver, rectum, œsophagus, suprarenal body, prostate, and ovary.

The posterior part of the uveal tract is more often affected than the anterior, and if the growth spreads to it by a process of permeation, the sheaths of the posterior ciliary arteries are the tracts along which it has presumably extended. Tumour cells encircling the posterior ciliary arteries, in their passage through the sclerotic, have

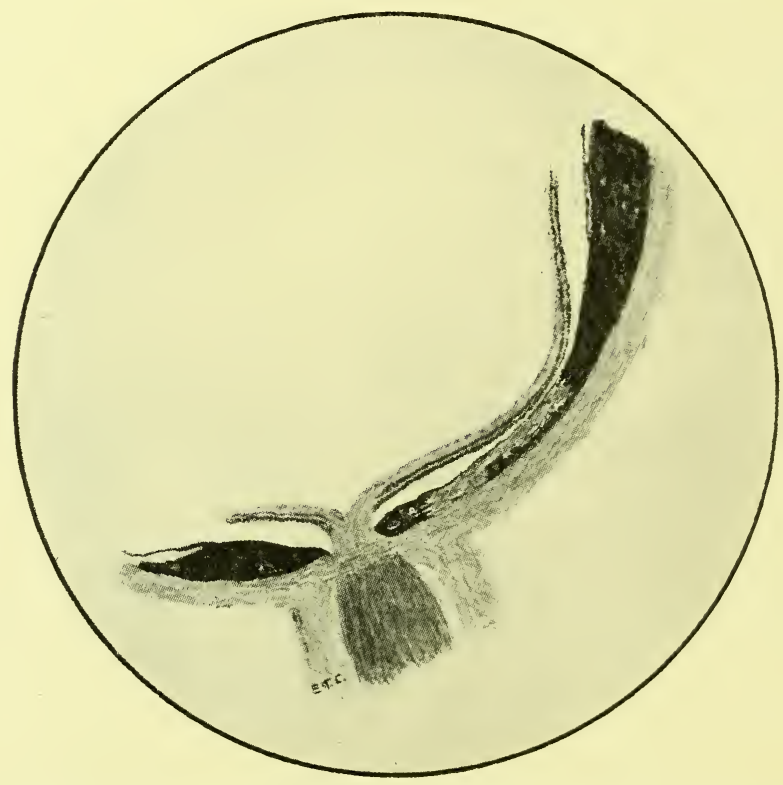

FIG. II 8.- Section of the posterior part of an eveball with a metastatic carcinoma of the choroid, secondary to a scirrhus cancer of the breast.

several times been seen. It has not, however, been definitely determined whether such encircling cells were extensions from without inwards or from within outwards. Cases of metastatic carcinoma beginning in the ciliary body and in the iris have been recorded, if these cases were due to permeation, one of the anterior ciliary arteries was probably the track along which the growth entered the eye. 
The carcinomatous cells having reached the lymphatic spaces of the choroid proliferate in them, thickening the choroid without forming any marked localised prominence, in which respect they resemble flat or diffuse sarcomata (see page I9I).

The thickest part of the growth, when the choroid is affected, is always at the posterior pole of the eye, and it

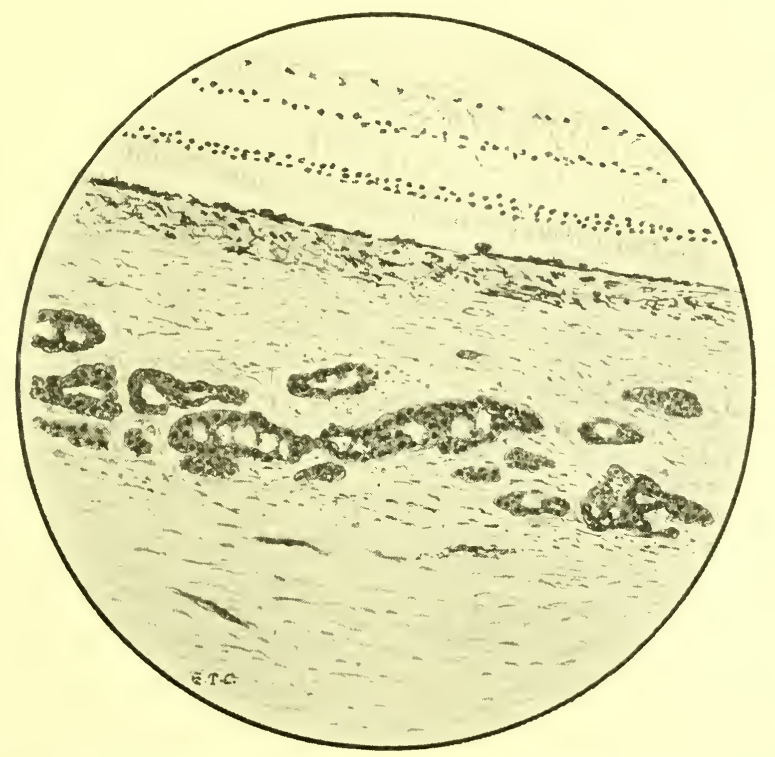

Fig. I I9.- Section of the growth shown in Fig. II 8 under higher magnification.

gradually tapers off as it approaches the ciliary body. It rarely breaks through the elastic lamina (Fig. II8). Ophthalmoscopically a grey area is at first seen, with an ill-defined margin and a mottled surface. This rapidly increases in size, and the growth interfering with the circulation of blood in the choroidal vessels, causes an effusion of serum from them which detaches the retina. In course of time this detachment is followed by an increase of tension.

The histological characters of a metastatic carcinoma of the uveal tract depend upon those of the primary growth, 
which it closely simulates. There are alveoli containing groups of glandular epithelial cells without any intercellular substance between them (Fig. I I9). The richness of the growth in cells and the amount of fibrous tissue bounding the alveoli varies in different cases. Scirrhus cancer originating in the breast is most frequently met with. Widespread degenerative changes in the cells are of common occurrence, they become vacuolated and break down into a hyalin or granular material.

Metastatic Thyroid Tumours in the Orbit. ${ }^{1}$-Metastases of adenocarcinoma of the thyroid occur in the bones and in the lungs. A few cases have been recorded in which they have been localised in the walls of the orbit, and given rise to the usual symptoms of an orbital neoplasm. They grow slowly and if cut into at an operation usually bleed freely. The primary growth in the thyroid may be so small as to escape detection clinically. The metastases may show normal thyroid adenomatous tissue.

Orbital Metastatic Lympho-sarcoma.-Secondary growths of a lymphoid character are met with in the orbit. They are composed of closely packed, small, round cells with a very delicate, scarcely discernible reticulum. Often there are symmetrical tumours in the two orbits, and the condition is frequently, though not always, associated with chloroma or leukemia (page 266). In these cases it has usually been found impossible to locate the seat of the primary growth. In cases which have ended fatally the autopsy has revealed a wide dissemination of growth, involving most of the viscera. The absence of any lymphoid tissue normally in the orbit would seem definitely to exclude it as the possible seat of the primary growth. Moreover, where the two orbits have been found affected the independence of the growths in them has been demonstrated.

' Arnold Knapp. Internat. Cong. of Ophth., Washington, I922, 573. 


\section{CYSTS}

Cysts arising in or about the eyeball may be parasitic or non-parasitic, the former are described with the parasites (see page 565). Non-parasitic cysts vary in the nature of the lining membrane and may be divided according to whether they are derived from (I) cuticular epiblast, (2) neural epiblast, or (3) mesoblast.

I. Cuticular Epiblastic Cysts.-The cysts lined with cuticular epithelium arise in different ways, and may be either $(a)$ congenital, (b) traumatic, or $(c)$ retention cysts.

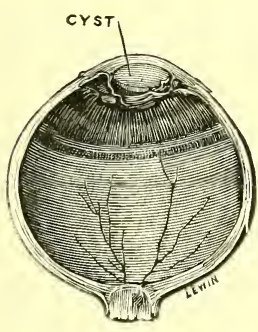

FIG. I 20.

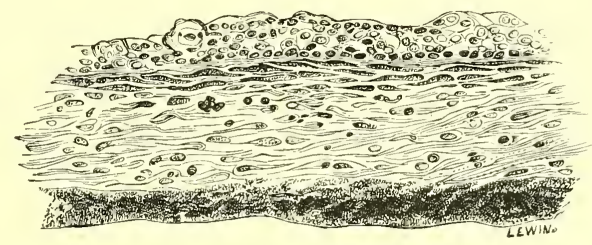

FIG. I 2 I.

FIG. I20.-Epithelial lined cyst which formed in the anterior chamber after extraction of cataract and gave rise to glaucoma.

FIG. I2I.-Microscopical section showing the lining epithelium on the surface of the iris of the cyst shown in Fig. I20.

(a) Congenital cysts lined by laminated epithelium are dermoid cysts (see page 135 ).

(b) Traumatic cysts lined by laminated epithelium may be produced by implantation of detached pieces of surface epithelium into subcutaneous parts, or by the extension of epithelium downward around the lips of an unhealed wound.

The cells of implanted detached epithelium when well nourished continue to proliferate. The result is that either a solid pearl-like tumour is formed composed of concentric layers of flattened cells, or, from degeneration of the central cells, a cystic growth lined by epithelium is produced, an implantation cyst.

A column of epithelium forming between the lips of a slowly healing wound may produce a cyst, either by the 
degeneration and liquefaction of the cells in the centre of it, or by its extension round a cavity, such as the anterior chamber (Figs. I 20, I 2 I).

The contents of traumatic cysts produced in any of the above ways vary according to the character of the lining epithelium. It may be fatty epithelial débris containing cholesterin crystals, or a clear, or slightly turbid, fluid.

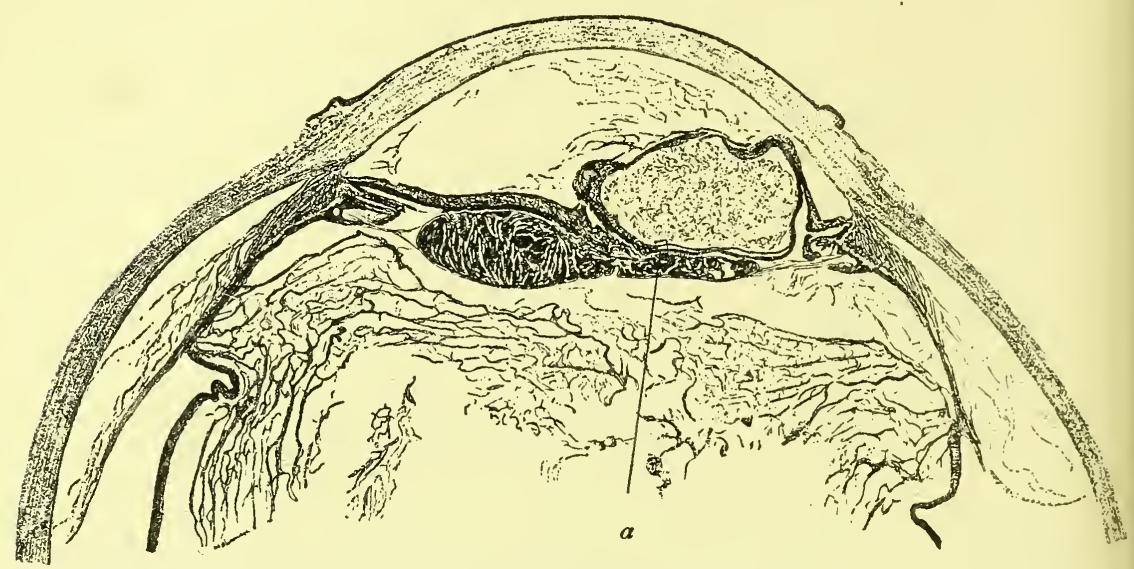

FIG. I22.- Section of the anterior half of an eye with a cyst in the iris, following on the implantation of an eyelash into the eye. The square to which $a$ points is the part shown in Fig. I 23 more highly magnified. $\times$ Io. Recorded in Trans. Ophth. Soc. of the U. K., XIII, I893, I99.

Implantation cysts have been produced experimentally in rabbits' eyes by the introduction into the anterior chamber of small pieces of skin, conjunctiva, or corneal epithelium. ${ }^{1}$. They usually occur in man as the result of punctured wounds. Epithelial pearl tumours may form around an eyelash when it becomes implanted in the interior of the eye; they arise from the cells of its root sheath (Figs. I 22 , I 23 ).

Traumatic cysts lined by epithelium have been met with in the orbit, conjunctiva, cornea, anterior chamber, iris, and vitreous chamber.

In the conjunctiva they form small blister-like elevations. In the cornea they are usually only discovered on

${ }^{1}$ E. Masse. "Kystes, tumeurs perleés et tumeurs dermoides de 1'iris," 1885. 


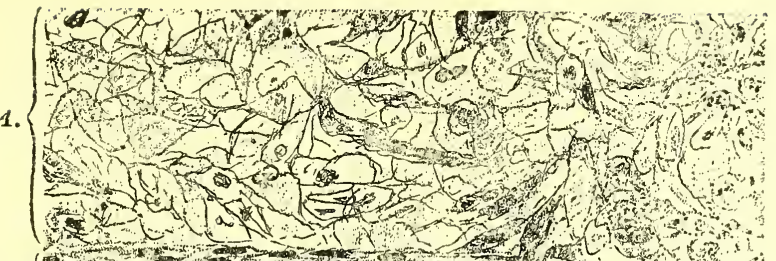

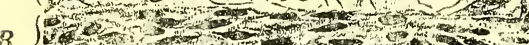

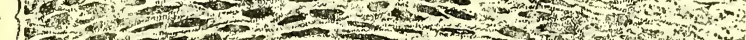

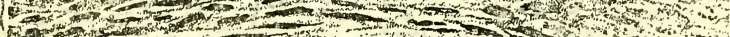

b.

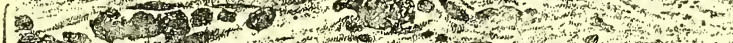

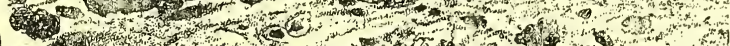

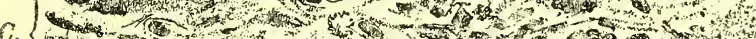

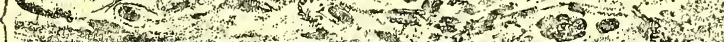

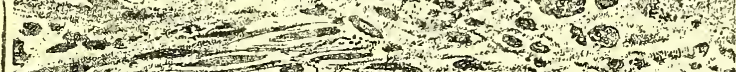
2.

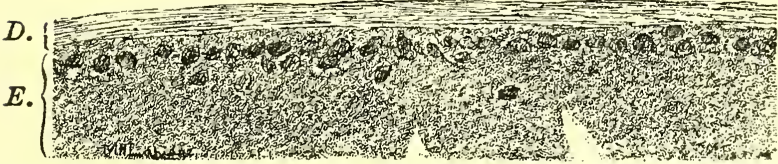

FIG, I 23.- Shows part of the wall of the cyst shown in Fig. I22, and included in the square marked $a . \times 300 . A$, Contents of cyst; $B$, epithelial lining of cyst; $C$, iris; $D$, lens capsule; $E$, lens.

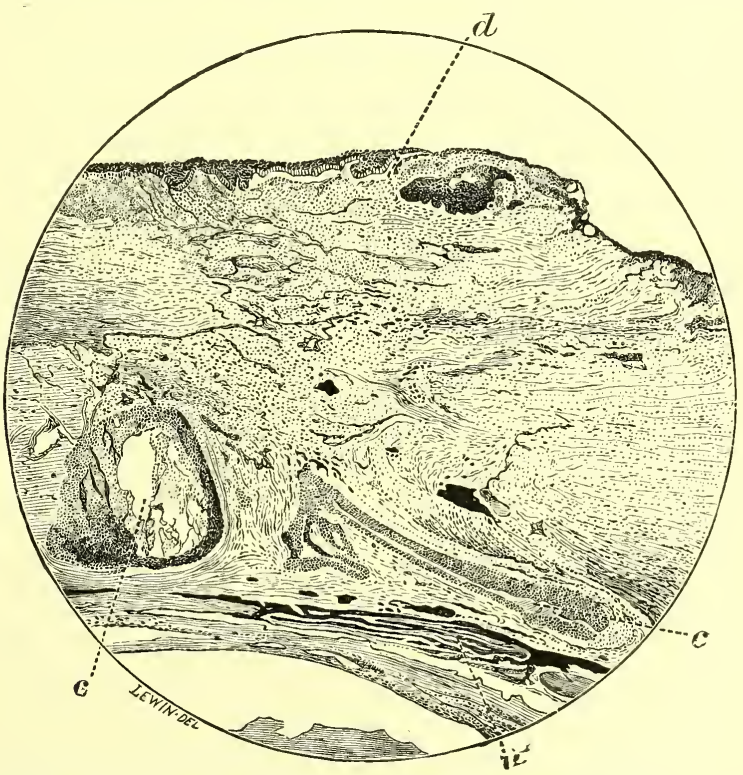

FIG. I24.-Epithelial implantation cysts in the cornea following a perforating wound with a shot. $c$, Cysts; $d$, Bowman's membrane; $u$, uveal pigment of iris. 
pathological examination (Fig. I 24), but may give rise to a protuberance resembling an anterior staphyloma (Fig. I 25). In the iris they form translucent round bodies resembling white currants. If the anterior chamber becomes com-

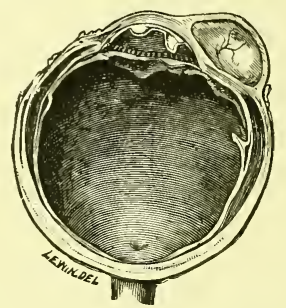

FIG. I25.- Lateral half of an eyeball, showing an epithelial implantation cyst in the cornea, following a wound with a piece of wood. Recorded in Trans. Ophth. Soc. of the U. K., XII, I892, 64.

pletely lined with epithelium it obstructs the exit of intraocular fluid and produces glaucoma. If left to itself, a cyst lined by epithelium slowly expands, causing by its pressure atrophy and destruction of surrounding parts. Thus in a

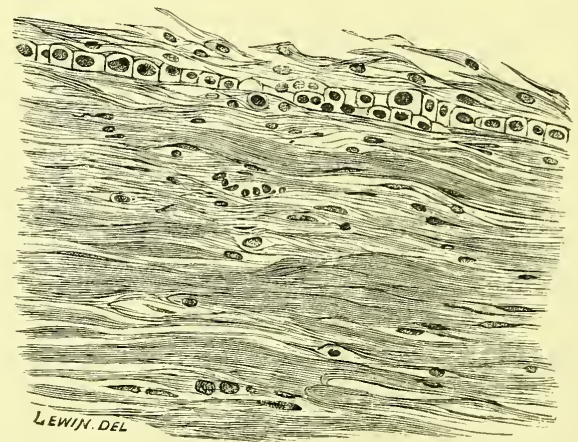

FIG. I 26.- Shows the laminated epithelium lining the cyst in the cornea depicted in Fig. 125 .

shrunken globe which had been wounded with a tip-cat twenty-eight years previously, a cyst lined by laminated epithelium was discovered measuring I $2 \mathrm{~mm}$. by I I. $5 \mathrm{~mm}$. and occupying the whole of the interior of the eye ${ }^{1}$ (Fig. I 27 ).

1 Treacher Collins. Trans. Ophth. Soc. of the U. K., I89I, XI, I33. 
The implantation cysts can be differentiated pathologically from those due to extension downward of the epithelium along a slowly healing wound, by the absence in the former of any continuity of the lining cells of the cyst with the surface epithelium, a condition which can only be determined with certainty by the examination of serial sections. ${ }^{1}$

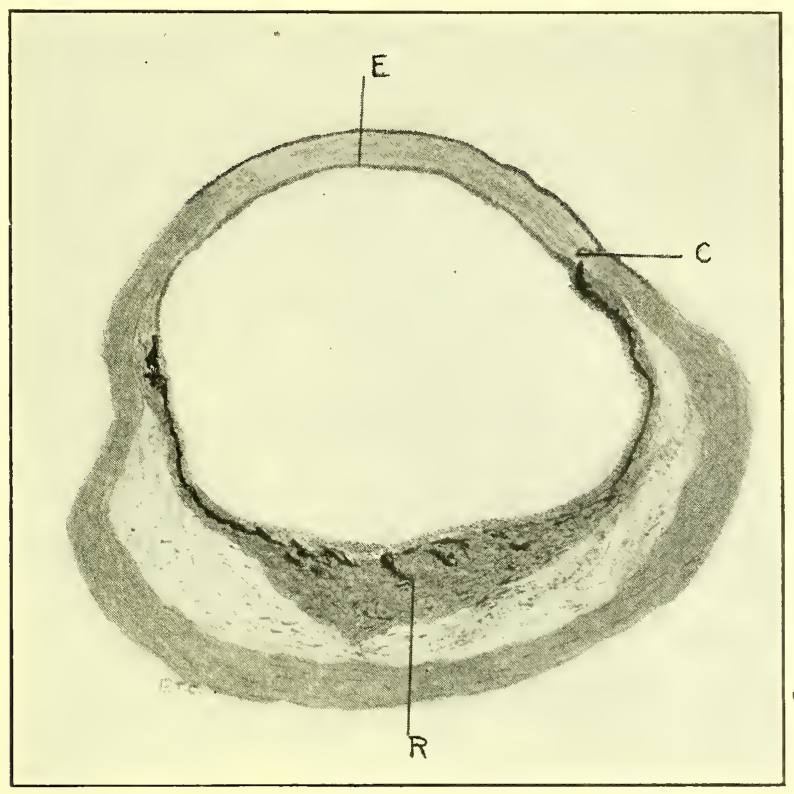

FIG. I 27.- Large epithelial lined cyst in a shrunken globe removed 28 years after a wound with a "tip-cut." $C$ points to the cicatrix of the wound in the cornea; $E$, to the lining epithelium of the cyst on the back of the corena; $R$, to the retina displaced by the expansion of the cyst.

(c) Retention cysts are met with in connection with the lacrymal gland and with the various glands of the eyelids and conjunctiva.

Cysts of the Lacrymal Gland.-Cysts of the 1acrymal gland, "dacryops," are of rare occurrence. They are probably caused by an inflammation of the conjunctiva which leads to obstruction of the gland's excretory ducts,

${ }^{1}$ M. S. Mayou. R. Lond. Ophth. Hosp. Reps., XVI, I905, 3 I8. 
either from a pericanicular sclerosis or a proliferation of their lining epithelium. A translucent, fluctuating swelling is formed beneath the conjunctiva at the upper part of the outer fornix, which may reach the dimensions of a pigeon's egg. ${ }^{1}$ Such tumours have been observed to enlarge when the activity of the gland is increased, either by exposure to cold winds or by emotional excitement.

These retention cysts of the lacrymal gland may be unior multilocular, the lining membrane is usually composed of cylindrical epithelium like that within the ducts of the gland; it may, however, consist of several layers of flattened cells. The fluid contents resembles the lacrymal secretion.

Cysts of Zeiss's Glands. - A retention cyst of one of the large sebaceous glands connected with the hair follicle of an eyelash may result from the occlusion of the orifice of its duct, due to hyperkeratosis. Clinically such a cyst forms a little rounded swelling in the skin at the margin of the eyelid, presenting much the size and appearance of a millet seed. Its contents is an opaque fatty substance like sebum.

Cysts of Moll's Glands.-The glands of Moll are modified sweat glands which open into the follicles of the eyelashes; if the orifices of their ducts become occluded little, blisterlike, cystic swellings are formed, situated along the free border of the eyelid internal to the roots of the eyelashes. When the distention occurs in the duct the cysts are unilocular and lined by a double row of cells. When the tubules of the gland itself are involved the cyst is multilocular, and lined by a single row of cells with unstriated muscle fibres external to them. The contents of these cysts is a clear fluid having much the same constituents as normal sweat.

Cysts of Meibomian Glands.-The Meibomian glands resemble in structure the sebaceous glands of the skin. Their orifices frequently become occluded either from a hyperkeratosis in the duct, or from the formation of fibrous tissue in the conjunctiva, as in trachoma, or after Burrow's

${ }^{1}$ Arnold Lawson. Trans. Ophth. Soc. of the U. K., XVII, I 897, 233. 
operation for entropion, when all the ducts of the glands are divided. The blockage of their ducts seldom leads to the formation of cysts due to the density of the fibrous tissue of the tarsus in which they lie embedded. It offers much greater resistance to their expansion than the comparatively loose tissue of the corium of the skin does to that of its sebaceous glands.

For the description of chalazion, often inaccurately described as a cyst of a Meibomian gland see page 494 .

Cysts of Krause's Glands.-Cysts due to obstruction of the excretory ducts of the glands of Krause usually occur in connection with the formation of fibrous tissue bands, and arise as sequelæ of trachoma or pemphigus of the conjunctiva.

These cysts are situated in the retrotarsal fold and protrude forward when the eyelid is everted. They have translucent walls and are lined by a single layer of epithelium, from which tuft-like projections sometimes protrude inward. Their contents is a clear fluid similar to the lacrymal secretion.

Cysts of Henlé's Glands. - The false glands of Henlé may become the seat of retained, degenerate, epithelial contents, and form small, yellowish, slightly raised areas in the conjunctiva at the upper margin of the superior tarsus. One of these glands at the inner angle, which is larger than the others, very frequently becomes affected.

The small epithelial retention cysts formed in chronically inflamed conjunctiva, in which there has been papillary formation, are described on page 442 .

ii. Neural Epiblastic Cysts.-Cysts in neural epiblastic portions of the eye are produced, either by an accumulation of fluid between the parts derived from the two layers of the secondary optic vesicle, or by the formation of new spaces in the substance of the retina.

Separation of the two layers of the secondary optic vesicle may take place in the region of the iris, ciliary body, or choroid. It may result from congenitally defective 
involution of the secondary optic vesicle; the contraction of organising exudate adherent to the inner surface of its inner layer; and the exudation of fluid between the two layers from the blood-vessels of the uveal tract.

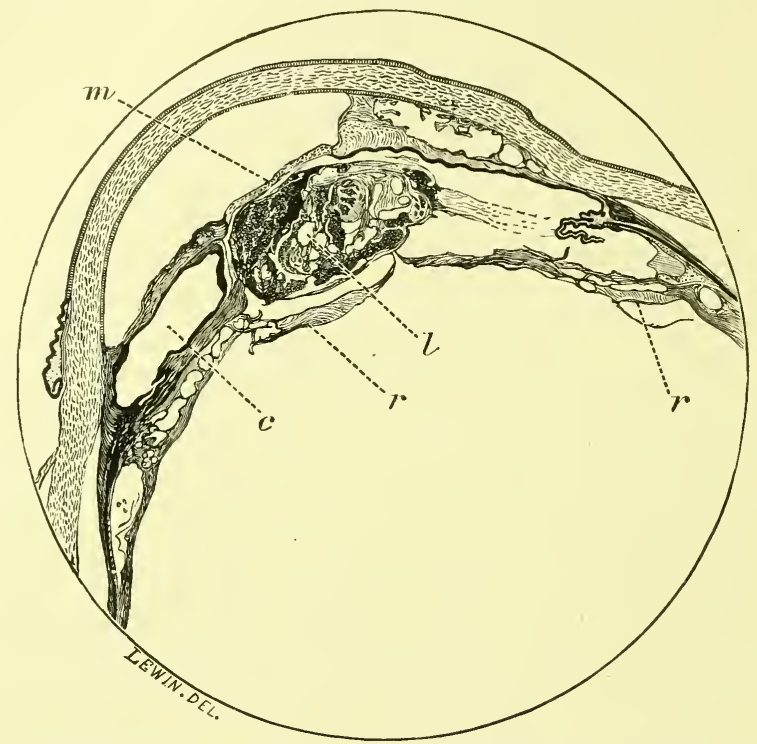

FIG. I28.- Section through the anterior half of an eye which had had plastic iritis after a perforating wound. A. cyst has formed between the two layers of pigment epithelium on the back of the iris. The posterior being adherent to inflammatory exudate. $c$, Cyst; $m$, inflammatory membrane in pupil; $l$, lens; $r$, detached retina.

Cysts due to congenital defect in the involution of the secondary optic vesicle are described under congenital aberrations (see page 18 ).

In inflammation of the iris, adhesion of the posterior layer of pigment epithelium to the lens capsule and the collection of serous fluid between it and the anterior layer, over a portion of its extent, is of common occurrence. Clinically the appearance produced is that of a localised condition of iris bombé (Fig. I28). If an iridectomy be performed in such a case, the stroma and anterior layer of pigment epithelium will be removed, but the posterior layer will be left adherent to the lens capsule, filling up the coloboma. 
The contraction of a cyclitic membrane, produced by the organisation of inflammatory exudate in the anterior part of the vitreous, is very liable to drag the non-pigmented layer of cells in the region of the pars plana of the ciliary body away from the pigmented layer, causing the formation of a cystic space between them filled with albuminous fluid (Fig. I 29).

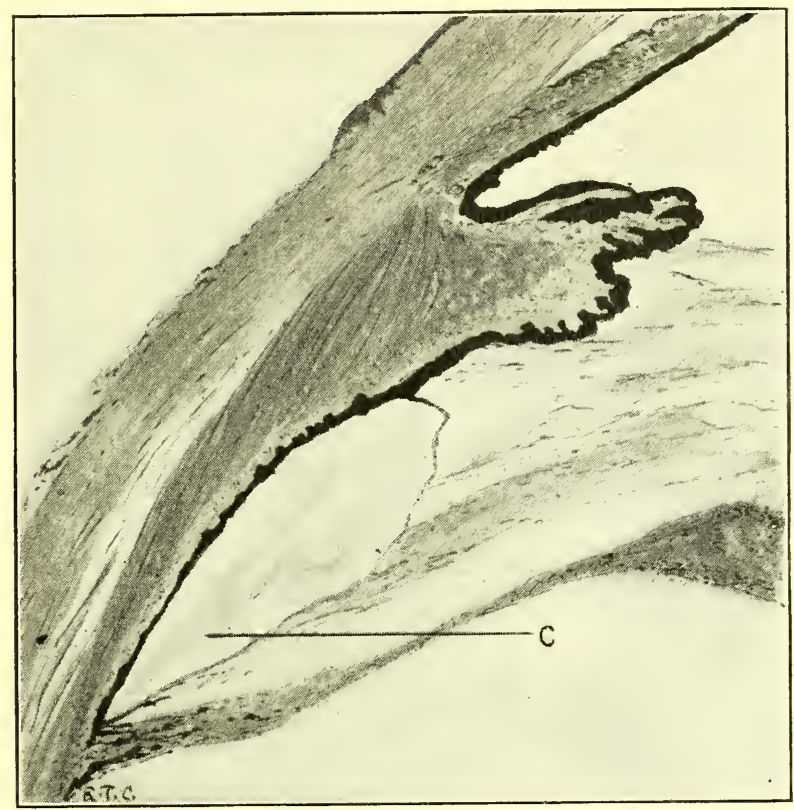

FIG. I29.-Cyst formed by the separation of the two layers of the pars ciliaris retinæ, the inner unpigmented layer from the outer pigmented one. $C$ points to the cavity of the cyst.

In a similar way the organisation of exudates further back in the vitreous, which have become adherent to the inner surface of the retina, drag it away from the pigment epithelial layer giving rise to one form of detachment of the retina (see page 469 ).

Edema of the iris, apart from inflammation, may result in localised accumulations of serum between the two pigment layers on its posterior surface. A cyst so formed 
has been found in a case where there was pressure on the root of the iris from a sarcoma of the ciliary body, which also produced swelling of the stroma (Fig. I30). A few cases have been recorded of cysts of the iris due to separation of its pigment epithelial layers where a pig-

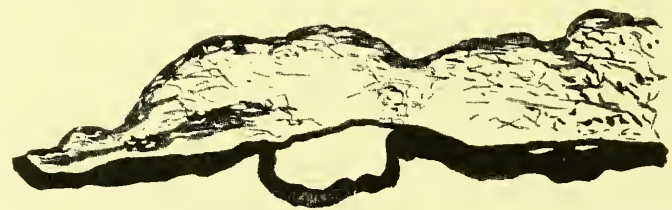

FIG. I30.- Shows a section through the iris from an eye which had a sarcoma of the ciliary body. Pressure on the root of the iris caused it to become edematous, and a small cyst formed between the two layers of pigment epithelium on its posterior surface.

mented protuberance has been seen clinically at the pupillary margin (Fig. I3 I). Such pigmented cysts are very liable to be mistaken for malignant melanotic growths.

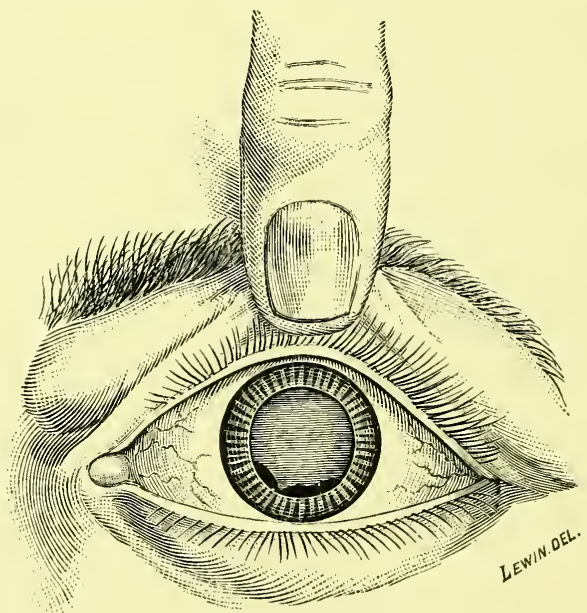

FIG. I3I.-Pigmented cyst of the iris due to separation of the two layers of pigment epithelium on its posterior surface. Recorded R. Lond. Ophth. Hosp. Reps., XIII, I890, 58.

The ciliary body being the source of the aqueous humor it is not surprising that occasionally a separation should occur of the two layers of cells lining it, which have to be 
permeated by the aqueous fluid in its transit from the ciliary blood-vessels to the interior of the eye. A sudden evacuation of the aqueous humour from trauma is the most usual cause of such a separation. A cyst thus formed has been mistaken clinically for a solid growth of the ciliary body.

These cysts have an outer wall of pigmented cells and an inner wall of unpigmented cells. This characteristic serves to differentiate them from two other forms of cysts lined by neural epiblast occurring in the same locality. Small cysts are sometimes found bounded entirely by pigmented epithelial cells, they are probably due to cystic distention of the tubular processes of cells which project outward from the pigment epithelial layer in the pars plana of the ciliary body, and are usually the sequela of cyclitis.

Another form of cyst of the ciliary body is lined entirely by unpigmented epithelial cells. ${ }^{1}$ It arises by multiplication and union of the cells on the inner surface of the ciliary processes, so that a portion of the posterior chamber becomes shut off and distended by secretion. The nature of such a cyst can only be definitely determined by the microscopical examination of serial sections.

Separation of the retina from the pigment epithelial layer due to effusion of serum from the choroidal vessels may take place. It has been met with in association with general dropsy due to nephritis. In the detachment of retina which occurs in association with choroidal sarcoma, the albuminous sub-retinal fluid is undoubtedly an exudate. These detachments occur in the lower part of the globe, owing to the gravitation of the fluid beneath, whatever may be the site of growth; they are frequently entirely isolated from the tumour the intervening retina being in situ. ${ }^{2}$ It is the first stage of the total detachment which eventually supervenes. The fluid beneath the retina is unlike the normal intraocular fluid, being highly albuminous, and

${ }^{1}$ A. R. Brailey. Trans. Ophth. Soc. of the U. K., XXVII, I907, 95.

${ }^{2}$ J. H. Parsons. Ophthalmic Review, XXIV, I905, I66. 
exudes from the choroidal vessels as the result of the engorgement of them from the presence of the growth.

Whether or not simple detachment of the retina (i.e., detachment occurring without any obvious cause, or in myopic eyes) is due to exudate from the choroid is a matter which has given rise to much discussion.

The two theories put forward as to the causation of a simple detachment of the retina are described as "the exudative theory" and "the traction theory."

The objection which has been raised to simple detachment of the retina being due to choroidal effusion is that, as the tension of the eye is not raised, it necessitates the disappearance of a corresponding quantity of the contents of the vitreous chamber. A sufficiently rapid disappearance of normal vitreous humour is exceedingly difficult to account for. The vitreous humour before a detachment takes place is, however, frequently either shrunken or liquefied, when the former, fluid like the aqueous humour fills the space between the hyaloid membrane and the retina.

The traction theory regards a rupture of the retina as a necessary precursor to the occurrence of detachment; such ruptures are supposed to be due to traction on it from bands which have formed in a shrinking vitreous. When the rupture takes place, fluid which has accumulated between the shrunken vitreous and the retina passes out through it, and raises the retina away from the choroid. The cause of the adhesion of the hyaloid of the vitreous to the retina, of sufficient firmness to tear the retina when the former shrinks, is left unexplained.

The occurrence of ruptures in detached retinæ was noted in the early days of opthalmoscopic research. The frequency with which they can be seen clinically has been estimated, by one observer, at 20 per cent. of the eyes examined with detachment of the retina, and by another, at 38 per cent.

These ruptures present different appearances, some are slit-like spaces in the membrane and others punched-out 
holes. The latter are doubtless produced in eyes where choroido-retinitis has preceded the occurrence of the detachment. The retina and choroid acquire firm adhesion at the seat of inflammation, and remain adherent when the former becomes detached elsewhere, a piece being torn out from the surrounding retina. In eyes with detached retinæ which have been examined pathologically isolated pieces of atrophied retina have been found adherent to the choroid.

Those in favour of the exudation theory attribute the slit-like spaces to rupture of the retina from the tension of the sub-retinal fluid.

A modification of the exudation theory of detachment of the retina is what is known as "the diffusion theory." It suggests that serous fluid exuded from the choroidal vessels becomes mixed with an abnormally fluid vitreous by a process of diffusion through the retina and hyaloid. The diffusion occurring according to the ordinary physical law, that is to say, a larger quantity of less albuminous liquefied vitreous humour passes outward into the subretinal space, than of more albuminous choroidal exudate inward into the vitreous chamber. In this way a detachment once started would tend to extend.

Small cystic spaces in the retina itself are of common occurrence; they are frequently met with immediately behind the ora serrata in the apparently otherwise healthy eyes of old people, and are probably the result of arteriosclerosis affecting the retinal capillaries.

They are also produced in various situations, not uncommonly at the macula, as the result of edema from inflammation or trauma.

In blind eyes with detached retinæ quite large cysts may be formed, producing protuberances on its outer surface, sometimes as big as currants and usually multiple (Fig. I32). The fluid contained in these cysts is, as a rule, highly albuminous. It may form a coagulated gelatinous material in hardened specimens. 
The primary condition seems to be always a disturbance in the vascular supply of the retina resulting in either atrophy of its nerve elements, or edema, or both. The spaces formed by the disappearance of the nerve elements, or by the effused fluid, open into one another, usually to

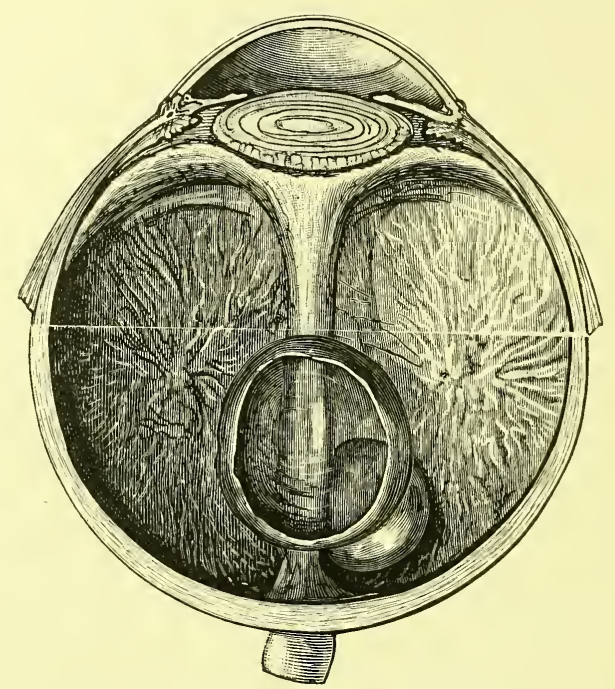

FIG. I32.- The lateral half of an eyeball showing a detached retina with cysts protruding from its outer surface. Specimen in the R. Lond. Ophth. Hosp. Museum.

the greatest extent in the internuclear layer, where oval or round cavities are formed bounded by the elongated fibres of Müller.

Attenuation from stretching of the fibres of Müller leads to a further running together of small cystic spaces and the formation of larger ones. In association with some of them there seems to be an hypertrophy of the supporting structures of the retina.

III. Mesoblastic Cysts.-The mesoblastic cysts are lined by a single layer of endothelial cells, and arise in connection with lymphatics, either in or outside the eyeball.

Extraocular lymphatic cysts occur in the conjunctiva and orbit. In both these situations, as already mentioned, they are met with in connection with lymphangiomata. 
On the ocular conjunctiva a lymphangiectasis may become converted into a cyst by the complete occlusion of the channel leading from it. Multilocular cysts may arise from the fusion of several dilated lymphatic channels. Such cysts have a clear fluid contents and transparent walls; when nipped between the eyelids they tend to become pedunculated.

Orbital cysts from the distention of Tenon's capsule, or portions of it forming the sheaths of the extraocular muscles, are of rare occurrence. Distention of Tenon's capsule causes proptosis and the formation of a fluctuating swelling overlying the sclerotic.

Intraocular cysts with an endothelial lining are met with in the iris and in the lamina suprachoroidea. In the iris they are due to blocking of the mouths of the crypts on the its surface. The result probably of thickening and fusion of the strands which are often seen crossing them, or the formation of an endothelial membrane overlying them. They have a clear fluid contents and thin transparent walls. Clinically they have much the same appearance as epithelial implantation cysts of the iris, and they may, like them, form after an injury, though not necessarily a perforating injury.

Cysts, from a dilatation and fusion of the lymphatic spaces of the lamina suprachoroidea, are occasionally met with in shrunken eyes, where the uveal tract has become drawn away from the sclerotic by contracting fibrous tissue in the vitreous chamber. The lamina suprachoroidea may then become hypertrophied forming a network of fibres of considerable thickness between the two outer coats. In this network cystic spaces sometimes develop with an endothelial lining. 
CHAPTER III

\section{DISTURBANCES IN THE CIRCULATION AND CON- STITUTION OF THE NUTRIENT FLUIDS OF THE EYE}

The circulating fluids which supply nutrition to the tissues of the eye are-the blood in the blood vessels, the lymph in the lymphatic spaces, and the intraocular fluid in the anterior, posterior and vitreous chambers.

The lymph and the intraocular fluid are derivatives of the blood, and disturbances in the circulation of the latter frequently involve disturbances in that of the two former. For purposes of description, however, the subject of this chapter will be divided up as follows:

I. Disturbances in the Circulation and Constitution of the Blood.

i. The blood supply to the interior of the eye.

ii. Complete obstruction to the central artery of the retina.

iii. Complete obstruction to the central vein of the retina.

iv. Complete obstruction of a ciliary artery.

v. Obstruction of a vortex vein.

vi. Impeded retinal circulation.

vii. Impeded choroidal circulation.

viii. Extravasation of blood.

ix. Dilatation of orbital vessels.

$\mathrm{x}$. Diseases due to changes in the constituents of the blood.

II. Disturbances in the Circulation of Lymph.

i. Serous detachment of the choroid and ciliary body.

ii. Serous detachment of the retina.

iii. Serous exudation from the iris.

iv. Serous exudation from the ciliary body.

v. Serous effusion into the head of the optic nerve.

vi. Serous exudation from the retinal vessels.

vii. Edema of the cornea.

ix. Serous effusion into the conjunctiva.

$\mathrm{x}$. Serous effusion into the orbit. 
xi. Serous effusion into the eyelids.

xii. Serous effusion into Tenon's capsule.

xiii. Serous effusions secondary to general diseases.

III. Disturbances in the Circulation of the Intraocular Fluid.

i. Mechanism for the maintenance of normal intraocular tension.

ii. The causes of hypertony or increased intraocular tension.

iii. The effects of hypertony on the various structures of the eye and their respective functions.

iv. The means employed for the reduction of hypertony.

$\mathrm{v}$. The causes of hypotony or decreased intraocular tension, and its effects.

\section{DISTURBANCES IN THE CIRCULATION AND CON- STITUTION OF BLOOD}

i. The blood supply to the interior of the eye is derived from the ophthalmic artery which is a branch of the internal carotid. This vessel gives off two sets of branches to the globe - the retinal, which supply the inner layers of the retina-and the ciliary, which supply the uveal tract.

The central artery of the retina enters the optic nerve some ${ }_{5} \mathrm{~mm}$. behind the globe. On its way forward it supplies small twigs to the nerve trunk, these communicate with the vessels derived from its pial sheath which entirely supply the optic nerve behind the entrance of the retinal vessels. As the artery passes through the lamina cribosa it breaks up into superior and inferior temporal, superior and inferior nasal, and macular branches. Around the optic nerve, just after its entrance into the eye, a circular anastomosis of vessels is formed by branches from the short ciliary vessels. This anastomosis is called the circle of Zinn. Branches are thrown off from this circle which pass into the papilla and anastomose with the central retinal artery. This anastomosis is of importance in that it is the only situation in which the choroidal and retinal circulations communicate. Occasionally a ciliary vessel may supply a portion of the retina (cilio-retinal artery or vein), (see page 64). In pathological conditions, such as 
embolism and thrombosis of the central retinal artery, a vessel of this nature may be of importance in carrying on the nutrition of part of the retina (see page 243). The capillary anastomosis between the vessels of the choroid and those in the optic disc is a favourite site for the lodgment of emboli of microörganisms. The branches of the central artery in the retina resemble those in the brain in that they do not anastomose freely with each other; they are what are known as end-arteries. The venous return is similar, but the communication with other retinal veins, and with the veins of the choroid near the disc, is freer than the arterial anastomosis. The vessels within the retina run in the nerve-fibre layer, and do not penetrate deeper than the inner nuclear layer, the outer layers of the retina deriving their nutrition from the choroidal vessels. In the anterior part of the globe, nearly the whole retina is so nourished. In many animals the retinal vessels are absent and the nutrition of the retina is entirely supplied by the choroidal circulation.

The capillaries in the retina form loops. These are absent from the macular region which derives its nutrition from lymph - a fact which is probably of importance in the production of central scotoma in toxic amblyopia (see page 273).

The blood-vessels of the uveal tract are the long and short posterior ciliary arteries which penetrate the sclerotic around the nerve, and the anterior ciliary arteries which penetrate the sclerotic in the neighbourhood of the insertion of the ocular muscles. The choroid is supplied by the short posterior ciliary arteries, and the ciliary body and iris by the anterior ciliary arteries and long posterior ciliary arteries, the latter pass forward in the choroid without division and then break up and anastomose with the former. From this anastomosis recurrent branches are given off which run backward to the anterior part of the choroid and are of importance in the nutrition of the anterior part of the retina. It also gives rise to the circulus arteriosus iridis 
major and minor which supply the iris, forming anastomosing capillary loops in two main situations - the latter near the pupillary margin, the former at the root of the iris.

The capillary anastomosis in the other parts of the uveal tract is extraordinarily free. Indeed, the whole uveal tract consists mainly of a spongy network of blood-vessels. In the choroid the chorio-capillaris is closely allied to cavernous tissue and in some fishes there is a rete-mirabile. In the ciliary body, around the so-called glands, the ciliary processes are mainly composed of capillary networks. This enormous blood supply not only gives nutrition to the uveal tract and overlying retina, but also supplies the aqueous humour and nutrient fluids of the vitreous and lens.

The major part of the blood derived from the uveal tract is returned through the venæ vorticosæ, some from the anterior part of the eye leaves by the anterior ciliary veins. From these vessels the blood passes into the ophthalmic vein and thence into the cavernous sinus. The orbital veins have a communication with the angular vein and with the pterygoid plexus.

\section{ii. Complete Obstruction of the Central Artery of the} Retina.-If a branch of the central $\operatorname{artery}^{1}$ of the retina be the site of complete obstruction, there is a sudden loss of sight corresponding to the area supplied by the vessel. If it be the main artery that is affected, total blindness results. When, however, a cilio-retinal artery is present the retina supplied by it remains functional. A portion of the temporal field may also retain perception of light for a short time, when the periphery of the nasal part of the retina derives its nutrition mainly from the choroidal blood-vessels. The obstruction to the circulation most commonly occurs behind the lamina cribrosa, partly because the vessel becomes constricted in passing through it, and partly on account of the vessel bifurcating in that region (Fig. I33). The immediate effect of the block in the artery is an intense anemia of the retina.

${ }^{1}$ G. Coats. R. London Ophth. Hosp. Rep., XVI, I905, 262. 
Ophthalmoscopically the retina has a white appearance, due to loss of transparency, from coagulation necrosis of its ganglion cells (see page 625 ). The macula appears as a cherry-red spot, owing to the red choroid being seen through the retina which is devoid of ganglion cells in this situation and much thinner than elsewhere. In a few

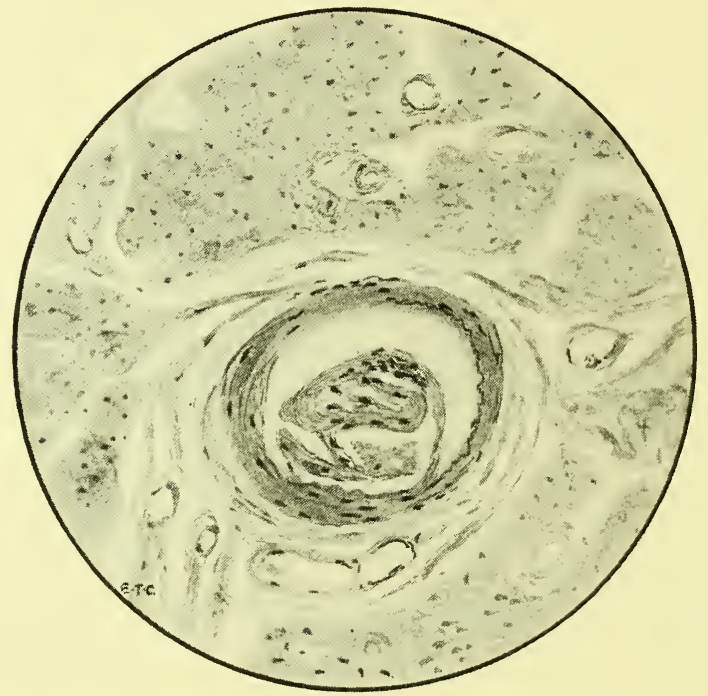

FIG. I33.- Transverse section of the optic nerve showing organising embolism of the central artery.

hours after obstruction the artery begins to refill with blood, as the anastomosis around the nerve head becomes opened up. At first the blood in the artery does not fill its lumen entirely. Drops of blood appear in the vessels with intervals between, giving it a "railway truck" appearance. This is due to a drop of blood being pumped in with each systole of the heart, and then the vessel collapsing under the intraocular pressure during the heart's diastole. After about forty-eight hours the artery becomes entirely filled, but it is always too late to restore the function of the retina, which becomes atrophic. Finally, the artery, as a result of endarteritis, becomes reduced to a mere thread and 
secondary atrophy of the optic nerve takes place, owing to death of the retinal ganglion cells.

Although during the early stages the central artery contains little or no blood, the central vein is always full. This is due either to regurgitant blood from the ophthalmic vein, or, more probably, to the blood being unable to leave the eye, owing to intraocular pressure producing a collapse of the central vein at the disc in the absence of the vis a tergo of the blood pressure.

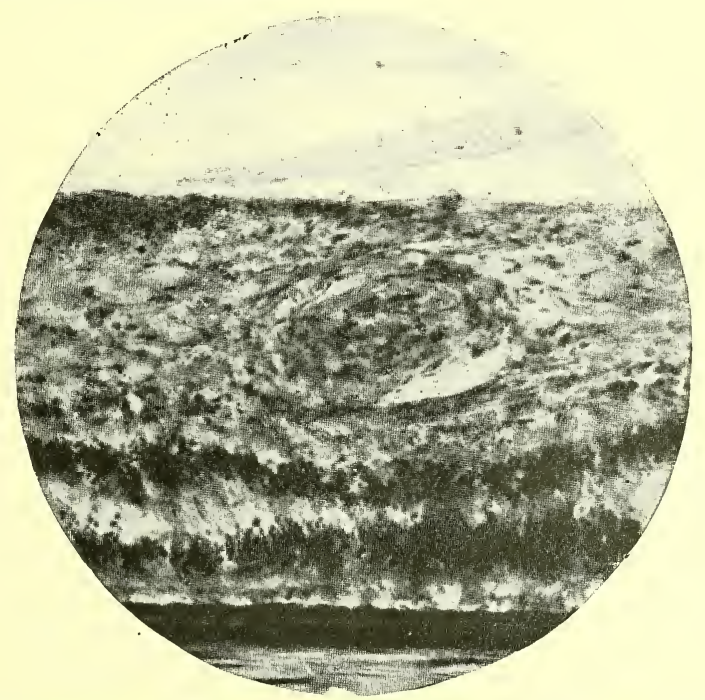

FIG. I34.- Shows a section through the retina with a thrombosed retinal vein cut transversely.

It is probable that the clot may sometimes shrink to one side of the artery and its lumen again become pervious. More frequently organisation with the formation of fibrous tissue completely obliterates the lumen of the vessel.

iii. Complete Obstruction of the Central Vein of the Retina. ${ }^{1}$ - This may occur in either the main vein or one of its branches (Fig. I34). If it affects the main vein the block usually occurs at the lamina cribrosa, where it is constricted and bent in passing out from the eye, and where it lies in close proximity to the artery which, when thick-

${ }^{1}$ G. Coats. R. London Ophth. Hosp. Reps., XVI, I904, 62. 
ened, may exert pressure on its walls. Occasionally it occurs in front of the lamina cribrosa, and the clot may extend so that it can be seen ophthalmoscopically as a reddish-black point on the disc. As the result of venous obstruction, blood brought by the central artery can only leave the eye by the anastomosis around the nerve head. This leads to intense engorgement of the retinal veins, the increased pressure in which causes either rupture of the capillaries or the transudation of their contents, so that the retina becomes covered with multiple hemorrhages. The fluid exuded also produces an intense edema of the retina and papilla. The edema is especially marked in the papilla, owing to the engorgement caused by the opening up of the choroidal retinal anastomosis. The immediate effect of thrombosis is loss of vision; this is not usually complete and it may subsequently be to a great extent restored, either from canalisation of the clot or from the establishment of a fresh anastomosis around the site of the thrombosis. Another sequela of thrombosis results from the stagnation produced in the already diseased artery, leading to thrombosis in that vessel also; the sight is then entirely destroyed and the clinical condition previously described under obstruction to the central artery will be superimposed on the clinical appearance of thrombosis. On looking at such a fundus an intense papilledema with hemorrhages and engorged veins is seen, while the arteries are reduced to mere threads and the retina appears whitish in colour. Finally there may be complete obliteration of all the retinal vessels.

The occurrence of thrombosis of the retinal vein causes the exudate of highly albuminous fluid into the vitreous chamber, and this, together with the sclerosis which is usually present in the pectinate ligament and ocular vessels, may lead, in the course of. some weeks, to acute glaucoma, without blocking of the angle of the anterior chamber by the root of the iris (see page 302). When the glaucoma has existed for some time new vessels appear on 
the surface of the iris - a point which is of diagnostic importance. They are situated in a thin endothelial membrane which is formed on the anterior surface of the iris, in such cases (Figs. I35, I36). They may be the fetal

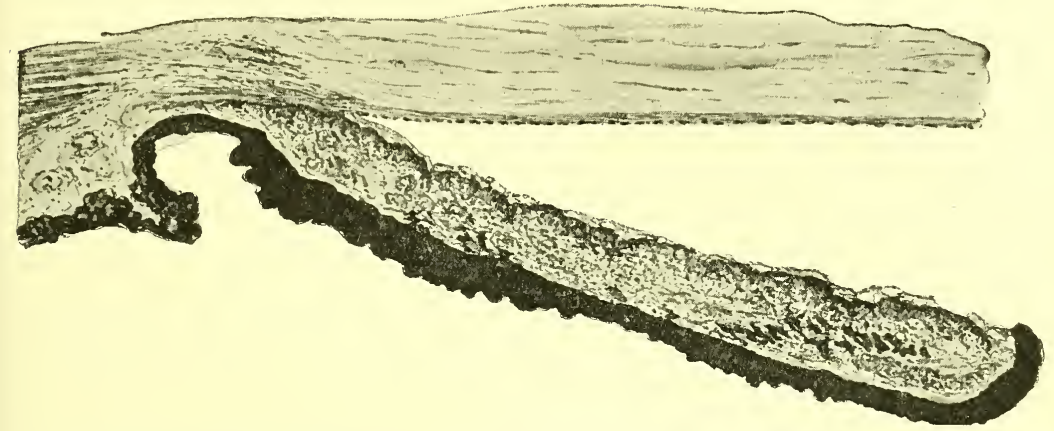

FIG. 135.- Section of iris in a case of glaucoma following thrombosis of the central retinal vein, showing a thickened endothelial membrane with new bloodvessels in it on the anterior surface. Case recorded in Trans. Ophth. Soc. XXXIII, I9I3, I 84 .

vessels of the irido-pupillary membrane which becomes reopened up.

The highly albuminous nature of the exudate into the retina, together with the extravasated blood, may produce

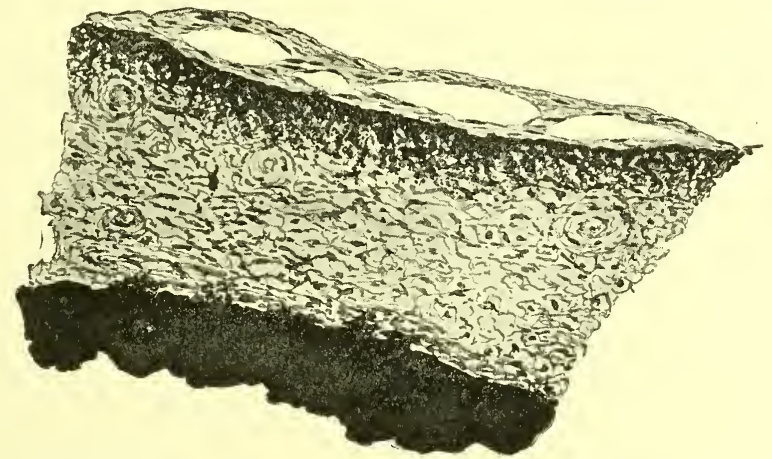

FIG. 136.-Piece of iris from the same case as shown in Fig. I35 under highmagnification.

a condition which is described as massive retinitis (see page 470). Subretinal exudate may cause detachment of the retina. Hemorrhage may also take place into the vitreous 
and produce a condition termed retinitis proliferans (see page 472 ).

iv. Obstruction of a Ciliary Artery.-Gradual obliteration of the ciliary vessels leads to the changes described under vascular sclerosis (see page 672); if one vessel is suddenly occluded the anastomosis is so free that the circulation is carried on by the others, but if more than one is affected blindness corresponding to the area of rods and cones supplied results.

v. Obstruction of a Vortex Vein.-Experiments have shown that the ligature of all the vortex veins leads to albuminous exudation into the subretinal space and aqueous chamber, and to secondary glaucoma. . If, however, only one vein is occluded no rise of tension is produced. It is probable that thrombosis of the vortex vein occurs more frequently than the records of it would lead us to suppose.

A case ${ }^{1}$ is recorded of a detached retina associated with glaucoma, choroidal hemorrhage and massive exudation following a doubtful injury in a boy aged 8, in which thrombosis of the venæ vorticosæ was found. Thrombosis of the venæ vorticosæ has also been found in several cases after glaucoma.

vi. Impeded Retinal Circulation.-The condition which gives rise to vascular sclerosis acts on all the ocular vessels impeding the circulation through them, but one set of vessels may be more affected than others; thus in albuminuric retinitis the retinal vessels are chiefly involved and in syphilis the choroidal. Even when one set of vessels is the chief seat of the disease some branches may be more extensively sclerosed than others. For pigmentary degeneration secondary to sclerosis of choroidal vessels see page 672.

Albuminuric retinitis ${ }^{2}$ is a condition which shows that renal disease is affecting the ocular vessels. It is most

${ }^{1}$ Mayou. Trans. Ophth. Soc. of the U. K., XXXV, I9I3, II 2.

${ }^{2}$ E. Nettleship. R. London Ophth. Hosp. Reports, XV, I903, 320. 
commonly associated with a contracted interstitial nephritis and less frequently with a large white or amyloid kidney. It occurs rarely in acute nephritis associated with specific fevers, and is sometimes met with in albuminuria of pregnancy. It generally occurs in patients over the age of forty, but has been known to commence as early as the age of five. The prognosis to life in those affected is

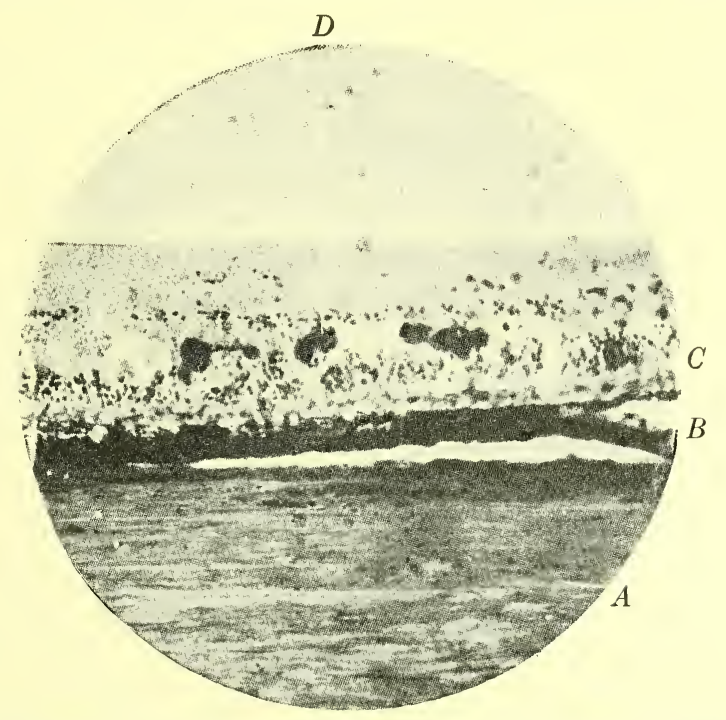

FIG. I37.- Shows a section through the coats of the eye in a case of albuminuric retinitis. In the retina there are albuminous coagula which produce the white patches seen clinically. $A$, Sclerotic; $B$, choroid; $C$, retina; $D$, albuminous coagulum.

usually bad, the patients dying within two years of the onset of the ocular symptoms. This is not the case, however, when the retinitis is associated with the albuminuria of pregnancy or in the rare cases when it occurs in connection with acute nephritis, which are due to toxemia and not to sclerosis. In the toxic cases the retinal changes may clear up entirely.

The changes in the retinal vessels associated with chronic nephritis are described under vascular sclerosis see page 669.

The exudate which occurs is highly albuminous and may be subretinal or intraretinal. When the exudate is sub- 
retinal it may be so extensive as to cause retinal detachment. Such detachments are more common than are usually supposed; recovery is frequent with considerable pigmentation of the retina from proliferation of the retinal pigment taking place.

If intraretinal it may occur in the nerve fibre layer, or intranuclear layers, and give rise opthalmoscopically to grey patches with ill-defined margins. At first the patches

A

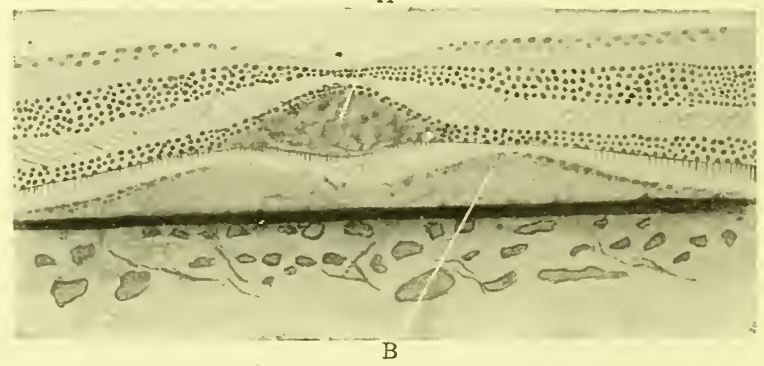

FIG. I38.- Shows a section through the macula lutea in a case of albuminuric retinitis in which a star-shaped figure was present. A points to intraretinal exudate and $B$, to sub-retinal exudate.

consist of a network of fibrin; after a time the fibrillæ cease to stain with Weigert's stain and form discreet globules, which may run together and become large masses of exudate, more particularly in the intranuclear layers (Fig. I37). Large globular phagocytic cells appear in the neighbourhood of the hyalin masses and help to bring about their absorption, the contents of these cells stains with osmic acid and is, therefore, of a fatty nature.

In the macular region the exudate takes a peculiar starshaped arrangement of sharply defined white patches. This is due to the lines of tension in the retina, produced by the peculiar attachment of it to the pigment cell layer in that region. The exudate is both sub- and intraretinal (Fig. 138). For retinal hemorrhage see page 257. Edema of the nerve head is also usually present and in rare instances may occur without other retinal exudation. 
Diabetic retinitis ${ }^{1}$ is characterised by a number of punctate hemorrhages and white patches around the macular region. The patches of hemorrhage and exudation are very much smaller than in albuminuric retinitis and are probably derived from the giving way of capillaries in the deeper layers of the retina. A star figure is extremely rare, and retinal detachment does not occur. The lesions are more frequently unilateral than in albuminuric retinitis.

Vascular sclerosis probably aids in their production as they occur only in old people. Young subjects with diabetes never showing such changes. The prognosis with regard to life in cases of diabetic retinitis is better than in albuminuric retinitis, as half the patients live more than two years. Albuminuria may accompany the diabetes, but diabetes alone may be associated with the retinal changes.

The rare condition of lipemia, which is met with in diabetes, gives rise ophthalmoscopically to a peculiar yellow colour of the blood vessels. It has been attributed to the fatty constituents present in the blood becoming adherent to the vessel wall. ${ }^{2}$

Before such changes manifest themselves the fat contents of the blood must reach at least 5 per cent., it may be as high as 25. Patients are always young, the oldest recorded case being 48 years of age. Death in these cases usually occurs rapidly but a few recoveries have been recorded.

vii. Impeded Choroidal Circulation.-Syphilitic vascular sclerosis resembles Retinitis Pigmentosa. The pigmentation of the retina in the former being a purely secondary change, due to the cutting off of the blood supply as the result of sclerosis of the choroidal vessels. In some cases it is extremely difficult to distinguish between the two conditions, but the family history and the presence of the Wassermann reaction is usually sufficient."

${ }^{1}$ E. Nettleship. R. Lond. Ophth. Hosp. Reps., XV, I903, 320; XVI, I904, I I. L. Heine. Klinisch. Monats. f. Augen., XLIV, I906, 45 I.

${ }^{2}$ Hale-White. Lancet, Oct. Io, I903. 
viii. Extravasation of Blood.-Hemorrhage from the uveal tract or retina may occur as the result of $(A)$ rupture of the vessel wall; $(B)$ diapedesis.

$A$. The causes of rupture of the vessels are

I. Increased intra-vascular pressure, local or general.

(a) Local, e.g., contusion of the eye, venous thrombosis, papilledema, optic neuritis, and all forms of retinitis.

(b) General, e.g., hypertrophy of the left ventricle, straining, coughing, cessation of menstruation, and high arterial tension.

2. Diminished support from sudden lowering of the intraocular tension; e.g., sub-choroidal hemorrhage after glaucoma iridectomy, or extraction of cataract.

3. Degeneration in the vessel walls, e.g., arterio-sclerosis.

$B$. Diapedesis, as the result of

I. Increased intravascular pressure.

2. Alteration in the constituency of the blood.

The damage done by an extravasation of blood depends first, on the nature and density of the tissue affected; thus delicate tissue, such as the retina, when torn up by a hemorrhage, is destroyed; second, on the amount of subsequent organisation that takes place in the clot leading to fibrocicatricial tissue, which by its contraction may press or pull on surrounding structures. The extent of the contraction probably depends on the rapidity with which blood coagulates, the hemolytic properties of the serum, and the absorbing power of the lymphatics in the tissues into which the hemorrhage takes place.

Hemorrhage from the iris may be extravasated into the anterior chamber (hyphemia). It is usually the result of injury but may occur when the iris is engorged with blood, as in acute iritis; also where there is much vascular degeneration, such as follows long standing cyclitis or glaucoma. If the angle of the anterior chamber is not blocked the blood is readily absorbed, but if retained a long time it may organise into a large pinkish-grey coloured mass at the bottom of the anterior chamber. These masses, microscopically, consist 
of endothelial cells enclosing spaces filled with cholesterin, crystals, and other dèbris of degenerated blood clot (Fig. I39).

After iridectomies, more especially in connection with glaucoma, and cataract operations, bleeding will sometimes

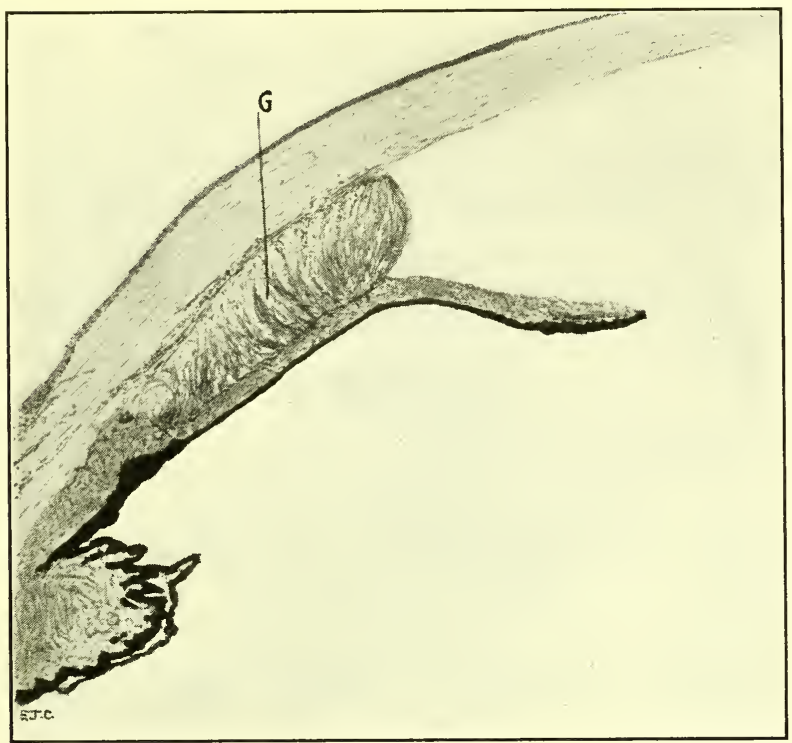

Fig. I39.- Shows a mass of retained and degenerate blood clot, $G$, at the angle of the anterior chamber. The slit-like spaces in it contained cholesterin crystals.

continue into the anterior chamber for a month or six weeks evidently coming from the cut surface of the iris. These secondary hemorrhages are often associated with a certain amount of iritis, the pupil tending to become updrawn as the result. It is probable that these secondary hæmorrhages are septic in origin and due to softening of the plug in the cut end of the vessels.

From blood long retained in the anterior chamber the hemoglobin may dissolve out of the red blood corpuscles, and pass into the substantia propria of the cornea by diffusion through Descemet's membrane, producing blood 
staining of the cornea ${ }^{1}$ (Figs. I40 and I4I) the condition frequently occurs in eyes in which the tension is increased

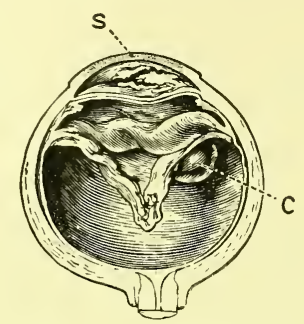

FIG. I40.- The lateral half of an eye which became blind from detachment of the retina. Four months previous to removal it received a blow from an open hand, which resulted in extensive hemorrhage into the anterior chamber and the staining of the whole cornea, with the exception of a narrow rim at its periphery, a reddish brown colour. $S$, Stained part of the cornea; $C$, cyst in detached retina. Recorded in Trans. Ophth. Soc. of the U. K., XI, I89I, 43.

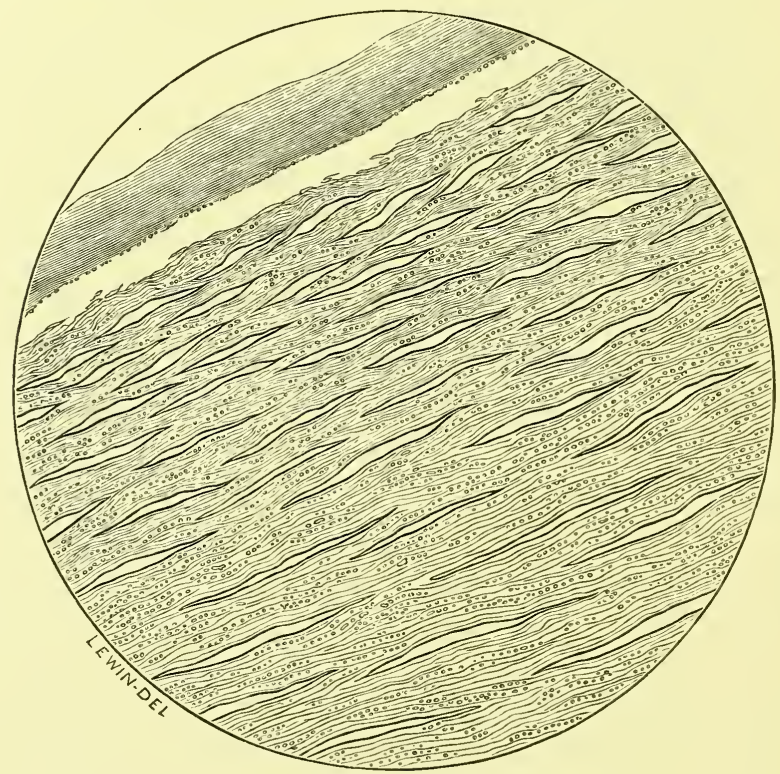

FIG. I4I.- Shows the microscopical appearances of the blood-stained area in the cornea depicted in Fig. I40. There are numerous granules of blood pigment scattered throughout the substantia propria.

It gives rise to a brown or green appearance of the cornea, depending on the time it has existed. The condition at first sight is liable to be mistaken for a dislocation of the lens

${ }_{1}$ Treacher Collins. Trans. Ophth. Soc. of the U. K., XV, I 895, 69. 
into the anterior chamber, because a narrow band at the periphery of the cornea in its entire circumference remains clear. Histologically, granules are found between the fibres of the substantia propria; these are of the nature of hematoidin (containing no iron) or hemosiderin (containing iron). The pigment is slowly absorbed from the periphery toward the centre of the cornea, often taking from eighteen months to two years to clear up completely.

Hemorrhage into the vitreous may arise from the ciliary body or retinal vessels, apart from any injury. In young people, more especially men, recurrent attacks take place, sometimes alternating with nose bleeding. In these cases the blood coagulability has been found in some to be raised, and in others lowered; it is probable that the hemorrhage is due to thrombosis occurring in a small vessel which subsequently gives way, from the vis a tergo. The blood may be derived from either the ciliary or retinal vessels; if from the retinal vessels the hemorrhage is at first sub-hyaloid, rupture of that membrane allowing it to pass into the vitreous. In older patients vitreous hemorrhage is usually associated with disease in the vessel wall or diabetes. The extent of the hemorrhage varies with the size of the vessel which gives way. Sometimes the whole vitreous is filled with blood which can be seen behind the lens; in other cases it merely constitutes a haze in the vitreous; between these two conditions varying amounts have been observed.

The subsequent change produced by the hemorrhage varies with the constituents of the blood extravasated, and the absorbing power of the intraocular lymphatic system. The blood being in contact with living tissues takes a long time to coagulate; if the blood coagulability of the patient be high, due to the blood containing an excess of calcium salts, it will coagulate more rapidly than normal, and then will not so readily undergo absorption. The red corpuscles discharge their hemoglobin, which is absorbed by the lymphatics, their stroma is broken up, and 
in the degenerative process cholesterin crystals are frequently formed. The fibrin in the vitreous, which sometimes can be seen on the posterior surface of the lens, either becomes absorbed or organised. The endothelial cells from the blood-vessels of the retina or ciliary body spread into it and bands of fibrous tissue develope-a condition known as retinitis proliferans (see page 472 ). These bands of fibrous tissue by their contraction may cause shrinking of the vitreous, detachment of the hyaloid, and, if the latter be adherent to the retina, detachment of that structure also.

Hemorrhage from the choroid occurs most frequently from the giving way of a vessel due to injury, to the sudden lowering of intraocular tension, or to thrombosis of a choroidal vein. In the latter two cases disease in the vessel walls plays an important predisposing part. The sudden reduction of intraocular tension, as after an iridectomy for glaucoma, may cause a rupture in a choroidal vessel. If this be a large artery in the outer layers of the choroid the blood is extravasated between the choroid and sclerotic, pushing the former inward. As a result the lens may be pushed forward and, if the bleeding continues, the lens, retina, and choroid escape through the wound. If a small vessel in the chorio-capillaris of the choroid gives way the blood may rupture through Bruch's membrane and hemorrhage take place into the subretinal space. Smaller extravasations of blood, such as occur at the macula in myopic eyes, may remain localised in the choroid. Such hemorrhages are followed by intense secondary pigmentation, the pigment being derived partly from the blood and partly from the proliferation of the retinal and choroidal pigment cells. Hematogenous pigment from subchoroidal hemorrhages deposited in the perivascular spaces around the short ciliary arteries, especially in the neighbourhood of the disc and circle of Zinn, produce the condition known as Angeoid Streaks ${ }^{1}$ (Fig. 142).

${ }^{1}$ Treacher Collins. Trans. of the Ophth. Soc. of the U. K., XIIII, $1923,273$. 
Hemorrhage from the Retina.-The amount of blood extravasated into the retina, and the damage done to it thereby, depends on the size and situation of the vessel which gives way. If the vessel be superficial the bleeding takes place beneath the hyaloid membrane of the vitreous

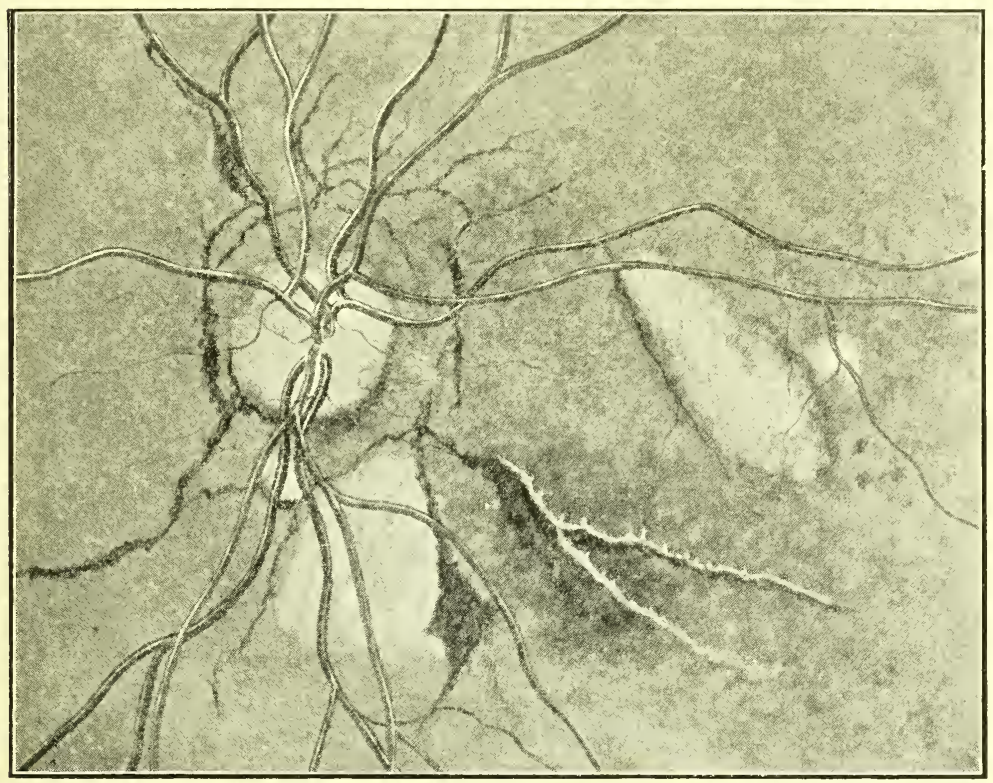

FIG. I42.-Angeoid streaks in the fundus oculi. Case recorded in Trans. Ophth. Soc. of the U. K., XLIII, I923, 286.

and is usually extensive. The blood sinking downward separates the hyaloid from the retina, and generally takes the form of a boat-shaped red area darkest in colour in the lower part, owing to the greater mass of red blood corpuscles present in that situation. This form of hemorrhage does not give rise to much destruction of the retinal elements, and on its absorption good vision may be restored (Fig. I43). Occasionally the hemorrhage may be so extensive as to cause rupture of the hyaloid and give rise to turbidity of the vitreous or ultimately a condition of retinitis proliferans (see page 472 ). 


\section{DISTURBANCES IN THE NUTRIENT FLUIDS OF THE EYE}

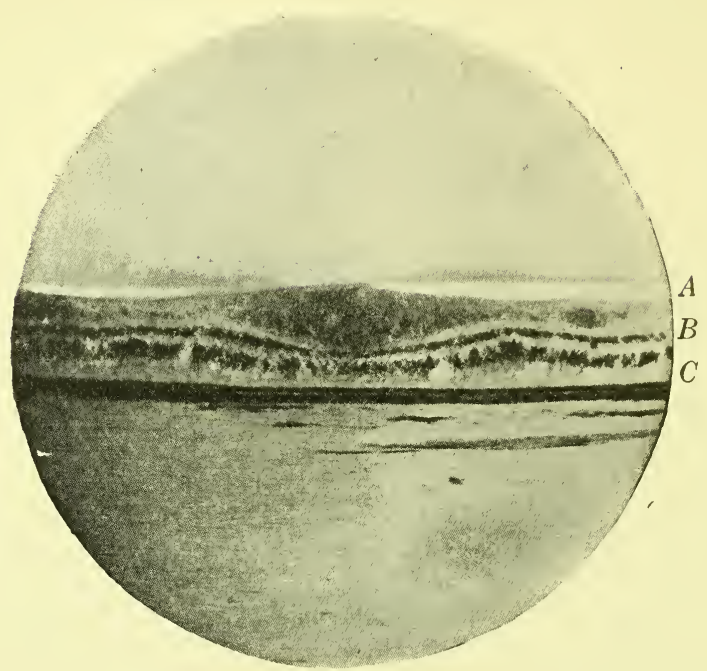

FiG. I43.- Section through the coats of the eye in a case of sub-hyaloid hemor. rhage. It shows the comparatively slight change caused in the retina thereby. $A$, Hyaloid membrane; $B$, hemorrhage; the blood has accumulated at the most dependent part; $C$, retina.

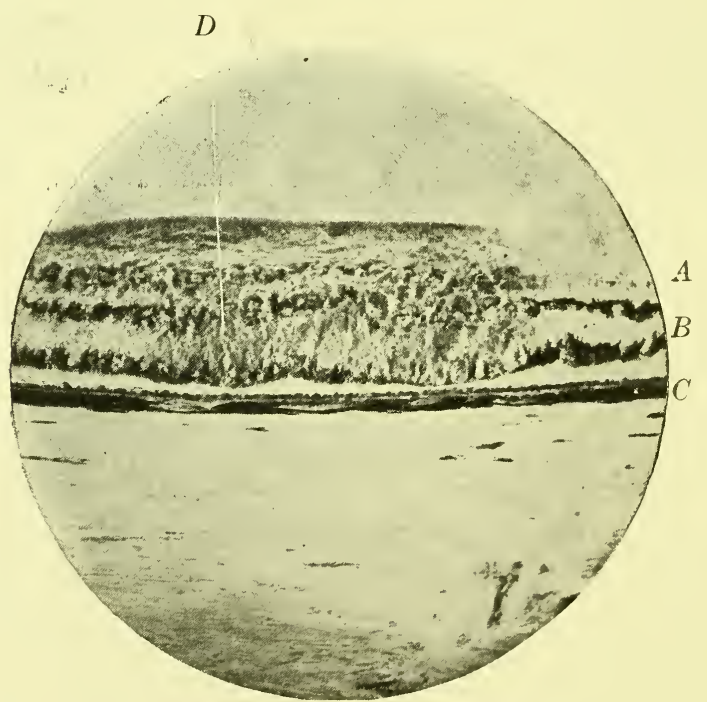

FIG. I44.- Shows a section through the coats or the eye in a case of albuminuric retinitis. The destruction to the retina caused by hemorrhage into it is seen. $A$, Sclerotic; $B$, choroid; $C$, retina; $D$, hemorrhage. 
Hemorrhages into the nerve-fibre layer, owing to its anatomical arrangement, usually have a flame-shaped or

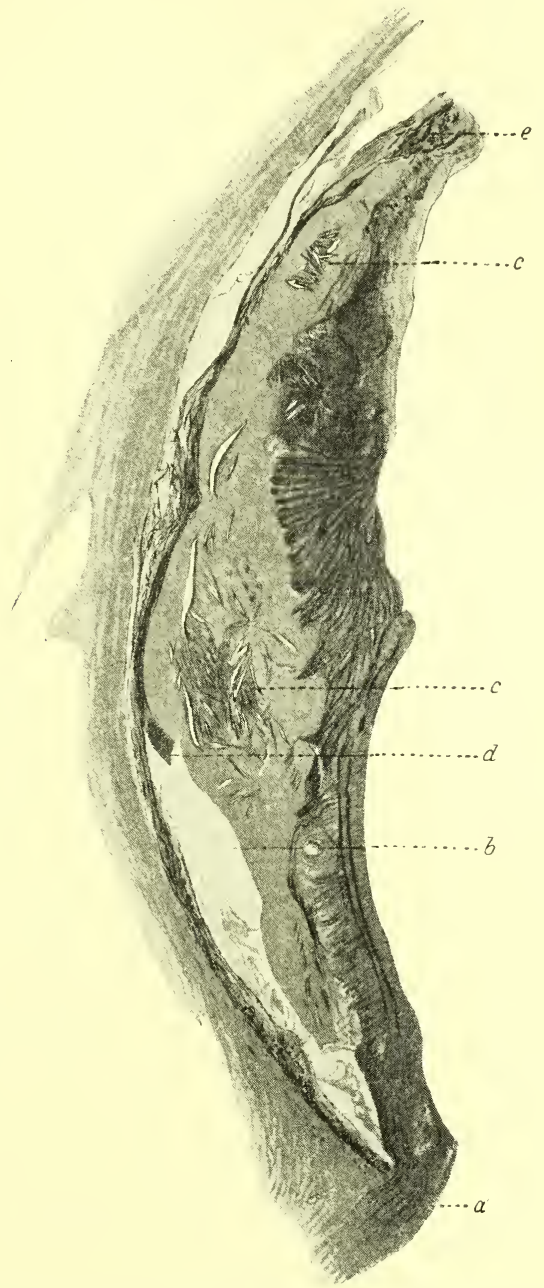

FIG. I45.- Section through the coats of the eye showing a mass of organising blood clot beneath the retina, so-called "massive retinitis." $a$, optic nerve; $b$, mass of fibrillated structure with fusiform cells in it; $c$, spaces which contained cholesterin crystals; $d$, mass of red blood corpuscles; $e$, hyalin excrescences from elastic lamina. Case recorded Trans. Ophth. Soc. of the U. K., IX, I889, I98.

striated appearance. Hemorrhages into the deeper layers of the retina cause immense destruction of tissue, the retinal 
elements being torn apart, with the result that a permanent scotoma remains in the field of vision (Fig. I44).

Retinal hemorrhages will persist for months without showing appreciable ophthalmoscopical change. As the blood disintegrates it gradually becomes darker in colour, and finally black so that clinically it cannot be distinguished from the retinal pigment. Microscopically the fact that the pigment derived from hemoglobin will for some considerable time after its formation yield the test for iron helps to differentiate it. The breaking up of the stroma of the corpuscles may give rise to crystals of cholesterin, which appear as glistening points in the fundus.

The diseases known as massive retinitis (Fig. I45) and retinitis circinata are due to retinal hemorrhage and are probably nearly allied to one another, the latter being a less severe type of the former, but up to the present time retinitis circinata has only once been submitted to microscopical examinations. There are two main groups of cases-(a) those associated with marked vascular degeneration of the intraocular vessels, and therefore occurring principally in old people, although in rare instances young people with chronic nephritis may be affected; and (b) those associated with nose bleeding, menstrual disorders etc., which are probably due to a high blood coagulability; these patients are usually young persons. In each instance it is probable that the determining factor in the production of the hemorrhages is thrombosis of some small venous radicle. Histologically the changes are situated principally around the macular region where large raised white areas may appear. The exudate is in the internuclear layers, the supporting structures of the retina being pushed to one side to form the walls of pseudocystic spaces which contain blood clot, cholesterin, albuminous fluid, or hyaline material with a certain amount of organising fibrous tissue. Occasionally the walls of these spaces can be found ruptured into the sub-retinal space. 
Hemorrhage into the optic nerve and its sheath may occur spontaneously or as the result of injury. The condition leads to a sudden loss of vision, owing to pressure on the nerve. If the extravasation be into the nerve sheath an edema of the papilla may be caused by pressure on the central vein. The recovery of sight depends on the amount of destruction caused by the primary extravastion, or subsequently, by the amount of organisation which takes place in the blood clot.

ix. Dilation of Orbital Vessels. Localised Dilatation of the Vessels Connected with the Orbit.-Either arteries or veins alone may be affected or a communication between the two may be established. If the vein is merely dilated, as the result of the communication with the artery, the condition is an aneurismal varix, but if aneurism forms between the artery and the vein the condition is a varicose aneurism.

The dilatation of the vessels gives rise to exophthalmos, and if a main artery takes part in the dilatation it causes pulsating exophthalmos. The affection is due either to trauma or vascular degeneration, which latter is frequently syphilitic in origin. The conditions found postmortem in the order of their frequency are: communication of the internal carotid artery with the cavernous sinus, aneurism of the ophthalmic artery in the orbit, aneurism of the ophthalmic artery outside the orbit, aneurism of the intracranial portion of the internal carotid. It is probable that a communication between the vein and artery in the orbit may also occur.

For description of the various angiomata see page 2I4.

General dilatation of the orbital vessels may occur as the result of local inflammation or obstruction to the venous return, as has already been described. It may also occur as the result of stimulation of the sympathetic nerve.

Exophthalmic Goitre.-The ocular features of the disease only require mention here. They are: retraction of the lid (Dalrymple's sign); the upper lid not moving down- 
ward in unison with the eye (von Graefe's sign); imperfect closure of the lids (Stellway's sign); defective convergence (Moebius' sign); pigmentation of the lids. The proptosis is usually bilateral but may be unilateral. There have been many explanations as to its cause, one suggests that it is due to a dilatation of the vessels of the orbit, as the result of the action of the increased thyroid secretion on the sympathetic. In favour of this is the fact that in most cases the exophthalmos disappears after death. It is probable that in cases where proptosis persists after death some increase of fat has been formed in the orbit as the result of the engorgement of the vessels.

Cavernous sinus thrombosis may arise from the spread of thrombosis from one of its communicating veins. These are; the ophthalmic veins through which it communicates with the angular vein, and the petrosal sinus connected with the veins of the ear; it also indirectly communicates with the pterygoid plexus and with the veins from the cerebrum, and the two sinuses communicate freely with one another. The cause of the thrombosis may therefore arise in the orbit, nose, ear or tonsil. The signs are enlargement of the mastoid emissary vein from venous obstruction, paralysis of the sixth nerve from pressure, and proptosis. When both sinuses are affected these signs are bilateral. The proptosis is due to engorgement and edema of the orbital tissue. There is no enlargement of the retinal veins unless the ophthalmic vein be thrombosed, since the latter vessel communicates freely with the angular vein and the pterygoid plexus. In other cases papillitis and retinal hemorrhage from obstruction of the central vein may be present. If the clot be septic death usually follows, but in aseptic cases the patient may live, the exophthalmos continuing and post-neuritic atrophy setting in. In septic cases the staphylococcus aureus, streptococcus and pneumococcus have been found in the clot.

$x$. Diseases Due to Changes in the Constituents of the Blood.- In the retina and choroid the appearances of 
the blood clinically can be better examined than in any other part of the body. Changes in the fundus of the eye are, therefore, of considerable assistance in the diagnosis of diseases of the blood.

The whole fundus may be altered in colour due to, a high percentage of leucocytes in the blood in the choroidal capillaries, a low percentage of red blood corpuscles, a low percentage of hemoglobin or an infiltration of the choroid with leucocytes.

The colour of the blood in the retinal arteries and veins may, in anemias and leucocythemia, become very similar.

Alterations in the character of the blood may lead to the extravasation of one or more of its constituents through the vessels walls. Extravasation of the fluid of the blood into the retina gives rise to edematous haze or opacity of that membrane, which may result from an alteration in the normal osmotic process or from some defect in the lining endothelium of the capillaries.

The lymphocytic enlargements met with in leukemia and chloroma are probably due to a diapedesis of leucocytes through the capillary walls, and their proliferation in the tissues. Some authorities, however, regard such swellings as primary overgrowths of the lymphoid elements in the affected part, and closely allied to round celled sarcomata.

Extravasation of the red blood corpuscles into the retina may occur from the formation of a thrombus in one of its veins, multiple hemorrhages then being found along the whole course of the blocked vessel. A deficiency in the mechanism which results in clotting of the blood also favours the occurrence of hemorrhages. The formation of the fibrin in a clot depends on the blood platelets, when these are reduced in number clotting does not take place. In hemorrhagic purpura the platelets are almost entirely absent from the blood, and purpura has been produced experimentally in animals by destroying them with antiplatelet serum. In pernicious anemia and in acute lymphatic leukemia, in which retinal hemorrhages are common, there is 
a marked deficiency in the number of the platelets. In chlorosis where retinal hemorrhages hardly ever occur, there is no such deficiency.

The diseases of the eye due to changes in the constituents of the blood may be divided into:

(a) Ischemia from loss of blood.

(b) Deficiency of Red blood corpuscles. Pernicious Anæmia.

Secondary Anæmia.

(c) Excess of Red blood corpuscles.

(d) Deficiency of Hemoglobin.

Erythræmia (Vaquez's Disease).

(e) Increased White blood corpuscles.

(f) Increased coagulability.

Chlorosis.

Leukemia.

Chloroma.

Thrombosis and Embolism. Retinitis Septica.

(g) Decreased coagulability.

(h) Deficiency of Vitamins. (see degenerations) Vitamin A.

Hemophilia.

Xerosis of Conjunctiva and Night-blindness.

Vitamin B. Vitamin C.

(i) Poisons in the blood.

Beriberi Neuritis.

Scurvy.

I. Endogenous.

2. Exogenous.

(a) Ischemia from Loss of Blood.-Loss of blood in large quantities may cause failure of vision, due to ischemia of either the retinal or cerebral blood vessels. The source of the hemorrhage is most frequently the stomach and intestines, and after that the uterus. It rarely occurs after wounds or operations, but patients who are actually dying from loss of blood often say that everything is black before them. The onset of blindness is usually rapid but may be delayed; it does not always occur in the two eyes simultaneously. Subsequently improvement of the vision sometimes takes place. Retinal ischemia may produce secondary degenerative changes in the retina, such as edema, punctate hemorrhages, and atrophy of the ganglion cells which leads to a permanent defect of vision. 
(b) Deficiency of Red Blood Corpuscles. Pernicious anæmia gives rise to pallor of the optic disc and fundus, and retinal hemorrhages. Retinal edema, and papilledema are common in the later stages of the disease. They produce secondary degenerative changes in the nerve elements of the retina. It has been stated that these changes do not occur until the red blood corpuscles have dropped to about 32 per cent of the normal.

I. Secondary Anæmia. Aplastic Anæmia.-Aplastic anæmia is due to degeneration of the bone marrow as the result of poisons such as benzol, trinitroluene and salvarsan, or prolonged exposure to x-rays. Retinal hemorrhages and neuro-retinitis have been recorded.

2. Subacute combined degeneration of the spinal cord gives rise to a severe secondary anæmia leading to small retinal hemorrhages and slight papilledema. Irregular contraction and sluggishness of the pupil together with nystagmus may also be present, and in some cases paresis of the external ocular muscles.

(c) Excess of Red Blood Corpuscles. Erythræmia ( Vaquez's Disease).-In this disease the red cells usually number 7-r 2 million; the cells being normal in appearance. The hemaglobin is seldom increased and there is slight leucocytosis. The patients have a florid cyanotic appearance, and there is an enlargement of the spleen and liver. The disease occurs in adults between 30 and 60 , and is probably due to a hyperplasis of the erythroblastic tissue of the bone marrow. The conjunctiva is often congested. Defects of vision of varying degrees of intensity may occur. In the early stages no ophthalmoscopic changes are found. In the later stages engorgement of the retinal veins with hemorrhage and papilledema occur.

(d) Deficiency of Hemoglobin. Chlorosis.-Beyond the light colour of the blood-vessels of the conjunctiva, and occasional pulsation of the central artery of the retina, it is doubtful if chlorosis produces any primary ocular changes. Optic neuritis and retinal hemorrhages have 
been ascribed to this disease, but they are probably due to some secondary condition.

(e) Increased White Blood Corpuscles. Leukemia.1Both lymphocytic and myelogenic forms of leukemia, acute or chronic, may affect the orbital tissues, eyelids or eyeball. In the lids, orbit and ocular conjunctiva, lymphomata may be produced (see page 224). The first changes inside the eye are, light yellowish pallor of the whole fundus and multiple flame shaped peripheral retinal hemorrhages. White spots bordered by hemorrhages are sometimes seen situated at the macula or periphery. In the latter stages the interior of the eye may become the seat of lymphomata situated either in the choroid or retina. When the latter is affected, the term "leukemic retinitis" has been applied.

There is peri-vascular infiltration of the veins as shown by the broad white lines which form along them. The disc and whole retina may be edematous, disorganisation with degeneration of the nerve fibre layer of the retina, and the formation of cytoid bodies subsequently taking place.

Chloroma. ${ }^{2}$ - Chloroma gives rise to rapidly growing tumours of a bright green colour which affect the skull and dura mater, later becoming desseminated in the viscera of the body. In the orbit they cause proptosis and in rare cases the retina and choroid have been secondarily affected. The disease is very closely allied to leukemia since profound changes are found in the blood, it has been regarded as a malignant disease of the bone marrow with the secondary changes in the blood. The cellular changes, as in leukemia, being usually of a lymphocytic but occasionally myelogenous type.

(f) Increased Coagulability. Thrombosis and Embolism. Thrombosis.-Alterations in the coagulability of the blood may give rise to thrombosis, especially if the ocular vessels are already diseased (see page 246). It is probable

${ }^{1}$ A. C. Hudson. Royal Lond. Ophth. Hosp. Reps., XVIII, I9I I, I 5.3.

${ }^{2}$ A. C. Hudson. Royal Lond. Ophth. Hosp. Reps., XVIII, I9I I, I8I. 
also that recurrent spontaneous vitreous hemorrhages which occur in young people, especially men, are due to this cause (see page 255 ).

It is possible that the hemorrhage which occasionally occurs in the macular region associated with menstruation may be due to raising of the blood coagulability.

Embolism.-A portion of a blood clot from a thrombus, or another embolism, fibrin from the surface of the cardiac valves, atheromatous plates from the inner surface of large vessels may be carried by the blood stream and impacted in the vessels of the eye. When the material contains pyogenic microörganisms it is followed by an inflammation which is known as pyemia (see page 484 ). When an embolism occurs in the central artery of the retina, the clot usually lodges in the vessel as it passes through the lamina cribrosa, probably because of the narrowing of its lumen in that situation, and possibly also because it is the common position at which it bifurcates, the embolism straddling the bifurcation (Fig. I33). Occasionally only one branch of the retinal artery is occluded. The clinical signs and subsequent changes have been described under obliteration of the central artery (see page 243). The onset is sudden, and without the premonitory symptoms of temporary loss of vision, as is present in some cases where the obstruction of the artery is due to vascular sclerosis.

Embolism in the Uveal Tract.-The uveal tract is a common site for the lodgment of septic emboli in the eye as will be pointed out in connection with pyemia (see page 484 ). There is no doubt also that aseptic thrombi, etc., lodge in the choroid, although the condition is not recognised clinically as such, probably because the anastomosis in the choroid is very free and the area affected, therefore, very localised. A case has been described pathologically, showing a patch of obliterated choroidal vessels with fibrosis and hematogenous pigmentation, which was probably of this nature, but more evidence is wanting as to its occurrence. 
Embolism in the ophthalmic artery may give rise to occlusion of both retinal and choroidal vessels. Such cases are usually associated with embolism of the common carotid artery. In this case the clot in the common carotid may extend by thrombosis to the ocular vessels, or, a portion of this clot become detached and carried into the ophthalmic artery. ${ }^{1}$ Cases have been recorded which seem to show that both these conditions can occur. Thus a hemiplegia, with embolism of the central artery and complete obliteration of the common carotid on the same side, the choroid being normal, seems to point to detachment of a portion of the embolism from the carotid vessel; while hemiplegia with complete obliteration of the common carotid, retinal and choroidal vessels, points to an extension of the clot from the carotid by thrombosis completely blocking the ophthalmic artery.

Endocarditis.-The diagnosis of malignant endocarditis is frequently made by finding retinitis septica. Small round or oval hemorrhages together with numerous white spots in the retina are characteristic of the disease. The disease has been found to be due to streptococcus viridans. Actual bacterial emboli have been found in the vessels in the retina and papilla to account for the hemorrhages, but probably not all the hemorrhages are due to emboli, some being due to the effect of bacterial toxins on the endothelium. The white spots have been attributed to swollen nerve fibres, but may also be caused by inflammatory exudation. Some small patches of inflammation found in the choroid are probably also embolic in origin.

(g) Decreased Coagulability of the Blood. Purpura.Retinal hemorrhages with yellowish patches sometimes occur in the nerve fibre layer of the retina in connection with purpura. They are apt to recur from time to time, but are not found in every case. In hemorrhagic purpura,

${ }^{1}$ Guthrie and Mayou. Trans. Ophth. Soc. of the U. K., XXVIII, I908, I04. 
as already mentioned, the blood platelets are almost entirely absent.

Hemophilia.-Hemophilia does not frequently give rise to hemorrhage in the eye, but wisps of blood are sometimes seen upon the iris in these cases. In cases of injuries to the conjunctiva, and removal of the eye, it has given rise to enormous loss of blood even resulting in death. Strictly speaking hemophilia is not a blood disease, the number of platelets in the blood is normal; it is probably due to the absence of a tissue coagulin which is usually present in the vessel walls.

(h) Deficiency in Vitamins.-The chemical nature of vitamins is, so far, obscure, but they may be differentiated by the source from which they are derived, their varying solubilities, their action, and the maladies which are produced when they are absent.

Though there are probably many of them only three are at present recognised, these it has become customary to allude to as $\mathrm{A}, \mathrm{B}$, and $\mathrm{C}$.

Vitamin A is contained specially in the green parts of plants, it is soluble in fatty material. Cows who eat grass pass it into their milk. Its absence is supposed to be one of etiological factors in the production of rickets. For its connection with xerosis of the conjunctiva and night blindness see page 593 .

Vitamin B is contained in the grains of cereals, and is soluble in water.

Beriberi, is a form of multiple peripheral neuritis and occurs most commonly in males, but with much greater frequency in females during pregnancy. The disease occurs in people who live on rice from which the pericarp has been removed. This pericarp contains the vitamin $\mathrm{B}$ the absence of which from diet causes the disease. There are two types, the dropsical and the paraplegic. The eye symptoms which the disease gives rise to are, decreased sensibility of the conjunctiva and cornea; retrobulbar neuritis which results in the formation of a scotoma varying in size from a 
dumbell-shaped area, including the macula and the blind spot, to merely a small central defect for colours; optic atrophy may ensue. Paresis of the ocular muscles, especially of the levator palpebræ producing ptosis, is frequent in infants who have been infected from their mothers.

Vitamin $\mathbf{C}$ is obtained from fruits and edible vegetables such as potatoes and turnips, it is soluble in water.

Scurvy.--Scurvy is a deficiency disease due to the absence of Vitamin C. It gives rise to a deficient secretion of the lacrymal gland, drying of the cornea and conjunctiva is apt to ensue and even ulceration. It is associated with night-blindness due to malnutrition. In children extensive sub-periostral ecchymoses frequently occur in the orbit, lids and conjunctiva, and retinal hemorrhages are met with more often than in the adult form.

(i) Poisons in the Blood.-A poison circulating in the blood may produce temporary or permanent blindness by its action on the nerve elements of the retina or cerebral centres for vision.

Poisons affecting the retina do so either by direct action on the ganglion cells or their synapses, or by the production of ischemia which interferes with their nutrition. The ischemia is produced either by the action of the poison on the sympathetic nerves causing constriction of the retinal blood vessels, or by dilatation of the vessels in the splanchnic area causing constriction of vessels elsewhere, including those of the retina.

Following the retinal degeneration atrophy of the whole or part of the optic nerve takes place, associated with a leukocytosis into the nerve to remove the débris of the broken up medullary sheaths; this leukocytosis has been considered by some observers to be a retrobulbar neuritis, which they regard as the actual cause of the defect of vision, the changes in the ganglion cells being secondary.

The poisons which may affect vision in the above ways are either endogenous, i.e., generated in the body itself from faulty metabolism, or exogenous. 
The endogenous toxins producing amblyopia are found in connection with diabetes and uremia.

The principal exogenous toxins which produce amblyopia are tobacco, methyl and ethyl alcohol, carbon disulphide, iodoform, quinine, ergot, nitrobenzol, anilin and arylarsonates, filix mas, lead, nitrate of silver and salicylic acid.

I. Endogenous Toxic Amblyopia. Diabetic Amblyopia.The toxin which produces amblyopia in diabetes is not known. The symptoms to which it gives rise are similar to those found in tobacco-alcohol amblyopia; namely, failure of vision, a central scotoma for colours, and in longstanding cases pallor of the temporal side of the optic disc. From the similarity of the symptoms it may be supposed that the action of the poison is, as in tobacco amblyopia, mainly on the ganglion cells or their synapses, possibly assisted by defective nutrition due to changes in the vessel walls. Some authorities attribute it to a retrobulbar neuritis. ${ }^{1}$

Uremia occurring in connection with acute or chronic nephritis may be associated with transient amaurosis without ophthalmoscopic signs, the pupil reacting freely to light. The onset of blindness is rapid and usually complete. Recovery may also be rapid and complete, but sometimes hemianopia is present for a short while. The activity of the pupils, the rapid recovery from the attack and the occasional presence of hemianopia, suggest that the amaurosis is due to the action of a toxic agent on the higher centres for vision. The view has been advanced that it is due to cerebral anemia following increased intracranial tension, but against this hypothesis is the fact that usually no optic neuritis is present. The poison which causes uremia has not so far been isolated.

The Puerperal State.-Amblyopia arising during pregnancy may be the result of uremia associated with kidney disease. There seems also to be another form of temporary

${ }^{1}$ Nettleship and Edmunds. Trans. Ophth. Soc. of the U. K., III, I883, 165. 
amblyopia unassociated with uremia which may come on before, during or after labour; ${ }^{1}$ albumin may or may not be present in the urine, but headache, convulsions, and edema of the face are frequently present. The amblyopia may last for some time, but after the birth of the child recovery gradually takes place. There are usually no ophthalmoscopic changes.

It has been demonstrated that in 89 per cent. of pregnant women, especially from the eighth to the ninth month of gestation, some contraction of the temporal fields of vision occur due to the normal enlargement of the pituitary body which takes place at that time. ${ }^{2}$ Occasionally a fleeting bitemporal hemianopsia has been observed.

2. Exogenous Toxic Amblyopia.-Exogenous poisons may act directly on the nerve-elements in the retina, like tobacco, or indirectly by producing ischemia, like quinine. They may also produce amblyopia by setting up optic neuritis, like lead, or by acting on the cerebral centres of vision, like salicylic acid.

Tobacco Amblyopia.-In man chronic poisoning with tobacco is usually associated with the abuse of alcohol and vascular sclerosis, probably for this reason tobacco amblyopia is rarely met with before the age of thirty-five years.

The tobacco used by such patients is usually a strong form, such as shag, smoked in a pipe. It produces failure of vision consisting of defective form-sense, light-sense and a central scotoma for colours (green and red). In severe cases there may be an actual central scotoma for white. It always affects both eyes and patients state they are able to see better in dull than in bright lights. The poison is probably not nicotine but some derivative, such as pyridine.

Histological examination has revealed chromolytic changes in the ganglion cells of the macular region. It is

${ }^{1}$ W. P. Herringham and S. Stephenson. Ophthaolmscope, VIII, I9io, I68.

${ }^{2}$ C. E. Finlay, W. B. Lancaster and Maude Carvell. Trans. Internat. Ophth. Congress, Washington, I922, I 44. 
suggested that the macular region is attacked in preference to other parts of the retina because it is poorly supplied by blood, partly as the result of vascular sclerosis from the alcohol, and partly because the retinal vessels are normally absent from that region; the nerve-elements at the macula are therefore less able to resist the deleterious influence of the toxin than those in other points. In cases in which there is much vascular sclerosis the recovery, which usually takes place on discontinuing the use of tobacco, may be delayed or not occur at all. The fact that improvement of vision is caused by dilatation of vessels following the use of nitrite of amyl lends support of the theory, that the vascular supply is an important factor in the causation of the condition.

Another theory attributes the affection of the macular region to the selective action of the poison on the cone ganglion cells or, as is more probable, on the synapses between them and the bipolar cells. The cones in the macular region have only one synapsis with their ganglion cells; while the rods, have several paths of conduction connecting them with several cells, so that if one is destroyed others will remain open. In support of the primary affection of the cones, or their connections, is the fact that patients with tobacco amblyopia see best in dull lights, the cones being particularly associated with form-sense, the rods with light-sense.

Degenerative changes in the papillo-macular bundle of the optic nerve have been demonstrated as far backward as the basal ganglia; these changes are probably secondary to the changes in the retina, although some authorities regard the condition as a primary retrobulbar neuritis and have demonstrated leukocytosis in the nerve. This latter if present is probably phagocytic in nature, owing to the presence of the degenerating fibres.

Alcohol amblyopia from acute alcohol poisoning is usually due to the methyl variety, commonly known as wood alcohol. The blindness is generally for a time complete. 
As recovery takes place a central or paracentral scotoma may be present in the field. Experimental investigations on animals have shown that the primary change is in the ganglion cells of the retina, principally in the neighbourhood of the macula, followed by corresponding secondary degenerative changes in the optic nerve. ${ }^{1}$ Previous to this the changes were thought to be due to a retrobulbar neuritis. ${ }^{2}$

The part played by chronic ethyl alcohol poisoning has already been referred to under tobacco amblyopia.

Carbon disulphide is used in rubber works and inhaled by the employés. Iodoform is used for surgical purposes. Both these substances may cause amblyopia with a central scotoma for colours. Probably similar changes in the retinal ganglion cells and their synapses are produced, as are found in alcohol-tobacco amblyopia. Experimental investigations on animals have shown changes in the cells of the central nervous system, but these have not as yet been demonstrated in the retina.

Quinine amblyopia occurs mostly in those suffering from malaria for which affection enormous doses of the drug are frequently administered. The smallest dose recorded as causing defect of sight is 2 grams given in thirty-eight hours.

The symptoms produced are deafness and blindness, of rapid onset and a very marked character. The former precedes the latter and is of only temporary duration.

The blindness affects both eyes and is more complete than in any other condition from which recovery takes place. In one recorded case the deafness and blindness were so marked that the patient could only be communicated with by the sense of touch. Ophthalmoscopically the appearances at first resemble those of embolism of the central artery of the retina. There is marked ischemia of the optic disc and retina, with a white haze of the latter

${ }^{1}$ Birch-Hirschfeld. Archiv. für Ophth., L, I900: LI, I90 I; LII, 1902.

${ }^{2}$ Uhthoff. Archiv. für Ophth., XXXIII, I887, i, 257. 
around the macula and disc, and a cherry-red spot at the macula. The pupils become widely dilated and are insensible to light. The complete blindness may last for several days. Some cases recover entirely, in others the only permanent defect is a contraction of the field for colours; more frequently the field for white is also permanently contracted, the patient being reduced to what is known as tubular vision; i.e., seeing well in the centre of the field but not in the peripheral parts. The pupils recover their mobility with the return of vision; the ophthalmoscopic appearances in the late stages of the disease resemble optic atrophy, the disc remaining very white, and retinal vessels narrow with white lines coursing along them.

Examinations of the retina and optic nerves of dogs, at periods ranging from two days to seven weeks after the administration $^{1}$ of toxic doses of quinine, have been made, and the changes found were constriction of the retinal blood-vessels, degeneration of the ganglion cells, and an ascending atrophy of the optic nerve.

It will be seen that the way in which vision is affected is just the reverse of what occurs in tobacco amblyopia; in the latter there is loss of central vision, the peripheral parts of the field being unaffected; in quinine amblyopia peripheral vision may be lost and central vision retained.

Ergot may cause transient amaurosis from constriction of the retinal vessels, but no case has been recorded which has gone on to optic atrophy.

Nitrobenzol has a similar action to quinine but the ischemia is not so severe or prolonged and hence recovery is more complete.

Anilin.-Amblyopia due to poisoning by anilin oil has frequently been recorded. ${ }^{2}$ The clinical changes are chiefly in the retinal blood-vessels; retinal hemorrhages are sometimes present; poisoning by this substance is of interest in view of the extensive use of the arylarsonates.

${ }^{1}$ W. A. Holden. Am. Ophth. Soc., VIII, I 898, 405. Birch-Hirschfeld. Archiv. für Ophth., L, I900, I66.

${ }^{2}$ Mellingnoff. Klinisch. Mon. f. Aug., XL.IV, I906, 35. 
Arylarsonates.-The chief of these are atoxyl, soamin, and arsacetin. These substances are compounds of anilin and arsenic. They have been administered subcutaneously in large doses in cases of spirochetal infection (syphilis, sleeping sickness, etc.). A number of these cases have been followed by optic atrophy. ${ }^{1}$ During the acute stage of the poisoning the fundus has seldom been observed. In one case there was intense retinal ischemia, the condition of the fundus resembling that found in embolism of the central artery; this was subsequently followed by optic atrophy. This case suggests that the action of the poison is mainly on the blood-vessels.

Although numbers of cases of arsenical poisoning have been recorded, no loss of sight or similar changes in the fundus have been found associated with it. The administration of arsenic in large doses in the form of dioxydiamidoarsenobenzol ("606"), and other similar compounds, has not yet been followed by primary optic atrophy; presumably therefore the harmful action is due to the anilin part of the compound in the case of the arylarsonates.

Filix mas amblyopia is probably somewhat similar to that due to quinine. There is a limitation of the peripheral field of vision associated in the early stages with constriction of the retinal vessels. Secondary changes in the ganglion cells and optic nerves follow. It may affect the eyes unequally; thus one eye may be blind while the other may recover nearly normal vision. Filix mas may also cause acute nephritis and, in a few recorded cases, optic neuritis and retinal changes, similar to those associated with that disease, have been found associated with the amblyopia.

Lead poisoning produces two forms of optic neuritis.

The first in people who have often only worked for a short time in a lead factory. This comes on with headache, vomiting and optic neuritis, which may be followed by optic atrophy. Albumin may be absent in the urine.

${ }^{1}$ E. Clarke. Trans. Ophth. Soc. of the U. K., XXX, I9Io, 240. 
It is often associated with acute encephalitis, which may or may not precede it. The optic neuritis is probably papilledema due to increased intracranial tension, caused by rapid effusion of fluid into the basal cisterns of the meninges (see page 287 ).

Cases of this sort are sometimes met with in young women who have taken diachylon pills to produce abortion.

The second form is met with in old standing cases of lead poisoning where the kidneys have become affected and there is albumin in the urine. The clinical appearance of the fundus is the same as that found in albuminuria (see page 248). Uremia may be associated with this condition and give rise to coma which must be differentiated from the coma caused by encephalitis associated with the more acute forms of poisoning.

Nitrate of silver in large doses has a similar effect to lead. If the patient survives or the poison is chronic the nitrate of silver becomes deposited in the intracellular tissue, and the action of the light turns it brown. Continuous local application to the conjunctiva causes a similar appearance in that membrane. The condition known as "argyrosis" (see page 395).

Salicylic acid in doses over I 5 grm. may cause temporary amaurosis. It is usually accompanied by tinnitus; recovery is rapid and complete and no ophthalmoscopic signs are present. The drug probably acts on the cerebral centres for vision.

Santonin administered for worms may cause yellow vision; this passes off without producing amblyopia.

\section{DISTURBANCES IN THE CIRCULATION OF LYMPH}

Under normal conditions the lymph which nourishes the coats of the eyeball must be clearly differentiated from the intraocular fluid which fills the anterior, posterior and vitreous chambers. Lymph contains proteins, nearly equal in amount to those in the blood, and lymphocytes, it coagulates 
on removal from the body. Intraocular fluid contains only 0.045 per cent. of proteins, no lymphocytes and is not coagulable.

Strictly speaking the term lymph should be confined to the fluid normally contained within the lymphatic vessels. The fluid in the tissues, in which nutritional and other changes mostly take place, being spoken of as tissue fluid; this latter, however, closely resembles lymph in its composition and is very different from the intraocular fluid. It escapes from the tissues mainly by absorption into the veins.

Lymphatic vessels vary considerably in their number and distribution in different parts of the body, their function is to absorb any excess of tissue fluid or any solid particles which may have gained entrance into the body. They can do this more readily than the venous capillaries because the spaces between the endothelial cells lining them are wider; whilst in some places stomata have been discovered through which foreign particles can be passed by the aid of phagocytosis.

In the eye the largest endothelial lined lymphatic spaces are those between the choroid and sclerotic, which communicate on the one hand with the intercellular spaces of the choroid, and on the other, by means of perivascular lymph channels surrounding the vortex veins, with the lymphatics of the orbit. The inner third of these veins as they pass through the sclerotic is surrounded by a definite endothelial lined space and the remainder by a kind of mesentry. There is a similar mesentry in the spaces surrounding the anterior and posterior ciliary arteries in their passage through the sclera, which probably form atypical lymph channels.

The retinal blood vessels are surrounded by lymphatic sheaths similar to those around the blood vessels of the brain. These are more clearly defined around the veins than the arteries. The subarachnoid space of the optic nerve communicates freely with a similar space around the 
brain and contains cerebro-spinal fluid, a fluid very different from lymph; it is formed by the secreting activity of the cells covering the choroidal plexuses, and is very similar to the intraocular fluid. Cerebro-spinal fluid seems a specially constituted vehicle by which nutriment is conveyed to the cells and fibres of central nervous system. The optic nerve and the retina, which are, from an anatomical point of view, prolongations forwards of the brain, have their nutriment provided for them in a similar fashion, the cerebro-spinal fluid extending down into the subarachnoid space of the optic nerve, and the intraocular fluid secreted by the ciliary body bathing the inner surface of the parts developed from the secondary optic vesicle. In connection with the optic nerve, as in the brain, there are true 1ymphatic sheaths surrounding its blood vessels.

The conjunctiva possesses true lymphatic vessels which form a small superficial and a larger deep plexus. At the margin of the cornea there is a minute plexus which is probably in communication with the lymphatic system of the cornea. The vessels from the deeper plexus, into which all the others drain, join those of the eyelids. Perineural lymph channels surround the corneal nerves, both in the substantia propria and where they pass forwards through Bowman's membrane to the epithelium.

The lymphatic vessels of the eyelids form two groups, a superficial and a deep, the former drains the skin and the orbicularis muscle and the latter the tarsal plates and conjunctiva. They join up into main trunks, those coming from the upper lid passing down to the pre-auricular lymphatic gland, and those from the lower lid and lacrymal region to the submaxillary lymphatic gland.

There is some uncertainty as to the nature of the $1 \mathrm{ym}$ phatic system of the orbit in man. There are no lymphatic glands in the orbit and no definite lymphatic vessels have been demonstrated. Tenon's capsule has been regarded as a large endothelial lined lymphatic space, but whether it is in communication with the lymphatic channels pro- 
ceeding from the eyeball and the optic nerve has not yet been definitely determined. Doubtless there are perivascular spaces surrounding the orbital blood vessels.

The lymph in the interior of the eye is formed by filtration through the vascular capillaries, and its amount depends on the pressure within them. If the intraocular pressure is higher than that of the blood in the capillaries, they become compressed and emptied, so that the flow of lymph from them is arrested. If the intraocular pressure is decreased they dilate and more lymph filters from them.

When the intraocular pressure becomes markedly subnormal, the lymph which filters from the capillaries, not only distends the spaces which it normally occupies, but is also effused into those occupied by the intra-ocular fluid (see page 284).

When the blood pressure in any of the intra-ocular capillaries becomes increased above the normal intraocular pressure, due to venous obstruction either local or general, what in the healthy eye are only potential spaces (like that of the secondary optic vesicle) may become opened out by an effusion of lymph.

\section{i. Serous Detachment of the Choroid and Ciliary Body.-} In the consecutive pathological examination of a series of 574 excised eyes I53 cases of detachment of the choroid and ciliary body, by fluid yielding a granular coagulum on fixation, were met with. ${ }^{1}$ From an analysis of these cases the following conclusions were arrived at:

I. That serous detachment of the choroid and ciliary body is the natural accompaniment of considerable reduction of the intraocular pressure, and that its occurrence is the rule in every case of sustained reduction of pressure.

2. That the degree of the detachment varies, more or less directly, as the degree of reduction of the intraocular pressure.

${ }^{1}$ A. C. Hudson. Royal Lond. Ophthal. Reports, XIX, I9I4, 303. 
3. That the fluid responsible for the detachment is derived probably from the choroidal blood vessels and not improbably from the veins.

4. That reduction in intraocular pressure is the chief factor in the causation of detachment of the choroid and ciliary body in shrunken eyes.

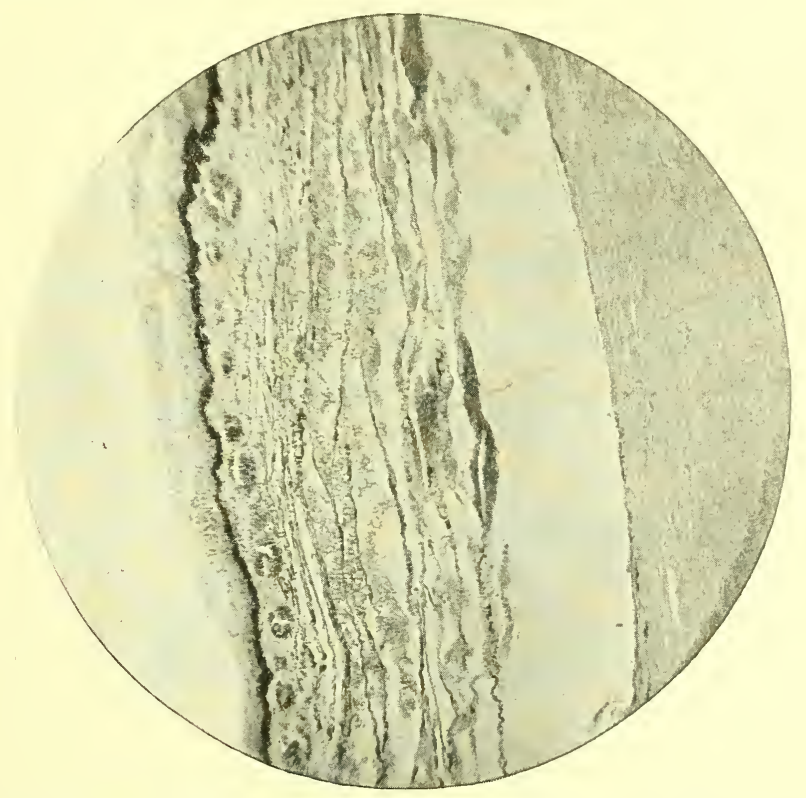

FIG. I46.- Section through the posterior part of the ciliary body showing separation of it from the sclerotic. Part of it may be due to postmortem changes, but the wide separation of the lymph-spaces of the lamina suprachoroidea which are filled granular coagulum is certainly ante-mortem. From photomicrograph taken by E. Collier Green.

Detachments of the uveal tract occur first, and become most marked, in the region of the ciliary body, the part of the eye least accessible to ophthalmoscopic examination (Fig. I46). It is not surprising, therefore, that clinically they frequently escape detection. When looked for systematically it has been estimated that detachment of the choroid and ciliary body is met with in 4 per cent. of cases after cataract extraction, in ro per cent. of cases after iridectomy for glaucoma, and in more than 22 per cent. of cases 
after sclero-iridectomies. As a general rule these postoperative detachments disappear when the normal intraocular tension becomes restored.

ii. Serous Detachment of the Retina.-The cavity of the secondary optic vesicle, between the pigment epithelium of the retina and the rods and cones, is in the normal eye only a potential cavity. As, however, the outer layers of the retina receive their nutriment from the capillary blood vessels of the choroid, lymph must be constantly passing from the latter, through the pigment epithelium, and across the potential cavity to the former.

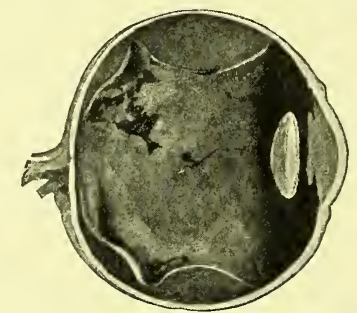

FIG. I 47.-Detached retina due to thrombosis of venæ vorticosæ (?) showing retinal and choroidal hemorrhages. Retina detached by coagulated albuminous exudate. Case recorded in Trans. Ophth. Soc. of the U. K., XXXV, I915, I07.

In some cases of detachment of the retina the potential cavity of the secondary optic vesicle is found to have been opened out by the effusion into it of fluid, which sets into a jelly like coagulum in hardened specimens (Figs. I47 and I48). It seems most probable that such an abnormal accumulation of lymph beneath the retina is due to an increase of the blood-pressure in the capillaries of the choroid above the normal pressure in the vitreous chamber. Associated with sarcoma of the choroid there is very frequently a detachment of the retina, apart from the elevation of it caused by the new growth, the subretinal space being filled by coagulated lymph. In such cases the growth probably causes obstruction to the flow of blood through the choroidal veins and so heightens the pressure in the capillaries. In cases of albuminuric retinitis, bilateral detachment of the retina is sometimes met with, 
the subretinal exudate being of a coagulable nature, in these cases there is a general venous obstruction affecting both choroidal and retinal vessels, and sometimes also a general edema. The detachment of retina met with in cases of orbital cellulitis is most likely due to venous thrombosis of the orbital veins, obstructing the return of blood from the choroid, and increasing the blood pressure in its capillaries.

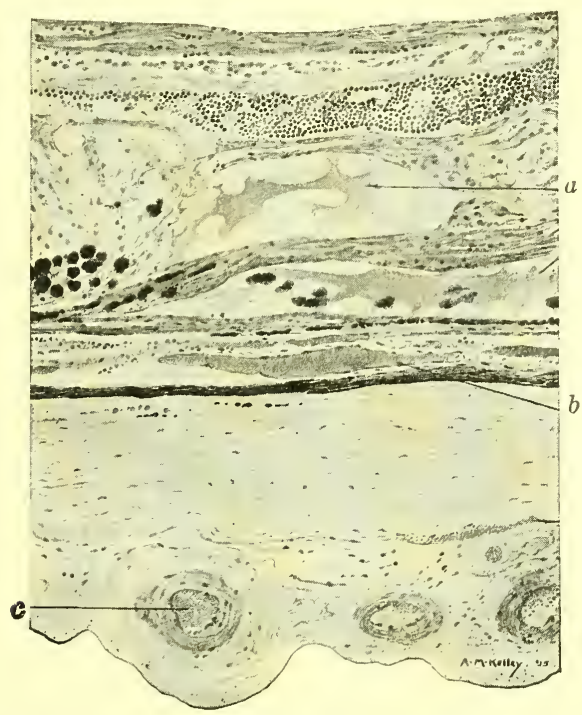

FIG. I48.- Section through the walls of the eyeball shown in Fig. I47. $a$, Albuminous fluid in the inter-retinal space in which are strands of fibrous tissue. $b$, Choroidal hemorrhages. $c$, Sclerosed posterior ciliary vessels.

An alteration of the relative pressure in the vitreous chamber and the blood in the choroidal capillary vessels may be brought about, not only by raised blood pressure in the latter, but also by decrease of the normal pressure in the former. Traumatic conditions which produce rapid decrease of pressure in the vitreous chamber, such as escape of fluid from it through a perforating wound of the sclera, or rupture of the suspensory ligament of the lens allowing displacement forward of the vitreous body through the circumlental space, are often followed by de- 
tachment of the retina. In such cases if the subretinal fluid is of a serous character it is probably a transudation from the choroidal vessels.

In many cases of so called simple detachment of the retina the tension of the eye is minus and the anterior chamber deeper than normal, whether these symptoms precede the onset of the detachment or are sequlæ of it cannot usually

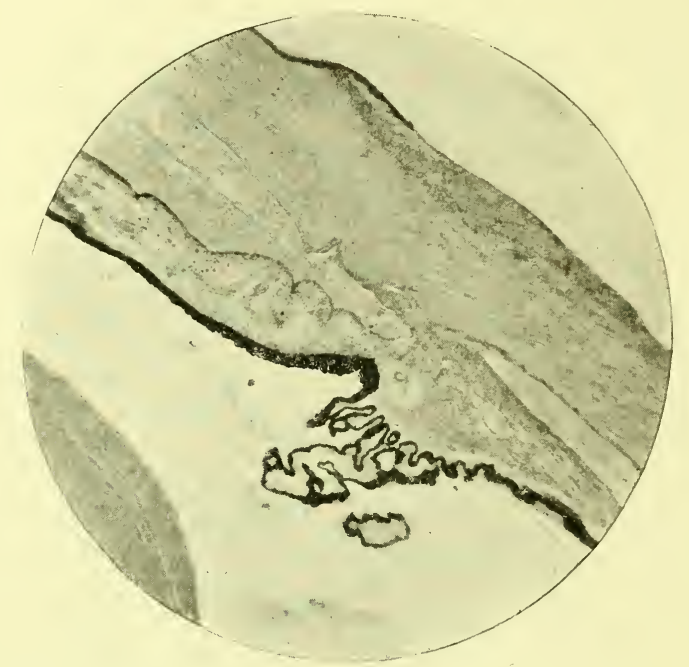

FIG. I49.- Shows coagulated serous effusion into the anterior chamber in an eye with hypotony following a concussion injury. There is no coagulum behind the iris or in the neighbourhood of the ciliary processes. Case recorded in Trans. Ophth. Soc. of the U. K., XXXVII, I9I 7, 28I.

be ascertained. If, however, in such cases the subretinal fluid is coagulable, it is more likely to have been a transudation of lymph from the choroid, than intraocular fluid which has passed through a rupture in the retina from the vitreous chamber.

iii. Serous Exudation from the Iris.-When the aqueous humour is evacuated through a wound in the cornea, the new fluid which is at first formed in the anterior chamber is not like normal aqueous humour, but is coagulable and resembles lymph (Figs. I49, I50). 
In rabbits' eyes in which the intraocular tension has been lowered, ${ }^{1}$ either by paracentesis of the anterior chamber or by letting fluid out of the vitreous chamber through a scleral wound, the anterior chamber becomes filled by fluid, which, fixed in Zenker's mixture, forms a firm clot present-

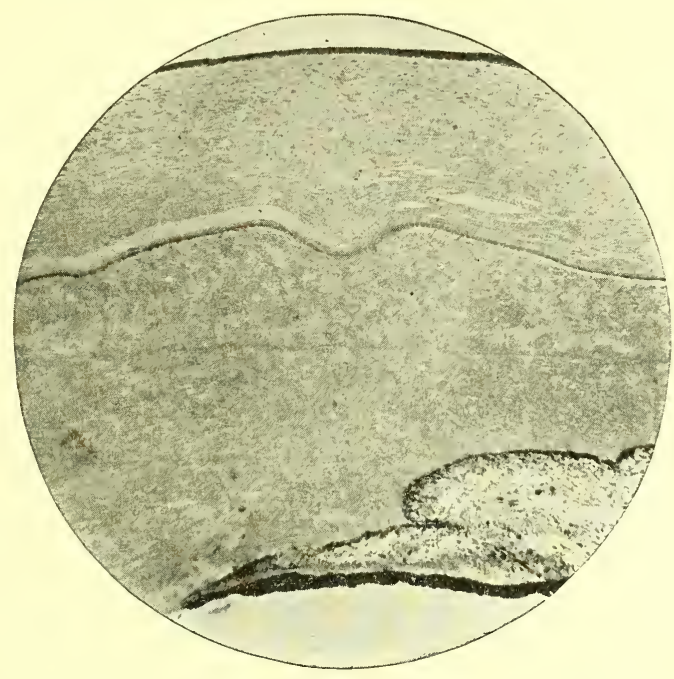

FIG. I50.- Shows the central part of the anterior chamber and the pupillary margin from the same case as depicted in Fig. I49. The coagulum fills the anterior chamber but does not pass backwards through the pupil.

ing microscopically a. granular appearance. A similar clot is found in the peripheral part of the posterior chamber, much smaller in amount when the tension is lowered by escape of fluid from the vitreous than when the anterior chamber has been opened. It seems impossible that this lymph filling the anterior chamber can come from the ciliary body, as there is no continuity between it and that in the posterior chamber, and the amount in the latter is so comparatively small.

Experiments of injecting Indian ink into the eyes of animals has shown, that under normal circumstances the veins of the iris act as excretory channels for the aqueous

${ }^{1}$ Treacher Collins. Trans. Ophth. Soc. of the U. K., XXXVIII, I9I8, 217. 
humour. When, however, by evacuation of the aqueous, or even by escape of fluid from the vitreous chamber, the pressure in the anterior chamber becomes markedly lower than that in the iritic blood vessels a transudation of lymph from them takes place, which by refilling the anterior chamber quickly aids in re-establishing the normal tension of the eye.

Conditions which lead to compression of the root of the iris, such as glaucoma or a new growth, if sufficient to obstruct the flow of blood through the veins without impeding its passage through the arteries, cause an effusion of lymph into the tissue of the iris so that it becomes swollen and edematous. Sometimes the lymph effused collects between the two pigment epithelial layers on the back of the iris giving rise to a cystic formation (see page 233).

iv. Serous Exudation from the Ciliary Body. - Sudden reduction of pressure in the posterior chamber in rabbits' eyes produced by the evacuation of the aqueous humour, causes the blood vessels of the ciliary processes to dilate, and transudation of lymph through their walls to take place. This effused lymph encounters some resistance in passing into the posterior chamber from the epithelial cells lining the ciliary processes. This epithelium becomes raised up in the form of vesicles instead of being permeated by it. ${ }^{1}$ When the vesicles have become greatly distended their inner walls rupture and the coagulable lymph then passes into the posterior chamber.

Detachments of the unpigmented layer of cells of the pars ciliaris retinæ from the pigmented layer, apparently produced in this way, are often met with in diseased conditions in human eyes.

In rabbits' eyes if the fluid from the anterior chamber is allowed to flow away through a canula for from twenty minutes to an hour, the protoplasm of the epithelial cells lining the ciliary body becomes gradually more and more

${ }^{1}$ Ibid. 
disintegrated, ${ }^{1}$ until they are only represented by a few scattered nuclei and scarcely any protoplasm. Together with this disintegrating process in the epithelial cells, a clot forms on the inner surface of the ciliary body, with which it becomes so intimately connected that it cannot be completely removed without injuring that structure.

If, in a rabbit's eye, the intraocular pressure is lowered by allowing fluid to escape from the vitreous humour through a wound in the sclera, the lymph effused comes mostly from the posterior part of the ciliary body into the vitreous chamber, there being only a small amount of exudate from the anterior part into the posterior chamber. These changes tend to confirm the suggestion which has been made, that the aqueous humour is mostly a secretion from the anterior part of the ciliary body, and that it is the posterior part which supplies the fluid which nourishes the vitreous body.

\section{v. Serous Effusion into the Head of the Optic Nerve.-}

Obstruction to the return of venous blood by the central retinal vein, due to pressure on the nerve at its entrance into the globe, may take place from fluid collecting in the nerve sheath, which is first distended on its dorsal surface near the globe. This causes intense edema of the papilla commencing at the upper margin of the disc-a condition which is usually associated with intracranial tension. The state of the disc used to be known as "choked disc," but is now termed papilledema. In this condition, although there is a very marked ophthalmoscopic change, in the early stages there is comparatively little disturbance of vision, which is in striking contrast to what occurs in the interstitial form of neuritis where there is early and rapid loss of sight. (For optic neuritis see page 473.)

vi. Serous Exudation from the Retinal Vessels.-An abnormal amount of exudation of lymph from the retinal vessels occurs, when the pressure in them becomes markedly higher than the pressure in the vitreous chamber. This

${ }^{1}$ E. E. Henderson and Janet Lane-Claypon. Royal Lond. Ophth. Hosp. Reps., XXII, I907, 97. 
may be brought about, either by local or general increase of blood pressure, or by decreased intraocular pressure.

A pathological transudation of lymph from the retinal blood vessels collects mostly in the tissue of the retina and in the optic papilla, so that they become swollen and edematous. Sometimes the fluid in the retina may break through the membrana limitans externa and collect beneath the retina, giving rise to a flat detachment. It may also, when the intraocular pressure is decreased, pass through the membrana limitans interna and collect between it and the hyaloid membrane of the vitreous, producing detachment of the hyaloid, which sometimes ruptures.

Effusions of lymph into the retina in connection with vascular sclerosis and thrombosis of the central vein have already been described on page 246 . In detached retinæ where the nerve elements have atrophied, and only a neuroglial network remains, the spaces in the network are filled with lymph; if, as often happens in such cases, fibrous tissue changes in the optic nerve obstruct the retinal venous circulation, the exuded lymph breaks through the partitions in the network and forms cysts (see page 237).

Cases have been recorded of prolonged hypotony due to traumatism, in which the head of the nerve has been swollen from effusion into it, and showing no change as long as the cases remained under observation.

In a case of long standing cerebellar tumour, with enormously dilated vessels and swollen nerve head, the swelling and engorgement remained I4 years after the removal of the tumour, probably as the result of lymphatic obstruction in the nerve head.

vii. Edema of the Cornea. ${ }^{1-T}$ The epithelium of the cornea is more sensitive than the conjunctiva to external irritants. Drugs affect it freely, thus cocaine readily produces a 'marked edema of the epithelium, so much so as to cause desiccation of the surface. Inflammation in the conjunctiva, as in the early stages of Ophthalmia Neona-

${ }^{1}$ E. Fuchs. Trans. Ophth. Soc. of the U. K., XXII, I902, I5. 
torum, may cause a hazy condition of the cornea from edema due to the presence of toxin.

Vesicular Keratitis is frequently associated with increased intraocular tension, it is also associated with deep inflammatory affections of the cornea and iridocyclitis. The change found on histological examination is a separation of the basal cells of the epithelium from each other by fluid, which causes a desquamation of the superficial cells of the epithelium, so that the surface of the cornea becomes irregular and presents a roughened appearance clinically.

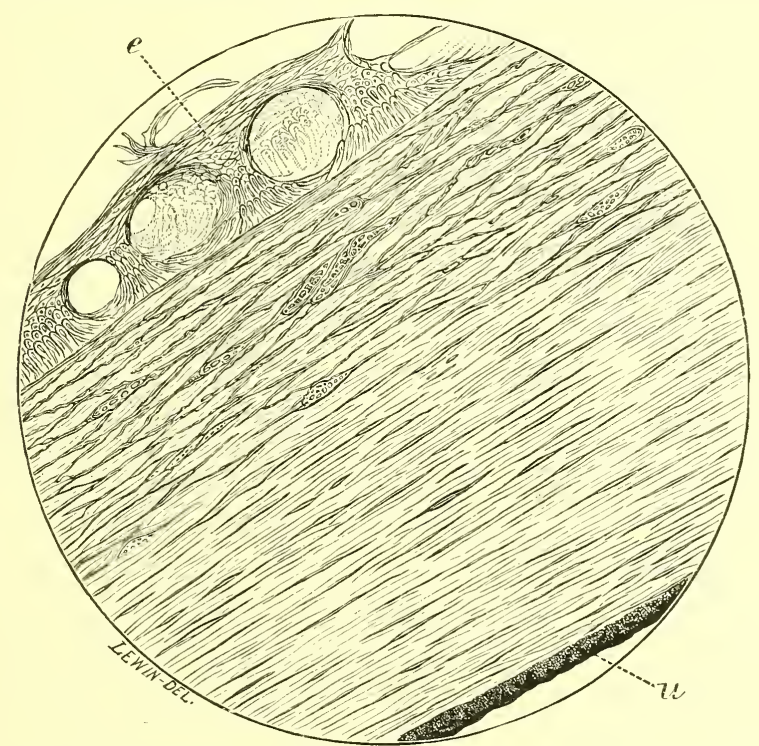

FIG. I5I.-Section through a staphylomatous cornea with increased tension, showing vesicles in the epithelium on its anterior surface. $e$, Epithelium; $u$, uveal pigment adherent to the back of the cornea. Case recorded in R. Lond. Ophth. Hosp. Reps., XIII, I890, 48.

The fluid, as it increases, lifts up the cells from Bowman's membrane, and droplets of it running together form vesicles or bullæ (Fig. I5I). In the early stages the basal cells occasionally show signs of vacuolation.

In cases where vesicular keratitis is associated with plastic cyclitis, the fluid gives rise to a deposit of fibrin between the epithelial cells and Bowman's membrane, into 


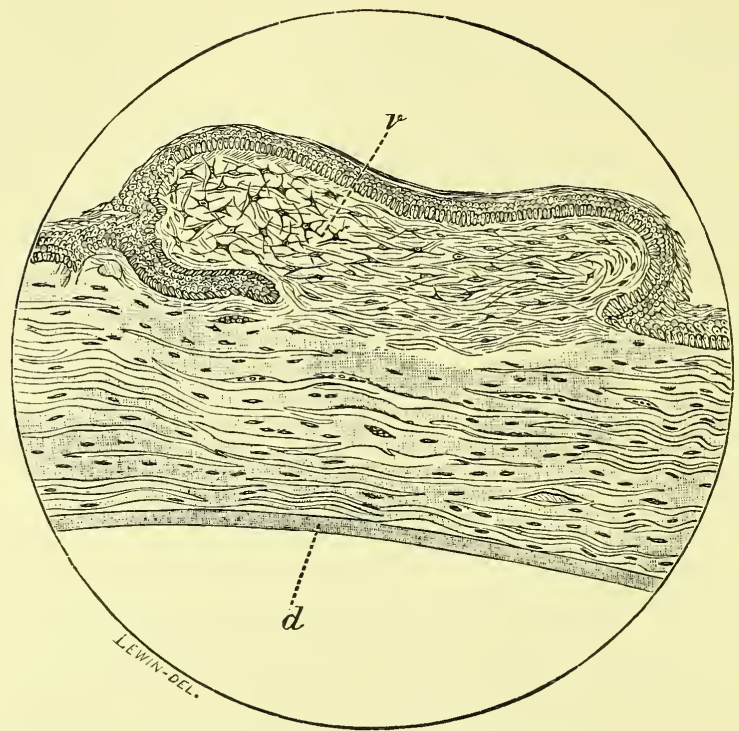

FIG. 152.- Section of the cornea from a case of hereditary syphilitic keratoiritis, showing collection of organising edematous exudate, $v$, beneath the surface epithelium; $d$, Descemet's membrane. Case recorded in R. Lond. Ophth. Hosp. Reps., XIII, I890, 50.

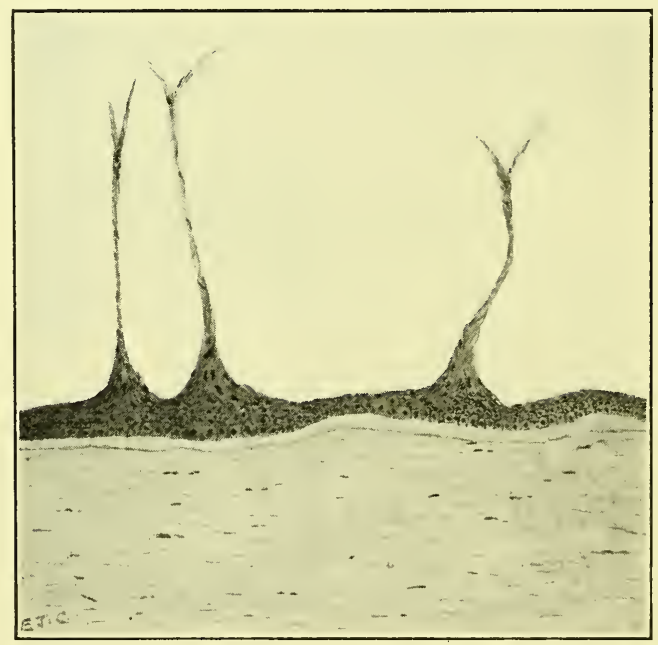

FIG. I53.- Section of the anterior part of the cornea in an eye which had had vesicles form in its surface epithelium, the vesicles have burst and given rise to the formation of filamentary processes. 
which vessels springing from the limbus may extend, causing it to become organised into a thick fibro-vascular membrane (Fig. I $5_{2}$ ).

A recurrent single bulla occasionally occurs at the site of an old wound in the cornea. Its recurrence seems to be due to an imperfect adhesion between the epithelium and the underlying scar tissue. The process of its formation is similar to that already described, the change being limited to the area affected. The vesicles connected with herpes zoster and herpes febrilis present the same histological characters.

Herpes Zoster has been attributed to atrophic changes in the cells of the Gasserian ganglion. It is frequently associated with chickenpox. Vesicles occur on the cornea, and nodules in the sclera and iris giving rise to an intense iridocyclitis and scleritis. ${ }^{1}$ A case of optic neuritis has also been met with in association with this disease.

After rupture of the vesicles in herpes febrilis, if the resulting minute abrasions remain discrete a condition known as superficial keratitis punctata is produced, while if they run together a branch-shaped ulcer is formed, dendritic keratitis. It is apt to recur with every febrile attack such as a cold and may eventually lead to a dense opacity of the cornea.

In either of these conditions the corneal abrasion may become infected with pyogenic organisms and a purulent ulcer be formed.

Occasionally in vesicular keratitis when two vesicles lie in contact with each other and both rupture, the partition of epithelium lying between them remains adherent to the cornea as an epithelial filament (Fig. I53). By the movements of the eye this filament becomes twisted and mucus collects around it. The epithelial cells at its base proliferating cause its elongation into a cord-a condition which is known as filamentary keratitis. ${ }^{2}$

\footnotetext{
${ }^{1}$ M. S. Mayou. Trans. Ophth. Soc. of the U. K., XXXVII, I9I7, I96.

${ }^{2}$ Treacher Collins, Lancet, Feb., I90o.
} 
viii. Serous Effusion into the Conjunctiva.-The conjunctiva, except where it is firmly adherent to the under surface of the eyelids, is a very loosely attached structure, and very liable to edematous swelling on the slightest provocation. Such swelling may be produced by:-

(a) Inflammation, varying considerably with the exciting cause.

(b) Irritation by foreign bodies.

(c) The use of lymphagogs, such as dionin.

(d) Certain animal and vegetable secretions, such as eel's blood and the secretion from the worm ascaris lumbricoides.

(e) Burns from chemicals, electricity or heat.

(f) Light, e.g., associated with snow blindness.

A permanent edema and thickening of the conjunctiva, due to blocking of the lymphatics, may occur in the ocular conjunctiva around the cornea.

A patch of edema of the ocular conjunctiva may occur opposite the site of a stye or suppurating meibomian gland.

Inflammation of the lacrymal gland gives rise to a characteristic triangular patch of edema of the ocular conjunctiva at the outer canthus.

Blocking of a lymphatic of the ocular conjunctiva gives rise to the appearance of a string of clear watery beads, which occasionally can be made to disappear with massage.

ix. Serious effusion into the orbit may be the result of injury or of inflammation. Inflammation in the accessory sinuses, especially the ethmoidal, leads to edema on the inner side of the orbit extending to the eyelids. Whilst inflammation of the superior maxilla or antrum leads to edema along the lower margin of the orbit.

Angio-neurotic edema of the orbital tissues is very rare as compared with the eyelids. In the case of a girl aged I 8 , who had recurrent attacks accompanied by proptosis, complete loss of vision, loss of pupillary reaction and papilledema, as the edema subsided, the field of vision gradu- 
ally returned from the periphery to the centre, full vision being ultimately restored.

x. Serous effusion into the eyelids may occur as the result of (a) Injuries, such as wounds or the cicatrices produced by them, especially at the outer canthus and over the malar bone. (b) Erysipelatous inflammation in the skin, in which case it is frequently bilateral. (c) Angioneurotic edema, which may affect both upper and lower lids, and may be transient or permanent; in the latter case the skin assumes a shiny appearance. (d) Any inflammation in the eye or orbit.

xi. Serous effusion into Tenon's capsule may be acute, but is usually chronic, it leads to a characteristic swelling around the eyeball and ocular conjunctiva, and sometimes to proptosis of the eye without any lateral displacement. The cause is often difficult to discover. It has been found associated with chronic sepsis and with syphilis. Effusion into Tenon's capsule also occurs when there is suppurative inflammation in the eye, but is then usually masked by the other changes.

xii. Serous effusion secondary to general diseases such as renal and heart disease gives rise to the puffiness of the lower lids so characteristic of the disorder.

Myxedema also gives rise to a puffiness of the eyelids, it is characterised by being associated with a very dry skin, sometimes hazyness of the corneal epithelium, and the general symptoms associated with the absence of the thyroid secretion.

Senile atrophy in the sub-epithelial tissues of the skin of the lids, associated with looseness of the sub-epithelial tissue and some blocking of the lymphatics, leads sometimes to edema.

\section{DISTURBANCES IN THE CIRCULATION OF THE INTRA- OCULAR FLUID}

i. Mechanism for the Maintenance of Normal Intraocular Tension.-The intraocular fluid, contained under 
normal conditions in the aqueous and vitreous chambers, is practically of the same consistency. As already stated it differs from lymph in being exceedingly poor in proteins, richer in salts, and containing no lymphocytes. It resembles in its composition, and mode of production, the cerebro-spinal fluid more closely than any other fluid of the body.

There is clinical and experimental evidence to show that the intraocular fluid is not stagnant and contained in a closed cavity, but that it is continuously being secreted and excreted. Experimentally it has been proved by injection of fluorescine into the circulation that it is secreted by the ciliary body. Clinically its circulation is made evident by the way in which lens matter is dissolved and removed from the eye, after a wound of the anterior capsule. The rate at which it is secreted has not yet been satisfactorily determined. Any attempted estimate in which the anterior chamber is opened must be fallacious, because the escape of aqueous by reducing the intraocular tension causes a transudation of lymph, which becomes mixed with any newly formed aqueous.

The intraocular fluid, forms with the cornea, the anterior portion of the dioptric apparatus; it also contributes to the nutrition of the lens and vitreous humour, and may afford some protective influence to the inner layers of the retina.

The intraocular fluid is not merely a transudation from the blood vessels of the ciliary body but a secretion from its lining epithelium. If it were simply a transudation it would be produced not only by the ciliary body but also from the choroid and iris, and in composition it would resemble lymph. If arsenical compounds are injected into the circulation they do not appear in the normal aqueous, but are present in the lymph which is effused into the anterior chamber after the normal aqueous has been evacuated. ${ }^{1}$

${ }^{1}$ H. Neame and J. Webster. Trans. Ophth. Soc. of the U. K., XLIII, I923, 38I. 
The folding of the lining membrane of the ciliary body, produced by the ciliary processes, provides a large epithelial covered secreting surface, overlying a dense plexus of blood vessels. There are also numerous tubular downgrowths of the pigment epithelial layer of a glandular character (Figs, I 54, I 55 , I 56 ). ${ }^{1}$

As the intraocular fluid is a secretion, it can be formed at a higher pressure than the blood in the intraocular veins. If, however, the intraocular tension from any cause reaches a higher pressure than is present in the capillaries they tend to become obliterated, less blood enters the ciliary body, and secretion is diminished or arrested; both of which

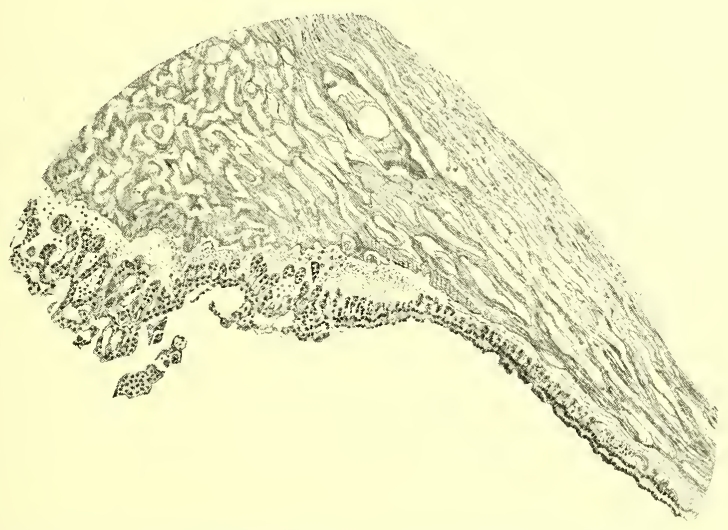

FIG. I54.-Shows a bleached section of the ciliary body of a normal eye. $X$ I20. Numerous cellular processes are seen projecting downward toward the ciliary muscle from the pigment epithelial layer which has been deprived of its pigment.

conditions tend to reduce tension. On the other hand, if, either from a rise in general blood pressure or local dilatation of the vessels supplying the eye, such as is produced by inhibition of the sympathetic nerve, the capillaries of the ciliary body become dilated, increase of secretion takes place, and the intraocular tension tends to rise.

There is as yet no proof that there is any mechanism controlling the secretion of the intraocular fluid beyond the

1 Treacher Collins. Trans. Ophth. Soc. of the U. K., XI, I891, 55. 
vaso-motor mechanism affecting the blood vessels which supply the interior of the eye.

The intraocular fluid, secreted by the posterior part of the ciliary body into the circumlental space, passes backward into the vitreous by diffusion through its hyaloid membrane, and from the anterior part of the ciliary body forward between the iris and lens into the anterior chamber.

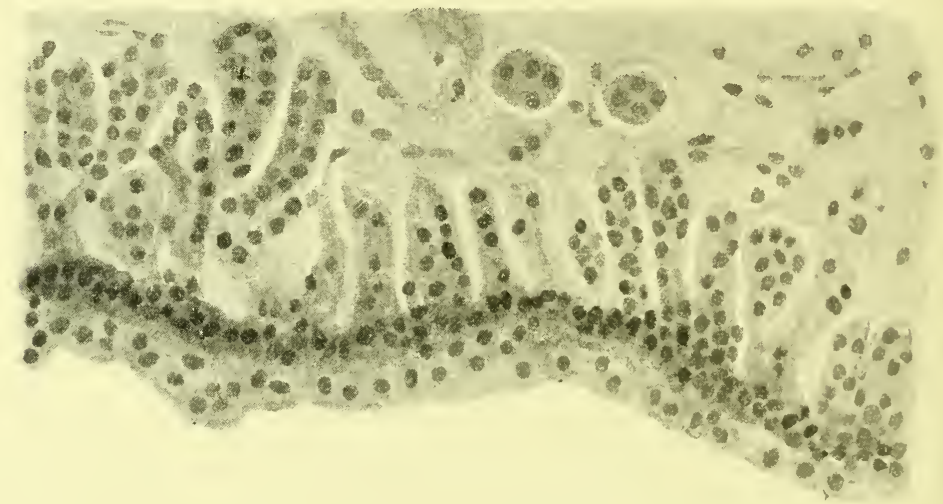

FIG. 155.- Shows the processes of cells which project downward from the pigment epithelial layer depicted in Fig. I54, more highly magnified. $\times 300$.

As yet no instrument has been devised which can demonstrate any difference in pressure in two parts of the eye, such as the vitreous and anterior chambers, but it is obvious that there must be a variation in pressure between the point of entry of the fluid and the point of excretion.

A small amount of the intraocular fluid may be drained away from the vitreous chamber by the lymphatics surrounding the arteria centralis retinæ, the greater part of it, however, after passing forward into the anterior chamber escapes at its angle into the vein known as the canal of Schlemm (Fig. I 57), and from it into the anterior ciliary veins. Particles of Indian ink when injected into the anterior chamber of rabbits' eyes have been found to pass, not only into the canal of Schlemm, but also through the crypts at the base of the iris into its veins, and from them 
into the vortex veins. Under normal conditions, therefore, the veins of the iris also act as channels of excretion.

The particles of pigment or Indian ink are carried into the canal of Schlemm by phagocytes, in their passage through the pectinate ligament, a certain amount of pigment is expressed from the phagocytes and remains behind in its meshes.

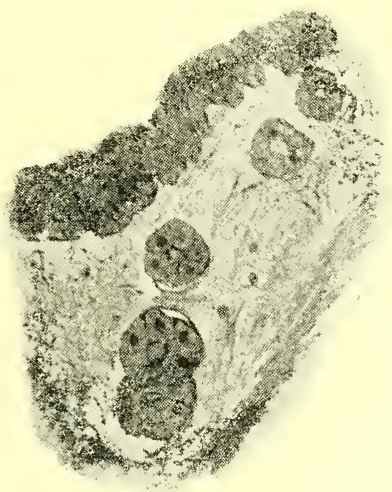

FIG. I56.--Shows a bleached section of the ciliary body in which some of the downgrowths from the pigment epithelial layer have been cut transversely. These transverse sections are seen to have a central lumen, so that the down growths are evidently tubular in character, and presumably glandular.

The canal of Schlemm unlike other veins is always found patent in sections of the eye, and under normal conditions never contains blood. It is held open by its fibrous tissue surroundings, and so kept from collapsing like the central retinal vein on the optic disc, when the intraocular pressure is increased at each systole of the heart.

The escape of fluid into the canal of Schlemm is further aided by the pump-like action of the ciliary muscle, ${ }^{1}$ which on contraction pulls back the scleral spur at the angle of the anterior chamber, so increasing the size of its lumen, and sucking fluid into it from the anterior chamber.

Experimentally it has been shown, in the eyes of various animals and of man, that Indian ink injected into the

${ }^{1}$ Arthur Thomson. The Ophthal'moscope, IX, I9I I, 470. 
vitreous passes forwards into the anterior chamber. As clinical evidence of the passage of fluid from the posterior to the anterior chamber we have the condition of iris bombé, in which the iris becomes ballooned forward by pressure from behind; in some cases with atrophic patches in the iris, the thinned areas can be seen to vary in their degree of

$A$

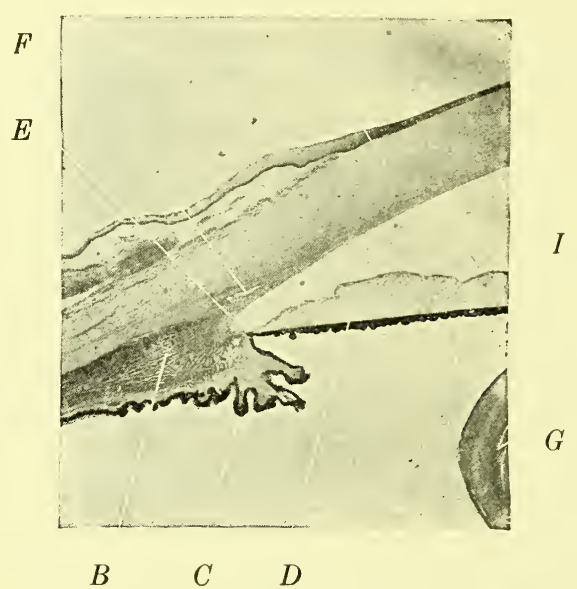

Fig. I 57.- Shows the normal angle of the anterior chamber. $A$, Cornea; $B$, ciliary processes; $C$, iris; $D$, ciliary muscle; $E$, pectinate ligament, to the right of which is the angle of the chamber; $F$, canal of Schlemm; $G$, lens; $I$, anterior chamber.

prominence with alterations in the degree of pressure in the two chambers. Alterations in the depth of the anterior chamber occur clinically with variations in the degree of pressure in the vitreous chamber; in glaucoma when the pressure in the vitreous chamber is increased the anterior chamber becomes shallow; when the pressure in the vitreous chamber becomes decreased, either by escape of fluid from it through an opening in the sclera or from shrinkage of the vitreous humour due to inflammation, the anterior chamber becomes deep.

The passage of fluid from the vitreous into the anterior chamber, though mainly a question of pressure, is probably also influenced by the osmotic properties of the fluid on 
either side of the anterior hyaloid membrane. Alterations in the consistency and structure of the membrane may result in some disturbed transudation.

There is a considerable difference in the apparent hardness of different eyes, and even of the same eye at different times, this normal variation lies within a certain range which can be estimated by the fingers of a skilled observer, or more accurately by means of a tonometer. When the tension is below this normal range it is called hypotony and when above hypertony or glaucoma.

The eyeball is not a closed sphere, its walls are slightly elastic, and its contents varies in volume both normally and when affected by disease. The rise or fall in volume of the intraocular contents depends on the amount of blood present in the vessels of the eye, the amount of lymph effused into its lymphatic spaces, and the balance between the secretion and excretion of the intraocular fluid. As these two latter fluids are both derivatives of the blood it is natural to inquire as to what relations exist between the intraocular pressure and the systemic blood pressure.

All observations show a want of correspondence between the variations in the arterial pressure and the pressure of the intraocular fluid. The use of drugs like nitrite of amyl, which produce marked lowering of the arterial pressure, also slightly lowers the intraocular pressure. Strophanthus, on the other hand, which causes a rise in arterial pressure also produces a lowering of intraocular pressure. The increase of arterial pressure met with in elderly people, and in connection with arteriosclerosis, is not particularly prone to be associated with glaucoma. It is probable that the thickening of the arterial coats in arteriosclerosis may tend to prevent exudation from the capillaries, and so favour a lowering of intraocular tension rather than an increase.

There is much evidence to show that there is some association between glaucoma, and obstructed venous return or high venous pressure. The pulsation in the reti- 
nal veins observed on the optic disc, is due to a slight increase in intraocular pressure during the systole of the heart, which temporarily arrests the flow of blood through them. From this we must infer that the intraocular pressure is slightly lower than the pressure in a vein, of the calibre of a retinal vein on the optic disc, during the diastole of the heart, and slightly higher during the systole. The vein, termed the canal of Schlemm, has a larger calibre than the retinal veins, and being always held open by its surrounding tissue, does not collapse like them when the intraocular pressure is increased. Intraocular fluid is, therefore, able to filter away through it so long as the pressure in the eye is higher than that in the veins which lead away from the eye. Should the pressure in veins outside the eye become greater than the intraocular pressure the escape of fluid from the eye is obstructed, and a tendency to the development of glaucoma is established.

Glaucoma is a symptom not a disease. When increased tension of the eye is the first symptom which manifests itself the condition is called primary glaucoma. When increased tension of the eye comes on in the course of some obvious disease the glaucoma is said to be secondary.

ii. The Causes of Hypertony or Increased Intraocular Tension.-The commonest cause of increased intraocular tension is the obstruction to the entrance of fluid into the canal of Schlemm at the angle of the anterior chamber. This may arise (a) from blocking of the angle of the chamber by the root of the iris being applied against the periphery of the cornea; (b) structural alterations in the channels of filtration, congenital or acquired; $(c)$ alteration in the consistency of the aqueous. (More than one of these factors may in some cases contribute to the production of glaucoma.)

(a) The Blocking of the Angle of the Chamber by the Root of the Iris.-Histologically the commonest change to find in glaucoma is occlusion of the angle of the chamber by the root of the iris (Fig. I 58 ). 
Shallowing of the anterior chamber frequently precedes the onset of primary glaucoma, due to the enlargement of the lens that takes place with advancing years, or to the swelling of it which occurs in the early stages of senile cataract. A shallow anterior chamber has necessarily a narrowed angle, and one which would easily become occluded by slight

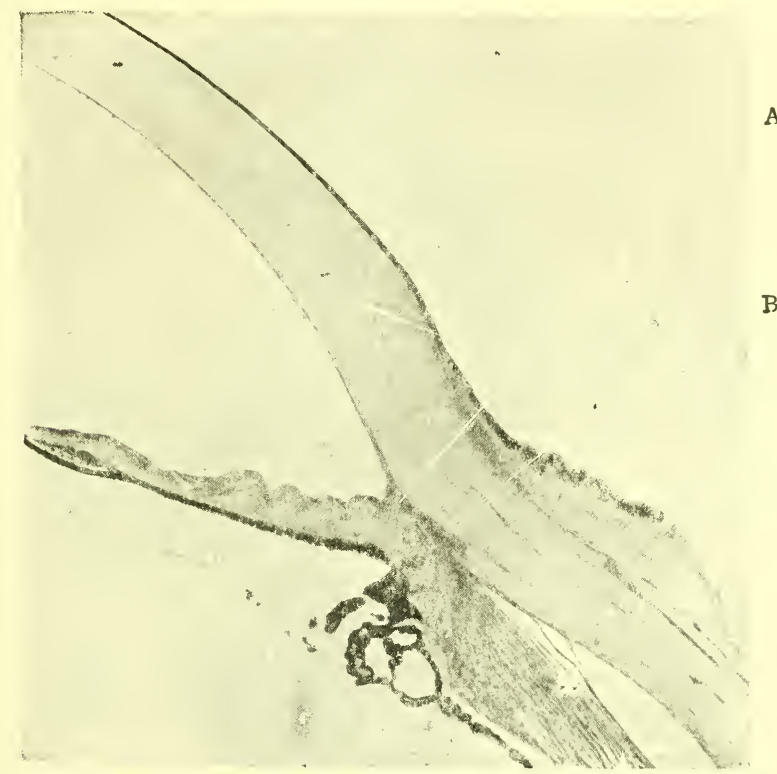

FIG. 158.- Shows the angle of the anterior chamber from a case of recent glaucoma, it is occluded by the base of the iris, $A$, which is adherent to the posterior surface of the cornea. $B$ points to the canal of Schlemm.

pressure on the root of the iris from behind. In primary glaucoma such pressure is brought about by congestion of the ciliary processes producing a displacement forwards and inwards of their apices.

At first, when the root of the iris is only just in apposition with the back of the cornea, some filtration from the anterior chamber may still take place through the veins of the iris. Later, when the iris becomes firmly adherent to the back of the cornea, as the result of a slight plastic exudation from its surface, the whole periphery of the anterior cham- 
ber becomes obliterated. Firm compression of the iris tissue against the back of the cornea completely occludes the spaces of Fontana preventing any filtration into them, and at the same time obstructs the vascular circulation through the tissue of the iris. The effect of cutting off its blood supply is shown by the atrophy which ensues.

$B$

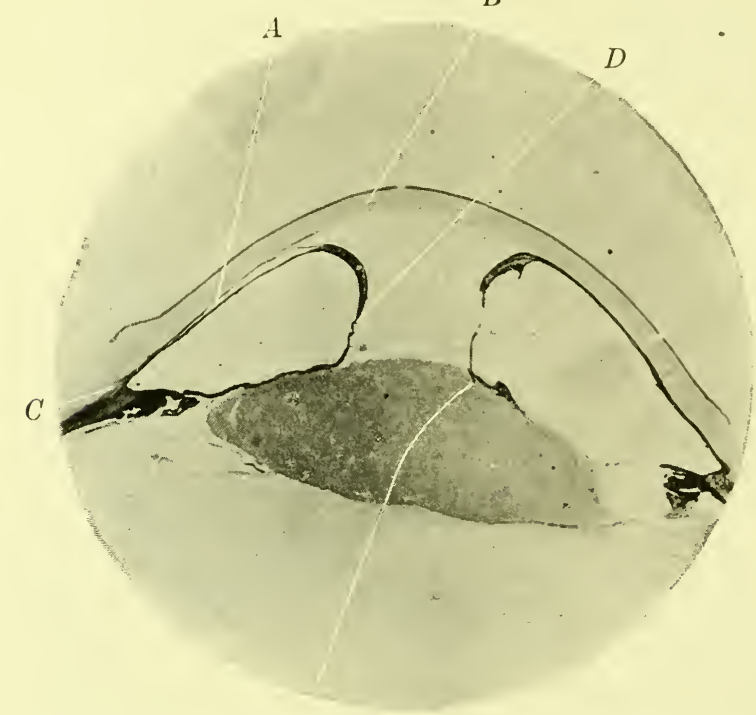

FIG. I59.- Section through the anterior part of an eye with a condition of iris bombé, which resulted in secondary glaucoma. $A$, The iris closely applied to the back of the cornea, $B$, occluding the angle of the anterior chamber, $C$. The pupillary margin of the iris, $D$, together with some of the pigment from the posterior surface of the iris, are united by inflammatory exudate to the anterior capsule of the lens.

In glaucoma secondary to sarcoma of the choroid the increase of tension is often produced, not by any direct pressure on the lens by the growth, but by the increase in the contents of the globe behind the lens forcing it and the iris forward, so leading to a closure of the angle of the anterior chamber. A similar condition results from hemorrhage or serous exudate beneath a detached retina.

In the causation of the glaucoma which follows thrombosis of the central retinal vein there are probably several 


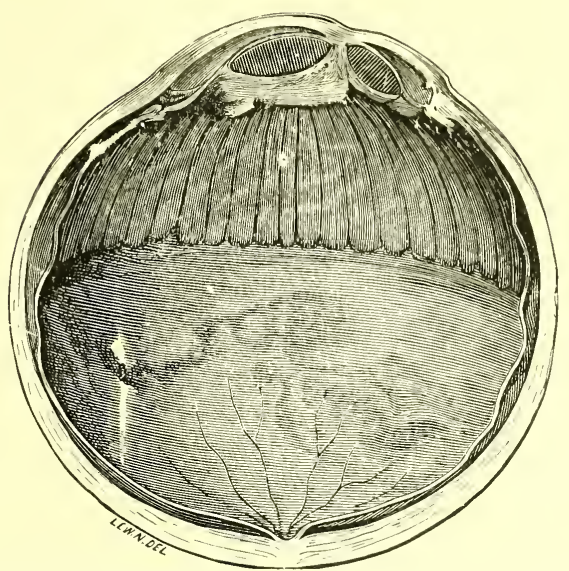

FIG. I60.-Shows the lateral half of an eye, the cornea of which had been wounded with a chisel. The whole of the iris and large part of the lens escaped through the wound. The lens capsule, with some remains of lens substance in it, is adherent to the cicatrix in the cornea. The tension of the eye at the time of its removal, I 2 years after the injury was +2 . Specimen in the R. Lond. Ophth. Hosp. Museum.

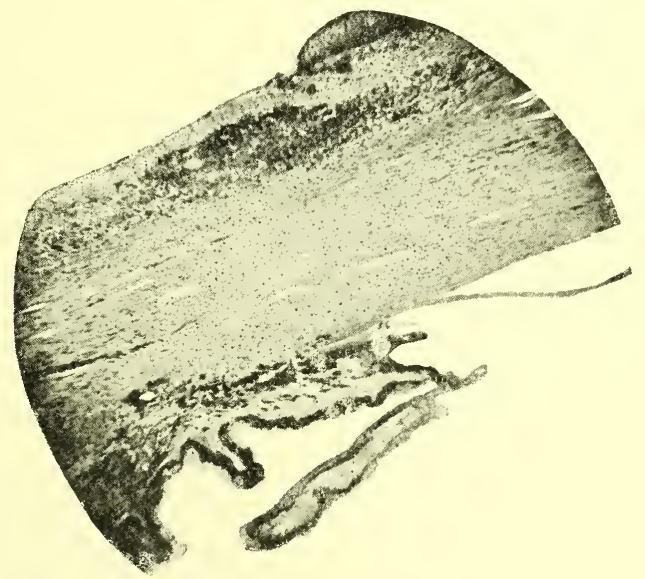

FIG. I6I.-Shows a section through the angle of the anterior chamber of the eye depicted in Fig. I6o. The iris has torn away at its root from the ciliary body. The most anterior of the ciliary processes is drawn forward by the adherent lens capsule into contact with the back of the cornea in the filtration area, thus blocking the exit of fluid through it, and accounting for the onset of glaucoma. 
factors. It is not necessarily an accompaniment of that affection. Vascular sclerosis is usually present and a sclerosis of the channels of filtration may have some influence in its production. The chief factor, however, is probably the exudate of lymph into the vitreous chamber from the retinal vessels, due to the obstructed circulation in the

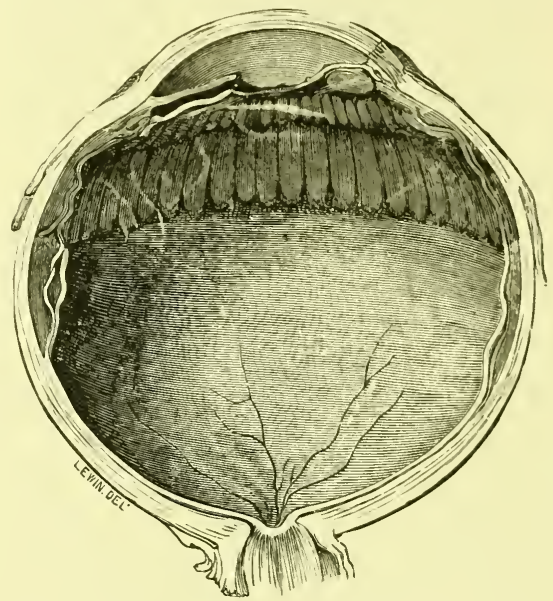

F1G. I62.- The latera1 half of an eye which has had a cataract extracted from it with iridectomy one year and eight months previous to its removal. A discission of the capsule was performed a year later. The sight became destroyed as the result of secondary glaucoma. The lens capsule is shown adherent to the extraction cicatrix at the sclero-corneal margin. Its advance in position has dragged forward the iris and occluded the angle of the anterior chamber. There is a large hole in the centre of the capsule as the result of the discussion operation. From the Museum R. Lond. Ophth. Hosp.

veins. An increase of albumen in the vitreous would cause more, of the less albuminous secretion of the ciliary body, to diffuse backwards through the anterior hyaloid membrane and so cause the vitreous to enlarge. Enlargement of the vitreous would push forward the lens, shallow the anterior chamber, and approximate the root of the iris to the cornea. Occlusion of the angle of the chamber is not found in every case, the angle may be widely open and filled with an albuminous coagulum, which no doubt accounts for the increase of tension in such cases..

${ }^{1}$ G. Coates. R. Lond. Ophth. Hosp. Reports, XVI, 1904-6, 62-5I6. Tatsuji Inouye. R. Lond. Ophth. Hosp. Reports, XVIII, 19I0, 24. 
Alteration in the pressure of the aqueous in the anterior and posterior chamber may arise in cases of occluded and secluded pupils (secondary glaucoma). The aqueous, secreted by the ciliary body, then collects behind the iris and being unable to pass through the pupil, balloons forward

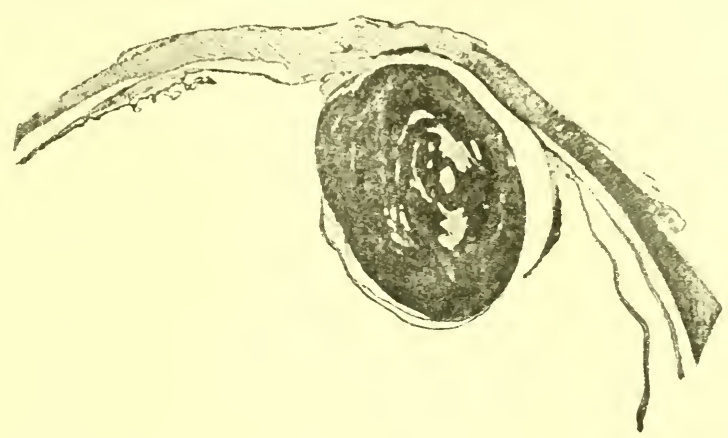

FIG. I63.- snows a section through the front half of an eye in which the sight had been destroyed by glaucoma secondary to dislocation of the lens. The angie of the anterior chamber is markedly narrowed in its entire circumference. The lens presses forward the iris and lies with its long axis antero-posteriorly. Case recorded in R. Lond. Ophth. Hosp. Reps., XIII, I890, 58.

the iris against the back of the cornea, so that the angle of the anterior chamber becomes blocked by its root, and a rise of intraocular tension ensues (Fig. I59).

Blocking of the angle of the anterior chamber by the root of the iris may also result from forward displacement of the iris produced by an adhesion of it to the cornea after a perforating wound or ulcer; by adhesion of the lens capsule or vitreous to the cornea, as occurs sometimes after extraction of cataract, ${ }^{1}$ (Fig. I62) or by pressure on the iris of a displaced lens (Fig. I63).

A congenital imperfect separation of the iris from the back of the cornea has been found in cases of congenital glaucoma or buphthalmos (see page 86, Fig. 54).

(b) Structural alterations in the channels of filtration may be congenital or acquired. A congenital absence of the canal of Schlemm is one of the causes to which congenital glaucoma or buphthalmos has been attributed. Congenital

${ }^{1}$ Treacher Collins. Trans. Ophth. Soc. of the U. K., X, I890, I08. 
adhesions at the angle of the anterior chamber as the result of remains of the pupillary membrane are particularly associated with buphthalmia, and with glaucoma in young people where the globe does not materially enlarge.

Sclerosis of the pectinate ligament offers resistance to filtration into the region of the absorbing vessels. ${ }^{1}$

(c) The aqueous humour may be so altered as to contain a large amount of albumen or more cellular constituents than normal. The amount of albumen is increased in intraocular inflammation, hemorrhage, or serous exudates due to the obstruction of the venous return. Its exit from the eye is then very slow, probably owing to the difficulty the fluid finds in passing through the walls of the venous radicals. Glaucoma from this cause, without blocking of the angle by the root of the iris, may occur. The accumulation of the fluid and increase of pressure in the anterior chamber, tends to push backward the iris and lens, making it abnormally deep. At the same time, the vitreous becomes compressed and some fluid is squeezed out of it. The increased tension associated with serous cyclitis, thrombosis of vena vorticosæ and following thrombosis of the central retinal vein, where the angle is found widely open, may be thus explained (see page 304).

The alteration in the aqueous may depend upon it containing cellular elements. The angle of the chamber may be blocked as the result of the exudation of leukocytes following cyclitis. The cells of a new growth may multiply in the meshes of the pectinate ligament and cause occlusion of the angle.

Occasionally epithelium carried through, or spreading down, a wound in the cornea may grow in and line the anterior chamber. When the angle becomes covered by these cells the tension of the eye is increased.

In traumatic cataract the aqueous becomes highly albuminous, owing to the globulin of the lens becoming dis-

${ }^{1}$ T. Henderson. Trans. Ophth. Soc. of the U. K., XXVIII, I908, 47. 
solved in the aqueous; fragments of undissolved lens matter may also be entangled in the mesh of the pectinate ligament.

In primary glaucoma there are many predisposing factors which help to bring about some of the changes above described. These may be divided into physiological and pathological conditions. Primary glaucoma is most common between the ages of sixty and seventy, and is rather more common in females than males. It rarely occurs before the age of thirty, and is then usually due to some congenital defect, such as is described under buphthalmia. The association between old age and glaucoma is probably due to general vascular sclerosis when it causes a rise in venous blood pressure, or localised sclerosis producing changes in the ligamentum pectinatum and the canal of Schlemm.

The size of the lens, like the tendency to glaucoma, increases with age, and it is held by some authorities that diminution of the circumlental space obstructs the passage of fluid through it, so that the lens becomes pushed forward and the angle of the chamber occluded. ${ }^{1}$ An increase in the antero-posterior diameter of the lens shallows the anterior chamber. An anterior chamber thus rendered shallow may be looked upon as one of the accompaniments of old age, and as a predisposing cause of glaucoma.

Small eyes, especially those with a small cornea, are predisposed to glaucoma to such an extent that some authorities assert that an eye with a cornea that measures less than ro $\mathrm{mm}$. will surely become affected. The fact that nearly all patients suffering from acute primary glaucoma are hypermetropic may be thus accounted for. In these eyes there is, moreover, generally hypertrophy of the ciliary muscle as a result of accommodative effort, which further favours blockage of the angle of the anterior chamber. Acute primary glaucoma in cases of high myopia is exceedingly uncommon.

1 Priestly Smith. "Glaucoma," I89I. 
Occasionally glaucoma shows a marked hereditary tendency; it is probable that the tendency is to a congenitally small eye rather than a glaucomatous one. For the same reason some races seem more affected than others, Jews, Egyptians, and Brazilian negroes being frequently the subjects of the disease.

The exciting causes of primary glaucoma are divided into two groups: namely (I) those which produce inequality in the pressure in the aqueous and vitreous chambers, and (2) those which tend to block directly the angle of the anterior chamber.

I. The exciting causes which produce alteration in the venous blood pressure and so variation in the intraocular tension are the emotions; thus grief, anxiety and worry play an important part. Females being emotional are more frequently affected. The disease commonly occurs in widows. It is possible that the alteration of the venous blood pressure connected with the menopause may sometimes be an exciting cause. The shock of an operation on one eye may bring on an attack in the other. Congestion of the eye as the result of injury or venous obstruction in febrile diseases may sometimes be a determining factor.

2. The exciting cause tending to block the angle of the anterior chamber is dilatation of the pupil, owing to the iris being retracted into the angle of the anterior chamber. This may be produced by the indiscreet use of mydriatics or by bandaging the other eye after operation.

iii. The Effects of Hypertony on the Various Structures of the Eye and Their Respective Functions.-The maintenance of the normal amount of intraocular tension is most essential, for the regular performance of the functions of the different structures composing the eyeball. The changes which are produced in an eye as the result of a disturbance in the intraocular tension are as follows:

Sclerotic and Conjunctiva.-A sudden onset of increased tension so disturbs the circulation of the blood in the eye 
as to cause a general congestion of the ciliary vessels in the sclerotic, and often also those of the conjunctiva. In the most acute cases this congestion is accompanied by edema of the conjunctiva (chemosis), and sometimes of the eyelids. The vessels being mostly engorged with venous blood, the injection has a characteristic dusky hue. The main exit of blood from the uveal tract is by the venæ vorticosæ; the channels in the sclerotic through which these pass run very obliquely, and when the sclerotic is stretched, as it is in glaucoma, they easily become closed and thrombosis sometimes takes place. The result of such obstruction is to cause considerable enlargement of the anterior ciliary veins, which normally give exit to only a small portion of the venous blood from the ciliary body, and which perforate the sclerotic more at a right angle than the venæ vorticosæ.

When the onset of increased tension comes on gradually, instead of suddenly, an adaptation of the intraocular blood circulation to the altered conditions is rendered possible, and the violent disturbance resulting in congestion and edema does not take place. Indeed, some cases of primary glaucoma develop so slowly that scarcely any alteration in the state of the ciliary blood-vessels is to be observed, or at most slight enlargement of the anterior perforating vessels.

The effects of increased tension on the sclerotic vary very much with the age of the patient. In early life the sclerotic is an elastic structure; as life advances, it becomes tougher and less expansile. Consequently, if increase of tension is met with in infancy or early childhood, the sclerotic will give and the whole globe become enlarged. After adolescence, the sclerotic being hard and, unyielding, little alteration in the shape of the globe is met with as the result of glaucoma; usually there is slight distention in the spaces between the recti muscles, so that the globe becomes somewhat square in shape. 
Should there have been, previously to the onset of the glaucoma, some weakening or thinning of the walls of the globe, as from a patch of choroiditis, then, when the tension is increased, that spot is likely to give and to become staphylomatous.

Cornea.-As the result of increase of tension, some interference may occur in the circulation of the lymph in the cornea. This is especially liable to take place where the onset of tension is sudden. Its effect is to cause edema of the anterior layers; the spaces between the anterior lamellæ of fibrous tissue are found enlarged and filled with albuminous fluid. Spaces are also met with between the surface epithelium and the anterior limiting membrane, between the epithelial cells themselves, and in the channels in the anterior limiting membrane through which the nerve fibres pass to the epithelium. Clinically, this edema gives rise in the later stages of the disease to a haze of the cornea, and in cases where it has been present for a long time small vesicles form on the surface. There is another form of corneal haze met with in glaucoma which comes on rapidly when the tension is increased, disappearing at once when it is decreased, and which cannot therefore be attributed to edema. It is probably due to an increase in the double refractive index of the corneal fibrillæ.

Anesthesia of the cornea is a common accompaniment of glaucoma, and has been attributed to compression of the nerve fibres going to the epithelium by the fluid in the lymph spaces around them, as they pass forward through channels in the anterior limiting membrane. It is more likely due to compression of the long ciliary nerve, from which the corneal nerves are derived, against the hard, unyielding sclerotic, as they pass forward on the outer surface of the choroid.

The haziness of the cornea when present, apart from other causes, tends to make vision misty. It is also the cause of another very characteristic symptom of glaucoma, viz., the appearance in the dark of halos of rainbow 
colours around lights, the red colour always being the outermost.

A precisely similar appearance of halos of rainbow colours around lights is produced by dropping a solution of the alkaloid erythrophleine, obtained from an African arrow poison, into the eye. ${ }^{1}$ This drug causes also a slight steami-

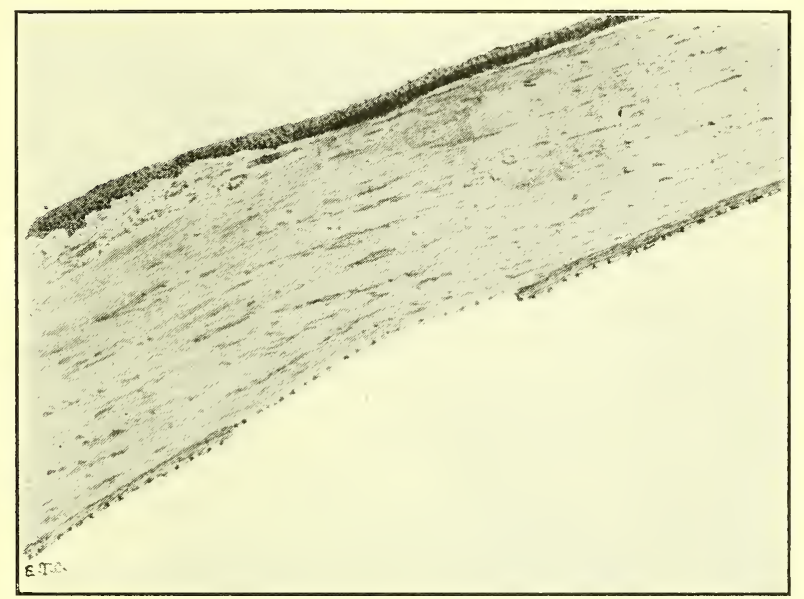

FIG. I64.- Section through the cornea near the periphery in a case of congenital glaucoma or buphthalmia. It shows a rupture which has taken place in Descemet's membrane.

ness of the surface of the cornea, slight anesthesia, and some blurring of vision, but does not increase the tension. Some specimens of it cause dilatation of the pupil and others contraction; with both the halos are seen. The effect can be produced in the eye of a patient who has undergone extraction of cataract. It is evident therefore that the halos are the result of the slight haze in the cornea, and not the effect of the dilatation of the pupil, change in the lens or pressure on the retina.

In primary glaucoma, which occurs late in life, no appreciable alteration in the shape or size of the cornea occurs. When, however, increase of tension is met with in infancy or early life, the cornea, like the sclerotic, being still very

${ }_{1}^{1}$ Treacher Collins. Ophth. Review, IX, I890, I96. 
elastic, enlarges and becomes globular in shape (buphthalmia). The stretching of the cornea thus produced frequently results in the formation of fissures in Descemet's membrane, ${ }^{3}$ which can be seen clinically as fine lines, sometimes branching dichotomously. Microscopically at the seat of the fissures a gap is found in the posterior elastic lamina but the endothelium on its surface is nearly always intact (Fig. I64). It is probable that when the rupture occurs the endothelium also gives way, but that proliferation of the cells soon fills up any opening in it. In some cases there has been a slight cloudy opacity of the cornea in the neighbourhood of the fissures, which is accounted for by deficiencies in the endothelium allowing of filtration of the aqueous humour into the substantia propria.

Anterior Chamber.-The condition of the anterior chamber in glaucoma varies with the position at which the primary obstruction in the circulation of the intraocular fluid takes place. In primary glaucoma it is shallow; this is due to an increase of tension occurring first in the vitreous chamber, the lens with its suspensory ligament being forced forward. A continued shallowing of the anterior chamber, and pressure of the ciliary processes against the root of the iris, results in adhesion of the latter to the back of the cornea and a narrowing of the angle of the chamber. This may be seen by examination either by contact illumination, direct ophthalmoscopy, or by a corneal microscope with an eye adhesion glass. The latter is the most serviceable method as by it the extent of the adhesion can be actually measured in millimeters.

In some cases of secondary glaucoma, and in cases of congenital glaucoma, the primary obstruction to the circulation of the intraocular fluid is at the angle of the anterior chamber, where it gains exit from the eye; the anterior chamber then becomes deepened.

Iris.-Pressure of the root of the iris against the back of the cornea leads to compression of both its blood-vessels

${ }^{1}$ G. Coats. Trans. Ophth. Soc. of the U. K., XXVII, 1907, 48. 
and nerves. If the increased tension is sudden in onset, the compression at first causes edema and venous engorgement, which alters the colour of the iris. Later on its vessels become empty and its stroma atrophies and shrinks.

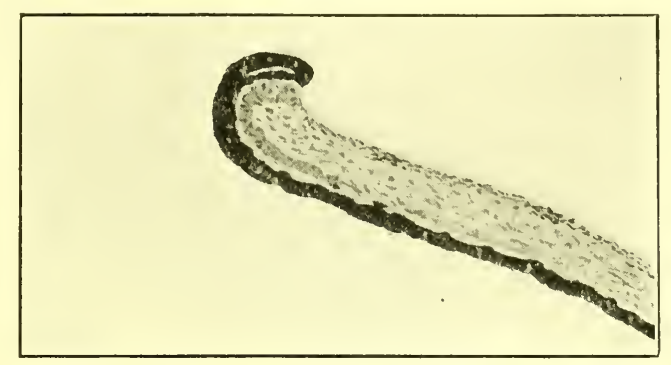

FIG. I65.-Ectropion of the uveal pigment at the pupillary margin of the iris. The pigment epithelium on the posterior surface of the iris is not involved in the atrophy. It normally ends at the pupil-

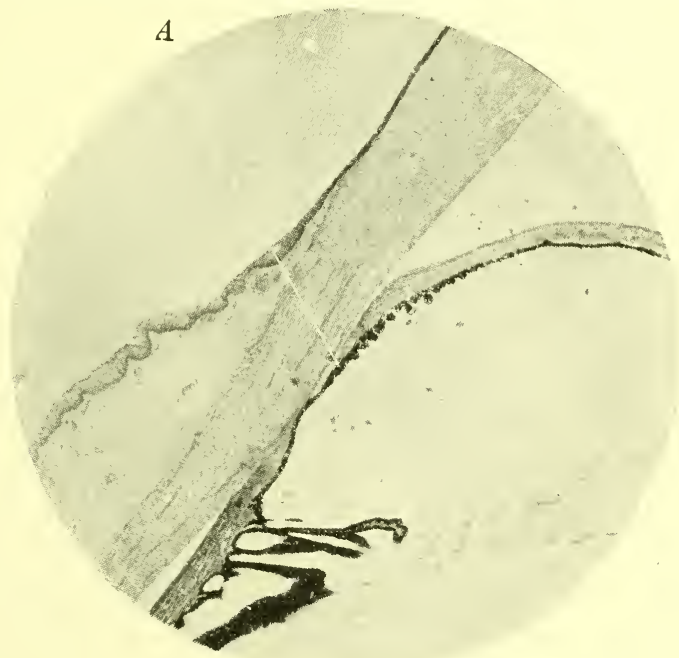

FIG. I66.--Shows the angle of the anterior chamber in a case of glaucoma of long standing. The root of iris $A$ is firmly adherent to the cornea and much atrophied.

lary margin; but in cases of glaucoma of long standing, by the shrinking of the stroma, the unatrophied pigment epithelium becomes drawn round on to the anterior surface, a 
condition known as "ectropion of the pigment epithelium" (Fig. I65). Clinically it is seen as a dark pigmented area on the surface of the iris at the pupillary margin, usually extending more in one direction than another. It is most marked where the iris has become most atrophied and where the dilatation of the pupil is widest.

Fine granules from the pigment epithelium often pass into the anterior chamber, and become deposited on the surface of the iris, giving rise to a fine peppery-like deposit which can be best seen with a corneal microscope, it is known as Koeppe's sign. It, however, is not pathonomonic of glaucoma, occurring also in inflammatory conditions of the uvea.

The pressure of the ciliary nerves against the sclerotic in acute cases of glaucoma paralyses the iritic muscles, so that the pupil becomes inactive and semi-dilated. If the tension is relieved before atrophy has set in, its activity returns. In long standing cases of glaucoma, permanent dilatation of the pupil may be brought about, through atrophy of the sphincter muscle and shrinking of the stroma. The amount of dilatation may not be equal in all directions, so that the pupil becomes oval or irregularly circular, and is displaced away from the centre.

When the cornea and root of the iris have been in apposition for only a short while, their separation is easily effected. After a time, however, plastic exudation takes place so that they become adherent, and in long standing cases of glaucoma most intimately adherent to one another (Fig. I66).

In chronic cases of glaucoma, when the onset of tension is gradual, and there has been time for compensatory changes to take place in vessels and nerves, the dilatation of the pupil and atrophy of the iris may be absent.

Ciliary Body.-Increase of tension early causes disturbance in the accommodative action of the ciliary muscle, due probably to compression of the ciliary nerves against the sclerotic. It manifests itself by the apparent rapid advance 
of presbyopia, the patient requiring stronger and stronger glasses for near work. In the early stages of primary glaucoma, more especially in acute cases, the ciliary processes are swollen and edematous, the veins are engorged, and the processes are pressed forward against the root of the iris. After increase of tension has been present for some time, the ciliary muscle and processes become atrophied and shrink, so that in cases of glaucoma of long standing the latter are no longer in contact with the back of the iris, and a considerable interval is left between them and the margin of the lens. The ciliary body receives an extensive nerve supply, from both the long and short ciliary nerves, which perforate the sclerotic posteriorly and pass forward in the lamina suprachoroidea, until they break up into a network of fine branches, which is known as the ciliary plexus. It is the sudden onset of pressure on this plexus against the unyielding sclerotic which is the cause of the excessive pain of acute glaucoma-pain which is not confined to the eye; but referred also to other parts supplied by the fifth nerve, especially those receiving branches from its first division. Reflex disturbances, such as vomiting, may also be set up.

When increased tension comes on gradually and is not very intense, the nerves, like the blood-vessels, have the power of adapting themselves to the changed conditions, and in the majority of cases of chronic glaucoma no pain is experienced by the patient.

Choroid.- The effect of increased tension on the choroid in acute cases of glaucoma, as on the other portions of the uveal tract, the iris and ciliary body, is first to produce a condition of venous congestion and edema; later on, emptying of its vessels and atrophy. In chronic glaucoma, on the other hand, no sudden disturbance of the circulation is set up, but the compression of the choroid against the sclerotic tends to empty the blood out of the capillaries. The fundus ophthalmoscopically in such cases loses its uniform red hue, and presents a tessellated appearance, due to exposure of the network of larger vessels in its outer layers. 
Atrophy of the choroid, the result of increased tension, is most marked at the parts where it has the firmest attachments to the structures external to it, viz., around the optic disc and at the seats of exit of the vortex veins. When increase of tension has existed for some time, the optic disc usually becomes encircled by a yellowish-white ring, which is due to the atrophied choroid allowing the sclerotic to be exposed to view.

Lens.-The displacement forward of the lens in primary glaucoma tends, if the eye is emmetropic, to make it myopic, as also does expansion of the globe in the antero-posterior axis. A drag on the suspensory ligament from displacement forward of the lens or expansion of the globe in the ciliary region would, on the other hand, lessen its refractive power and tend to make an emmetropic eye hypermetropic.

In glaucoma in the adult hardly any expansion of the globe takes place, but it is stated that during attacks of glaucoma the refraction is usually increased.

In glaucoma in early life, where considerable enlargement of the globe in all its meridians is met with, the lengthening of the globe is compensated for in part by the flattening of the lens, and the amount of myopia is not as much as might otherwise have been expected.

In glaucoma of long-standing the nutrition of the lens sometimes suffers, and it becomes cataractous. The opacity of the lens occasioned by glaucoma usually presents a bluish metallic lustre.

Optic Nerve and Retina.-Increase of tension in the eye constricts the vascular supply of the optic papilla and retina, resulting in pallor and atrophy of the former. The atrophy of the nerve fibres exposes the lamina cribrosa which can be seen as a white area with grey markings, the latter being the openings for the nerve fibres.

The foramen in the sclera and choroid, through which the nerve passes, and across which the lamina cribrosa extends, is a weak spot in the walls of the globe. When the intraoc- 
ular pressure becomes increased it tends to give and become stretched.

The cupping of the optic disc which occurs in glaucoma is, therefore, due to two things, atrophy of the optic papilla and stretching of the lamina cribrosa. The amount of the cupping varies considerably not only in different cases, or in the two eyes of the same case, but in different parts of the

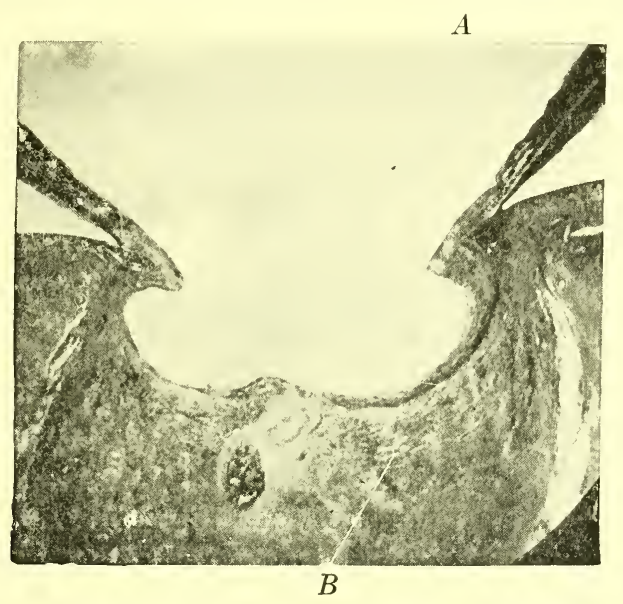

FIG. I67.- Shows a section through the optic nerve at its point of entrance into the eye in a case of long-standing glaucoma. There is deep cupping of the optic disc. The overhanging edge to the cup, $A$, is what causes the broken appearance of the retinal vessels, which is seen ophthalmoscopically at the margin of the disc. The lamina cribrosa, $B$, is displaced backward.

same disc. This is accounted for by variations in strength of the transverse lamellæ composing the lamina cribrosa, and variations in the amount of atrophy of the nerve tissue.

The retinal vessels in the early stages of the affection become displaced to the nasal side of the disc, due probably to stretching of the posterior pole of the globe causing traction on the retina. Atrophy of the nerve precedes the appearance of any very definite signs of cupping.

As the depression backwards of the lamina cribrosa increases the cup deepens and its sides become steeper. The sclerotic at the margin of the optic disc is tough and unyielding, and in advanced cases remains projecting out as a promontory whilst the cup expands laterally behind it. 
The cup in section then presents a flask-shaped outline, and the retinal blood vessels, which adhere to its margins, when viewed ophthalmoscopically seem to have a break in their continuity, those at the bottom of the cup not appearing to join those at its margins (Fig. 167 ).

The immediate effect of increased tension on the retinal blood-vessels is to obstruct both the entrance of blood by the arteries and its exit by the veins. Consequently the latter become enlarged and the former smaller than normal. The intraocular pressure and the pressure of the blood in the retinal vessels are so balanced, under normal conditions, that no pulsation is to be observed in the retinal arteries. When the intraocular pressure is much increased, or the arterial pressure much diminished, this balance is disturbed; blood can then force its way into the retinal arteries only during contraction of the heart, and pulsation in them becomes visible in the vicinity of the optic disc. If the increased tension in a case of glaucoma is not sufficient to give rise to pulsation of the retinal arteries, it may readily be elicited by slight pressure on the globe with the finger. Under normal conditions a considerable amount of pressure on the globe is required to produce pulsation.

Pulsation of the retinal arteries has been observed in cases of aortic regurgitation and of syncope, without increased tension of the eye, due to a sudden fall in the blood pressure during the heart's diastole.

Strong venous pulsation is sometimes noted with glaucoma, but is of no diagnostic importance.

A disturbance of the function of the retina, as the result of increased tension, may be due either to diminished blood supply or to atrophy of its nerve elements. Loss of vision due solely to the first cause is recoverable; that due to the second is permanent.

If the tension of a healthy eye be increased by pressure from without, as with the finger upon the eyelid, vision may be completely abolished, disappearing last in the region of the macula. This may be attributed to arrest of the 
circulation in the retinal vessels, and in the choroidal capillaries, from which the outer layers of the retina receive their nutrient supply. Directly the pressure is removed, the circulation is re-established and vision returns.

In the same way in acute glaucoma, vision may, in the course of a few hours, be reduced to mere perception of light or completely abolished. If normal tension is re-established before sufficient time has elapsed for organic changes in the nervous tissue to set in, vision will be restored.

The branches of the retinal artery which go to the periphery of the retina on the temporal side have a longer course to pursue than those distributed to other parts, because the point of entrance of the optic nerve into the eye is situated to the nasal side of the middle line. It is the capillaries, therefore, from the temporal branch which are affected first by any increase of tension.

In eyes blinded by glaucoma atrophy of the nerve elements of the retina occurs, the ganglion cells become vacuolated and after a time disappear; the outer limbs of the rods and cones become flattened by the intraocular tension against the pigment cell layer, and after a time become much bent, but do not atrophy. Small cystic spaces are very commonly met with in the anterior portion of the retina, in the vicinity of the ora serrata.

The failure of the field of vision which occurs in chronic glaucoma, though it may to some extent be nutritional in origin, is certainly largely due to damage to the optic nerve fibres from the stretching and pressure to which they are exposed, in the formation of the ectasia at the optic disc. The anatomical distribution of the nerve fibres to different portions of the retina helps considerably in explaining the various defects, which are revealed by perimetric measurements with large and small objects. In describing their anatomical arrangement it will be well to apply names to the different groups of nerve fibres.

The fibres which emerge from the nasal side of the optic disc extend evenly, in fan-like fashion, to the nasal half of 
the retina, and may be termed the nasal fan (Fig. I68).

The fibres which go to the macular region occupy about a third of the circumference of the optic disc, and proceed from the central part of its outer half, these are known as the papillo-macular fibres.

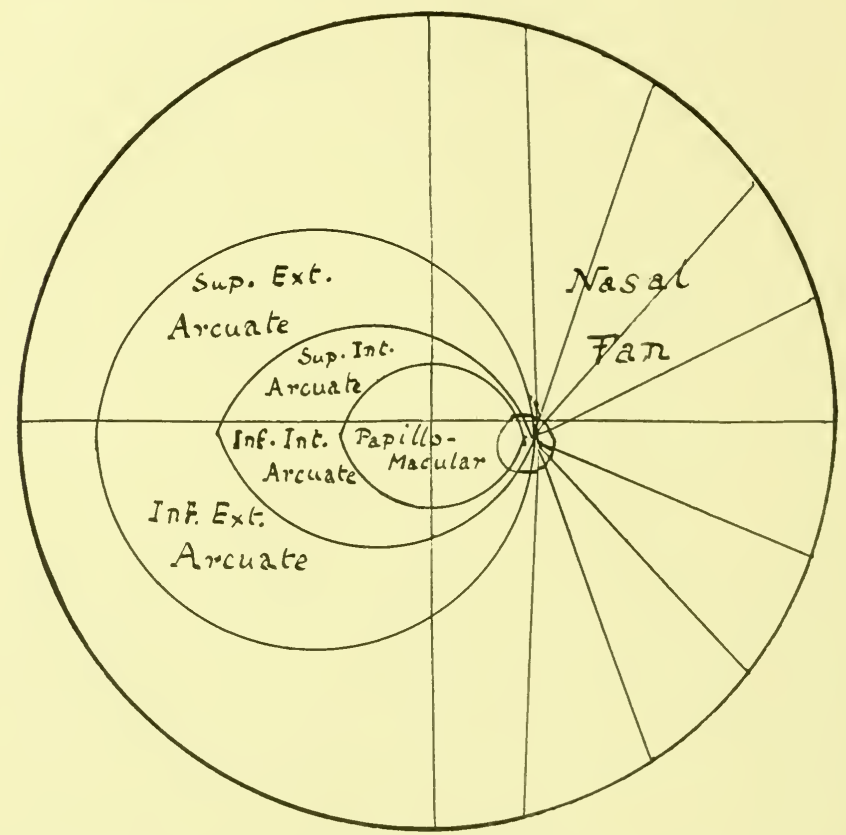

FIG. I68.-Diagram showing the distribution of the nerve fibres in the retina.

From above and below the papillo-macular fibres, others proceed from the outer side of the optic disc to the temporal half of the retina. These arch round the papillo-macular bundle, those coming from above meeting those coming from below in a central raphé. The arched fibres may conveniently be divided into four sets. The superior internal arcuate fibres which lie in close contact with the upper border of the papillo-macular bundle, and which meet, immediately external to the macula, the lower internal arcuate fibres which run a similar course below 
the papillo-macular bundle. From the upper border of the optic disc other arched fibres proceed, external to the superior internal arcuate bundle, to the extreme temporal part of the median longitudinal raphé, these are the superior external arcuate fibres. Fibres running a similar course pass from the lower border of the optic disc and meet at the lower margin of the central raphé the superior external arcuate fibres, these are termed the inferior external

\section{arcuate fibres.}

Another point in connection with the distribution of the nerve fibres is, that the fibres which enter the eye nearest the centre of the optic nerve probably go to the most peripheral parts of the retina, and therefore run the longest course; whilst those which enter the eye at the sides of the nerve go to the more central parts of the retina, and run a shorter course.

The defects in the field of vision met with, as the result of increased tension, vary in different cases according to which of the above different sets of fibres become involved. The fibres affected correspond to the part of the disc which is most cupped, and that, as already stated, is dependent largely on the strength of different portions of the lamina cribrosa. The blood vessels after passing through the lamina cribrosa also afford some support to the nerve fibres; they are mostly situated on the nasal side, the temporal is, therefore, generally the weaker, and that in which cupping most frequently begins. Pressure backwards of the lamina cribrosa causes the nerve fibres to become stretched, and those which run the longest course, passing from the centre of the disc to the periphery of the fundus, are stretched the most. It is for these reasons that the commonest defect to meet with in the field of vision in glaucoma is contraction of its periphery, commencing generally in the upper or lower quadrant, according to whether the cup commences nearer the upper or lower margin. If it begins at the upper margin the superior external arcuate fibres are the first involved, and the lower temporal part of the field is 
lost, a sharply defined defect of vision occurring along the horizontal meridian on the nasal side, which is known as Roenne's nasal step. If the cupping begins at the lower margin the inferior external arcuate fibres become first involved, a similar step like defect is produced, but it is the upper temporal part of the field which is lost.

If the tension is not relieved a general contraction of the field continues, until a small portion of its centre alone remains, giving a tube-like vision; the last fibres to become affected are those of the nasal fan which enter the eye at the margin of the disc, so that the last fragment of vision to go is usually an area to the outer side of the fixation point.

Pressure on the nerve fibres, as they curl round the edge of the disc against the tough unyielding spur of the sclerotic, is liable to disturb their conductive power in the same way as stretching of them appears to do. Such pressure affecting the superior or inferior internal arcuate fibres causes a defect in the field of vision which spreads from the upper or lower border of the normal blind spot. Wing-like extensions of the blind spot in an upward and downward direction may be the very first sign of the presence of glaucoma in an eye, it is known as Seidel's sign. In more advanced cases of glaucoma, in which the superior and inferior internal arcuate fibres become more extensively affected, scotomata starting from the normal blind spot sweep round the central fixation area in comet-like fashion; these are best revealed by testing with small objects and are known as Bjerum's sign. The two blind areas above and below the fixation spot may extend until they meet at the horizontal meridian on its temporal side, a ring scotoma being so produced. The part of the retina surrounding the fixation spot, to which the superior and inferior internal arcuate fibres are distributed, is that which is most acutely sensitive to light; when patients have developed a ring scotoma in connection with glaucoma night blindness often becomes a prominent symptom. The extension of a scotoma to 
the temporal side of the fixation spot tends very materially to interfere with a patient's reading capacity, even though the central acuity of vision may remain perfectly good. To read with ease it is essential to be able to follow on quickly to the next word, this a patient with a blind area to the outer side of the fixation spot is unable to do.

In one of the bundles of nerve fibres already named, it may happen that some of the fibres become affected sooner than others; this is revealed by the presence of isolated areas of defective vision, or by the presence of a ragged toothed edge to a scotomatous area, known as Elliot's sign. It can be best demonstrated by the use of that writer's scotometer in which a small test object is used on a rotating screen.

The papillo-macular bundle of fibres, which only run a short distance from the nerve to the retina, are as a rule among the last to become affected. In exceptional cases due to some unusual weakness in the lamina cribrosa where the macular fibres perforate it, those fibres are early involved, and a central scotoma or a marked defect in the central acuity of vision appears early in the disease.

Due to the late involvement of the nerve fibres going to the macula, where the cones are most abundant, defects of colour vision are rarely complained of by those suffering from glaucoma. The colour fields are found to be relatively as large as the white fields in glaucoma, a point often of diagnostic importance, as in other forms of atrophy of the optic nerve, the colour fields are generally more affected than the white.

\section{iv. The Means Employed for the Reduction of Tension} in Primary Glaucoma.-For the relief of tension in primary glaucoma it is necessary either to $(a)$ open up the normal channels of escape of fluid from the eye, or to (b) make fresh channels.

(a) The opening up of the normal channels of escape of fluid from the eye may be effected temporally by the use of myotics, which by contracting the pupil narrows the width of the iris, and draws it away from contact with the back of 
the cornea at the angle of the anterior chamber. To open up the angle of the anterior chamber permanently some operative procedure is required. Those employed for this purpose are iridectomy, iridectomydialysis, and cyclodialysis.

Iridectomy.-In a simple iridectomy a piece of the iris is removed without any attempt being made to drag it away from its periphery. In eyes in which it has been

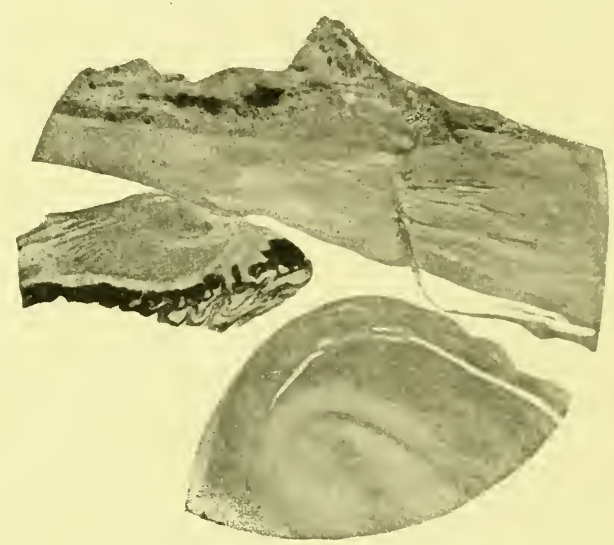

FIG. 169.- Shows a section through the angle of the anterior chamber of an eye upon which an iridectomy had been performed for primary glaucoma of two months' duration. The tension was relieved by the operation, but the eye had to be excised five weeks later on account of ulceration of the cornea. The iris has been removed right up to the ciliary body and the angle of anterior chamber opened up.

performed successfully for acute glaucoma, and which have subsequently had to be removed for some intercurrent malady, the root of the iris was found to have become dislodged from its faulty position. ${ }^{1}$ Evidently in such cases sufficient time had not elapsed for it to have become adherent to the cornea. The escape of the aqueous, and consequent relief of pressure in the vitreous chamber, together with the local escape of blood and manipulation of the iris, must have sufficed to dislodge it from apposition with the cornea, and to have reopened the normal excretory channels.

\footnotetext{
${ }^{1}$ Treacher Collins. R. Lond. Ophth. Hosp. Rep., XIII, I89I, Io6.
} 
In eyes where an iridectomy has failed to relieve tension in glaucoma a portion of the root of the iris has been found to have been left behind, either adherent to the back of the cornea or held in contact with it by an entanglement of the cut end between the posterior lips of the sclero-corneal incision (Fig. I7I).

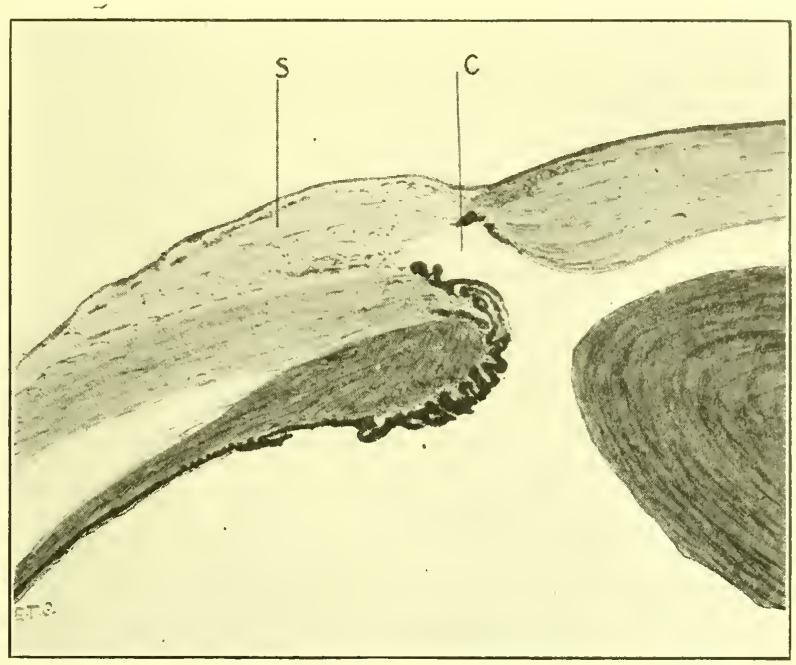

FIG. I70.- Section of an eye in which a cystoid cicatrix formed after an iridectomy operation for chronic glaucoma. Eight months later severe iritis set in, and the eye was ultimately removed for the relief of pain. $C$ points to the fistula formed in the sclero-corneal tissue at the seat of the operation; it is partly lined by the ciliary processes and partly by atrophied iris; $S$ points to the edematous conjunctiva due to escape of aqueous into it.

Iridectomydialysis. - In an iridectomydialysis an attempt is made to tear a piece of the iris away from its junction with the ciliary body, in the course of its removal. If the root of the iris has not previously become too intimately adherent to the back of the cornea, this is easily effected because the thinnest part of the iris is at its extreme periphery (Fig. I69). Should, however, firm adhesion have become established between the root of the iris and the cornea previous to the operation, as is often the case in long standing glaucoma, then the iris, instead of tearing away at its point of junction with the ciliary body tears through where 
it ceases to be attached to the cornea, a piece being left behind blocking the filtration area so that the channels for the escape of the aqueous remain closed.

Another way in which an iridectomydialysis sometimes fails to relieve tension is the result of delayed reformation of the anterior chamber. From such delay the lens may become united to the posterior surface of the wound by

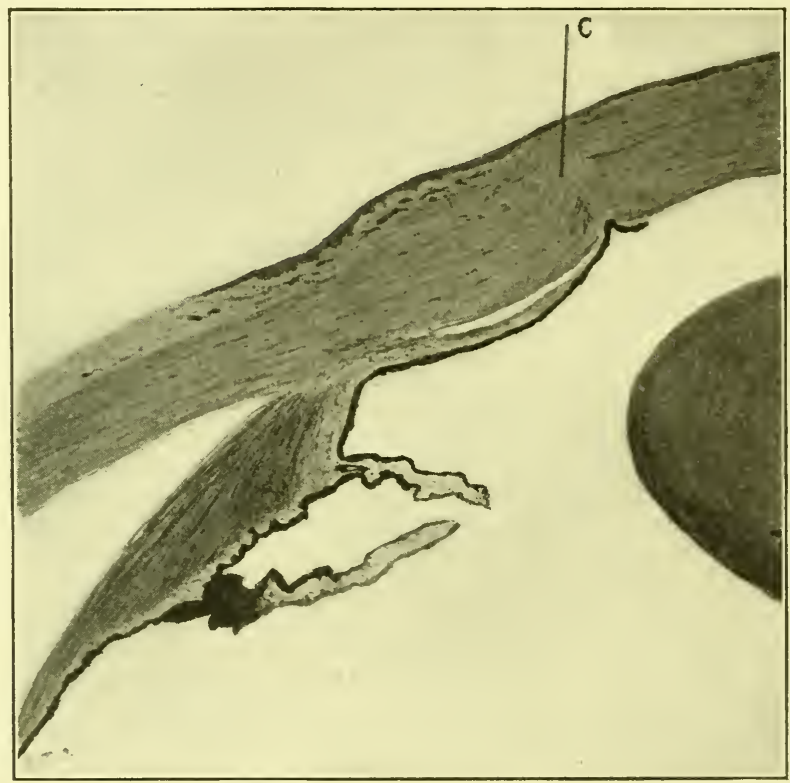

FIG. I 7 I. - Section of an eye in which an iridectomy failed to relieve the tension in glaucoma. The iris has been cut off level with the line of cicatrix in the cornea to which $C$ points. If dragged upon it would doubtless have torn away more peripherally at its junction with the ciliary body. A large piece of it would not then have been left blocking up the angle of the anterior chamber and securely fixed there by adhesion of its cut end to the corneal cicatrix.

plastic exudation, then when the anterior chamber does reform, the lens becomes drawn forwards, its adherent margin pressing the anterior of the ciliary processes into contact with the filtration area in the region of the coloboma (Fig. I 72 ).

Cyclodialysis. - In the operation of cyclodialysis the ligamentum pectinatum is torn through, and the ciliary body 
and root of the iris are separated from the sclerotic and cornea. The contraction of the ciliary muscle then tends to draw back the ciliary body, and with it the iris, away from the filtration area in the cornea. It also opens up a communication between the anterior chamber and the suprachoroidal lymph space. It has been estimated that in 30 per cent. of the cases in which this operation is performed a permanent relief of tension was obtained. The operation fails in some cases because the ciliary body gains a fresh attachment to the sclerotic and the iris again comes into

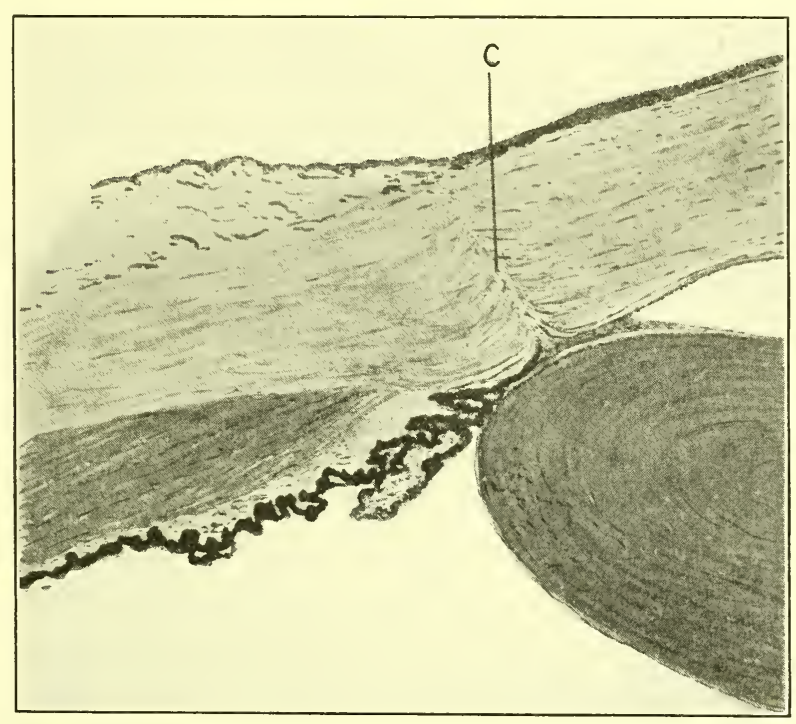

FIG. I72.- Section of an eye in which an iridectomy was performed for absolute glaucoma; the increased tension returned after the operation and the eye was subsequently $y_{3}$ removed for relief of pain. $C$ points to the iridectomy cicatrix in cornea. The lens is united by organised exudate to its posterior surface. The filtration area is blocked by the anterior part of the ciliary body and root of the iris, held forward by the adherent lens.

contact with the periphery of the cornea. If a piece of the iris were removed at the time of the operation, or before the new adhesions formed, this difficulty might be overcome. Another cause of failure is due to the root of the iris having become too intimately adherent to the periphery of the cornea to allow of its separation, at the time of the operation. 
(b) The Formation of New Channels for the Escape of Fluid from the Eye.-A new channel may be established for the escape of fluid from the eye, by the formation of a filtration scar or cystoid cicatrix at the periphery of the cornea, or by the formation of an opening in the uveal tissue which facilitates the access of the aqueous humour to its veins.

Filtration Scars and Cystoid Cicatrices.-The experiment has been performed by tying a freshly removed cornea over the end of a glass tube, and forcing water through the tube against its posterior surface. As long as the endothelium lining Descemet's membrane remained intact no fluid could be forced into the cornea, but when the endothelium was removed, it readily penetrated into the substantia, propria, making it opaque, and causing the surface epithelium to become raised in the form of vesicles.

From this experiment it would seem, that the first essential factor in the formation of a permanent filtration scar is to establish a permanent gap in the endothelium lining Descemet's membrane.

After a simple perforating wound of the cornea, the divided ends of the posterior elastic lamina retract, carrying with them their endothelial lining. Whilst the opening in the endothelium exists, aqueous humour filters through it, causing the lips of the wound to swell and a fibrinous coagulum to from between them. The divided endothelium usually proliferates, and extends over the posterior surface of the coagulum. When it is again intact further filtration becomes arrested.

In a perforating wound of the eye the restoration of the continuity of the endothelium of Descemet's membrane may be impeded in various ways, under which circumstances the filtration of the aqueous through it is prolonged or rendered permanent: ${ }^{1}$

r. The inclusion of a fold of iris, of a piece of lens capsule or of a piece of silk thread, between the posterior lips

${ }^{1}$ Treacher Collins. Ophthalmoscope, XII, I9I4, 589. 
of a wound interferes with the extension across it of the endothelium.

2. The endothelium at the margins of the wound may be so extensively damaged, either mechanically or chemically (as by the use of strong antiseptics), that its reformation is less easily accomplished.

3. The gap formed in the tissue at the seat of the wound may be so wide that the endothelium is unable to extend across it.

Sometimes, after an iridectomy operation for glaucoma, what is termed a cystoid cicatrix forms, usually at one angle of the coloboma. In such cicatrices a clear gap or fistula is seen in the sclero-corneal tissue, overlying which the conjunctiva is swollen, boggy and edematous (Fig. I 70). Pathological examination has shown, that the fistula in such scars results from the prolapse of a fold of iris which prevents the two sides of the wound in the fibrous tissue uniting, but over which the conjunctiva heals. At first the iris tissue lining the wound offers an impediment to the passage of fluid out of the eye, but this being a weak spot in the walls of the globe, as the tension rises, it bulges and the lining iris tissue atrophies, so that ultimately a complete fistula is established for the passage of the aqueous into the subconjunctival tissue.

In some of the early sclerotomy operations a fold of iris was deliberated allowed to prolapse and produce a cystoid cicatrix, the amount of bulging to which such a procedure sometimes gave rise was enormous.

Although the production of cicatrices of this description may prove beneficial in the relief of tension, they are attended with a certain amount of risk. Practically an adhesion between the conjunctiva and iris is formed, any inflammation of the former may then readily spread to the latter, and a general uveitis ensue. A recent writer ${ }^{1}$ claims that this risk can be overcome by fibrosis taking

${ }^{1}$ H. Herbert. Trans. of the Ophth. Soc. of the U. K., XII, I9I4, 239. 
place in the stroma of the prolapsed iris and in the covering subconjunctival tissue.

The operation in which a strand of silk thread is inserted between the lips of a sclero-corneal wound, so that one end lies just in the anterior chamber and the other in the subconjunctival tissue, has been found to be effectual in the relief of tension. ${ }^{1}$ It has not, however, been general practised due to the difficulty in keeping the thread in exactly the right position, and to the risk of infection spreading down the thread to the interior of the eye.

That filtration scars at the sclero-corneal margin can be obtained, free from any entanglement of the uveal tract, and without the insertion of any foreign substance, has been definitely proved. ${ }^{2}$ Such scars are occasionally seen after sclerotomy operations, but occur more frequently after some form of sclerectomy. Their filtering character is due to the failure in formation of an endothelial lining on the posterior surface of the scar, either from the wide retraction of the divided ends of Descemet's membrane or from a piece of it having been cut away.

In Herbert's small rectangular flap Sclerotomy operation, three small rectangular incisions are made through Descemet's membrane which facilitate the retraction of their margins, and sometimes lead to the formation of a permanently filtrating scar.

It might have been anticipated that where a piece of the scleral tissue was excised, as in Lagrange's sclerectomy or in Elliot's sclero-corneal trephining, a formation of granulation tissue would take place and fill up the gap; when, however, the wound is kept thoroughly aseptic this does not occur. The conjunctival flap heals over the surface of the gap, but its two edges remain unclosed and uninfiltrated. Apparently uninfected wounds of tissue, kept bathed by the aqueous humour manifest no tendency to

${ }^{1}$ M. S. Mayou and Zorab. Ophthalmoscope, X, 1912, 254 and 258.

${ }^{2}$ Verhoeff. Arch. of Ophth. XLIV, 1915, I 29. 
the development of granulation tissue, and remain open indefinitely.

It is obviously desirable in a sclerectomy operation for glaucoma, that the tissue removed should include a piece of Descemet's membrane. This is ensured in Elliot's trephining operation by dissecting up the conjunctival flap well forwards, so as to expose fully the sclero-corneal margin, and then placing the trephine astride it. Sections of the disc removed under such circumstances are found to be either completely lined by Descemet's membrane or lined partly by it and partly by a small piece of the pectinate ligament. ${ }^{1}$

One of the chief drawbacks to the trephining operation is the occasional occurrence of intraocular infection, some months or years after what has apparently been a very successful operation. Pathological examination of eyes in which this has occurred has revealed the presence of an entanglement of uveal tissue, passing round the peripheral edge of the opening, and in contact with the conjunctiva. This entanglement was presumably the track along which infection from the conjunctiva had spread into the interior of the eye.

Openings in the Uveal Tissue Which Facilitate the Access of the Aqueous Humour to Its Veins.-The anterior surface of the iris is covered by a single layer of flattened endothelial cells, except opposite the openings of its crypts. It is through these crypts that solid particles, such as grains of Indian ink, have been found to gain access to the iridic veins. Presumably the endothelial cells covering the remainder of the iris prevent their passage into its tissue elsewhere.

Wounds of the iris, like wounds of the sclero-corneal tissue, if kept aseptic and bathed with aqueous humour, manifest no tendency to form granulation tissue or to cicatrise. The cut margin of the iris after an iridectomy operation, when examined microscopically years later, pre-

${ }^{1}$ R. H. Elliot. Trans. Ophth. Soc. of the U. K., XXXVIII, I918, 227. 
sents the same ragged appearance as when the wound was first made; the endothelium covering the surface of the iris shows no tendency to spread over its cut ends. ${ }^{1}$ It seems probable, therefore, that such an open wound in the tissue of the iris like the crypts in the iris, would afford a means of access by which the aqueous might pass into the iridic veins. The beneficial results of an iridectomy for glaucoma has been attributed to the opening up of such a new channel for excretion.

In the operation of cyclodialysis a communication is established between the anterior chamber and the suprachoroidal lymph spaces, the fluid in these spaces for the most part drains away along the perivascular lymph channels surrounding the venæ vorticose. It has been suggested that the reduction of tension, which is sometimes produced by a cyclodialysis, may be due to the fresh means of access which it affords for the aqueous humour to reach the vortex veins or the lymphatic channels around them.

\section{Detachment of Choroid and Intraocular Hemorrhage} after Glaucoma Operations.-After any operation for the relief of increased intraocular tension detachment of the choroid, either by serous exudation or by hemorrhage, is liable to occur. Serous detachment of the choroid is extremely common, especially after trephining operations. It has been met with in 70 per cent. of the cases carefully examined by contact illumination during the first two or three days after operation; as a rule the fluid becomes absorbed and the choroid returns to its normal position without any evil results. It sometimes occurs suddenly a few days after operation, its presence being made manifest by the disappearance of the anterior chamber, which had reformed after the operation.

Intraocular hemorrhage may take place after an operation for glaucoma from either the choroidal or retinal

\footnotetext{
${ }^{1}$ Thomson Henderson. Ophth. Rev., XXVI, I907, 19I.
} 
vessels. When it occurs from the choroidal vessels the choroid becomes detached, and it may be so extensive as to give rise to a stony hardness of the eye or to protrusion of the contents of the globe through the wound. Retinal hemorrhages are usually small, and unless occupying the macular region, do not interfere much with vision. Occasionally hemorrhage may take place into the vitreous giving rise to a defect of vision which tends to improve as it becomes absorbed.

These intraocular hemorrhages after operations for glaucoma, result from a rush of blood into its sclerosed vessels due to the sudden relief of intraocular pressure. Every means should, therefore, be taken before operation to lower the intraocular tension, and to decrease the general blood pressure. For the relief of the intraocular pressure eserine should be used; in some cases of very acute glaucoma scleral puncture has been resorted to as a preliminary measure. The general blood pressure is best lowered by the use of a watery purgative before operation. Leeching and venesection may also be employed with advantage.

v. The Causes of Hypotony, or Decreased Intraocular Tension, and Its Affects.- Hypotony or decrease in intraocular tension in contradistinction to hypertony or glaucoma, is a condition which is frequently present from a variety of causes. Naturally penetrating injuries give rise to hypotony which lasts as long as the wound in the globe leaks, either on the surface or beneath the conjunctiva. Apart from penetrating injuries hypotony is associated with concussion injuries, iridocyclitis, detachment of the ciliary body and choroid, and detachment of the retina. Hypotony may arise either as the result of:

(a) Diminished secretion, due to (I) nerve inhibition, (2) vascular disturbance, (3) injury to the ciliary epithelium.

(b) Increased excretion either through (I) normal or (2) newly formed channels.

(a) Diminished Secretion.-(I) Diminished secretion from nerve inhibition has been proved beyond all doubt. 
Section of the cervical sympathetic in animals, and men leads to reduction in tension of the eye, sometimes lasting as long as five months. Gunshot injuries causing nerve concussion have been frequently recorded with diminished tension. The injury to the ciliary nerves probably affects the vaso-motor system, rather than the secretory epithelium of the ciliary processes, but as yet more proof is wanting on this point.

(2) Vascular Disturbance. Division of the posterior ciliary arteries does not give rise to any diminution in tension, but division of the anterior ciliary vessels seems to do so. Cases of hypotony have been recorded in which they have been found divided after an injury; and it is possible that the reduction in tension due to the operation of cyclo-dialysis is also partly due to this cause.

(3) Injury to the epithelial lining of the ciliary body may possibly give rise to decreased tension as the result of direct damage to the cells, or as the result of detachment of the pars plana. Such injuries are always associated with others to which the minus tension may be attributed, so that more evidence is required before any definite conclusion as to what part they play in the production of hypotony can be arrived at.

(b) Increased Excretion. ${ }^{1-}$ - ( I) Through the normal channels, by perssure upon the eye as the result of an injury, a compressive bandage or massage. It is probable that the reduction in tension so produced is brought about by the expression of intraocular fluid from the eye, rather than the diminution of the blood supply.

(2) Through newly-formed channels. This occurs; after the operation of sclero-corneal trephining where a piece of Descemet's membrane has been removed; through a cystoid scar the result of iris inclusion; as the result of internal rupture of the sclera in the neighbourhood of the canal of Schlemm which has thereby been opened up; and in cyclo-

${ }^{1}$ Treacher Collins. Trans. Ophthal. Soc. of the U. K., XXXVI, I9I6, 206. 
dialysis, either the result of an operation or a concussion injury, in which in addition to the opening up of the canal of Schlemm, the anterior ciliary arteries may have been divided.

The sequelæ of hypotony may be classified into:1$A$. Those due to relaxation of the tissues usually kept taut.

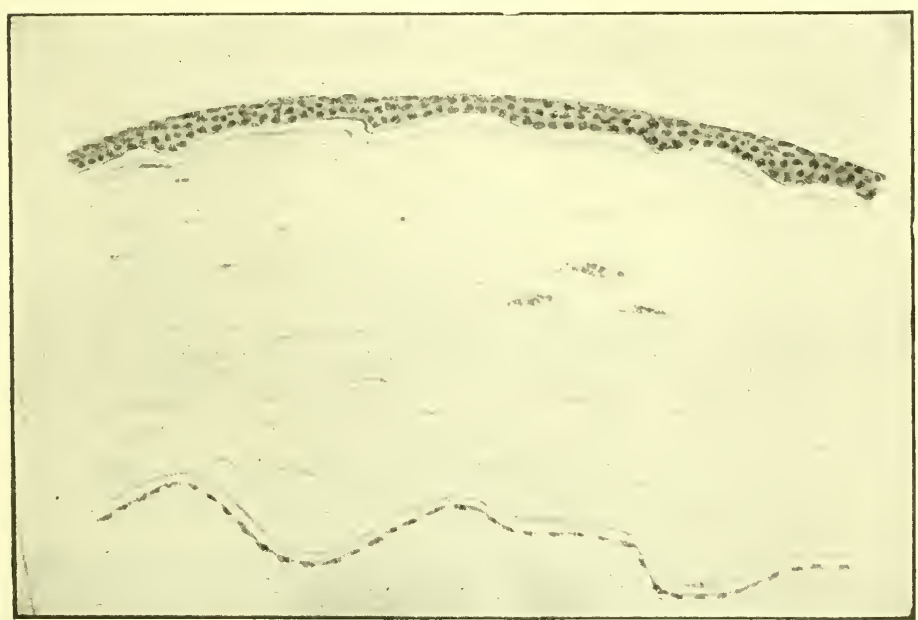

FIG. I73.- Section of cornea from an eye with hypotony showing wrinkling of Bowman's and Descemet's membranes.

(I) Wrinkling of the anterior elastic lamina of the cornea; producing superficial double contoured lines (Figs. I 73, I 74).

(2) Wrinkling of the posterior elastic lamina of the cornea and layers of the substantia propria immediately adjacent to it; producing deeply seated striated opacities (Fig. I 73).

(3) Thickening of the sclerotic and the production of puckers in it behind the insertions of the recti muscles (Fig. I 75).

(4) Wrinkling of the elastic lamina of the choroid, and heaping up of the pigment epithelium on its inner surface where the folds are deep (Figs. I76, I 77).

${ }^{1}$ Treacher Collins. Trans. of the Ophth. Soc. of the U. K., XXXVII, 1917, 28r. 
(5) Displacement forwards of the lens and slackening of its suspensory ligament; both of which tend to increase the refraction of the eye.

$B$. Those due to production of relatively higher bloodpressure compared with intra-ocular pressure, than exists under normal conditions:-

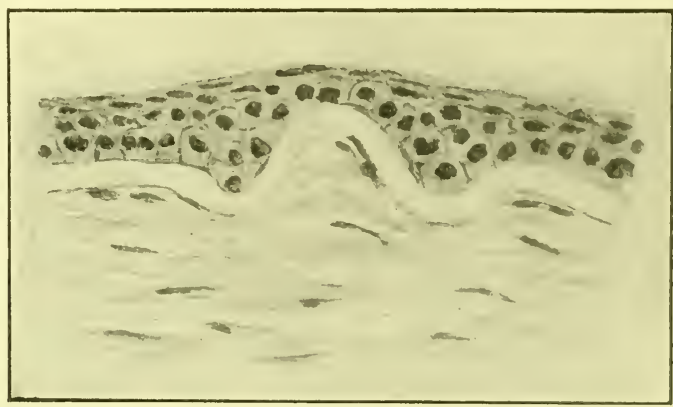

FIG. I74.- Section showing the wrinkling of Bowman's membrane the result of hypotony.

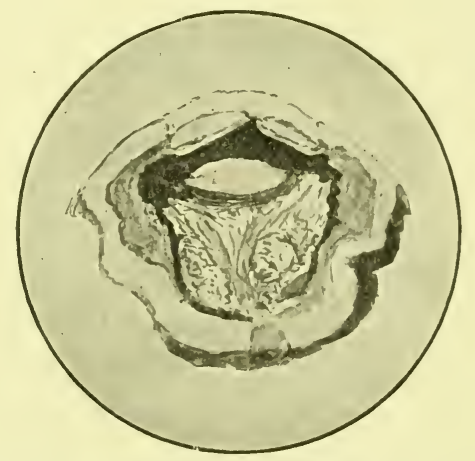

FIG. I 75. - Lateral half of a shrinking eyeball showing puckering of the sclerotic behind the insertion of the recti muscles.

(I) In the choroidal veins the normal excretion of lymph through them is arrested and instead an exudation of lymph takes place, giving rise to detachment of ciliary body and choroid.

(2) In the veins of the iris the normal excretion of aqueous humour through them is arrested, and instead an exudation 
of lymph into the anterior chamber takes place; this exudation alters the normal consistency of the aqueous by increasing its percentage of proteins and rendering it spontaneously coagulable.

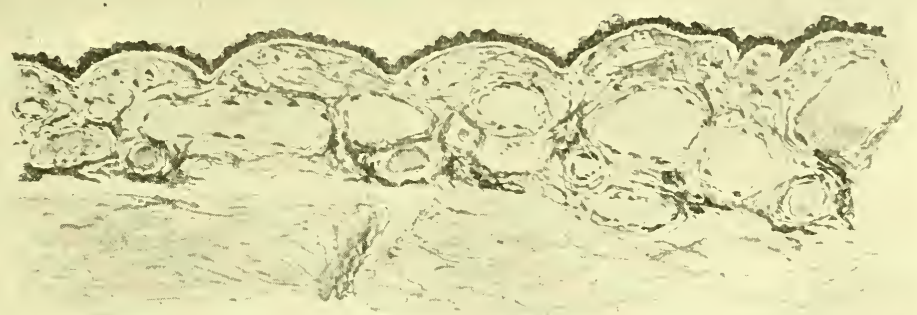

Fig. I76.-Wrinkling of the membrane of Bruch in an eye with hypotony.

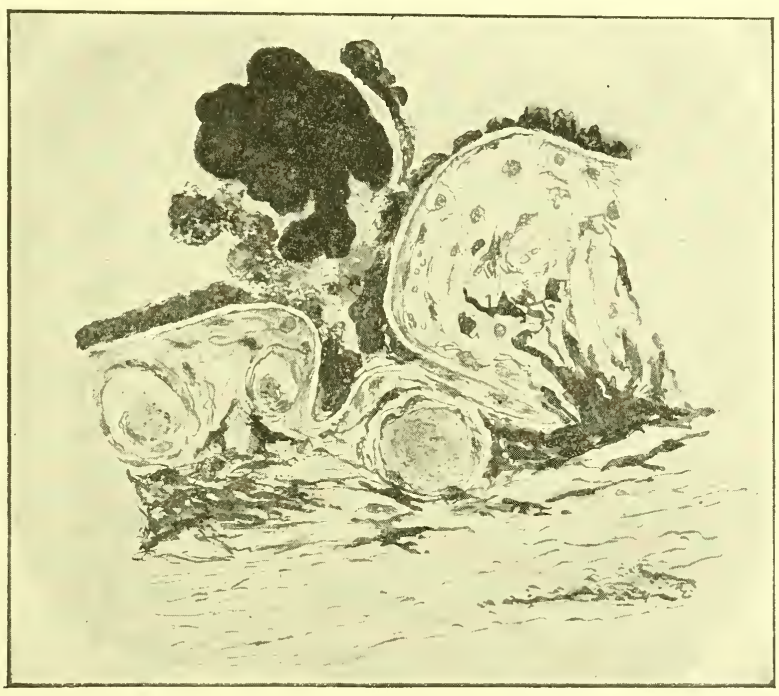

Fig. 177.-Wrinkling of the membrane of Bruch and heaping up of the pigment on its inner surface in an eye with hypotony.

(3) In the capillary vessels of the ciliary body the secreting function of that structure is quickened, and an exudation of lymph takes place, the albuminous constituents of which, 
338 DISTURBANCES IN THE NUTRIENT FLUIDS OF THE EYE

being unable to permeate the epithelial lining of the part raise it up in the form of vesicles.

(4) In the capillary vessels of the choroid the normal exudation of lymph is stimulated, which lymph, if it permeate the pigment epithelium, may collect beneath the retina causing detachment of the membrane.

(5) In the retinal veins of the optic disc the normal excretion of fluid from the vitreous is arrested, and an exudation of lymph into the head of the optic nerve takes place, giving rise to the appearance of choked disc. 


\section{CHAPTER IV}

\section{INJURIES}

Injuries of the eyeball and its appendages may.be divided into those affecting: I. Eyeball, II. Eyelids, III. Orbit.

\section{INJURIES OF THE EYEBALL}

Injuries of the eyeball will be described under the following headings: (i) Ruptures, (ii) Concussions, (iii) Wounds, (iv) Foreign Bodies, (v) Effects of Heat and Cold and Chemicals, (vi) Effects of Excessive or Deficient Light, Electricity and X-rays.

A rupture is the result of a blow from a blunt object which causes the outer fibrous tissue wall of the globe to give way, due to a sudden increase in its tension from compression.

The changes which take place in the structures in the interior of the eye from blows by blunt objects, in which no rupture of the outer fibrous tissue coat occurs, are described under concussions. The blow in these cases is usually received on the front of the eye, but in gun-shot injuries of the orbit it may be inflicted posteriorly. Injuries to the eye from the pressure by forceps at birth are also included under this heading.

Wounds of the eye may or may not perforate the globe. When perforation takes place the changes produced vary considerably according to the position, depth, and size of the wound, they are therefore divided up under several sub-headings.

Foreign bodies may become lodged in the external coats of the eye, penetrate them and become located in its interior, or pass right through the globe into the orbit. 
The changes which ensue vary considerably according to the nature of the foreign body.

Burns of the eye may be produced by excess of heat and cold, and caustic effects of chemical substances. Undue exposure to ultra-red rays produces cataract.

Under the effects of excessive and deficient light the following affections are described: eclipse-blindness, nightblindness, erythropsia, snow-blindness, and miners' nystagmus. Electrical injuries proceed either from lightning or industrial electricity. The effects on the tissues of the eye from exposure to $\mathrm{x}$-rays and radium are also described under this heading.

\section{i. Ruptures}

The sudden compression of an eyeball, between the bony wall of the orbit and a blunt object, may so increase its

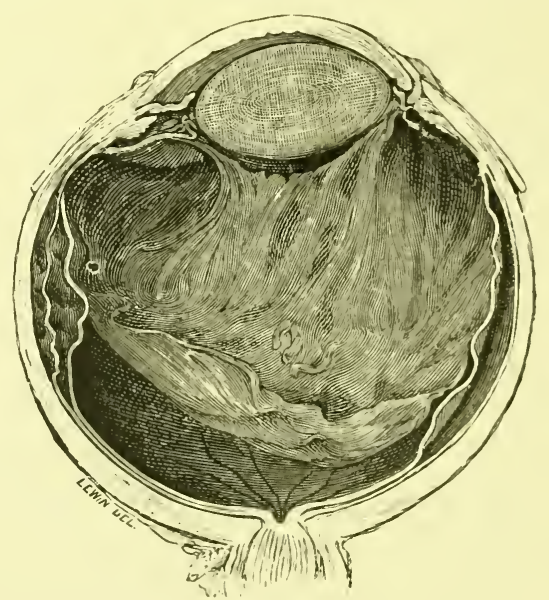

Fig. I78.-The lateral half of an eye ruptured by a blow from a fist. It is situated in the usual position and extends into the extreme periphery of the anterior chamber. The iris has prolapsed and the lens become displaced forward and upward. Specimen in the R. Lond. Ophth. Hosp. Museum.

tension as to cause its fibrous tissue outer wall to rupture. Such compression of the eye is most frequently occasioned by something forced into the orbit from one side, as for example a prod with a cow's horn. A blow forcing the 
eye straight back in the orbit, against the pad of fat behind, does not cause it to rupture.

A rupture of the fibrous tissue outer wall is always in the anterior part of the eye, usually 2 to $3 \mathrm{~mm}$. from the corneal margin, passing through the canal of Schlemm and ligamentum pectinatum into the extreme periphery of the

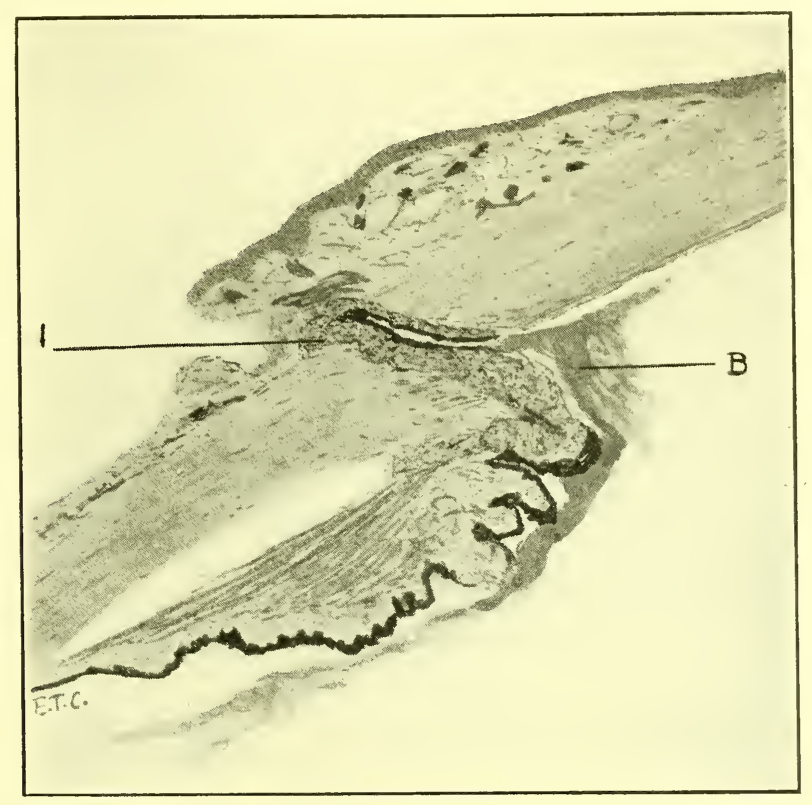

FIG. I79.-Rupture of the globe showing a prolapse of the iris $I$, into the wound. The lens has been extruded and the anterior chamber is filled with blood, $B$.

anterior chamber (Fig. I78). This is not the thinnest part of the sclerotic, which is situated at the equator just behind the insertion of the recti muscles. It is, however, the thinnest part unsupported externally by the orbital muscles and internally by the other coats of the eye. It is also weakened by the presence of the loose tissue composing the filtration area.

Ruptures of the cornea are exceedingly rare; occasionally in an eye with the scar of an old perforating wound of the cornea, instead of the sclerotic rupturing, the cicatrix 
gives way. A rupture of the fibrous tissue outer coat of the eye occurs on the side of the globe which is brought into contact with the wall of the orbit, and on the opposite side to which the blow is inflicted. As the eye is protected on the inner side by the nose, and above by the brow, injuries are most often received from the outer side or below, hence ruptures of the sclerotic most often occur upward or inward, rarely downward or outward.

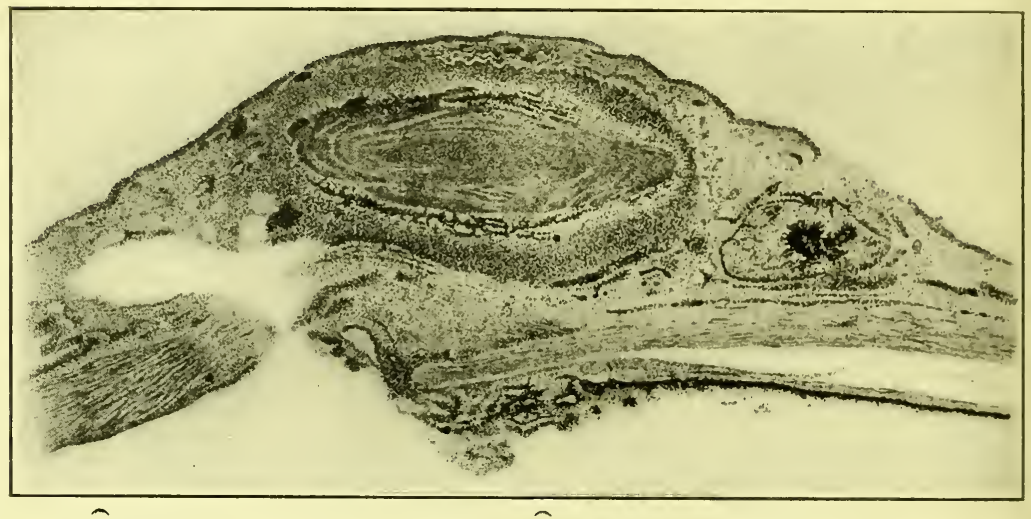

FIG. I80.- Section through a rupture in the sclerotic produced by a blow with a fist. The rupture extends into the extreme periphery of the anterior chamber, and the iris and lens have escaped through it. They are shown lying beneath the conjunctiva external to the sclerotic. The pigmented tissue on the righthand side of the figure is the iris. Around the lens there is much cellular exudation.

The rupture takes place from within outward. Incomplete ruptures occur in which the inner fibres in the vicinity of Schlemm's canal are alone torn, the outer ones remaining intact. Sometimes the whole thickness of the sclerotic is torn through, the conjunctiva overlying it remaining intact.

In recent ruptures the lips of the wound are always everted; as the wound heals the cicatrisation which takes place causes them to become inverted, sometimes to such an extent that a puckered depression is formed at the seat of the lesion. 
The force of a blow sufficient to give rise to rupture of the sclerotic is nearly sure to cause derangement of some of the other structures of the eye. Various portions of its contents may escape either partly or entirely through the opening. If the conjunctiva remains intact, the escaping structures lie beneath it. The iris in the neighbourhood of the rupture frequently becomes folded and prolapsed (Fig. I79). Sometimes it becomes torn away from the ciliary body in its entire circumference and escapes from the eye with the aqueous, or lies entangled in the wound. The suspensory ligament of the lens is frequently torn through, the lens becoming displaced forward toward the rupture or escaping through it in its capsule. Occasionally the capsule bursts and remains in position while its contents is extruded. When the lens escapes through the rupture, and lies beneath an intact conjunctiva, a large disc-shaped prominence is seen on the surface of the globe just behind the wound, a condition spoken of as sub-conjunctival dislocation of the lens (Fig. I 80).

A tear in the suspensory ligament of the lens allows the vitreous humour to protrude forward, prolapsing or escaping to a great or less extent. Following displacement of the vitreous the retina may become detached and even prolapse through the opening in the sclerotic.

Hemorrhage, in cases of rupture of the sclerotic, may be found in all parts of the globe, subconjunctivally, in the anterior or vitreous chambers, and beneath the retina or choroid.

The ultimate outcome of a rupture of the sclerotic varies with the amount of the contents of the globe which escapes. Some cases regain useful vision, even after the lens has become extruded. A subconjunctivally dislocated lens if not removed becomes surrounded by phagocytes and slowly absorbed; traces of it may remain years after the injury. Where the vitreous has prolapsed, shrinking of the eye generally ensues. Infective organisms from the conjunctival sac may gain entrance through the wound and 
excite an intraocular inflammation. Cases of sympathetic ophthalmitis following rupture of the eye have been recorded.

\section{ii. Concussions}

As the result of a blow on the front of the eye from a blunt object, various lesions of the intraocular contents may occur without any rupture of the sclerotic being produced, these may be conveniently classed as concussion injuries.

The tendency of a blow from a blunt object on the front of the eye is to flatten out the cornea, which has a greater

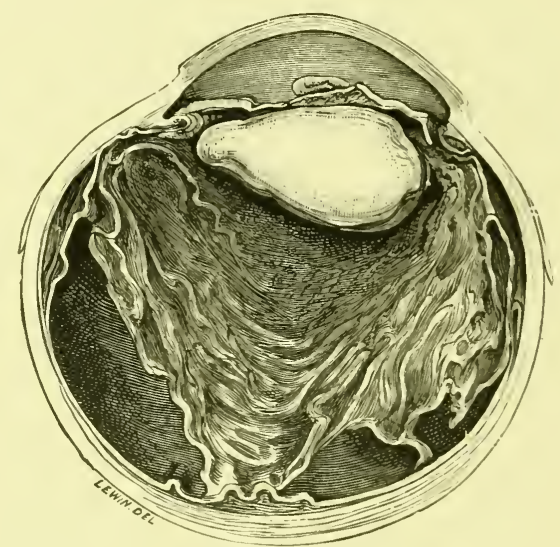

FIG. I8I.-The lateral half of an eye injured by a blow from the stick of a catapult. On the right side iridodialysis has occurred, and the suspensory ligament has ruptured, the vitreous extending forward between the lens and the ciliary body. On the left side there has been a partial rupture of the ligamentum pectinatum and of the ciliary muscle, prolonging the angle of the anterior chamber outward. The lens is misshapen, due to rupture of its capsule. Specimen in the R. Lond. Ophth. Hosp. Museum.

curvature than that of the sclerotic and projects from its surface. Flattening out of the cornea forces the aqueous humour backward and laterally. Backward displacement of the aqueous humour presses on the iris and lens, and its lateral displacement widens out the angle of the anterior chamber. The receding iris finds more support centrally from the lens than peripherally from the suspensory ligament. The strain produced by such an injury is, theretore, 
felt chiefly at the periphery of the iris, the angle of the anterior chamber, and the suspensory ligament of the lens.

Iridodialysis. - The thinnest part of the iris is at its junction with the ciliary body. One of the commonest results of a concussion injury of the eye is for it to be torn through in this situation, in a portion of its circumference, a condition termed iridodialysis being produced (Fig. I8I). The gap left where the iris becomes separated from the ciliary body may be seen clinically as a black area, like the pupil. The contraction of the sphincter muscle widens the gap, and in so doing alters the shape of the pupil, destroying its normal rotundity.

The extent of an iridodialysis varies considerably in different cases. Two separate gaps may be present in the same eye. The iris never becomes reunited to the ciliary body, though a band of fibrous tissue, probably formed from organised blood clot, has been found to bridge across the gap.

Rupture of the Pigment Epithelium of the Iris.-In concussion injuries of the eye the iris, when it is forced backwards, becomes kinked. The occurrence of such sudden and abrupt kinking sometimes leads to rupture of the pigment epithelial layers on its posterior surface. The stroma of the iris in front of them remaining intact (Fig. I82). Such ruptures are clinically liable to be overlooked because the anterior surface of the iris remains unchanged. The gap in pigment epithelium only becomes apparent on examination by reflected light or on contact illumination.

Rupture of the Sphincter Pupillæ, Mydriasis and Cyloplegia.-The sudden dilatation of the pupil which occurs in concussion injuries, from the displacement backward of the periphery of the iris, may cause rupture of its sphincter muscle. One or more notches are then seen in the pupillary border of the iris and the pupil itself remains semi-dilated, failing to react normally to the usual stimuli.

Mydriasis and immobility of the pupil are also met with after concussion injuries without any apparent lesion of the 
sphincter, probably the result of inhibition in conduction by the ciliary nerves. Cycloplegia without any obvious injury to the ciliary muscle, occurring under similar circumstances, may be attributed to a like cause.
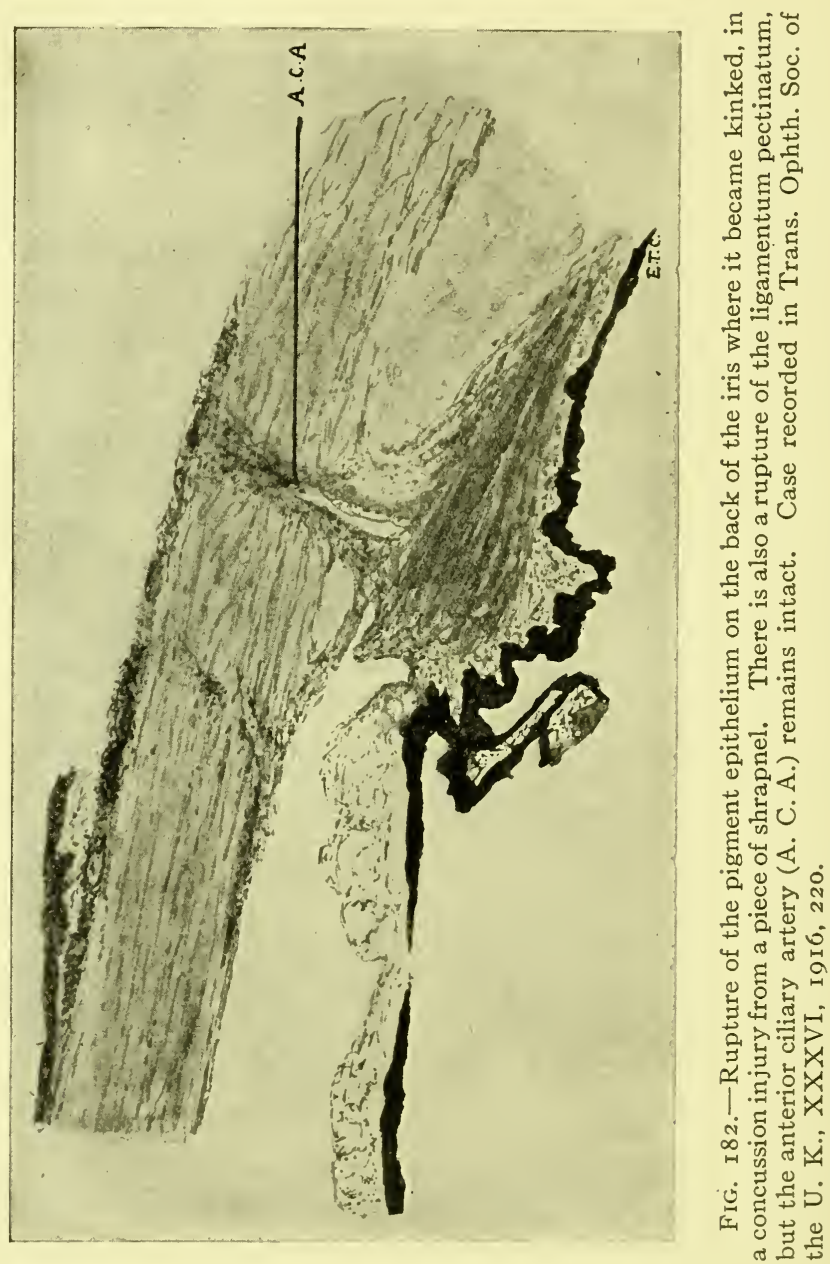

Rupture of the Ligamentum Pectinatum and Ciliary Muscle.-From the lateral dispersion of the aqueous humour a partial or complete rupture of the ligamentum 
pectinatum may result. ${ }^{1}$ In a partial rupture of the ligamentum pectinatum it is those fibres which curve around the angle of the anterior chamber to go to the root of the iris, the so-called pillars of the iris, which become torn. The rupture may also extend into the ciliary muscle

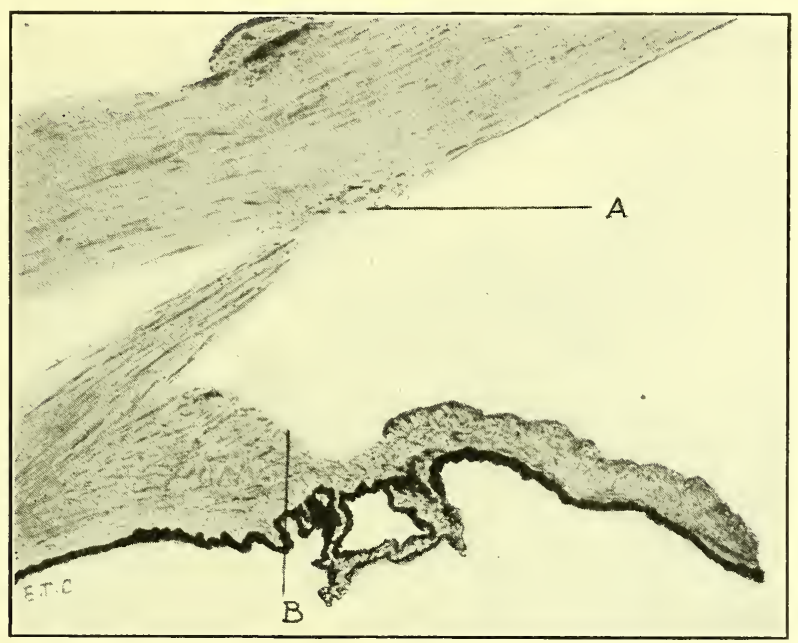

FIG. I83.-Partial rupture of the ligamentum pectinatum and ciliary muscle in the eye of a boy aged six, who was struck with a stone shot from a catapult. $A$ points to the ruptured fibres of the ligamentum pectinatum which were formerly united at $B$, forming the angle of the anterior chamber.

for a short distance separating its circular fibres away from the longitudinal (Fig. I83). Partial rupture of the ligamentum pectinatum and ciliary muscle is a difficult condition to recognise clinically, the only symptom it gives rise to being deepening of the anterior chamber, most marked at the periphery in the locality of the lesion.

When the ligamentum pectinatum is completely ruptured, not only are the pillars of the iris torn through, but also those fibres which give origin to the ciliary muscle (Fig. I84); that structure then loses its most fixed point of attachment, and by its contraction the ciliary body and iris become displaced outward and backward.

${ }^{1}$ Treacher Collins. Trans. Ophth. Soc. of the U. K., XII, I 892, I 80. 
If an anterior ciliary artery, as it passes from the sclerotic to the ciliary muscle, also be torn, there is considerable hemorrhage into the anterior chamber. When the blood has become absorbed a portion of the iris may be found to have disappeared from view, or its pupillary margin in a portion of its circumference may alone be visible.

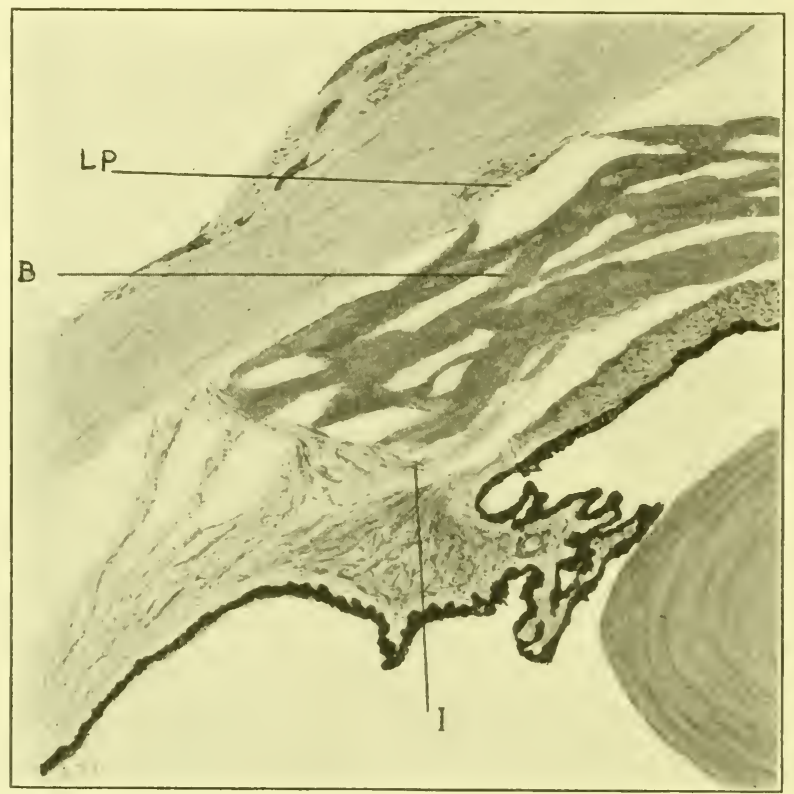

FIG. I84.-Complete rupture of the ligamentum pectinatum. LP points to its torn fibres which were formerly united to the ciliary body at $I$, forming the angle to the anterior chamber; $B$ is blood clot in the anterior chamber.

This disappearance of the iris is due to its retraction by the ciliary muscle, which has become deprived of its most fixed point of attachment. The depth of the anterior chamber also becomes altered, often to varying degrees in different parts.

When the retracted ciliary body remains in contact with the sclerotic they may become reunited by the formation 
of fibrous adhesions ${ }^{1}$ (Fig. I85), sometimes they become widely separated by blood clot and remain ununited.

Concussion Injuries in the Pars Plana of the Ciliary Body.-The pars plana of the ciliary body is a part of the eye which cannot be examined clinically, for this reason. lesions met with in it due to concussion, though not infre-

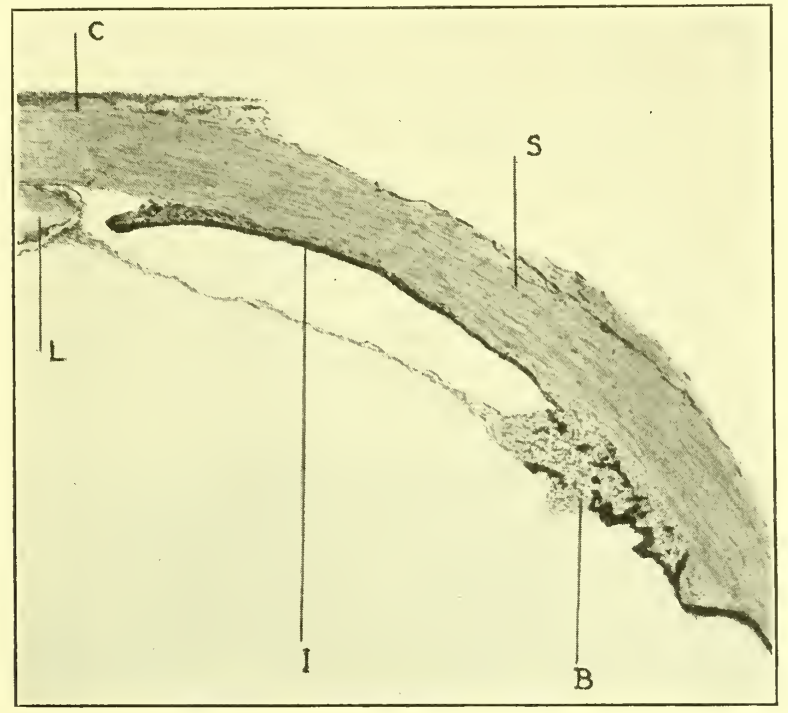

FIG. I 85.-Concussion injury to eye 30 years previous to removal. Complete rupture of the ligamentum pectinatum with retraction of the ciliary body and iris, $I$, which have become readherent to sclerotic $S$. $C$, Limbus; $L$, lens; $B$, ciliary body with a mass of fibrous tissue on its surface extending to the lens.

quent, appear to have escaped description. Pathological examination in different cases has revealed the following changes.

I. Rupture of both the pigmented and non-pigmented layers of epithelium with hemorrhage into the anterior part of the vitreous (Fig. I 87).

2. Detachment of the non-pigmented from the pigmented layer of the pars ciliaris retinæ (Fig. I88).

${ }^{1}$ L. Buchanan. Trans. Ophth. Soc. of the U. K., XXIII, I903.

M. S. Mayou. Trans. Ophth. Soc. of the U. K., XXIX, I909. 
3. Rupture of the retina from the non-plicated part of the ciliary body at the ora serrata (Fig. I 89).

It is the sudden strain to which the firmly adherent structures composing the pars plana of the ciliary body are exposed, when the eyeball is flattened from before backwards, which causes these changes.

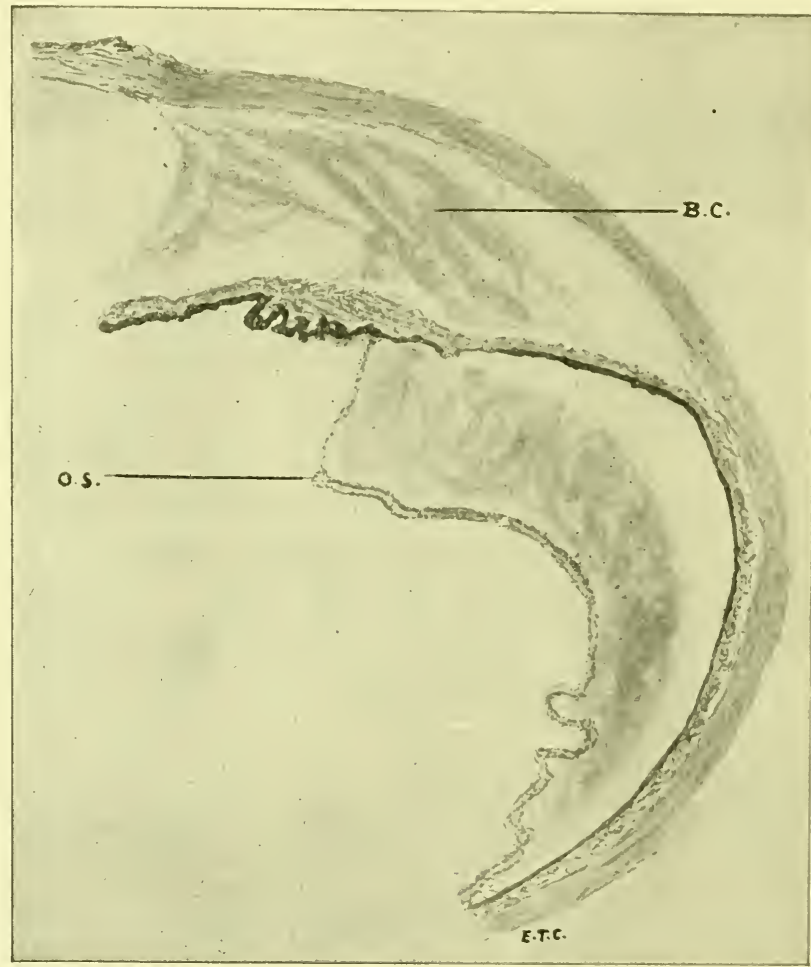

FIG. I 86.-Wide separation of the ciliary body from the sclerotic due to a concussion injury, and the formation of blood clot (B. C.) between them. The retina is also detached together with pars ciliaris retinæ as far forwards as the ciliary processes. O.S. marks the position of the ora serrata. Case recorded Trans. Ophth. Soc. of the U. K., XXXVI, I9I6, 2 II.

Hemorrhage into the vitreous chamber as the result of blows on the eye from blunt objects is of common occurrence. Such hemorrhage may come from the retinal vessels, the vessels of the choroid if its elastic lamina and the 
retina are ruptured, or the ciliary blood vessels. In many of the cases the hemorrhage is situated in the anterior part of the vitreous and can be seen coming from the direction of the ciliary body. For blood to reach the vitreous from the ciliary blood vessels there must necessarily be a lesion

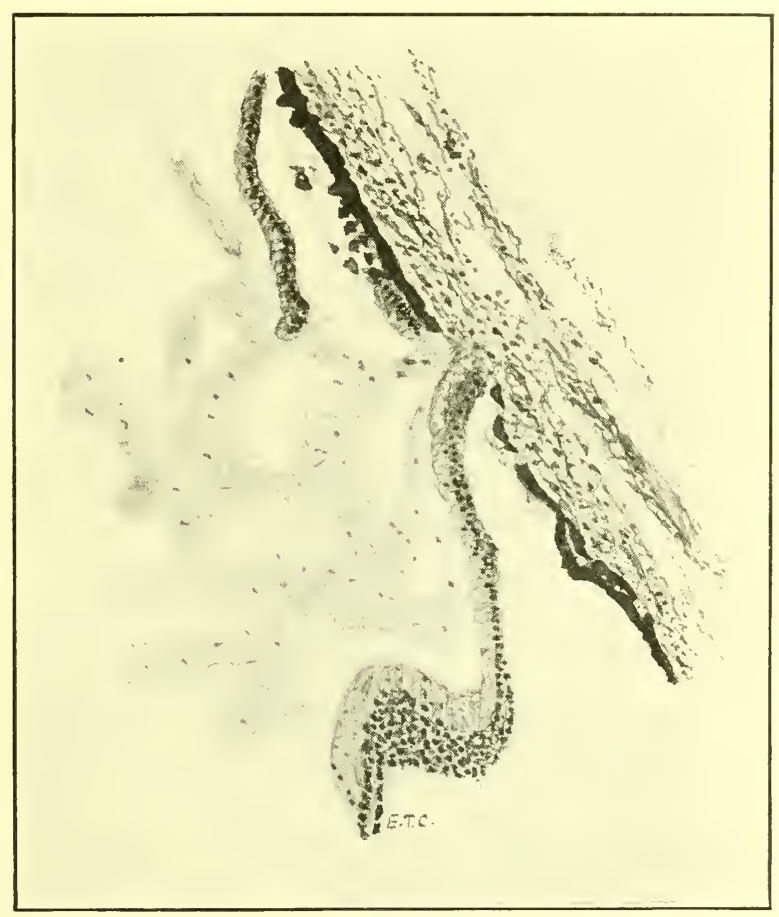

FIG. I 87.- Rupture of the pigmented and unpigmented layers of the pars plana of the ciliary body, with hemorrhage into the vitreous, as the result of concussion.

of the two layers of the pars ciliaris retinæ. Pathologically such lesions are met with in contused eyes in the posterior part of the ciliary body near the ora serrata (Fig. I 87).

Detachment of the non-pigmented layer of epithelium from the pigmented layer is of interest in connection with contusion hypotony (see page 334). A lesion of that character in the secreting area of the ciliary body being a possible cause of diminished tension. 
Rupture of the retina at the ora serrata and its consequent detachment has been met with both clinically and pathologically (Fig. I 89).

Clinically it gives rise to very characteristic ophthalmoscopic appearances. The choroid becomes directly exposed to view beyond the detached retina, as far forwards as can be seen peripherally.

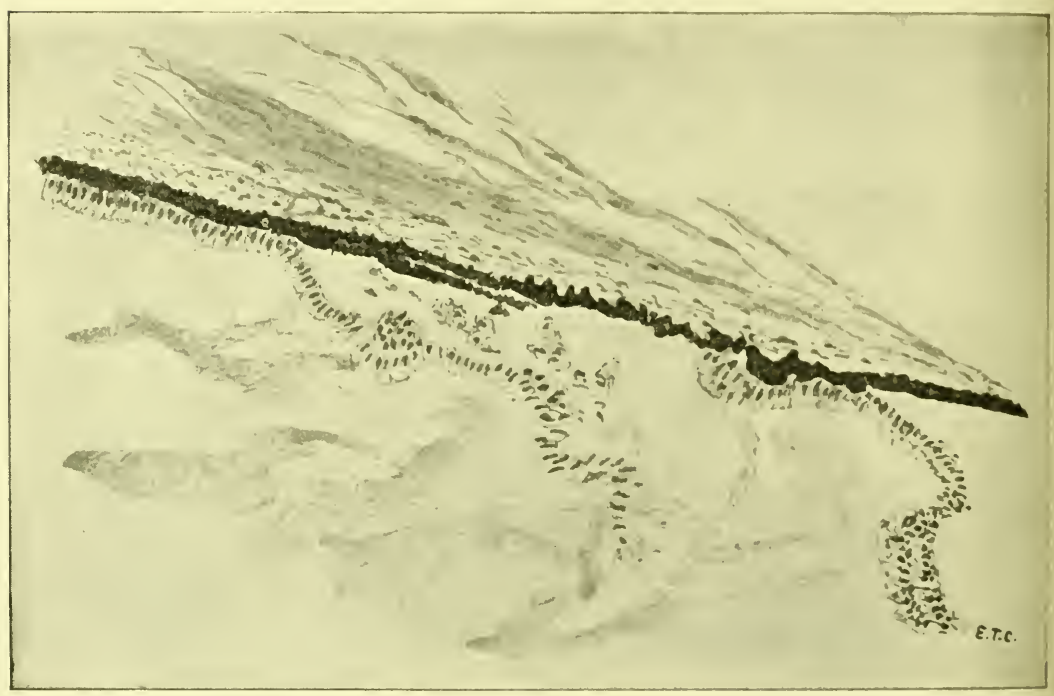

FIG. I88.- Shows the appearances of the pars plana of the ciliary body in an eye which was contused by a blow from the stick of a catapult. The unpigmented layer of epithelium is detached from the pigmented layer. Case recorded Trans. Ophth. Soc. of the U. K., XXXVI, 1916, 216.

Dislocation of the Lens.-A rupture of the suspensory ligament of the lens occurs in concussion injuries, either from the iris being pressed back against it or from undue strain occasioned by the tilting forward of one edge of the lens.

If only a part of the suspensory ligament is torn, the lens becomes drawn away from the injured side, producing lateral dislocation. On the side toward which the lens is displaced the iris becomes pressed forward, and the anterior chamber shallowed; its root sometimes being brought into 


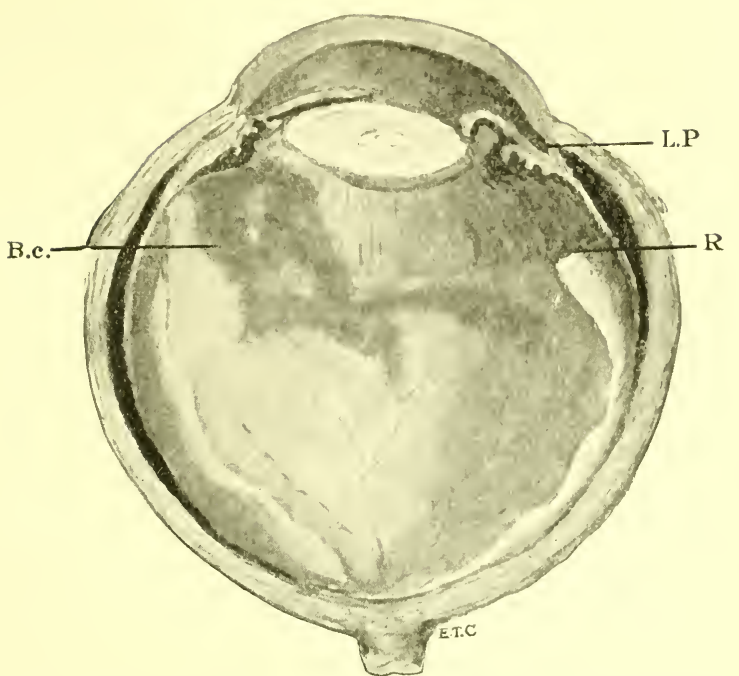

FIG. I89.-Eye injured by concussion with a piece of shrapnel. $L . P$ points to ruptured fibres of the ligamentum pectinatum. The fibres of the suspensory ligament of the lens on that side are also ruptured, so that the lens is displaced to the opposite side.

$R$ points to the free edge of the retina where it has been torn away at the ora serrata.

$B$. c. points to blood clots in the anterior part of the vitreous. Case recorded Trans. Ophth. Soc. of the U. K., XXXVI, гог6, 220.

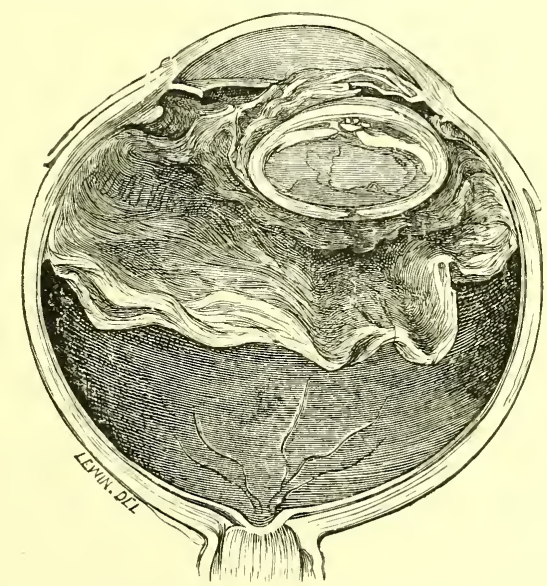

FIG. I90.-The lateral half of an eye with lateral dislocation of the lens, due to a blow from a piece of wood. The vitreous humour passes forward between the side of the lens and the ciliary body into the anterior chamber. The optic disc is cupped as the result of secondary glaucoma. The angle of anterior chamber is closed by the root of the iris which is pressed forward into contact with the cornea. Specimen in the R. Lond. Ophth. Hosp. Museum. 
contact with the back of the cornea, so as to block the filtration area.

On the side away from which the lens is drawn the iris falls back and the anterior chamber is deepened. The withdrawal of support from behind the iris in this position causes it to be tremulous on movements of the eye, a condition termed iridodonesis.

The vitreous humour at the site of the rupture in the suspensory ligament may protrude forward to the back of the iris, and even pass through the pupil into the anterior chamber (Fig. I90).

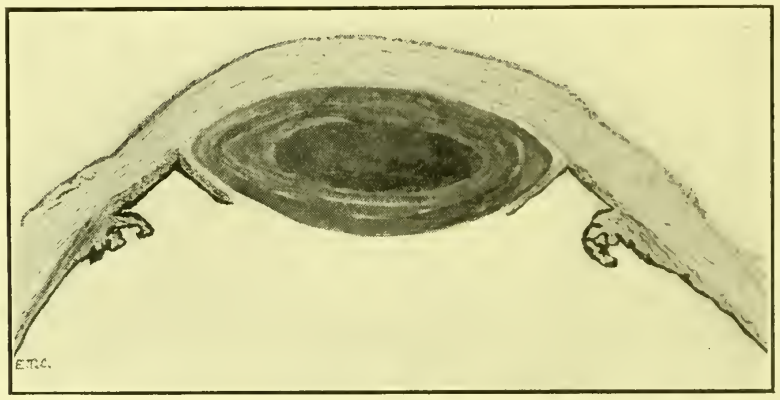

FIG. I9I.-Section through the anterior part of an eye in which the lens has become dislocated into the anterior chamber as the result of blow. Secondary glaucoma ensued.

Occasionally, when the lens is much drawn over to one side, and the iris much pushed back on the other, its pupillary margin will there slip around the edge of the lens to its posterior surface, a partial dislocation of the lens through the pupil being the result. In some cases the backward displacement of the iris round the side of the lens is so extensive that part of it clinically disappears from view; this is called retroflexion of the iris.

When, as the result of the pressure backward of the iris against the suspensory ligament, that structure becomes torn through in its entire circumference, the lens may be dislocated forward into the anterior chamber (Fig. I9I) or 
backward into the vitreous chamber. The former is brought about by the displacement of the periphery of the iris backward, causing such a wide dilatation of the pupil that its margin slips round the border of the lens in all directions. A lens dislocated into the anterior chamber may remain clear for a time, but will ultimately become opaque.

A lens displaced backward may be forced, either between the anterior hyaloid of the vitreous and the retina, or through a rupture in the anterior hyaloid membrane into the vitreous. In the old operation for displacement of cataract (couching) the lens was either "depressed" into the former position or "reclinated" into the latter. A clear lens, dislocated in its capsule, may retain its transparency in the vitreous chamber for many years. On movements of the eye, a lens dislocated into the vitreous, usually floats up so as to allow of its crescentic edge being seen ophthalmoscopically. All forms of dislocation of the lens are liable to be followed by secondary glaucoma, especially lateral dislocation and dislocation into the anterior chamber.

Concussion Cataract.-The flattening of the eyeball from before backward, when struck anteriorly by a blunt object, increases its transverse diameter, so putting suddenly into a state of extreme tension the suspensory ligament and lens capsule. In this way a rupture, not only of the suspensory ligament, but also of the lens capsule may be produced. ${ }^{1}$

The commonest position at which the lens capsule ruptures is its equator; occasionally it bursts at the posterior pole $^{2}$ (Fig. I92). The margins of the capsule at the seat of rupture roll outward; if it is situated at the equator, the lens substance is exposed to the action of the aqueous humour and rapidly becomes opaque. The whole of the lens substance may become dissolved by the aqueous and be removed from the eye after a rupture of the capsule, leaving

${ }^{1}$ J. B. Lawford. Ophthalmic Review, VI, I887, 28 I.

2 Treacher Collins. Trans. Ophth. Soc. of the U. K., XI, I89I, I 26. 


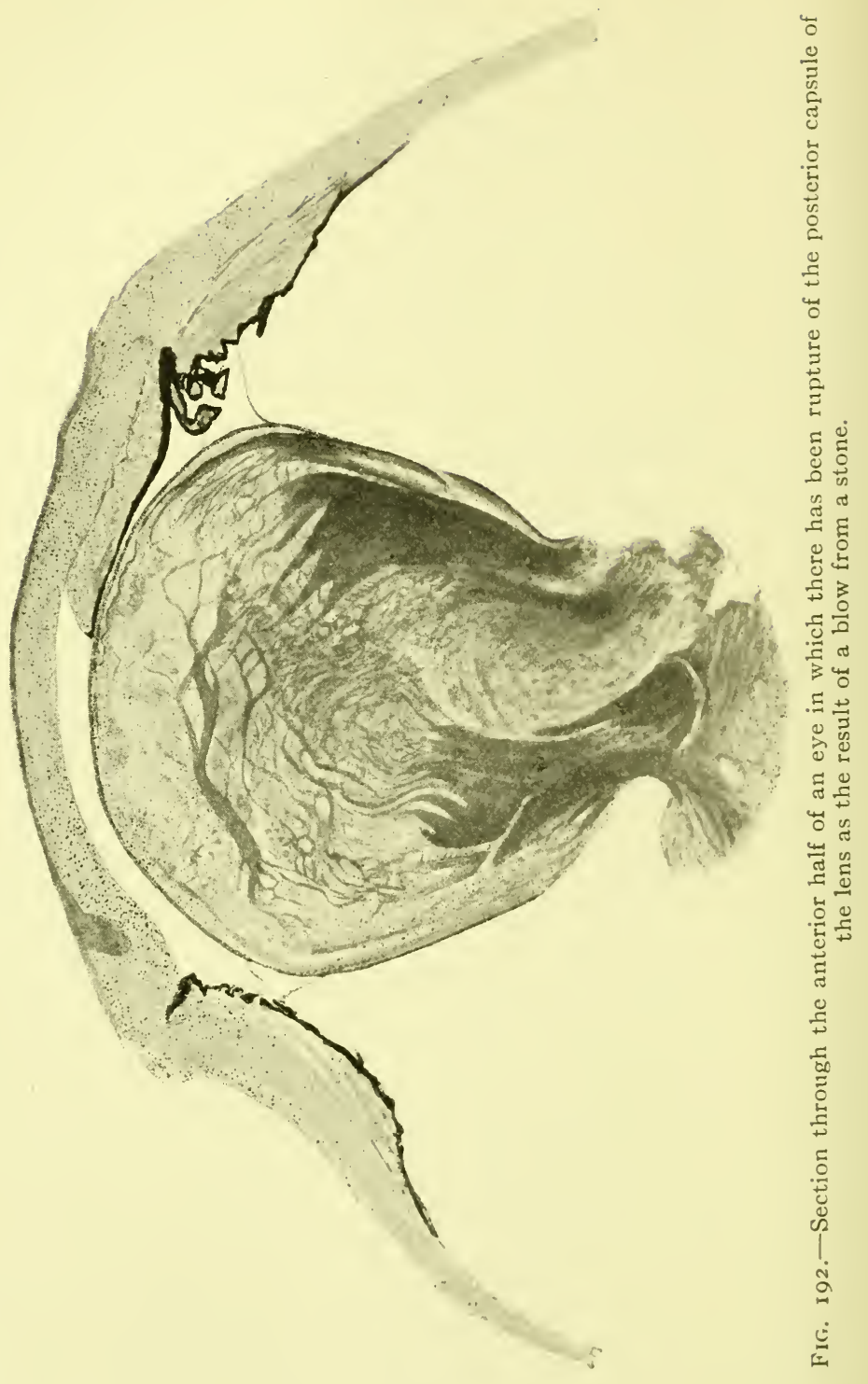


a clear black pupil. The lens matter which protrudes through a rupture in the posterior capsule is exposed to the vitreous humour, and the formation of cataract is then a much slower process.

Annular or disc-shaped opacities, ${ }^{1} 3$ to $4 \mathrm{~mm}$. in diameter. of a brownish hue, are sometimes observed on the anterior surface of the lens after concussion injuries. As a rule they disappear after a few weeks duration. Examination of these opacities by slit-lamp microscopy, definitely shows that they are due to a deposition of pigment granules on the

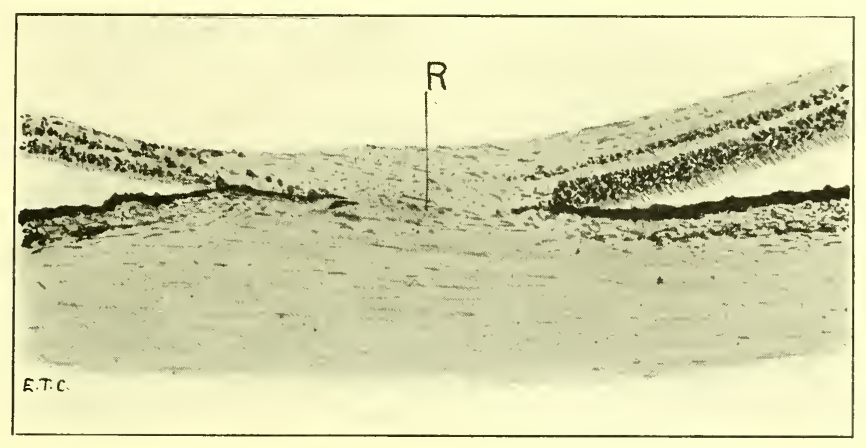

FIG. I93.- Section through the coats of an eye at the seat of a rupture of the choroid. $R$, points to fibrous tissue filling the gap in the choroid to which the retina has become firmly united.

outer surface of the anterior capsule, and not to any disturbance in the lens itself. Whether the granules are squeezed out of the pigment epithelium on the posterior surface of the iris into the pupillary area, or are a deposit of blood pigment, has not yet been certainly determined.

Rupture of the Choroid.-Another effect of flattening the globe from before backward and increasing its transverse diameter in concussion injuries, is to suddenly put on the stretch its two internal coats, the choroid and retina, either or both of which may rupture.

${ }^{1}$ Vossius. Arch. of Ophth., I906.

M. H. Whiting. Trans. Ophth. Soc. of the U. K., XXXVI, I9I6, I67 
Rupture of the choroid is more frequent than rupture of the retina, probably from the presence in it of the comparatively brittle elastic membrane of Burch. It is not, however, only this elastic lamina which tears, but also the pigment epithelium attached to it internally, and all or part of the vascular layers externally. The usual position for such a rupture is in the centre of the membrane, midway between the optic disc and yellow spot, and in a vertical direction. Multiple ruptures may occur more or less concentric to one another. Immediately after the occurrence of a rupture in the choroid, there is so much hemorrhage from its vessels, that the characteristics of the lesion are obscured from view. As the blood becomes absorbed the gap in the choroid, filled with newly formed fibrous tissue, becomes visible ophthalmoscopically as a yellowish-white crescentic slit. The concavity of the crescent is turned toward the optic disc, and its extremities are tapering or forked. Its borders are at first usually free from pigmentation, becoming blackened later from proliferation of the pigment epithelium. In course of time the retina itself, at the seat of rupture atrophies, becomes adherent to and incorporated in the fibrous tissue filling the gap (Fig. I93).

Rupture of the Retina.-As a rule, the retinal vessels where they cross a rupture in the choroid have no break in their continuity, showing that the retina has remained intact. Exceptionally a rupture of the retina occurs in the same position as a rupture of the choroid, and its vessels are seen torn across. In other cases a rupture of the retina may be met with independent of any rupture of the choroid. Slit-like openings are frequently seen in detached retinæ. When the detachment has followed a concussion injury, and shows such a slit-like opening, it is probable that a rupture of the retina has occurred, through which fluid passed from beneath the hyaloid membrane of a shrunken vitreous, separating the retina from the choroid.

Commotio Retinæ. Pigmentation of the Retina. Hole in the retina at the Macula.-In commotio retinæ there is 
produced by a concussion injury of the eye, a milky white opacity of the retina with an ill defined margin, which after a few days duration entirely disappears. Such opacities are most frequentix met with in the macular region, but often in addition to the patch at the macula there is a separate one, of a similar character, in the region in which the blow was inflicted. ${ }^{1}$ When the blow which gives rise to commotio retinæ is received on the cornea the opacity is most marked around the optic disc and macula, the fovea standing out abnormally red in contrast to the surrounding pallor. The vision is usually markedly affected, more especially the central vision, though the field may also be restricted. Generally in the course of a few days the opacity clears and the vision returns, but sometimes after the opacity disappears pigmentation of the retina gradually takes place over the affected area, and the sight remains permanently defective.

Commotio retinæ has been produced experimentally in rabbits' eyes by striking them with an elastic stick. ${ }^{2}$ Pathological examination of these eyes showed an extravasation of blood between the choroid and sclerotic in the region of the opaque retina. Such subchoroidal hemorrhages in an intact eyeball produces ischæmia of the choroid from compression of its vascular layers $^{3}$ (Fig. I94). It is from the capillary layer of the choroid that the outer layers of the retina receive their nutrient supply. Experiments have shown that if a single ciliary artery be divided, in the course of half an hour, an opacity of the retina similar to that met with in commotio retinæ, makes its appearance Microscopically the changes in the retina are most marked in its outer layers; they consist of serous effusion, with molecular haziness and swelling of the tissue elements.

Compression of the choroidal vessels, by the effusion of blood between the choroid and sclerotic, soon arrests the

${ }^{1}$ Haab's "Atlas of Ophthalmoscopy," Figs. 49 and 50.

${ }^{2}$ Berlin. Klin. Monats., XI, I873, 42 .

${ }^{3}$ Treacher Collins. Trans. Ophth. Soc. of the U. K., XXXVII, I9I7, II 2. 
hemorrhage from them. As the blood becomes absorbed, or fluid becomes squeezed from the vitreous body, the circulation in the choroidal vessels is re-established, the changes in the retina disappear, and its function is restored. When, however, the circulation is arrested so long that the bacillary layer becomes damaged, other changes ensue. Atrophy of the rods and cones involves atrophy of the whole of the nerve tissue of the retina, so that it becomes reduced to a mere network of supporting neuroglia. The pigment epithelial cells, which apparently

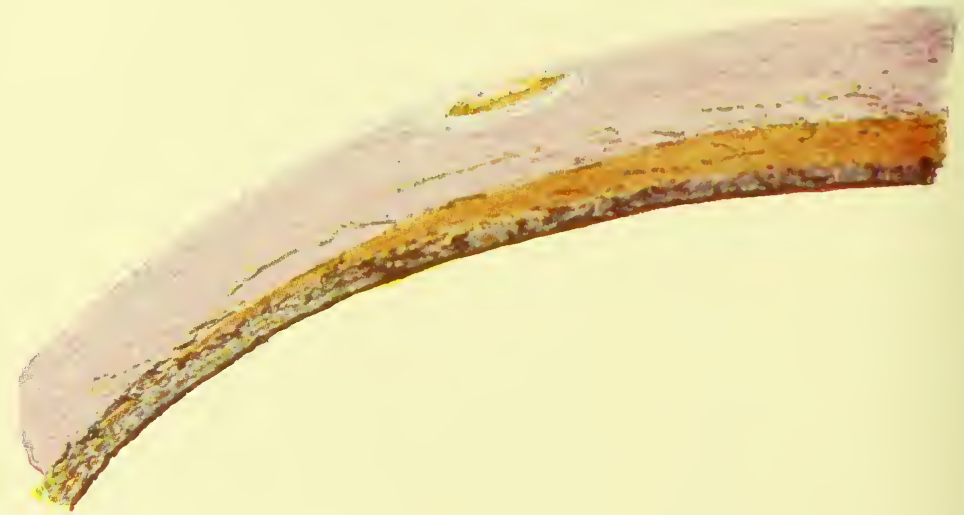

FIG. 194.- Subchoroidal hæmorrhage, the result of a concussion injury, compressing and producing ischæmia of the choroid.

are less easily starved than the nerve elements of the retina, then proliferate and grow forwards into the fibrous network; their migration in that direction being promoted by the phototactic powers which they are known to possess. This atrophy of the nerve elements of the retina and migration of the pigment epithelial cells, account for the symptoms of permanently impaired vision and pigmentation of the retina, which are sometimes produced by concussion injuries.

If the macula is affected, though the impairment of vision is very marked, the amount of pigmentation is often very slight, consisting only of a little mottling or a few fine specks in the centre of the fovea. When other regions 
of the fundus are involved there is a corresponding loss in the field of vision, and in the affected area dense circular or branching patches of pigmentation gradually form. ${ }^{1}$

There are cases in which after a concussion injury of the eye a central permanent defect of vision is met with, and where ophthalmoscopically an appearance suggesting a hole in the retina at the macula is found. ${ }^{2}$ A circular or slightly oval patch of deep red colour is seen surrounded by a grey area. It varies in size in different cases, but is usually about half that of the optic disc. The grey edge appears slightly raised above the level of the red area, and frequently has some irregularities in it. In many of the cases the defect is associated with an absolute central scotoma, but not in all.

A condition apparently quite similar is sometimes seen in association with iridocyclitis or retinal vascular disease, where there has been no injury to the eye.

In many of the cases where it has been met with after an injury, some considerable time has elapsed before its presence was recognised.

It has been found ${ }^{3}$ that the primary condition in these cases is edema of the retina (commotio retinæ in the traumatic cases). The walls of the fluid-containing spaces rupture and larger spaces become formed. Finally rupture of the inner wall of a large space produces the appearance of a hole in the membrane. When a hole involves the whole thickness of the retina at the macula there is necessarily an absolute central scotoma.

\section{Rupture of the Lamina Cribrosa and Evulsion of the} Optic Nerve.-Sudden forcible stretching of the optic nerve, either by displacement forwards of the globe, or traction on the nerve behind in some injury of the orbit,

${ }^{1}$ Jon. Hutchinson (Jun). Trans. Ophth. Soc. of the U. K., IX, I 889, I I6. R. E. Bickerton. Trans. Ophth. Soc. of the U. K., XXIV, I904, 258.

${ }^{2}$ F. M. Ogilvie. Trans. Ophth. Soc. of the U. K., XX, I900, 202.

${ }^{3}$ G. Coats. R. Lond. Oph. Hosp. Rep., XVII, I907, 69. 
may result in rupture of the lamina cribrosa and evulsion of the fibres of the optic nerve from the scleral foramen.

The rupture of the lamina cribrosa takes place at its margin, ${ }^{1}$ where it joins the sclerotic, and may involve a portion or the whole of its circumference (Fig. 195). In the evulsion of the fibres of the optic nerve ${ }^{2}$ they become separated from the retina, and are retracted in the nerve sheath, to varying degrees in different cases. The retinal tissue around the optic disc, and sometimes also the vitreous humour, become drawn backwards for a short distance into the scleral foramen. The retinal blood vessels become torn across, in partial ruptures of the lamina cribrosa it may be only one vessel, in complete ruptures all the vessels are severed and the retina is left entirely avascular. Usually the rupture of the retinal vessels causes so much vitreous hemorrhage that the ophthalmoscopic changes in the fundus are for a time obscured from view. In some cases the blood clot in front of the optic disc becomes the seat of a mass of fibrous tissue, seen ophthalmoscopically as a white raised area in that region. The real nature of the lesion can then only be diagnosed by the complete loss of sight and the absence of the retinal vessels. When the hemorrhage in the vitreous clears away without the formation of fibrous tissue, if the lamina cribrosa is ruptured in its entire circumference and the whole of the nerve is evulsed, the optic disc is replaced by an apparently bottomless pit, and there is a complete absence of retinal vessels. If only a portion of the lamina cribrosa is torn across, there is a localised pit in the optic disc in that situation, and a loss of continuity in one or more of the retinal vessels.

In these cases it seems that the sheath of the nerve being elastic stretches without tearing, but that the less elastic nerve fibres contained within it give way.

${ }^{1} \mathrm{~W}$. Lang. Trans. Ophth. Soc. of the U. K., XXI, I90I, 98.

${ }^{2}$ Lister and Hine. Trans. Ophth. Soc. of the U. K., XXXIX, 1919, 190. 
Concussion Hypertony and Hypotony.--Increase of tension following concussion injuries of the eye is brought about by obstruction to the exit of the intraocular fluid, which may be due to dislocation of the lens, either laterally or forwards into the anterior chamber.

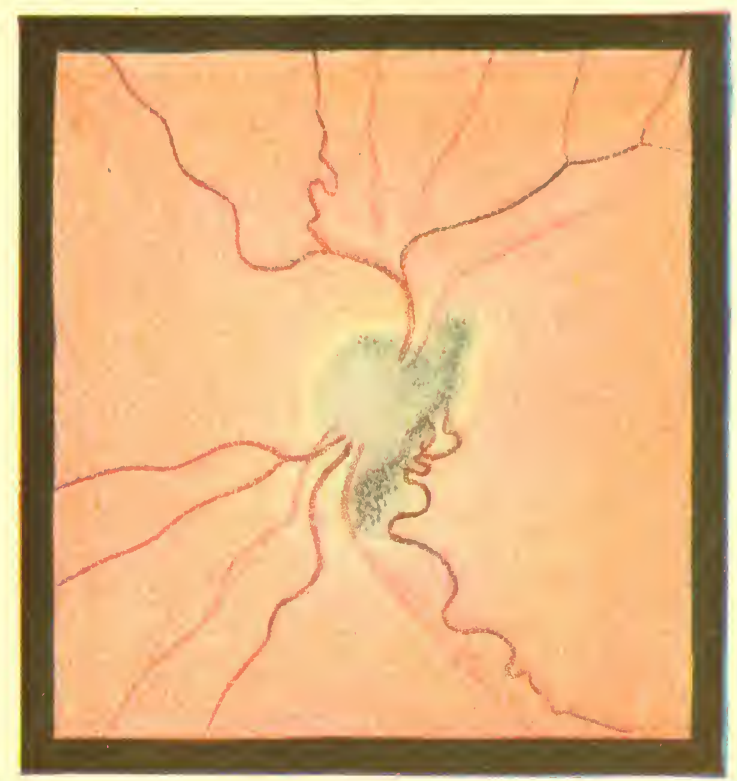

FIG. I95.-Rupture of lamina cribrosa in the lower and outer part of the optic disc, due to a blow from a bat in a girl aged I2. Case recorded in Trans. Ophth. Soc. of the U. K., XXXIV, I9I4, I90.

In lateral dislocation the root of the iris is pressed forwards into contact with the cornea at the angle of the anterior chamber, on one side by the displaced lens and on the other by the displaced vitreous body. A lens displaced into the anterior chamber may so completely block the pupil as to prevent the passage of fluid through it; the accumulation of fluid in the posterior chamber will then press forwards the root of the iris into contact with the cornea, thereby effectually obstructing the exit of the aqueous humour from the eye. 
Diminished tension the result of concussion is often of only short duration, but may be permanent; it is due either to (I) diminished secretion of intraocular fluid, or to (2) increased facilities for its exit. ${ }^{1}$

I. Diminished secretion occurs when the blood supply to the ciliary body is cut off by rupture of one or more of the anterior ciliary arteries. Also probably when the secreting epithelium becomes damaged, as by separation of the nonpigmented from the pigmented layers, or by rupture of the pars plana (see page $35 \mathrm{I}$ ).

It is possible that a traumatism might produce some inhibition in function of the nerves of the ciliary body. Though there is no evidence of the existence of any special secreting nerves, the presence of vaso-constrictor fibres to the intraocular vessels has been proved by manometric measurements. A paresis of these fibres may lower the blood pressure in the ciliary body and so decrease its secretion and lower the intraocular tension.

2. Increased facilities for exit of intraocular fluid, as the result of concussion, may be due to enlargement of the normal channels of exit or the formation of new ones.

The lateral displacement of the aqueous humour, produced by a blow on the cornea from a blunt object, tends to expand the angle of the anterior chamber, and may possibly force more fluid through its lymph spaces than escapes under normal circumstances. The temporary decrease of tension which follows pressure on the eye in the use of Schiötz tonometer, or from the use of a compressive bandage, may be best explained in this way.

A rupture of the ligamentum pectinatum, or partial internal rupture of the sclera in the region of the canal of Schlemm, as the result of concussion; would form new channels of drainage for the intraocular fluid and so tend to cause a permanent diminution of tension.

Intraocular Hemorrhage.-Intraocular hemorrhage the result of concussion injuries is of frequent occurrence and is

1 Treacher Collins. Trans. Ophth. Soc. of the U. K., XXXVI, I916, 204. 
found in various situations in the eye, for the changes produced by intraocular hemorrhage see page 252 .

Birth Injuries.-Injuries to the eyes from compression during parturition are of more frequent occurrence than is generally supposed. Several conditions which have been regarded as developmental defects are probably attributable to them.

One observer ${ }^{1}$ has noted the following lesions as due to pressure by forceps: excoriation, ecchymoses, edema, exophthalmos, fracture of the orbit, paralysis of the lid muscle, corneal troubles, retinal hemorrhages, optic atrophy, paralytic strabismus and hypermetropic astigmatism.

Intraocular hemorrhage, most frequently retinal, may occur in cases of normal labour where no instruments have been used. Probably in some cases it is due to increased blood pressure apart from any direct pressure on the eye.

The corneal lesions ${ }^{2}$ may result in temporary or permanent opacity. The temporary opacities which are of comparatively frequent occurrence are due to edema. In the cases with permanent opacity there is a rupture of Descemet's membrane, and sometimes of the fibrous lamellæ adjoining it. The rupture is generally linear and vertical; it is undoubtedly caused by the direct pressure of the instrument on the eyeball. Following on the rupture there is edema of the cornea and often inflammatory reaction.

Paralysis of the sympathetic nerve on one side with failure in the development of pigment in the stroma of the iris of the eye supplied by it, causing heterochromia iridis, has also been attributed to injury at birth. ${ }^{3}$

\section{iii. Wounds}

Wounds of the Cornea.-A wound of the cornea may vary in extent, from an abrasion of its epithelium to a perforation of its whole thickness.

${ }^{1}$ H. Truc. Ann. d'oculist., CXIX, I898, I6r.

${ }^{2}$ W. E. Thomson and Leslie Buchanan. Trans. Oph. Soc. of the U. K., XXIII, 1903, 3I2.

${ }^{3}$ M. S. Mayou. Trans. Ophth. Soc. of the U. K., XXX, I9Io. 
Perforating wounds may be simple or compound, the former only involving an escape of the aqueous humour, the latter being associated also with some entanglement or adhesion of the intraocular contents.

In a case of simple abrasion of the epithelium repair is soon affected without any nebula being left behind. Karyokinesis in the surrounding cells commences about twentyfour hours after the receipt of injury, the newly formed cells spreading laterally over the denuded area.

The value of the surface epithelium, as a protection to the substance of the cornea from the deleterious action of external influences, is shown by the risks to which it is exposed when an abrasion has taken place.

When a drop of a solution of fluorescine is instilled into a healthy eye with the epithelium intact no staining of the cornea takes place. When, however, there is an abrasion of the epithelium present the fluorescine is able to penetrate to the fibrous tissue of the cornea and stain it green. This staining lasts only a short time, not producing any deleterious effect. If a solution of subacetate of lead (lead lotion) is dropped into an eye with abraded corneal epithelium, the white carbonate of lead becomes deposited in the substantia propria at the denuded area, producing a permanent white porcelaine-like opacity.

In cases of obstruction of the lacrymal duct the lacrymal sac may be inflamed and distended with discharge containing pneumococci. This germ-laden fluid will from time to time regurgitate from the distended sac over the surface of the cornea; as long as its epithelium is intact it remains unharmed. Should, however, an abrasion of the epithelium occur the pneumococci will attack the substantia propria and give rise to an infective ulcer.

In rare cases the new epithelium which forms over an abraded area fails to acquire its necessary firm attachment to Bowman's membrane. In the course of a few weeks it will become raised in the form of a vesicle, which on rupturing leaves a fresh abrasion, one form of so-called bullous 
keratitis. In the formation of the vesicle the filaments of the corneal nerves, which break up and terminate in the epithelium, become stretched and the patient complains of much pain. This disappears as soon as the vesicle ruptures and the tension on the nerve filaments is relieved. The formation of such vesicles is liable to recur, causing relapsing attacks of pain.

Vesicles in the corneal epithelium, due either to an abrasion or some other cause, may rupture and lead to the development of filamentary processes. Such a condition is often spoken of as filamentary keratitis though it is not necessarily accompanied by inflammation (see page 29I).

In a simple wound of the anterior surface of the cornea, a gap is formed due to retraction of the anterior elastic lamina and the anterior laminæ of the substantia propria. This soon becomes filled with epithelium, which has proliferated from the margins, and polymorphonuclear leuckocytes. Beneath this primary plug of cells others, produced by the proliferation of the corneal corpuscles, subsequently appear and develop into fibrous tissue. This new formed fibrous tissue gradually forces up and reduces in thickness the primary plug of epithelium, until its becomes again equal to that normally covering the cornea. No new formation of the anterior elastic lamina takes place, and the newly formed fibrous tissue has not the regular laminated arrangement of the normal lamellæ of the cornea. It is on account of this that a permanent opacity becomes formed, termed a nebula or leucoma according to its density.

In peripheral wounds of the cornea there is an extension of blood-vessels, either conjunctival or scleral, into the affected area, which aid in the development of the granulation tissue which fills the wound.

In a simple perforating wound of the cornea the anterior and posterior elastic laminæ with the fibrous tissue layers immediately adjacent to them retract, so that when the lips of the wound come together again, there is contact 


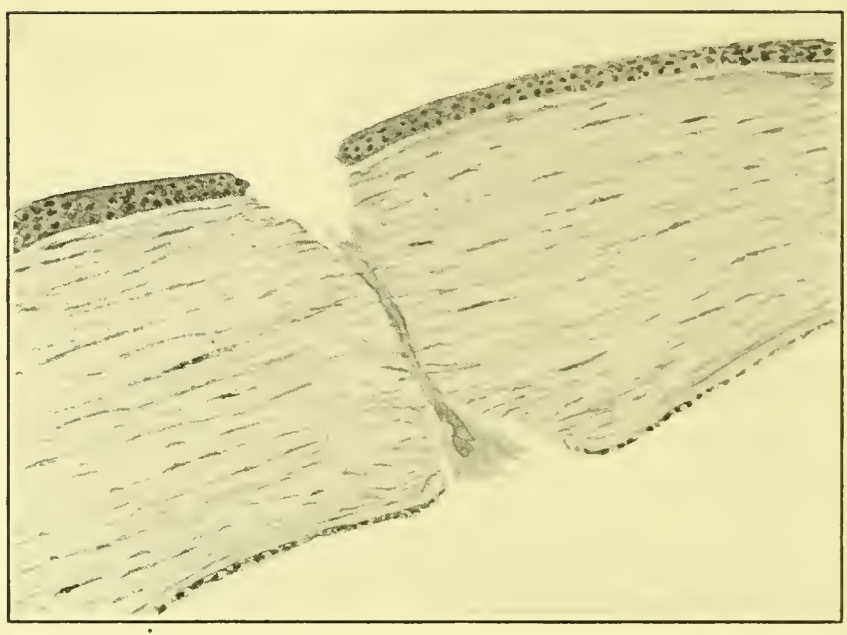

FIG. I96.-Recent perforation wound of the cornea showing retraction of its lips anteriorly and posteriorly. A coagulum has formed between the margins of the wound.

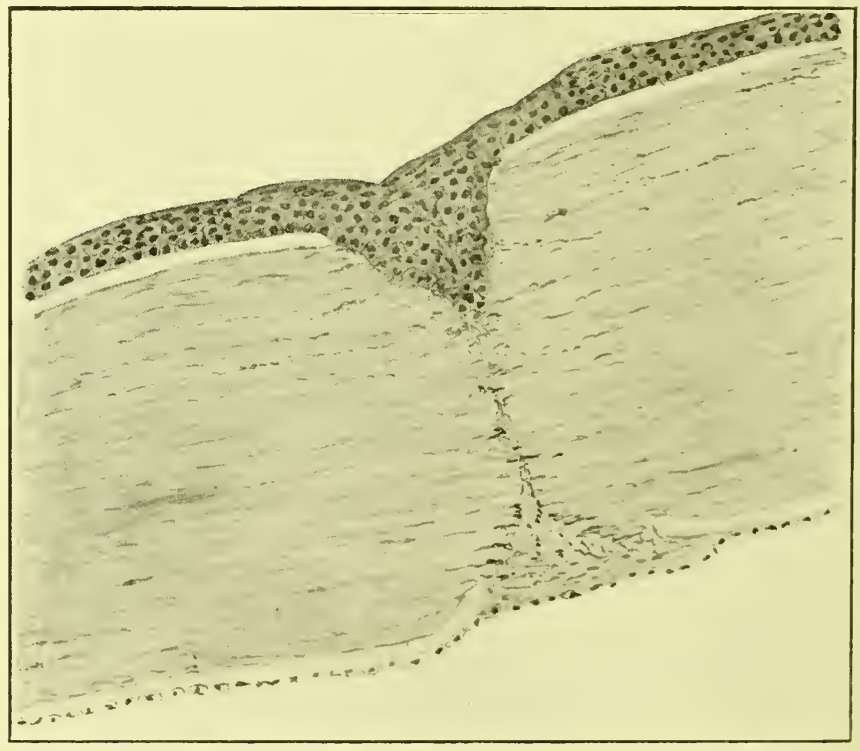

FIG. I97.- Perforating wound of the cornea commencing to heal. Epithelium filling up the wedge-shaped gap on the anterior surface, and organising fibrous tissue covered by endothelium that on the posterior surface. 
of the central layers of the substantia propria and wedge shaped gaping spaces left anteriorly and posteriorly (Fig. 196).

Through the gap in Descemet's membrane the fluid in the anterior chamber permeates, causing swelling of the lips of the wound. The newly formed fluid in the anterior chamber is more albuminous than the normal aqueous humour. From this albuminous fluid, or from serous exudate from the corneal tissue, a fibrinous coagulum is formed, which agglutinates the edges of the wound and serves as a matrix for the subsequent development of cicatricial tissue.

The wedge-shaped gap anteriorly soon becomes filled with a plug of epithelium, which extends into it from the sides (Fig. 196).

The wedge-shaped gap posteriorly becomes filled with fibrinous coagulum, over the posterior surface of which the divided endothelium of Descemet's membrane gradually extends. When this is again intact any further filtration of aqueous humour into the wound ceases.

The cells of the cicatricial tissue which replace the fibrinous coagulum are derived from the corneal corpuscles. As this cicatricial tissue develops it extends upwards into the anterior wedge-shaped area, gradually reducing the thickness of the plug of epithelium, and downwards into the posterior wedge-shaped area, which ultimately it fills right down to the endothelium.

The two margins of the posterior elastic lamina, after a perforating wound, become more retracted than those of the anterior, besides being retracted they are usually slightly everted forwards. Though these two edges never reunite, in course of years a new hyaline layer may be formed which bridges across the gap between them; this new hyaline membrane is produced, like the normal Descemet's membrane, as a kind of secretion from the endothelial cells lining it (Fig. I98).

Opacity of the cornea, due to imbibition of fluid, is not uncommonly met with after extraction of cataract, either 
in the form of a greyish haze mapped out into a number of squares by dark lines, or of vertical streaks extending down from the wound. The latter is due to rucking of Descemet's membrane by the swollen tissue, it is often inaccurately spoken of as striated keratitis, it is not an inflammatory condition and should be termed striated

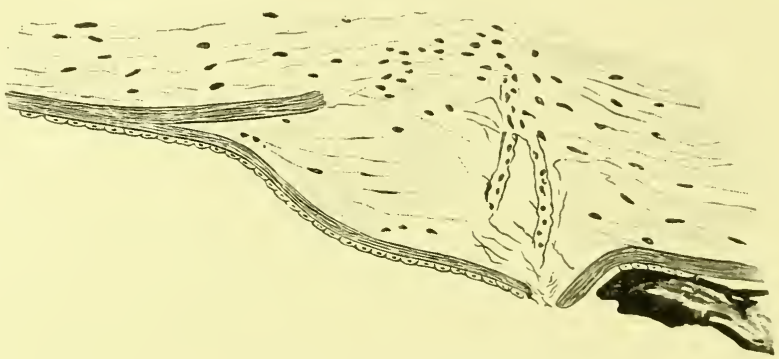

FIG. I98.- Shows a new formation of hyaline membrane lined by endothelia cells on the posterior surface of the scar in the cornea of an incision made $21 / 2$ years previously for iridectomy. The divided ends of Descemet's membrane are separated by cicatricial tissue, that on the left side turning forward and that on the right backward. Case recorded in R. Lond. Ophth. Hosp. Reps., XIV, I 896,3 Io.

opacity. As the endothelium at the back of these wounds usually soon reforms, and then checks any further filtration of aqueous into them, the opacity to which it gives rise is generally of only temporary duration.

Wounds at the sclero-corneal margin in which a piece of Descemet's membrane is cut out, as in sclerectomy and sclero-corneal trephining, or where the divided edges of that membrane become very widely retracted, as in some forms of sclerotomy, never get their posterior surfaces recovered by endothelial cells and persist, therefore, as permanently filtering cicatrices.

If the anterior margins of a corneal wound are widely separated the epithelium at its borders may spread round the lips of the wound, but not become of sufficient thickness to bridge across the gap, so that a groove is for a time left on the surface.

Should the lips of a corneal wound fail to become agglutinated by the temporary coagulum, or having become 
agglutinated, should they subsequently become reopened, there is a tendency for the surface epithelium to extend inwards more deeply than usual. It may even extend through the whole length of the wound and pass into the anterior chamber (Fig. I99). In some cases the whole of the anterior chamber has become lined by epithelium proceeding down from the surface in this way.

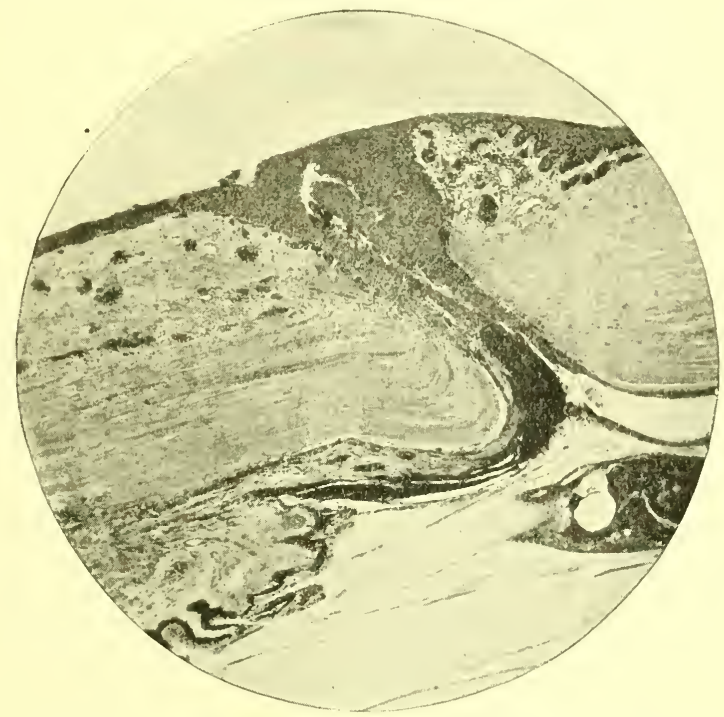

FIG. I99.- Shows an extension down of the surface epithelium along the lips of an extraction wound. It passed into, and completely lined the anterior chamber. The condition resulted in glaucoma, which necessitated the removal of the eye. From a photomicrographtake by E. Collier Green.

Delayed formation of endothelium over the posterior surface of a corneal wound, allows of a continued filtration of aqueous humour into it. Such filtration gives rise to swelling of the lips of the wound, and opacity from distention of the lymph spaces. In clean incised wounds the endothelium soon reforms, and there is but slight opacity or swelling at their margins. In jagged wounds, where the lining endothelium is much damaged, extensive swelling takes place and a grayish opacity forms. In a wound producing a $\mathrm{V}$-shaped flap there is considerable opacity, 
and the flap becomes so swollen that when it is fitted into position it is raised to some distance above the level of the surrounding cornea.

In complicated wounds of the cornea there may be adhesion or entanglement of the iris, lens capsule, vitreous humour, or retina.

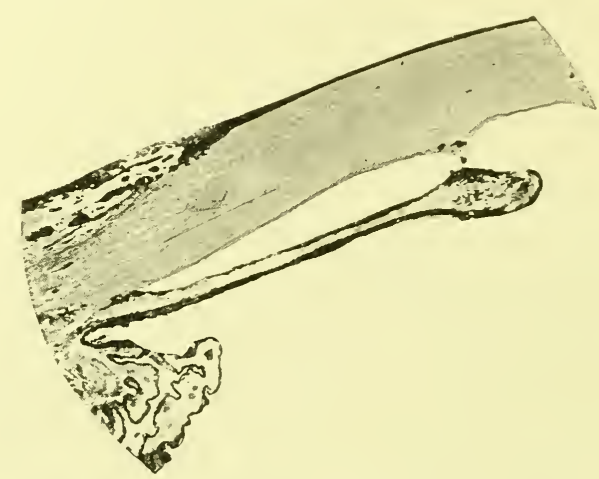

FIG. 200.-Section showing an anterior synechia of the iris near its pupillary border, which resulted from a wound of the back of the cornea with the point of a keratome in the performance of an iridectomy for glaucoma, on an eye with a shallow anterior chamber. Case recorded R. Lond. Ophth. Reps., XIII, I891, 193.

If the iris lies in contact for a short time with the back of the cornea at the seat of a wound, a fibrinous exudate is thrown out which brings about adhesion of the two structures, an anterior synechia (Fig. 200). This at first simple adhesion becomes in time transformed into a cicatrix with atrophy of the iris stroma.

The presence of the adherent iris arrests the extension inward of the endothelium on the back of the cornea across the gap in Descemet's membrane. Often it extends at the margins of the wound on to the anterior surface of the iris, and may there secrete a hyaline layer like the posterior elastic lamina. ${ }^{1}$

Entanglements of the iris in a corneal wound may be divided into three classes:

I. Where the cut end of a piece of iris lies in a gap in the cornea.

1 Treacher Collins. Erasmus Wilson Lectures. Lancet, Feb. I7, I900. 
2. Where a fold of iris prolapses through the whole thickness of the cornea.

3. Where a fold of iris prolapses between the posterior layers of the cornea only.

I. The cut end of a piece of iris lying between the lips of a corneal wound causes more cell exudation and formation of granulation tissue than in an uncomplicated wound.

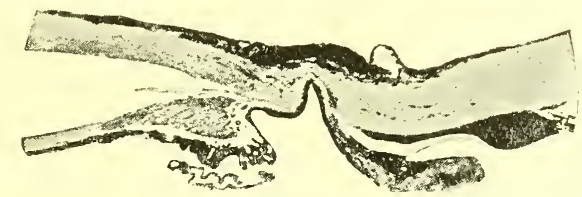

FIG. 20I.-Section showing the prolapse of a knuckle of iris into a wound at whe sclero-corneal margin. The eye had had an iridectomy performed for glaucoma a fortnight previous to its removal. The prolapse was at one margin of the coloboma. It represents the first stage in the formation of a cystoid cicatrix.

Ultimately the stroma of the entangled iris atrophies, its pigment epithelium remaining incorporated in the cicatricial tissue.

2. When a fold of iris prolapses through the whole thickness of the cornea an obstruction is imposed to the reunion of the fibrous tissue on the two sides (Fig. 20I). A fibrinous exudate, which is afterward transformed into fibrous tissue, first agglutinates it to the sides of the wound, the stroma of the iris atrophies, and its pigment epithelium alone remains. A piece of iris left protruding through a wound develops granulation tissue on its surface. Over this granulation tissue the epithelium from the sides of the wound spreads, and it itself becomes transformed into fibrous tissue. If the wound is at the periphery of the cornea the prolapsed iris may be covered by conjunctiva to which it will become adherent. A permanent gap will then be left in the outer wall of the eyeball covered by conjunctiva and lined by the pigment epithelium of the iris; this as the result of intra-ocular tension will tend to stretch and expand, forming what is termed a cystoid cicatrix. As the scar stretches, gaps form in the continuity of the pigment epithelium lining it, and the aqueous humour 
is then able to filter into the subconjunctival tissue, giving to it an edematous appearance (Fig. 202). ${ }^{1}$

3. Where a fold of iris prolapses between the posterior layers of the cornea only, a bulging scar, not a cystoid scar, is produced. The anterior layers of the cornea unite in the usual way and a permanent gap in the posterior layers lined

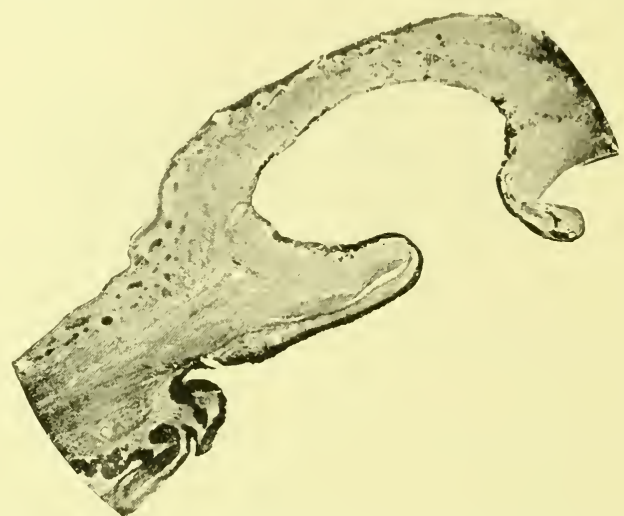

FIG. 202.- Shows a section through a fully developed cystoid cicatrix, which occurred in an eye lost from glaucoma following extraction of cataract. The gap in the fibrous tissue at the corneal margin is lined by the iris, much stretched and atrophied, and with many breaks in the pigment epithelium on its posterior surface. The subconjunctival tissue around the gap is much swollen due to the filtration of aqueous humour into it.

by iris is formed. As the stroma of the iris atrophies this lining of iris comes to consist only of pigment epithelium. A scar of this description like the one previously described is a weak spot in the walls of the globe, and may expand before the intraocular tension, especially if it becomes abnormally high. It, however, never expands to such an extent as to become translucent like a cystoid scar. Nor does it allow of filtration through it of the aqueous humour. It tends, in bulging, to alter considerably the curvature of the cornea in one of its meridians, and so gives rise to marked astigmatism.

An adhesion of the lens capsule to a wound of the cornea may occur while the capsule remains intact or after it has been opened. An entanglement of the lens capsule in a

${ }^{1}$ Treacher Collins. Royal Lond. Ophth. Hosp. Reps., XIII, I89I, I7I. 
corneal wound can only take place after it has been perforated.

Anterior synechiœ of the lens capsule besides occurring after accidental injuries are sometimes met with after opera-

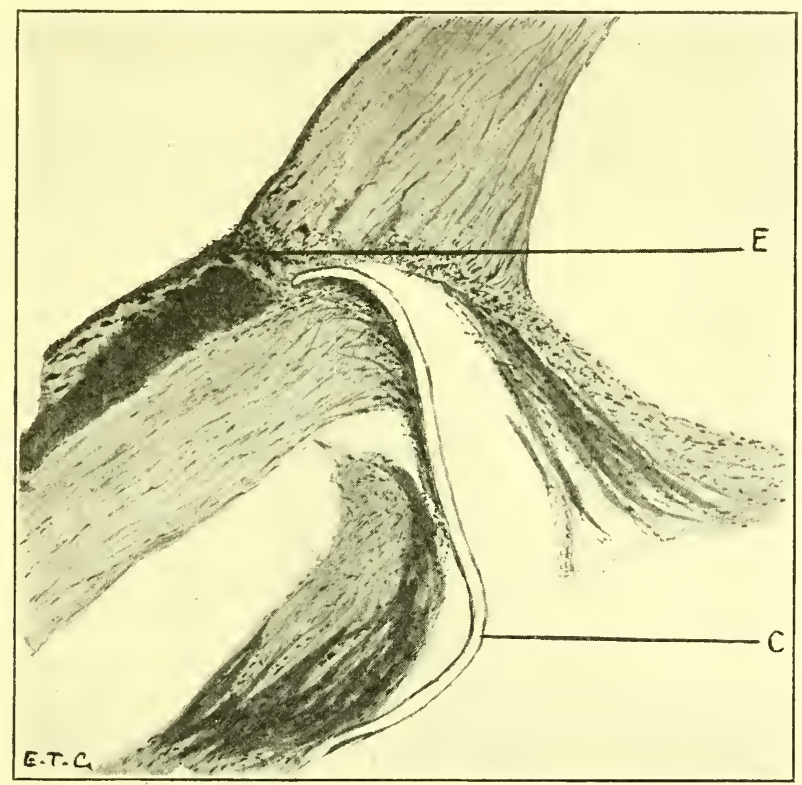

FIG. 203.-Eye removed five months after extraction of cataract on account of serous iritis and increased tension. Drawing shows a section through the extraction wound, the external part of which has alone united. E points to a downgrowth of surface epithelium. The peripheral portion of the anterior capsule of the lens $C$ lies in the widely gaping posterior portion of the wound, the lips of which show much cell infiltration.

tions. The delay which not infrequently occurs in the reformation of the anterior chamber after iridectomy for glaucoma, may, for some time leave the lens lying in contact with the cornea at the seat of the incision. The coagulum which unites the edges of the wound may then unite the lens capsule to the cornea, at the gap formed by the retraction of Descemet's membrane. Later a permanent cicatricial adhesion between the two structures becomes established. 
An adhesion of the lens capsule to the back of the cornea causes a considerable advance in the position which it normally occupies in the interior of the eye. This advance in its position draws forward the iris, shallowing the anterior chamber and narrowing its angle. Adhesion of the lens capsule to the back of the cornea after extraction of cataract sometimes gives rise to glaucoma (Fig. 206).

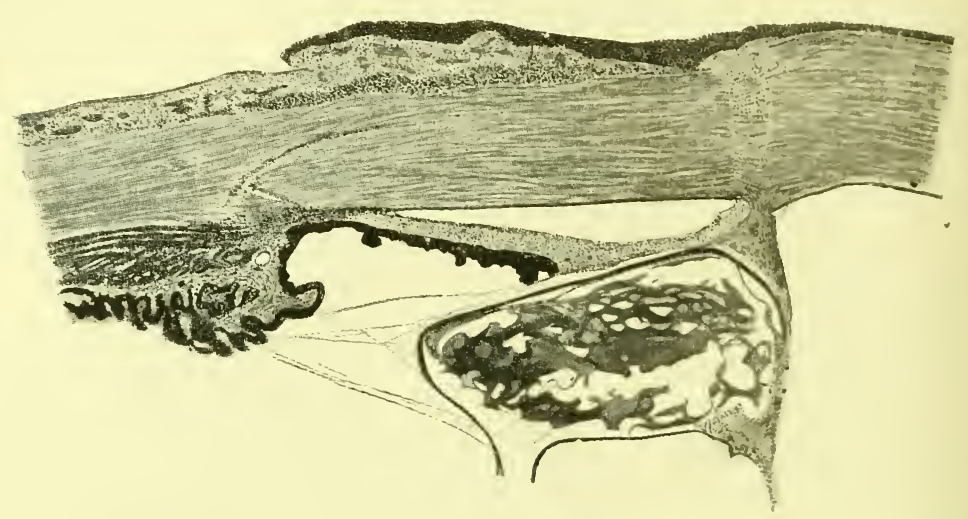

FIG. 204.- Section showing an adhesion of the lens capsule to the posterior surface of an extraction scar. Increased tension ensued, and ultimately destroyed the sight of the eye. Case recorded Trans. Ophth. Soc. of the U. K., X, I890, I24.

A piece of lens capsule entangled in a corneal wound acts very similarly to a foreign body. It delays healing, sometimes for months, and excites in the tissue around considerable cell accumulation (Fig. 203). Close to the capsule giant cells are found of the Langhan's type, also epithelioid cells and outside them a zone of lymphocytes. The delay in the healing of the wound renders it very prone to infection. The drag which the entangled capsule keeps up on the ciliary processes causes congestion of them. Hence eyes with wounds of the cornea complicated with an entanglement of the lens capsule often become affected with iridocyclitis.

An entanglement of the vitreous in a corneal wound may take place when, after a perforation of the cornea, the lens has escaped in its capsule or been displaced (Fig. 205). 
An adhesion of the vitreous to the cornea, even more than an adhesion of the lens capsule, advances the position of the iris, shallowing the anterior chamber and restricting its angle. An adhesion of the anterior hyaloid of the vitreous to the cornea, after an extraction of cataract in its capsule, has caused glaucoma.

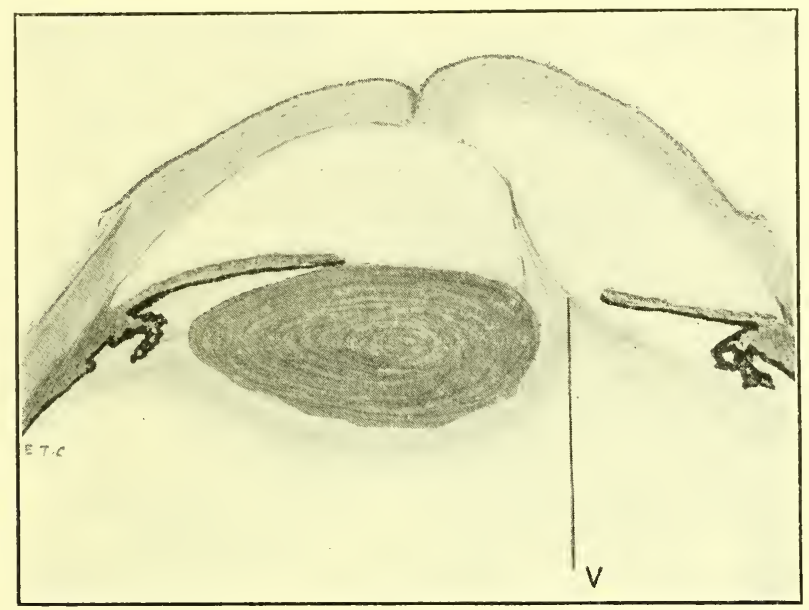

FIG. 205.-Wound of the cornea and suspensory ligament of the lens. Im perfect union owing to the vitreous, $V$, having prolapsed and become adherent to the wound. The eye was removed one month after the injury for chronic cyclitis.

An anterior synechia of the vitreous may also occur after removal of the lens when an opening has been made through the two layers of its capsule. Such entanglements have been met with after operations of needling of the lens capsule, a tag of vitreous being carried into the needle puncture in the cornea on the withdrawal of the instrument. If it protrudes through the puncture, it forms a filamentary tag on the surface of the cornea which will stain with fluorescine. It may remain and delay healing for a considerable time, forming a track along which infective organisms can gain entrance to the eye.

In eyes where there has been an extensive escape of vitreous through a corneal wound; and where the lens has escaped or been displaced, the retina may be detached and 
forced forward so as to prolapse or become adherent to the wound.

Operation wounds and perforating wounds which pass into the periphery of the anterior chamber, owing to the

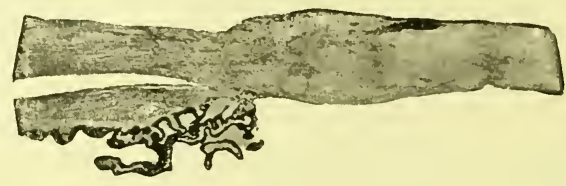

FIG. 206.- Section showing the cicatrix left after an incision through the cornea with a keratome for iridectomy for glaucoma. The increase of tension had not been of long standing and the iris was removed right up to the ciliary body.

obliquity of the line of junction between the cornea and sclerotic, are wounds of both those structures and also of conjunctiva. In the operation of anterior sclerotomy the incision passes partly through the cornea and partly

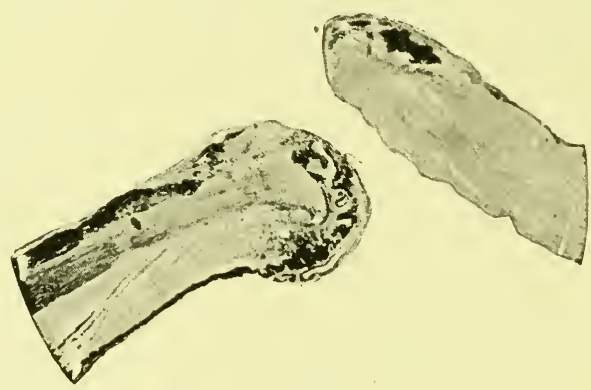

FIG. 207.- Shows a gaping wound at the sclero-corneal margin. The cornea is on the right and the sclerotic on the left. The ciliary body is prolapsing into the wound round the edge of the latter. A very peripheral incision had been made in performing an iridectomy for glaucoma and the lens escaped from the eye the day after the operation.

through the sclerotic. It is very exceptional to meet with any sclerotomy or iridectomy incisions which divide the ligamentum pectinatum; they nearly always divide Descemet's membrane internal to it. ${ }^{1}$ A Graefe's knife if introduced r. $6 \mathrm{~mm}$. external to the apparent sclero-corneal margin penetrates the tissues obliquely, and enters the anterior chamber through Descemet's membrane. An incision with a keratome passes even more obliquely

${ }^{1}$ Treacher Collins. Royal Lond. Ophth. Hosp. Reps., XIII, I891, 166. 
through the fibrous tissue external coat of the eye than that made with a Graefe's knife (Fig. 206). The obliquity of the incision facilitates its early closing. The danger of making an incision for an iridectomy so peripherally, as to pass through the ligamentum pectinatum, is that prolapse of the ciliary body may occur. If the anterior part of the

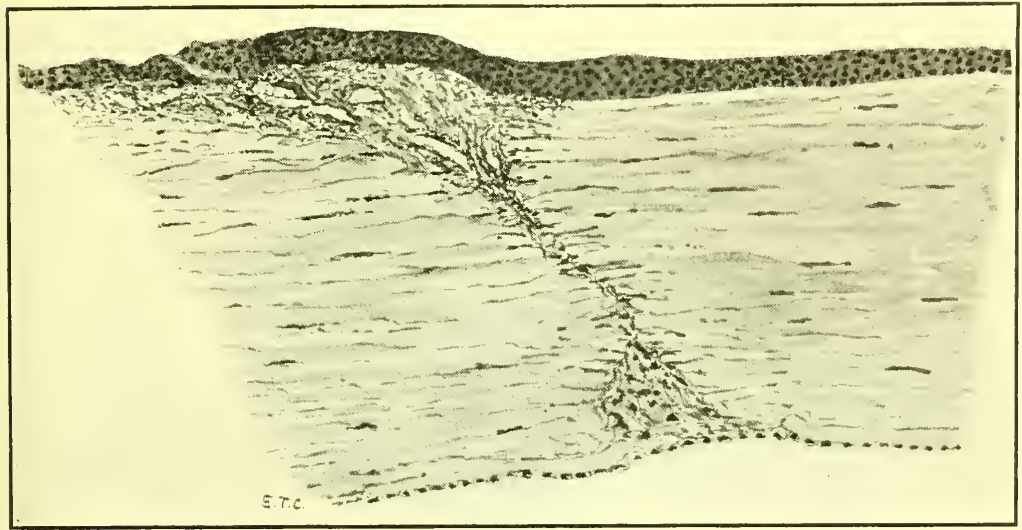

FIG. 208.- - Healing perforating wound at the limbus with a conjunctival flap. Subepithelial vascular tissue shown filling the wedge-shaped gap left by retraction of tissue on its anterior surface.

ciliary body prolapses into a wound, through drag on fibres of the suspensory ligament which are attached to it, the edge of the lens becomes tilted forward, resulting sometimes in the escape of that structure from the eye (Fig. 207).

A wound passing through the limbus differs from one passing through the cornea, in having the vascular conjunctiva external to it. The presence of the overlying conjunctiva in such wounds prevents any preliminary downgrowth of a plug of epithelium between the gaping anterior lips of the fibrous tissue. Its place is taken by vascular subconjunctival tissue, which is later replaced by newly developed fibres (Figs. 208, 209).

It is in wounds through the limbus where folds of iris most frequently prolapse beneath the conjunctiva, and where cystoid scars make their appearance. 
Wounds of the Conjunctiva.- Superficial wounds of the eyeball behind the limbus, involving the conjunctiva and anterior layers of the sclerotic, are often followed by much subconjunctival hemorrhage. The blood effused will readily permeate the loose subconjunctival tissue and extend up to the attachment of the conjunctiva at the corneal margin. Sometimes blood pigment will diffuse from a subconjunctival hemorrhage into the substance of the cornea, staining its margin a straw-colour.

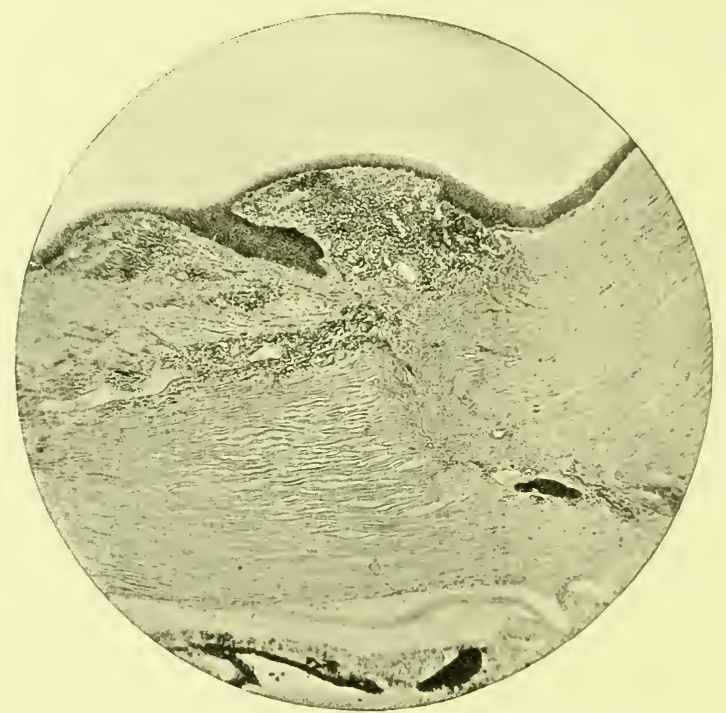

FIG. 209.-Shows a section through a limbal cicatrix with a conjunctival flap in an eye removed nine months after extraction of cataract. The surface epithelium is seen extending down into the subconjunctival tissue, but ceases at the surface of the sclera. From a photomicrograph taken by E. Collier Green.

Sections of a series of wounds made experimentally in the ocular conjunctiva at varying intervals, from 6 to i 70 hours, have been examined microscopically. ${ }^{1}$ They show that the edges of the wound retract and expose the episcleral tissue. The epithelium at its margins turns in, some cells swelling up and degenerating. Usually hemorrhage occurs,

${ }^{1}$ M. S. Mayou. Changes Produced by Inflammation in the Conjunctiva. 1905, 47 . 
and in the blood clot organisms from the conjunctiva may grow. In the first twenty-four hours the blood-vessels around dilate and there is an exudation of polynuclear leukocytes brought by the blood. These have marked phagocytic powers and destroy the organisms lying in the wound. At the end of twenty-four hours proliferation of the epithelium commences; it gradually spreads and fills up all the irregularities in the surface of the wound, and in so doing produces several papilla-like processes. If the wound remains aseptic, at the end of twenty-four hours the exudate consists of mononuclear leukocytes and plasma cells only, both of which make their appearance in the vicinity of the blood-vessels, and are probably derived from the endothelium and perithelium. At the end of forty-eight hours new blood-vessels begin to appear, and there is active proliferation of the deep connective-tissue cells, which continues for some time. These cells lengthen out and develop into new connective tissue and the plasma cells disappear, undergoing degeneration. At the end of I 70 hours the new formation of connective tissue is well advanced, and giant cells derived from the endothelial cells are presient.

Wounds of the conjunctiva rarely become septic, but occasionally considerable polypoid-like nodules of granulation tissue form in connection with them. A little nodule of granulation tissue is not infrequently seen after the operation of excision of the eye at the apex of the orbit, where the edges of the conjunctiva have not come into good apposition. Masses of granulation tissue sometimes form after operations for strabismus, and on the inner surface of the eyelid after operating on a chalazion.

\section{Perforating Wounds of the Globe behind the Limbus.-} A perforating wound of the globe behind the limbus, and anterior to the insertion of the recti muscles, penetrates conjunctiva, sclerotic and ciliary body. One situated behind the insertion of the recti muscles, in addition to the conjunctiva, Tenon's capsule, and sclerotic, may go through 
one of the extraocular muscles, and will also penetrate the retina and choroid.

The amount of gaping of such wounds, and the amount of intraocular hemorrhage to which they give rise, depends on whether they run parallel to the corneal margin or at right angles to it. The edges of a wound through the sclerotic at right angles to the fibres of a rectus muscle may, by the contraction of the latter, be made to ride one above the other. In a wound which runs parallel to the fibers of a rectus muscle the edges fall more evenly into position.

The blood-vessels of the choroid and retina run mostly antero-posteriorly, and a wound running in that direction, divides fewer of them than one crossing them at right angles.

The phagocytic exudation met with about the margins of a perforating wound through the sclerotic varies in amount according to the character of the wround, and the presence or absence of any irritant in connection with it. In a clean cut aseptic wound, such as that made in the operation of posterior sclerotomy, it is very slight.

The cicatricial tissue which forms after a perforating wound of the sclerotic is largely produced by proliferation of the cells of the vascular episcleral tissue, aided to some extent by the fixed cells of the sclerotic itself and the vascular tissue of the choroid.

The cicatricial tissue which forms in the opening left by a perforation of the ciliary body or of the choroid and retina, is mainly produced by the proliferation of the cells about the blood-vessels of those structures. In the retina the nerve elements in the region of a perforating wound are destroyed and never restored. It is unlikely that the epiblastic portions of that structure take any part in the reparative process. The fibrous tissue formed in the opening, and on the inner surface of the membrane, is probably entirely derived from mesoblastic tissue composing the retinal and choroidal blood-vessels. 
Through a perforating wound of the globe into the vitreous humour, some escape of fluid from that structure usually occurs. Occasionally a portion of its framework may prolapse through the wound, and lying in the conjunctival sac, form a channel along which infective organisms travel into the eye, there setting up intraocular inflammation.

Wounds of the Iris.-Aseptic wounds of the iris, such as are made in a surgical iridectomy, show no tendency to the formation of granulation tissue or to cicatrisation. The appearances of a wound of the iris years after its infliction, except for the absorption of the blood, may be precisely the same as when it was first made. ${ }^{1}$ The ragged edge left remains a ragged edge, there being no new formation of fibrous tissue to fill it in, and no extension around it of the endothelium from the anterior surface, or of pigment epithelium from the posterior surface. This is probably accounted for by its suspension in the aqueous humour preserving it free from all irritation. There is ample evidence that the iris is capable of producing granulation tissue, and of becoming sclerosed, when irritated by toxic substances. When the iris becomes prolapsed, either through a wound or a perforating ulcer, a granulation grows from it which subsequently develops into fibrous tissue.

After some wounds of the iris a large extravasation of blood takes place into its tissue, constituting a hematoma.

Wounds of the Lens.-After a wound of the lens capsule the divided ends retract and roll outward. In small experimental aseptic wounds a layer of fibrin has first been found to cover the gap, then the epithelial cells lining the capsule proliferate, and extend across it. These first formed cells lengthen out into fibres, similar to those met with in anterior polar cataracts. Later, underneath these fibres a single row of cubical cells form, continuous with and similar to those lining the normal capsule. From this single row of cells a new hyaline layer, like the capsule, is slowly secreted. ${ }^{2}$

${ }^{1} \mathrm{~T}$. Henderson. Ophthalmic Review, XXVI, I907, I9I.

${ }^{2}$ O. Schirmer. Archiv. f. Ophth., XXXV, I899, I, 220. 
After a wound of the lens; the aqueous or vitreous humour extends into its substance rendering it opaque. The fluid causes the lens fibres to separate, become swollen, and break up into globules or irregular shaped masses (Fig. 210). Through a wound in the anterior capsule the lens substance will usually protrude forward into the

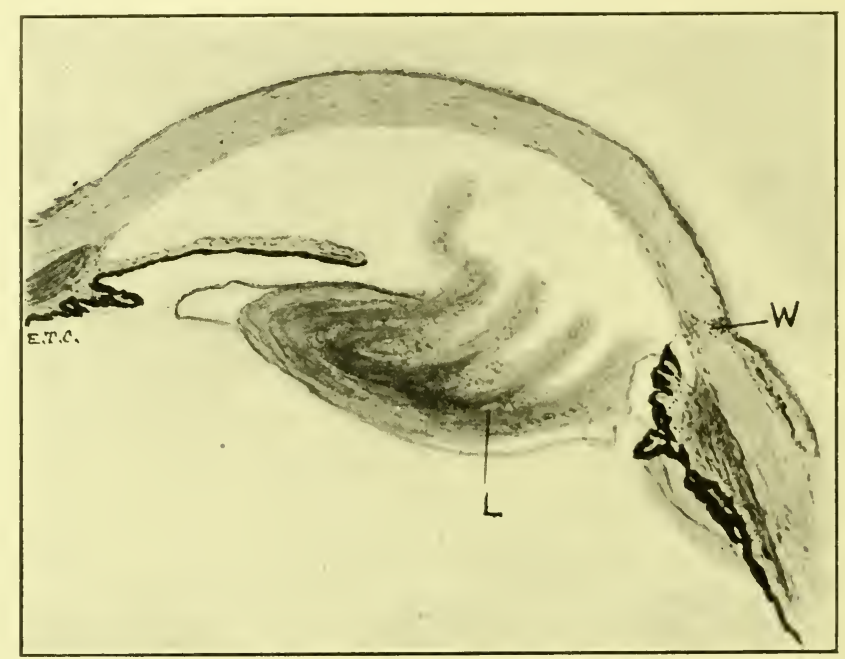

FIG. 210.-Perforating wound of cornea $W$ and lens $L$. The iris prolapsed and has been cut off. The swollen lens fibres are shown breaking up and protruding through a gap in the anterior capsule into the anterior chamber.

anterior chamber. The lens fibres are mainly composed of an albuminous substance called globulin. The aqueous humour consists of a weak solution of chloride of sodium in which globulin is soluble. Lens matter, therefore, projecting into the anterior chamber and exposed to the action of the aqueous humour gradually becomes dissolved by it. The aqueous humour is constantly filtering out of the eye and being secreted, so that as long as a wound in the lens capsule remains open fresh lens matter will become dissolved and be carried away in solution. In this way the whole lens may become absorbed and removed from the eye.

The lens substance forms an excellent nutrient medium for the growth of microörganisms. After septic wounds of 
it polymorphonuclear leukocytes are rapidly thrown out, pass through the opening in the capsule, and penetrate between the lens fibres.

In wounds of the lens with less violent inflammation of the surrounding parts, mononuclear leukocytes and fibroblasts from the iris pass into the capsule, forming fibrous tissue which unites the two structures.

When the lens is removed through an opening in its capsule, as in the operation of extraction of cataract, some of the cortex is nearly always left behind. The amount varies in different cases; usually in complete cataracts the whole of the lens substance opposite the pupillary area is removed, the anterior and posterior capsule being there left lying in contact with one another. Very commonly a ring of lens substance in the extreme periphery of the capsule remains. In incomplete cataracts some cortical matter may be left in the pupillary area, its presence owing to its transparency being unrecognized at the conclusion of the operation. Later, as the result of the action of the aqueous humour, this cortical substance becomes opaque. The opening in the capsule through which the nucleus was removed, in time, becomes closed by a proliferation of the capsule cells, and the opaque cortex is then shut off from the solvent action of the aqueous. It may remain in the capsule indefinitely, and sometimes become the seat of calcareous deposit. A secondary opacity appearing soon after an extraction of cataract is accounted for in this way.

A secondary opacity appearing later, causing vision which for a time has been good to slowly deteriorate, is due to proliferation of the epithelial cells lining the anterior capsule. They, like the epithelial cells on the surface of the body from which they are derived, have a tendency to proliferate throughout life. When the lens is present the capsule cells are transformed laterally into lens fibres. The proliferative activity of the cells is kept in check, as years advance, by the intracapsular tension to which they become exposed. After extraction of the lens this tension is 
relieved and the rate of multiplication of the cells increases. The newly developed cells accumulate in several layers on the inner surface of the capsule, and either lengthen out forming a kind of fibrous tissue (Fig. 2II), as in anterior

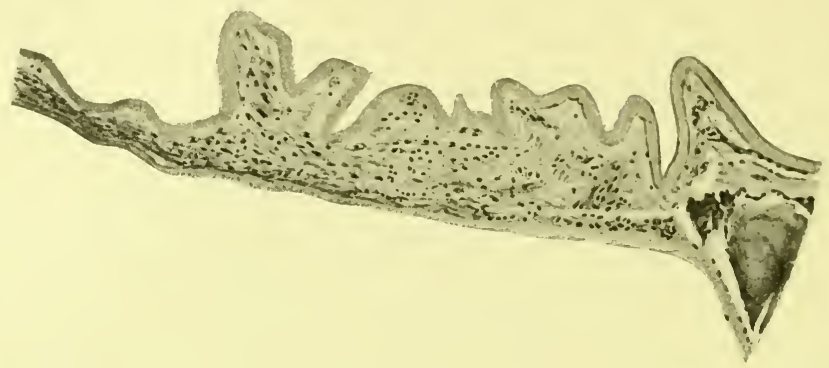

FIG. 2II.- Shows the two layers of the lens capsule after extraction, with laminated tissue lying between them, formed by proliferation of the lining epithelium subsequent to the operation.

polar cataracts, or swell up into large globular cells (Fig. 2I2). The opacity which such new formations give rise to is often not very dense, but the globules and irregularities

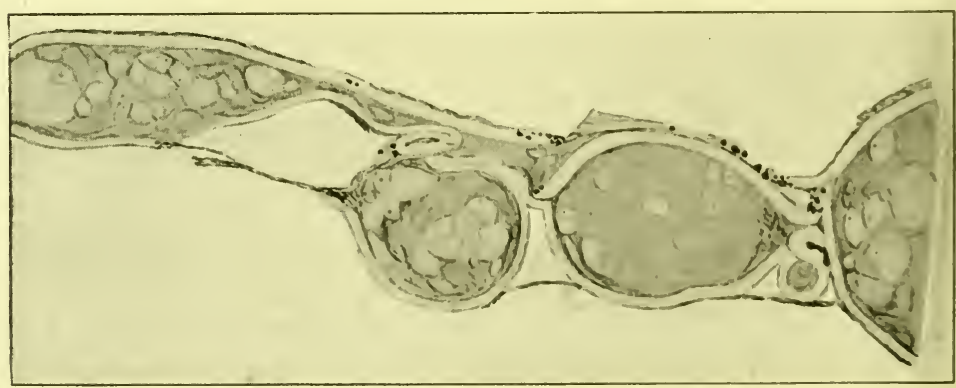

FIG. 2I2.- Shows the two layers of lens capsule after extraction of cataract. with large bladder-like cells, of subsequent formation, lying between them.

in it break up the rays of light as they pass through, and cause considerable disturbance of vision.

A third form of secondary opacity is due to the development of true fibrous tissue inside or outside the lens capsule in the way already mentioned, such opacity is always the outcome of iritis. 


\section{iv. Foreign Bodies}

Cases in which a foreign body is implanted in the eye may be divided into $(a)$ those in which it has lodged in the cornea, conjunctiva or sclerotic, and $(b)$ those in which it has penetrated the outer wall of the globe and become located in the interior.

(a) Foreign Bodies in the Cornea, Conjunctiva or Sclerotic.-The foreign bodies which have been met with implanted in the cornea, conjunctiva or sclerotic are fragments of iron, stone, coal, glass, porcelain, wood, copper, grains of gun-powder, particles of lime or sand, eyelashes, and hairs of caterpillars. Foreign bodies are more often found in the cornea than in the conjunctiva or sclerotic. A foreign body implanted in any of these structures usually causes considerable irritation and pain, though cases are met with in which the amount of reaction has been exceedingly slight. A foreign body in the sclerotic beneath the conjunctiva is more readily tolerated than in the cornea. If septic organisms are implanted with the foreign body suppuration around it ensues. Some foreign substances, e.g., pieces of copper, are capable of exciting inflammation by the chemical action to which they give rise. Fragments of iron rapidly cause a rust staining of the tissue around them due to oxidation.

Ophthalmia Nodosa.-The hairs of the caterpillar, of the fox moth, the Bombyx rubi, popularly known as the woolly bear, implanted into the front of the eye is the cause of most cases of ophthalmia nodosa, though in some cases the hairs from other species of caterpillars have been found.

There is always an interval of some weeks between the receipt of the injury with the caterpillar and the formation of the nodules. An examination of the hairs shows them to be sharp-pointed with an imbricated arrangement of cells on their surface; this probably accounts for their remarkable capacity of penetrating the tissue, which is doubtless assisted by the patient's rubbing the eye on 
account of irritation experienced, and by the friction of the eyelids in winking.

It seems probable that the pernicious effect produced by the hairs is to some extent the result of a specific poison contained in them, though it may be in part due to mechanical irritation caused by their toothed edge.

Around the implanted hairs semi-transparent, round or oval nodules form, about I to $2 \mathrm{~mm}$. in diameter, grey or yellowish in colour; when in the conjunctiva they feel firm to the touch. In the cornea the hairs give rise to infiltration and vascularity. They have been known to penetrate as deeply as the iris, causing inflammation and the formation of nodules in it. The nodules in the conjunctiva if left to themselves ultimately shrink and leave scars behind; they never break down and ulcerate.

Microscopically the caterpillar's hair is seen in the centre of the nodule with a grouping of cells around it very similar to that met with in a tubercular nodule. There are giant cells with peripherally arranged nuclei, epithelioid cells, and dense round cell infiltration.

(b) Foreign Bodies in the Interior of the Eye.-Any hard substance, provided it is propelled with sufficient force, may penetrate the coats of the eye and become lodged in its interior. Sometimes foreign bodies, such as an eyelash, may be carried into the eye with the wounding instrument and be left there after its withdrawal.

Injured eyes have been found to contain chips and scales of steel and iron, fragments of stone and marble, pieces of wood, lead, coal, glass, copper, percussion caps, shot, eyelashes (Fig. 2 I3), and a tin tack.

A foreign body which has penetrated to the interior of the eye may lie in the anterior chamber, be entangled in the iris or embedded in the lens, float free in the vitreous chamber, pass through the vitreous and become entangled in the retina and choroid, or pass through them and be embedded in the sclerotic. Sometimes a foreign body after passing through the vitreous chamber to its posterior wall 
has not sufficient force to penetrate the coats of the eye a second time, but after striking them, rebounds, falling down to its lowest part. In such an eye the aperture of entrance is seen and the track can be followed to the point of impact with the retina, where there will be either a bruised spot or an area of atrophy, while the foreign body itself will be found in the lowest part of the vitreous chamber.

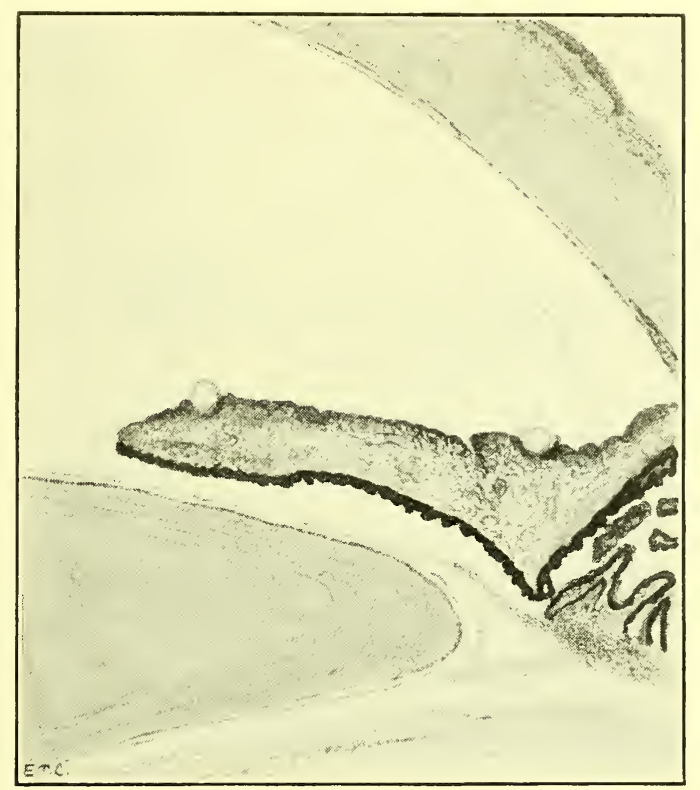

FIG. 213.- Section of iris with two eyelashes cut transversely lying on its anterior surface.

Foreign bodies located in some structures of the eye are more readily tolerated than in others. Cases in which foreign bodies have remained embedded in the lens for more than twenty years, without exciting irritation, have been recorded. Foreign bodies embedded in the sclerotic, after passing through the eye, have also frequently remained for many years without causing any disturbance. It has been shown experimentally that pieces of sterilised copper which rest on the iris or inner surface of the ciliary body 
always excite suppuration around them. A similar piece introduced into the lens produces no purulent exudation; it becomes enveloped by a thick coating of albumen which prevents it from exciting an irritating chemical action.

The amount of reaction excited by an implanted foreign body depends not only on its situation and its chemical nature, but very largely on the organisms which may be carried in with it.

Where virulent septic organisms are introduced with a foreign body, suppuration rapidly ensues. Not uncommonly the chips of metal which enter the eye accidentally have become sterilised by heat from being hammered before

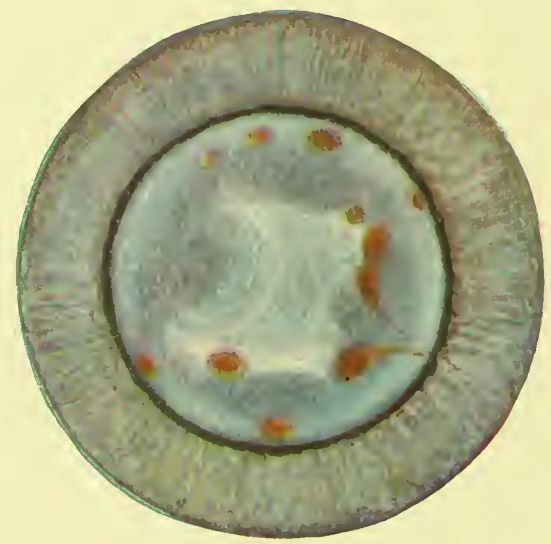

FIG. 214.-Patches of siderosis in the lens capsule in a man aged 29. Case recorded Trans. Ophth. Soc., XXXVI, I9I6, I40.

striking the eye. Experiments on animals have shown that different substances introduced into the eye produce very different effects according to the chemical reactions to which they give rise. ${ }^{1}$ Thus pieces of sterilised gold or glass introduced into a rabbit's eye excite no inflammation, but slowly become encapsuled by a delicate covering of connective tissue or giant cells. A drop of sterilised quicksilver or a piece of sterilised copper introduced in the same way

${ }^{1} \mathrm{~T}$. Leber. Bowman Lecture, Trans. Ophth. Soc. of the U. K., XII, 1892,4 . 
gives rise to a localised suppuration, while croton oil or cantharides introduced aseptically cause no formation of pus but a fibrinous exudation with necrosis of tissue.

Accidents in which pieces of copper enter the eye used to be of common occurrence from the explosion of percussion caps; the pieces are frequently sterile but give rise to suppuration around them. This suppuration is unlike that due to microbic infection in that it does not tend to spread. If the piece of copper is in the anterior chamber, the suppuration does not extend to the vitreous. If it is in the vitreous the suppurative inflammation excited may result in phthisis bulbi, the cornea remaining transparent and the anterior chamber unaltered.

Siderosis Bulbi.-Eyes in which pieces of iron have remained embedded for a long time undergo certain degenerative and pigmentary changes, which have been termed siderosis bulbi. Night-blindness and contraction of the field of vision are observed, and sometimes detachment of the retina. Various tissues become stained a rusty brown colour. Minute brown granules are found in the cornea. A ring of reddish-brown patches forms in the anterior part of the lens at the margin of the pupil. The iris, if blue or grey, changes to a brown or greenish-brown colour (Fig. 2I4). The retina becomes pigmented. The condition is due to a solution of the iron being formed in the intraocular fluid, its diffusion throughout the eye, and re-deposition in some of its tissues.

The soluble bicarbonate is probably formed by the action of the carbonic acid in the tissues. The cause of the re-deposition of the iron is doubtful; it has been suggested that it is brought about by its oxidation by the blood, and the way in which it is found in the vicinity of the retinal vessels favours this view. Its deposition in the capsular epithelium of an avascular structure like the lens, is against it. Another theory, and one which has much in its favour, is that the deposition is due to a special affinity which some cells have for iron. 
In sections of eyes with siderosis, which have been stained by the ferrocyanide of potassium method, (see page 709) a deposition of iron is chiefly found in the following situations: the tissue immediately around the foreign body; the unpigmented epithelial cells of the ciliary body; the retina; the cells lining the lens capsule; the iris and cornea, particularly at the angle of the anterior chamber about the fibres of the ligamentum pectinatum. If the foreign body is removed the siderosis tends slowly to disappear.

A brown discolouration of the cornea and lens may sometimes be due to their impregnation with pigments containing iron derived from the blood. After an intraocular hemorrhage, the retinal pigment epithelium will also sometimes take up iron, derived from it. The staining of the tissues with iron containing pigments derived from the blood has been termed hematogenous siderosis, to distinguish it from that derived from a foreign body which is spoken of as xemogenous siderosis.

Leaden shot implanted in the eye are usually aseptic and set up very little irritation; they soon become encapsuled with a covering of connective tissue.

Around some foreign bodies which are lodged in the eye, giant cells, very similar to those met with in tubercular systems, make their appearance. They are most probably derived from the endothelium of the blood and lymphatic vessels, and have a phagocytic function. They are usually to be seen about foreign bodies of an absorbable nature and less often around pieces of metal. The formation of nodules with giant cells around the hairs of caterpillars when they penetrate to the iris has already been referred to (see page 388). Giant cells are also met with around cilia implanted in the eye.

Cilia have been met with lying in a corneal wound, also in the anterior, posterior, and vitreous chambers. They have been introduced into the anterior chamber of a rabbit's eye experimentally. If the root sheath of the hair is carried in, the cells composing it proliferate and form a pearl-like epi- 
thelial tumour, or a cystic growth lined by laminated epithelium (see page 226).

\section{v. Effects of Heat, Cold and Chemicals}

Injuries of the eye from heat usually arise from splashes of hot metal, from accidents with curling tongs, or from explosions of either gunpowder, dynamite, or match heads. Undue exposure to ultra-red rays produce lenticular changes.

Injuries from cold occur in connection with use of carbon dioxide snow for remedial purposes, to the conjunctiva in trachoma, and to the eyelids in cases of rodent ulcer or nævi. There are no recorded cases of frost bite of the cornea or eyelids from exposure to a low degree of atmospheric temperature.

Injuries with chemicals are produced by splashes of strong acids or alkalies, the fumes of irritating gasses, and by lime, either unslaked in the form of calcium oxide or as motar, that is, slaked lime mixed with sand.

The general effects produced by burns, frost bites, and the corrosive action of caustics are so similar that they may be dealt with together.

The most superficial injuries involve only the epithelium, that of the cornea becoming opaque and white.

The abraided area formed soon becomes covered by proliferation of the cells around and no permanent opacity results. If the deeper parts are involved a slough forms which separates by a process of suppuration leaving a granulating surface. The cicatrisation which takes place at the site of this granulating area leads to considerable contraction. When both cornea and conjunctiva are involved the latter, owing to its loose attachments, becomes drawn over part of the former, giving rise to a condition which simulates a pterygium and is termed false pterygium.

If the opposing surfaces of the eyeball and eyelid become burnt, the two granulating surfaces readily unite. A 
cicatricial band joining these two structures is termed symblepharon.

In the most severe cases of burn, perforation of the cornea or sclerotic may take place, with escape of some of the contents of the globe and the other sequelæ of a perforating lesion.

After lime burns of the conjunctiva or cornea particles of lime may remain in the tissue, as calcium chloride, and give rise to a permanent dense porcelain-like opacity, a condition which is termed calcareous incrustation.

The fumes of irritating gasses most likely to produce damage to the eyes in civil life are those of ammonia. Cases have been met with due to the explosion of cylinders containing ammonia in ice factories, ${ }^{1}$ and also in connection with other industrial pursuits in which it is employed. The amount of irritation or destruction produced varies with the length of the exposure and the degree of saturation of the vapour. Ulceration of the cornea following burns with ammonia, either in the form of vapour or liquid, are peculiarly intractable, often taking months to heal and even then leaving behind a vascularised nebula. In the most severe cases the ulceration may extend so deeply as to cause perforation and loss of sight.

In the late war two forms of poisonous gas were employed by the Germans, the so-called "lacrymatory gas" and the "mustard gas" (dichlorethy-sulphide). ${ }^{2}$ The former gave rise to only a temporary conjunctivitis which was usually recovered from within two weeks, the latter produced more serious lesions. There was considerable redness and swelling of the skin of the eyelids with the formation of bullæ, and chemosis of the conjunctiva, especially in the region of its cul-de-sacs. The ocular conjunctiva opposite the palpebral fissure often presented a swollen white appearance due to granulomatous infiltration. The cornea was

${ }^{1}$ Denig. Zeit. f. Angenh., XI, I904.

${ }^{2}$ G. S. Derby. Trans. Amer. Ophth. Soc., XVII, I9I9, 90; and M. S. Mayou. Trans. Ophth. Soc. of the U. K., XXXVIII, I9I8, I48. 
affected to different degrees in different cases. In the mildest cases the superficial layers of the epithelium were alone destroyed giving rise to a roughness of the surface. In others the whole thickness of the epithelium exfoliated and staining followed the application of fluorescine. In the worst cases the substantia propria of the cornea was also involved, it turning a greyish or porcelain white colour in the region of the palpebral fissure. Secondary to such destructive changes infection was liable to take place leading to ulceration, and even panophthalmitis with loss of the eye.

Another change in the tissues of the eye which may be produced by chemicals is that of staining. This most commonly takes place in the conjunctiva either from prolonged local application of silver salts, organic or inorganic, in the treatment of ophthalmia, or as part of a general staining of cutaneous structures from the prolonged internal administration of nitrate of silver. The condition produced is brownish discolouration of the membrane, both ocular and palpebral, which, microscopically, is found to be due to the deposition of granules of black pigment chiefly around the fibres of elastic tissue, but also in the cement substance of the endothelial cells of the capillaries and smaller blood-vessels. The surface epithelium, is unaffected, its constant renewal not allowing time for any deposition to take place.

A reddish-brown or rusty colour of the upper and lower limbus of the cornea has also been described ${ }^{1}$ as resulting from the prolonged use of copper sulphate in cases of trachoma.

Ultra-red ray Cataract.-Experimentally the rays from an arc lamp of 30 amperes, ${ }^{2}$ filtered through running water and a solution of iodine and iodide of potasium, have been concentrated on the eyes of rabbits by a rock salt lens, and found to produce a complete opacity of the crystalline lens

\footnotetext{
${ }^{1}$ Sydney Stephenson. Trans. Ophth. Soc. of the U. K., XXIII, I903, 25 .

${ }^{2}$ A. Vogt. "Atlas," I 92 I.
} 
after three hours exposure. The filters absorb the ultraviolet rays, and all the luminous rays except the outer red. So that the changes produced must be mainly due to the short red rays. The subcapsular cortical layers, anterior and posterior, were found to be the first affected.

Glass-workers who in their trade are exposed to incandescent molten glass are very prone to develop cataract. The appearances of glass-worker's cataract in its early stages are very characteristic. It commences as a circular central opacity in the posterior cortical layers of the lens, lying entirely in the pupillary area. It may remain in this condition for several years or extend to other parts of the cortex, ultimately becoming a complete cataract. It is then indistinguishable from a cataract due to other causes. In glass-blowers, owing to the position in which they work, the left eye is usually affected first and the skin of the face on the left side acquires a peculiar reddish-brown colour.

Cases with similar lens opacities have recently been recorded ${ }^{1}$ among iron-workers, puddlers, chain makers and tinplate millmen.

Clinical evidence seems to point to the ultra-red rays as being the most likely cause of glass-blowers and ironworkers cataracts. The tinplate millman are exposed to practically no ultra-violet rays, but they are exposed constantly to excessive amounts of ultra-red rays.

\section{vi. Excessive or Deficient Light, Electricity and X-rays}

Various affections of the eye may be produced by undue exposure to bright light. A central scotoma, positive or negative, may be produced by looking for a long time at the sun, as in watching an eclipse without the precaution of using a tinted glass.

In some cases the scotoma in course of time completely disappears, in others it remains permanent. The condition has been termed eclipse-blindness.

\footnotetext{
${ }^{1}$ B. Cridland. Brit. J. of Ophth., V, I92 I, I93; and I. I. Healy. Ibid., 194 .
} 
The ophthalmoscopic changes which accompany it are usually very slight, and appear disproportionate to the defect in vision. A few small white dots or a little pigmentary disturbance is usually all that is to be seen. Edema of the head of the optic nerve, of the retina in the macular region, and retinal hemorrhages have been met with. A pallor of the macular fibres in the optic disc has been noted where the central scotoma has remained permanent.

The change is probably of a chemical nature brought about by a too prolonged concentration of the ultra-violet, chemical active rays on the retinal elements at the macula. Any damage from a concentration of the heat rays is probably prevented from a reduction in their potency by passage through the media.

Experiments on animals have shown that exposure to the ultra-violet rays produce a chromolytic action in the retinal cells which, if prolonged, results in injurious effects.

A prolonged exposure to reflected light from the sea or desert plains, etc., may in badly nourished individuals cause night-blindness. This affection is more commonly met with in the tropics than in temperate regions. It is unaccompanied by any ophthalmoscopic changes, recovery soon takes place by protection of the eyes from bright light and an improvement of the nutrition of the patient. It is probably due to some failure in the reformation of the visual purple after it has been destroyed by bright light, the condition being a prolongation of the normal temporary failure of sight which is experienced on passing from a strongly lighted place to a dark one.

Bright reflected light, like that from snow, in persons who have undergone the operation of extraction of cataract, may excite a condition called erythropsia or red vision. The crystalline lens probably absorbs rays of light, which when it is absent, are able to pass into the eye and cause this affection. It has been known, however, to occur in normal eyes with a widely dilated pupil, which have been purposely exposed to glare from snow in the mountains. 
The red vision is not observed until the affected person passes into a dark place, then all objects assume a roseate hue. It may last for only a few minutes or for several days. Its cause has not yet been definitely determined, though several different explanations have been suggested. The fact that uniocular cases are met with shows that the condition must be due to a change in the eye itself, and not in the central nervous system. One hypothesis attributes it to fatigue of the retina and another to hyperæsthesia. The former supposes that the retina tires sooner of the more refrangible rays in diffuse light than of the less refrangible; the latter that the red percipient elements in the retina become over sensitive. It has been suggested that the ultra-violet rays, which produce changes in the ganglion cells of the retina, may also produce an alteration of the hypothetical substance of Hering. Other theories ${ }^{1}$ regard the condition as due to entoptic vision of blood in the retinal vessels, an abnormal hyperemia of the retina being excited by exposure to bright light in the same way as a hyperemia of the skin is produced.

Besides affections of the retina, undue exposure to ultraviolet rays may produce changes in the front of the eye, conditions which are termed clinically snow-blindness and ophthalmia electrica. The symptoms occur suddenly after a latent period of some hours, and consist of great photophobia, lacrymation, edema of the eyelids, conjunctival hyperemia, slight erosions of the surface of the cornea, and contraction of the pupil. A considerable number of ultraviolet rays are reflected by the snow, and emanate from electric lamps, especially arc lamps and mercurial vapour lamps.

Experimentally the above symptoms, together with some cloudiness of the cornea and iritis, have been produced in rabbits by exposure to ultra-violet rays.

Nystagmus.-In feeble illumination the central acuity of vision becomes lowered, and no longer forms the pre-

${ }^{1}$ W. H. R. Rivers. Trans. Ophth. Soc. of the U. K., XXI, I90I, 296. 
dominating factor in determining the stability in the movements of the eyes that it does in bright lights.

There can be no doubt that the absence of a high degree of central acuity of vision forms an important factor in the production of various forms of nystagmus. In congenital malformations and infantile diseases of the eyes, in which the central acuity of vision is affected, nystagmus is almost sure to develop.

There is some evidence to show that cases of nystagmus associated with head-nodding in infancy (spasmus nutans) are the result of insufficient illumination. Cases have been met with in infants who have been confined to dark rooms, and in a large proportion of the recorded cases the onset of the symptoms occurred during the darker months of the year, October to March. ${ }^{1}$ The nystagmus may be vertical, lateral or rotatory; and the head-nodding antero-posterior, rotatory or lateral. The head-nodding is probably secondary and compensatory to the nystagmus. The onset of the symptoms is always quite early in life, never later than the end of the first year, as a general rule they disappear entirely after a few months. It would seem that delayed development of the predominating central acuity of vision at the macula is the cause of the affection, and that the absence of an adequate amount of illumination tends to retard its development.

A Committee of the Medical Research Council on Miners' Nystagmus, in a report recently issued, arrived at the following conclusions:

"I. The essential factor in the production of miners nystagmus is deficient illumination. Other factors, such as position during work, accidents, alcoholism, infections, malnutrition, hereditary predisposition, and errors of refraction, are of secondary importance only, while depth of workings, thickness of seams, and the ordinary gaseous impurities in mine air have no direct influence on the disease.

${ }^{1}$ W. I. Hancock. Roy. Lond. Ophth. Hosp. Reps., XVII, I907, I04. 
"2. The deficient illumination is due to the low illuminating power of the safety lamps generally used by coal miners, to the distance at which these lamps have to be placed from the objects which the miner has to look at, and to the great absorption of light by the coal and the coal-dust covered surfaces. In addition to the effect of the coal-dust or dirt in obscuring the lamp glasses, the choking of the wiregauze chimneys, and the presence of moisture or low oxygen percentage in mine air, all reduce the light given by oil lamps, while failing voltage, poor bulbs, or lack of proper attention, have similar effects on the illumination given by electric lamps.

"3. Workers at the coal face are more affected than other underground workers, and this appears to be due to the unrelieved blackness of the coal and the greater need for accurate vision.

"4. Distinct signs of nystagmus are present in a large proportion of coal miners, though only in a small proportion do the symptoms ever become so severe as to cause even temporary incapacity for work underground."

Those who acquire nystagmus, like miners, after they have learned to correlate the movements of their eyes in accordance with a high degree of central acuity of vision, attribute the displacement of the retinal images which takes place to movements in the objects they look at. Whilst those in whom the nystagmus dates from early life, the result of defective central acuity of vision, in projecting their retinal sensations take the movements of their eyes into account, and see no oscillations of objects to and fro.

Miners when they return to the normal conditions of illumination, and the central acuity of vision again becomes predominant, soon loose their nystagmic movements. The symptoms, however, rapidly recur if work in the mines is resumed.

Injuries from Lightning.- -Lightning may injure the eye in three different ways: 
I. Through its ultra-violet rays. (2) Through its heat rays. (3) By electrolytic action or concussion.

I. Through its ultra-violet rays it produces symptoms similar to those just enumerated in connection with snowblindness and ophthalmia electrica. In some cases these may be the only result of a lightning injury.

2. Through its heat rays burns of the skin of the lids, eyelashes or front of the eye may occasionally occur.

3. The injuries resulting from electrolytic action are very various. Most of them have been produced experimentally in animals by electric explosions from a Leyden jar made in close proximity to their heads. ${ }^{1}$

The commonest changes are circulatory disturbances resulting in intraocular hemorrhages or opacities of the transparent media, cornea, lens, or vitreous. Nerve lesions may occur causing paralysis of the extra- or intraocular muscles, also atrophy of the optic nerve. Detachment of the retina has also been recorded.

Injuries from $\mathrm{X}$-rays. - Experiments have shown that the $\mathrm{x}$-rays have no power of bleaching the visual purple. On healthy living tissue they are known to cause a superficial irritation with dilatation of capillaries and, according to the amount of the exposure, anything from a mild leukocytosis to an actual gangrene. They have been used in the treatment of trachoma, the leukocytosis which ensues tending to cause absorption of the lymphoid follicles characteristic of the disease. Short exposures over a prolonged time produce an accumulative effect, in the case of a medical man who had been using them for several years a xerotic condition of the ocular conjunctiva was set up.

The case of a woman has been recorded ${ }^{2}$ who was treated with $\mathrm{x}$-rays for lupus of both cheeks, having twenty exposures on the right side and eighteen on the left, the eyes being protected with rubber. Nine months afterward her

${ }^{1}$ C. Hess. Bericht d. VII. Internat. Ophth. Kongress, Heidelberg, I888. K. Kiribuchi. Arch. f. Ophth. L., I900, I, I.

${ }^{2}$ Lesile Paton. Trans. Ophth. Soc. of the U. K., XXIX, I909, 37. 
sight had considerably failed. When examined some years later with dilated pupils, there was found to be in each eye a dense greyish-white granular plaque at the posterior pole of the lens, and also some granular opacity in the cortex. The appearances of the opacities were very similar to those met with in glass-worker's cataract.

\section{INJURIES OF THE EYELIDS}

Injuries of the eyelids may be divided into (i) Contusions; (ii) Wounds; (iii) Burns.

i. Contusions. - The skin of the eyelids is exceedingly loosely attached to the underlying tissues; this loose attachment, whilst facilitating the movements of the lids, also favours the collection of exudates. The eyelids are one of the first parts of the body to become edematous in advanced kidney disease. In contusion injuries the loose attachment of the skin of the eyelids allows of extravisated blood to collect beneath it, more readily than elsewhere. It is for this reason that "black eye" is of such common occurrence as the result of pugilistic encounters. In resolution of the extravasated blood the hemoglobin becomes dissolved out of the red blood corpuscles, and the blood crystals which become formed undergo different degrees of oxidation, affording a variation in the colour in the skin which is reminiscent of the play of colours seen in the familiar Gmelin's test for bile pigments.

In contusion injuries of the eyelids, which are accompanied by a fracture of the inner wall of the orbit, considerable swelling of the eyelids may be produced by air being forced into them through the fracture from the nasal sinuses. Such a condition is readily recognised by the presence of emphysematous crackling on palpation.

ii. Wounds of the eyelids vary much in their severity according to the extent to which their muscles are involved. When the palpebral margin is cut across, and the fibres of the orbicularis muscle are divided transversely, wide 
gaping of the edges of the wound occurs; and unless care is taken in bringing them accurately together a permanent unsightly notch remains. In inserting stitches to reunite the lips of the wound, it is important to remember the part which the fibres of the muscle of Riolani play in the control of the edges of the eyelid in its relation to the eyeball. These fibres are situated at the extreme inner edge of the free border of the tarsus, and internal to the ducts of the Meibomian glands. By their contraction the sharp free border of the eyelid is kept closely pressed against the surface of the eyeball, over which it passes in a sort of squeegee fashion. Unless a stitch be inserted through the tarsus, within the line of the eyelashes, perfect co-aptation of the edges of the wound at the lid margin will not take place, and the normal action of the muscle of Riolani will not be restored.

Transverse wounds of the upper eyelid may cut across the levator, palpebræ superioris muscle, producing drooping of the eyelid, traumatic ptosis.

Perforating wounds of the eyelid, involving also the surface of the eyeball, are liable to result in cicatricial adhesion of the two structures, symblepharon.

Lacerated or septic wounds, of the skin of the eyelids or of surrounding parts, in the course of cicatrisation, tend to draw the edge of the eyelid away from the eyeball, cicatricial ectropion.

Wounds, on the other hand, involving much cicatricial contraction of the conjunctiva will produce in-turning of the lid margin, cicatricial entropion.

Deeply-seated cicatrices near the attached margins of the eyelids are liable to interfere with the lymph circulation in them, a permanent swollen, edematous condition being established.

Wounds passing through the border of one of the eyelids near the inner canthus may cut across a canaliculus; subsequent cicatrisation, by obliterating the lumen in its divided ends, causes an over flow of tears or epiphora. 
iii. Burns of the eyelid may be due to excessive heat or cold, or to the caustic effects of chemical agents. They vary much in degree according to the duration and intensity of the destructive influence. In the slight cases, there may be only a little redness of the skin followed by desquamation of the surface epithelium, in the severe cases, the whole thickness of the eyelid may be destroyed, or the cicatrisation which follows may be so extensive that the eyeball is left uncovered, and the cornea exposed to the risks of ulceration.

\section{INJURIES OF THE ORBIT}

Injuries of the orbit may be divided into (i) Concussions; (ii) Wounds; (iii) Foreign bodies.

i. Concussion injuries of the orbit, due to falls or blows on the head, may result in fracture of its bony walls, or lesions of the structures contained within it.

Fractures of the roof of the orbit not uncommonly occur in connection with fractures of the base of the skull, and their occurrence may be recognised clinically by the appearance of sub-conjunctival hemorrhage extending from behind forwards.

Fractures extending through the optic foramen may so injure the optic nerve as to cause complete loss of sight in the one eye. In such cases, the nerve being damaged behind the point of entrance into it of the arteria centralis retinæ, there are at first no ophthalmoscopic changes. That the failure of vision is due to some loss of conductive power in the visual path, and not to some physic disturbance, is made evident by the loss of the pupillary reaction to direct light. In the course of some weeks, as the atrophy extends downwards from the seat of the lesion, palor of the optic disc makes its appearance.

Fracture involving the inner wall of the orbit may open up connection with the frontal or ethmoidal sinuses. In such cases air will sometimes become forced into the 
tissues of the orbit, on blowing the nose or other violent expiratory efforts, producing proptosis. If the air extends fowards to parts which are accessible to palpation its presence may be recognised by emphysematous crackling. If by fracture of the wall of the orbit a communication be established with an air sinus, in which there is some septic inflammation, orbital cellulitis is likely to result from the injury.

A depressed fracture of the floor of the orbit has been known to lead to some sinking of the contents of the orbit into the antrum of Highmore, with backward displacement of the eyeball, producing one form of traumatic enophthalmia.

Fracture of the margin of the orbit is of rare occurrence, and is only met with after direct blows from hard missiles propelled with considerable force.

Lesions of the contents of the orbit due to concussion, apart from those of the eyeball which have already been dealt with, may produce exophthalmos, enophthalmos, or ocular paresis. Hemorrhage from rupture of the blood vessels causes exophthalmos, as a rule it soon becomes checked by the tension in the tissues to which it gives rise. In rare cases the protrusion of the eye becomes so marked that the eyelids are unable to close over its surface, and the safety of the cornea is endangered. Such a condition has been met with as the result of a blow on the brow in a lad who was the subject of hemophilia. ${ }^{1}$

In blows on the head causing intracranial hemorrhage, either with or without fracture of the skull, the blood may extend forward between the dural and arachnoid sheaths of the optic nerve. ${ }^{2}$ This distension of the sheaths becomes most marked near the eyeball, and due to compression of the central retinal vein where it passes into the nerve, gives rise to papilledema.

${ }^{1}$ Priestly Smith. R. L. Ophth. Hosp. Reps., XII, I888, 70.

${ }^{2}$ Priestly Smith. Trans. Ophth. Soc. of the U. K., IV, I884, 27 I. A. Q. Silcock. Ibid, 274 . 
Enophthalmia may occur as the result of concussion, unassociated with any fracture or any perforating wound. It has been suggested that it might be due to a lesion of the cervical sympathetic nerve, it seems more likely that it is produced by rupture of one or more of the check ligaments allowing the unrestricted retracting action of the recti muscles to come into play.

Various forms of ocular paresis may be produced in concussion injuries either from rupture of the muscle fibres or damage to the nerves supplying them.

ii. Wounds of the orbit may be due to thrusts with sharp pointed instruments or gunshot injuries. The disturbances produced vary according to which of the different structures contained within the orbit are involved. The orbital wounds which are most likely to be attended with fatal consequences are thrust wounds which penetrate the roof of the orbit. If the brain is penetrated death is almost certain, when it is not wounded cerebral or meningeal irritation is liable to be set up, either by a piece of the injuring instrument breaking off in the cranial cavity or by sharp splinters of broken bone. Orbital cellulitis, meningitis, delirium, coma and death may then ensue. It is probable that Harold of England and Henry II of France both lost their lives from injuries of this description.

Compared with these exceedingly fatal thrust wounds of the orbit it is remarkable what little risk to life is involved in some of the gunshot injuries. A bullet in a gunshot injury may enter the orbit on the temporal side, pass right through it, across the ethmoidal sinuses, through the orbit on the opposite side and emerge from its outer wall without in any way jeopardising the life of the patient; and in some cases even, without the patient loosing consciousness.

The missile in these gunshot injuries, unlike that in the thrust wounds, becomes sterilised by heat. In these "through and through wounds" of the orbit, as they are called, the brain and its meninges are not involved. The 
sequelæ, therefore, in such injuries are not those which result from septic inflammation or cerebral irritation, but simply those incident to the damage directly produced. The most serious are those in which the back of the eyeball is perforated so that it collapses, the vision being irretrievably destroyed.

A shot or other projectile may strike the outer surface of the sclerotic, and without perforating it produce changes in the interior of the eye. In gunshot wounds of the orbit the vibration caused in the soft tissues around the globe, by the passage of the projectile through them, may affect the intraocular contents, without it coming in contact with the sclerotic. ${ }^{1}$

In gunshot wounds of the orbit the ciliary arteries or the optic nerve may be injured, and it is often difficult to differentiate changes in the eye produced by such lesions from those the result of concussion.

Rupture of the choroid or retina, detachment of the retina and extensive intraocular hemorrhage may certainly result from concussion in these cases. The following changes have been shown experimentally and clinically to result from section of the optic nerve and the surrounding ciliary vessels: swelling of the head of the nerve with subsequent atrophy; white opacity of the retina followed by pigmentation; atrophy of the retina and choroid with intimate adhesion of the two membranes.

Sudden forcible stretching of the optic nerve, which sometimes occurs in gunshot wounds of the orbit, may, as has already been pointed out (see page $36 \mathrm{I}$ ) result in rupture of the lamina cribrosa and evulsion of the nerve fibres.

Ocular palsies may result from wounds of the orbit due either to injuries of the recti or oblique muscles, or to lesions of the nerves supplying them.

Gunshot wounds of the orbit, punctured wounds, or a blow or fall sufficient to occasion a fracture of the base of

${ }^{1}$ Nettleship. Trans. Ophth. Soc. of the U. K., XXI, I90I, Io2. 
the skull, may bring about a communication beween the carotid artery and the cavernous sinus which results in the formation of an arterio-venous aneurism.

There is usually an interval of some months between the receipt of the injury and the establishment of the characteristic symptoms of this affection. At first there is some swelling and redness of the conjunctiva, with slight proptosis, due to venous obstruction. As soon as the arterial blood passes freely into the cavernous sinus, it is propelled into the ophthalmic veins giving rise to pulsation of the eyeball, buzzing noises in the head, a distinct bruit on auscultation, and in course of time to soft, compressible, pulsating tumours at the margin of the orbit. Ophthalmoscopically swelling of the optic disc, dilatation and pulsation of the retinal veins, and retinal hemorrhages may be met with. In advanced cases the dilatation of the veins may extend to those of the forehead, the bridge of the nose and the inner angle of the opposite orbit. The conjunctiva also becomes more and more congested and chemosed. Should rupture of one of the pulsating tumours occur arterial blood escapes, and immediate ligature of the carotid artery becomes necessary.

Punctured wounds of the orbital tissues are often followed by the formation of bands of cicatricial tissue, the contraction of these, and the absorption of fat by which they are accompanied, are liable to give rise to some retraction of the eyeball in the orbit or traumatic enophthalmia.

iii. Foreign bodies in the Orbit have been found lodged; (a) entirely in the tissues contained within its bony walls; (b) partly in the orbit and partly in one of the nasal sinuses; (c) partly in the orbit and partly in the cranial cavity. In the first two classes the foreign body may be so completely embedded, and give rise to so little disturbance, that its presence for a time remains undetected. Sometimes the history of the accident is very misleading. For instance, in one case a man presented himself with a small wound beneath his right eyebrow, which he attributed to having been thrown from his horse on to a rubbish heap. It was 
only after some weeks, during which time the wound persisted as a discharging sinus, that on exploration with forceps a piece of wood an inch in length was detected and removed.

In another case a boy, aged six, fell downstairs and injured his eye, there was some conjunctival injection, and the doctor called in treated the case as one of conjunctivitis. Six weeks latter, when the boy was brought to the hospital, a little bud of granulation tissue was seen at the outer canthus, with a slight discharge of lymph from its depressed centre. On exploration of this sinus with forceps a piece of slate pencil two inches in length was removed. The lad afterwards remembered that he was carrying a slate pencil at the time of his fall.

A case has been recorded in which an iron hat-peg was impacted in the orbit and the antrum of the opposite side, and remained there for a number of days without the patient being aware of its presence. It was extracted and the man made a rapid recovery.

The above cases are examples of those in which no permanent damage had been caused by the foreign body, but others occur in which serious orbital cellulitis is set up, or in which blood vessels or nerves entering the eyeball have been injured, so that the sight becomes lastingly impaired.

In the third class of cases, in which the foreign body lies partly in the cranial cavity as well as the orbit, a fatal termination is almost sure to ensue from meningitis, as in the thrust wounds of the orbit already described. 


\section{CHAPTER V \\ INFLAMMATION}

The inflammatory changes in the eye will be discussed under the following headings:

I. The changes which are common to all inflammatory processes.

II. The modifications they undergo in the various structures of the eye.

III. The method of infection of the ocular tissues.

In the next chapter the specific microörganisms and the changes they give rise to in the eye will be dealt with.

\section{THE CHANGES IN THE TISSUE WHICH ARE COMMON TO ALL INFLAMMATORY PROCESSES}

The tissues of the eye, being capable of accurate clinical and pathological examination, have been largely used as the field of experimental and histological investigations of the many problems which have arisen in connection with inflammation.

The cornea, being free from blood-vessels and not very cellular, allows, in the early stages of an inflammation, a study of the manner in which the cellular exudate from the blood-vessels of the limbus makes its way in the tissue toward the site of inflammation. The transparency of the cornea allows of the effect of the introduction of various substances into the anterior chamber being observed.

The aqueous, especially that freshly formed, after evacuation of the anterior chamber, contains the protective and toxic bodies found in the bood; it is a means of obtaining fluid for examination of these substances, and for the diagnosis of disease by the Bordet-Gengou and other 
reactions. In inflammation of the uveal tract the aqueous contains organisms and toxins, which may be obtained therefrom and examined. Cultivations may also be made for the preparation of vaccines.

In the conjunctiva the development of lymphoid tissue can be examined and the formation of the cellular elements found therein, which are so important as protective agents against infection, and in the process of repair after inflammation. The accessibility of its position allows it to be employed for testing the effects on the tissues of various toxins.

The fundus allows the examination of blood-vessels under magnification, and the changes they undergo when the retina, optic nerve, or choroid become inflamed. The blood-vessels being the only mesoblast contained in the retina, afford a position in which to study the part played by endothelium in the process of repair and formation of fibrous tissue.

Without going deeply into the controversies which surround the various theories put forward to explain the phenomena connected with inflammation, it is not out of place to give here an outline of the changes which take place, and the theories which are supposed to govern them, also to show to some extent the part played by the ocular tissues in solving some of these problems.

Inflammation may be defined as the first of a series of changes which occur in the tissues as the result of an injury, provided the injury is not of sufficient violence to cause instant death of the part. The injury may be either chemical, bacterial, or mechanical in its nature. The severity of the change depends on the intensity of the injury, its duration, and on the length of time the cause is active.

Bacteria are by far the commonest cause of inflammation. Microörganisms growing in the tissues set up a local reaction of varying severity. They themselves or the poison they produce enter the blood stream, and thereby set up profound alteration in the serous and cellular elements of 
the body, which give rise to general symptoms known collectively as fever.

It may be possible to recognise the cause of the inflammation by one of the following means:

(a) The clinical change set up by the organism may be characteristic and distinctive; e.g., angular conjunctivitis, due to the diplobacillus of Morax-Axenfeld.

(b) The organisms may be found on microscopical examination of the secretion; e.g., gonorrheal conjunctivitis.

(c) The histological appearances of the affected tissues may be so characteristic as to reveal its nature, e.g., tubercle.

(d) The biochemical examination of the changes produced in the blood-serum as the result of inflammation, e.g., Widal reaction for typhoid, Wassermann sero-diagnosis of syphilis.

(e) The reaction produced by the introduction into the blood stream of small doses of the dead organisms (vaccine) similar to that causing the lesion; this may be seen locally at the site of the lesion; in the blood it may be gauged by the opsonic index or other biochemical reaction; in the whole organism by the production of fever.

$(f)$ The local reaction of the tissues to vaccine by surface inoculation, e.g., the ophthalmo-tuberculin reaction; hypersensitiveness.

(g) The inoculation of the diseased tissue into animals will reproduce the disease in a more typical form if the animal be susceptible to it; e.g., the inoculation of tubercle into guinea-pigs.

The earliest changes which occur in the tissue of the affected area as the result of inflammation are dependent upon the blood, the changes in the blood being again dependent on the general reaction of the whole body to the infection, the blood stream merely conveying to the inflamed part the products of the tissues. The later changes, more especially those associated with the reparative processes which follow the inflammation, are dependent on the local tissues of the part. 
The first change which takes place in an acute inflammation is dilatation of the vessels and an increased flow of blood to the part, as is well demonstrated in any acute inflammation of the conjunctiva; the increased flow of blood associated with increased chemical activity raises the local temperature, as has been shown by introducing specially devised thermometers into the conjunctival sac in acute conjunctivitis. After a time the blood stream is slowed, and complete stasis may occur, which, in severe cases, may go on to coagulation of the blood and exudate, frequently causing necrosis of the affected area (coagulation necrosis).

The slowing of the blood stream in the capillaries allows an exudation of their contents. This consists of the plasma, which passes through their walls by a process of osmosis, and of cellular elements. Leucocytes make their way through the walls by their ameboid movement, a process known as diapedesis. Certain influences (such as the toxin of the Koch-Weeks bacillus) cause the red cells also to pass out into the tissues.

This exudation of the fluid and cellular elements of the blood gives rise to swelling in the tissue, which, in the conjunctiva, owing to its laxity, may be very extensive, causing the condition known as chemosis.

In addition to the local pain, redness and swelling, there is a loss of function of the part, as for example the inactivity of the pupil in iritis, and the failure of vision in retinitis and inflammation of the optic nerve.

The plasma exuded from the blood-vessels, by virtue of certain bodies it contains, acts on the bacteria and either destroys them or renders them inert, while the leucocytes, especially the polymorphonuclear variety, by their ameboid movement, or more probably by some alteration in the surface tension, or electrical condition of the organism, surround and then digest them.

The blood-serum plays a most important part in inflammation, and as the future diagnosis and therapy of many diseases of the eye will be based on its examination and use, 
it is necessary to give a brief outline of some of the general principles upon which our present knowledge of humoral pathology is based.

Immunity.-Immunity is the power of an animal's body to resist the attack of organisms which gain entrance through a breach in the continuity of the mechanical defenses of skin or mucous membrane. Absolute immunity is extremely rare, and it necessarily follows that the resistance is only a question of degree. Immunity may be natural or acquired.

Natural Immunity may be (a) "Species" immunity such as the difficulty of inoculating animals with gonorrhœa or syphilis.

(b) Racial Immunity. The diseases to which some races are liable and others are immune.

(c) Individual resistance, some individuals are less prone to infection than others. This form of immunity largely depends on the state of health and metabolism of the body. Some immune bodies such as lentocytolysin (see page 2) and diphtheria antitoxin (see page 5 I8) may be transferred from the parent to the child through the placental circulation.

Acquired Immunity.-When an individual suffers from some disease, such as typhoid, or scarlet fever, an immunity is conferred on him by the attack. This is called an acquired immunity. Another form of acquired immunity is artificial immunity which may be of two types active or passive.

I. An active immunity may be produced by inoculation with an attenuated culture of an organism which has been subjected to heat, drying or some chemical processes; (2) by the injection of a sub-lethal dose of a fully virulent organism; (3) by the injection of emulsion of dead bacteria (the organism with its endotoxin), by the injection of bacterial products e.g. toxins.

Passive Immunity is produced by the administration of the sera of animals which have been inoculated with 
sub-lethal doses of organisms. The tissues of the animal protected with these sera take no part in their own defence, and the immunity thus produced is called passive.

Antibodies and Their Derivatives. - The first light thrown on the question of immunity was the discovery of the normal power of the blood serum to kill bacteria. This power is destroyed by heating it to $56^{\circ} \mathrm{C}$. The thermolabile substance of the blood serum possessing this power is called alexin. The next discovery was that the blood sera of animals actively immunized against diphtheria or jequirity will protect normal animals. This body in the serum is called antitoxin. If organisms are injected in to the peritoneal cavity of animals, and the fluid abstracted from time to time examined, it is found that the bacteria rapidly swell up and become digested. The constituent of the blood serum which gives rise to this phenomenon is known as bacteriolysin. It is further found that when certain bacteria are brought in contact with the immune serum they are deprived of their motility and firmly clump together, the body in the serum which gives rise to this phenomena is known as agglutinin.

When filtrates of a culture of certain organisms are mixed with their specific immune sera precipitates are produced, the body in the serum which gives rise to this phenomenon is known as precipitin. It has been found that these phenomena are not limited to bacteria. Sensitising, agglutinating and precipitating effects may likewise be produced by a large number of different protein substances. Amongst these are the red blood corpuscles. It has been shown that the serum of animals, repeatedly injected with the defibrinated blood of another species, exhibits the specific power of dissolving the red blood corpuscles of these species, a reaction known as hemolysis. These bodies being so numerous the generic term antigen has been given to them.

If an antitoxin is present in an animal's serum, and a dose of toxin be administered, the antitoxin enters into a bio-chemical combination with the toxin and renders it inert. This action is specific. If a toxin be injected into an animal 
and at some interval the antitoxin be administered the animal may or may not recover, the result depending on the length of time which has elapsed between the administration of the antitoxin and the toxin. The toxin enters into union with cells of the animal; the antitoxin, if administered late in the course of the disease, is unable to dissociate this combination, the action of the toxin continues, and death ensues. Hence the importance of the early administration of antitoxin.

To explain the formation and reaction of the various antibodies Ehrlich propounded his "side-chain" theory, on the principle analogous to the benzine ring in chemistry, which although useful for explanatory purposes is not strictly accurate. He supposed that every cell has figurative arms by which they attach to themselves nutrition, toxins, etc., these arms he called receptors. The ultimate

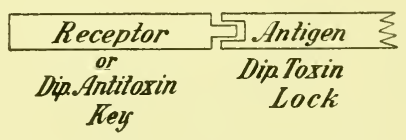

effect on the cell of the injection of a sub-lethal dose of the poison is an overproduction of these receptors which are thrown off into the blood, and there confer on the animal an immunity (antitoxin). Thus if a sub-lethal dose of diphtheria toxin be administered there would be an overproduction of receptors or antitoxin formed in the blood; the serum from this animal would contain receptors and when such serum is administered to a patient with diphtheria they would combine with the toxin (antigen) and so render the latter inert. The action of the antitoxin is specific; that is to say, the receptor must belong to the same group as that of the antigen. The receptor has been compared to a key and the antigen to a lock. Before the lock can be opened the wards of the keys must fit the lock.

In the course of the standardisation of diphtheria antitoxin it was found that after five months a toxin solution would lose half its power, but in spite of this it still retained 
the power of neutralising antitoxin, therefore, if the neutralisation of the antitoxin is a purely chemical one, there can be but one explanation namely, that the toxin consists of two atoms, one of which has the power of combining with the antitoxin which Ehrlich designates as the "haptophore" group, the other which causes the poison which he calls the "toxophore" group.

Bordet on the other hand believes that the toxin and antitoxin mixture is not a true chemical combination, but like reactions with colloidal substances the antitoxin is distributed evenly over the toxin leaving them partially saturated, not as in the purely chemical reaction where part of the toxin would be left entirely free in the serum.

Lysins (Bacteriolysin, Cytolysin).-Bacteriolysin is a substance present in an "immunised serum" which dissolves bacteria. This immunised serum is produced by the injection into an animal of sub-lethal doses of the organism. Immunised serum, on heating to $56.5^{\circ} \mathrm{C}$. for half an hour, loses its power of dissolving bacteria, but if a little fresh serum, which may come from an unimmunised animal, be added this power is regained. Therefore two bodies are present in this serum: (I) a heating-resisting substance, and (2) a substance destroyed by heat which is known as the Complement or Alexin. The receptor which resists the action of heat in this instance is double ended and is therefore known as the Amboceptor. By the one end it is capable of attaching to itself the antigen or bacteria, and by the other the complement or substance which is destroyed by heat. The amboceptor combines first with the antigen and afterwards with the complement. Immunised serum, which has been treated by heating it to $56.5^{\circ} \mathrm{C}$. for half an hour, is known as inactivated, because the serum cannot exert its lytic power, but can be activated again by the addition of a small quantity of serum from any unimmunised animal as it contains the complement.

The union of the amboceptor with the antigen is specific, but with the complement is not specific; from whatever 
serum the latter is derived it will act on any form of amboceptor so as to produce union with the specific antigen. To apply the simile again, the key can be turned in the lock by any hand, the hand representing the complement. Although this is Ehrlich's view Bordet has pointed out that there are many uncertainties about it. It is certain that alexin or complement does not enter into union with the unsensitised antigen, but the action of the complement upon the antigen

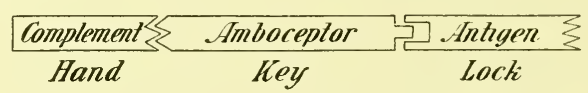

is made possible only by preliminary sensitisation by union with the specific antibody, and it is not justifiable to assume that the sensitising antibody is an amboceptor or a bridge between the antigen and the complement; therefore both sensitiser and complement refer to the same substance.

It is probable that the sensitising antibody is the same whether the process is one of lysis, agglutination or precipitation.

Toxins combine more readily with some cells of the body than others and are neutralised by them to a certain extent; thus if a lethal dose of tetanus toxin be mixed with fresh brain substance, and then injected into an animal, little or no result will follow; in other words toxins possess a selective action on certain tissues. This probably accounts for the fact that both eyes are liable to be attacked by the same disease, as in tubercular or sympathetic uveitis. No doubt the soil on which the organism is originally grown makes it more liable to attack similar tissues, thus tubercle may attack only joints or glands, and it frequently attacks the other eye after one has been infected.

Agglutination.--It was found that when immune serum from bacillus coli or typhoid was added to boullion cultures of these organisms, the cultures lose their turbidity and the organisms sink down in clumps to the bottom of the tube. The same clumping of organisms can be observed in hanging drop preparations under the microscope. 
Upon this re-action Widal based the diagnosis of typhoid from the serum of the patient. The actual clumping is no doubt a purely physical phenomenon owing to the presence of electrolytes, but whether the agglutinins are identical with the immune body has not yet been decided. Agglutinins are produced in the serum by the introduction of microörganisms into the body, they can be produced by all the known bacteria and appear in the animal's blood three to six days after inoculation. Agglutination, like other immune phenomena, is a manifestation of a biological law and not limited to bacteria. Thus, as hemolysins are produced by the injection of red blood corpuscles, so hemagglutinins, or substances which clump the red blood corpuscles together, are similarly formed.

Precipitation.-When the serum of animals immunised against certain organisms, such as the typhoid and cholera bacillus, is mixed with a clear filtrate of boullion cultures of the respective organisms avisible precipitate is produced.

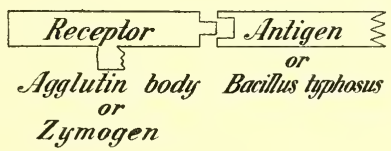

The precipitate is only produced when the filtrate and immune serum are homologous. It is evident, therefore, that a specific antibody is produced in the serum of an animal so treated, hence its name "precipitin." Like agglutination it is not limited to bacteria, a similar reaction being produced by the injection of the sera of other animals or even milk.

Precipitins and agglutinins are both inactivated by heating to $65^{\circ} \mathrm{C}$. and cannot be reactivated by the addition of normal serum. Like toxin they are probably built up of two atom groups, a haptophore and a precipitophore. The specificity of precipitins is important as it gives us the means of differentiating animal proteins. More particularly the distinguishing of one species of blood from an- 
other, thus blood spots can be recognised by this reaction as originating from man or from animals, even after months of drying. Unlike agglutinins, precipitins have not been demonstrated in normal sera.

In Ehrlich's side-chain theory precipitins and agglutinins are of a different order. The receptor requires no complement, it contains a single haptophore group for the anchorage of the ingested material, and at the same time a constantly attached zymophore group, by means of which the anchored substance is transformed preparatory to its absorption by the cell protoplasm. In other words this form of receptor can be compared to a bacteriolytic or hemolytic amboceptor with a premanently attached and inseparable complement.

Bordet on the other hand has shown that the antigen and the agglutinin body first combine, and are subsequently agglutinated by the presence of salt (electrolyte) in the solution.

Hemolysins.-Emulsions of cells provided they be of an animal of another species; animal and vegetable fluid such as abrin; when injected into animals produce antibodies in the serum called cytolysins. It has been already mentioned that if the blood cells of one animal are injected into another animal of the same species, a hemolytic substance is produced in the blood of the second animal which is specific for the variety of cells injected. Hemolysins in one animal against the blood cells of a different animal are called heterolysins. It has also been proved, that when an animal is injected with the red blood cell of a member of its own species a hemolytic substance is produced, called isolysin. Such a hemolysin will not produce hemolysis with the red blood corpuscles of all animals of its species, but only with certain classes. Upon this, and isoagglutination which is produced in a similar way, depends the classification or grouping of bloods for the purposes of transfusion, as it is obvious that blood if not transfused from the same series will undergo a hemolytic process. 
So far it has not been possible to produce autolysins, that is to say substances in the blood serum which will cause hemolysis of the individuals own corpuscles. Antiisolysins have been produced by the injection of isolysins. They are absolutely specific in their action.

Deviation of the Complement (Alexin).-It has already been pointed out (see page 4I 7 ) that an immunised serum contains two bodies, a heat resisting substance or amboceptor and a substance which is destroyed by a temperature of 56.5 C. for half an hour (complement). According to Ehrlich's conception the amboceptor has a double end, by one end it is capable of attaching itself to the antigen or bacteria and by the other end to the complement. The amboceptor first combines with the antigen and afterwards with the complement. "Inactivated" serum can be activated again by the addition of a small quantity of serum from an unimmunised animal, as it contains the complement. The union of the amboceptor with the antigen is specific, but the complement is not specific, from whatever serum it is derived it will act on any form of amboceptor so as to produce the union with the specific antigen.

The biochemical union which takes place between the amboceptor and the complement on the one hand, and the antigen on the other, is a very firm one. Upon it Bordet and Gegnou have introduced a reaction which has gone far to revolutionise clinical medicine, and probably may help very considerably in the diagnosis of many diseases of the eye. If the complement is engaged with its complementophile affinity of an amboceptor, developed as the result of bacterial infection, it cannot be dissociated, and therefore cannot act upon the complementophile affinity of hemolytic amboceptor, such as is developed as the result of immunising one animal against the red blood corpuscles of another. The complement being "fixed" to the bacterial amboceptor cannot act on the hemolytic amboceptor and so no hemolysis is produced. Thus, if we take an emulsion of typhoid organisms and "inactivated" serum (the com- 
plement being destroyed by heat) obtained from a typhoid patient (and therefore containing the typhoid amboceptor), and the serum of a normal guinea-pig which is rich in complement, and mix them together, the complement will combine with the typhoid amboceptor. If, then, to this be added a mixture containing the "inactivated" serum of
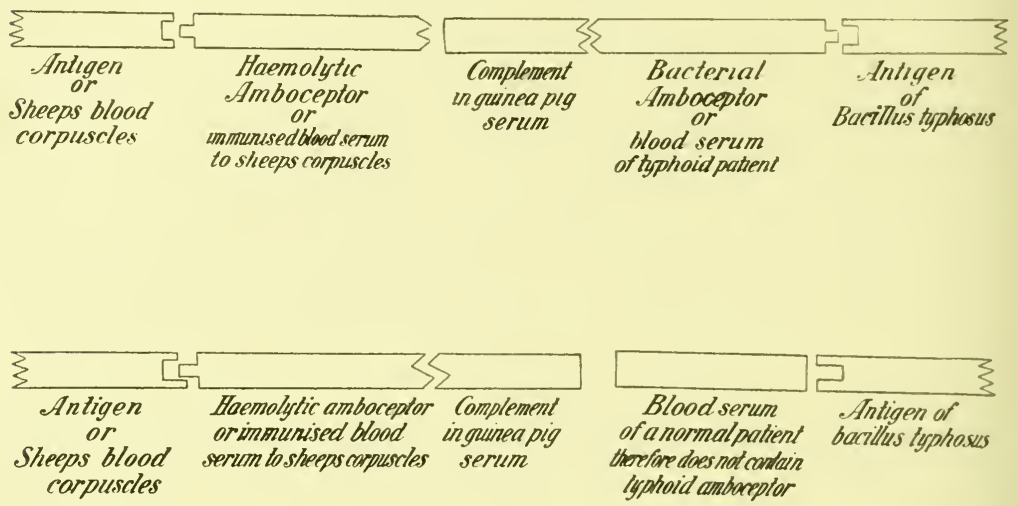

a rabbit immunised to sheep's blood corpuscles (which therefore contain a hemolytic amboceptor) and sheep's blood corpuscles in suspension, no hemolysis takes place, because the complement in the guinea-pig's blood-serum has been already "fixed" to the typhoid amboceptor, and is no longer free to act upon the hemolytic amboceptor attached to the sheep's blood-corpuscles. If, on the other hand (the experiment be repeated, substituting the serum of a normal person for that of the typhoid patient, no typhoid amboceptor being present), hemolysis will take place, because the normal serum contains no typhoid amboceptor, the complement will not be "fixed" to it, but will be free to combine with the hemolytic amboceptor.

The test in this form is only applicable to organisms which can be cultivated outside the body, it has been already successfully applied to tubercle, cerebro-spinal meningitis, and gonorrhœal infections. Upon these principles depends the sero-diagnosis of syphilis introduced by 
Wassermann; that is to say, the diagnosis of syphilis by the fixation of the complement. It is carried out as in the previous experiment except that instead of using the bacillus typhosus, the liver or spleen of the syphilitic fetus is ground up so as to get at the spirocheta pallida and employ it as the antigen. The supposed syphilitic serum (containing the specific amboceptor) is obtained from the blood serum of the suspected patient, while the other ingredients are the same as in the previous experiment. If the syphilitic amboceptor be present in the serum no hemolysis takes place, but if it be not present hemolysis takes place owing to the complement not being "fixed" but free to combine with the hemolytic amboceptor.

Although the method given above was the original way the test was carried out, it has since been found that the syphilitic antigen may be replaced by alcoholic extracts of syphilitic or even normal liver or heart muscle, indeed any lipoidal extract. This might raise some suspicion as to the value of the test, but as a matter of practical experience it has proved reliable. In undoubted syphilitic lesions of the eye, a positive reaction has been obtained in 92.2 per cent., in interstitial keratitis 83.3 per cent., in iritis 33.3 per cent., in choroiditis and retinitis 28.0 per cent.

Using the aqueous as the amboceptor instead of the blood-serum the same results were obtained, as the antibody is found in it.

Having described some of the principal changes which take place in the serum during inflammation, it is next necessary to describe the part played by some of the cells which are found in the inflamed area.

Phagocytosis.-Unicellular animals take into their protoplasm particles with which they come in contact, if the particle is of an inorganic nature and indigestible it will be extruded, but if it be such as can be utilised for nutrition it is surrounded by a small vacuole and finally dissolved. The digestion is due to a proteolytic enzyme 
which acts in a weakly alkaline fluid, and is probably of a trypsin-like nature, this process is called phagocytosis.

In man and higher animals this process is limited to certain cells of the body, leucocytes, of the polymorphonuclear variety; large endothelial cells, especially those derived from the blood vessels and serous cavities, and to the so-called phagocytic "wandering cells" in the connective tissue.

For studying the activity of the cells, the corneal tissue being free from blood vessels has been largely utilized. In a localised area of infection of the cornea the leucocytes can be seen passing up to the infected spot as a grey area of infiltration. When the infected area is directly attacked in this manner it is known as a positive chemotaxis. In some instances around the point of infection a clear area may be seen, due to the paralytic action of the toxin on the leucocytes, which condition is known as negative chemotaxis. The toxic bodies which give rise to this paralytic action are known as aggressins. This condition of negative chemotaxis is often seen in the early stages of corneal infection (see page 440).

There is no doubt that phagocytosis is a very important factor in the protection of the body from infection, whether the serum or the cells are the more potent is not at present known. There is also no doubt that there is a direct attack by the leucocytes upon the organisms before they are destroyed by the serum, for it has been shown that living organisms taken into the cells may remain alive and active. It has been further proved that phagocytosis is most active against microörganism when an animal has a high natural or artificial resistance to disease. Metchnikoff claims that immune bodies and complements are derived from leucocytes.

Wright discovered a substance in the serum which he called Opsonin, which apparently is not identical with the lytitic bodies, but has an action on the bacteria which renders them subject to phagocytosis. It has been 
shown that this body is specific, and can be taken out of the serum by treating it with the various species of bacteria. Opsonin like other antibodies can be increased in the serum by the injection of sub-lethal doses of organisms. At first after injection there is a slight decrease in opsonin (negative phase) which is soon followed by a considerable increase in its production (positive phase). The opsonic index is determined by mixing in a capillary tube the blood serum from the patient, the bacteria emulsion and washed leucocytes; incubating for 30 minutes, then making a smear preparation from the contents of the tube, and staining and counting the number of bacteria taken up by the cells. The result must be compared with a control experiment made with normal serum. It is upon the outcome of this work that the present vaccine therapy has largely arisen.

At the present time vaccines are made from a large number of organisms. The early work was performed on staphylococci, tubercle bacilli and typhoid bacilli, and these three organisms still afford the most satisfactory results. If the organism is obtained from the patient the vaccine is known as autogenous vaccine. Such vaccines in the case of some organisms, give far the best results, but with others, like the staphylococci, a mixed strain acts equally well. To form a vaccine the organism is grown on culture media and an emulsion made from the growth. It is then standardized either by counting or by weight, sterilised at $60^{\circ} \mathrm{C}$. for an hour on several days, and after being tested on an animal, it is ready for use. The second dose is given at the highest point of the positive phase, which is generally about I 4 days after the first dose. There is a consensus of opinion that the opsonic index is a poor guide to treatment and for that purpose has fallen into disuse.

Vaccine treatment is most frequently employed in diseases of the eye due to staphylococcal infection. It has been used successfully in cases of pustular blepharitis, multiple chalazion, styes, corneal ulceration, iridocyclitis 
following operation, and iridocyclitis of metastatic origin, such as is associated with boils, pyorrhea alveolaris and gonorrheal infection.

The preparation of a vaccine for use in iridocyclitis is somewhat difficult, owing to the frequent absence of a satisfactory focus, apart from the eye, from which a culture can be obtained. It may be obtained from the aqueous by performing paracentesis, when it is desirable to use a sterile hollow needle to avoid contamination from the conjunctiva.

The dose of staphylococcus vaccine is usually $500,000,000$ which may be increased to $2,000,000,000$ or more, given in gradually increasing doses.

In gonorrheal infections the first dose should not exceed $20,000,000$ as the organism is more toxic in its effect.

In the cases of tubercle, as the inoculation of dead tubercle bacilli causes far too much reaction and even suppuration, Tuberculin T. R. (Tuberculin Ruchstand) is most frequently employed. It consists of an aqueous extract of the ground up tubercle bacillus killed by heating at $60^{\circ} \mathrm{C}$. for an hour. The first dose should not exceed $1 / 2000$ of a milligram of this substance. It may be gradually increased up to $1 / 250$ or more. Recently a defatted vaccine has been used with success. Inoculation should not be performed more than once in two weeks, otherwise the negative phase may be unduly prolonged, it should be continued for a long time, often more than a year.

In ophthalmic diseases, the effect of the vaccine can be estimated so accurately by observing the local reaction in the eye, that no other control is necessary.

Leucocytic Endolysins. - If the pleural cavity of a rabbit is injected with a sterile irritant large quantities of leucocytes are poured out into it. These can be separated by centrifugalisation and after being tested for sterility, used as injections to produce an increased leucocytosis, they have a slightly beneficial effect on an infective condition. 
The bactericidal substance in the leucocytes is known as an endolysin, it is a different substance from serum bacteriolysin, not being rendered inactive by temperature under $80^{\circ} \mathrm{C}$. and not, when once destroyed, being reactivated by addition of fresh serum. These endolysins are not increased by immunisation, and are probably only the material required by the individual leucocyte for the digestion of bacterial substances.

Non-specific Protein Therapy.-When bacterial and other proteins are injected into a patient suffering from an acute infection a sudden drop of temperature occurs, followed by a rise with rigors, and a beneficial effect on the course of the disease. One of the protein substances which have been injected, especially for gonorrheal lesions, is milk. Treatment with it is as yet on its trial, but in gonorrheal ophthalmia neonatorum, the results have proved very far from satisfactory. The beneficial effects when they occur may probably be explained by the increased leucocytosis which is produced.

Virulence.-Certain bacteria may be injected into the body in large quantities and only produce a slight local reaction. On the other hand small doses of other organisms, such as anthrax, produces a fatal septicemia. The degree of susceptibility varies considerably in the same species and in the same patient. This variation in susceptibility Bail attributes to "aggressins." He found that animals which were highly susceptible to anthrax often possessed marked bactericidal powers against the bacillus, but in spite of this died if inoculated with a minute dose of organism. From this he inferred that the microörganism possess some power to counteract the animals protective powers. This substance which he regarded as being given off by the bacteria to protect them from phagocytosis he called aggressin. He has separated it from the bacteria, and successfully immunised animals by its use. It is very doubtful if this substance is a separate entity, or whether its apparent properties may not be due to exudates con- 
taminated with bacterial receptors. It is also possible that Bail's "aggressin" may be of the nature of anaphylatoxin, which added to bacteria will convert a sub-lethal dose into a lethal one, or that the reaction between the lytic antibodies and the bacterial protein produce toxic substances which prevent a positive chemotaxis taking place. There is little doubt that encapsuled organisms resist phagocytosis, better than those without a capsule.

Hypersusceptibility.-A human being or animal suffers from hypersusceptibility when the administration of a substance produces an injury, which, administered by the same method and in the same amount to a normal member of the same species, produces no injurious effect. In some instances, as is probable in food idiosyncrasies, etc., this hypersusceptibility may be hereditary or it may be acquired as the result of previous contact with the substance, as in the injection of horse serum or atropin irritation. There seem, therefore, to be two forms: (I) that in which it cannot be shown to be due to a union between the antigenantibody, and (2) that in which it can be proved that such union has occurred.

Taking first the hypersusceptibility due to antigenic substance to which the term true anaphylaxis has been applied. It was first noticed by Behring that an animal highly immunised against diphtheria toxin would occasionally, when given a small dose of toxin, show a marked reaction. If eel serum is injected into dogs, instead of the animals becoming immune to the injections, they become more sensitive; the same applies to the injection of horse serum into rabbits. The action of the serum has no relation to its toxin or anti-toxin constituents. Sensitisation is at its height about ten days after the first injection. This sensitisation is not limited to serum but can be produced by other animal proteins, such as milk, egg albumin, pepton and bacterial extracts.

For true anaphylaxis to be present (I) the toxic symptoms from the injected material must depend upon the 
sensitisation of the animal, that is the substance must not produce similar symptoms in a non-sensitised animal. (2) It must be possible to induce passive sensitisation by the injection of the serum of a sensitised animal, in other words a true anaphylaxis upon the meeting of the antigen with its homologous antibody.

Anaphylactic antigens are always of a protein nature, any substance which can incite the formation of true antibodies may also be an anaphylactic antigen.

Sensitisation of animals may be either active or passive. Active sensitisation may be produced by the injection of the smallest quantity of the antigen. Guineapigs, dogs, rabbits and monkeys are readily sensitised. Sensitisation in man is manifested by an immediate skin reaction, and serum sickness, followed by acute shock, which latter is rarely fatal.

Passive sensitisation is carried out by the injection of normal animals with serum taken from anaphylactic animals. If 23 to 48 hours after sensitisation the protein used for sensitisation is given, the animals so treated die with symptoms of hypersusceptibility; simultaneous injections of the two substances does not produce this effect, a certain time therefore must elapse before the protein is given to produce the symptoms. The precipitates which are formed in a test tube between an antiserum and its antigen, when injected, produce passive sensitisation. Acquired anaphylaxis may be transmitted by a mother to her offspring; it is not conveyed by the mother's milk. Its nature is probably identical with the sensitising antibody and is amenable to agglutination, precipitation or complement fixation.

It has already been pointed out that time must elapse before an animal becomes sensitised, this is probably due to the fact that an interval is required for the anchoring of the antibodies to the cells of the tissues. It has been proved that the change does not take place in the blood by replacing the blood of the animal so injected by that of another. The 
site of the anaphylactic reaction therefore takes place in the tissue cells.

The symptoms of anaphylaxis are a fall in blood pressure, temperature, a diminution of leucocytes in the blood and an increased flow of chyle. Other symptoms vary in different animals. Guineapigs die as the result of respiratory troubles, rabbits show asthmatic symptoms, dogs show hepatic trouble from obstruction of the hepatic veins, due to contraction of the smooth muscle in its wall. Asthmatic human beings are particularly liable to anaphylactic symptoms, due to contraction of the muscles in the bronchioles which are already hypertrophied.

If a sensitised animal recovers from anaphylactic shock, it is for a time immune to injections of the same substances which produce it. This is known as "anti-anaphylaxis." The same condition may be produced if the animal, after receiving the first sensitising dose, is injected with a large quantity of the same substance. during the incubation period, the immunity is of short duration. Various theories of the formation of anaphylatoxin have been advanced.

The most feasible seems to be that antibodies are formed by the first injection, which on subsequent reaction with the antigen of the second injection, lead to poisonous protein-splitting products. Thus when the antibody and antigen meet in the circulation, the union renders the antigen amenable to a complement action, and the action of the complement upon this complex splits off from it a poison which is called anaphylatoxin. In support of this theory, there is a diminution of the complement in the serum of animals suffering from the acute shock which accompanies anaphylaxis. It has been suggested that the action of the anaphylatoxin is due to the alteration of the colloidal conditions in the blood, and it is in support of this theory that if serum treated with kaolin, or substances in fine suspension, be injected into the blood symptoms similar to anaphylaxis are produced. 
The injection of horse serum or foreign protein into human beings is followed by what is known as "serum sickness." It consists of an eruption of an urticarial form at the site of the injection soon after its administration, a rise in temperature, albuminuria, and pains in the joints, often associated with low blood pressure and decreased coagulability of the blood. If a second injection is given an increased reaction follows. These symptoms are probably of the nature of true anaphylaxis.

The skin reactions may be immediate or delayed. In a sensitised human being the reaction may occur locally in a few minutes to an hour with a large urticarial swelling at the site of the injection, or after a period of 24 to 48 hours, as in the tuberculin tests of Von Pirquet or on the mucous membrane as in Calmette's tests. It is probable that these latter reactions are not true anaphylaxis, but are due to a substance of a proteose nature and differ from true anaphylactic antigen in being more permeable, and penetrating into the cells of the part.

It is possible to desensitise human beings who are to be injected with antitoxic substance. The hypersusceptibility of the patient may be tested by using the skin test. It should always be done in asthmatic people or people who suffer from chronic pulmonary trouble, as anaphylaxis produced in these patients is very dangerous. To perform this 0.02 c.c. of a solution of $\mathrm{I}$ in ro horse serum is injected subcutaneously as a test. If an urticarial wheal occurs in about 5 or ro minutes, increases, and lasts for 4 or 5 hours, the patient is evidently anaphylactic. The antigen serum should then be given in minute doses such as .0025 c.c. of serum at half an hours interval, doubling the dose each time until 25 c.c. have been given; three or four days are then allowed to elapse before a further dose is given; the dose must necessarily be decreased if anaphylactic symptoms appear.

Local and general hypersensitiveness play an important part in chronic infectious diseases. After such an infection 
as tuberculosis there is no doubt that an anaphylactic phenomenon participates in the general symptoms and pathological appearance of the disease. The sensitiveness to tuberculin in minute doses in some cases is due to this cause, and the local skin reactions, although probably not actually due to the specific hypersensitive substance, are due to a substance of a less complex molecular structure which is absorbed by the cells and reacts upon them. The skin reaction is also applicable to proteins, and has been used to test for idiosyncrasies against food stuffs.

After the removal of a cataract, inflammation of a varying amount sometimes occurs in the eye associated with soft lens matter in the anterior chamber. A bacteriological examination of the fluid in the anterior chamber in these cases has proved it to be sterile. The inflammation has been attributed to the soft lens matter, which is said to increase in its toxicity with the age of the patient, due to chemical changes in the tissues, the result of an acquired or congenital hypersensitiveness. It has been suggested that all cases of cataract should be tried with a dermal inoculation of lens protein before being operated on, and if stronglypositive intra-capsular extraction performed.

Idiosyncrasies to Drugs.-There are many drugs which in certain people give rise to poisoning or local reaction in minute doses, such as atropin. The hypersusceptibility is generally specific for their chemical group, the symptoms produced are not the exaggerated symptoms of the physiological effect of the drug, and are the same for all drugs of the group.

Drug idiosyncrasies cannot be transmitted passively, and no successful experiments on artificial hypersensitisation in animals have been made. It is probable that the drug enters in some form of combination with the proteins of the serum, which acts as an antigenic substance. When applied to the skin in hypersensitive subjects they produce a local reaction. 


6
60

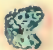
A.
B.
C.
D
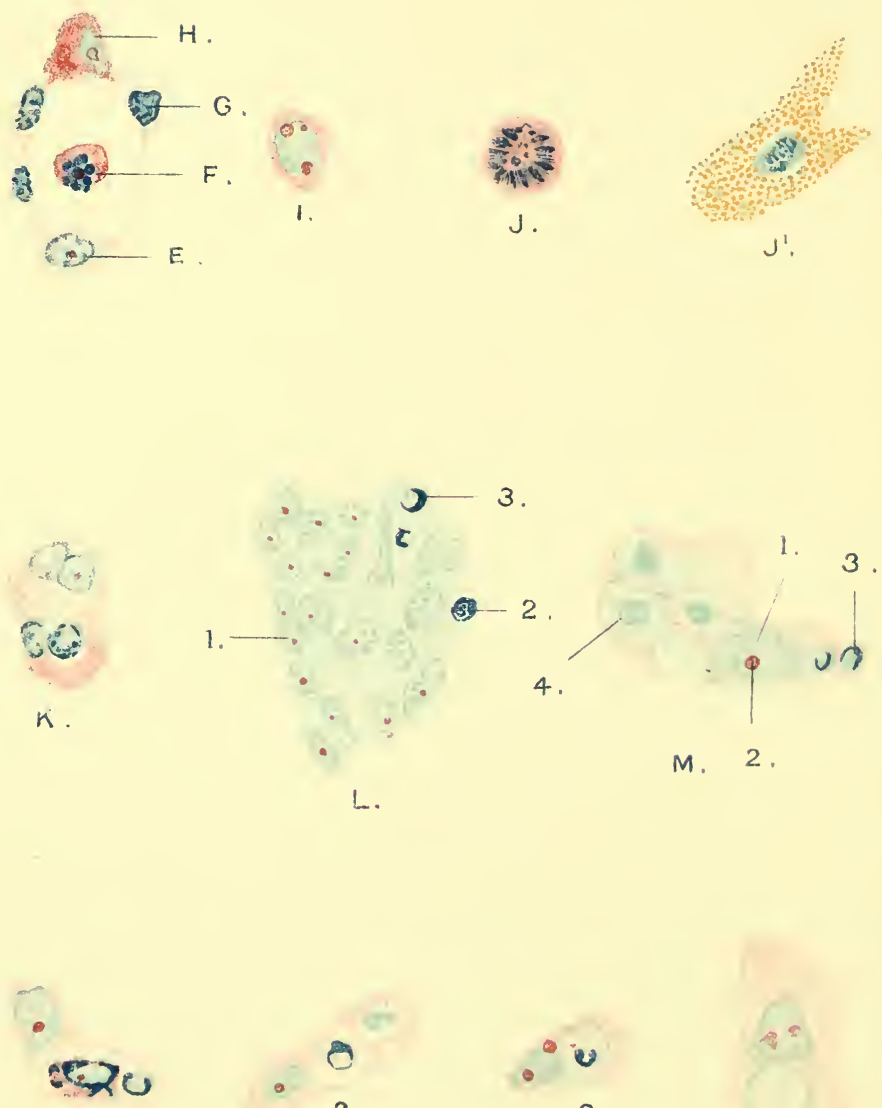

1.

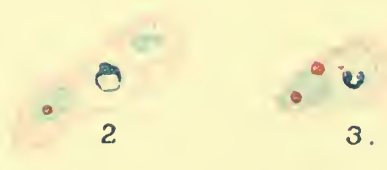

N

4.

Cells found in inflammation: A, Lymphocyte: B, transitional; C, large mononuclear leucocyte ; I) poly. morphonuclear neutrophile; $\mathrm{E}, \mathrm{F}, \mathrm{G}, \mathrm{H}$, showing the origin of the rells from a suall vessel within a follicle; $\mathrm{F}$, endothelial cell which has recently proliferated; F, plasmil cell: ( $i$, ? L.vmphorvte, endothelial cell; H, clasmatocyte; I, clasmatocyte ; J, mitosis in a clasmatocyte, or lar.e plasma cell ; J', mast cell; K, chorioplaque ' yiant coll, plasma cell (ype); L, yiant cell, endothelial tvpe-1) nucleus-like endothelial cell, (2) inclusion (nucleus of lymphocyte), (3) coccoid body of Leber (disintegrating cell inclusion); .11, large phagocytie endothelial cell-(1), nucleus, $(2)$ nucleolus, (3) letser's coccoid body, ( 4 ) disintegrating lymphocyte; $N$, showing the various stages of digestion of a plasma cell by an endothelial phagocytic cell-(1) an inclusion of a plasma cell, (2) plasma cell breaking up, (3) cuccoid body formed of nucleus, ( $\downarrow$ ) vactulisation. (Pappenheim's stain.) 
Having described the principal changes which take place during inflammation, it is necessary to describe some of the cells which are found in the inflamed area and the source from which they are derived. ${ }^{1}$ The manner and order in which they appear in an uncomplicated wound is given under Wounds of the Conjunctiva, page $38 \mathrm{I}$.

Polymorphonuclear leucocytes are cells having a bi-lobed or horseshoe-shaped single nucleus with a fair amount of cytoplasm (Plate I, D). This cytoplasm contains fine granules, the intersections of the reticulum. In some cells these do not stain strongly with either acid or basic dyes, and are known as neutrophile cells, while some have large granules which stain strongly with acid dyes, and are known as eosinophiles; others stain strongly with basic dyes, and are known as basophile or mast cells (Plate $\mathrm{I}, \mathrm{J}^{1}$ ).

Their shape varies very considerably owing to their ameboid movements. When they are killed by the process of "fixing" for microscopical examination they are usually rounded in outline, but when imprisoned in the layers of a dense tissue like the cornea they are irregular in shape, assuming that of the intercellular space along which they are making their way, and then are sometimes known as "spider" cells.

The irregularity in shape of the nuclei is also due to their ameboid movements, the denser protoplasm of the nucleus on death not assuming so rapidly the round form of the cytoplasm. These cells form 70 per cent. of the white cells in normal blood and are derived from the bone marrow. In inflammatory affections the percentage in the blood becomes considerably increased, owing to the increased call for their production, due probably to the effect of the toxin circulating in the blood stream acting on the bone marrow. They appear at the site of injury almost immediately after its infliction and rapidly increase in number. In most inflammatory infections the toxin produces an exudation

${ }^{1}$ M. S. Mayou. Hunterian Lecture, 1905. 
of neutrophile cells, but in rare instances an exudate of eosinophile cells occurs; this is a great help in diagnosis as, for example, in differentiating between vernal catarrh and trachoma.

The cellular elements of pus consist nearly entirely of these polymorphonuclear leucocytes, either alive, or in the process of disintegration caused by the bacterial toxins. At one time it was thought that pus was only produced by microbial irritation. It has, however, been shown that a hypopyon is formed when aseptic pieces of copper are introduced into the anterior chamber. This, and other experiments, prove that a purulent exudate can be excited by chemical agents alone.

Large mononuclear leucocytes are found in small numbers round inflammatory foci (Plate I, C). Like the polymorphonuclear leucocytes the cytoplasm may contain neutrophile, eosinophile, or basophile granules; those containing the latter are frequently present in the conjunctiva, especially around resolving phlyctenulæ. They are also met with in chronic inflammation of the iris.

They are probably a less highly developed form of the polymorphonuclear leucocytes, and are found in large numbers in the bone marrow, in which structure they are seen developing into the latter cell.

Large and Small Lymphocyte and Plasma Cells.-The remaining cells which are found round the site of inflammation appear at a later date, and are the product of the local activity of the tissue. The derivation of the large and small lymphocyte (Plate I, A and B) and plasma cells (Plate I, $\mathrm{F}$ and $\mathrm{J}$ ) from the local endothelium of the part can be well seen in the conjunctiva. Before birth, when the conjunctiva is free from irritation, no lymphoid tissue is present, and the rate at which it develops after birth is proportionate to the amount of irritation to which the membrane is exposed. When continued irritation is present, follicles are formed. In the conjunctiva the cells within these follicles are derived from proliferation of 
endothelium. They thus resemble newly formed lymphatic glands.

These cells are probably slightly ameboid but are nonphagocytic, their presence is usually connected, in an open wound, with the process of repair or granulation. Hence when they make their appearance in a wound the inflammation is usually becoming chronic, the system having been shut off from general septic infection.

The appearance of the plasma cell in large numbers is usually associated with local immunity, since the cytoplasm of this cell becomes easily broken up in the presence of a toxin.

The relation of these cells to each other is very close. The large lymphocyte is evidently a small one which has increased in size by growth of cytoplasm. The plasma cell is probably the result of the division of the endothelial cell, which is the parent cell of them all.

The relationship of this group of cells to the large mononuclear and polymorphonuclear leucocytes is still somewhat doubtf.ul. During early fetal life all the white cells found in the fetal blood are lymphocytes, which somewhat suggests that the one group may develop into the other.

The role played by the lymphocytes and plasma cells in chronic inflammation is far from being understood. Experimentally it has been shown by the injection of Indian ink and carmine that the lymphocytes are non-phagocytic, that they do not take particles or organisms into their protoplasm for digestion like the polymorphonuclear cells, but they undoubted present a barrier to the spread of inflammation, both from a local lesion and after the poison has reached the lymphatic glands. These observations have been confirmed by all observers. The barrier which is produced is probably due to the breaking down of the cells setting free antibodies (leucocydin). This particularly applies to the cytoplasm of the plasma cells which is very easily broken up in the presence of the toxin. The tendency of the lymphocyte and plasma cell to run into 
nodules is well seen in the formation of follicles, and also in the formation of small masses on the back of the cornea known as Keratitis Punctata. It is probable that this massing together of cells, especially in the latter condition, is due to an agglutinating body present in the aqueous. It has already been shown that red blood corpuscles are agglutinated by such poisons as Ricin, and it is probable that toxic substances may produce agglutination of comparatively non-ameboid cells such as lymphocytes and plasma cells. Indeed the different types of Keratitis Punctata appear to be produced by the different types of toxins which are present in the aqueous as the result of cyclitis. The pigment granules which are set free from the uveal tract also become agglutinated, but in all probability it is more the electrical condition of the particles than the antizen of the agglutinating body which causes them to run together. ${ }^{1}$

The endothelial cells (Plate I, E, H and G) occurring in an inflamed area are almost entirely connected with the reparative process. They are derived from the endothelium of the blood-vessels, serous membrane, and the wandering cells in the connective tissue. Their proliferation becomes most marked as the appearance of the lymphocytes subsides. They are strongly phagocytic (Plate I, $\mathrm{M}$ and $\mathrm{N}$ ) and are found containing portions of other cells, organisms, etc. They probably finally organise into fibrous tissue, which is always the outcome of any inflammation that has gone on to necrosis without producing total destruction of the part.

A number of other cells of less importance appear at the site of inflammation, especially when chronic. These are modifications of the cells already described, and are probably monstrosities produced by the action of the toxins upon them.

Giant cells (Plate $\mathrm{I}, \mathrm{L}$ ) are large cells having multiple nuclei. They are derived from endothelial cells by division

${ }^{1}$ M. S. Mayon. Trans. Ophth. Soc. of the U. K., xliv, 1924. 
of the nucleus without division of the cytoplasm. Like endothelial cells they are strongly phagocytic, and in tubercular foci often contain the bacillus of that disease. In this case the nuclei of the cells frequently have a peripheral arrangement or are grouped together at one side of the cell.

Chorioplaques (Plate I, K) are giant cells formed from plasma cells by division of the nucleus without division of the cytoplasm.

Epithelioid cells are large cells found around foci of chronic inflammation, more especially of a tubercular nature. They probably have their origin in the surrounding lymphocytic exudation.

Degeneration in all varieties of cells takes place in one of two ways, depending on the osmotic properties of the cell wall.

I. The cell may lose fluid and shrink and the granules in the cytoplasm become more marked (pyknosis); these have been mistaken by some observers for organisms in the cells.

II. The cell may imbibe fluid as a result of which it becomes vacuolated, swells up, and finally bursts distributing its contents (hyperchromatosis).

Intrauterine Inflammation.-Infection of the fetus by microörganisms can undoubtedly occur. This may take place through the maternal blood via the placenta, as in syphilis, or through a minute aperture in the membranes via the liquor amnii, as in children born with fully developed ophthalmia neonatorum.

Formerly congenital defects were frequently attributed to intrauterine inflammation, many of these are now known to be aberrations in development. Intrauterine inflammation, however, does occur and may produce lesions which sometimes can only be distinguished with difficulty from faulty development. It is easier to recognise, both clinically and histologically, that intrauterine inflammation was the cause of an abnormality the nearer to the time of birth of the fetus the inflammation occurred. 
The infection of the fetus through the maternal blood may take place at any time while the fetus is attached to the placenta. Undoubted evidence in the eye of its early occurrence is that there are children born with occluded pupils and iris bombé, together with other signs of congenital syphilis corresponding to the late secondary stage.

Infection of the eye through the membranes can only take place while the lids are ununited; they close at the seventh week and separate at the end of the fifth month of fetal life. Children have been born by Cæsarian section and unruptured membranes with gonorrheal ophthalmia fully developed; the organism having gained an entrance through a small opening insufficient to allow the liquor amnii to escape.

\section{MODIFICATIONS OF THE GENERAL PROCESSES OF INFLAMMATION IN THE VARIOUS STRUCTURES OF THE EYE}

The conjunctiva ${ }^{1}$ is the mucous membrane lining the eyelids and is reflected on to the globe. For purposes of description it is divided into the palpebral conjunctiva, which is firmly attached to the posterior surface of the eyelids; the bulbar conjunctiva, which is attached to the globe, firmly at the limbus and more loosely elsewhere; and the conjunctival fornices which are two folds upper and lower, where the membrane is reflected from the lid on to the globe.

The epithelium of the normal conjunctiva differs from that of the skin in that it is thinner and shows no keratinisation, except under unusual conditions (see Xerosis). As the basal cells proliferate some of the superficial cells are thrown off from the surface (desquamation) while others undergo mucoid changes. These latter cells are known as goblet cells and supply the normal mucus secretion. The presence of mucin and its predecessor mucinogen in a cell causes it

1 M. S. Mayou. Hunterian Lecture, 1905. 
to swell up and the nucleus to be pressed to one side. The granules present in the degenerating epithelial cells have

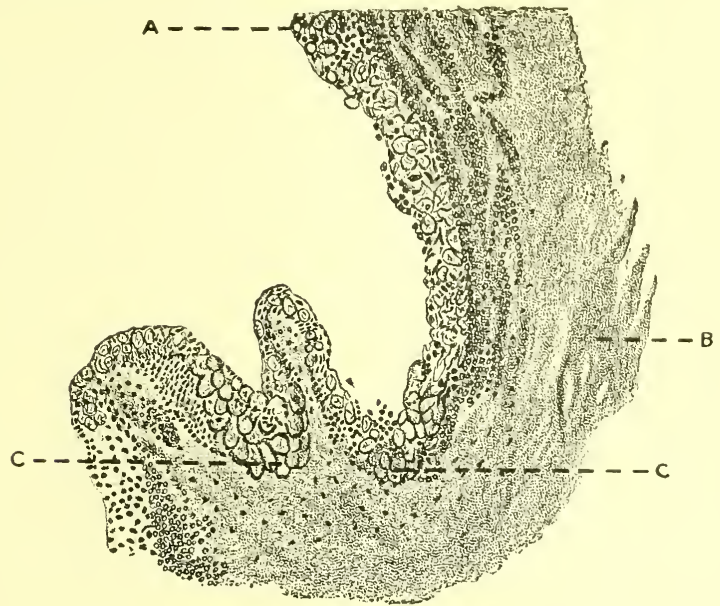

FIGS. 2 I5 and 2I6.- Sections of a polypus of the plica semilunaris showing the process of new gland formation by simple folding.

FIG. 2I5.- $A$, Epithelium undergoing mucoid change; $B$, subepithelial tissue; $C$, fold of the conjunctiva showing mucoid change at the bottom of the 'fold, with irregularity of the basement membrane.

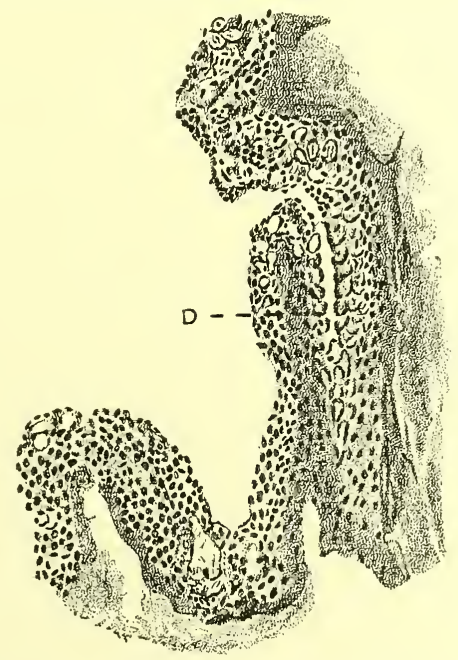

FIG. 2I6.-The fold completed, forming the new gland $(D)$.

been mistaken by various observers for microörganisms, and it is possible that the bodies found in connection with 
trachoma may be of this type. The normal conjunctiva is smooth, and contains no papillæ except at the limbus, where also there are a few pigment cells.

The epithelium of the conjunctiva in the new born infant differs considerably from that of the adult conjunctiva-a point which is of importance in connection with ophthalmia neonatorum. The epithelium, with the exception of that near the lid margins, is thinner, and not so flattened as in the adult. In the fornices it undergoes

C

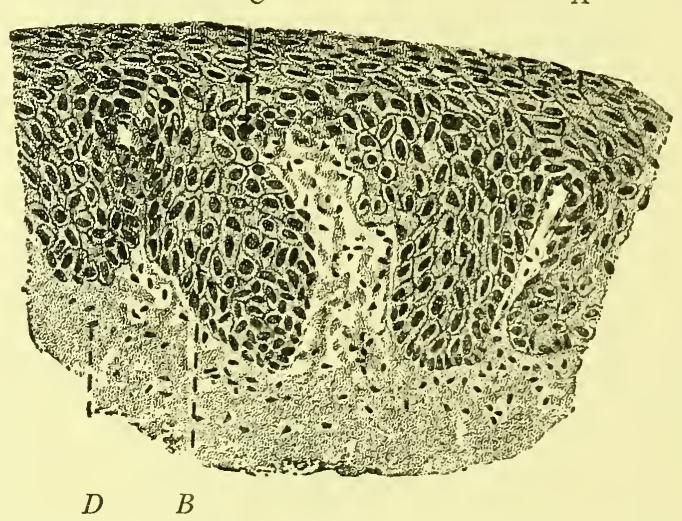

FIG. 2I 7.-Epithelium from a case of ectropion, showing the changes due to exposure. $A$, Surface epithelium; $B$, downgrowth of epithelium; $C$, leucocytes between the epithelial cells; $D$, leucocytes in the subepithelial tissue.

considerable mucoid and desquamative changes, laying bare the basement membrane, and so affording a passage through which microörganisms can easily gain entrance.

The changes in the epithelium produced by inflammation depend on whether it be kept moist or dry. If moist, the cells proliferate and undergo greatly increased mucoid changes. The passage between them of enormous numbers of polymorphonuclear leucocytes, together with the softening action of the serous exudate, cause them to be cast off in large numbers in the discharge. The exudation into the subconjunctival tissue causes the formation of papillæ from rucking of the surface epithelium; if two epithelial surfaces lie in contact with each other, it is the 
cells of these surfaces which show the most extensive mucoid change, commencing at the bottom of the folds, causing some irregularity of the basement membrane, and giving the appearance of a new gland formation (Figs. 2 I5, 2I6). The glands of Henlé are of this type, the regularity of their formation along the upper margin of the tarsus being due to this being the situation in which

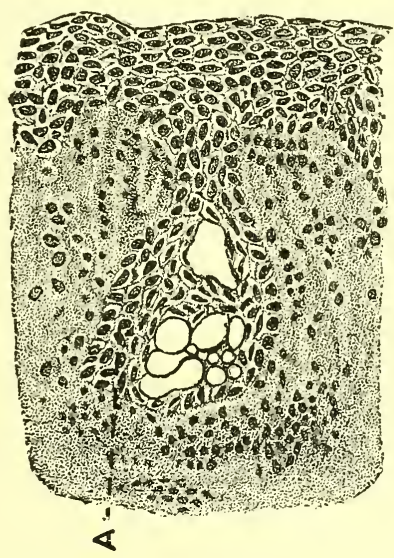

FIG. 2I8.-New gland-formation. Commencing mucoid change; portion of the epithelium from a case of ectropion from near the junction of the moist and dry surface; $A$, epithelial downgrowth with commencing mucoid change.

folding is most likely to take place, because the fixed palpebral conjunctiva there joins the loose conjunctiva of the fornix. A large gland of this type is often seen at the inner end of the tarsus, and is the one which most frequently becomes cystic.

Although this is the commonest way in which these false glands of the conjunctiva are formed, they may be observed, arising in a different manner, near the junction of the moist and dry epithelium due to exposure or to chronic inflammation, as in ectropion, secondary xerosis, and papillomata of the conjunctiva which have been exposed in the palpebral fissure. As the result of this exposure the epithelium becomes thickened and dips down into the subepithelial tissue in the form of papillæ (Fig. 
2I7). Mucoid degeneration of the epithelial cells in the centre of these papillæ takes place, with the formation of flask-shaped mucous glands (Figs. 2 I8, 219). The openings of these false glands are often occluded with epithelial débris, with the result that the lower end becomes distended to form a cyst. These cysts on the palpebral

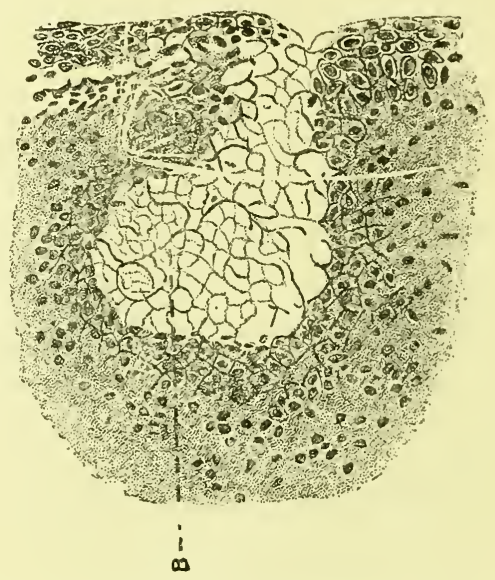

Fig. 219.-New gland-formation. $B$, Mucoid change completed. Section taken from a polypus of the plica semilunaris.

conjunctiva are extremely common, and one conjunctiva may contain hundreds of them. They are lined by flattened cells, three or four deep, and contain epithelial débris, which is sometimes calcified. In acute inflammatory conditions of the conjunctiva these cysts, if present, often become filled with pus, appearing as brillant yellow points, which subsequently rupture. Cysts of the same nature in the bulbar conjunctiva are comparatively rare, but when they occur are usually single, and transparent, containing clear fluid; they are lined by epithelium having many tuft-like processes, the cells of which, undergoing mucoid change, supply the fluid contents.

If the conjunctival epithelium becomes dry, as in cases of ectropion, a process of keratinisation takes place, the surface cells remaining longer attached and the formation 
of keratin, keratohyaline, and prickle cells takes place. The basement membrane becomes irregular, and processes of epithelium dip down into the subconjunctival tissue, forming papillæ. In fact, the epithelium of the conjunctiva which is exposed resembles very closely in structure the true skin with the exception of the absence of glands, hair follicles, etc.

If the keratinized epithelium be again moistened, as after ectropion operations, it tends to resume its former state, in consequence of the softening of the superficial layers, with subsequent mucoid change in the deeper layers. From this it seems that the presence of moisture supplied by the lacrymal fluid is necessary to the formation of mucin by the epithelial cells.

The condition known as xerosis ${ }^{1}$ is due to drying of the epithelium (see page 59 ).

The subepithelial tissue consists of a loose reticulum of connective tissue with fine elastic fibres in it. In the fornices it is filled with lymphocytes, thus producing a layer of lymphoid tissue. This layer of lymphoid tissue is absent at birth. It develops during the first four weeks, its rate of formation depending on the amount of irritation to which the membrane is exposed. If nitrate of silver be applied to the conjunctiva at the time of birth, this layer of lymphoid tissue is fully produced by the end of the first week. Its presence no doubt plays an important part in the prevention of infection.

Acute inflammation of the conjunctiva involving the whole membrane may be due to organisms, growing in the epithelium and also in some cases in the superficial layers of the subepithelial tissue. They give rise to toxins which are absorbed, causing changes in the subepithelial tissue. As a result of their absorption the vessels become enormously dilated, and the exudation produces intense swelling of the ocular conjunctiva which is known as chemosis. The histological character of the changes will be ${ }^{1}$ M. S. Mayou. Trans. Ophth. Soc. of the U. K., XXIV, I904, 9 and 39. 
described under the headings of the various forms of infection.

A localized acute inflammation of the ocular conjunctiva gives rise to the condition known as a phlyctenule. In its early stages a phlyctenule is a small opalescent swelling about the size of a millet seed, usually seen in the ocular conjunctiva in the region of the limbus with a leash of dilated vessels leading up to it. As a rule, on increasing in size the epithelium on the surface of the eminence gives way leading to the formation of a greyish ulcer. Occasionally the nodules of exudation disappear without the

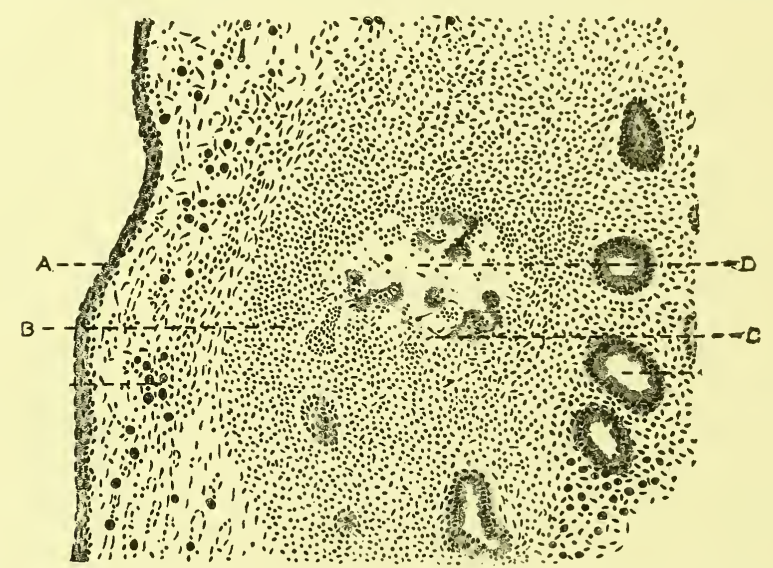

Fig. 220.- Shows a section through a phlyctenule of the conjunctiva in an early stage of its formation, before it has ruptured. $A$, Epithelium; $B$ and $C$, cellular exudation; $D$, cavity containing pus.

formation of an ulcer. The disease is often preceded by some other form of conjunctivitis of a muco-purulent character which may be very slight.

The position of a phlyctenule is not limited to the limbus; it may occur in other parts of the bulbar and occasionally on the palpebral conjunctiva. The frequency of its occurrence at the limbus is probably due to the fixed condition of the conjunctiva in that position, the friction of the lid causing the organism to penetrate deeply into the tissues. Occasionally a phlyctenule on the 
ocular conjunctiva is accompanied by another on the palpebral conjunctiva exactly opposite to it, showing its infective nature.

Ulcers produced by phlyctenules may spread to the cornea from the limbus, and when lasting for some time, become vascularized by the budding out of the vessels of the limbus.

In the early stages a phlyctenule ${ }^{1}$ consists of an exudation of leucocytes in the deeper layers of the conjunctiva, in the centre of which is found an area of necrosis (Fig. 220).

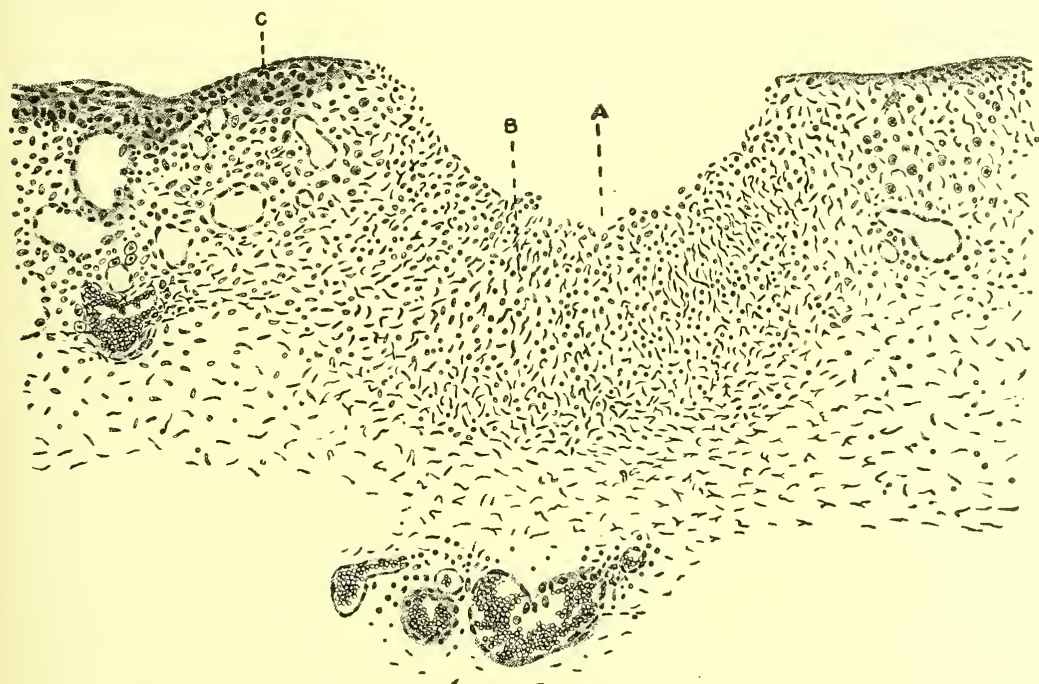

FIG. 221.--Shows a section through a phlyctenule of the conjunctiva in a later stage than in Fig. I59, after rupture has taken place. $A$, Base of the ulcer; $B$, cellular exudation; $C$, epithelium.

The leucocytes toward the centre are principally polymorphonuclear, while those around are chiefly of the mononuclear type. The blood-vessels in the neighbourhood are dilated and the endothelium proliferates; in fact the condition is a minute abscess in the deeper layers of the conjunctiva. Resolution or rupture of the phlyctenule may take place. If rupture takes place the exudation, making

${ }^{1}$ M. S. Mayou. Hunterian Lecture, 1905. 
its way along the paths of least resistance, breaks through the epithelium and an ulcer is formed.

At this stage the polymorphonuclear leucocytes have enormously increased in number, infiltrating the walls of the recently evacuated phlyctenule (Fig. 22 I). The vessels are dilated, their endothelium has proliferated, and a large number of plasma and mast cells have made their appearance.

When the ulcer begins to heal the epithelium spreads over its surface, and numbers of fine new vessels with proliferating endothelium make their appearance in the subepithelial tissue. Scattered among these are large numbers of mast cells with remains of the mononuclear and polynuclear exudation.

Occasionally, instead of an ulcer forming, the cavity becomes lined by epithelium growing down over its surface, and a cyst is formed.

In primary chronic inflammation of the conjunctiva, and in acute processes which are subsiding, lymphoid cells make their appearance. These at first are evenly spread beneath the epithelium, but if the inflammation continues, and becomes very chronic, these cells increase in number and from aggregations which are known as follicles. They resemble in structure and function those found in lymphatic glands, in which the cells are similarly produced. The formation of these follicles is mainly limited to the fornices where there is already an existing lymphoid layer, but they may occur elsewhere; on the tarsus and at the limbus, as in trachoma. Before inflammatory diseases of the conjunctiva were classified, according to the nature of the infection, this follicular formation was known as follicular conjunctivitis (Plate 2, Figs. I and 2).

In the cornea the process of inflammation is considerably modified by its structure. The substantia propria is closed between two membranes, the anterior known as Bowman's membrane is covered by about ten layers of epithelial cells, while the posterior known as Descemet's 


\section{Fig. 1}

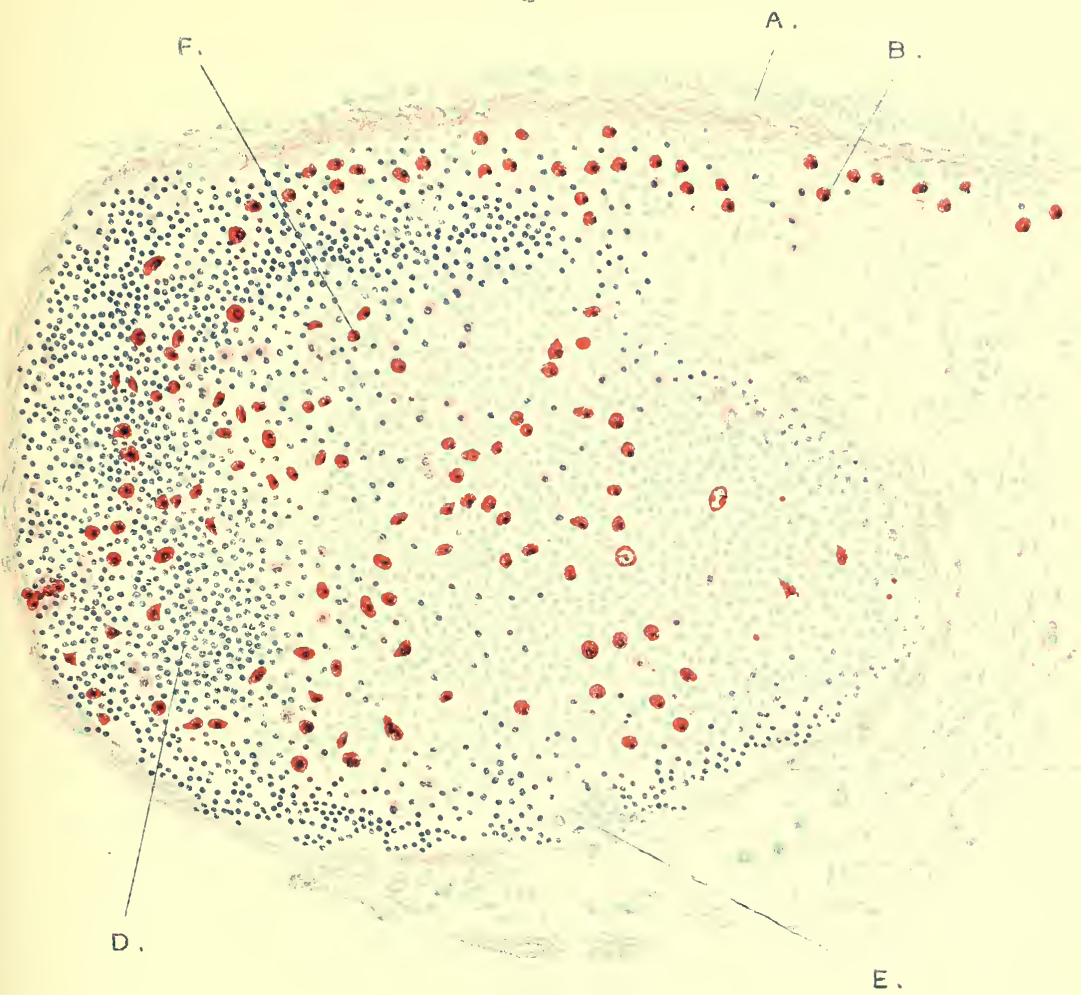

Fig. 2 .

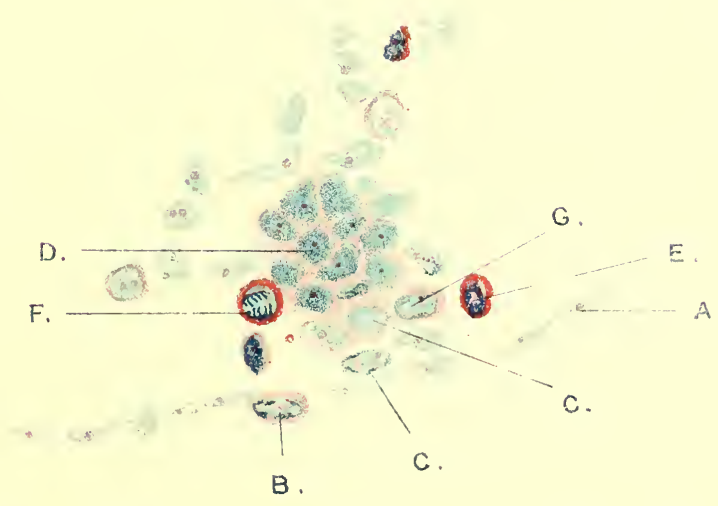

FIs. I. This section was made from a patient suffering from so-called follicular conjunctivitis. A, Epithe lum which is undergoing proliferation and mucoid change; 1 , plasma ceris beneath the epithelium; $C$, endo thelium of the follicle, which is deficient towards the surface; $D$, an endofollicle well developed: $E$, an early follicle, more highly magnified in fig. 2; F. plasma cells within the forllicle, numbers of which are undergoing mitosis. (Pappenheim staining.) $\frac{1}{6}$ olj. No. 4 eyepiere.

Fici, 2.-An early endofollicle. This figure also shows the probable origin of the cells within the follicle (E, fig. I). A, Endotheiial lining; B, proliferating endothelial cell; C, transition cell between éndothelial cell and mononuclear leucocyte; 1 ), mononuclear leucocyte; $E$, plasma cell ; F, plasma cell undergoing nitosis; Gi, transition cells between mononuclear leucocytes and plasma cells, or between endothelial cells and plasma rells. (Pappenheim staining.) it. obj. No. 4 eyepiece. Long tube. 

membrane is covered on its posterior surface by a single layer of endothelial cells. Both these membranes are impervious to microörganisms and cellular exudations, but they allow the diffusion of fluids through them. Bowman's membrane with the epithelium affords protection against infection of the substantia propria, while Descemet's membrane prevents the passage of microörganisms into the eye from the cornea. Fluids containing toxins are able to

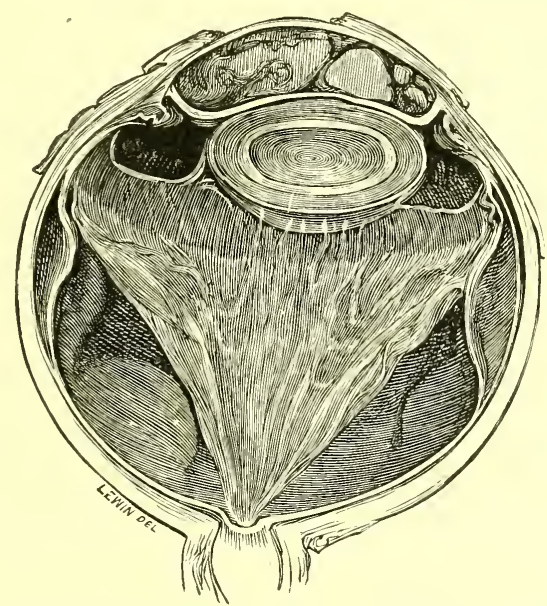

FIG. 222.- Shows the lateral half of an eye with an extensive suppurating ulcer of the cornea. There is mass of purulent exudate, which has coagulated in the hardening of the specimen, in the anterior chamber. The vitreous humour is shrunken and detached antero-laterally and posteriorly. Specimen in R. Lond. Ophth. Hosp. Museum.

pass through both these membranes. For instance, the toxin produced by pneumococcal infection of the cornea, and even of the conjunctiva, may pass into the anterior chamber setting up an inflammation of the iris and ciliary body. The presence of the toxin in the anterior chamber causes an exudation of polymorphonuclear leucocytes into it (hypopyon, Fig. 222). These cells, being unable to make their way forward to the site of infection, owing to Descemet's membrane being impervious to their passage, fall to the bottom of the anterior chamber where they form a layer of pus which contains no organisms. 
Infection of the cornea by microörganisms arises from without, as in corneal ulceration; through the blood stream, or by spread from the conjunctiva or ciliary body.

In acute inflammation, due to infection from without, the organisms must gain entrance through a wound of the epithelium, and probably Bowman's membrane. Prolonged contact with discharge containing the gonococcus or KlebsLoeffer bacillus is supposed by some authorities to produce ulceration without an abrasion. In rare instances micro-

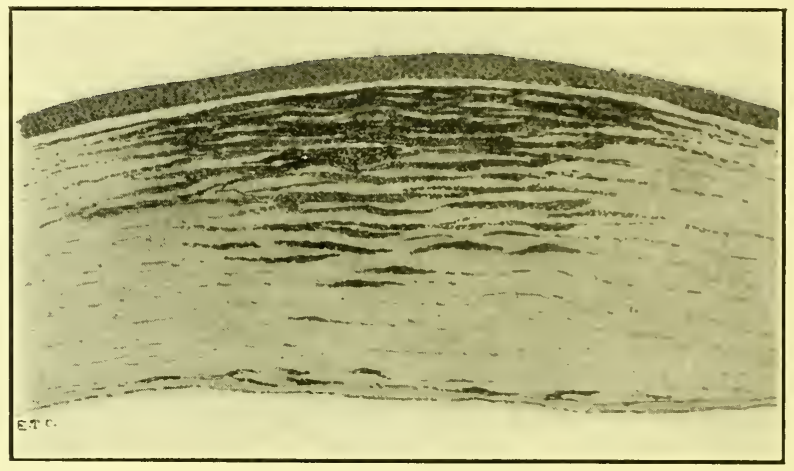

FIG. 223.- Section through the cornea showing inflammatory cells infiltrating the anterior layers of the substantia propria.

örganisms implanted in the cornea produce an abscess or onyx between its layers. If the surface of an infective corneal ulcer be scraped, and the débris examined microscopically, it will usually be found to consist of an almost pure colony of microörganisms together with a few leucocytes and epithelial débris. Histological examination shows that the organisms do not spread deeply into the tissue but laterally along the lamellæ; hence the importance of applying the cautery to the spreading margin.

The cornea being free from blood-vessels, lymph containing protective bodies and cells, must in the early stages be derived from the blood-vessels of the limbus in inflammation of the superficial layers, and from the blood-vessels of 
the ciliary body in inflammation of the deeper layers. The polymorphonuclear leucocytes appear at the limbus within fifteen minutes of the injury; they make their way between the layers of the substantia propria, of which there are about sixty, along potential spaces between its fibres (Fig. 223).

The exudation may appear as a circular grey area round the site of inflammation and may reach right up to it (positive chemotaxis), or there may be a ring of clear cornea round the site of inflammation (negative chemotaxis), and outside that again a "ring infiltration." The latter condition must be distinguished from "peripheral annular infiltration" (see page 455 ).

As the ulcer progresses, masses of polymorphonuclear leucocytes collect between the lamellæ just beyond the spreading margin, this gives rise to the yellowish infiltration which is seen clinically in serpiginous ulceration.

As the deeper layers of the cornea become involved the exudation from the ciliary vessels may cause Descemet's membrane to bulge inward toward the anterior chamber, become softened, split up, and even rupture before the ulceration from the surface has reached it, a condition which is known as an internal ulcer.

If the ulcer perforate the cornea, the aqueous is lost, and the tension of the eye is lowered. This allows a freer circulation of lymph and cells between the corneal lamellæ, as a result of which the healing of the ulcer usually takes place. This, in some instances, may be promoted by the surgeon performing paracentesis or cutting through the base of the ulcer (Sæmisch's section).

The process of repair in the cornea, owing to its non-vascularity, is slow as compared with that which takes place in connective tissue generally. New vessels which vascularise the base of the ulcer, bud out from the limbus and lymphocytes appear at the end of the fourth day.

Granulation tissue is formed slowly in the base of the ulcer, which is finally filled in by fibrous tissue; this causes an opacity in the cornea which is known as nebula, macula, 
or leucoma, depending on its density. The new connective tissue is very opaque at first, but as it becomes less cellular and the fibres are stretched out by cicatricial contraction, the opacity becomes less marked.

In place of the ordinary connective-tissue cell there are in the cornea large cells known as corneal corpuscles; these are both fixed and wander cells. They are easily distinguished from the leucocytes, being larger, having a different type of nucleus, and cytoplasm free from granules.

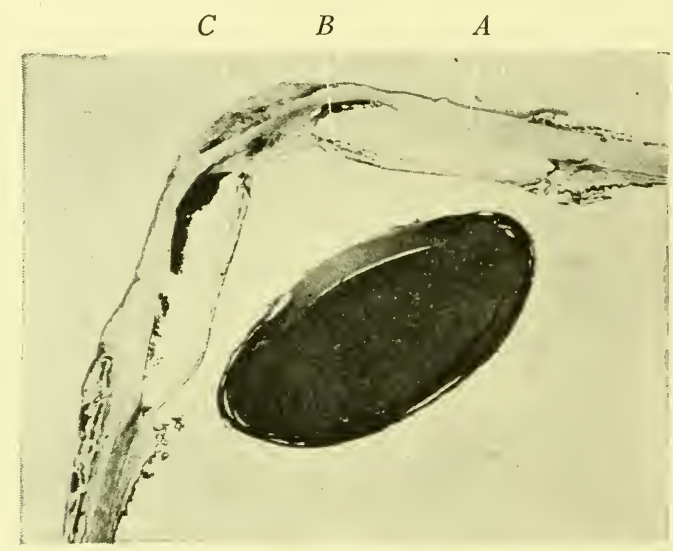

FIG. 224.- Section through the anterior part of an eye showing an anterior synechia of the iris following perforation of a corneal ulcer. $A$, cornea; $B$, iris adherent to the base of the older ulcer; $C$, leucoma.

To all intents and purposes they fulfil the same function as the large endothelial cell. In the child the cornea is thicker and softer in consistency than in the adult and, therefore, allows of much freer circulation of lymph and more rapid cellular changes; hence opacities in quite young children clear more thoroughly than in the adult.

The epithelium grows down over the sides of a healing ulcer into all its irregularities, and then thickening helps to fill in the gap in the cornea until it is on the same level as the surrounding normal tissue. The repair of Descemet's and Bowman's membrane is similar to that in wounds of the cornea (see page 369 ). 
If the ulcer stops just short of perforation Descemet's membrane may bulge into its base producing a keratocele. This prevents the proper filling in of the base of the ulcer with fibrous tissue, so that a weak place in the cornea is left which, under the normal intraocular tension, may continue to expand.

If the ulcer perforates, the organism may spread into the interior of the eye and produce panophthalmitis. When the anterior chamber is evacuated the lens may be extruded;

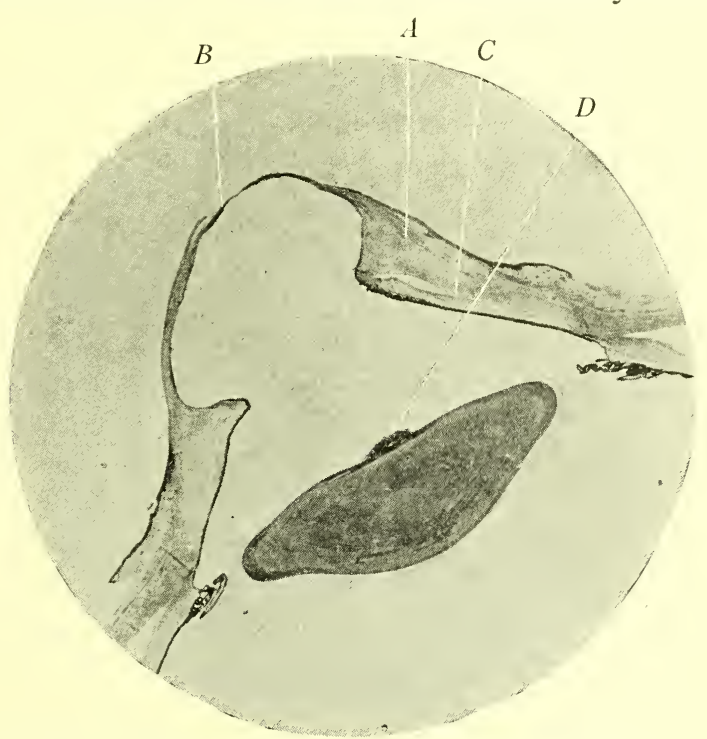

FIG. 225.-Section of the anterior part of an eye which had a perforating ulcer of the cornea and incarceration of the iris. Glaucoma ensued, and the cornea in the region of the cicatrised ulcer stretched and became staphylomatous. $A$, substantia propria of the cornea; $B$, staphyloma; $C$, iris, which is adherent to the base of the old ulcer; $D$, anterior polar opacity in lens.

if the perforation be large, the vitreous and even the retina may prolapse. The lens may come in contact with the back of the cornea and the exudate adhere to the anterior capsule. The contact of the lens with the base of the ulcer causes proliferation of the cells lining the anterior capsule and a subcapsular opacity of the lens is the result (acquired anterior polar cataract) (see page 596). When the ulcer heals the anterior chamber is re-formed, and a band of fibrous 
tissue is sometimes to be seen passing from the surface of the cataract to the back of the cornea beneath the nebula, thus showing the origin of the cataract.

The iris may become incarcerated in the base of the ulcer and adherent thereto. After healing is completed the condition is known as a leucoma adherens (Fig. 225). The iris may also prevent the proper filling in of the ulcer with fibrous tissue by bulging into the gap. The result of this is that the normal intraocular tension causes the scar to bulge, and an anterior staphyloma is produced.

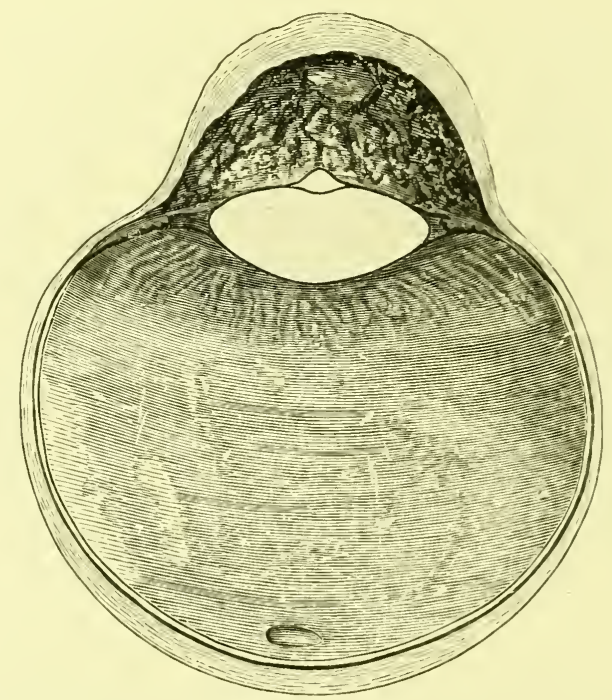

FIG. 226.- Shows the lateral half of an eye in which the whole cornea has been destroyed by ulceration and replaced by a mass of fibrous tissue, which is lined by a network of the uveal pigment. This pseudo-cornea has developed from granulation tissue on the surface of the iris. The tension of the eye was increased; the pseudo-cornea has become staphylomatous and the optic disc is cupped. There is a pyramidal-shaped opacity at the anterior pole of the lens. Specimen in the R. Lond. Ophth. Hosp. Museum.

Anterior Staphyloma.-When the perforation is small, the iris becoming incarcerated gives rise to the formation of granulation tissue on its anterior surface where it lies in the base of the ulcer. This granulation tissue fills in the gap in the cornea, becomes covered with epithelium and converted into fibrous tissue. The scar tissue yielding to 
the normal intraocular tension gives rise to a local bulging of the cicatrix, which is known as a partial staphyloma.

When the perforation is extensive a greater surface of the iris is exposed, and a much larger granuloma is formed; this may protrude above the level of the cornea. When the anterior chamber re-forms and the scar yields, an irregularly bulging cicatrix is the result. In some situations this may be very thin, being little more than the thickness of

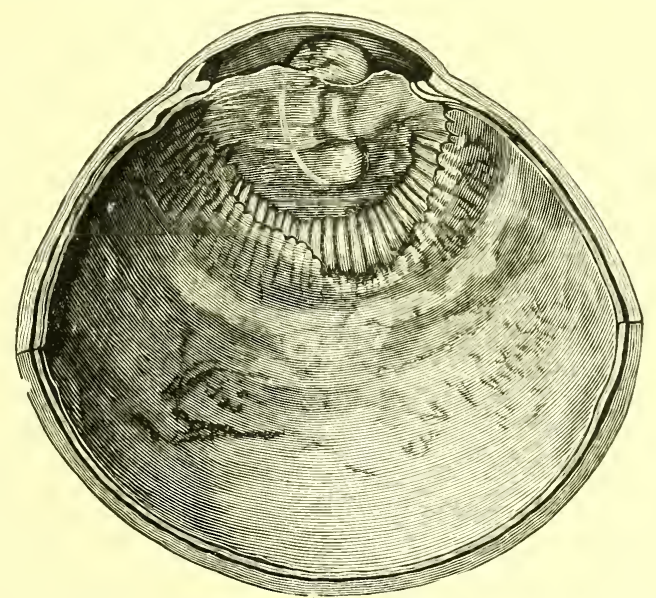

FIG. 227.- Shows the front part of an eye in which the lens capsule is adherent to the posterior surface of a staphylomatous cornea. A fold of retina is seen to have been drawn forward from the region of the ora serrata over the inner surface of the ciliary body. Specimen in the R. Lond. Ophth. Hosp. Museum.

the normal iris, while in others it may be thicker than the normal cornea. When the whole cornea is destroyed the tissue replacing it, or pseudocornea, bulging forward constitutes a total anterior staphyloma (Fig. 226).

The epithelium which covers the surface of the staphyloma is irregular, thickened and much papillated, due to its tendency to fill up all inequalities of the surface. If the staphyloma protrude between the lids the epithelial cells on the surface become keratinised, due to their drying.

The iris, which is always incarcerated in the perforation, becomes converted into fibrous tissue, the pigment cells becoming distributed throughout the newly formed cica- 
tricial tissue in the neighbourhood. In the early stages the scar tissue is very vascular, but in time the blood-vessels disappear and secondary degenerative changes are liable to make their appearance. Hyalin and calcareous changes may take place beneath the epithelium and in the scar tissue. Erosions of the epithelium and atheromatous ulceration may follow.

The lens sometimes escapes through a perforating ulcer of the cornea, the lens capsule being left adherent to it; then, if the cornea becomes staphylomatous, the traction produced on the fibres of the suspensory ligament elongates the ciliary processes, and draws forward a fold of retina from the region of the ora serrata over the inner surface of the ciliary body (Fig. 227).

The iris being pulled forward by the incarceration in the base of the ulcer the angle of the anterior chamber may be blocked, and increased intraocular tension produced.

Infection of the cornea by means of the blood stream must take place from the limbus, so that diseases of it which have their origin in this way must start in that position. For the changes which take place see page 559 .

Inflammation of the substantia propria may occur also as the result of inflammation in the interior of the eye, due to diffusion of the toxins through Descemet's membrane. In non-suppurative iridocyclitis, such as is associated with some septic trouble, e.g., pyorrhea alveolaris, the toxin in the aqueous diffuses through Descemet's membrane and then sets up an inflammation in the deeper layers of the cornea-a condition known clinically as keratitis profunda, which is frequently associated with a wrinkled condition of the membrane giving rise to striation, one form of striate keratitis. ${ }^{1}$

A more severe interstitial inflammation occurs in tubercle of the iris from the same cause. It is also possible that some cases of interstitial keratitis of syphilitic origin may be due to the diffusion of toxin from the aqueous. In ${ }^{1}$ W. T. Holmes Spicer. R. Lond. Ophth. Hosp. Reps., XIV, I 895, 33. 
severe intraocular infections leading to suppuration, such as occur in metastatic panophthalmitis and occasionally with punctured wounds, an "annular infiltration" occurs, which may go on to the formation of a "ring abscess" (Fig. 228) or ulcer situated about $4 \mathrm{~mm}$. from the limbus in its entire circumference. These conditions result from

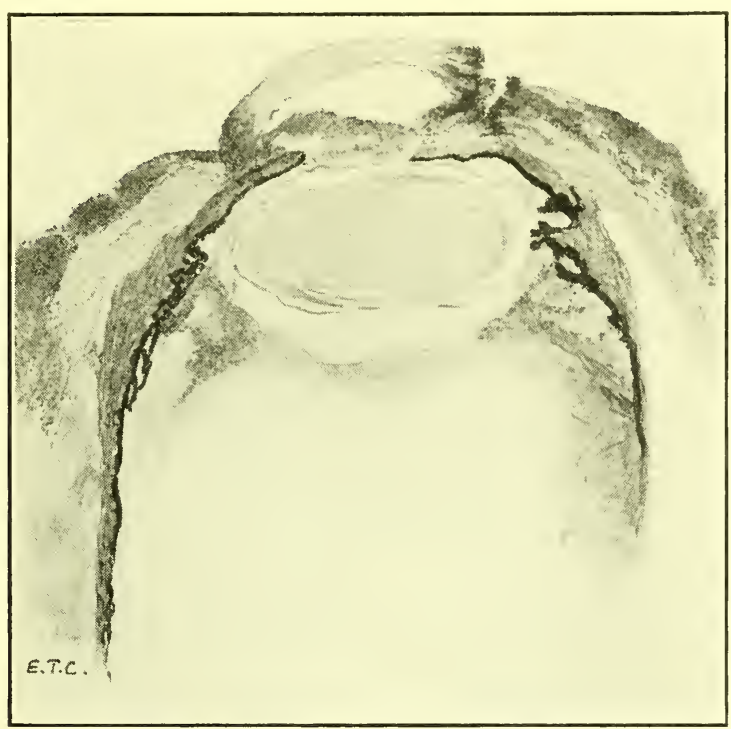

FIG. 228.- Section of the front half of an eye showing a ring abscess of the cornea secondary to intraocular inflammation.

toxins causing death of the central portion of the cornea, the "annular infiltration," or "ring abscess," being the line of demarcation along which the slough would subsequently separate.

Keratectasia is the yielding of the cornea without incarceration of the iris. The pathogenesis of a keratocele has already been explained. Occasionally after interstitial keratitis, especially in those cases which have been associated with increased tension, the whole cornea softened by the inflammatory process may yield, with the result

${ }^{1}$ E. Fuchs. Archiv. für. Ophth., LVI, I903. 
that the anterior chamber is deepened. Occasionally in cases where the inflammation is mainly limited to the centre of the cornea, a condition of conical cornea may be produced. This yielding of the cornea may give rise to splits or ruptures in Descemet's membrane (see Buphthalmia).

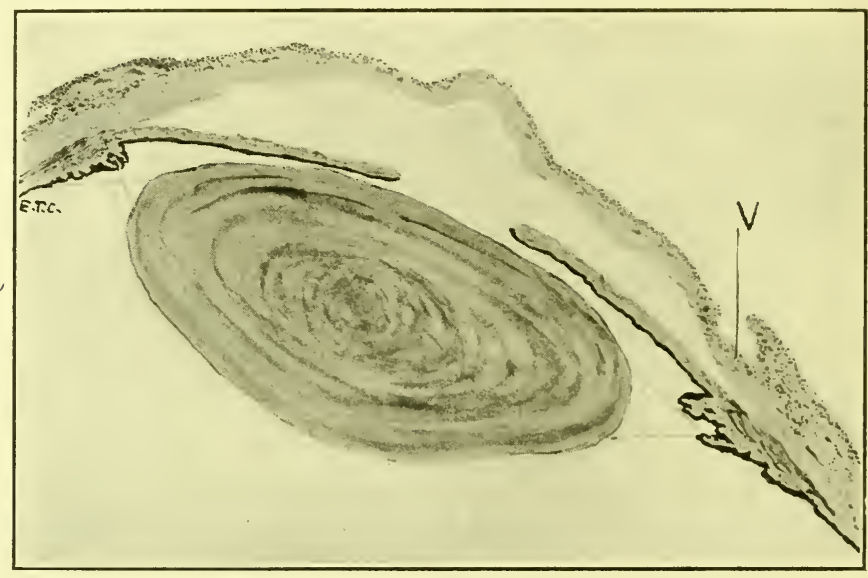

FIG. 229. - Section through the anterior half of an eye in which a Mooren's ulcer has involved the whole cornea and reduced its thickness by about half. At $V$ a small secondary ulcer has formed.

Mooren's Corneal Ulcer.-This disease is characterised by a slow necrosis of the cornea involving about half its thickness. It is frequently bilateral, and when comparatively aseptic there is little or no ciliary injection. The ulcer usually starts at the corneal margin, frequently in the upper segment, and ultimately involves the whole cornea (Fig. 229). It is greyish in colour, with the spreading margin deeply undermined, and having an overhanging edge of proliferating epithelium; there is no. discharge unless infection with pyogenic organisms takes place. As the disease spreads, the portion which has been involved heals, the epithelium growing over its surface. There is little or no formation of new fibrous tissue, as after the healing of an ordinary corneal ulcer, so that when it has entirely healed the substantia propria of the cornea is 
reduced to about half the normal thickness. Cicatricial tissue not being present, the amount of opacity which follows is comparatively slight, so that fair vision may be restored. In rare cases, after the whole cornea has been involved and has healed, it may again break down. The slow character of the necrosis; the fact that it always involves the whole cornea and never perforates; together with the absence of inflammation, when free from pyogenic organisms, suggest that the disease is due to a slow necrotic process in the superficial layers of the substantia propria from defective nutrition by the lymph or blood. This is further borne out by the fact that there is little or no attempt at reformation of fibrous tissue to replace the loss. Organisms have been found in the ulcer, but none has been identified as the cause of the disease, and they are probably only saprophytic in nature.

The sclerotic, being composed of interlacing fibrils of dense connective tissue which run antero-posteriorly and concentrically, offers a strong resistance to the spread of infection, either from without inward or from within outward. It is poorly supplied with blood-vessels, derived from the branches of the ciliary vessels, and from vessels which run along the tendons of the ocular muscles inserted into it. In the neighbourhood of the entrance of the optic nerve it derives further blood supply from the circle of Zinn. Due to this scanty blood supply the changes produced by inflammation are slow both to form and to resolve. Infections of the sclerotic, apart from wounds, are always metastatic in origin, the anterior part being most commonly affected.

Inflammation of the superficial portion of the sclerotic is known as episcleritis. It consists of a bright red patch of injection with lymphocytic infiltration, but comparatively little swelling.

When the deeper layers are affected the term scleritis is used. The latter disease may start either at the outer or inner surface of the sclerotic. Upon the outer a definite 
vascular swelling is formed, which in tubercular cases may undergo caseation, giving its centre a yellowish appearance. In this stage the disease is comparatively superficial and can be removed by excision; as it progresses it may spread outwards involving the conjunctiva, but ulceration is extremely rare. It usually extends laterally around the cornea (annular scleritis).

Inflammation in the inner surface of the sclerotic (deep scleritis), starts either in the deep layers of the sclerotic, or more commonly in the suprachoroidal lymph space. From this latter situation the disease may spread into the sclerotic and appear on the outside of the globe, or it may make its way forward in the mesh of the ligamentum pectinatum and involve the cornea, producing a condition known as sclerosing keratitis. It may also involve the ciliary body and choroid, giving rise to vitreous opacities.

The uveal tract consists of the iris, ciliary body, and choroid, which are continuous with each other. Hence inflammation starting in one portion of the tract is liable to spread to the others. The primary source of infection may be exogenous or endogenous. Apart from wounds infection is usually endogenous, but inflammation may spread from the cornea, sclerotic, or retina. Inflammation of the uveal tract may be divided into suppurative, plastic, and serous, but there is no true line of demarcation between the processes. Taken as a general rule the more virulent the infection the more acute the inflammation, the greater the amount of exudation and the larger the area of the tract involved.

Suppurative uveitis may be produced by the infection of any part of the tract with virulent pyogenic organisms, acute inflammatory changes rapidly spread to all the other portions, and thence to the retina, producing a condition of panophthalmitis. Less virulent pyogenic infections give rise to local suppuration which may resolve, and not lead to panophthalmitis. If the choroid is primarily affected, it is called suppurative choroiditis (abscess in the choroid); 
if the ciliary body, suppurative cyclitis; if the iris, suppurative iritis.

Non-suppurative uveitis is produced by infections of a less virulent character giving rise to a plastic or serous inflammation. In the former there is an exudate of fibrin and lymphocytes, with a marked tendency to the formation of connective-tissue membranes; in the latter the exudate consists of albuminous fluid in which the cellular elements are reduced to a minimum.

The uveal tract being extremely rich in blood-vessels inflammatory changes usually occur in it with great rapidity. The amount of blood passing through these vessels, and therefore the amount of tissue change, depends very largely on the state of the intraocular tension in its relation to the blood pressure. If the intraocular tension is high the vessels are in a state of collapse and little tissue change goes on; on the other hand, if the tension be low, the vessels are capable of undergoing enormous distention, especially in the choroid which varies greatly in thickness according to the amount of blood passing through it. This is seen clinically in cases of plastic irido-cyclitis with increased tension, the amount of exudation on the surface of the iris after paracentesis is very marked, appearing 24 to 48 hours after operation.

Chroiditis.- The inner surface of the uveal tract is lined by the membrane of Bruch. This membrane, which is thickest at the posterior part of the globe, allows the diffusion through it of fluid containing toxins in the early stages of an inflammation, but presents considerable resistance to the passage of cellular exudation and microörganisms. It follows that inflammation occurring in the choroid, unless very acute or prolonged, will not cause destruction of the overlying retina, producing only an edema, by which its function is temporarily inhibited. The exudation may be beneath or within the retinal substance. The subretinal exudate may contain granules from the pigment cells, or even the pigment cells themselves. which 
become loosened from their underlying membrane; the proliferation and migration of these cells gives rise to the pigmentation which is seen in the fundus after the inflammation subsides.

A softening of the membrane of Bruch, with destruction of it, may occur in long standing or very acute inflammation, in much the same way as Descemet's membrane is affected

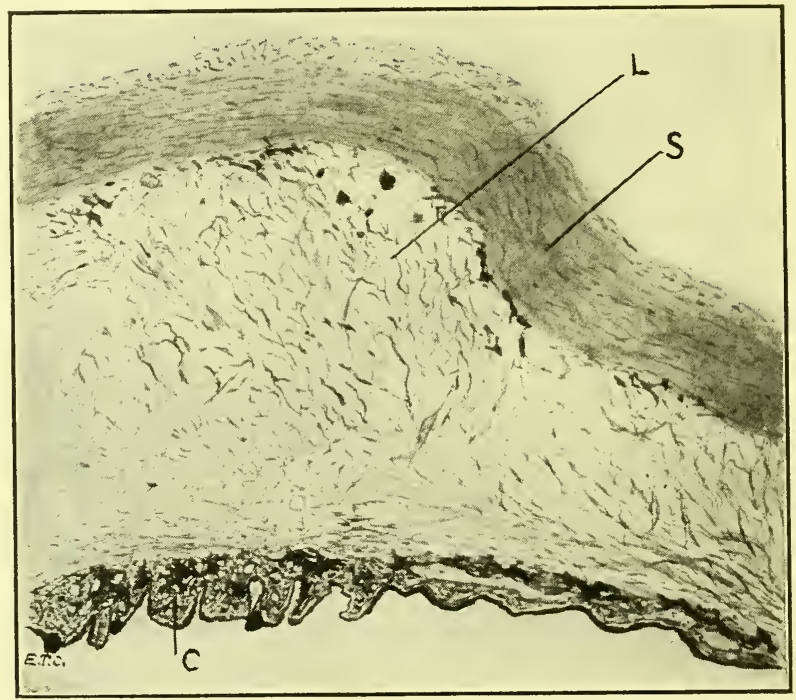

FIG. 230.- - Section showing new formation of connective tissue $L$ between the sclerotic $S$ and choroid $C$ in a shrunken eyeball which has been the subject of suprachoroiditis.

in corneal ulceration; the overlying retina then becomes involved in the inflammatory process, its nerve elements necrose, and it is deprived of its functional activity, being converted ultimately into fibrous tissue. In cases of subretinal exudation where Bruch's membrane has given way, the exudate may become organised by the extension of endothelial cells into it from the choroid. As the result of the contraction which follows the formation of the fibrous tissue, the retina may become rucked and folded upon itself, so as to form a prominent white mass with some pigmentation. Such eyes have been excised under the 
idea that the surgeon was dealing with a new growth. As this scar tissue becomes less vascular secondary degeneration occurs; fatty, hyaline, calcareous and bony changes taking place in it. The formation of colloid nodules on the membrane of Bruch is discussed under degenerative processes (see page 644). Occasionally in shrinking eyes, or in eyes that have been the subject of subchoroidal exudation or hemorrhage, the subchoroidal exudation may organise into a laminated fibrous tissue membrane lying between the choroid and sclerotic (Fig. 230); this membrane is firmly attached to the choroid but not to the sclerotic. Pigmented cells from the choroid may migrate into it but secondary calcification and ossification does not take place, as it does in the membranes on the inner surface of the choroid. The condition is known as suprachoroiditis.

Iridocyclitis.-Although Bruch's membrane can be traced forward covering the ciliary body and iris, it is extremely thin in these situations, and does not offer much resistance to the passage through it of either fluid or cellular exudate.

In the anterior part of the globe the epiblastic covering of the uveal tract consists of two layers of cells - in the ciliary body an external or pigmented layer, and an internal or non-pigmented layer. The pigmented epithelium over the ciliary body dips down into the underlying tissue in processes which resemble short tubular glands ${ }^{1}$ (Figs. I 54, I 55). There is little doubt that under normal conditions the epithelium covering the ciliary processes plays an important part in the formation of the aqueous humour from the blood. Although not in its strictest sense a true secretion of the cells, in that there are no precursory granules in them, and that the amount of aqueous secreted is dependent on the blood pressure in relation to the intraocular pressure, the epithelium under normal conditions has the power of keeping back the albuminous portion of the serum. Similarly if arsenical compounds are introduced into the circulation they do not appear in the aqueous, but directly

${ }^{1}$ Treacher Collins. Trans. Ophth. Soc. of the U. K., XI, I89I, 55. 
the epithelium becomes damaged by inflammation, or the intraocular tension is reduced, transudation of the albuminous portion of the blood-serum occurs, and arsenic if present in the circulation makes its appearance. This process has its analogy in the epithelial cells lining the kidney glomeruli which exert the same function on the blood circulating

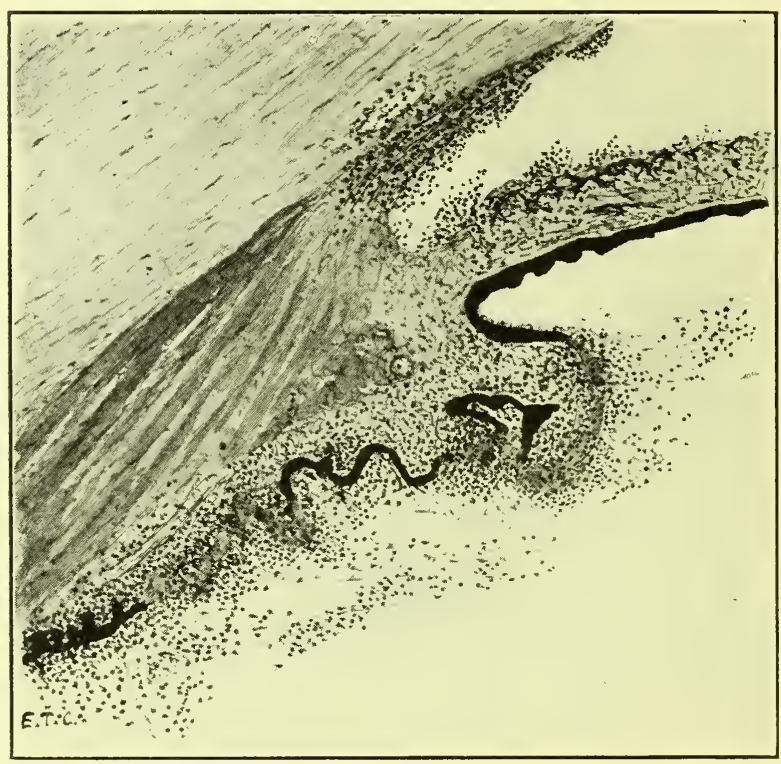

FIG. 23I.-Section showing the ciliary body and angle of the anterior chamber in a case of serous cyclitis. The vascular tissue on the inner surface of the ciliary muscle is much infiltrated with inflammatory cells. The pigment epithelium lining the ciliary body shows areas of degeneration. Cellular inflammatory exudate is shown on the inner surface of the ciliary body and about the angle of the anterior chamber. The mesh of the ligamentum pectinatum is filled with cells, accumulations of them have formed on the back of Descemet's membrane and on the anterior surface of the iris.

through it, the secretion also being dependent on the local blood pressure. In inflammation of the ciliary body the cells become swollen and proliferate, the tubular processes becoming more marked. Increase in tension of the eye during the early stages of iridocyclitis is due to the albuminous character of the secretion, and to an accumulation 
of cellular elements in the mesh of the ligamentum pectinatum (Fig. 23I).

It is probable that the beneficial result of paracentesis in cyclitis with increased tension is due, first, to the evacuation of some of the toxic products of the inflammation, and

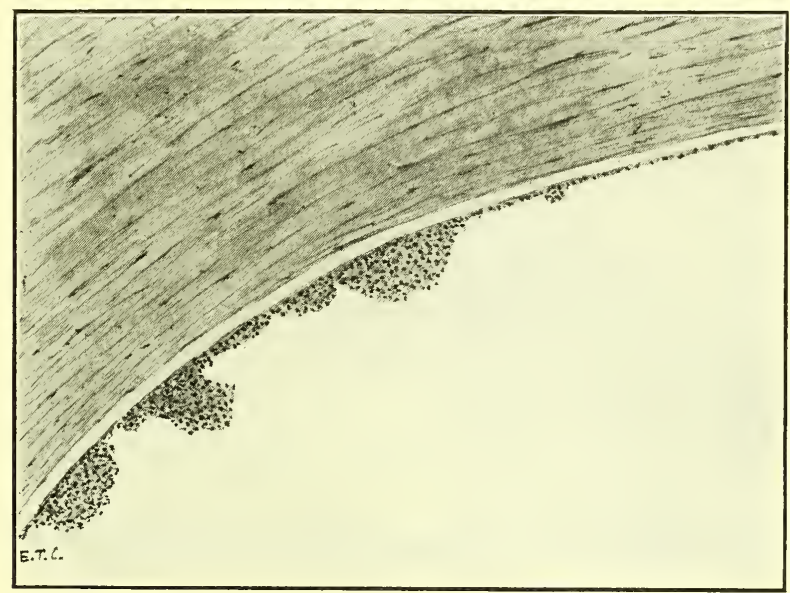

FIG. 232.- The posterior surface of the cornea in an eye with "Keratitis Punctata." The groups of cells which have become precipitated on the back of Descemet's membrane are shown.

second, to the diminution of the intraocular tension allowing a fresh filtration of fluid containing protective bodies to come from the blood-vessels.

Iridocyclitis is divided into serous, plastic, and suppurative. In serous Iridocyclitis ${ }^{1}$ the fibrinous exudate is reduced to a minimum, while the cellular exudate consists entirely of lymphocytes and plasma cells. These cells which are derived from the iris, or ciliary body, pass forward in the circulation of the intraocular fluid through the circumlental space into the anterior chamber, where they are agglutinated and deposited on the posterior surface of the cornea (see page 436). They form collections of cells which stain well and give rise to dotted opacities on the back of the cornea (Fig. 232) sometimes arranged in a tri-

${ }_{1}^{1}$ M. S. Mayou. Brit. Med. Journ., Oct. 28, i91o. 
angular form base downwards. These clumps of cells are known as keratitis punctata. They readily adhere to the back of the cornea owing to the change in its lining endothelium, the cells composing which become swollen, irregular and loosened; even some actual desquamation may occur, so that the posterior surface of the cornea is roughened. The change in the endothelium is more marked in the lower than in the upper part of the cornea, probably due to the albuminous toxin present having a higher specific gravity than the normal aqueous, and so tending to sink to the lower part of the anterior chamber. Pigment granules are frequently set free from the uveal tract, and these like the cells become agglutinated. They are never found within the mononuclear leucocytes which are non-phagocytic, but are often picked up by the endothelial cells on the back of the cornea, where they may remain permanently long after the leucocytes have disappeared. They are also picked up by wandering endothelial phagocytic cells and carried into the canal of Schlemm. Sometimes in their passage through the pectinate ligament the pigment granules may become filtered out from the phagocytes and remain in its meshes.

Prolonged chronic cyclitis may produce other changes in the eye. The exudation from the ciliary body may appear as opacities in the vitreous. Round-celled infiltration, with secondary pigmentation, is frequently present in the anterior parts of the choroid, from the disease spreading to it from the ciliary body. As the result of osmosis of the toxic bodies the lens after a time may become opaque. The iris may become thin, blanched and atrophic. Occasionally, if the disease begin in a subacute attack of iritis, an atrophic patch may appear in the iris marking the probable site of a septic embolism which gave rise to the inflammation.

Plastic Iridocyclitis. ${ }^{1}$-This form of iridocyclitis, when accompanying wounds in the globe, may give rise to sympathetic inflammation.

${ }^{1}$ L. Buchanan. Trans. Opth. Soc. of the U. K., XXI, I90I, 208. 
The feature of this disease is the marked tendency that the exudate shows to the deposit of fibrin, with the formation of inflammatory connective tissue membranes. The cellular exudation in the tissue consists of nodules of lymphocytes and plasma cells. These are present in the iris, ciliary body, and choroid. Occasionally giant cells and large endothelial cells may be present in these follicles.

The exudation into the vitreous causes the formation of fibrous tissue which may subsequently contract and de-

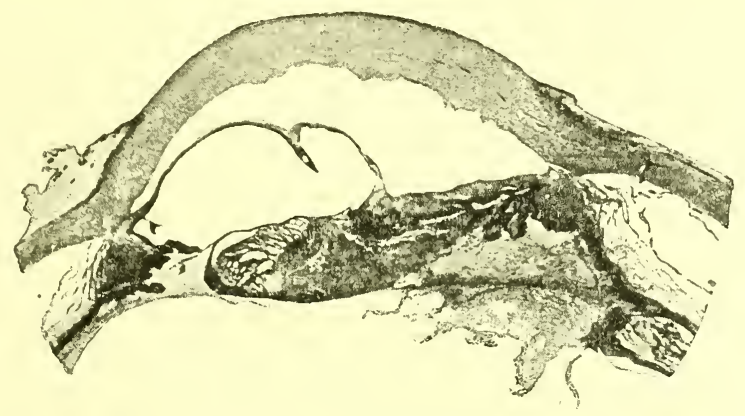

FIG. 233.-Shows a section through the front part of an eye which had plastic iritis as a result of a perforating wound of the cornea and lens. The iris on the right side is adherent in its whole length to the lens capsule. On the left side it is adherent at the pupillary margin but bowed forward, by fluid unable to pass through the pupil, elsewhere. Case recorded R. Lond. Ophth. Hosp., Reps., XIII, I 890,55 . From a photograph by E. Collier Green.

tach the retina. The exudation from the iris causes occlusion of the pupil, total posterior synechiæ and its sequelæ.

Thick fibrinous membranes may occur on the surface of the iris, ciliary body, and choroid. Keratitis punctata occurs in all cases, and occasionally a thick fibrous membrane may be found on the posterior surface of Descemet's membrane. If bullæ occur on the cornea, the fluid they contain is rich in fibrin, and a fibrous deposit forms which may organise into a thick, fibro-vascular membrane lying between Bowman's membrane and the epithelium. Finally shrinking of the eye may result.

In sympathetic cyclitis the microscopical changes in the sympathising eye are similar to those in the exciting eye. 
In iritis, the stroma of the iris is infiltrated and the exudate appears principally on its anterior surface. The fibrinous exudate and swelling of the stroma tend to obliterate the crypts on the surface of the iris, causing obscuration of its pattern and discolouration. Fibrinous coagula may also form and collect at the bottom of the anterior chamber. As the inflammatory process subsides the exudate gradually becomes absorbed, but the iris, especially after many attacks, becomes thin and atrophic and in some instances permanently discoloured.

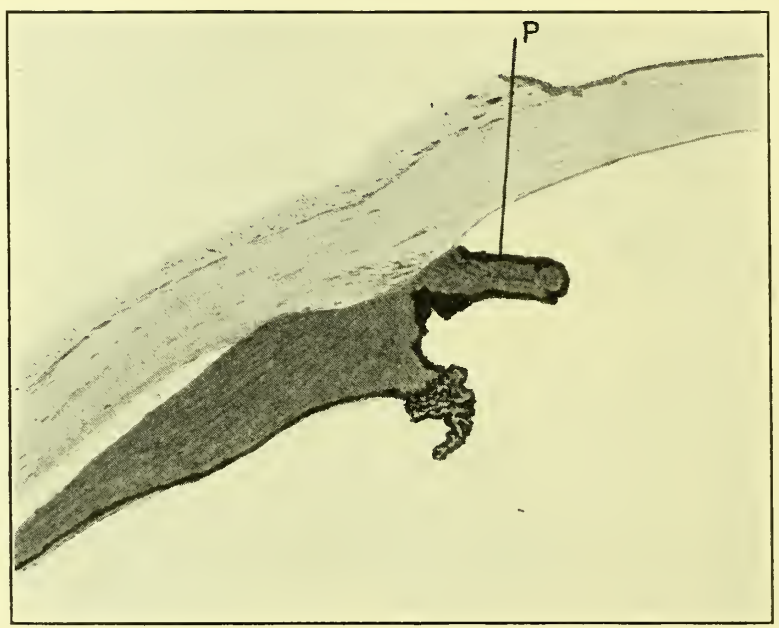

FIG. 234.- Shows the iris drawn back into the angle of the anterior chamber, from the organisation of fibrous tissue on its anterior surface, the result of iritis following a perforating wound. $P$ points to the pigment epithelium which has become drawn round the pupillary margin, and covers the whole of what there is left free of the anterior surface of the iris.

The exudation may cause adhesions between the posterior surface of the iris and the anterior capsule of the lens (posterior synechiæ). If this occurs at the margin of the pupil in its entire circumference it is known as a secluded pupil, while if the lymph cover also the anterior capsule of the lens in the pupillary area it is known as an occluded pupil. The aqueous secreted into the posterior chamber, bulges forward the iris, producing a condition 
known as iris bombé which may lead to secondary glaucoma (see page 305). Cysts between the two pigment epithelial layers on the posterior surface of the iris are of frequent occurrence, producing a condition resembling iris bombé. After very severe plastic or suppurative inflammation the whole posterior surface of the iris may be stuck down to the anterior capsule of the lens-a condition known as total posterior synechia (Fig. 233).

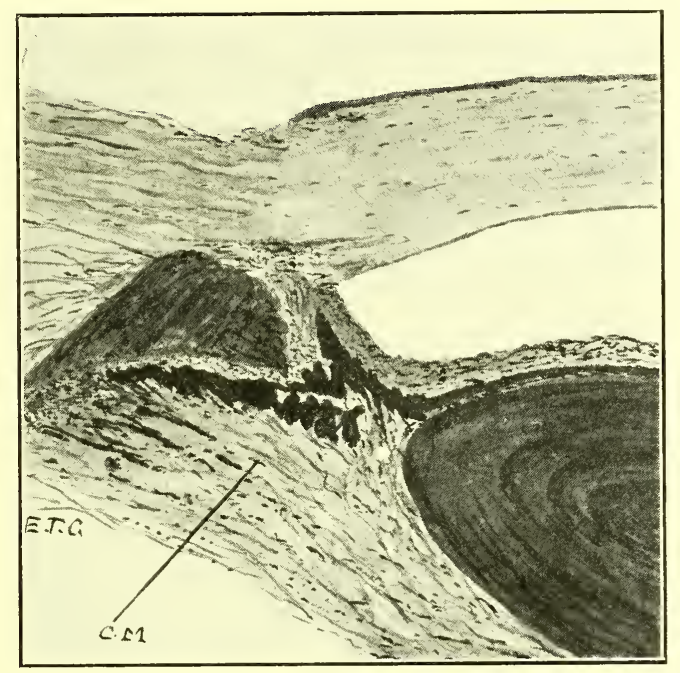

FIG. 235.- Section of an eye which had had plastic cyclitis. A cyclitic membrane, $C . M$., has formed, the organisation and contraction of which has drawn the ciliary body away from the sclerotic and retracted the root of the iris, so deepening the periphery of the anterior chamber.

The exudate on the surface of the iris may organise into a membrane of varying thickness; it may be so thin that it can only be distinguished microscopically as a single layer of cells, or so thick as to conceal the iris. In both forms new vessels may be present. In cases where the iris is not bound down to the anterior capsule of the lens, the cicatricial contraction of this connective tissue on its surface may cause retraction of the iris, and ectropion of the pigment epithelium round the pupillary margin. The retrac- 
tion, in rare cases, may be so great that the iris becomes drawn up into the angle of the anterior chamber, and clinically an appearance of aniridia be produced ${ }^{1}$ (Fig. 234).

In suppurative iridocyclitis, polymorphonuclear leucocytes may not only collect at the bottom of the anterior chamber, and give rise to hypopyon, but also be exuded

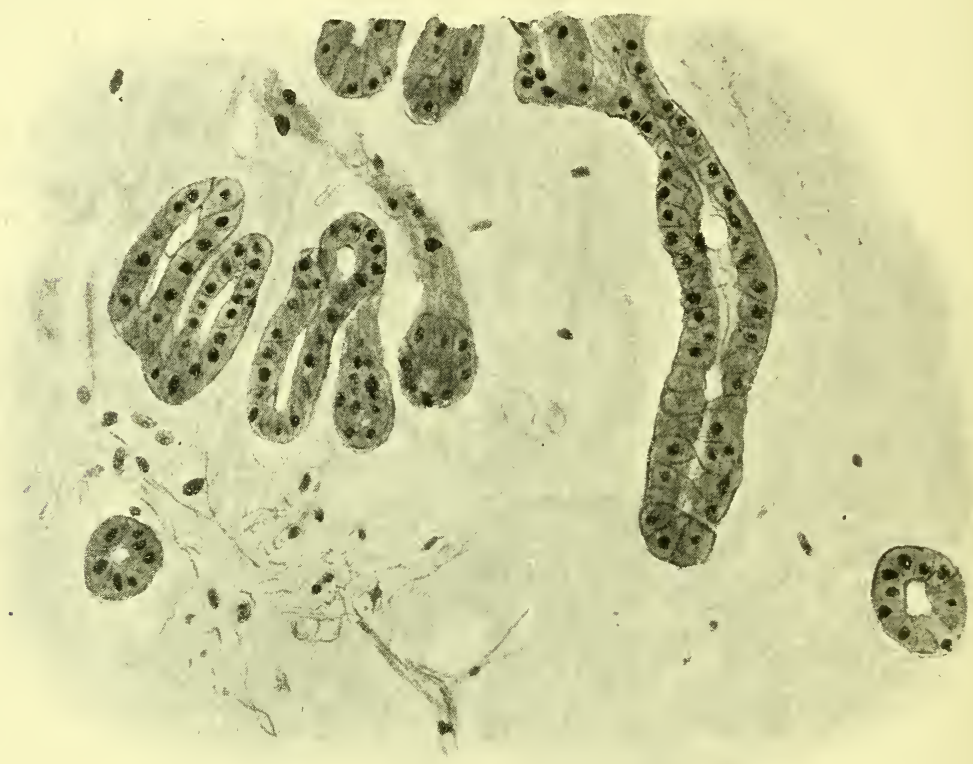

FIG. 236.- Shows a bleached section of tubular processes of epithelial cells which have extended into a cyclitic membrane, the result of plastic cyclitis. $\times 300$.

from the ciliary body into the vitreous behind the lens. In the early stages such exudation is seen clinically as "greyish streaks or dust-like particles in the anterior part of the vitreous. As the inflammation progresses pus forms in the vitreous and a condition of panophthalmitis may result. If this takes place, the blood-vessels of the retina and choroid participate in the formation of the exudate. Occasionally, even when pus is formed in the vitreous, the inflammation may subside and organisation in the exudate

${ }^{1}$ Mayou. Trans. Cph. Soc. of the U. K., Vol. XXIX, p. 204. 
takes place. A fibrous tissue membrane (cyclitic membrane) then forms behind the lens giving rise to a yellowish reflex - a condition which may clinically resemble glioma of the retina, and hence has received the name of pseudo-glioma.

The formation of fibrous tissue in the vitreous causes secondary changes in the eye. The vitreous may shrink, and if adherent to the retina, cause detachment of that membrane. In pseudoglioma, the retina becomes matted up with the exudate from the ciliary body, to form a large

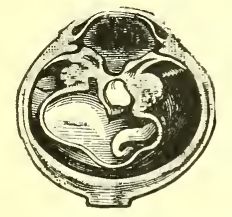

Fig. 237.- Shows the lateral half of an eye which has a mass of organised inflammatory exudate in the anterior part of the vitreous humotrr, "pseudoglioma." By its contraction it has detached the retina, retracted the root of the iris, and produced a projection backward at the posterior pole of the lens. Case recorded in R. Lond. Ophth. Hosp. Reps., XIII, I892, 39 I.

mass in which the pigment cells proliferate and migrate. The choroid, ciliary body, and ciliary processes are pulled inward, opening up the suprachoroidal lymph spaces. The root of the iris is drawn backward into the circumlental space, deepening the periphery of the anterior chamber (Fig. 235). The epithelium covering the inner surface of the ciliary body often extends into the cyclitic membrane in the form of pigmented, or partially pigmented, tubular processes (Fig. 236).

Atrophy of the ciliary body causes the eye to become soft and a buckling inward of the sclerotic beneath the recti muscles takes place. This general shrinking of the whole globe is known as phthisis bulbi (Fig. I75). Fatty degeneration takes place and lime salts may become deposited, principally around the vessels in the choroid, to a less extent in the posterior part of the ciliary body, and in the cyclitic membrane. Large cells of the nature of osteo- 
blasts, probably derived from endothelial cells, make their appearance and true bone is deposited. This may go on to such an extent that the entire circumference of the choroid becomes converted into a cup of bone, which is usually thickest posteriorly. A round hole in the cup is present where the nerve passes through it. Bony changes never take place in the tissue of the iris.

The lens, the posterior surface of which is embedded in the cyclitic exudation, is at first clear but after a time becomes opaque; its posterior capsule becomes wrinkled up by contraction of the membrane, and vacuolation takes place in the lens fibres. The pressure of the exudate may produce a molding of the lens into a posterior lenticonus (Fig. 237). Secondary calcareous changes may take place in it, but as long as the capsule is intact there is no bone formation.

Inflammation of the Retina.-Before the pathology of the diseases affecting the retina was properly understood many of them, such as albuminuric retinitis and retinitis pigmentosa, were ascribed to inflammation, but these are in reality degenerative processes.

The epiblastic constituents of the retina do not undergo any active changes in inflammation of it. In what is termed retinitis it is the blood-vessels and their contents which are primarily affected; the other parts of the retina merely undergoing secondary changes. Of these changes perhaps the commonest is edema, which may be either associated with the inflammatory process or secondary to vascular sclerosis, a sequela of the inflammation. The edema may be diffuse or localised. Diffuse edema occurs in the early stages of retinitis. The exudate being highly albuminous and opalescent, it gives rise to a general retinal haze when seen with the ophthalmoscope. In localised edema the exudate may collect in the layers of the retina or beneath the hyaloid membrane of the vitreous. Most commonly it is situated in the internuclear layer, where it forms cystic spaces which displace the nuclear layers. It may burst 
through the membrana limitans externa into the subretinal space; a collection of fluid in that situation giving rise to detachment of the retina.

As a result of prolonged edema the retina tends to become disorganised, the ganglion cells lose their Nissel's granules, become vacuolated, and after a time disappear; the rods and cones become swollen and break down into a finely granular débris. The fluid which accumulates in the cystic spaces may give rise to sharply defined white areas ophthalmoscopically, and later become changed into the hyalin material which is so frequently found on histological examination. After a severe inflammation the nervous elements become entirely destroyed, its fibrous tissue supporting elements alone being left.

Ophthalmoscopically the situation of a retinal exudation can only be told by its relation to the retinal vessels, which lie in the nerve fibre layer, and whose terminals do not penetrate deeper than the external molecular layer. Exudation seen to lie in front of the vessels must be situated in the nerve-fibre layer of the retina or beneath the hyaloid membrane, and exudation beneath the vessels in the deeper layers. The retinal vessels are surrounded by perivascular lymph spaces, in which the exudate may lodge, producing the white lines so often seen with the ophthalmoscope along the vessels in retinitis. Organisation of the exudate is more liable to take place in the region of the optic disc than elsewhere, as that is the most vascular portion of the retina. After the inflammation has subsided the retinal blood-vessels may undergo secondary sclerosis, and degenerative changes may occur in the retina, similar to those found in primary vascular sclerosis (see page 669).

It is not uncommon after inflammation of the retina to find a thin membrane, composed of endothelial cells, lying between its inner surface and the hyaloid membrane of the vitreous. The contraction caused by the organisation of these cells into connective tissue produces folds in the hyaloid membrane. The white bands sometimes seen on the 
surface of the retina may be accounted for by such changes. When a similar condition occurs over the optic disc, the cicatricial contraction may pull a fold of retina inward over the papilla.

The part played by the membrane of Bruch in inflammatory processes has already been discussed under inflammations of the uveal tract, and it has been shown how its continuity prevents the spread of endothelial cells from the choroid to the retina. The continuity of the hyaloid membrane of the vitreous plays a part similar to the membrane of Bruch in preventing the spread of endothelial cells from the retinal vessels to the vitreous. Leucocytes and toxins can, however, pass through it, the former constituting the vitreous opacities which usually accompany retinitis. When gaps in the continuity of the hyaloid membrane occur, endothelial cells may spread into the vitreous and cause the formation of bands of fibrous tissuea condition known as retinitis proliferans. ${ }^{1}$ The subsequent contraction of these bands often causes detachment of the retina from its pigment cell layer.

The part played in retinal inflammation by the pigment cells, although very prominent from an ophthalmoscopic point of view, is in reality purely a secondary one, and is no direct evidence of the extent or severity of the inflammation. They receive their nutrition from the choroid and may survive when all the nervous elements of the retina have perished from inflammation. The changes in the pigment cells are most prominent as the inflammation begins to subside; at first they are loosened from the membrane of Bruch and degenerate; the granules of pigment are taken up by the leucocytes or lie free in the exudation. Granules of pigment, which are thus set free by the breaking up of the cells, may be carried into the lymphatics where they become deposited and form pigment streaks in the retina. As organisation of the inflammatory exudate begins to take place the pigment cells proliferate, the new ones mi-

${ }^{1}$ P. Flemming. Trans. Ophth. Soc. of the U. K., XVIII, I898, I54. 
grating, either by their ameboid movements or by multiplying in continuity, into the retina and any newly formed connective tissue which may be present. The sharply defined masses of pigment seen with the ophthalmoscope are thus produced. Hemorrhages due to changes in the vessel walls are a usual accompaniment of retinal inflammation. For the changes they undergo (see page 257).

Purulent retinitis may arise from direct infection through wounds, secondary to traumatic or metastatic infection of the uveal tract, and from septic embolism in the retinal vessels. The retina becomes infiltrated with polymorphonuclear leucocytes which make their way inward, collecting in large numbers between the retina and the hyaloid membrane, and in the neighbouring vitreous. The disease progressing may end in panophthalmitis or a condition of "pseudo-glioma," which condition has already been described (see page 469). Septic emboli may also give rise to a non-suppurative form of retinitis, which is known as retinitis septica, in the same way as septic emboli may produce non-suppurative forms of inflammation of the uveal tract. In retinitis septica white areas appear in the neighbourhood of the disc, usually associated with retinal hemorrhage, and occasionally optic neuritis. In the early stage of the disease the patches consist of round-celled infiltration, in which microörganisms are found.

Inflammation in the Optic Nerve.-Inflammation may take place in the optic nerve in any part of its course, but the ophthalmoscopic appearances of optic neuritis are only present when the inflammation involves the nerve below the entrance of the retinal vessels, i.e., about $\mathrm{I} 2 \mathrm{~mm}$. behind the globe. Inflammation behind this area (retro-bulbar neuritis) produces subjective symptoms, such as loss of vision and tenderness on pressing the globe back into the orbit, but no changes in the optic disc, although a descending atrophy may appear later.

In inflammation of the nerve, the nerve fibrils, the medullary substance, and the neuroglial sheaths take no 
active part, but later secondary degenerative changes occur in them. The inflammation may affect the connective tissue between the nerve bundles (interstitial neuritis) or the nerve sheath (perineuritis).

In interstitial neuritis the leucocytosis and exudation is into the connective tissue between the nerve bundles, where it presses on the nerve fibres and may lead to a temporary

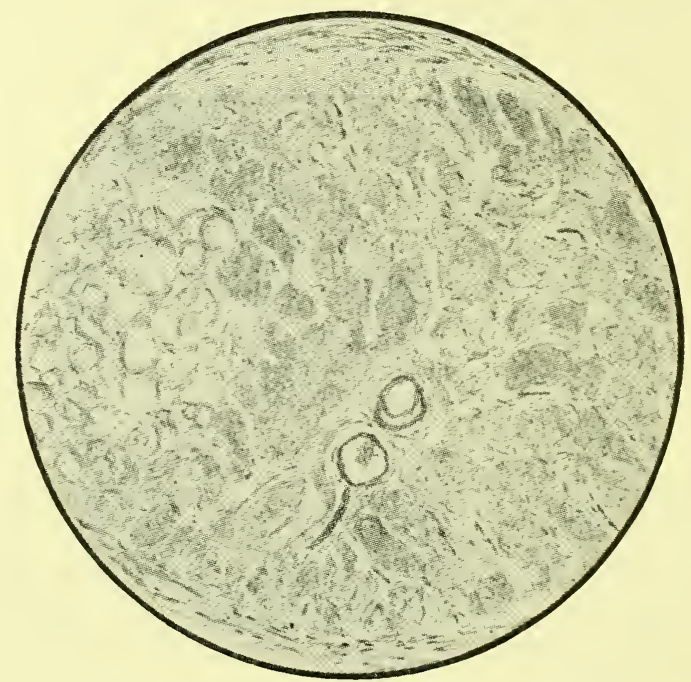

FIG. 238.-Transverse section of the optic nerve showing degeneration of the macularłfibres after an attack of retrobulbar neuritis.

or even permanent loss of vision. The cicatricial contraction following the inflammation causes compression of the nerve fibrils, and may give rise to a shrinking atrophy of the whole nerve. More frequently, islands of inflammation may occur, so that certain bundles of nerve fibres are affected; thus the macular bundle is not infrequently implicated (Fig. 238). The nerve fibres undergo degeneration, their medullary sheaths becoming broken up into fatty globules; these are taken up by the leucocytes and endothelial cells, which may become enormously distended. The axis-cylinders become varicose and swollen, and finally disintegrate. 
In perineuritis exudation takes place into the nerve sheath. The optic nerve is covered by membranes which are continuous with the membranes covering the brain, namely, the dura mater, the pia mater, and the arachnoid. Perineuritis may therefore be divided into pachymeningitis and leptomeningitis, according to the membrane most affected; both forms are usually the result of inflammation spreading from the meninges, which may be of a purulent or non-purulent variety.

The purulent cases usually end in death before organisation can take place. In the non-purulent variety, or in the cases which recover, cicatricial contraction with secondary degenerative changes ensue (optic atrophy). Although perineuritis does occur associated with meningitis, the changes at the disc in cases of meningitis are most frequently due to the increased intercranial pressure distending the nerve sheath, causing venous obstruction and edema of the head of the nerve.

Inflammation in the nerve, below the entrance of the retinal vessels, causes pressure on the retinal vein where it lies in the scleral foramen, the engorgement and tortuosity of the vein so produced is followed by edema of the nerve head and surrounding retina (Fig. 239). This is frequently accompanied by hemorrhages into the retina. The margins of the disc become obscured by the exudation, which by its expansion laterally pushes outward the retina so that ophthalmoscopically the disc appears to be enlarged. The exuded fluid collects between the nerve fibres forming cystic spaces, which in section contain granular débris with a few lymphocytes. These spaces may reach a considerable size, and the exudate undergoing hyaline change give rise to a permanent swelling of the optic disc. The neuroglia stands out prominently in sections owing to the surrounding fluid separating its fibres, but it does not seem to undergo proliferation. As the disease progresses the axis-cylinders in the neighbourhood of the papilla become broken up into finely granular material and disappear. A physiological 
cup when present becomes filled in by the inflammatory exudate, and a fine endothelial membrane may form over the optic disc, derived from the endothelium of the retinal vessels. This organised membrane over the disc tends to conceal the lamina cribrosa, when viewed ophthalmoscopically; an important point in diagnosis when the inflamma-

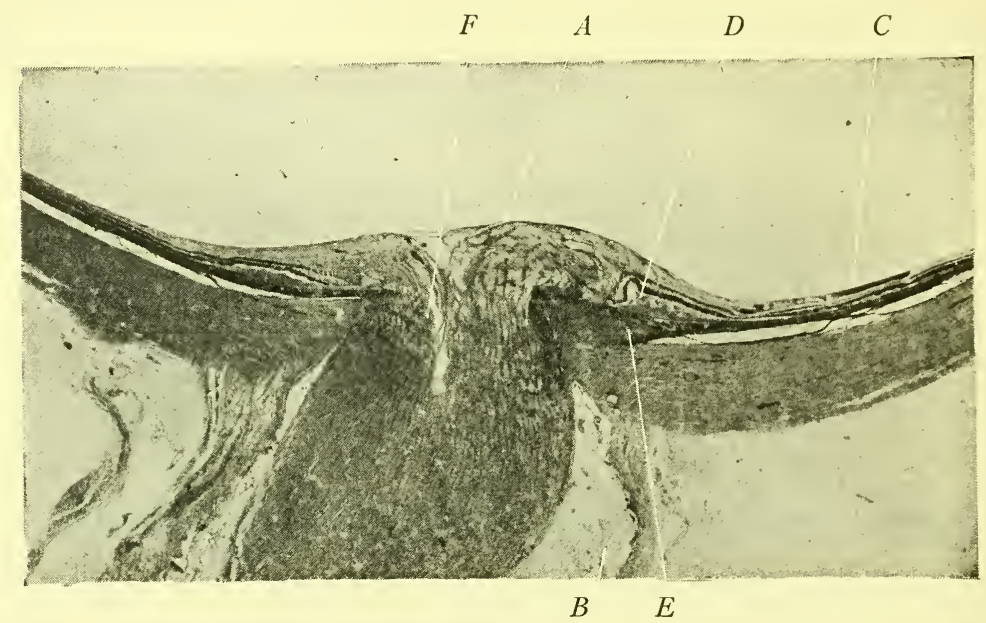

FIG. 239.- Section through the optic nerve at its point of entrance into the eye, showing optic neuritis secondary to a tuberculous mass at its margin. $A$, Swollen edematous nerve head; $B$, distended nerve sheath; $C$, retinal hemorrhage; $D$, sub-retinal edema; $E$, mass of tubercle involving the nerve pressing on the retinal vessels $F$.

tion subsides, between postneuritic and simple atrophy. The exudate may spread over the surrounding retina, and also along the perivascular lymph spaces, leading to the formation of white lines along the retinal vessels. Secondary sclerosis in the retinal vessels may result and retinal degeneration follow (see page $67 \mathrm{I}$ ).

Degeneration of the fibres of the optic nerve takes place in both an upward and a downward direction from the site of a lesion. This is unlike the degeneration which takes place in the peripheral nerves, which occurs only in the downward direction, the upper end being left in connection with the ganglion cells from which they derive their nutrition. The optic nerve in reality is an intercentral nerve. 
The nerve fibres in it are derived both from the retina and the brain. Although the medullary sheaths are developed from the brain toward the eye, degeneration of these sheaths takes place both upward and downward. After division there is never any tendency to regeneration of the nerve fibres.

Inflammation of the lacrymal gland (dacryoadenitis) is the result of infection, either through the ducts of the gland which open on to the upper and outer fornix of the conjunctiva, or through the blood stream, endogenous infection. The disease starts in the interstitial connective tissue of the gland and may be acute or chronic. The acute form may be suppurative or non-suppurative, the latter is more frequent, especially in children. ${ }^{1}$ The palpebral portion of the gland is the part usually affected. The interstitial tissue in the gland becomes swollen and infiltrated, the normal number of lymphocytes becomes considerably increased, and polymorphonuclear leucocytes make their appearance. The swelling is communicated to the surrounding ocular and palpebral conjunctiva from which there is usually some discharge, a triangular patch of edematous ocular conjunctiva on the outer side being very characteristic of the disease. If suppuration takes place, which is more common in inflammation of the orbital than the palpebral portion of the gland, the abscess may burst into the conjunctival sac or open through the skin of the eyelid.

In chronic dacryoadenitis there is an inflammation with the formation of cicatricial tissue in the substance of the gland, as a result of which degenerative processes occur in the secreting epithelium. As yet only a few cases have been examined pathologically. Such changes in the glandular epithelium lead to diminution in the secretion. It is probable that a similar change may be found in connection with essential shrinking of the conjunctiva, and secondary

${ }^{1}$ W. Inman. R. Lond. Ophth. Hosp. Reps., XV, I903, 379. 
xerosis following trachoma, as in both these diseases the lacrymal secretion is absent or very deficient.

Inflammation in the Course of the Lacrymal Canals. In the normal state the cilia of the ciliated epithelium work toward the nose and play an important part in removing organisms, débris, etc., which may gain entrance to the lacrymal sac and duct. They also prevent the spread of organisms upward from the nose on the surface of the mucous membrane.

Inflammation of the lacrymal sac (dacryocystitis) is always accompanied by obstruction of the duct, which may be situated: (a) in the duct, due to epithelial débris and foreign bodies; $(b)$ in the wall, to inflammatory affections in the mucous lining and surrounding bone walls; and $(c)$ outside, to inflammation of the nasal sinuses or new growths in the nose, which either fungate into the duct or involve its walls.

The common causes of lacrymal obstruction at the various times of life are: in infants, epithelial débris blocking the lower end of the duct, sometimes associated with malformations of its walls - congenital lacrymal obstruction $;^{1}$ in older children, periostitis produced by the snuffles in congenital syphilis; in adults, inflammatory affections of the mucous lining spreading from the nose. Lacrymal obstruction leads to distention of the lacrymal sac with tears and mucus (mucocele). This forms a suitable pabulum for the growth of organisms, which are found in large numbers in the fluid, more especially the pneumococcus. The epithelium lining the mucocele may lose its cilia and desquamate, thus leaving the subepithelial tissue open to infection. If this takes place an acute peridacryocystitis is the result, with the formation of an abscess around the lacrymal sac, which may rupture externally. If this opening does not heal the epithelium growing downward from the skin lines the tract, so that a permanent fistula is formed.

${ }^{1}$ M. S. Mayou. R. Lond. Ophth. Hosp. Reps., XVII, I908, 246. 
A more chronic inflammation also occurs around the sac, when there is a lymphocytosis, and occasionally the formation of actual follicles in the subepithelial tissue. The subsequent organisation following this chronic inflammation leads to permanent fibrous thickening of the sac wall. The epithelium lining the sac in these cases is often several layers thick, and devoid of cilia-indeed it resembles epithelium of the squamous variety.

Inflammation of the Skin of the Eyelid.-The pathology of inflammation of the skin of the eyelid, such as eczema, impetigo, etc., is not within the scope of this book.

Inflammation affecting the margins of the lids (blepharitis) is of either a seborrheic or pustular type. Seborrheic blepharitis merely affects the glands at the margins of the lids. Pustular blepharitis is an infective process of the glandular structures and hair follicles of the skin of the lid margins (see page 494). As a result of marginal blepharitis the hair follicles may become distorted so that the lashes turn inward (trichiasis), or they become so involved in the cicatricial tissue as to be destroyed (madarosis). Thickening of the lid margin may occur (tylosis), the puncta become everted and epiphora result. This, together with the fact that the patient wipes away the tears downward, tends to produce permanent ectropion of the lid.

Tenon's capsule is a closed serous sac wrapped around the posterior part of the globe to allow of its free movements on the orbital tissues. Inflammation in the capsule may follow injury, metastatic infection, or the spread of inflammation from the interior of the globe, as in panophthalmitis. In very acute cases suppuration may take place, and if the inflammation is primarily in the capsule the abscess may burst either into the globe or externally. Usually in secondary cases, such as those following panophthalmitis, the two layers become adherent to each other by plastic exudation-a point which is very manifest when enucleation is performed on a globe which has suppurated. A serous inflammation of the capsule gives rise to proptosis owing 
to its distention by fluid, the eye being pushed straight forward with pain and limitation of movement. See also page 203 .

Inflammation of the Orbit.-Inflammation affecting the orbit may occur as the result of infection from punctured wounds, or from inflammation spreading from the surrounding nasal sinuses, more especially the ethmoidal sinus as its bony orbital wall is very thin. ${ }^{1}$ Inflammation in the sphenoidal sinus may affect the optic nerve at the apex of the orbit either by pressure or by the direct spread of the inflammation. Suppuration in the orbit may be associated with necrosis in the upper jaw, especially in infants. Endogenous infection may occur associated with pyemia.

Orbital cellulitis may or may not be accompanied by suppuration. The infiltration of the orbital tissue gives rise to proptosis, the eye being pushed away from the main site of inflammation. Thrombosis of the cavernous sinus may follow. Vision may be interfered with either by pressure on the optic nerve, or subsequently by cicatricial contraction around it. An abscess may form and point either at the upper or lower orbital margin.

\section{THE METHOD OF INFECTION OF THE OCULAR TISSUE}

An infectious disease is one that is transmitted by the multiplication of organisms.

The term infectious includes contagious, since the difference between the two is only one of degree of contact with infective material.

Before an organism can be definitely recognised as the cause of a disease the following points must be demonstrated:

The organism must be found in the tissues.

It must be isolated and cultivated.

Inoculation into animals or man must produce the same disease in which the organism is again found.

${ }^{1}$ St. Clair Thompson. Practitioner, 1909. 
Similar chemical products should be found on cultivation of the organism on media as in the body.

A specific serum reaction, agglutination or bacteriolytic reaction, should be obtained if the blood of an infected person be allowed to act on the specific organism producing the disease.

The dissemination of infectious diseases of the eye depends on many factors which may be divided into those which are connected with the microörganism, and those connected with the individual affected.

Those connected with the microörganism are:

I. The quantity of discharge from the eye together with the number of organisms it contains.

2. The number of organisms gaining entrance to the eye.

3. The resistance of the infective material to the deleterious effects of drying, light, heat and oxygen; thus in gonococcal infections of the conjunctiva the activity of the gonococcus is destroyed or much inhibited by drying.

4. The ability or inability of the organism causing the disease to grow outside the infective tissues, either in other hosts or inanimate material (facultative saprophyte).

5. The ability of the organism to grow in the skin or mucous membrane before the onset of the disease, a lowered resistance, either local or general, on the part of the patient being required before the organism can gain a foothold in the tissues. Thus the staphylococcus and pneumococcus can grow in the conjunctiva without causing infection, while the diplobacillus of Morax-Axenfeld is often present in the nose and occasionally in the conjunctiva without causing symptoms. The Klebs-Loeffler bacillus may be present in the throat and nose and probably also in the conjunctiva without causing diphtheria.

Those in connection with the individual affected depend on prophylactic precautions taken with regard to its spread. Most of the infective diseases of the eye are affections of the conjunctiva. The manner in which they spread is by the discharge from the site of infection being conveyed to the 
eye by the fingers, handkerchiefs, towels, etc., or by washing water. The latter method of conveying the disease is not so frequent as the former, since most of the organisms, with the exception perhaps of the Klebs-Loeffler bacillus, are killed by exposure to the influence of cold water. Most of the organisms which cause conjunctivitis are pure parasites and do not long survive outside the body. The Morax-Axenfeld bacillus will, however, withstand drying for several days, and hence may be conveyed to the eye by the air.

The organisms which give rise to conjunctivitis are often present in the nose, and occasionally in the throat and mouth, during the attack, where they may or may not set up inflammation. Many of the organisms, such as the pneumococcus and staphylococcus, are constant inhabitants of these cavities, while others, such as the KlebsLoeffler bacillus, Morax-Axenfeld bacillus, may be present when epidemics of the disease are prevalent without producing any symptoms, and yet be responsible for the spread of infection.

There is no doubt that the discharge from the nose in cases of conjunctivitis must be looked upon as being infectious, and it is probable that the spraying from sneezing, coughing, etc., plays a part in the dissemination of the disease. Indeed, the use of nasal lotions or ointments is very important from a prophylactic point of view; this is especially the case in infection by Morax-Axenfeld bacillus as, although the disease is comparatively easily eradicated from the eye for a time, it is very liable to recur due to reinfection from the nose where the organism may persist.

The method of the entrance of organisms into the body, causing endogenous infection, such as tubercle, syphilis, etc., is not within the scope of this book.

\section{An infective process may be divided into:}

An incubation period, which is the time the organism takes to form sufficient toxin in the tissue to produce a clinical effect. The duration depends on the amount of in- 
fected material introduced, its nature, its virulence, and its power of reproducing itself, together with the initial resistance of the tissue inoculated. In the conjunctiva, during the incubation period, the organisms cannot be found by bacteriological methods. Probably this is because they are present in such small numbers.

The period of greatest activity, during which the organisms are most numerous in the tissues, giving rise to an inflammation which may be either serous, e.g., serous iritis; fibrinous, e.g., plastic iritis; purulent, e.g., gonorrheal conjunctivitis; croupous, e.g., diphtheritic conjunctivitis; hemorrhagic, e.g., Koch-Weeks conjunctivitis; necrotic, e.g., kerato-malacia.

A Period of Subsidence.-This may be gradual (lysis) as is usually the case, e.g., in gonorrheal conjunctivitis, where the discharge and the organism gradually disappear from the conjunctival sac, or comparatively rapid (crisis), e.g., in pneumococcal conjunctivitis, where the disease and organisms sometimes disappear in twenty-four to fortyeight hours.

Infection of the eye and its adnexa may take place from without by direct infection (ectogenous infection), by the spread of inflammation from the surrounding nasal cavities, or by being implanted in the eye from the blood stream from a focus of the disease elsewhere in the body (endogenous infection).

Ectogenous infection is the commonest form of infection for the outer coverings of the eye, namely, the lids, conjunctiva and cornea, while endogenous infection is more usual in the case of the uveal tract, retina and optic nerve.

Ectogenous Infection.-The infection may be pyogenic (pus-producing) or non-pyogenic. Ectogenous infection with pyogenic organisms is the most frequent, but as has already been pointed out they sometimes fail to produce any reaction that can be clinically regarded as suppuration. Besides the nature of the organism much depends on its 
point of entry into the eye; thus the staphylococcus epidermidis albus, when introduced into the conjunctival sac or. even the anterior chamber, produces no symptoms, but when introduced into the vitreous may produce suppuration.

Endogenous or Metastatic Infection.-In this form of infection the organisms are brought by the blood stream from some focus of disease elsewhere in the body, and deposited in the eye. There is yet no evidence that inflammation which is primary and local in any part of the eye can be produced only by toxins circulating in the blood. When the organisms are of a pyogenic nature the disease is known as pyemia. Pyemic infection may be acute or chonic.

Acute Pyemia.-In acute pyemia, suppuration always follows embolic infection. The veins in the neighbourhood of the original lesion become thrombosed; a piece of the clot, softened by the growth of organisms in it, becomes detached, is carried by the circulation until it becomes lodged in one of the capillaries in the eye where it sets up suppuration, e.g., middle-ear disease with thrombosis of the jugular vein sometimes becomes associated with metastatic panophthalmitis.

Chronic pyemia more frequently gives rise to ophthalmic complications. It is probable that the organisms alone, free from any clot, may circulate in the blood and lodge in the eye causing inflammation, but not necessarily giving rise to suppuration. For example, chronic iridocyclitis associated with boils, pyorrhea alveolaris, and apical tooth abscesses, etc., is in reality a chronic pyemia without suppuration, the organisms circulating in the blood without producing general symptoms. The probable reason why the primary lesion is of a suppurative nature, and the other non-suppurative, is partly that the organisms become considerably decreased in virulence from their contact with the serum, and partly that the number which lodge in the eye are insufficient to produce suppurative inflammation in such a vascular tissue as the uveal tract. 
The fact that bacteria cultivated in a certain soil always grow best on the same soil, possibly due to an anaphylactic condition of the tissue, explains why tubercle may affect all the joints of the body and not affect any other tissue, and also the frequency with which tubercle affects both eyes. Going further, if we regard sympathetic ophthalmitis to be due to septic infection of the exciting eye, it may be inferred, that a chronic pyemia is set up which causes an infection of the other eye by the blood stream, without producing other infective lesions, because the organism has no suitable similar soil elsewhere in which to grow. The reason why sympathetic ophthalmitis does not follow suppurative inflammation of an eye, is probably that the acuteness of the inflammation produces sufficient antibodies in the serum to destroy any organism which may gain entrance to the blood stream.

Wound Infection.-After operations on the eye, although in most cases, organisms probably gain entrance to the wound since the conjunctiva cannot be sterilised, suppuration is rare. In the pre-antiseptic days post-operative suppuration was less common in the eye than elsewhere. Non-suppurative iridocyclitis following operation must. however, be regarded as septic in nature.

The normal conjunctiva, ${ }^{1}$ as has already been stated, is practically never free from microörganisms; although cultivations taken from it with a loop may show no growth at one time they may do so at another, or a growth on media may be obtained by using different methods of obtaining the cultivation. The ordinary test applied to the conjunctiva, of taking a cultivation from it with a platinum loop, is not a true test of sterility, but useful in that if many organisms be present a growth will be obtained. The number of organisms found in the normal conjunctiva varies with the surroundings in which the patient lives, his personal habits, and with the methods of obtaining the

${ }^{1}$ M. S. Mayou. Ophthalmoscope, VIII, I9ro, 554. 
cultivation. Although the tears are only feebly bactericidal in their action they play an important part in the mechanical cleansing of the conjunctival sac. The upper part of the conjunctival sac contains fewer organisms than the lower, whilst at the inner canthus, in the neighbourhood of the caruncle, they are usually most numerous.

The organisms most frequently found in the normal conjunctiva are the bacillus xerosis 94 per cent., staphylococcus albus 79 per cent., pneumococcus 9 per cent., streptococcus 5 per cent., diplobacillus 6 per cent., staphylococcus aureus 6 per cent. The xerosis bacillus and staphylococcus albus are almost constant inhabitants of the conjunctiva; although these organisms are non-virulent and rarely give rise to inflammation in the conjunctiva they may, when introduced into the interior of the eye, cause inflammation; indeed, the staphylococcus is by far the commonest cause both of suppurative and non-suppurative iridocyclitis following operations.

Incisions into the anterior part of the globe seem highly resistant to infection. This is partly due to the mechanical action of the newly formed aqueous washing away any organisms that may have obtained entrance into the wound, and partly to the newly formed aqueous containing protective bodies from the blood serum. The closure of a wound in the eye from the conjunctival sac is accelerated by making a conjunctival flap.

The vitreous humour has less power of resisting infection than the anterior part of the eye; indeed, the introduction of saprophytes into it, such as the bacillus subtilis and even the bacillus xerosis, seems capable of causing suppuration.

It has been pointed out that the organisms found in the normal conjunctiva, although they produce no inflammation in it, being largely saprophytic in nature, when introduced into the globe excite inflammation. It is, therefore, of the utmost importance to reduce their number 
as far as possible before operating upon the eye. Theoretically the conjunctiva should be tested for organisms in all cases. At any rate this should be carried out whenever there is a suspicion of discharge from the conjunctiva.

The number of organisms can be considerably reduced by mechanically washing out the conjunctival sac, either with saline solution or a mild antiseptic. In urgent cases the use of perchloride of mercury I in 3000 causes desquamation of the epithelium carrying with it some of the organisms.

Bandaging the eye considerably increases the number of organisms in the conjunctival sac. Wounds in the globe which have a conjunctival flap are usually shut off from it in forty-eight hours, unless there be a prolapse of the iris or other complications. The closure of wounds in the globe is facilitated by restraining its movements, and it is more important to effect a rapid shutting off of the interior of the globe from the conjunctival sac, than to decrease the number of organisms. Thus, although post-operative conjunctivitis is not uncommon, wound infection is rare.

The lacrymal sac, if there be obstruction in the duct, is a frequent source of sepsis after operations; in such cases the pneumococcus is usually found; the organism developing in the stagnant fluid in the lacrymal sac regurgitates into the conjunctival sac, with the result that infection of the wound takes place. In these cases the lacrymal sac should first be removed and the operation on the eye performed at a later date.

The eyelashes when free from blepharitis are not a common source of infection, since they are comparatively sterile and lie entirely outside the conjunctival sac. It is, however, possible that instruments coming in contact with them might become contaminated and cause septic infection.

The glands of the lid margin may be infected with staphylococci, in which case it is probable that the conjunctival sac will contain organisms of the same nature. 
The frequency of the staphylococcus, and according to some authorities of the pneumococcus, as a cause of sepsis has led to the use of vaccines and serums for the production of an artificial immunity against septic infection, before performing major intraocular operations, such as cataract extraction. It has been suggested that this method of producing artificial immunity should always be carried out where these organisms are found in any number in the conjunctival sac. The use of staphylococcal vaccine after acute infection has taken place has been attended with good results. Serum containing protective bodies has not as yet succeeded in reducing inflammation in these cases.

In rare cases after major intraocular operations, such as cataract extraction, the eye will progress favourably, the wound healing with only slight infection, and then some weeks after the operation a hypopyon forms or even panopthalmitis sets in. In these cases it is probable that an organism of low virulence has gained entrance into the eye without causing immediate suppuration, but later, when the number of organisms has increased or for some reason the resistance of the patient's tissues has become lowered, then suppuration has set in. A pure culture of the bacillus xerosis from one such case was obtained, and in another of the staphylococcus albus. The wound in each instance was firmly healed. This prolonged incubation period might be termed latent sepsis.

Endogenous Infection of Wounds.-There is no doubt that injury plays a part in determining the lodgment of organisms from the blood stream. As general examples we may take the onset of tubercle in a joint which has been the subject of an injury. The onset of interstitial keratitis of syphilitic origin is not infrequent in an eye which has received an injury. This affection has arisen after needling the lens for high myopia. There is little doubt that in rare instances endogenous infection of an operation wound can occur if there be some septic focus, such as 
pyorrhea alveolaris or apical tooth abscess in the bod y indeed, panophthalmitis has been known to occur in an eye three months after a cataract extraction which had no sign of inflammation after the operation, obtained good vision, and where the wound was found firmly healed on microscopical examination. 


\section{CHAPTER VI}

\section{PARASITIC DISEASES AFFECTING THE EYE}

The parasites causing diseases of the eye are divided into I. Bacteria. II. Fungi. III. Animal Parasites.

In Section IV are described diseases which are probably due to microörganisms but in which the specific organism has not yet been ascertained.

\section{BACTERIA (Schizomycetes)}

The following are the ways in which all bacteria should be investigated to discover their characteristics:

I. Examination of a smear preparation;

(a) To determine its staining reactions.

(b) To determine its motility by making a hanging-drop preparation.

2. Cultivation on fluid and solid media either in a tube, plate, or hanging-drop form; to determine whether it is aerobic or anaerobic and, if the latter, in what form; thus by the exclusion of air, exhaustion of air, the absorption of oxygen, or the displacement of air by another gas.

3. Isolation brought about by using different media, by incubation at different temperatures; by sterilisation, at different temperatures; by differential atmosphere cultivation; or by animal inoculation.

4. Morphology.

5. Biology.

6. Chemical products of growth.

7. Pathogenicity.

To differentiate between microörganisms all available means are required, as bacteria which strongly resemble 
each other are frequently found on the same tissue, probably because they require the same conditions for their growth; some of these may be pathogenic, while others are non-pathogenic. In some cases a group may give rise to two different forms of infection; thus, in the "influenza group," the bacillus of Koch-Weeks and the bacillus of influenza both give rise to conjunctivitis exhibiting different characters. Further, pathogenic microörganisms lose their pathogenicity after cultivation, and at the same time may slightly alter their characteristics. Organisms may be exalted and made virulent by. inoculation, but it is doubtful whether a non-pathogenic organism can be converted into a pathogenic one of the same group. As the pathogenicity of an organism is the most important point we have to consider, those in which it is absent can hardly be regarded as identical with those in which it is present, and therefore every means must be employed to differentiate them.

The bacteria are divided into (i) Cocci, (ii) Bacilli:

\section{(i) The Cocci}

The cocci are (a) Staphylococci, (b) Streptococci, (c) Diplococci.

(a) Staphylococcus Pyogenes.-The group of organisms known as the staphylococcus pyogenes is divided into the albus, aureus, and citreus, depending on the colour which they produce when they are cultivated outside the body. They are round in form and vary considerably in size. The staphylococcus albus is usually the largest, especially the form which is known as the epidermidis albus which is so frequently found in the skin, margins of the eyelid, and conjunctival sac. They stain readily with aniline dyes, have no capsule, are non-motile, and do not form spores. They retain the stain in Gram's method. On cultivation they grow readily on nearly all media in round colonies which increase in size and run together, especially in sub- 
cultivations. When grown on gelatine they all produce liquefaction of that media, with the exception of the epidermidis albus which occasionally does not do so. It is on this media when grown in the cool incubator that the characteristic colour is best developed.

Microscopical examination of a cultivation shows the colonies to consist of masses of organisms arranged in the form of bunches of grapes.

Resistance to heat is high. The thermal death point is about $58^{\circ} \mathrm{C}$. but may be as high as $100^{\circ}$ if the organism has been kept in a dry state. Desiccation is well borne, the organisms being kept alive up to fourteen weeks on dry paper. Inhibition of growth is produced by $\frac{\mathrm{I}}{327}$ boracic acid, $\frac{I}{80,000}$ carbolic acid and $\frac{\mathrm{I}}{\mathrm{I}, 000}$ thymol. Staphylococci are killed by $\frac{\mathrm{I}}{\mathrm{r}, 000}$ corrosive sublimate in ten minutes; 3 per cent. carbolic in two minutes. Ethyl alcohol is not efficient. Nascent iodine is very powerful in destroying the organism.

Their virulence varies considerably, being greatest in the staphylococcus aureus and least in the epidermidis albus; in fact the latter organism may almost be regarded as saprophytic in nature, and can only produce inflammation when the resistance of the tissues is lowered, or when it effects an entrance into a tissue which has naturally a low resistance, e.g., the vitreous humour.

Hemolysins. - It has been shown that the staphylococci possess a strong hemolytic action, and that the hemolytic substance which is secreted by the staphylococcus can be demonstrated in broth cultures. This hemolytic property is strongest in the staphylococcus aureus and least in the albus; indeed it roughly corresponds to the virulence of the organism. Absolutely non-virulent staphylococci do not produce hemolysis. The hemolytic action of the staphylococcus takes place in the blood stream as well as 
in the test tube. Staphylo-hemolysin is readily destroyed by heat of $56^{\circ} \mathrm{C}$. in twenty minutes, and it is not possible to re-activate it. Anti-staphylohemolysin can be artificially prepared by injecting staphylohemolysin, and is sometimes found normally in the blood.

Leucocidin.-It has been shown that the filtrate from cultures of staphylococcus aureus, when grown on blood serum, contains a substance which causes death and partial solution of leucocytes, both in the test tube and in exudate in the body. The various strains of staphylococcus do not produce the same amount of leucocidin. The aureus group produce the most, and the albus hardly any. Leucocidin is destroyed by heating to $58^{\circ} \mathrm{C}$. and is thus distinguished from staphylohemolysin. Antileucocidin has been produced by treating rabbits with leucocidin. It has been shown that leucocidin is not specific, antileucocidin being capable of neutralising the toxic properties of all the different groups of staphylococci. Leucocidin should not be confused with leucotoxin, which is a substance obtained in the serum by treating animals with leucocytes, that is a cytotoxin, and nothing to do with staphylococci. The antistaphylolysin and antileucocidin both possess distinct protective power when given at the same time as the staphylococci, but if the staphylococci are given first and an interval allowed to elapse between the administration of the antistaphylolysin or anti-leucocidin, the latter is not efficacious.

Agglutinins of one of the pathogenic staphylococci will agglutinate all virulent staphylococci, but will not agglutinate any group of a non-virulent type.

Vaccine therapy is most successful in treatment of staphylococcal infections; leucocytic extracts have also proved of great service. The transfusion of blood, from people immunised to staphylococci, to patients suffering from chronic staphylococcal infections, has been followed by success.

An extraocular lesion due to infection by staphylococci tends to become multiple owing to the discharge from it 
infecting the glands, hair follicles, conjunctiva, etc., in the neighbourhood. These organisms can grow in the secretion of the glandular structure of the skin and of the eyelids. Acne vulgaris is frequently due to the infection of the sweat glands of the skin with this organism. It is often associated with styes, chalazion, phlyctenulæ of the conjunctiva, and marginal corneal ulcers. ${ }^{1}$ The staphylococcus albus and occasionally the staphylococcus aureus is found in the discharge.

In pustular blepharitis the staphylococcus albus is generally found. It is sometimes associated with the bacillus of Morax-Axenfeld, suppuration taking place in the hair follicle. If infection of Zeiss glands takes place and suppuration follows, a hordeolum externum or stye is produced. If an eyelash is affected the hair usually falls out and the follicle may be entirely destroyed or a finer hair grow in its place, which is often maldirected, owing to the distortion of the follicle from the formation of cicatricial tissue. The disease being liable to spread from one follicle to another, it may cause considerable loss of the lashes.

The infection of the Meibomian glands causes them to be inflamed with the result that the secretion from them is semi-purulent in character. ${ }^{2}$ When the epithelial cells lining them proliferate, and do not break down into secretion, the lumen of the gland may become blocked. A periadenitis occurs around the gland in the tarsus which becomes softened. The lumen of the gland above the obstruction is then filled with inflammatory infiltration and epithelial débris. When the inflammation is not very acute the exudation consists principally of lymphocytes with giant cells. This granuloma, known as a chalazion, may remain chronically inflamed, and after a time become organised from the periphery, so that the wall becomes thickened and converted into fibrous tissue.

In more acute inflammation suppuration takes place and the exudation consists of polymorphonuclear leuco-

${ }^{1}$ M. S. Mayou. Ophthalmoscope, I908, 566.

${ }^{2}$ R. W. Doyne. Trans. Ophth. Soc. of the U. K., XXX, I910, 85. 
cytes. The abscess formed may point toward the skin or conjunctiva, more frequently the latter, it being usually the line of least resistance, since in front of the tarsus there is the skin and orbicularis muscle while behind it there is only the conjunctiva. When it points through the conjunctiva it is known as hordeolum internum. The disease is liable to spread from one gland to another, so that many chalazia may be present at the same time or the disease may recur.

The bacillus xerosis is found in all Meibomian secretion and it is therefore usually present in the contents of a chalazion, but probably takes no part in its formation, being merely saprophytic in nature. Other organisms, such as diplobacillus of Morax-Axenfeld, pneumobacillus and the streptococcus have been found in the pus, but are rare.

The importance of the staphylococcus being a normal inhabitant of the conjunctival sac has already been pointed out under the infection of wounds (see page 486).

Lesions produced by the staphylococcus in the conjunctiva occur in people of all ages. Clinically they differ very considerably, according to the virulence of the strain which has caused the infection. An abrasion of the epithelium seems necessary for the organism to grow in the tissue. Usually a muco-purulent conjunctivitis of moderate severity is set up, which is frequently accompanied by phlyctenulæ, and the appearance of follicles in the fornices toward the end of the attack. The staphylococcus aureus is associated with one of the more virulent forms of ophthalmia neonatorum.

Staphylococci ${ }^{1}$ are often found in Parinaud's disease, which is characterised by the formation of large follicles all over the palpebral conjunctiva and in the fornices. Some purulent discharge is usually present, and there is enlargement of the preauricular gland, which in rare cases may ${ }^{1}$ Sinclair and Shennan. Trans. Ophth. Soc. of the U. K., XXVIII, 1908, I3. 
suppurate. Enlargement of the submaxillary glands is also of common occurrence.

Histologically, the epithelium shows the usual changes found in subacute inflammation; the subepithelial tissue is densely infiltrated with lymphocytic exudation, which in the early stages contains comparatively few plasma cells; as these cells undergo necrotic changes fragments of them are found free in the tissue, and within the phagocytic endothelial cells.

Streptococci and a special bacillus have also been described as having been found in this disease.

Staphylococcal infection of the cornea is usually the result of an injury. It gives rise to a purulent ulcer of moderate severity, which may be situated in any position, and be associated with hypopyon. As a rule the organisms do not penetrate very deeply into the tissue, and hence the disease usually yields readily to treatment with the cautery. The staphylococcus aureus may give rise to a more severe form of ulceration which ends in sloughing of the entire cornea.

A phlyctenular form of ulceration associated with acne vulgaris, due to the staphylococcus, sometimes occurs.

Staphylococcal infection of the uveal tract may occur as the result of ectogenous or endogenous infection. In ectogenous infection it follows penetrating wounds inflicted accidentally or in the performance of operations. The infection may result in panophthalmitis, as has already been described (see page 458 ), or in chronic iridocyclitis which may give rise to sympathetic ophthalmitis (see page 464 ). It is probable, though by no means established, that this organism is a cause of this latter disease, since it seems sometimes to have the power of circulating in the blood of patients without producing much general disturbance. In endogenous infection the common primary lesions, to which the ocular complications are secondary, are pyorrhea alveolaris, boils, otitis media. ${ }^{1}$ The favourite sites for ${ }^{1}$ M. S. Mayou. Brit. Med. Journ., I9ro, Oct. 28. 
the lodgment of organisms in the eye are the ciliary body and the choroid in the neighbourhood of the optic disc. ${ }^{1}$ When the infection is virulent panophthalmitis occurs.

In less virulent cases there is a chronic cyclitis with keratitis punctata, or a local patch of choroiditis is produced, which may also be associated with cellular deposit on the back of the cornea.

Staphylococcal infection of the retina may also take place by ectogenous or endogenous infection, giving rise to suppuration ending in panophthalmitis. If suppuration does not take place it may produce local patches of inflammation such as are seen in retinitis septica (see page 473).

Staphylococcal infection of Tenon's capsule, both by ectogenous and endogenous infection has been described. Suppurative or non-suppurative inflammation may follow.

Staphylococci are occasionally the cause of orbital cellulitis.

They are the commonest organisms found in the pus of a lacrymal abscess.

(b) Streptococci.-The streptococci pyogenes are round in form and vary much in size, the smaller forms being usually most virulent. They are arranged in chains some of which are of considerable length (streptococcus longus) while others are comparatively short (streptococcus brevis).

The latter are less frequently pathogenic than the former, a better classification recently introduced is based on their hemolytic properties; these are (I) Streptococcus longus or hemolyticus, the most virulent produces hemolysis in blood media. (2) Streptococcus viridans, less virulent are in shorter chains and produce greenish non-hemolytic colonies upon blood media. (3) Streptococcus mucosus, which is now generally regarded as belonging to the penumococcal group. Further differentiation of the hemolyticus group has been made by agglutination tests. There seems to be little doubt that the scarlet fever group is

${ }^{1}$ W. T. H. Spicer. Trans. Ophth. Soc. of the U. K., XXVII, I907, 235. 
definitely separated from those of erysipelas, mastoiditis, orbital cellulitis, etc.

The viridans may be found in apical abscesses of teeth, accessory sinuses of the nose, in the tonsillar crypt and in the conjunctiva. Some forms are absolutely saprophytic; others cause a general infection of the blood stream and may give rise to sub-acute vegetative endocarditis, to these rheumatic articular lesions and irido-cyclitis have also been ascribed.

Streptococci stain well with aniline dyes and retain the stain by Gram's method. They have no capsule and do not form spores. On cultivation the streptococcus is a facultative anaerobe and grows on broth, ascitic fluid and milk, the latter of which it coagulates. On agar it forms very small colonies which do not show any tendency to run together. The organism is non-motile. In cultivation difficulty may arise in differentiating it from the pneumococcus, which loses its capsule on cultivation. The elongated form of the latter, the fact that it again develops a capsule on inoculation into mice, and that it does not grow on gelatine so readily as the streptococcus serve as distinguishing features. Further the presence of a small amount of taurochlorate of soda in the media prevents the growth of the pneumococcus but does not inhibit that of the streptococcus (see also page 50I).

The virulence of the streptococci varies considerably. They may produce the most acute infections which end in the death of the patient, or only comparatively innocuous lesions. The variations in the different strains of streptococci make the use of antiserum of little value, unless by accident a similar strain be used in its preparation. Immunisation with a large variety of streptococci gives rise to a "polyvalent serum" which exerts highly protective qualities when injected with the streptococcus. Clinically it has been used with somewhat varying success.

In the skin of the eyelids the organism is found in impetigo. 
Affections of the conjunctiva due to streptococci vary according to the virulence of the organisms. In mild cases, such as are associated with impetigo, a muco-purulent conjunctivitis of moderate severity is produced, phlyctenulæ often being present. In severe cases a membranous conjunctivitis is set up. This may be even more severe than that due to the Klebs-Loeffler bacilli. In the early stages there is intense chemosis, and the eyelids are much swollen; there is often comparatively little discharge; the cornea frequently becomes ulcerated and destroyed, when panophthalmitis may ensue. In the most acute cases there is a rise of temperature with the general symptoms of fever. Orbital cellulitis, thrombosis of the cavernous sinus and death from pyemia are possible sequelæ.

In mild cases of streptococcal infection of the cornea, such as are associated with impetigo, small grey ulcers are present, while in the severe cases sloughing of the whole cornea may result in twenty-four hours. This is especially liable to occur in badly nourished children, the condition being known as keratomalacia.

Infection of the uveal tract always ends in panophthalmitis.

The streptococcus is the commonest organism found in a lacrymal abscess.

Infection of the orbit by streptococci, either from facial erysipelas, the conjunctiva, or by direct injury, may lead to a most acute form of orbital cellulitis, of which disease it is one of the common causes.

(c) Diplococci are divided into those which retain the stain in Gram's method (Gram-positive), and those which do not (Gram-negative).

Gram-positive Diplococci. Pneumococcus (Fraenkel). This organism is a small oval coccus about $\mathrm{I} \mu$ long, occurring in pairs or arranged in chains of four to ten. It is lanceolate in shape, and in the body is surrounded by a capsule (Fig. 240, D). It stains with all the aniline dyes and retains the stain in Gram's method-a most important point in 
differentiating this organism from the gonococcus. The capsules usually appear as clear areas around the organism, but they can be stained by a special capsule stain, such as MacConkey's.

Cultivation.- - It is a facultative anaerobe, non-motile, and grows best on slightly alkaline media at a temperature of $37^{\circ} \mathrm{C}$.; it will not grow below $23^{\circ} \mathrm{C}$. and is destroyed by

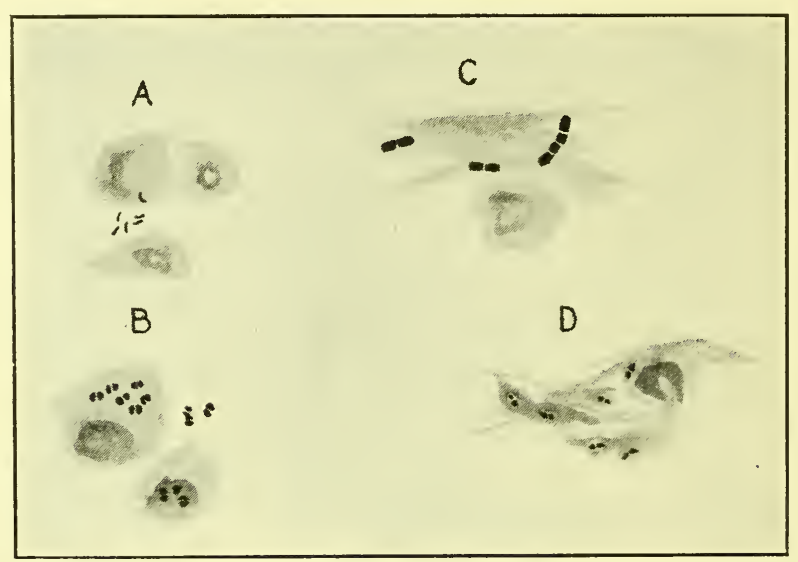

FIG. 240.- Shows the microörganisms found in smear preparations of the conjunctival secretion in different forms of ophthalmia. A, Koch-Weeks, bacilli; $B$, gonococci; $C$, Morax-Axenfeld diplobacilli; $D$, pneumococci.

a temperature of $42^{\circ} \mathrm{C}$. On blood-serum and blood-agar it appears in twenty-four hours in fine colonies scattered along the streak. The colonies are round and resemble those of the streptococcus pyogenes, from which organism in culture it is sometimes difficult to distinguish.

It will also grow on agar and in broth, but not so readily on gelatine, and then only after sub-cultivation; as a rule, there is no growth on potato. Milk is coagulated by its growth.

On whatever media the pneumococcus is grown its capsule disappears, except in a few organisms found in the water of condensation after the primary culture on bloodserum. The capsule reappears however on inoculation. 
The most important differentiation between pneumococci and streptococci is that if I per cent. inulin be added to a medium of beef serum, which has been coloured by blue litmus, the pneumococci acidify the mixture and coagulate the serum, but streptococci do not cause fermentation of the inulin. Pneumococci are soluble in dilute solutions of sodium taurochlorate whereas streptococci are not. The various forms of pneumococci have been separated into four groups, according to their capacity for agglutination and protection, as tested in mice. In groups I and 2 the organisms are only agglutinated by $\mathrm{I}$ and 2 sera respectively. Group 3, is the so-called "streptococcus mucosus," which is really a pneumococcus, as is shown by the inulin and bile tests, etc. Group 4 is a composite group of pneumococci which cannot be placed in any of the above three groups.

Inoculation.--Like staphylococci and streptococci, the virulence of pneumococci varies enormously, some specimens killing mice with septicemia in twenty-four hours and others producing no reaction at all. A non-pathogenic form has been isolated from the mouth and conjunctiva, which is characterized by slowly liquefying gelatine and growing on potato. Rabbits are less susceptible than mice, these latter animals being immune to the Friedlander bacillus. The most virulent forms are rarely present in the eye, those of moderate severity being found in such lesions as corneal ulceration.

Inoculation into the human conjunctiva has produced results which vary considerably. In many cases a mucopurulent conjunctivitis has been set up forty-eight hours after inoculation, the organisms being principally found outside the leucocytes. Inoculation of the conjunctiva with the pus from an empyema did not in one case produce any reaction for seven days. In eight cases similarly inoculated no conjunctival reaction was produced.

The variability of these results, both as regards the human conjunctiva and that of animals, is probably due to 
the variability in virulence of the organisms. It has also been suggested that it may be due to the varying power of resistance of the tissues inoculated, but before any conclusion can be arrived at as regards this point, standardised cultures must be used and more than one person inoculated. On cultivation the virulence of the organism is not maintained. Mice are peculiarly susceptible, and the virulence of the strain is raised by inoculation into them, but the organism becomes non-pathogenic to man-a point of importance in the preparation of antiserum. There is practically no phagocytosis on the part of the leucocytes toward the pneumococcus in the early stages of infection. It has been shown that agglutination of the cocci in the presence of the antitoxin takes place, and it has been found that normal leucocytes become phagocytic toward the organism in the presence of the antitoxin (opsonin?) - all points in favour of the use of vaccines and antisera in the early stages of ocular infection. ${ }^{1}$

Unfortunately the toxin, which is probably of the nature of an endotoxin, has not been properly isolated and, therefore, the prepared antitoxins are as a rule not very efficient; it is absolutely essential in giving antitoxic serum that the group to which the pneumococcus belongs should be first determined by the agglutination test.

Infection of the conjunctiva gives rise to a conjunctivitis, the clinical signs of which are frequently sufficiently characteristic to render its diagnosis possible. The incubation period is forty-eight hours. There is a muco-purulent discharge from the conjunctiva and much lacrymation. There is little or no chemosis but a bright red injection of the vessels of the ocular conjunctiva, both superficial and deep. Frequently the ciliary vessels are also engorged which sometimes makes the diagnosis from iritis somewhat difficult. Indeed this organism is the cause of the only form of conjunctivitis which can give rise to iritis by the

${ }^{1}$ P. Römer. Archiv. für Ophth., LIV, I902, I, 99. 
absorption of toxins into the anterior chamber, without involving the cornea; the iritis produced is rarely severe unless the cornea is involved. Hemorrhages and phlyctenulæ are rare, frequently the affection is confined to one eye, these characteristics help to distinguish it from that conjunctivitis due to Koch-Weeks bacilli, which in other respects it resembles.

The disease terminates by crisis, the organism and frequently the discharge disappearing in forty-eight hours. This rapid termination accounts for the fact that follicles are rarely formed. In exceptional cases, especially in young and badly nourished children, the pneumococcus produces so severe an inflammation in the conjunctiva that a membrane is formed.

Although pneumococcal infection of the cornea is common in connection with lacrymal obstruction, the cornea is rarely affected in pneumococcal conjunctivitis. This is due to the fact that the toxin has little effect on the corneal epithelium, the organism being unable to gain an entrance unless it is abraded.

Although the discharge is undoubtedly contagious, the susceptibility of men and animals for the same strain of pneumococcus varies very considerably, which probably accounts for the frequency of sporadic cases in this country; in rare instances, however, epidemics have occurred. As a rule it is neither associated with, nor the cause of, pneumococcal lesion in other parts. A local immunity of short duration seems to be conferred on the patient as the result of an attack.

Infection of the Cornea.-The pneumococcus is the common cause of the typical serpens ulcer. This form of ulcer is not seen in children, when pneumococcal infection of the cornea occurs in them it produces a deep and more rapid necrosis, probably owing to the substantia propria not being so dense. In adults the disease is very frequently associated with lacrymal obstruction, the organisms growing in large numbers in the sac. For the pneumococcus to 
gain entrance to the corneal tissue an abrasion of the epithelium is necessary, which accounts for the fact that lacrymal obstruction may be present for years without infection taking place. The incubation period is twenty-four hours. In the very earliest stage the ulcer has the appearance of a colony of organisms on the cornea surrounded by slight infiltration. The cornea being infected the organisms spread laterally between the lamellæ of the substantia propria beyond the margin of the ulcer. They are found in groups surrounded by a dense infiltration of polymorphonuclear leucocytes. After a time as the deeper layers become involved a perforation may take place. Before this happens a severe iritis is usually set up due to the absorption of the toxins. For the same reason the ocular conjunctiva becomes swollen and edematous. For further complications of corneal ulceration see page 449 .

Pneumococcal infections within the globe may also give rise to ring abscess or ulcer of the cornea (see page 449).

Panophthalmitis due to the pneumococcus may follow penetrating wounds and ulcers. Endogenous infection of the globe is usually associated with pneumococcal meningitis. A condition is then produced which ultimately ends in what is known as "pseudoglioma" (see also page 469).

Streptococci and staphylococci are the common causes of peridacryocystitis, rarely the pneumococcus; the latter, however, is the organism which is usually found in the discharge from a mucocele, both purulent and non-purulent. The organism is probably not derived from the nose but makes its way into the lacrymal sac from the conjunctiva, growing readily in the fluid there.

The pneumococcus is the organism most frequently found in orbital cellulitis. Suppuration in the nasal sinuses is frequently caused by it, and that disease is the commonest cause of orbital cellulitis.

The Gram-negative Diplococci.-These are the gonococcus, diplococcus intracellularis meningitidis, and micrococcus catarrhalis. 
The gonococcus is a kidney-shaped diplococcus. It varies in size from $.8 \mu$ to r. $6 \mu$ long and $.6 \mu$ to $.8 \mu$ broad. The flattened sides of each coccus are opposed to each other; they often appear in tetrads and are frequently found within the cells of the discharge (Fig. 240, B). It stains strongly with aniline dyes. A good method of staining both the gonococcus and other non-Gram staining organisms is by means of Pappenheim's stain which consists of pyronine and methyl-green, the organisms being picked out in the red colour of the pyronine while the polymorphonuclear leucocytes are stained green. It is rapidly decolourised in Gram's method. It has no capsules and does not form spores. On cultivation it is a facultative anaerobe and grows best at the body temperature, it cannot be cultivated below $20^{\circ} \mathrm{C}$. A medium containing human blood-serum should be used; the organism will, however, grow readily on agar if some of the pus is smeared over the surface, but sub-cultivations from such a specimen as a rule do not grow. It appears in small round colonies in about twenty-four to forty-eight hours. These have a peculiarly transparent slimy appearance. They reach their maximum size in four to six days, after which they die out. They rapidly lose their pathogenicity on cultivation. When examined serologically, the gonococcus can be split up into several groups.

Inoculation on to the mucous membranes of animals produces no effect, although if a quantity be applied to the conjunctiva of a young rabbit a slight discharge may be set up. Injection into the peritoneal cavity of animals of large numbers of the organism produces death from septicemia. The toxin is best obtained by cultivation in ascitic fluid. When dropped into the human eye it produces acute inflammation in the conjunctiva. The onset of this is about twenty-four hours after it has been instilled.

The differential diagnosis of the gonococcus from other Gram-negative diplococci may be difficult, but as a rule it is not of much importance in ocular diseases since the other organisms do not produce severe lesions. The organisms 
found in the eye which resemble the gonococcus are the micrococcus catarrhalis and the diplococcus intracellularis meningitidis. The micrococcus catarrhalis, which is commonly found in the nose and bronchial secretion, may cause a mild conjunctivitis. It is larger and rounder than the gonococcus, both points being specially marked on cultivation where the colonies are also larger and non-translucent; it will grow on milk and gelatine.

The diplobacillus intracellularis meningitidis is more a long oval in form, and is not so readily decolourised by Gram's method. Agglutination yields a satisfactory means of distinguishing the three organisms. They can also be distinguished by the fermentation test. By using different varieties of sugar in a fluid medium, to which litmus has been added, it is found that in the case of the gonococcus an acid reaction is produced only when dextrose is present; in the case of the meningococcus when dextrose or maltose are present; in the case of the micrococcus catarrhalis no acidity is produced with any of them.

The conjunctiva and cornea are the parts of the eye most frequently affected by the organism.

The conjunctiva may be affected at any age (gonorrheal conjunctivitis).

In ophthalmia neonatorum the gonococcus is the cause of 66.6 per cent. of all cases. ${ }^{1}$ The source of infection is the vaginal discharge of the mother.

Infection of the child's eyes may take place before, during, and immediately after birth of the child or at an interval of some days.

Infection before Birth.-Children have been born with fully developed ophthalmia neonatorum in which the membranes have only been ruptured three-quarters of an hour before birth. A child has been removed by Cesarean section with fully developed ophthalmia neonatorum. It therefore seems probable that infection of the child's eyes

${ }^{1}$ S. Stephenson. "Middlemore Prize Essay," I908. M. S. Mayou. Practitioner, I9I0. Trans. Ophth. Soc. of the U. K., XL, I920, 57. 
can occur either through the membranes, or through an opening in them insufficient to produce the onset of labour. This infection must take place after the end of the fifth month as the lids are united up to that time.

Infection at Birth.-As a rule at birth the lids are tightly closed, the junction having been made water-tight by the fatty Meibomian secretion at the margins, but it has been proved that they may be open. During the act of birth the discharge from the cervix and vagina may enter the eyes of the child; this is especially likely to occur in the cases where from pressure the upper lid overlaps the lower. The vaginal discharge may be carried into the eyes by forceps or, in the case of face presentation, by the finger of the accoucheur. A tightly stretched perineum may also carry the infected material into the eye.

Infection Immediately after Birth.-When the child first opens its eyes, is probably the time at which infection most frequently occurs. It may also take place during the washing of the infant, some of the secretion being carried into the eyes.

Infection some time after birth is known as "secondary infection" and may take place from want of cleanliness on the part of the midwife, from the diapers, contaminated water at first bath, sponge or towel, etc.

New-born children are especially prone to infection of the conjunctival sac with microörganisms for the following reasons: (a) The absence of lacrymal secretion. (b) The thinness of the epithelium of the conjunctiva compared to that of the adult's, and the absence of any superficial flattened layer of cells. Numbers of the cells, especially in the fornix, are lost owing to the mucoid change, which in places leaves the basement membrane almost bare. The absence of any leucocytes from the lymphoid tissue.

In gonorrheal ophthalmia neonatorum the length of the incubation period varies from one to three days, and has a definite relation to the severity of the attack; the more acute the symptoms the shorter it is. 
In the adult form infection usually takes place from the urethral discharge being conveyed to the eye by the fingers, towels, handkerchiefs, etc. In the case of nurses and doctors it may be conveyed from the eye of an infant afflicted with ophthalmia neonatorum, by the squirting out of the discharge on separation of the lids.

Endogenous infection of the conjunctiva with the gonococcus has been reported, but more evidence is required before this can be accepted.

The organism is found free in the discharge, within the leucocytes and epithelial cells, and occasionally in the subepithelial tissue.

In the early stage of the disease (infiltration stage), the discharge contains gonococci in great quantities and is generally free from other organisms. In the later stage (pyorrhea stage) the discharge often becomes contaminated with other organisms. As the attack subsides the organisms decrease in number, but virulent organisms have been found in the conjunctival sac for at least a month after the discharge has subsided.

The microscopical examination of the tissues shows much papillary formation. There is desquamation, and lateral separation between the epithelial cells in which organisms can be found. There is a large amount of exudation into the sub-epithelial tissue of polymorphonuclear leucocytes; toward the end of the attack lymphocytes and plasma cells make their appearance, the latter breaking up as they approach the epithelium in which the organisms are growing.

Undoubtedly an immunity to the gonococcus seems to be produced in the conjunctiva, although it may not last very long. The evidence that the gonococcus does produce such immunity is that cases do eventually get well and the discharge ceases if left to themselves, although the sight may have been destroyed.

A chronic gleet of the conjunctiva has never been recorded, but systemic infection from the conjunctiva occasionally occurs. 
If one eye be attacked some time before the other, the second eye is not so severely affected as the first. Occasionally one eye only is infected, although no precautions are taken to prevent the other from becoming involved.

Gonorrheal urethral discharge has been inoculated into an eye without producing ophthalmia (natural immunity).

Corneal infection with the gonococcus is always secondary to infection of the conjunctiva. The cornea is affected in 27 per cent. of all cases of ophthalmia neonatorum. These cases are nearly always of gonorrheal origin.

The cornea in the infant can be saved from infection if the disease be treated properly, and in this respect it differs from gonorrhea in the adult where, in spite of every care, the cornea may become involved. The reason of this is that in the latter there is much more chemosis of the ocular conjunctiva, so that a sulcus forms around the cornea in which the discharge collects, and lying in contact with it gives rise to marginal ulceration. In the cornea of a newborn infant the epithelium has no flattened layer on the surface as in the adult; this renders it more prone to attack. The substantia propria is, however, thicker in the newly born than the adult. The lymph spaces are also more readily opened up allowing a freer circulation of lymph containing protective bodies, through its substance, and better paths for the migration of leucocytes, hence better protection against infection. The subsequent clearing up of opacities in the cornea is due to the free supply of lymph, and the subsequent flattening of the scar by the growth of the cornea.

Corneal Haze.-A slight corneal haze may be present due to edema of the epithelium, if the haze becomes more localised it is the early stage of infiltration of the cornea by polymorphonuclear leucocytes as the result of infection; if this infection be arrested, resolution takes place; if not, necrosis follows.

Corneal ulcerations are of two types-either small and localised or large, involving the whole cornea. Both these 
forms may lead to perforation, the first frequently, the second always; the first resulting in a leucoma with a portion of clear cornea left, and possibly an anterior synechia of the iris or an anterior polar cataract; while the second leads to total anterior staphyloma. Granulomata at the site of perforation more frequently form in infants than in adults.

Hypopyon seems to be comparatively rare but, owing probably to the rapidity with which perforation takes place, it is not always noticed.

Infection of the iris may follow a perforating ulcer but the toxins alone do not seem capable of producing iritis; hence no posterior synechiæ form unless the ulcer perforates.

Gonorrheal iritis is the result of an endogenous infection. It is usually preceded or accompanied by gonorrheal arthritis; indeed, if metastases in the joints do not occur it is probable that gonorrheal iritis cannot take place. Whether this is merely coincident with the blood infection, or whether the organism gains a special proclivity to affect the iris after affecting the joints, is not known. Whichever it may be the organism has the power of remaining latent a considerable time; an iritis may start years after the urethral discharge has diappeared and tend to relapse. In these cases the prostatic secretion frequently shows the presence of the organism. The inflammation is of the serous type and does not as a rule give rise to many adhesions. In some very acute cases a characteristic gelatinous exudate forms in the anterior chamber. It is always accompanied by intense injection of the ocular conjunctiva which possibly may be due to the toxins.

The gonococcus is occasionally found in the distended lacrymal sac in cases of congenital lacrymal obstruction but does not give rise to peridacryocystitis.

Diplococcus intracellularis meningitidis is a round or somewhat lanceolate shaped coccus, found in pairs arranged end-to-end but rarely in tetrads. It stains well with thionine blue and is decolourised in Gram's method but not 
so readily as the gonococcus. It has no capsule, is nonmotile, and does not form spores. On cultivation it grows best on blood-serum at the body temperature in small round colonies, which are about the same size as colonies of the gonococcus but are whiter in colour. It will grow on serum and glycerine agar. The differential diagnosis between this organism and the gonococcus has already been discussed.

In the conjunctiva a purulent conjunctivitis of moderate severity is set up. It is rare and has no distinguishing clinical characteristics so far as has at present been observed. It may or may not be associated with meningitis. Ophthalmia neonatorum has been found to be due to this organism.

Infection of the cornea has been recorded in a child. The ulcer produced was of a purulent variety and went on to perforation; it was not associated with meningitis.

Infection of the uveal tract and retina ectogenously has never been recorded. Endogenous infection is always associated with meningitis. ${ }^{1}$ The organism is conveyed to the eye by the blood stream, and not by a direct spread from the meninges. This has been shown by the examination of a case in a very early stage, in which an embolus had lodged in the ciliary body setting up a purulent iridocyclitis with hypopyon. After about the end of the first week the hypopyon usually disappears but the exudate into the vitreous persists, subsequently organising into a fibrous cyclitic membrane producing the appearance termed "pseudoglioma." An embolus containing the organism may lodge in the choroid and retina producing a similar condition.

\section{(ii) Bacilli}

(a) The Influenza Group.-The bacilli belonging to this group of organisms are found on mucous membranes. The following occur in the eye. The Koch-Weeks bacillus, the bacillus of Müller, and the influenza bacillus.

${ }^{1}$ M. S. Mayou. R. Lond. Ophth. Hosp. Reps., XVI, I906, 565. 
The Koch-Weeks bacillus is a narrow, straight or slightly curved bacillus (Fig. 24I, A) and measures from I $\mu$ to $2 \mu$ in length; it occurs singly or in groups; it is non-motile, and non-flagellated. Occasionally thickenings of one end of the bacillus occur. It stains badly with all aniline dyes, prolonged staining with thionine blue, and subsequent decolourisation with alcohol, being the best method of demonstrating the organism; it is decolourised rapidly in Gram's method-an important point in distinguishing this bacillus from the xerosis and diphtheritic bacilli, with the former of which it is frequently associated.

On cultivation it grows best at a temperature of $35^{\circ} \mathrm{C}$., but, like the gonococcus and other parasitic bacteria, it is extremely difficult to cultivate; it is essential for its growth that the medium should be slightly alkaline.

On human blood-serum the organism will grow well.

On animals' inspissated blood-serum the organism does not grow or will only do so occasionally.

Human blood agar is the best of all media for its growth; over roo generations of the organism have been obtained on it. On other media, e.g., 5 per cent. agar, only three generations can be obtained, except from the most virulent cases. Pig's serum may be substituted for human serum, but it is not so satisfactory.

On glycerine pepto-agar with pig's serum the growth is also satisfactory; five generations have been obtained on this medium.

On broth, to which hydrocele fluid has been added, the organism will grow well, causing a slight cloud in the medium.

The organism was first cultivated on agar 0.5 per cent. At $35^{\circ} \mathrm{C}$., which is the best temperature for growth, this medium is nearly fluid; therefore inoculations are best made into flat bottles or petri dishes.

The organism will not grow on potato or gelatine.

At the end of from twenty-four to forty-eight hours colonies appear on serum agar as semi-transparent dew-like 
drops; they are fixed very lightly to the surface and are easily removed with the platinum loop. Examined with a lens, the colonies are seen to be round in outline and have, when magnified eighty times, slightly crenated edges. As growth takes place they are not actually limited to the streak of blood on the surface agar, but spread out into the medium beyond, and run together to a slight extent. Growth continues for about 5 days, after which the culture still remains active for another sixty hours, and then usually dies.

In culture the organism varies somewhat in length, and long thread-like involution forms appear; conical forms, can also be seen which have been ascribed to spore formation, but this is very doubtful; these involution forms retain their virulence for some considerable time.

The organism is killed in three minutes at a temperature of $60^{\circ} \mathrm{C}$., but will live for more than ten minutes at a temperature of $50^{\circ} \mathrm{C}$. It is not killed by a temperature of $7^{\circ} \mathrm{C}$. for one and one-half hours.

Drying of the discharge seems to kill the organism, so that infection is probably not carried in the air or dust. The bacillus will live in distilled water for seven hours, and it may therefore be conveyed from one person to another by washing in the same water.

All animals are immune, both to local inoculation into the eye or to subcutaneous inoculation. The bacillus is pathogenic to man only.

In human inoculation into the conjunctiva an increasing number of organisms are generally found up to the third day, when the symptoms appear. These continue up to the ninth day, the case being untreated. On the tenth day the symptoms begin to disappear, as well as the organisms from the conjunctival sac.

Primary cultures of $\mathrm{I} 20$ hours produce positive effects.

The toxins alone will produce inflammation. Dead cultures, dropped into the human conjunctival sac, produce reaction, but this is greatly lessened if the culture is 
previously filtered, from which it may be concluded that the toxin is contained principally within the organism.

A person may be inoculated several times with positive results, the shortest interval at which a positive reaction can be obtained being four weeks; there is, therefore, only a short period of local immunity after an attack. By experiments, it has also been proved, that inoculation from the same culture produced conjunctivitis of different intensity in different persons. Previous scarring of the conjunctiva, either prevents, or mitigates the attack.

In the conjunctiva the organism produces an inflammation which is usually so typical, except in infants, that the disease can be in most cases recognised clinically.

The bacillus of this disease is one of the most common causes of purulent conjunctivitis, and has been found in connection with it in all parts of the world. The disease, though of common occurrence both in adults and in children, is more frequent in the latter, but is rarely met with in the new-born. It occurs in sporadic and epidemic forms, often running through a whole school, as it is extremely infectious.

It almost invariably affects both eyes, and is characterised clinically by intense injection of both the palpebral and ocular conjunctiva. The injection in the ocular conjunctiva is very marked, there are numerous hemorrhages scattered over its surface, but very little chemosis. Toward the end of the attack follicles form in the fornix which persist for some time after the disease has subsided. Phlyctenulæ are often associated with this disease.

In young badly nourished children a membrane may be formed on the conjunctiva.

Histologically the changes in the epithelium are those common to all acute inflammatory affections. Although the organisms have only been found in the epithelial cells changes also occur in the sub-epithelial tissue. Deep localised changes account for the phlyctenulæ and hemorrhages that occur in the ocular conjunctiva, and for the 
follicular formations in the retrotarsal folds after the acute attack has subsided.

In the early stages the exudation consists largely of polymorphonuclear leucocytes; later of lymphocytes and plasma cells of which the follicles are formed.

In the cornea a break in the epithelium is necessary for infection to take place. A marginal phlyctenular corneal ulcer may be associated with conjunctivitis due to this organism. A superficial purulent ulcer due to it also arises apart from conjunctivitis. Care must be taken to distinguish it from the ulcer produced by the bacillus of Zur Nedden. Although the ulcer may be extensive it rarely penetrates deeply into the substantia propria, and usually heals readily under treatment. Hypopyon is usually present.

Intraocular infection has never been recorded.

The bacillus of Müller was discovered in the discharge from trachomatous conjunctivæ, but was not found constantly in all cases. It is of interest, since it fills the small gap in the morphological chain, between the influenza bacillus on the one hand, and Koch-Weeks bacillus on the other. It exhibits all the characteristics of the influenza bacillus with which organism it is supposed by most observers to be identical. It differs from Koch-Weeks bacillus in being able to grow on inspissated pigeon's blood.

Influenza Bacillus (Pfeiffer).-Mild conjunctivitis occurs in influenza, the discharge containing the influenza bacillus which exhibits very close if not identical characteristics, with the Koch-Weeks bacillus; indeed, it is possible that these organisms are identical. That is to say that KochWeeks conjunctivitis is a local inoculation of a general disease, standing in the same relation to influenza as pneumococcus conjunctivitis does to pneumonia; but as yet proof is wanting that the discharge from the bronchial tubes in influenza will produce a typical Koch-Weeks conjunctivitis. 
The differences between the two organisms are slight:

\section{B. Koch-Weeks}

Length $\mathrm{I} \mu$ to $2 \mu$ (not constant).

Occurs singly or in pairs.

Does not grow on gelatine.

Colonies. Small slightly crenated edge when magnified eighty times.

Short virulence (five days).

Blood streak on agar not limited to the blood area but spreads on to the agar.

Non-pathogenic to animals. No Pathogenic to monkeys, hares, toxic symptoms when injected.

\section{B. Influenza (Pfeiffer)}

Length $5 \mu$.

Generally in chains or networks.

Grows on liquid gelatine at $37^{\circ} \mathrm{C}$.

Colonies larger than Koch-Weeks. Homogeneous smooth edge.

Longer virulence (ten days or more).

Limited to the blood streak on agar.

guinea-pigs, with toxic symptoms.

Epidemics of these two diseases do not noticeably occur together.

(b) Diphtheroid Group.-Belonging to this group occurring in the eye are the bacillus Klebs-Loeffler and the bacillus xerosis.

Klebs-Loeffler Bacillus is a straight or slightly curved organism, measuring about $3 \mu$ in length and is about the

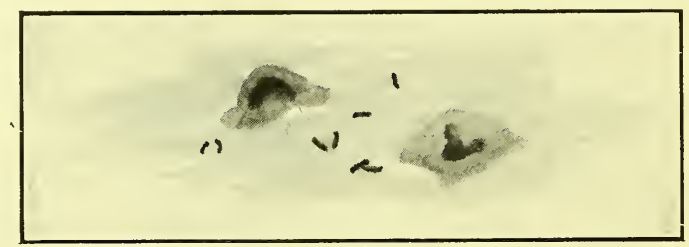

FIG. 24I.-Shows a smear preparation from discharge from the conjunctiva containing Klebs-Loeffler bacilli.

same thickness as the tubercle bacillus (Fig. 24I). It is non-motile and non-spore bearing.

It stains with thionine blue and other aniline dyes. Occasionally dots can be seen in the body, and the ends are somewhat enlarged. It retains the stain in Gram's method. Twenty-four hours culture on Loeffler's blood-serum shows polar staining by Neisser's method. 
On cultivation the Klebs-Loeffler bacillus grows best at $37^{\circ} \mathrm{C}$. and ceases to grow entirely at $20^{\circ} \mathrm{C}$. The media most suitable for its growth are Loeffler's inspissated bloodserum, blood agar, or even ordinary agar.

On blood-serum, growths appear in twenty-four hours as small circular opaque discs of whitish colour, which reach their largest size, of about $3 \mathrm{~mm}$., on the third day. On agar the growth is somewhat slower. It will grow on gelatine, but does not liquefy this medium. Broth containing glucose is rendered acid in from two to three days. It grows well on milk which becomes coagulated by the formation of acid.

Involution forms, with curious club-like ends, appear in from three to four days.

On inoculation, guinea-pigs are the most susceptible to this organism, being killed within forty-eight hours after inoculation, the exact time depending on the virulence of the organism. There is always local necrosis at the site of inoculation. As a rule after death the organisms are found at the site of inoculation in large quantities, but they are not present in the blood, showing that the disease is essentially a local infection, with general symptoms due to the absorption of toxins from the site of inoculation. In the exceptional more severe hemorrhagic forms of the disease the organism has been demonstrated in the other tissues of the body.

In the guinea-pig, the suprarenal capsules are found in a state of acute inflammation, no doubt due to the toxins. Rats and mice are immune to the organism. If animals inoculated with the organisms or toxins are kept alive, they develop the paralysis seen in the human subject.

To develop the toxins of the Klebs-Loeffler bacillus it is best to use a medium which contains a large percentage of peptones, and at the same time to have a free supply of oxygen. The medium should be free from glucose, as the formation of toxins is inhibited by the production of acid. A toxin of which .or c.c. would kill a guinea-pig in 
twenty-four hours has been obtained. The toxin is an unstable compound and can only be kept for any length of time by the total exclusion of light and air. It is destroyed by a temperature of $58^{\circ} \mathrm{C}$. and can be precipitated by alcohol. Instillation of the toxin into the conjunctival sac produces reaction in about twelve hours; this is more intense, with the formation of a pseudo-membrane, if injected subconjunctivally.

The antitoxin (see page 4I 5) is produced by the administration to a horse, of gradually increasing doses of toxin, until a stage is reached when the animal can stand enormous doses of a virulent toxin without reaction. The strength of this antitoxin is standardised into units by finding the amount of toxin it will neutralise when they are injected together into a guinea-pig.

Small quantities of antitoxin are present in the majority of people, and it is probably conveyed from the mother to the child before birth through the placental circulation. This antitoxin accounts for the resistance which normal individuals show against diphtheria, as the organisms are present in many healthy persons' throats; such persons may become "carriers" and disseminate the disease. The presence of this normal antitoxin is determinated by Shick's reaction. A standard diphtheria toxin diluted with saline solution .I c.c. containing $1 / 50$ of a minimum lethal dose for a guinea-pig, is injected subcutaneously. If the blood contains less than $1 / 30$ of a unit of antitoxin per cubic centimeter, a positive reaction, consisting of a slight thickening of the skin surrounded by a red areola appears in twenty-four hours. A negative reaction shows that the individual is protected against diphtheria.

The differential diagnosis of the Klebs-Loeffler bacillus from the bacillus xerosis is often extremely difficult (see page $52 \mathrm{I}$ ), if in a membranous conjunctivitis, many organisms exhibiting the characteristics of this group are found in the discharge from the eye, the case will almost certainly prove to be one of true diphtheritic infection. 
In the conjunctiva, infection with the Klebs-Loeffler bacillus is characterised by the formation of a membrane on its surface. This is generally most marked on the palpebral conjunctiva, to which it is firmly adherent and which when it is peeled off, bleeds readily. It consists of coagulated fibrin situated, not only on the surface of the epithelium, but also between the epithelial cells themselves. Throughout this membrane the Klebs-Loeffler bacilli are found scattered irregularly. Beneath the membrane the tissues are in a state of acute inflammation, the reticulum of the conjunctiva being greatly distended with lymph, also containing fibrinous exudation and leucocytes.

Diphtheritic conjunctivitis may or may not be associated with a membrane in the throat or nose (fibrinous rhinitis). When found it is a point of considerable assistance in diagnosis. It is well to emphasize here that all membranous conjunctivitis is not due to the Klebs-Loeffler bacillus; streptococcus, staphylococcus, and even gonococcus may give rise to a membrane indistinguishable from that produced by the Klebs-Loeffler bacillus. Further, toxic bodies, such as jequirity, nitrate of silver, and snake poisons may give rise to a membranous conjunctivitis.

The effects on the conjunctiva vary considerably according to the severity of the attack, which may end either in complete resolution, with perhaps some slight scarring, or in gangrenous sloughing of the conjunctiva, with the formation of adhesions (complete or partial symblepharon), and destruction of the cornea by ulceration.

It occurs most frequently in children from two to eight years old and in the severe type is associated with general symptoms. The temperature varies considerably; in mild cases and in the early stages it is not raised at all or only to about $100^{\circ}$, but in the more severe cases the temperature is often $101^{\circ}$ to $103^{\circ} \mathrm{C}$.; while again in the most severe cases of all the temperature is often subnormal at the commencement of the attack. Albumin is usually present in the urine in severe cases. 
Infection of the cornea apart from the conjunctiva does not occur. Ulceration occurs in about ro per cent. of all the cases. It may lead to complete destruction of the cornea or to small sloughing ulcers.

Xerosis Bacillus.-This organism has been the cause of many fallacies; it is purely a saphrophyte growing in the Meibomian secretion and bears no relation to xerosis or to diphtheria. ${ }^{1}$

It appears in the Meibomian secretion in infants soon after birth, and is probably identical with the bacillus which is found in the vaginal secretion whence it is derived. ${ }^{2}$

It is a straight or slightly curved bacillus $\mathrm{I} .75 \mu$ long and $0.5 \mu$ broad. It is a non-motile, facultative anaerobe, and does not form spores.

It stains well with all aniline dyes and exhibits thickenings similar to those of the Klebs-Loeffler bacillus and, like this latter organism, shows Neisser's polar staining in twenty-four-hour blood serum cultures. It retains the stain in Gram's method.

Cultivated on blood-serum it does not make its appearance till from thirty-six to forty-eight hours. It appears in round whitish colonies. As a rule it will only grow as a primary culture on a medium containing blood. After the primary culture it will grow on agar and gelatine, it does not liquefy the latter.

The differential diagnosis between this organism and that of diphtheria is only made by carefully following out its general characteristics, no single one, except inoculation, being sufficient to make a correct diagnosis.

The chief points in the differential diagnosis are:

${ }^{1}$ M. S. Mayou. Trans. Ophth. Soc. of the U. K., XXIV, I904, 9.

${ }^{2}$ M. S. Mayou. Trans. Ophth. Soc. of the U. K., XLI, I92I. 


\section{Klebs-Loeffler Bacillus}

The bacillus is more or less uniform in size through out its length.

On blood-serum it appears in twelve to twenty-four hours.

Primary culture on agar will grow.

In glucose, broth, and milk it produces acid.

Sub-cultures grow well on potato.

On gelatine (ro per cent.) it appears in twelve to twenty-four hours.

On media containing dextrose and dextrin, acid is produced, but no acid is produced in a medium containing saccharose.

Inoculation (perhaps most important) kills a guinea-pig in fortyeight hours with local necrosis.

\section{Pseudo-Diptheritic Xerosis} Bacillus

Slightly more thickened toward the ends, seen best in Gram staining.

Does not appear at all till from thirty-six to forty-eight hours.

Does not grow or only very badly in primary culture.

No acid is produced.

Does not grow on potato.

On gelatine forty-eight hours.

No acid is formed with dextrin media, but acid is formed with dextrose and saccharose. Bacillus Hoffmann forms no acid with either sugar.

No effect on guinea-pig.

The agglutination test is not always reliable.

(c) Diplobacilli.-Belonging to this group are the MoraxAxenfeld bacillus and the bacillus of Petit.

The Morax-Axenfeld Bacillus.-The bacillus is a large one. Its average measurement is $2 \mu$ long and $\mathrm{I} \mu$ broad. It varies somewhat in size and shape and the ends are usually thickened; they are arranged end-to-end in pairs (Fig. 240, C). Sometimes long chains are formed. Although, strictly speaking, they are non-capsulated, an area is occasionally seen around the bacillus which does not stain. They are non-motile, non-flagellated, non-sporing, obligate aerobes.

They stain strongly with all aniline dyes, but are decolourised in Gram's method. They take pyronine well in Pappenheim's method.

On cultivation they grow best at $30^{\circ}$ to $40^{\circ} \mathrm{C}$., but are killed in fifteen minutes by exposure to $56^{\circ} \mathrm{C}$. They 
require for their growth a slightly alkaline medium containing blood.

On Loeffler's blood-serum they begin to show growth in twenty-four hours, and are well developed in forty-eight hours. The surface of the medium becomes pitted, the pits having a shiny appearance. This is due to liquefaction of the medium, which is a very characteristic feature of the group of organisms to which the bacillus Morax-Axenfeld belongs. On serum agar they form small colonies resembling pneumococci. On serum bouillon they form a cloud in twentyfour hours, and fall to the bottom as a deposit. In this medium involution forms are not common during the first few days. On agar they do not grow, thus differing from the bacillus of Petit.

Involution forms appear on cultivation. On bloodserum in forty-eight hours long chains of organisms, together with some distorted bacilli, appear. They rapidly undergo degeneration.

The organisms usually die out on cultivation in about ten days. Dried organisms are still active after four days -an important point in the spread of the disease by dust, etc.

The action of sulphate of zinc is of interest, seeing the efficacy of this drug in the disease. Although the solution as used for the eye does not kill the organisms, traces of zinc sulphate in the medium prevent their growth.

The organism is non-pathogenic to animals. Inoculation of the healthy human conjunctiva with a twentyfour-hour bouillon culture produces a typical diplobacillary conjunctivitis. A cultivation of four days old failed to do so; hence it may be supposed that the pathogenicity dies out on cultivation. Vaccines have been prepared and are said to have been administered with good effect.

In the conjunctiva the inflammation produced occurs at all ages but is rather more common in adults than children. The palpebral conjunctiva is intensely red, the caruncle and plica semilunaris participating in the inflammation. 
The secretion accumulating at the outer and inner canthi causes excoriation and redness of the skin of the lids in those regions - a characteristic feature of the disease, hence the name angular conjunctivitis. Occasionally this redness may spread along the whole margin of the lid. The disease, unless treated, becomes chronic and in such cases is often associated with staphylococcus albus, especially when marginal blepharitis is produced.

The Morax-Axenfeld bacillus is a non-pyogenic organism, the discharge being merely a sticky secretion consisting of mucus, epithelial débris, organisms, and an occasional leucocyte.

Histologically the greatest change takes place in the epithelium, which undergoes increased desquamation and mucoid degeneration. In the later stages of the disease it dips down into the subepithelial tissue and new glands are formed; the orifices of these new glands are very liable to become occluded, with the result that retention cysts are produced.

There is no increase in the polynuclear or mononuclear leucocytes in the subepithelial tissue, except in the regions where the epithelium dips down into it, or where there are cystic formations. Elsewhere the change consists in a large increase in the number of plasma cells; directly beneath the epithelium the cells are somewhat broken up, no doubt due to the presence of the toxin. Owing possibly to the low vitality of these cells the disease is not eradicated spontaneously. There is also a considerable increase in the mast cells of the underlying tissue. In long standing cases hyaline degeneration of plasma cells takes place.

Corneal ulceration due to the Morax-Axenfeld bacillus is always associated with angular conjunctivitis. The ulcers are usually situated near the corneal margin, and have a somewhat serpiginous outline with sharply cut edges and a grey infiltrated base. There is no purulent discharge. 
Purulent ulceration with hypopyon has been ascribed to this organism, but in these cases it is probable that the organism is associated with others having a pyogenic character.

The diplobacillus of Petit presents the same characteristics as the bacillus of Morax-Axenfeld. The only difference between the two organisms is that the diplobacillus of Petit, unlike the Morax-Axenfeld bacilli, will grow on agar and media which do not contain blood. The diplobacillus of Petit has only been found in purulent ulcers with hypopyon, and does not produce conjunctivitis. Whether it be a non-virulent form of the bacillus Morax-Axenfeld, and not the actual cause of the hypopyon ulcer in which it has been found, has not yet been fully demonstrated.

No case of intraocular infection has been recorded from this group of organisms.

(d) Capsulated Bacilli.-Belonging to this group of organisms are Friedlander's pneumobacillus, the bacillus mucosus capsulatus, and the ozena bacillus.

Friedlander's pneumobacillus is a large organism surrounded by a thick capsule which stains readily. It stains well with thionine blue, but is decolourised in Gram's method. It is a non-motile facultative anaerobe.

On cultivation it grows well on all media. It does not liquefy gelatine.

On inoculation into animals it produces corneal ulceration. When inoculated into the peritoneum the animal dies.

In the human conjunctiva the organism produces conjunctivitis of varying severity. Some of the recorded cases have been of the pseudomembranous type, while others have not been so severe. The organism has been found in ophthalmia neonatorum.

Corneal ulceration with hypopyon has been ascribed to it. It has been found in one case of metastatic panophthalmitis following pneumonia, and in one case of orbital cellulitis. It has also been found in chalazion and in the secretion of the lacrymal sac in dacryocystitis. 
The bacillus mucosus capsulatus and the ozena bacillus have never been recorded as giving rise to ophthalmic lesions, although they have been found in the conjunctiva and in the contents of the lacrymal sac in dacryocystitis.

(e) The coli group of bacilli which affect the eye are the bacillus coli communis and the bacillus typhosus.

The bacillus coli communis is a short flagellated rod about $\mathrm{I} \mu$ to $3 \mu$ long and $.7 \mu$ broad. It has rounded ends, stains well with thionine blue, but is decolourised in Gram's method. It is non-capsulated, motile, and does not form spores. On cultivation it is a facultative aerobe, growth taking place on media in the cool incubator. It appears in dirty white, circular patches with crenated edges. In fluid media acidity is developed and milk is rapidly coagulated. If grape sugar be present in the media gas is evolved. If potassium nitrate and sulphuric acid be added to a twenty-four-hour culture a red colour is produced-nitroso-indol reaction.

In the conjunctiva the organism has been described in rare instances as the cause of purulent ophthalmia in the newborn or in young children. One case has been described in an adult. It has also been found in corneal ulceration with hypopyon and in the pus of dacryocysitis.

Irido-cyclitis both acute and chronic have been attributed to this organism, although the actual organism has not been obtained from the aqueous, it has been found in other lesions in patients suffering from irido-cyclitis.

The bacillus typhosus exhibits the same appearance as the bacillus coli communis, but has the following distinguishing characteristics. It is more motile, having eight to ten flagellæ, whereas the Bacillus coli has only three to four; it does not coagulate milk; no gas or indol is formed on cultivation. The fermentation, agglutination; and Bordet Grengou tests are reliable in distinguishing between the two organisms.

The lesions of the eye associated with typhoid fever are: 
I. The local conditions associated with the typhoidal state, which are not directly due to the typhoid bacillus; viz. : xerosis, conjunctivitis, and corneal ulceration.

2. The general conditions associated with the typhoid state, which are due to changes in the condition of the blood leading either to thrombosis of the cavernous sinus and retinal vein, and embolism of the central artery or to hemorrhage; Local into the eyeball, or into the optic nerve sheath; General into the intestine causing optic atrophy from anemia.

Cases of paresis of accommodation and punctate cataract have also been attributed to typhoid fever.

3. The only ocular lesion caused by the bacillus typhosus is panophthalmitis due to embolic infection. ${ }^{1}$

(f) The Acid-fast Bacilli.-The organisms belonging to this group affecting the eye are the bacilli of tuberculosis and leprosy. Saprophytic organisms resembling these, such as the butter bacillus, do occur but are not of importance in ophthalmic bacteriology.

Tubercle Bacillus.-The bacillus is a straight or slightly curved rod measuring from 2.5 to $3.5 \mu$ long and $.3 \mu$ thick. It is non-motile and non-flagellated. It is extremely doubtful if it forms spores, but the older members of a group show irregularities in staining which the younger do not; this is probably due to the degenerative changes taking place in the bacillus. Old cultures also show large clubbed and braided forms, which are supposed to be due to involution changes.

The tubercle bacillus is stained after long exposure to aniline dyes. It stains best with strong acid dyes, such as carbo-fuchsin, and it has the property of retaining that stain after the tissue has been decolourised in weak acid. This property is known as "acid-fast" and is limited to a small group of organisms of which the tubercle bacillus is one.

The tubercle bacillus is Gram-positive. Where the bacilli are only. sparsely scattered, as in sputum, they can

${ }^{1}$ M. S. Mayou. R. Lond. Ophth. Hosp. Reports, XVI, 1906, 565. 
be concentrated by adding liquor sodæ chlorinatæ with a I 5 per cent. solution of caustic soda. This dissolves out the mucus etc., and the bacilli are concentrated in the residue.

Tubercle bacilli in young cultures are often not acid-fast and it is not, therefore, always possible to demonstrate the organism in granulomatous masses. It was found by Much that there were Gram-positive granules in tubercular tissue, lying singly or in short chains and irregular clumps, which are non-acid-fast tubercle bacilli. Similar granules are found amongst the bacilli in pure cultures of the organism. Unfortunately "Much granules" are of not great diagnostic importance, as other bacilli form granules of similar appearance.

For cultivation of the tubercle bacillus the best temperature is $37^{\circ} \mathrm{C}$. The growth appears on blood-serum in ten to fourteen days as a whitish raised "scab-like" growth of rounded irregular outline. On glycerine agar it grows best of all, but it will only do so as a sub-cultivation. On glycerine broth it forms a cloud which falls to the bottom of the tube. No growth takes place on ordinary agar or broth. The presence of glycerine strongly facilitates its growth. On potato and other vegetables, especially when glycerine is present, growth takes place. Primary cultivation of the organism is often very difficult owing to the presence of other more rapidly growing organisms. By the addition of erycholine, 2 per cent., to an egg-albumen medium the other organisms are destroyed, but growth of the tubercle bacillus is not hindered.

The resistance of the tubercle bacillus is great; it is still active in dried sputum at the end of two months. It is killed by $I$ in 20 carbolic in five minutes, and also killed by direct sunlight.

The effect of inoculation on the tissues varies considerably according to the method employed. Guinea-pigs are peculiarly susceptible to the organism. If it be inoculated subcutaneously a mononuclear leucocytosis is first produced 
around the site of inoculation. About the fifth day epithelioid cells appear which are developed in all probability from the existing leucocytes. Later giant cells are formed, probably from the endothelial cells by division of the nuclei without division of the cytoplasm. The nuclei are arranged either to one side or at the periphery of these cells. Hyaline degeneration takes place in the epithelioid cells and caseous masses may be formed. The newly formed capillaries, which result from inflammation around the nodule, do not invade the mass, probably owing to the presence of the toxins.

These changes are very characteristic of tubercle, so much so that it is possible to make the diagnosis from the histological appearance of the tissue. At the same time the presence of a giant cell alone or epithelioid cells alone is insufficient, the general characteristics of the lesion must be taken into account. Sympathetic inflammation of the choroid much resembles microscopically a tubercular one, and may be mistaken for it unless a careful examination of the giant cells and the rest of the specimen be made.

When inoculated into the cornea the tubercle bacillus occasionally produces ulceration, which in some cases is followed by a general infection.

When introduced into the anterior chamber typical nodules are formed on the surface of the iris. These appear in from two to four weeks and are followed by general tuberculosis. A rabbit is the animal generally used for such an experiment. The tissue suspected of containing the tubercle bacillus is taken from a part which is likely to be free from other organisms, and is either introduced through an incision made with a keratome, or is first formed into an emulsion and then injected into the anterior chamber. A negative result from such an inoculation does not exclude the possibility of the tissue being of a tuberculous nature.

Intraperitoneal inoculation produces a form of tabes mesenterica, while intravenous inoculation may be followed by general tuberculosis. 
Dead tubercle bacilli, when injected, may produce a nodule in the conjunctiva which stimulates a phlyctenule. The dead bacillus retains its staining reaction for some time after injection.

Diagnosis.-The bacilli of tubercle and leprosy, unlike other pathogenic organisms, are "acid-fast." The clinical characteristics of the latter disease are usually sufficient to distinguish it from the former. In the tissues the tubercle bacillus is found between the cells or within the giant cells. It occurs either singly or in groups of two or three.

Inoculation or cultivation from the tissues may be successful.

The Bordet-Grengou reaction may be obtained either by using the blood serum or the fluid from the anterior chamber.

In cases where it is not possible to obtain a piece of tissue for microscopical examination or fluid from the anterior chamber, reliance must be placed on subjective tests due to the alteration of the tissues from the growth of the tubercle bacillus in the body. The chief of these is the reaction produced in a patient who is suffering from tuberculosis by the introduction of small doses of the tuberculous toxin into the tissue. The reaction which follows the introduction of tuberculin (toxin), may manifest itself either by a general disturbance or by a local reaction (anaphorexis).

The toxin of the tubercle bacillus is principally an endotoxin, although a small amount is no doubt secreted by the bacillus.

The forms of tuberculin in general use are: (i) old tuberculin (Koch), which consists of the filtrate of the fluid media in which the organisms have been grown, and is standardised by estimating the lethal dose for a guinea-pig; the human dose is $1 / 2000$ to $1 / 250$ of a milligram; (ii) tuberculin T. R., which is the aqueous extract of the ground up tubercle bacillus, the dose of which is $1 / 2000$ to $1 / 200$ milligram; (iii) bacillary emulsion, which is the ground 
up organism in suspension; many other forms of bacillary emulsions have been used.

The bacillus contains a quantity of fatty material and its removal makes the tuberculin more efficacious.

A general reaction, for which the old tuberculin is mostly used, is manifested by a rise of temperature which is at its greatest height about twenty hours after the injection. Great care must be exercised before its use to exclude extensive lesions, such as phthisis, otherwise the activity produced in the lesion may cause dissemination of the organisms and general tuberculosis.

A local reaction, for which tuberculin T. R. is usually employed, manifests itself in 48 hours by increased injection and in cases of cyclitis by increase of the keratitis punctata, ciliary flush, with increased haze in the cornea if the latter be affected.

The local application of tuberculin will also produce reaction. The ophthalmo-tuberculin reaction (Calmette) is obtained by dropping into the conjunctival sac of an eye (not affected with tuberculosis) a glycerine-free extract of the tubercle bacillus ( $\mathrm{I}$ in Ioo). The reaction produced varies so much in severity as to make its significance somewhat unreliable, and occasionally it is so severe as to be a danger to the eye. Von Pirquet's reaction is obtained by scarifying the skin and rubbing in tuberculin ( $\mathrm{I}$ in Ioo). A local redness is produced in some tuberculous patients. Control scarification should be made; the method is not always reliable.

The complement fixation test has also been applied to tuberculosis, and it appears to be reliable in about 70 per cent. of the cases.

Of all these tests the local reaction in the lesion is by far the most satisfactory, as by its means the amount of tuberculin to be given for treatment can be regulated.

Varieties.-The bacillus in Bovine tuberculosis differs somewhat from the human type. In size it is a little thicker, and when grown on glycerine broth, its final reac- 
tion is neutral, whereas that of the human bacillus is acid. The Bovine bacillus does not grow as readily as the human bacillus and its growth is not aided by the addition of glycerine to the media. The Bovine bacillus is more virulent for animals, particularly rabbits. The avian form of the bacillus grows more readily and at a higher temperature than the human form. Guinea-pigs are very susceptible to the human form, but are very refractory to the avian type; whilst rabbits are resistant to the human type, but are readily killed by the avian form. There is also a special form of tubercle bacillus which infects cold blooded animals.

Ocular ${ }^{1}$ lesions due to tubercle bacilli occur either as a part of a general tubercular infection e.g., tubercular meningitis, or as a local lesion, which may be due to ectogenous or endogenous infection, the latter being by far the most common.

The predisposing causes of ocular tuberculosis are divided into three sets of influences.

I. Those which predispose the individual to tuberculosis. There is frequently a history of tuberculosis in the family, which may imply either a want of resisting power to the specific organism or an increased risk of exposure to infection.

2. Those which predispose to the location of the disease in the eye. Of all periods of life childhood is by far the most liable. Side and sex have no influence on its etiology. Injury, although occasionally causing direct infection, comparatively rarely acts as a predisposing factor. If one eye is affected with tuberculosis the other eye is more liable to be involved than other parts of the body, probably because the organism growing in a certain tissue, when gaining access to the blood stream, is more likely to set up a fresh focus of inflammation in a similar tissue (see Infection, page 485).

3. Those which predispose certain parts of the eye to be starting-points of the affection. The tubercle bacillus, carried by the blood, is most likely to be arrested where the

1 Treacher Collins. Ophthalmoscope, V, I907, 2, 63, I I6. 
anastomosis of the vessels occurs, and where the capillary plexuses are finest. Hence it is not surprising to find, that in tubercle of the iris, the nodules characteristic of the disease generally appear first near its pupillary or ciliary margins, i.e., in the regions of the anastomosis of its bloodvessels known as the larger and lesser circle of the iris.

In the ciliary body the copious vascular plexus of the ciliary processes, situated between their epithelial covering and the ciliary muscle, is the site at which the affection most frequently starts. In the choroid the close capillary plexus forming its inner layer is the seat of primary election. Not infrequently this is near the disc at the anastomosis between the vessels of the papilla and the choroid. Tubercle commencing in the retina is rare. It usually starts near the disc or in the nerve head just inside the lamina cribrosa, these being the portions of the retina most freely supplied with blood-vessels.

In the conjunctiva it has been shown that for ectogenous infection to take place there must be damage to the epithelium. A frequent site is the sulcus subtarsalis, which is also the most common situation for the lodgment of foreign bodies. In this case a nodule forms beneath the epithelium, which subsequently ulcerates, the base of the ulcer being covered with large granulations. A septic condition of the ulcer may follow leading to infection of the cornea.

Another form of ectogenous infection is the follicular, in which multiple follicles form over the fornix and tarsus some of which break down and form minute ulcers. These cases usually occur in quite young children (see P1. 4, Fig. 2).

The commonest way, however, in which tubercle of the conjunctiva manifests itself is by the formation of a coxcomb excrescence in the fornix. Such cases are frequently associated with tuberculosis of the lacrymal sac. Histologically they exhibit very characteristic giant cell systems, but caseation in all conjunctival tubercle is very rare. The tubercle bacillus can frequently be found in and around the giant cells. 
Lupus may spread directly to the conjunctival sac from the face, or it may be secondary, due to a local inoculation. In the case of infection directly from the face, lupus invades first the outer surface of the lids, causing ectropion, finally spreading to the conjunctiva. The conjunctiva becomes injected; lupus nodules forming in the subepithelial tissue run together and ulcerate; cicatrisation subsequently bringing about obliteration of the fornices, with the formation of bands and pouches. As a result, partly of the cicatrisation and partly of the drying caused by the exposure, a condition is produced in the epithelium similar to that described under secondary xerosis. In the subconjunctival tissue a large number of typical lupus nodules with giant cells and epithelioid cells are formed, but as a rule the tubercle bacillus cannot be found in them. A condition strongly resembling the pannus of trachoma occurs in the cornea, with infiltration of the limbus. Sooner or later the cornea becomes infected with pyogenic organisms, resulting in perforating ulceration and destruction of the globe.

Direct infection of the cornea is usually the result of injury. This is followed by ulceration with a large amount of infiltration of the substantia propria. If the disease progresses the anterior chamber becomes invaded and multiple nodules form on the iris; indeed, the disease is not often diagnosed until this takes place.

The cornea may become secondarily infected from a tubercular ulcer of the conjunctiva, or from the spread of infection from the ciliary body along the fibres of the ligamentum pectinatum (see page $45^{8}$, sclerosing keratitis) and from nodules in tubercular iritis. These latter have a marked destructive effect on elastic tissue, and coming in contact with the posterior surface of the cornea cause a softening and disintegration of Descemet's membrane; the disease then spreading into the substantia propria. If resolution takes place an anterior synechia is left. An interstitial opacity of the cornea may occur in tubercular iritis without the formation of an anterior synechia, this 
probably results from toxin in the aqueous diffusing into it (see page 454).

Tubercular scleritis has already been described (see page 457 ).

The infection of the uveal tract with tubercle sets up an inflammation which frequently spreads from one portion of the tract to those adjoining it. Toxin diffusing from one focus of infection in the eye may set up inflammation in other parts of it; thus tubercle of the nerve head and choroid have set up an iritis which on microscopical examination presents none of the typical appearances of a tubercular lesion. In a similar way deep infiltration of the cornea may take place without bacillary invasion. The tubercle bacillus is not the only organism which generates a toxin which will produce such changes. Intraocular lesions, suppurative and non-suppurative, due to pyogenic and nonpyogenic organisms (e.g., staphylococcus, S. pallida), will also cause changes in other parts of the globe away from the site of the lesion, from a diffusion of their toxins.

Tubercular iritis is clinically divided into three classes:

r. Cases in which miliary disseminated nodules are present.

2. Cases in which there is a circumscribed mass made up of conglomerate or confluent nodules.

3. Cases secondary to tubercle occurring in some other parts of the eye.

It may be pointed out that these different classes often merge into one another. Thus, in cases beginning with disseminated nodules, a conglomerate mass sometimes results as the affection progresses. Where one large mass, springing from the iris is seen, several small satellites often develope around it. An iritis of variable amount is frequently met with in association with miliary disseminated nodules, or a conglomerate mass.

The miliary disseminated nodules appear first, usually, as previously stated, at the ciliary margin of the iris and 
extreme periphery of the anterior chamber (Fig. 242), or at its pupillary border (Fig. 243); the lower part of the iris is more often involved than the upper.

The nodules measure usually from 2 to $3 \mathrm{~mm}$. in diameter. They are of a greyish colour and semi-transparent, or of a yellowish hue and more opaque. The yellowness and opacity is probably accounted for by necrotic change

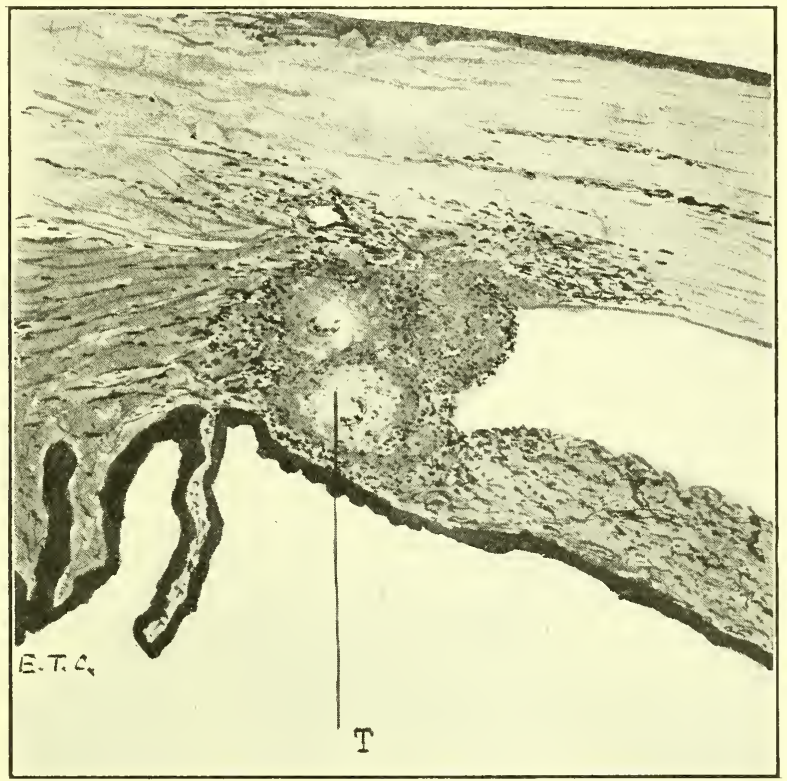

Frg. 242.-Tubercular nodules $T$ involving the periphery of the anterior chamber and root of the iris. Eye removed from a patient aged eighteen years, in whom the first symptom noted was episcleritis, the nodules appearing in the iris later. Four and one-half years after removal of the eye she was in good health and showed no other signs of tubercle.

commencing in the nodule. Some difference in the appearance of the nodules may also be due to the difference of depth in the iris at which they are situated. In microscopical sections of an affected iris nodules are sometimes seen situated at its anterior surface, projecting some considerable distance forward into the anterior chamber, the deep layers of the iris including the pigment epithelium passing beneath them and showing scarcely any sign of disturbance. 
In other cases the nodules are met with deep in the stroma of the iris, often breaking up and destroying the pigment epithelium on its posterior surface, so that no continuous line of pigment can any longer be traced, only scattered

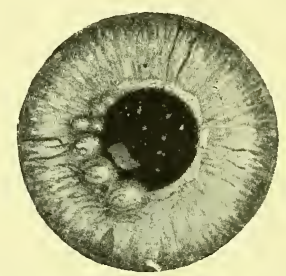

FIG. 243.- Shows the iris with tubercular nodules at its pupillary margin. The dots seen in the pupillary area are spots of keratitis punctata.

particles of pigment being seen (Fig. 244). Evidence is sometimes afforded clinically of the depth of a nodule in the iris tissue, apart from its colour, by the presence of blood-vessels on its anterior surface.

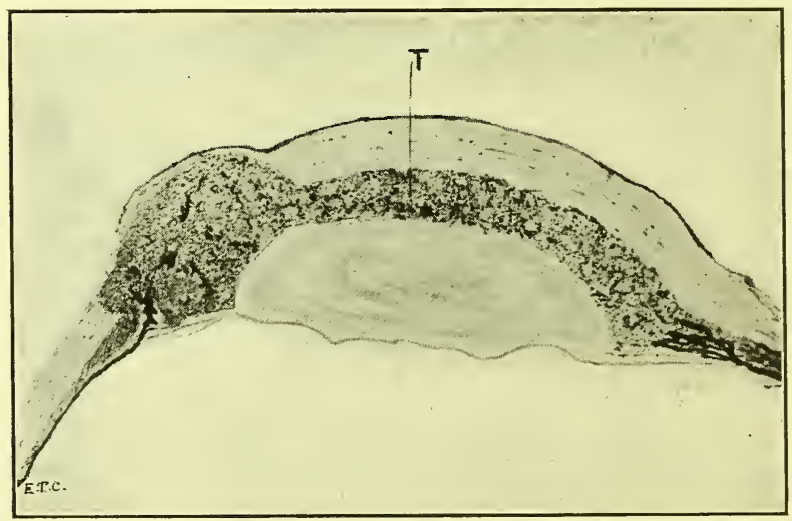

FIG. 244.-Tubercle of the iris. The whole of the iris has become involved in a conglomerate mass of tubercular nodules $T$, which fill the anterior chamber. Typical giant cell systems are shown. The cornea at its periphery has become invaded on the left side of the section.

In association with the nodules in the iris there is usually some ciliary injection, but very little photophobia or pain. Frequently deposits of a grey colour are seen on the back of the cornea; these may be of various sizes, some of them large and constituting what has been termed the "mutton 
fat" variety of "keratitis punctata" (Fig. 245). Microscopical examination of these deposits show them to consist of collections of mononuclear leucocytes on the inner surface of the endothelium of Descemet's membrane (see page $46_{3}$ ).

Disseminated miliary tubercular nodules may be met with in the iris without marked signs of iritis and with the formation of few, if any, posterior synechiæ. Microscop-

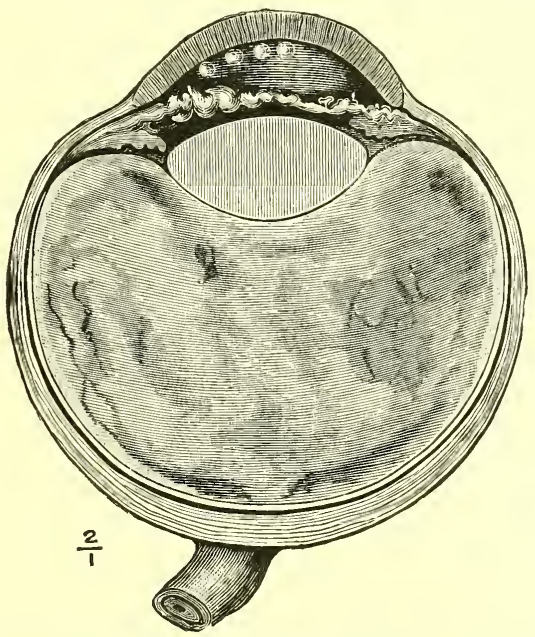

FIG. 245.-The lateral half of an eye of a child aged two years showing the iris thickened with tubercular nodules. There are deposits on the back of the lower part of the cornea, the so-called "mutton-fat" variety of keratitis punctata. Specimen in the R. Lond. Ophth. Hosp. Museum.

ically, the lines of demarcation of the nodules may be seen to be very sharply defined with but little cell infiltration of the surrounding tissue.

The mode of termination of a case of disseminated miliary tubercle of the iris varies. The nodules may disappear and leave but little permanent damage behind them, constituting what has been termed attenuated tubercle. The disease may spread backward, involving the ciliary body, and by destroying the source of supply of the intraocular fluid bring about an atrophic or shrunken condition of the globe. The miliary nodules may run into one 
another, form a conglomerate mass which invades the cornea, and terminate in the way to be described later under the heading of conglomerate tubercle of the iris.

In some cases the affection remains localised in one eye, in others both become affected. A general diffusion of tubercle throughout the body may precede or succeed a miliary tuberculosis of the iris.

These different modes of termination may be accounted for by the varying powers of attack on the part of the invading organisms, or by the varying powers of resistance of the host. On the one hand, the attacking organisms may vary in number or in the virulence of their type; on the other, invaded individuals may vary in their phagocytic power or in their capability of generating antitoxins.

A circumscribed conglomerate mass of tubercle in the iris presents the appearance of a yellowish neoplasm of that membrane, often commencing without any sign of iritis. The mass continues to enlarge, secondary nodules sometimes forming around it until the anterior chamber becomes partly or completely filled by the growth (Fig. 244).

The cornea usually becomes first invaded in the region of the ligamentum pectinatum, for it is there that the protuberant mass most frequently first comes into contact with it, but Descemet's membrane may be destroyed by direct contact, owing to the softening influence of the tuberculous mass on the elastic tissue of the membrane. As destruction of the substantia propia of the cornea takes place, the tuberculous mass with the iris from which it springs protrudes forward into the gap left, and a staphylomatous condition is seen clinically in that situation. Perforation then follows and a fungating, ulcerated vascular mass forms, which undergoing caseation, disappears, leaving a shrunken globe.

Tubercular Cyclitis.-Tubercle may attack the ciliary body either on the outer or inner side of the ciliary muscle. When affecting its outer surface it usually invades the cornea and sclerotic (see page 457). When affecting the 
inner surface of the ciliary muscle it may be in the form of small scattered miliary nodules or of a large conglomerate mass. The latter rapidly spreads inward destroying the pigment epithelium, and filling up completely the circumlental space in its vicinity. There seems a much greater tendency for tubercular growth starting in this position to extend forward and inward, than backward. It is remarkable how completely the space bounded by the back of the iris, side of the lens, and anterior hyaloid of the vitreous may be found filled with tubercular tissue, without the vitreous being involved; the fibres of the suspensory ligament being entirely destroyed, but the anterior limiting membrane of the vitreous remaining quite intact.

Where the ciliary body is affected with tubercle, the vitreous generally contains fibrinous exudate and excess of cells, but is not invaded by the tubercular nodules. If, however, in association with tubercle of the ciliary body there is much cyclitis, as is sometimes the case, there will be a more plastic exudate into the vitreous humour which goes on to the formation of fibrous tissue.

In conglomerate tubercle of the choroid, the ciliary body, like the iris, may be affected by plastic inflammation without being the seat of tubercular nodules, in which case the vitreous generally becomes shrunken and fibrous, a condition of "pseudo-glioma" being produced.

Tubercular Choroiditis.-There are two forms which tubercle of the choroid is definitely known to assume, either scattered miliary nodules which are seen ophthalmoscopically as grey patches, or a conglomerate mass which gives rise to the symptoms of an intraocular tumour.

The scattered miliary nodules are met with in cases of acute miliary tuberculosis, and as they generally develop shortly before death, frequent opportunities are afforded of comparing the ophthalmoscopic appearances with the histological alterations in the acute stage of the affection.

The patches are mostly met with in the posterior part of the globe in the vicinity of the optic disc and yellow spot 
(Fig. 246). They are circular and vary in size, seldom exceeding that of one-third of the optic disc. Their actual measurement has been estimated at .5 to $2.5 \mathrm{~mm}$. They have a greyish or greyish-yellow colour with a soft illdefined edge which shades off gradually into the surrounding fundus. There is no pigmentation about them; a slight arching forward of the overlying retinal vessels shows the larger ones to be raised a little above the level of the surrounding choroid.

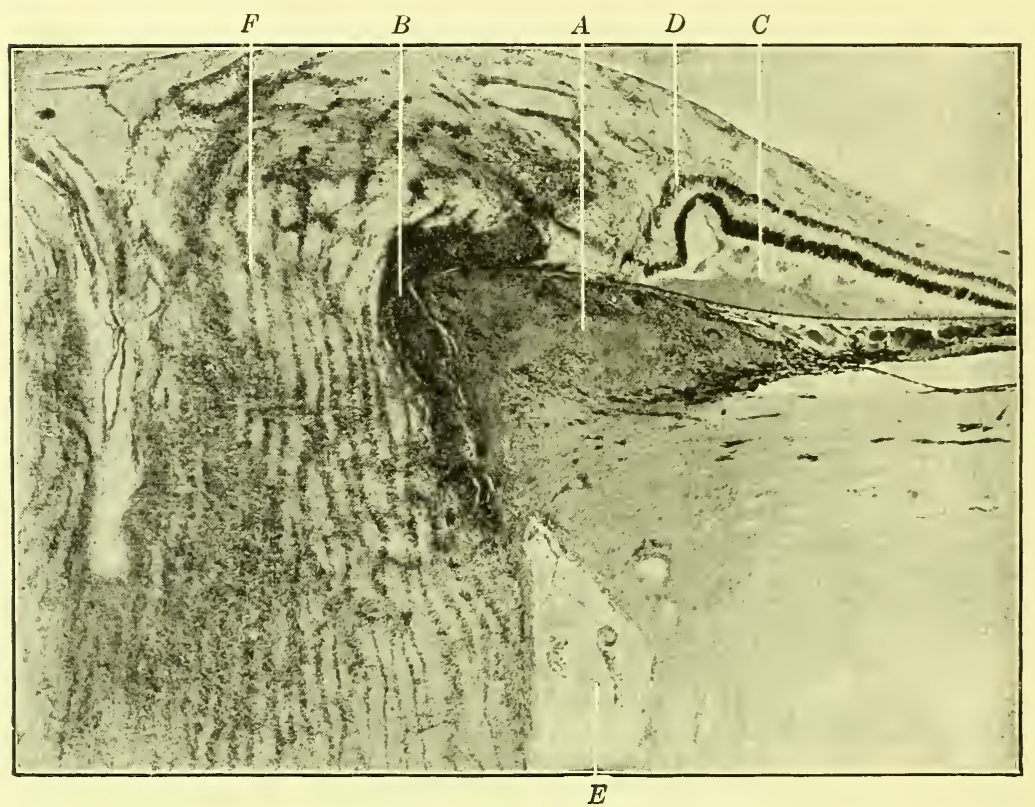

FIG. 246.- Section showing tubercular mass in the choroid pressing on and invading the optic nerve and causing an intense neuritis. $A$, Caseating tubercle; $B$, round-cell exudation in the nerve; $C$, sub-retinal exudation; $D$, retinal edema; $E$, part of the distended nerve-sheath; $F$, edema of the optic nerve within the lamina cribrosa. Case recorded in Trans. Ophth. Soc. of the U. K., XXVI, I906, Ioo.

Histologically the patches are seen to be composed in an early stage of one or more typical giant-cell systems situated in the vascular layers, or when small, not extending into the lamina suprachoroidea or up to the sclerotic (Fig. 247). The effusion, however, nearly always reaches up to the 
lamina vitrea, which is usually arched slightly inward together with the pigment epithelium lining it.

In the largest patches, in which necrotic changes are seen, the lamina vitrea will sometimes be found to have disappeared, the pigment epithelium cells to have been destroyed, and the granules of pigment widely scattered.

In conglomerate tubercle of the choroid a large area, if not the whole of it, is involved in the affection. The

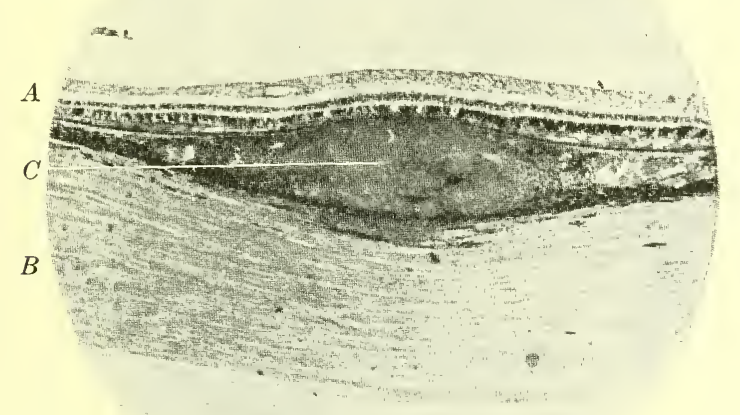

FIG. 247.-Section through the coats of the eye showing a tubercular mass $C$ in the choroid. $A$, Retina; $B$, sclerotic.

confluence of the nodules causes the choroid to become considerably thickened, large caseating patches forming in the centre of the mass (Fig. 248). The membrane of Bruch with the pigment epithelium lining it soon becomes destroyed. The retina becomes detached. Sometimes, owing to invasion of the retina in the neighbourhood of the disc, it undergoes extensive necrosis so that hardly any of its elements can be recognised microscopically. Where the retina is detached the sub-retinal fluid is of an opaque grumous consistency, and is seen microscopically to contain a quantity of fatty globules in suspension. 
Secondary foci of the disease may appear in the anterior portion of the uveal tract, and the whole tract be converted into a tubercular mass. More frequently a plastic iridocyclitis is excited by the toxins liberated from the disease in the choroid. The root of the iris may be found in contact with the periphery of the cornea, and glaucoma may be set up. As the choroid becomes thickened the sclerotic is invaded, and may later be perforated.

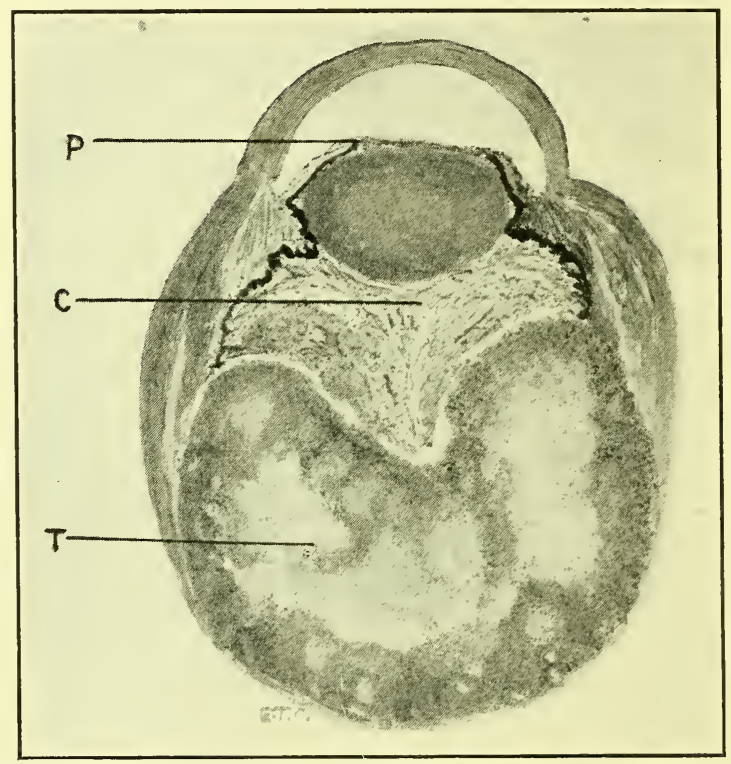

FIG. 248.- - Section of the eye of a child aged two years. containing a conglomerate mass of tubercle $T$, starting in the choroid and undergoing caseation. The retina is detached in front of it and the vitreous converted into a fibrous membrane $C$, stretching across the ciliary region. An inflammatory membrane also fills the pupil $P$.

Tubercle of the choroid, when not involving the ciliary body, does not give rise to exudation into the vitreous as long as the membrane of Bruch is intact, even when this latter structure is destroyed and the retina involved, the vitreous opacity is not marked.

Proliferation of the pigment cells of the retina occurs in the late stages. In miliary tuberculosis the patients as a 
rule do not live long enough for it to become manifest, but in cases where patients survive, a certain amount of pigmentation is usually seen.

Tubercle of the lens cannot occur primarily but opacity may be caused in it due to nutritional changes. The contact of a tubercular focus softens the elastic tissue of the capsule to such an extent as to lead to perforation. Occasionally, owing to the destruction of the suspensory ligament, dislocation of the lens occurs in tubercular iridocyclitis.

Tubercular Retinitis. ${ }^{1}$ - Primary tubercle of the retina is very rare, but secondary involvement of the retina following tubercle of the choroid is more frequent. In primary tubercular retinitis the clinical appearance is that of a white or yellowish-white swelling, usually in the neighbourhood of the optic disc. If the swelling is situated in the papilla, it may be so marked as to be mistaken for an intraocular tumour. It is sometimes accompanied by an iritis due to toxins. Cases which have been examined pathologically exhibited round-cell infiltration with giant cells and epithelioid cells, but the tubercle bacillus has not usually been demonstrated.

Tubercle of the Optic Nerve. ${ }^{2}$ - Miliary deposits in the pial sheath, chiasma and optic tract may occur associated with meningitis and general tuberculosis. Papilledema may also occur in meningitis owing to the increased intracranial pressure (see page 287). Localised tubercular masses may affect the nerve and its sheath anywhere along its whole course. When large masses are present in it, especially when situated near the globe, they may give rise to symptoms similar to optic nerve tumours. In these cases caseation is frequently very marked. The optic nerve may also be secondarily invaded by tubercle starting in the retina or choroid. In these cases the swelling of the nerve head is a prominent feature, partly due to the inflammation, and partly due to the edema caused by pressure on

${ }^{1}$ W. I. Hancock. R. Lond. Ophth. Hosp. Reps., XVI, I905, I50.

${ }^{2}$ G. Coats. R. Lond. Ophth. Hosp. Reps., XVI, I905, 38I. 
the central vein as it leaves the eye, which pressure may be so great as to cause trombosis. ${ }^{1}$

The lacrymal sac may be the seat of tubercle from ectogenous or endogenous infection, but infection secondary to lupus of the nose or disease of the conjunctiva is by far the most frequent cause. At the same time it must be remembered that lupus of the nose may cause lacrymal obstruction and dacrocystitis without tubercle being present in the sac wall. Histologically tubercle of the lacrymal sac exhibits typical giant-cell systems with epitheloid and round-celled infiltration of its wall. Considerable fibrosis may take place leading to great thickening of the sac wall-a condition which is characteristic of the disease. The epithelial lining of the sac is often deficient, and as other microörganisms are generally present, an abscess may form which ruptures externally; the sinus becoming infected with tubercle will not heal and a fistula follows.

The lower orbital margin is the most frequent seat for tubercular infection of the orbit. Occasionally in children the anterior surface of the frontal bone is the seat of the disease. In both instances the periosteum is the first portion of the bone to be affected, an abscess forms which ruptures externally with the formation of a sinus; the cicatricial contraction around may lead to a depressed scar and varying degrees of ectropion.

Leprosy bacillus ${ }^{2}$ is a small bacillus slightly shorter and rather thinner than the tubercle bacillus. It may becurved, straight, or somewhat thickened at the ends. It stains more readily than the tubercle bacillus and is acid-fast. Like the tubercle bacillus it shows inequalities in its staining which have been attributed to spore formation, but which are probably due to commencing disintegration. It has probably never been cultivated or inoculated, even in man, and it is only from its constant presence in the disease that

${ }^{1}$ Cargill and Mayou. Trans. Ophth. Soc. of the U. K., XXVI, I906, IoI.

2 Borthen and Lie. Die Lepra des Auges, Leipzig, 1899.

Treacher Collins. Trans. Ophth. Soc. of the U. K., XXIX, 1909, 323. 
it is considered to be the cause. It is easily found in the expressed secretion from the nodules, but it is important to obtain the smear preparation free from blood. The nasal discharge affords a ready means of obtaining the organism.

Leprosy in the eye usually manifests itself in the tubercular form, although the anesthetic variety does occur.

When the disease attacks the eyelids or eyebrows, in which situation it commonly begins, it commences in the middle layer of the corium, most frequently in the wall of a blood or lymphatic vessel. The bacilli multiply and in the early stages are found in enormous quantities. Around the clumps are found round-celled infiltration and epithelioid cells. There are also enormous cells distended with bacilli which are known as "globi" or lepra cells, the whole constituting the nodule which is so characteristic of the disease. The disease may invade the hair follicles and sweat glands so that the eyelashes and eyebrows become destroyed. It attacks the nerves and, spreading along their sheaths, destroys them, so causing anesthesia and paralysis. The organism does not seem to invade readily the muscular or elastic tissue.

In the conjunctiva nodules form which frequently ulcerate. They may also spread deeply and affect the tarsus causing ectropion and trichiasis.

The globe may be affected either by a direct spread of the disease from the conjunctiva, or possibly by metastatic infection. The neighbourhood of the limbus is a common situation for the disease to commence. Nodules are formed in the episcleral tissue, and from these the disease may spread inward through the sclerotic involving the ciliary body or iris, in either of which nodules may arise. In the cornea it causes a vascular interstitial opacity, resembling in its appearance tubercular sclerosing keratitis, and like it fails to resolve. Occasionally, when the bacillus lies directly beneath Bowman's membrane, a condition resembling superficial punctate keratitis may be produced. 
The uveal tract may also be involved by metastatic infection. The disease almost invariably begins at the angle of the chamber, spreading forward to the iris, and backward to the ciliary body and choroid. Nodules are formed in the iris and ciliary body which may lead to shrinking and atrophy of the globe. Choroiditis occurs in the anterior part of the globe in direct continuity with the disease in the ciliary body, but in the posterior part of the globe it is extremely rare. The retina is usually only affected secondary to the choroid and then only in the anterior part of the globe near the ora serrata.

Chaulmoogra oil by intramuscular injections has been successfully used in the treatment of the disease. In one case in which the eyes were affected, it was used without success.

(g) Unclassified Bacilli.-The bacillus mallei (glanders) (farcy) is a slender rod and measures about 2 to $3 \mu$ long. It is stained irregularly and feebly by aniline dyes, and is not stained in Gram's method. It is non-flagellated, nonmotile, and does not form spores. On cultivation it grows well on all media; on potato it forms a brown honey-like mass on the surface, which is very characteristic. Inoculation on the conjunctiva of animals has proved fatal. When introduced into the anterior chamber nodules are formed on the iris and general infection frequently follows. Guineapigs are peculiarly susceptible to the disease. The usual source of infection is from the horse, the organism being frequently carried in the discharge from the nose and mouth.

The disease is an infective granuloma, somewhat allied to tuberculosis only more rapid in its course.

When it affects the eyelids or conjunctiva nodules are formed which break down and ulcerate; there is usually early enlargement of the preauricular gland and those of the neck.

From the conjunctiva the disease may spread to the lacrymal sac and nose. 
The globe is only invaded secondarily to corneal ulceration. Metastatic deposits have never been recorded.

The strepto-bacillus of Ducrey is found in soft sores which occasionally affect the lids and conjunctiva. The organism can be found in the secretion scraped from the surface of the ulcers. The bacilli are about I. $5 \mu$ long and $.5 \mu$ broad, slightly curved and tend to lie side by side in pairs or chains. It stains irregularly and badly with aniline dyes and does not stain by Gram's method. On cultivation it grows on agar forming whitish colonies which, when inoculated into apes, produce a typical soft chancre. It can be easily distinguished from the diplo-bacillus of MoraxAxenfeld by the faint irregular staining with aniline dyes and by its growing on agar; from the Bacillus Mallei by not producing a brown growth on potato which is a typical characteristic of the latter organism.

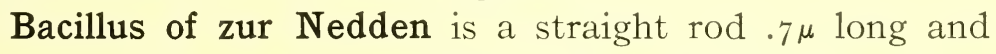
$.6 \mu$ broad, with rounded ends. It is about the same size and shape as the xerosis bacillus. It stains well with thionine blue and is decolourised by Gram's method. These bacilli have a superficial resemblance to the Coli group 'but are non-flagellated, non-motile obligate aerobes.

On cultivation they grow on all media and thus differ from the Koch-Weeks bacillus. Milk is coagulated. No gas is formed. On agar they form colonies 2 to $7 \mathrm{~mm}$. in diameter in about 24 hours which have a bluish transparent appearance.

Inoculated into the cornea of rabbits they produce a severe corneal ulceration with hypopyon, but if inoculated into the vitreous merely produce a transient reaction probably due to the want of a free supply of oxygen.

$\mathrm{Up}$ to the present time the organism has only been found in corneal ulceration. The ulcer produced is a severe grey infiltration usually situated near the corneal margin. Hypopyon is rarely present.

Bacillus subtilis is a large rod of about $2.5 \mu$ long and $.5 \mu$ thick. It varies considerably in length. It stains well 
with thionine blue and retains the stain in Gram's method. It is a flagellated motile aerobe. It forms spores situated about the centre of the organism.

On cultivation it grows best at body temperature, and forms on solid media a yellowish-brown skin raised above the medium which has a very characteristic appearance.

Neither the organism nor the toxin produces any change when instilled into the conjunctival sac, but when rubbed in after scarification it produces reaction. Inoculation of the organism into the anterior chamber produces a severe iritis, and when inoculated into the vitreous panophthalmitis ensues.

In the conjunctiva no case has been recorded in which the organism could be said to have caused inflammation. It is of importance, however, as occasionally it occurs as a saprophyte in the conjunctival sac, wound-infection and panophthalmitis having been attributed to it, but more evidence is yet required before this can be regarded as proved.

Bacillus aerogenes (perfringens) (emphysematous gangrene).-This bacillus somewhat resembles the bacillus subtilis in its appearance. It stains irregularly with aniline dyes, but is well stained in Gram's method. A gaseous envelope formed by the bacillus causes a retraction of the medium surrounding it, and gives rise to the appearance of its being encapsuled. It is non-motile. On cultivation it will only grow anaerobically, free gas being formed in a medium if it contains sugar. It does not grow on gelatine. Inoculated into the interior of the eye of an animal it causes panophthalmitis. It has only been found in cases of panophthalmitis following wounds of the globe and it is usually associated with other organisms, more especially the streptococcus. One of the chief characteristics of the panophthalmitis caused by this organism is that bubbles of gas appear beneath the conjunctiva, the inflammation being of a very violent character. ${ }^{1}$

${ }^{1}$ R. R. James. Trans. Ophth. Soc. of the U. K., XXX, I9I O, 179. 
Bacillus pyocyaneus (bacillus of blue pus) is one of the chromogenic bacteria. It is a slender rod of about I $\mu$ long armed with a terminal cilium. It stains moderately well with aniline dyes, but not in Gram's method. It is a motile facultative aerobe, growing readily in the cool incubator in all media. On gelatine, which it rapidly liquefies, it causes a greenish-blue fluorescence by its growth. It gives rise to free toxin in the media in which it is grown. Its virulence varies considerably, but all forms are pyogenic. In the conjunctiva one case of muco-purulent conjunctivitis attributed to it has been recorded.

In the cornea it causes severe serpiginous corneal ulceration. Intraocular infection generally follows wounds in the globe. It gives rise to a panophthalmitis which is frequently associated with ring abscess of the cornea, probably owing to the presence of the free toxin diffusing into it.

\section{FUNGI}

It is rare for the eye to be affected by fungi.

Hyphomycetes. - The hyphomycetes are divided into two large groups, the true molds and the trichomycetes. These are subdivided into the leptothrix, which are simple threads without branches; cladothrix, in which the simple thread is broken up at the end; streptothrix, in which there are numerous branches with spores and chain formation (Fig. 249), and actinomyces, which are characterised by club shaped ends and stellate arrangements of the threads.

Concretions may occur in the lower canaliculus due to the growth of a fungus. Much discussion has arisen as to whether the organisms found in these concretions are leptothrix or streptothrix fosterii, but the balance of evidence, in those cases in which cultivations have been obtained, is entirely in favour of the streptothrix; indeed, it is extremely doubtful if the leptothrix ever occurs. Leptothrix can be distinguished from streptothrix fosterii by not usually exhibiting branching ends on cultivation, and 
by not yielding the iodine reaction (turning blue on the addition of Gram's iodine solution owing to the presence of starch-like material).

Actinomyces does not occur in the canaliculus but may occur as a rare affection of the eyelids.

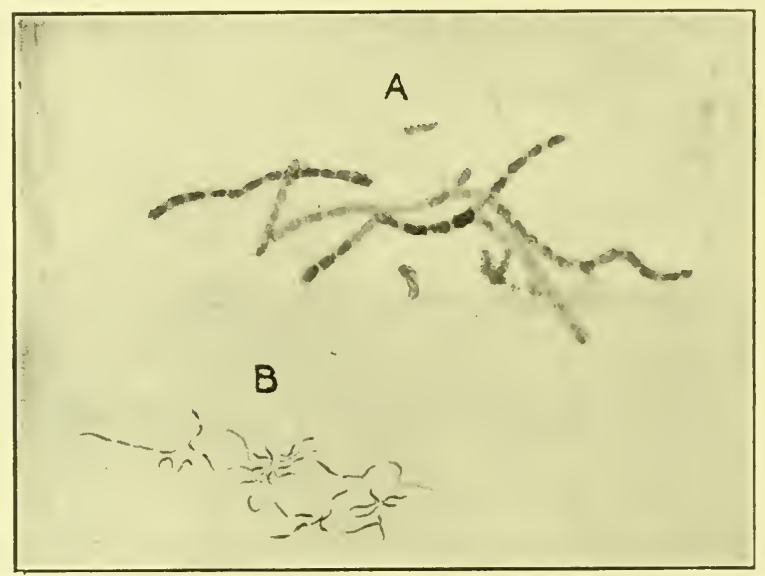

FIG. 249.-Two different specimens of the streptothrix fosterii removed from the canaliculus. $A$, Thick filaments; $B$, much narrower filaments.

The streptothrix fosterii is a mold with branching ends. It is found in concretions in the canaliculi together with other organisms and calcareous paricles. It stains badly with all aniline dyes but well with Gram's method, and is also turned blue by Gram's iodine. Irregularities in the filaments and variations in thickness are frequently seen (Fig. 249). On cultivation it can only be grown anaerobically, and is difficult to obtain in pure culture owing to the presence of other microörganisms. These will sometimes die out at the end of two or three weeks, allowing the streptothrix to be obtained pure on recultivation. Peptone-glycerine agar (acid) is the best medium for its growth. A stab culture should be made. It appears in from 4 to 6 days as greyish-white smooth granules at the bottom of the stab. In broth it forms a woolly mass at the bottom of the tube. Polymorphism is very marked on cultiva- 
tion. On inoculation large doses injected intra-peritoneally will sometimes produce suppuration.

Aspergillus or mold fungus occurs in many varieties. The chief of these are the aspergillus fumigatus, flavescens, niger, ficum, wenti, candidus, all of which are pathogenic to animals; and the aspergillus glaucus, ostranus, minimus, clavus, varians, and novus which are non-pathogenic. The one which has been most frequently described as causing ocular lesions is the aspergillus fumigatus.

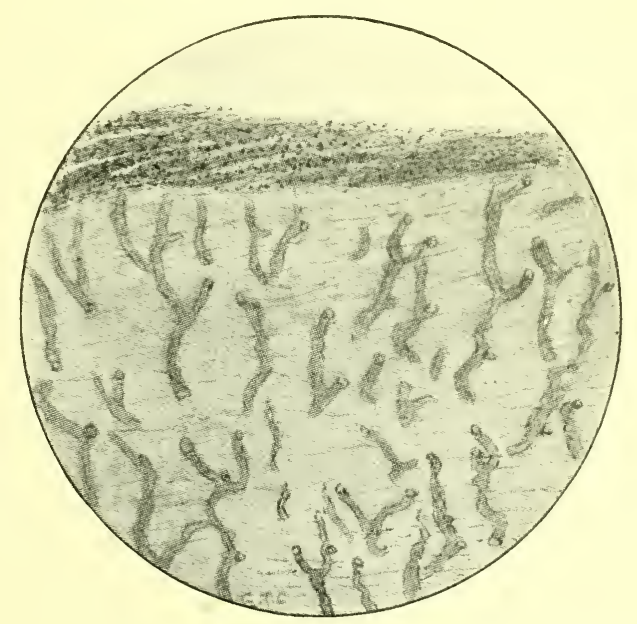

FIG. 250.-Aspergillus or mold fungus growing in the cornea.

It consists of filaments (mycelium) attached to which are the reproductive organs consisting of radial arranged sternigia and conidia (spores). These molds are best examined without staining in a hanging-drop glycerine preparation. They stain badly with aniline dyes, but are well stained with Gram's method. On cultivation they grow well on all media, especially when acid. They give rise to a white, soft, furry mass which after a time becomes greenish toward the centre (A. fumigatus).

In the cornea the aspergillum will only grow if a foreign body is implanted with it. It gives rise either to small 
vascular ulcers or to larger lesions causing destruction of the entire cornea. The appearance is very characteristic. The ulcer is a dry slightly raised area surrounded by a ring of demarcation and infiltration. Occasionally there is a leash of vessels running to it. The central area may sometimes be thrown off as a slough.

Microscopical examination of a scraping from the ulcer is usually sufficient to make a diagnosis; the mycelium being easily recognised though the reproductive organs are rarely present (Fig. 250). If the ulcer perforates, the fungus may in rare cases spread to the interior of the globe.

Infection of the vitreous and sclerotic accompanied by suppuration, due to the aspergillus, has been recorded.

Penicillium glaucum (Fig. 25 I) has been described as the cause of keratitis with hypopyon in one case. Though it is pathogenic to animals it is somewhat doubtful if it occurs in the human cornea.

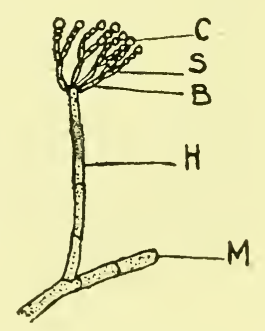

FIG. 25I.-Penicilium glaucum. $\times$ 400. $C$, Conidia; $S$, sterigmata; $B$, basidia; $H$, hyphia; $M$, mycelium.

Botryomycosis and Hefæ have also been described in corneal ulceration with hypopyon. Botryomycosis has also been met with in a lesion of the lid resembling a stye.

Favus is due to the Achorion schönleinii. The organism is sometimes found affecting the eyelashes and eyelids. ${ }^{1}$ It is recognised, by examining the unstained preparation after the addition of acetic acid, by the ovoid spores, and by its trichotomus branching mycelium; for method of

${ }^{1}$ Treacher Collins. Trans. Ophth. Soc. of the U. K., XXIII, I903, I. 
staining see page 700. The organism grows best in a culture on maltose agar.

Ringworm may be due to:

The microsporon audouini (9o per cent. of cases), a small spore fungus with mycelium principally in the hair and spores arranged in mosaic pattern outside; this destroys the outer sheath of the hair, a fact which serves to distinguish it from the other forms of ringworm and from favus.

The trichophyton endothrix distinguished from the above by the chain-like arrangement of the spores.

The trichophyton ectothrix which attacks the hair only on the outside.

\section{ANIMAL PARASITES}

Animal parasites are divided into (i) unicellular and (ii) multicellular organisms.

(i) Unicellular organisms (protozoa).

The diseases affecting the human eye and its appendages caused by this group are syphilis (spirochæta pallida), malaria (malarial parasite).

Although the spirochætæ are placed under this heading, it is probable that they should be classified as being between the protozoa and bacteria.

Trypanosomata have been demonstrated in animal's eyes but not in man's.

Syphilis.-That the spirochæta pallida is the cause of syphilis there is now little doubt, but as yet insufficient time has elapsed since its discovery for demonstration of the organism in the various intraocular lesions due to this disease. Up to the present time it has been found in primary chancre of the eyelids and conjunctiva, in the aqueous in acute iritis, and in the cornea of syphilitic infants. It is extremely difficult to find in gummatous lesions.

The spirochæta pallida is a protozoon from ro to I $_{5} \mu$ long and about $1 / 4 \mu$ thick. It appears in spirals of about 
eight to ten sharp, regular and short turns, which serve to distinguish it from the spirochæta refringens in which the turns are less numerous and longer. Each end of the organism is pointed and provided with a cilium, by means of which it moves freely. To demonstrate the organism, the dark ground illumination method, the indian-ink method, or prolonged staining by Giemsa's method, of smear preparations of scrapings from the tissue are the most reliable. By the latter method the organism is of a pink colour while other spirochætæ have a bluish tinge. The silver impregnation method can be used for staining them in the tissues, but fibres of the tissues, especially nerve fibrillæ, may be mistaken for the spirochæta (see page 702).

In infected persons the presence of mercury in the system causes the disappearance of the spirochæta pallida from the lesions.

The spirochæta has been grown on diluted horse serum into which a piece of infected material was introduced. The serum was covered with sterile paraffin oil and placed in an anaerobic jar.

Inoculation has proved successful by injecting the infected material into the testicles of rabbits, but inoculation of the spirochæta into apes produces the most reliable results. In making an inoculation on the surface a piece of infected tissue should be implanted under the skin of the eyelid or eyebrow in apes. Injection into the anterior chamber of an emulsion from a primary chancre has produced an interstitial keratitis of the cornea in rabbits. There is little doubt that the presence of the spirochæta pallida in the body gives rise to immune bodies, which help to eradicate the disease, since patients with syphilis cannot be reinfected. That this immunity does not last is shown by the fact that if the disease be completely eradicated from the system the person can again become infected with syphilis. But as long as the organism is still present the patient cannot be infected with syphilis, even if it gives rise to no symptoms as, for instance, in the mother of a 
syphilitic child. This is evident from the fact that as long as the spirochæta pallida is present in the body the blood of the patient will give the Wassermann reaction. Although the immunisation of animals with a cultured S. pallida produces antibodies, similar to bacterial antibodies, the biological change which takes place when the S. pallida is cultivated prevents the antibodies having any action on either man or animals, who have been infected by the disease. Although the Wassermann reaction is dependent on the presence of syphilitic infection, it is probably produced indirectly by a change in the physical state of the globulins present in the serum. Whatever the syphilitic virus may be it will not pass through a filter.

The spirochæta pallida and its toxins during the secondary stages have the power of exciting a chronic inflammation, which especially affects the vessel walls, due to the organism circulating in the blood. This endarteritis is also very marked in the local manifestations of the disease. It is followed by organisation and sclerosis of the vessel walls, a condition which is frequently seen clinically in the choroid and retina.

The infection is usually divided into three stages which are purely arbitrary, one stage merging into the other. The infection is limited for a short time to the primary sore; this is rapidly followed by the infection of the nearest lymphatic gland. The organisms then gain entrance to the blood stream, general dissemination of them taking place. This is the secondary stage, and is manifested by general symptoms of slight fever, followed by local eruptions due to aggregations of the organisms in the skin, mucous membrane, and uveal tract. The reaction of the tissues to the organism may produce an immunity so that no further symptoms arise. If, however, they be not completely eradicated, they lie latent in the tissue and again become active as the general immunity subsides. The inflammation which is then excited is of a more localised character, producing masses of exudate known as gummata, in 
which necrosis frequently takes place. In this, the tertiary stage, the disease is supposed to be non-contagious, partly because the organisms are very scanty, and partly because they are entirely limited to the local lesion.

A primary sore (Hunterian chancre) may affect the eyelids or conjunctiva. The infection is sometimes caused by the removal of foreign bodies from the surface of the eye by the tongue, a practice not uncommon among workmen. The incubation period is from two to four weeks; an ulcer with sharp-cut edges and an indurated sloughing base is formed; when near the limbus the cornea may be involved. Toward the end of the first week the preauricular and submaxillary glands begin to enlarge, and may reach a considerable size. There is usually a large amount of conjunctival discharge due to the ulcer becoming infected with pyogenic organisms. The spirochæta pallida has been found in scrapings from such ulcers. Microscopical examination of the ulcer shows a new formation of round cells and epithelioid cells, some of which are undergoing necrosis. In the later stages there is much formation of fibrous tissue. The vessels in the neighbourhood show intense sclerosis and are often thrombosed. A primary lesion of the eye is frequently followed later by interstitial keratitis in the same eye. ${ }^{1}$

Secondary syphilis rarely affects the conjunctiva and eyelids. Mucous patches may occur at the angles of the eyelids in infants, and ulceration of the palpebral conjunctiva may take place.

The uveal tract is peculiarly liable to be affected in this stage of the disease, usually about the time the rash begins to disappear from the skin. The lesions in the iris and choroid are no doubt of a similar nature to those of the skin but more diffuse. The inflammation is of a plastic type and usually affects both eyes (see page $4_{4}$ ).

In the iris vascular nodules may be formed which are most frequently observed, in blue irides, as rust coloured

${ }_{1}^{1}$ Treacher Collins. R. Lond. Ophth. Hosp. Reps., XVI, 1904, I6. 
elevations near the pupillary margin where the capillary anastomosis is finest (Fig. 252). Extensive posterior synechiæ are common and may be followed by complications (see page 466). The spirochæta pallida has been found in the aqueous humour, but not as yet in the nodules. The ciliary body may be slightly involved, but this structure is more frequently affected in tertiary syphilis.

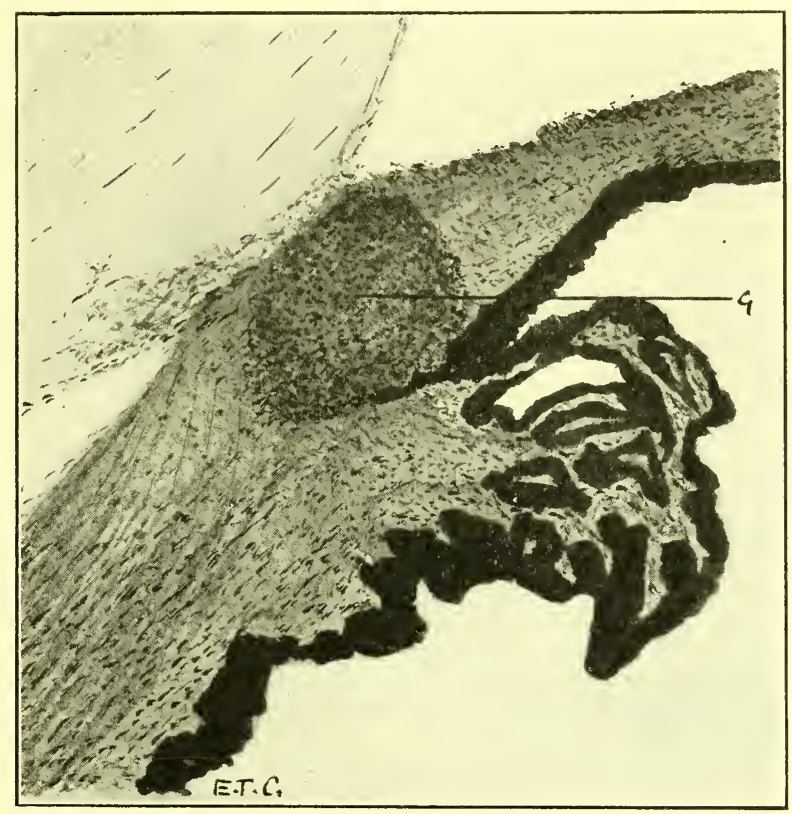

FIG. 252.-G, Gumma in the root of the iris in a child aged fourteen years, the subject of inherited syphilis.

The choroid is affected by multiple localised patches or more or less diffused round-celled infiltration, situated especially in its capillary layer. The overlying retina frequently becomes involved (chorio-retinitis). This inflammation is subsequently followed by organisation and vascular sclerosis (Fig. 253). If the outer layers of the retina are destroyed by the inflammation a loss of function corresponding to the area involved is the result, but if the nerve-fibre layer is also affected the retina to which these fibres are dis- 
tributed will likewise be rendered functionless. Marked secondary pigmentation of the retina follows, from the proliferation and migration of the pigment cells. The white areas surrounded by pigment seen ophthalmoscopically are due, partly to exposed sclerotic from atrophy of the chorio-capillaris, and partly to newly formed fibrous tissue.

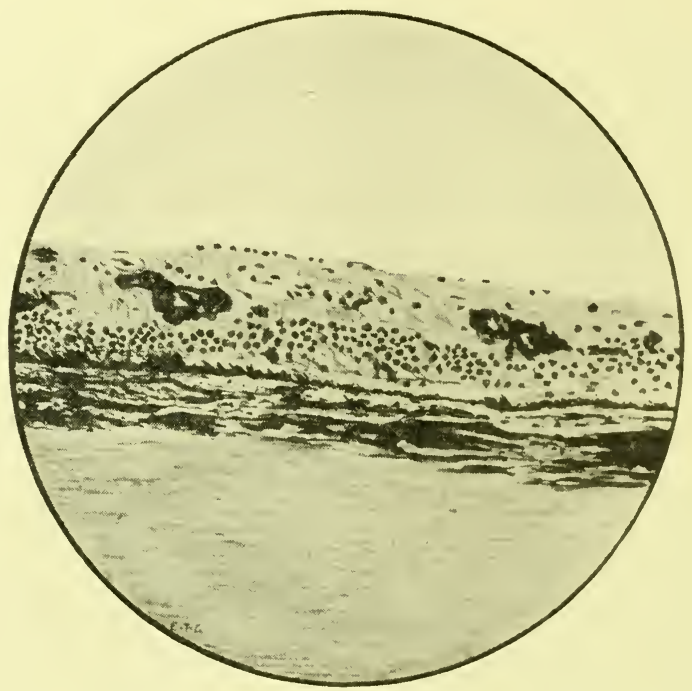

FIG. 253.-Section of the coats of the eye showing the degenerative and pigmentary changes left in the retina as the result of a patch of syphilitic chorio-retinitis.

The retina may be affected secondarily to the choroid as has already been described, or the spirochæta pallida may be primarily deposited in it. As in all metastatic lesions of the retina, this is more likely to take place in the neighbourhood of the disc where it is most vascular. The papilla also is usually involved, producing a condition of neuro- retinitis. The swelling and pressure of the exudation in the papilla, accompanied by inflammation in the vessel walls, will sometimes cause secondary thrombosis of a retinal vein.

Lacrymal obstruction is a frequent sequela of the rhinitis of congenital syphilis, which causes periostitis and thickening of the bony wall of the lacrymal canal. 
Tertiary syphilis rarely affects the lids and conjunctiva. Occasionally gummatous ulcers may form on the eyelid.

The cornea is affected by a gummatous infiltration of the deep layers of the substantia propria (interstitial keratitis). The disease usually occurs in children, the subjects of congenital syphilis, between the ages of ten and twenty, but it may occur as early as two and a half, or as late as

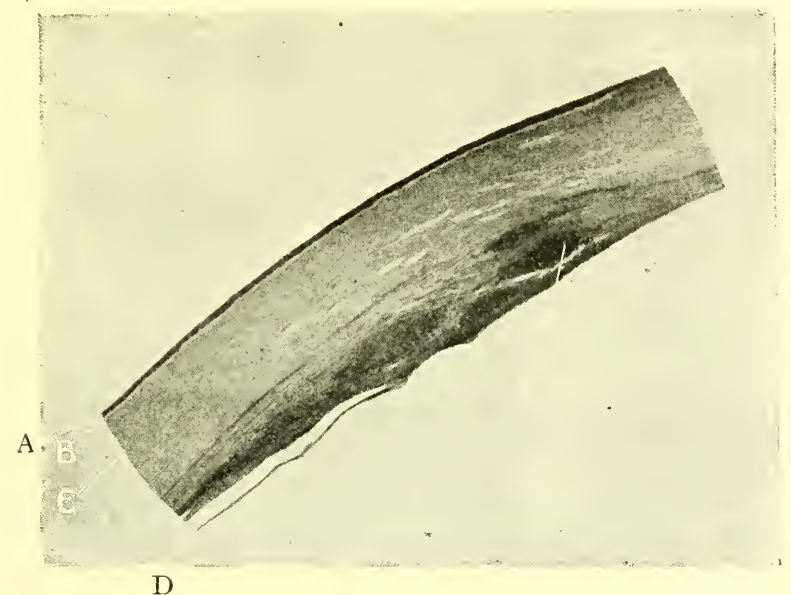

FIG. 254.- Shows a section through a cornea affected with interstitial keratitis. $A$, Corneal epithelium; $B$, Bowman's membrane; $C$, substantia propria; $D$, Descemet's membrane; $E$, round-cell infiltration in the deeper layers of the cornea.

forty years. It affects both eyes, though one is frequently involved before the other. Probably most cases are accompanied by a gummatous infiltration of the ciliary body and iris. In adults in the acquired form it is often unilateral.

Since the disease in the cornea is due to endogenous infection, it begins usually at the corneal margin. There are, however, rare cases with but little opacity and no vascularity, which commence near the centre of the cornea and are always accompanied by cyclitis; it is probable that they are due to diffusion of toxin from the aqueous through Descemet's membrane.

The spirochæta pallida has not been demonstrated in interstitial keratitis, partly because so far very few cases have been examined, and partly because the organism is 
very scanty in gummatous lesions. Histologically the cornea presents a round-celled infiltration in the deeper layers (Fig. 254) of its lamellæ, which are sometimes separated from each other by quite large masses of cells. Epithelioid and giant cells may also be present. The cells, especially toward the centre of the gummatous masses, undergo necrosis. After a time the ciliary vessels from the sclerotic bud out and vascularise the cornea. These are

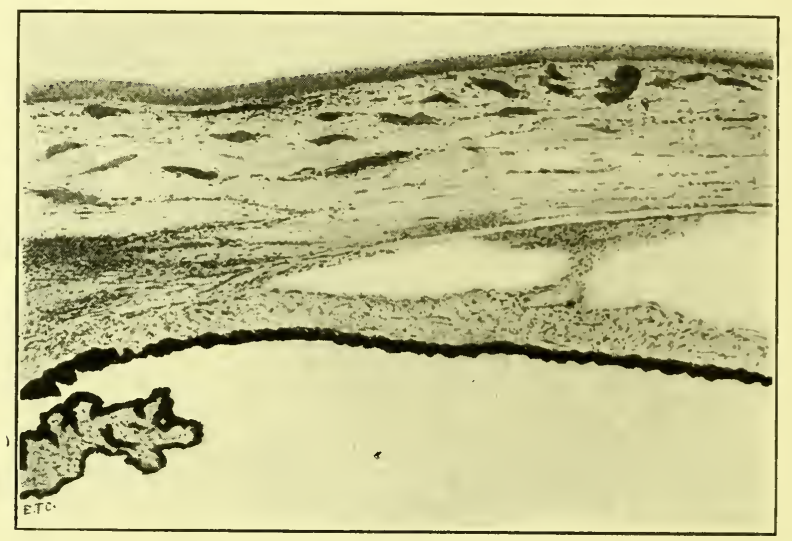

FIG. 255.-Shows a section through a cornea which had become vascularised as the result of interstitial keratitis of hereditary syphilitic origin.

fine vessels running a straight course, and may be so numerous as to give the vascularised area a uniform red appearance, a condition which is known as "salmon patch." The superficial layers of the cornea may also occasionally be affected and then vessels from the conjunctiva extend into it (Fig. 255). The epithelium on the surface is always edematous. In rare cases, where the whole circumference of the cornea is affected and becomes vascular, the central portion of it may break down and ulcerate. ${ }^{1}$ The disease usually involves the whole cornea spreading from the margin toward the centre, the lower and central portion of the cornea being the last to clear. The infiltration of the cor-

${ }_{1}^{1}$ Treacher Collins. R. Lond. Ophth. Hosp. Reps., XI, I887, 338. 
nea leads to softening and the normal intraocular tension may give rise to a local ectasia, or general yielding, and deepening of the anterior chamber. Clinically the former condition must be distinguished from the swelling due to infiltration, which is most marked when the anterior layers are involved. Yielding of the cornea may be so great as to produce a staphyloma. Occasionally in very rare cases the condition of conical cornea is produced; such cases usually have an opacity at the apex of the cone.

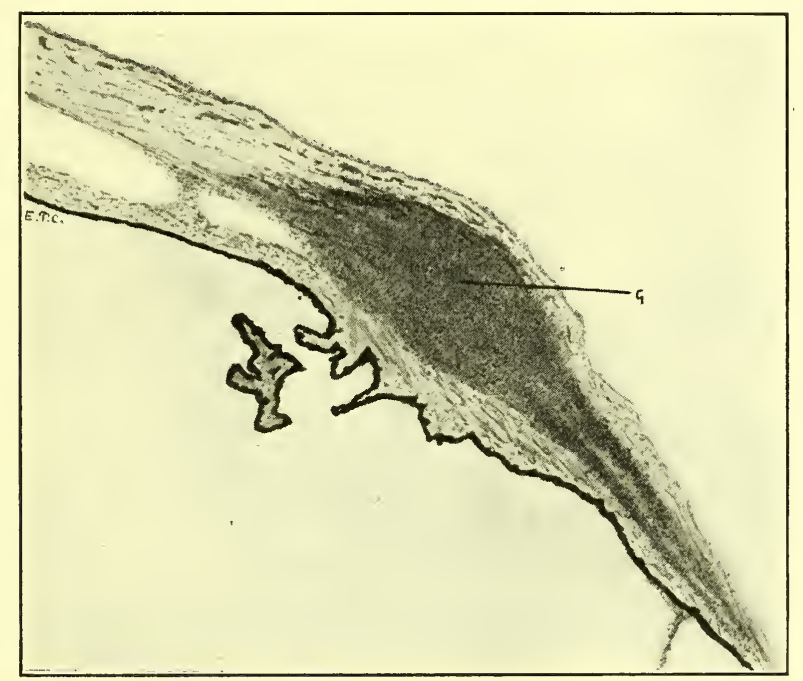

FIG. 256.- Shows a section through a gumma starting on the outer surface of the ciliary body and invading the sclerotic.

The amount of permanent opacity which follows the disease varies very considerably according to the amount of the necrosis which has taken place. In extreme cases the cicatrical contraction may give rise to flattening of the cornea; the opacity may be so dense as to leave the patient with nothing more than a bare perception of light. In the mild cases the opacity left may be little more than a slight haze in the cornea, the origin of which can usually be recognized by the straight hair-like remains of the vessels in 
the cornea which after the disease has subsided, become so sclerosed as to contain no blood.

Another cause of opacity of the cornea is complete destruction of the endothelium on Descemet's membrane over the lower part, associated with punctate keratitis which is so extensive as to undergo organisation into a membrane. Other complications, such as are associated with iridocyclitis may also arise (see page 466 ).

Localised gummata of the iris, ${ }^{1}$ are rare. They occur near the root of the iris, and form pigmented comparatively non-vascular tumour of slow growth, which are liable to be mistaken for melanotic sarcoma. The differential diagnosis is easily made by the examination of the blood or aqueous.

Localised gummata of the ciliary body may occasionally occur apart from interstitial keratitis. The inflammation starts either on the inner or outer surface of the ciliary muscle. A gumma on the inner side of the ciliary muscle, which usually is unaccompanied by much iritis, may be seen by focal illumination protruding inwards behind the lens as a yellowish-red mass. One starting on the outer side of the ciliary muscle invades the sclerotic (Fig. 256) and may cause perforation. In both instances the ciliary muscle becomes involved, but it is rare for its attachment to the sclerotic to be destroyed. If the disease spreads forward it involves the cornea; if backward, the anterior part of the choroid.

Localised gummata of the choroid are rare except as a result of direct spread of infection from the ciliary body or optic nerve. A diffuse gummatous infiltration is more common and a fibrous membrane may form between the retina and choroid. The disease usually starts in the capillary layer in the choroid, the retina often being secondarily involved. Histologically fatty degeneration of the inflammatory exudate is often a marked feature.

Gummata of the optic nerve occur most commonly in connection with meningitis in the neighbourhood of the

${ }^{1}$ R. A. Greeves. Trans. Ophth. Soc. of the U. K., XXXV, I9 I5, 277. 
chiasma, in which case they may give rise to symptoms resembling an intracranial tumour with a defect in the field corresponding to the portion of the nerve involved.

In rare cases the disease may attack the neighbourhood of the optic papilla, giving rise to a swelling of the nerve head and symptoms which resemble an intraocular tumour.

Gummata of the orbit affect the periosteum, especially of the upper and outer, and lower and inner orbital margins. The disease starts in the deeper layers of the periosteum, causing a thickening of that structure. As the mass increases in size it undergoes necrosis, a glairy yellow fluid being formed in it. The mass softening may extend forward so that the skin becomes involved, and destroyed, a sinus being formed. From the supervention of sepsis an extensive necrosis of the bone may result. Gummata in the neighbourhood of the optic foramen give rise to symptoms of pressure on the optic nerve (central scotoma) and later signs of an intraorbital tumour.

Malaria.-In a patient who died comatosed after failure of sight from malignant malaria,${ }^{1}$ red blood corpuscles infected with malarial parasites, which contained coarse melanin granules, were found in the vessels of the retina, iris and optic nerve, without any perivascular exudation. The brain showed numerous thrombosed vessels and hemorrhages, the thrombi consisting of infected cells, masses of dot parasites, particles of melanin and mononuclear cells.

Retinal hemorrhages of two types are met with in connection with malaria, small peripheral ones which occur during the time the temperature is raised; and large central ones associated usually with the cachexia of malignant malaria. They are doubtless due to thrombosis caused by the parasites, very dense pigmentation often resulting from them.

It seems very doubtful if any inflammatory affection of the eye can be attributed to the presence of the parasite;

${ }^{1}$ L. S. Dudgeon. Trans. Ophth. Soc. of the U. K., XLI, I92I, 236. 
in malarial districts there is a tendency to attribute all maladies to that disease; so-called malarial keratitis resembles herpetic or dendritic keratitis as met with in other febrile affections. The amaurosis sometimes occurring with malaria is probably in many cases due to quinine.

Trypanosomiasis. ${ }^{1}$ - The diseases of the eye known to be due to animal parasites, which have not already been described, are nagana, surra mobori, souma, mal de caderas, dourine and sleeping sickness.

The ocular lesions in man which have been observed in sleeping sickness are iridocyclitis, choroiditis, and edema of the eyelids; the disease is most prevalent in South Africa, being conveyed to the sufferer by the tsetse fly. In animals experimental inoculations have been found to give rise to similar lesions, and the trypanosomes have been found in the tissues. It is also of interest, in view of the fact that syphilis is due to an organism belonging to the same group, that animals affected by these diseases develop an interstitial keratitis in which the organism has been demonstrated. Bayer's 205 is the most successful drug in the treatment of this disease.

The keratitis and iridocyclitis in human beings is generally not so severe as the syphilitic form. A peculiar form of choroiditis has been described, in which there is a superficial choroidal atrophy in each macula region with some pigmentary disturbances and some light coloured disseminated patches throughout the periphery of the fundus.

Entamœbæ coli ${ }^{2}$ have been found associated with relapsing iritis in non-dysenteric patients. So far it has not been discovered histologically within the eye.

(ii) Multicellular organisms affecting the eye are divided into entozoa (worms) and insectivora.

Entozoa-Tapeworms.--The eye may be the seat of development of the intermediary stage of a tapeworm. The common forms which occur in the eye are the cysticercus

${ }^{1}$ C. W. Daniels. Pro. Roy. Soc. of Med., Ophth. Sec., IX, I9I5, 2.

${ }^{2}$ Lloyd Miles. Arch. of Oph., Nov., 1923. 
(tænia solium) and hydatid (tænia echinococcus). The adult worm, living in the intestine of its host, from time to time throws off the last segments (prologittis) containing ova; these being swallowed by the intermediary host the envelope is dissolved and the embryo (proscolex) which they contain set free. They make their way through the wall of the intestine and gaining access to the blood stream are carried to the eye.

Cysticercus $^{1}$ (T. solium) consists of a head with suckers, proboscis, and six hooklets. Attached to it by means of a segmented neck is a small bladder or cyst about the size of a pea; the head and neck are usually found inverted into the centre of the cyst.

The head of the T. mediocanellata is provided with four suckers but has no proboscis or hookets. The duration of life of the embryo in the tissues may be as long as three years.

Nearly all parts of the eye may be the site of their development.

In the conjunctiva the fornices are the most frequent site for the cyst, which appears translucent and in one situation may show an opaque area corresponding to the scolex.

The sclerotic was affected in one recorded case.

The iris, or rather the anterior chamber into which the organism makes its way, may be the site of a cyst, in which case it is usually attached to the iris by a pedicle. If the organism is alive it may be seen to alter its shape from time to time and also protrude its head. It gives rise to a severe plastic iritis and occasionally a hypopyon may be formed.

In the posterior part of the globe the cyst may begin in the retina or choroid, subsequently making its way into the vitreous. The retina usually becomes detached. Severe inflammation and even suppuration may result.

Hydatid (T. echinococcus). ${ }^{2}$ - The embryo of this worm when it escapes from the ova into the intestine is provided

${ }^{1}$ Hill Griffith. Trans. Ophth. Soc. of the U. K., XVII, I897, 220.

${ }^{2}$ L. Werner. Trans. Ophth. Soc. of the U. K., XXIII, I903, I93. 
with hooks. On gaining access to the eye it is converted into a cyst containing clear fluid in which hooklets may be found. The cyst consists of an outer laminated elastic layer and an inner parenchymatous layer containing granular matter, cells, etc.,

In this sterile state it may remain or it may be reproduced by the development of small cysts with scolices, suckers and hooklets (brood capsules). This is the only way by which reproduction has been recorded in the eye, but ectogenous or endogenous cyst-formation (daughter and grand daughter cysts) is met with elsewhere.

Cases of hydatids have been recorded affecting the vitreous, and also situated between the retina and choroid. The eyes inolved were removed for absolute glaucoma.

Filaria (thread worm).

The following worms have been found in the eye: Platyhelminthes, Cestodes, Sparganum Mansoni, Nemathelminthes, Filaria Bancrofti, Loa Loa, Filaria Inermis, Filari Oculi Humani, Onchoceriasis, Thelaziasis, and Ankylostomiasis.

Platyhelminthis.-Its primary seat is in the lung, but it may find its way into the iris and cause an inflammatory iritic tumour.

Filaria Inermis.-Specimens of this parasite have been removed from a cystic swelling in the conjunctiva of a native of the Balkan States.

Filaria Oculi Humani.-Filariæ are a common occurrence in the aqueous chamber of horses and oxen in India, and the presence of the epizoa in oxen, camels and turkeys has also been observed in Southern Europe and Northern Africa. In these animals the worms can be seen swimming about in the aqueous humor. In men they lie in the anterior chamber and give rise to iritis, diffuse keratitis and turbidity of the aqueous; they may be also found in the lens, vitreous and subretinal space and can be seen with the ophthalmoscope. In one case one was removed from the latter situation by a puncture with a keratome. 
Thelaziasis.-Filaria belonging to this group have been removed from the conjunctival fornix of a Chinese. They are hardly perceptible, small thread-like objects.

Ankylostomiasis. (Hook-worms). - There are two kinds namely, Ankylostomum duodenale and Necator americanus. The former is largely sub-tropical and the latter tropical. They produce intense anemia from loss of blood, and toxic poisoning. The eye symptoms to which they give rise are blurring of vision, diplopia, mydriasis, nightblindness, nystagmus, amblyopia and restriction of the fields. All these conditions may be explained as the result of the loss of blood or toxæmia. The retina is pale and frequently has small hemorrhages, and arterial pulsation similar to that met with in glaucoma is said to occur. The amblyopia is attributable to retrobulbar neuritis.

Sparganum Mansoni.-This worm in its larval form inhabits the urethra and has been found lying beneath the conjunctiva producing a tumour about the size of a small pea.

Nemathelminthes. Filariæ Bancrofti.-Occur in the Pacific Islands and the lesions attributed to them are edema, itching and a granular condition of the lids, and retinal hemorrhages.

Loa Loa or Filaria Oculi.-The fly which conveys the worm is the Chrysops dimidiata, it inoculates both the native and white people giving rise to what are known as Calabar swellings. The fly is yellow in colour and bites usually around the ankles without causing pain.

The worm has been compared in appearance to a piece of fishing gut, being round, firm, transparent and colourless, but it has minute bosses on its surface. The male measures from 25 to $30 \mathrm{~mm}$. long and $3 \mathrm{~mm}$. broad, the female being somewhat larger. ${ }^{1}$ The head end is furnished with a mouth without armature. There is no neck, though there is a sort of shoulder.I $5 \mathrm{~mm}$. behind the mouth. The tail end is sharply incurved and provided with five lateral

${ }^{1}$ Argyll Robertson. Trans. Ophth. Soc. of the U. K., XV, I895, I37. 
alæ, the three in front of the anus being very large. A characteristic feature of the worm is a number of round smooth hyaline cuticular protuberances upon its surface, which vary in number and are more numerous in the female. The worm is found in the skin of the eyelid and beneath the conjunctiva, it gives rise to great irritation which becomes intense when the worm is under the conjunctiva, but instant relief is obtained when the parasite goes into the deeper tissues. Installation of cocaine relieves the symptoms and there is reason to believe that sometimes it paralyses the worm. There is no definite case on record in which it has been found within the eyeball. The region around the eye is frequently affected, probably on account of the laxity of the tissue. The worm has been observed to travel from one eyelid to the other. It has been said that warmth will bring the worm to the surface.

The larval form of the parasite occurs freely in the blood in the later stages, more particularly in the day time, it produces a marked eosinophilia.

Insectivora may affect the eye in various ways:

I. By stinging; a mosquito or tick bite of the skin of the eyelids sometimes gives rise to extensive swelling.

2. By their irritating secretion; thus the great ant of Senegal has an acid secretion which when deposited in the conjunctiva excites an acute conjunctivitis.

3. By infesting the eyelashes. Pediculosis of the eyelashes is due to the pediculus pubis or crab louse. It is I $\mathrm{mm}$. long and I mm. broad, having an almost square body and six long legs and claws. The eggs or nits are firmly attached to the bases of the hairs. It differs from the head louse in being smaller and square in shape. The latter does not infest the eyelashes.

4. By burrowing beneath the epithelium. In San Domingo there is a variety of scabies which affects the lids.

Ocular Myiasis.-The principal flies which have been met with causing damage to the eye by the deposition of larvæ are the Musca or common house-fly, the Calliphora or 
Blue-bottle, the Lucilia or Green blue-bottle, the Chrysomyia or gold-fly from tropical America, the Cordylobia from Africa, the Estrus found in the nasal passages of sheep, the Hypoderma bovis, the Dermatobia or Macaw worm and the Sarcophagidæ, which are like blow-flies, viviparous, giving rise to small maggots at birth instead of eggs. Ocular Myiasis is most commonly met with in the Tropics, and relatively frequent in Russia. The condition varies considerably according to the species of insect and the number of eggs implanted. The Muscidæ, Estridæ and Sarcophagidæ producing the most severe symptoms. The emaciated and bedridden subjects are most frequently attacked. The fly striking against the eye deposits its eggs in the conjunctiva where they remain and hatch out. When the larvæ are born, they either remain in the conjunctiva or enter the eye.

(a) The extra ocular type may be very mild, all that can be seen on examination are several dark spots hidden in the deep folds of the conjunctiva which can be removed under cocaine, which paralyses the larvæ. Cases of this type have been reported as the Estrus ovis from Sicily, the Rhinœstrus purpureus from Russia. The screwworm larva (macellaria) is frequently found in India. Muscoid fly larvæ have also been found in Europe.

(b) The severe cases of extra ocular myiasis may produce a most horrible condition. The larvæ multiply and burrow in the conjunctival sac and lachrymal passages; some of the parasites eat their way into the surrounding structure so that the orbit may be nothing but a huge suppurating mass filled with crawling maggots. Most of these cases are due to the Muscidæ. Some rather less severe cases have been due to the Lucilia hominivorax. In the Argentine the larva of the Dermatobia cyanoventris has been found in tumours of the upper lid, and the larva of the larynetes has been removed from a scleral nodule.

(c) Intraocular myiasis. Cases which have been reported of this disease in Europe have nearly all been due 
to larvæ of the Hypoderma bovis, and have all occurred in children under 9 years of age. Some doubt exists as to how they enter the eye, whether directly through the sclera or via the blood stream, but the fact that the cases occur in children, where the sclera is soft is rather in favour of direct penetration of the eye. In one case the larvæ of a blow-fly, or Sarcophagous, was found in the anterior chamber giving rise to an intense irido-cyclitis with detachment of the retina. In another similar case the maggots were found beneath the retina.

6. By irritating hairs. Ophthalmia nodosa (see page $387)$.

7. Some flies in South America deposit an anterior horn in the cornea when they strike the eye.

8. By transference of discharge by flies. This is apparently a common method by which the infection of purulent ophthalmia and trachoma is carried in such countries as Egypt.

9. By transference of irritating pollen. In Cuba a fly lives on the euforbia ferox, the pollen of which, when transferred to the human conjunctival sac, causes an acute conjunctivitis with a vesicular eruption.

\section{THE DISEASES WHICH ARE PROBABLY DUE TO MICROÖRGANISMS}

Recent observations have shown that there are a considerable number of infections, which may be caused by organisms too small to be held back by filters, through which the smallest known bacteria cannot pass. The term filtrable virus has been applied to these. In some of these diseases minute globoid bodies, which are known as Chlamidozoa, have been discovered in the cells. They have been found in cases of trachoma, smallpox and molluscum contagiosum.

Trachoma.-Trachoma is a specific infective disease of the subepithelial tissue of the conjunctiva, characterised by 
infiltration and the formation of lymphoid follicles, which necrose. In the later stages of the disease there is a formation of fibrous tissue of variable amount; it may be so extensive as to cause obliteration of the conjunctival sac.

The term trachoma has been in use since the time of Hippocrates, and was formerly applied to any condition in

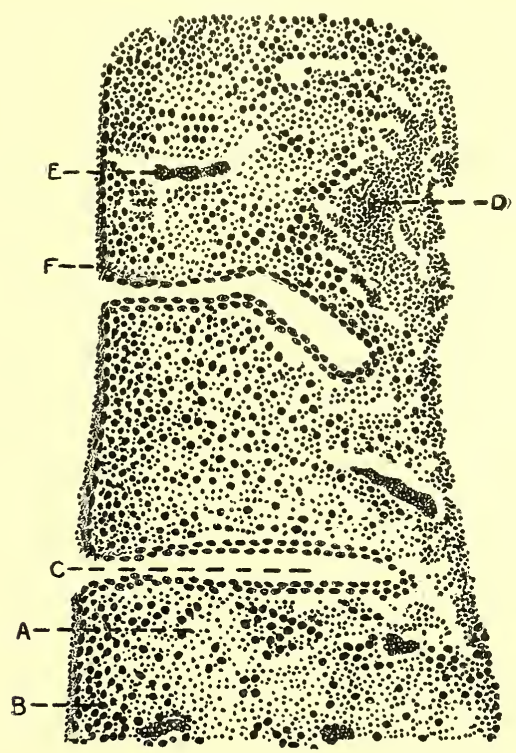

FIG. 257.- Section showing papillary formation in early trachoma. $A$, Papilla; $B$, plasma cells; $C$, crypt; $D$, portion of a trachomatous lymph follicle; $E$, blood-vessel; $F$, epithelium.

which there was an undue roughness of the inner surface of the eyelids. The specific disease to which it is now applied has only been differentiated from other inflammatory affections of the conjunctiva in comparatively recent times.

It has been proved by inoculating one patient with the discharge from another that the disease is directly contagious, and also that the different clinical forms which it presents are but different stages of the same disease.

So far no organism has been proved to be the cause of the disease. It has been attributed by different obser- 
vers to a coccus, a bacillus, and to ultra microscopic organisms. Bodies which it was thought might be the specific organisms were found in endothelial cells, but subsequently they proved to be fragments of cells formed by necrotic changes in the tissue.

More recently minute ovoid bodies known as Chlamidozoa, ${ }^{1}$ considerably smaller than any known cocci, have been found, by the use of Giemsa's stain, in the epithelial cells. They occur massed together near the nucleus in the form of a cap, but separated from it by a clear space. In the early stages they appear as fine granules in the chromatin of the cell, after a time they cause an extrusion of the plastin from the nucleus so that it envelopes the initial granules and forms the cap. Sometimes the nucleus is actually invaded by the granules. It has been claimed that they have been inoculated into the higher apes producing a mild form of the disease. That they are parasites is still doubtful; very probably they are merely thickenings of the chromatin filaments in the cytoplasm produced from degeneration of the cell, either by pyknosis or hyperchromatosis. This is further supported by the fact that they have been found in the epithelial cells of the normal urinary tract and conjunctiva.

The disease, beginning in either the upper or lower fornix, appears first in the form of gelatinous-looking round swellings or follicles, which become confluent, and then resemble grains of boiled sago or frog spawn. When large, these follicles easily rupture and their gelatinous contents is extruded.

The first sign of the disease in the tarsal conjunctiva is in the form of small, circular, pale, grey areas due to follicles embedded in the fibrous tissue; later these enlarge and form elevations on the surface which, like the follicles in the fornices may rupture.

\footnotetext{
${ }^{1}$ Halberstädter and Prowazek. Deusch. med. Wochenschrift, 1907, No. 32. Greeff. Lancet, April 17, 1907. Noguchi. Jour. Exp. Med. N. Y., XXII, 1915, 304.
} 
In association with the follicular developments there is always hyperemia of the blood-vessels and papillary formation, the amount of which varies considerably in different cases. Where it is excessive it is known as the papillary form of trachoma (Fig. 257). Where, on the other hand, the follicular formation is a more striking feature clinically than the papillary enlargement, the case is classed as the follicular variety.

In the later stages of the disease the subepithelial lymphoid tissue becomes replaced by newly formed fibrous tissue, which is seen as irregular white streaks on the inner surface of the lids; this is spoken of as the cicatricial stage. A band of fibrous tissue frequently forms along the line of the sulcus subtarsalis; it is termed Arlt's streak and the contraction caused by it is the commonest cause of entropion.

In some cases the infiltrate around the follicles undergoes hyaline degeneration and a peculiar waxy-like appearance is then seen in the conjunctiva, termed Stelwag's brawny edema.

In severe cases of trachoma the cornea is affected. It becomes opaque and vascular, pannus. The upper half is most frequently involved, a sharp line of demarcation separating the affected from the unaffected parts. In some cases the whole cornea is implicated.

The new blood-vessels in the cornea are derived from those of the conjunctiva, and are located in its superficial layers. In the early cases, where there is not much thickening and only slight vascularity, it is spoken of as pannus tenuis: where the vascularity is very marked, as pannus vasculosus. When the trachoma becomes cured the pannus as a rule disappears; in some cases, however, a permanent opacity and a few vessels are left; this is termed pannus siccus.

The amount of discharge in cases of trachoma varies considerably. In the disease which spread widely in Europe on the return of the troops from Egypt after the Napoleonic wars, there was much purulent discharge; it 
was doubtless due to mixed infection of trachoma and purulent ophthalmia. The two diseases are still prevalent in association with one another in Egypt and other Eastern countries.

Muco-purulent discharge may be present in trachoma but is not an essential feature, in some cases a mucoid secretion only being met with. Acute exacerbations may occur when the discharge is muco-purulent, it being at other times mucoid.

It would seem that trachoma is essentially a chronic disease, and that when these exacerbations occur there is a mixed infection, as pyogenic organisms are at these times always found in the discharge. The trachoma no doubt lowers the vitality of the tissue, and provides a means of entrance to the organisms by the rupture of the follicles.

Histology. ${ }^{1}$ - In the earliest stage of trachoma the epithelium shows little change, but directly any discharge appears it becomes infiltrated with leucocytes. These leucocytes, unlike those in the deeper tissue, are largely of the polynuclear variety; and no doubt their presence is due to pyogenic organisms growing in the epithelium, since without infection they do not usually appear in any number. As the disease advances the epithelial cells undergo increased mucoid change. Over the tops of the follicles they at times become almost entirely destroyed. In the crypts between the follicles, and in the folds produced by the swelling of the subepithelial tissue, they give rise to new pseudo-glands (see page 439). In long standing cases of trachoma, if there is any deficiency in lacrymal secretion, keratinisation of the epithelium may take place (see page 588 , secondary xerosis). This is specially liable to occur on the prominences produced by the newly formed fibrous tissue.

In the subepithelial tissue the changes are of two main types: (r) The formation of follicles; (2) Infiltration. They

${ }^{1}$ M. S. Mayou. Hunterian Lectures, 1905. 


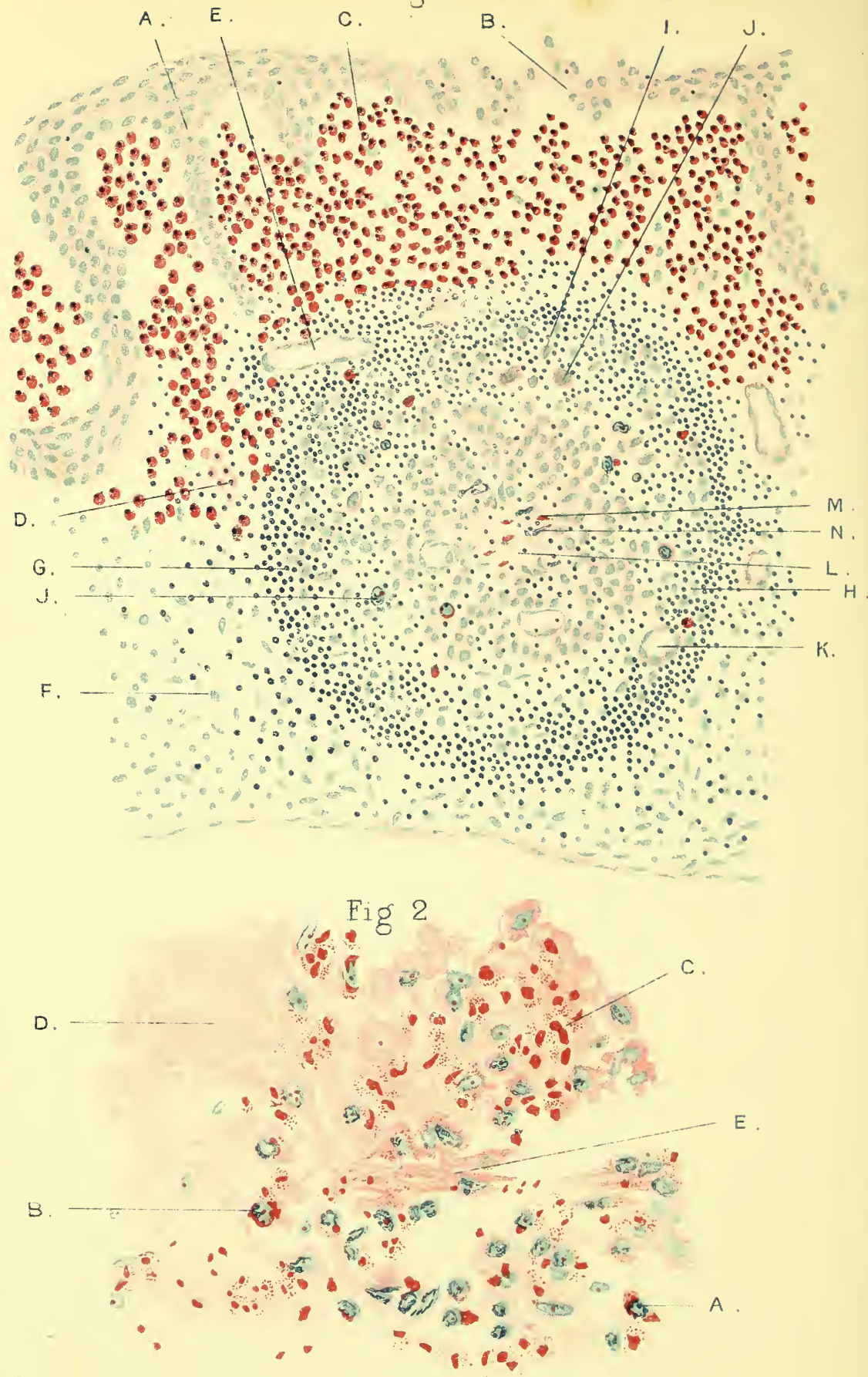

Fic. 1,-A fully-forned follicle infected with trachoma. A, Epithelium with papilla-like processes B, epithelium becoming thinned over the surface of the follicle; C, papilla packed with plasna iclls : D, deoenerating plasma cells; $\mathrm{E}$, blood-vessel ; F infiltration of sub-epithelial tissue ; G, outer "radzone "of mononuclear leucocytes (mainly lymplocytes); H, epithelioid-like cells (degenera:ing leucocytes): l, large mononuclear leucocytes; J, endothelial cell containing the coccoid bodies of Leber (clasmatocyte) ; $k$, vessel with proliferatıng endothelium, with a plasma cell in its neighbourhood; L, central area of broken-down leucocytes; $\mathrm{M}$, hyaline material; $\mathrm{N}$, connective tissue rell.

Fici, 2-Trachoma (Stelwag's brawny edema) showing the necrosis of the plasma cells in the infiltration. A, Plasma cell; B, plasma cell breaking up; $C$, hyaline material due to the breaking up of the cells; 1 ), fibrous tissue, staining slightly on account of the presence of hyaline; $\mathbb{E}$, young connective tissue fibre. (Pappenheim staining.) 采 obj. No. 4 eyepiece. 
always occur together, but one may be in excess of the other, producing the different clinical conditions previously described.

The follicles in trachoma differ considerably in structure from healthy follicles in lymphatic glands, and those met with in follicular formation due to other forms of conjunctivitis (Plate 2). They are usually found in the lymphoid layer, but may be more deeply situated. Their structure varies with their age.

In a newly infected trachoma follicle there is externally a single layer of somewhat elongated flattened cells, which appear to be of endothelial origin; their continuity is often much broken up, more so than in the follicles due to other forms of conjunctivitis. Within this external covering are other cells supported by an ill-defined reticulum. The outermost cells in the follicles are chiefly darkly staining lymphocytes. Toward the centre are a number of slightly larger cells, epithelioid in character, probably derived from the outer ones. They stain slightly, suggesting that degenerative changes have taken place in them due to the action of the toxin. Scattered in this central area there are also a few large endothelial cells, chiefly of the phagocytic variety.

Well-formed plasma cells are rarely found within a trachomatous follicle, probably because they tend to disintegrate rapidly in the presence of the toxin.

An older follicle (Plate 3, Fig. I) consists of a capsule of connective tissue considerably infiltrated with lymphocytes, especially if the disease be spreading. As a rule no regular endothelial lining to the capsule can be made out. The connective-tissue cells are mostly well formed, many of them showing signs of proliferation. Numerous mast cells are present. The amount of stroma in the follicle varies considerably with its age, but it is only in the old degenerated ones that it is at all a conspicuous feature, and consists of delicate fibres which proceed from the true connectivetissue cells. 
Numerous blood-vessels are found in the periphery of the stroma of the older follicles, they spread inward toward the centre as the follicle becomes organised.

The cells found in the older follicles, like those in the more recent ones, consist externally of darkly staining lymphocytes, within which again are larger more faintly staining cells of an epithelioid type. The central endothelial cells, however, have large oval nuclei and a quantity of cytoplasm which often contains fragments of necrotic cells.

Plasma cells are absent from the follicle, although they are occasionally found in the outer zones and in the neighbourhood of the vessels. They seem to disintegrate very quickly, and near the centre of the follicle rounded masses of hyaline material are sometimes found, which appear to be principally derived from the broken up cytoplasm of the plasma cells.

A trachoma follicle may finally become extruded, or undergo organisation and absorption.

Extrusion of the follicle may occur: $(a)$ As the result of operation. (b) From contraction of surrounding fibrous tissue.

(a) The results of extrusion of the follicle are seen in sections made from the conjunctiva after expression has been performed.

In sections through follicles shortly after expression the thick fibrous wall of the follicle encloses a space, which communicates with the surface of the conjunctiva. The wall of the follicle and the epithelium in the neighbourhood of the rupture are infiltrated with polynuclear leucocytes (spider cells), no doubt owing to the presence of septic organisms. Within the follicle are a number of blood-corpuscles, mononuclear leucocytes, plasma cells, etc.

Later fine bands of connective tissue stretch from one wall to the other, evidently derived from the proliferation of the connective-tissue cells of which they are composed. None of the original contents of the follicle remains, it 


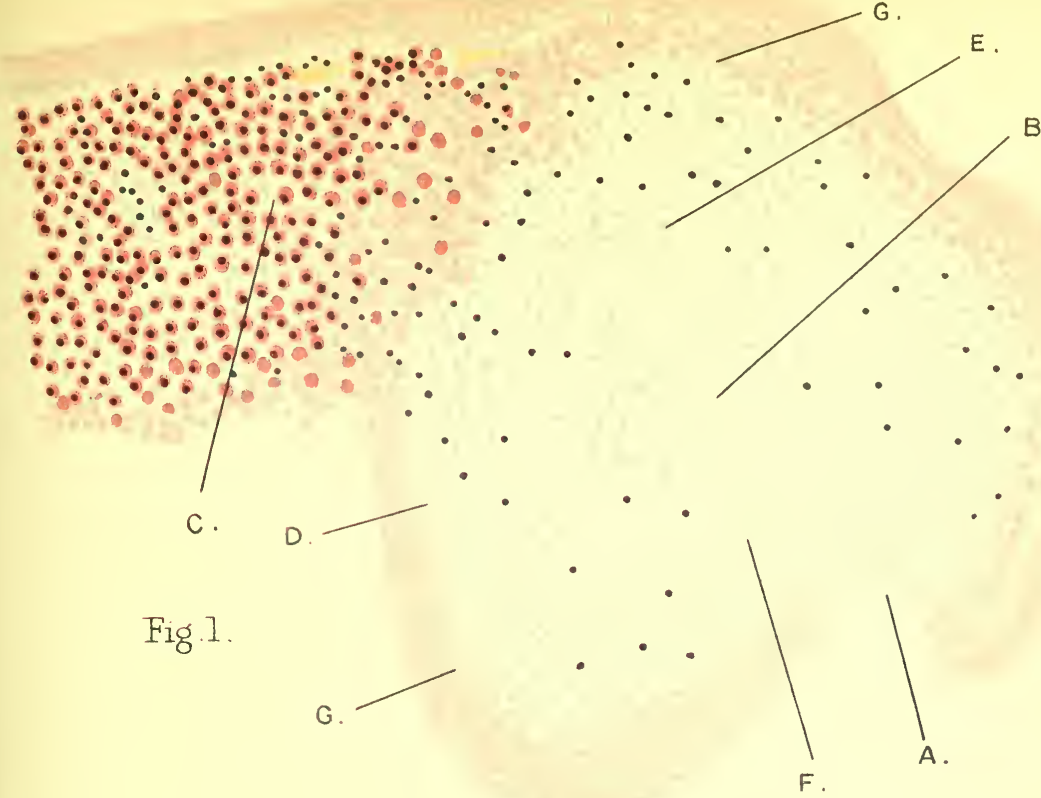

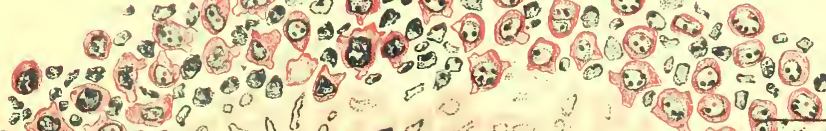

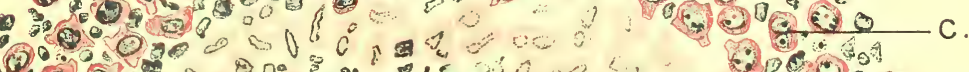
- 0 (1) 10 \% $0200 \%$

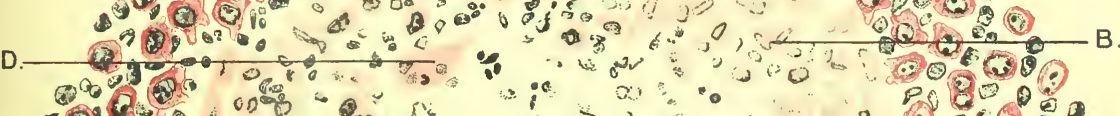

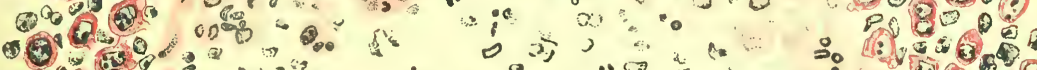
- (2) (C.

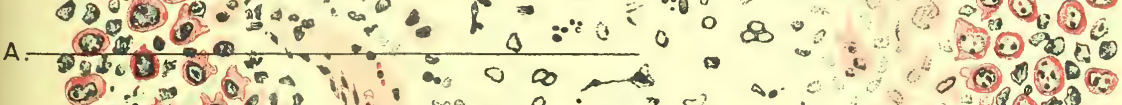

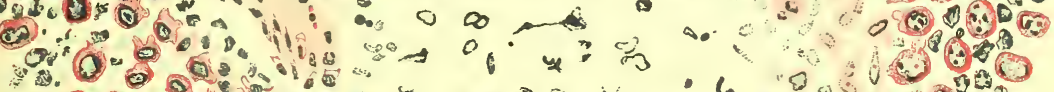

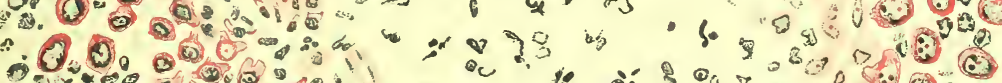

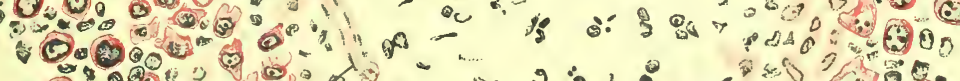

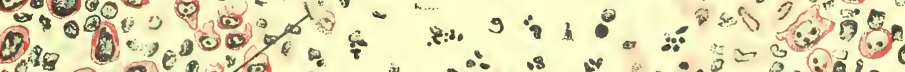

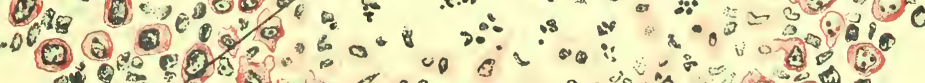

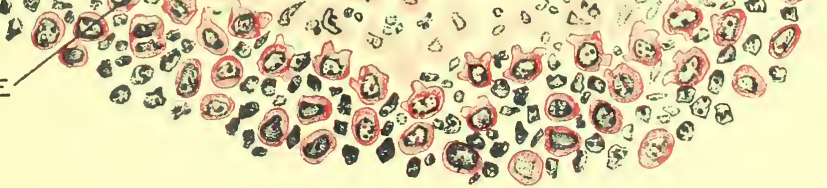

John Bale Sons \& Damelsson. Ltd $^{\text {td }}$

Fig. 2 .

Fig. 1.- Trachoma. Section through the bulbar conjunctiva and limbus, in a case of trachoma with severe pannus, showing a trachomatous follicle at the limbus. A, position where the limbus joins the cornea ; B, necrotic area in centre of the follicle; $\mathrm{C}$, well-formed plasma cells of the bulbar conjunctiva; $\mathrm{D}$, degenerating plasma cells around the follicle: E, epithelioid cells within the follicle; F, blood-vessels within the follicle. (Pappenheim staining.) th obj. No. 4 eyepiece.

FIG. 2. - An early deposit of tubercle in a lymphoid follicle showing necrosis of the plasma cells. A, Area of cellular necrosis; B, ring of degenerating plasma cells C, well-formed plasma cells; D, endothelial phagocytic cell; E, blood-vessel. (Pappenheim staining.) 1 obj. No. 8 eyepiece. 
Printed in Great Britain 
having either been thrown off or destroyed by the polynuclear leucocytes.

(b) As the result of contraction of the newly formed fibrous tissue around a follicle the contents is compressed and made to protrude. Between the follicle and the epithelium is a thin layer of plasma cells which do not develop into fibrous tissue; the epithelium overlying the follicle, from the friction of the lids and the pressure produced by its protrusion, becomes gradually thinned and ultimately the follicle ruptures, its contents being partly extruded. What remains becomes septic and is removed by the invading polynuclear leucocytes.

Absorption and Organisation.-There is no doubt that large numbers of the lymphocytes in the follicles find their way into the blood stream and lymphatics, since small venous radicles in the neighbourhood of the follicles are often found packed with these cells. When pyogenic infection of the whole conjunctiva takes place the polymorphonuclear leucocytes are at first chiefly found in the walls of the follicles, and do not readily invade it. Later, when its cells degenerate, the polynuclear cells enter the follicle and either remove its contents by phagocytosis, or filling the cavity rupture through the surface and discharge its contents. No doubt the beneficial effect of copper sulphate, jequirity, or an attack of gonorrheal ophthalmia, on trachoma is due to the polymorphonuclear leucocytosis attacking the disease, not only in the follicle but also in the infiltration.

The formation of fibrous tissue around and within the follicle has already been described.

The infiltration, like the follicles, is due to the disease setting up a chronic inflammation in the tissue. Unlike other forms of chronic conjunctivitis, it is by no means limited to the layer lying directly beneath the epithelium, but spreads to the deeper layers of the tissue of the conjunctiva and varies in character according to its depth. 
Situated directly beneath the epithelium and in the newly formed papillæ are masses of plasma cells. Comparatively few are seen between the epithelial cells, notwithstanding which numbers are found in the discharge. Doubtless many make their way through breaks in the epithelium, indeed, the best way to obtain these cells in the discharge is by rubbing a cover-glass over the surface of the palpebral conjunctiva. In this superficial layer of plasma cells a number of new blood-vessels form, the endothelial cells of which are undergoing active proliferation. Somewhat deeper the infiltration consists chiefly of lymphocytes, a few large proliferating endothelial cells, and a few scattered plasma cells, which become increased in cases of long standing and undergo disintegration. Deepest of all in long standing cases is a quantity of dense fibrous tissue, which tends to grow up into the more superficial parts around the follicles. It is extremely rich in mast cells.

In long standing trachoma the upper palpebral conjunctiva is sometimes converted into a pale gelatinous-looking tissue - brawny edema (Stelwag). It is usually associated with much scarring and represents one of the final changes in the infiltration.

The epithelium overlying it is thickened and often keratinised. The surface of the conjunctiva in the affected region is quite smooth.

The subepithelial layer consists entirely of infiltration much separated by bands of fibrous tissue; practically no follicular formation is left. The infiltration consists of a few mononuclear cells, and a large number of plasma cells in all stages of degeneration, their cytoplasm being much broken up, and converted into a hyaline material (Plate 3 , Fig. 2). Sometimes this hyaline material may go on to secondary amyloid and calcareous changes.

There is no doubt that the changes at the limbus, and the subsequent pannus, are due to a true infection of that region with trachoma, since follicles are found in them which show typical necrosis (Plate 4, Fig I). As the disease 
spreads to the cornea the cellular infiltration is at first superficial to Bowman's membrane (Fig. 258), but this structure after a time becomes destroyed and the substantia propria invaded. In the thick fleshy pannus follicles are formed. Small ulcers are not infrequent in connection with the pannus, and polymorphonuclear leucocytes are then present.

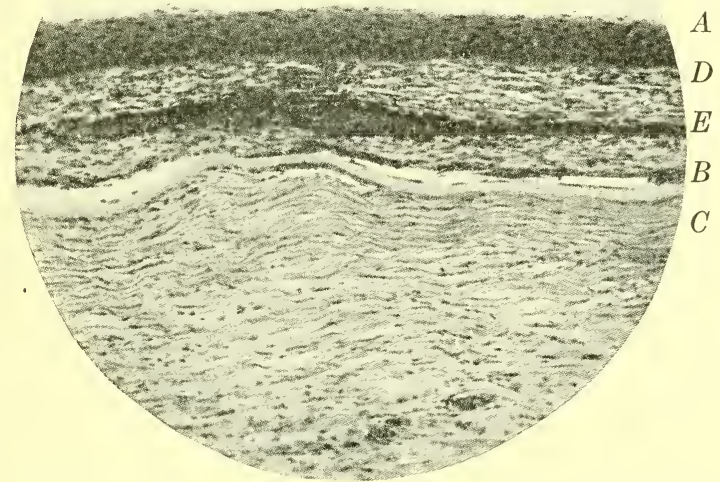

FIG. 258.- Shows a section through the front part of a cornea affected with trachomatous pannus. $A$, Epithelium; $B$, Bowman's membrane as yet undestroyed; $C$, substantia propria; $D$, cellular infiltration and fibrous tissue forming the pannus; $E$, blood-vessel.

Vernal catarrh is a disease which has been so named because its symptoms make their appearance, or become accentuated, during' warm weather, while they subside or disappear altogether during the cold seasons.

The patient complains of irritation, photophobia, and lacrymation. Broad flat papillæ, which have been compared to cobblestones, form on the tarsal conjunctiva, and the surface of the membrane presents a characteristic milky appearance. In cases of long standing the limbus is also involved, becoming thickened and nodular. It encroaches on the periphery of the cornea and may cause that structure to appear considerably reduced in size. 
There is but little conjunctival discharge. A thin layer of mucoid secretion usually covers the surface of the palpebral conjunctiva, which becomes increased if it is subjected to the least irritation. In this discharge there are immense numbers of eosinophile cells-a point of considerable

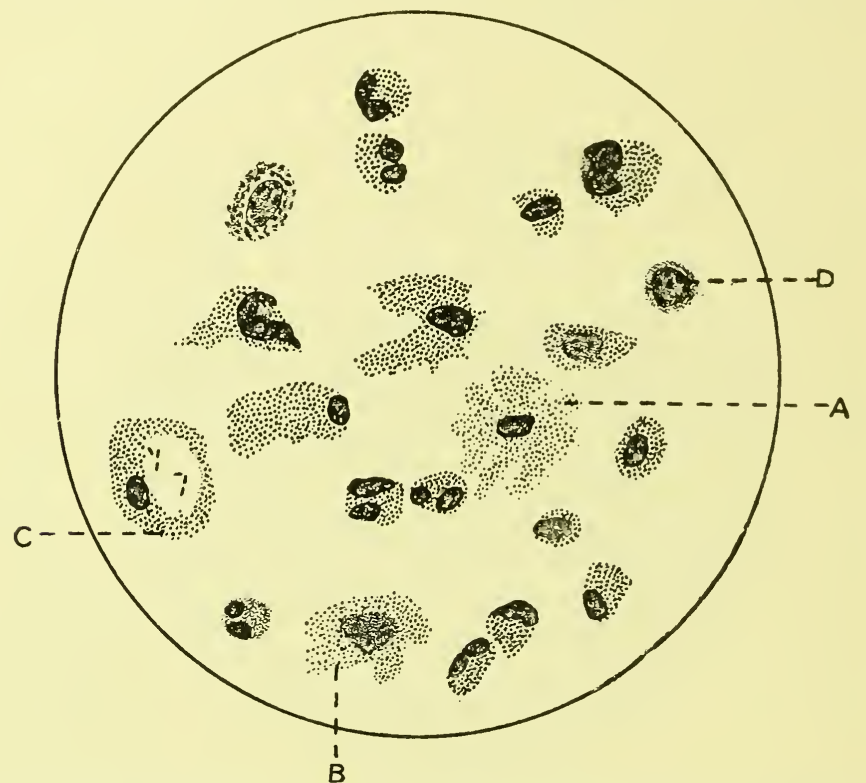

FIG. 259.-Discharge from a case of vernal catarrh showing eosinophiles. $A$, $B, C$, Eosinophiles; $D$, lymphocyte.

importance in the diagnosis of the affection. They are best demonstrated by staining with Leishmann's eosin-methylene-blue compound. The whole microscopic field under a $1 / 12$ objective may be found to consist exclusively of these cells, which are both polymorphonuclear and mononuclear (Fig. 259). They sometimes rupture, the granules in them becoming scattered.

There is no evidence that this disease is infective, and so far no microörganisms to which it may be due have been discovered.

Microscopical examination of the affected tissue shows the condition to be one of enormous papillary overgrowth of the conjunctiva. 
The epithelium covering the tops of the papillæ is much thickened, and appears to send finger-like processes down in the depressions between them. Comparatively little mucoid change takes place in the cells composing these processes.

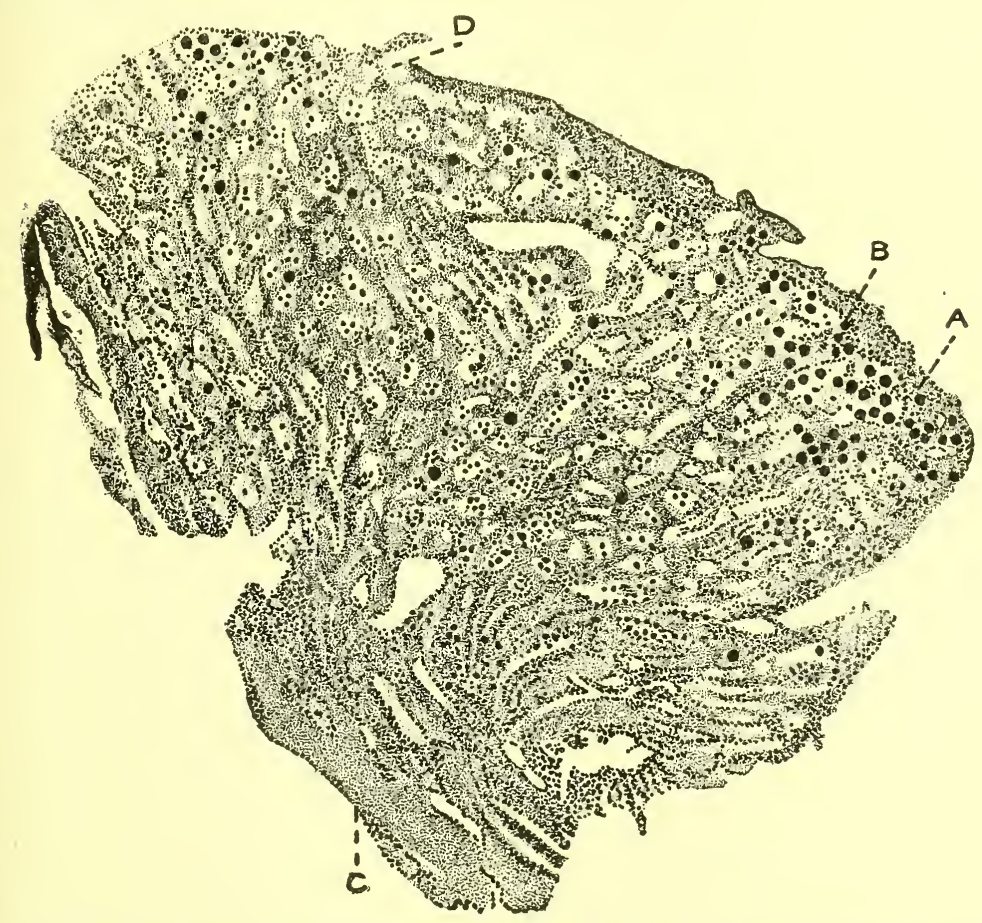

FIG. 260.-Shows a section of one of the papillæ from a case of vernal catarrh. $A$, Epithelium; $B$, eosinophiles; $D$, erosions of the epithelium; $C$, fibrous tissue.

The papillæ, which are very hard, consist of a quantity of newly formed dense connective tissue. The cells found in it are the ordinary connective-tissue cells, leucocytes, and plasma cells.

Some of the leucocytes are eosinophiles, but they are proportionately less numerous in the tissue than in the discharge. They are derived from the blood, in which they are also slightly increased in number, and are seen principally in the small vessels lying beneath the epithelium, 
often making their way through the walls and through breaks in the surface epithelium or between the cells (Fig. 260). The appearance of such large quantities in the discharge is due to their great power of ameboid movement.

Plasma cells are present in considerable numbers in the tissue, most of them showing signs of necrosis, due probably

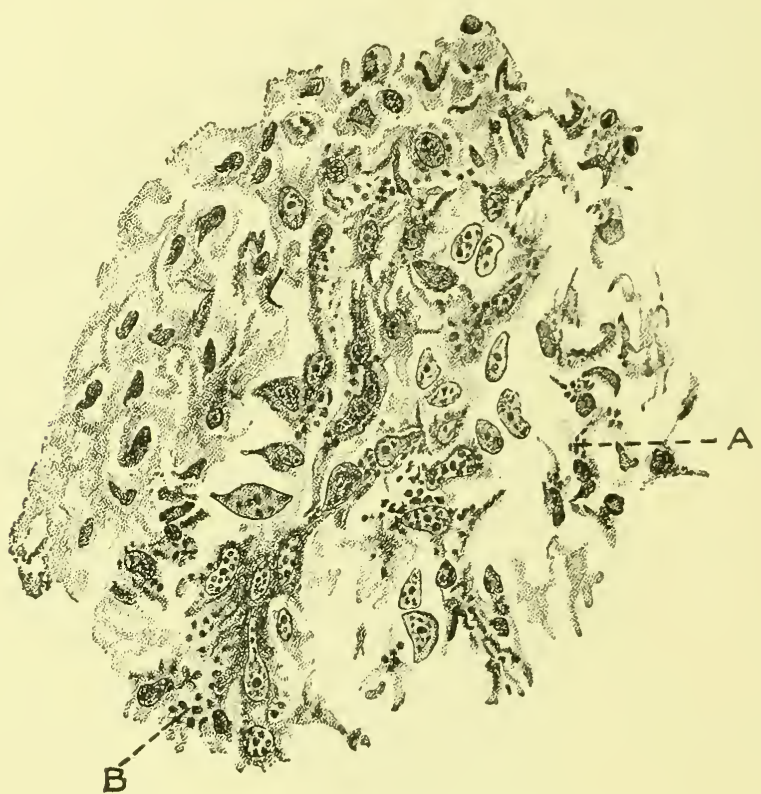

FIG. 26I.-Vernal catarrh, showing the necrosis in the plasma cells. $A$, Plasma cell showing fragmentation of its cytoplasm; $B$, hyaline material free in the tissue. (Pappenheim staining.)

to the presence of some toxin. Necrosis of the plasma cells results in the formation of a hyaline material (Fig. 26I), which is the cause of the milky appearance of the conjunctiva. ${ }^{1}$

Sympathetic Ophthalmitis.-Sympathetic ophthalmitis is an inflammatory granulomatous infiltration of the uveal tract started by a lesion of one eye, and after a variable interval affecting the second eye. The first eye affected is

${ }^{1}$ Mayou, M. S. Hunterian Lectures, 1905. 
termed the exciting or primary eye, and the other, which is subsequently involved, the sympathising or secondary eye.

The lesion which starts the affection in the exciting eye is almost always a perforating wound in which the uveal tract is involved. Wounds of the ciliary region are more frequently followed by the disease than those situated in other parts of the globe. It occasionally follows an opera-

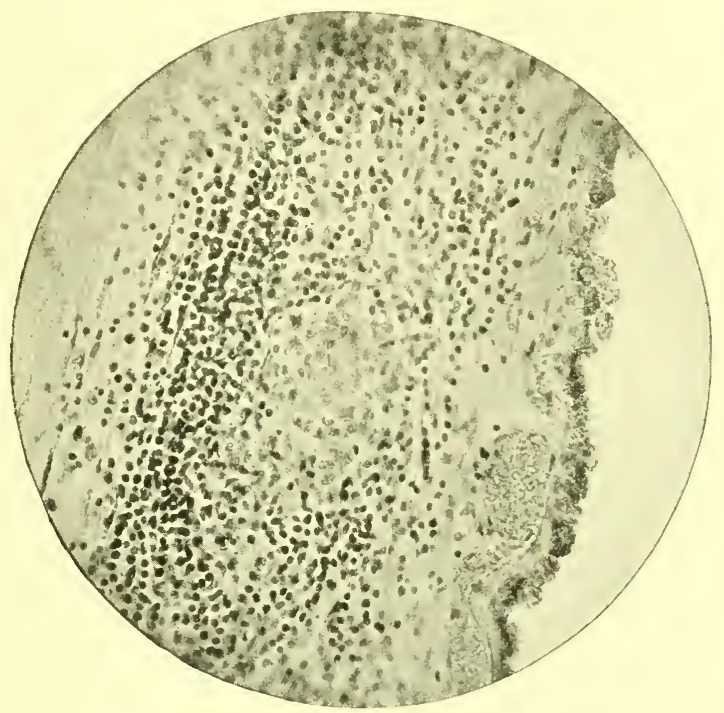

FIG. 262.--Section through a patch of proliferative choroiditis in an eye which excited sympathetic ophthalmitis after extraction of cataract. Reproduced from a photomicrograph taken by E. Collier Green.

tion such as extraction of cataract, or iridectomy for glaucoma. It has also been known to follow sub-conjunctival ruptures of the sclerotic, perforating ulcers of the cornea and sarcoma of the choroid.

The length of time which usually elapses between the receipt of injury in the exciting eye, and the appearance of the disease in the sympathising eye, is from four to eight weeks. It has, however, been met with as early as two weeks, and in some cases has not made its appearance until after an interval of twenty years. When there has been a very long interval, inquiry will frequently elicit a history of 
some fresh attack of inflammation, or irritability, in the exciting eye, shortly before the onset of the sympathetic disease. The inflammation in the sympathising eye may commence after the exciting eye has been removed, but not later than five weeks after its removal. The disease may be acute or chronic, or acute in one eye and chronic in the other.

The histological character of the inflammation in both the exciting and sympathising eye is the same, and involves all three divisions of the uveal tract-iris, ciliary body, and choroid. Scattered throughout it are numerous nodules composed of mononuclear lymphocytes, plasma cells, epithelioid cells and giant cells (Fig. 262). ${ }^{1}$ In some cases these nodules are discrete and separated from one another by healthy tracts of tissue. In others the nodules are in continuity with one another giving rise to a nearly uniform thickening of the uveal tract. The nodules are histologically very similar in appearance to those of commencing tubercle, but they do not, as in that affection, tend to necrose or caseate; nor do they, like tubercular nodules, invade structures outside the uveal tract. Together with these nodules there is, in sympathetic ophthalmitis, much exudate of a serous or plastic character. The clinical manifestations of the affection are similar to those of other forms of serous or plastic inflammation of the uveal tract, which have already been described (see page $46 \mathrm{I}$ ).

The fact that the disease seems always to result from a perforating lesion of the exciting eye suggests that the cause of the inflammation is ectogenous. Microörganisms have been found in eyes which have excited sympathetic ophthalmitis, but so far no specific microörganism has been discovered. In some exciting eyes, which after excision have been most carefully examined, no microörganism could be detected.

The mode of transference of the cause of the inflammation from one eye to the other is probably by the blood

${ }^{1}$ E. Fuchs. Archiv. für Ophth., LXI, 1905, 2, 365. 
stream. Hematogenous changes are produced, there being an increased mononuclear leucocytosis, but as yet sufficient investigation of the blood in these cases has not been carried out.

The staphylococcus (see page 496) is the common cause of ocular infection after penetrating wounds, and it has been found in cultivations taken from the exciting eye in some cases of sympathetic disease. This organism seems to have the power of circulating in the blood without producing much general change; it may possibly, therefore, be the cause of sympathetic ophthalmitis. The reason that the second eye is affected, rather than the other tissues of the body, is that the organism has a preference for a similar soil as that on which it originally grew.

The sensitisation of the tissue of the other eye may also be due to an anaphylactic condition of the uveal tract. In support of this theory, it has been shown that if a mixture of serum and uveal tract substance is injected into the blood stream, hemorrhages into the iris and choroid occur. It is probably due to anaphylaxis that so many of the diseases of the uveal tract are bilateral.

Suppurating eyes do not give rise to sympathetic ophthalmitis, probably because sufficient antibodies are produced in the serum, due to the severity of the inflammation, to protect the blood stream from infection by an organism of a comparatively low virulence. 


\section{CHAPTER VII}

\section{DEGENERATIONS}

In the degeneration of a tissue it becomes transformed into material of a lower chemical composition, i.e., less remote from inorganic matter. Some tissues of the eye undergo physiological degeneration, that is a degeneration which goes on throughout life and forms part of the normal routine of the cells concerned. Thus the epithelial cells of cornea are constantly proliferating, shrinking and being cast off, and some of the epithelial cells of the conjunctiva are constantly swelling from imbibition, and rupturing.

Degeneration of a tissue may be regarded as pathological when it serves no useful purpose and occurs prematurely.

The commonest cause of degeneration is malnutrition. The tissues of the eye are all nourished by the tissue fluids which are derived from the blood; whatever interferes with their circulation will cause a defect in the tissue metabolism which will be followed by degeneration of its cells, what may be termed circulatory degeneration.

If the cutting off of the nutrition is sudden and complete necrotic changes result in all the cells of the part (gangrene). When the degenerative process takes place slowly the cells gradually disintegrate and become absorbed (atrophy). The cells first affected are those which are most highly specialised and require most tissue fluid for their nutrition, such as the retinal ganglion cells. The parts last affected are the supporting structures, such as the sclerotic, which require but a small amount of nutriment and therefore rarely degenerate.

The way in which degeneration manifests itself in the cells depends on the osmotic properties of their walls. 
There is either a loss of fluid from the cell with a considerable condensation of the cytoplasm, the granules and nucleus staining more distinctly, or an imbibition of water by the cell causing it to swell up, lose its granules, and finally burst. After disintegration of the cell the débris may be absorbed or remain in the tissues; if the latter takes place, the colloid material may subsequently undergo hyaline, amyloid or calcarious change.

For the maintenance of normal nutrition it is essential, not only that there should be an adequate circulation of the tissue fluids, but also that they should contain the materials necessary for the normal metabolic activity of the cells. With a perfectly free circulation, if one of the vitamines be absent, or one of the ductless glands is not secreting sufficient of an essential hormone, degeneration of tissue occurs which may be termed deficiency degeneration.

Certain substances may find their way into the tissue fluids which have a selective action on the cells of some tissue of the eye, causing them to lose their vitality and degenerate. Such degeneration is called toxic degeneration: the poison may be derived from without (exogenous), as for example tobacco, and quinine; or from within the body itself (endogenous), the outcome of some faulty metabolism.

Involution and degeneration are normal processes in living tissues, just as much as evolution and development. In some tissues of the eye degeneration is frequently met with in the later years of life which is termed senile degeneration, e.g., arcus senilis in the cornea, presbyopia and senile cataract in the lens, and senile degeneration of the pigment epithelium of the retina.

Just as sometimes we meet with precociousness in development, so sometimes we meet with precociousness in degeneration. A degeneration due to a premature failure in , the vitality of the cells affected is termed abiotrophy. The characteristics of these abiotrophies are, that they arise apart from any inflammation or disturbance in nutrient 
supply, that they are always bilateral and usually hereditary.

Under this heading have been grouped; hereditary post-natal cataract; hereditary ocular palsies; retinitis pigmentosa; symmetrical macular pigmentary degeneration; amaurotic family idiocy; Leber's optic atrophy; Doyne's family choroiditis; nodular and lattice-like degeneration of the cornea.

The degenerations which take place in the tissues of the eyeball and its appendages will here be divided into those occurring in parts derived from epiblast and those derived from mesoblast.

The degenerations in structures derived from epiblast may be further divided into those of (I) cuticular epiblast; (2) lenticular epiblast; (3) neural epiblast.

\section{CUTICULAR EPIBLASTIC DEGENERATIONS}

It is characteristic of cuticular epithelium that its cells continue to proliferate throughout life. Mitosis occurs chiefly in the basal layers and the new cells as they form gradually get pressed forward toward the surface, where they undergo degenerative changes and are ultimately cast off. In some situations they undergo keratinisation and in others break down and form a secretion.

In the process of keratinisation the surface is dry, the cells as they are pressed forward toward it gradually lose their watery consistency, becoming first denticulated (prickle cells) and afterward flattened. The granules in the cytoplasm become more marked and composed of keratohyalin. As the loss of water progresses the condensation of the cytoplasm leads to its transformation into keratin, by that time the nucleus of the cell has undergone such changes that it is no longer recognisable. If these dried cells, as they reach the surface, instead of being cast off remain adherent, a condition of hyperkeratosis is produced. 
When an epithelial surface is kept continually moist keratinisation does not occur, the cells are either thrown off from the surface or elaborated into a secretion. If the latter takes place, granules form in the cytoplasm of the cells which are the precursors of the secretion. As they accumulate the cell enlarges and its nucleus becomes pushed more and more to one side. The cell may then either break down and form the secretion or discharge its contents without being destroyed.

The nutrition of cuticular epithelium is maintained by lymph which passes into it by a process of osmosis from the underlying tissue. In laminated epithelium there are delicate intercellular channels passing outward from the basal to the central and superficial layers.

Pathological degenerations of the cuticular epiblast may be grouped under the following headings: (a) Atrophy; (b) edema and vesiculisation; (c) keratinisation and hyperkeratosis; (d) mucoid, fatty, hyaline and calcareous changes.

(a) Atrophy of Epithelium.-The skin of eyelids in elderly people becomes atrophied, assuming a thin, shiny, silky appearance due to a decrease in number of the prickle cells, and flattening out of papillæ. It is also often darkened in colour from an accumulation of pigment in the basal cells.

Atrophy of the epithelium of the cornea, with diminution in both the number and size of the cells, occurs over cicatrices in it, especially when they are the seat of calcareous deposits. ${ }^{1}$ The superficial flat cells lose their nuclei and become thin scales, the middle polygonal cells are changed into flat ones, which lie directly on the cylindrical basal cells. Later on these latter also decrease in size, become flat, and in extreme atrophy entirely disappear. Atrophy of the epithelium exposes the underlying fibrous tissue to the risk of invasion by bacteria. It is in this way that the destructive form of ulceration occurs in old leukomata which

${ }^{1}$ E. Fuchs. Trans. Ophth. Soc. of the U. K., XXII, 1902, 25. 
is termed atheromatous ulceration or sequestrating cicatricial keratitis.

(b) Edema, Vesiculisation and Desquamation.-Edema of the corneal epithelium is met with in glaucoma, iridocyclitis, and keratitis. It gives rise to a superficial haze which presents a stippled appearance on magnification. It is often associated with small vesicles forming little rounded elevations on the surface which, after rupturing, leave behind shallow depressions. Sometimes when a vesicle bursts a small clear dark spot is left in the surrounding haze, clearly showing that the opacity is limited to the epithelium. Small drops of fluid collect first between the basement cells and later also in the more anterior layers. The accumulation of fluid in the interspaces between the cells in the central layers causes the intercellular bridges, which stretch from one cell to another, to become more pronounced so that they resemble the prickle cells of the skin. If the edema extends, the intercellular bridges give way and the spaces open into one another, small vesicles being formed; these are common in advanced cases of glaucoma. The cells themselves may become swollen by imbibition and have vacuoles form in them. Sometimes they burst on the surface and cause slight irregularities in it. In the deeper layers the swollen cells may be changed in places into a nearly homogeneous mass. The fluid effused between the cells loosens their coherence and favours the occurrence of desquamation. Single cells alone may become separated or whole layers of cells.

For further description of vesicle-formation and socalled filamentary keratitis, see page 289 .

(c) Keratinisation and Hyperkeratosis.-The epithelium of the cornea and conjunctiva under normal conditions does not become keratinised. This is due to its being kept constantly moist by secretions, and to the protecting influence afforded by the eyelids.

The surface cells of the cornea though flattened do not lose their nuclei or have keratohyaline form in them; 
they become rubbed off by the friction of the margins of the eyelids before any such changes occur.

In late life the superficial epithelial cells of the ocular conjunctiva, opposite the palpebral aperture, may show a few granules of keratohyaline, especially when it becomes raised up by the formation of a pinguecula.

When from some arrest of secretion, or displacement of the eyeball or eyelids, the surface of the conjunctiva or cornea ceases to be kept moist, keratinisation occurs, which clinically is termed xerosis. It is met with under the following conditions (Fig. 263):

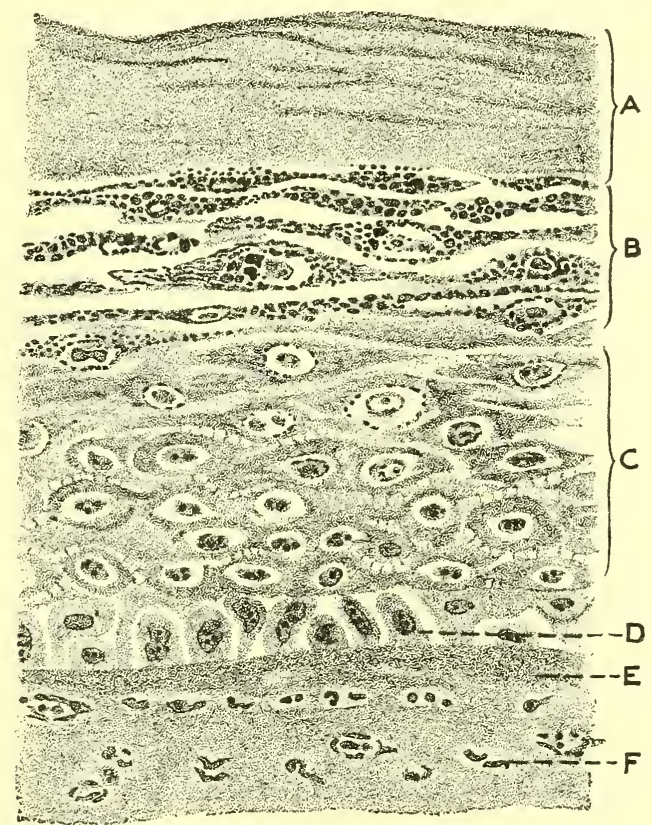

FIG. 263.- Section through the conjunctiva in a case of xerosis. $A$, Keratinised layer; $B$, layer of cells with keratohyaline granules. $C$, Prickle cells; $D$, basement cell layer; $E$, basement membrane; $F$, sub-epithelial tissue.

I. When there has been extensive fibrous-tissue formation in the conjunctiva cutting off the secretion of the glands opening into it, as in connection with trachoma and pemphigus. 
2. In ectropion of the eyelid the exposed portion of the conjunctiva or cornea, under the desiccating influence of the atmosphere, tends to assume epidermoid characters. Not only does keratinisation take place but the epithelium extends down in processes into the subjacent tissue, giving the appearance of papillary formation (Fig. 264).

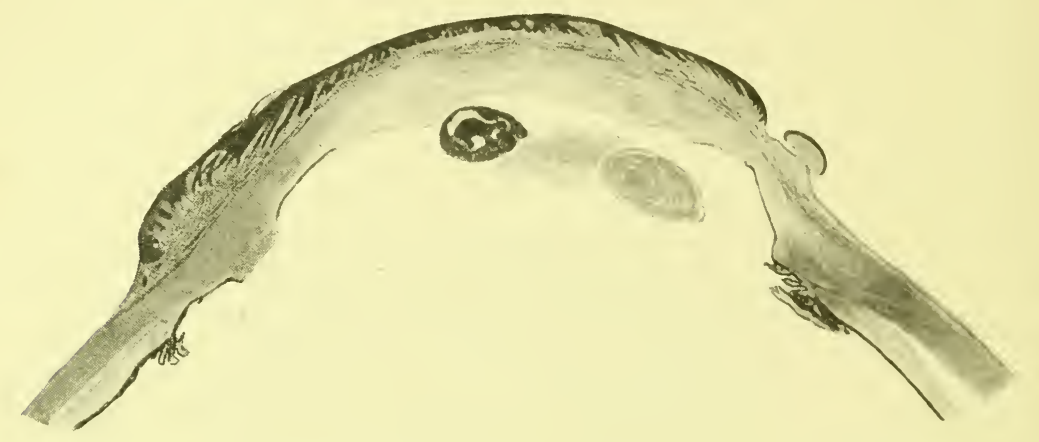

FIG. 264.- Section through the front part of the eye of a patient aged nineteen, who for many years had had a shrunken dry conjunctiva. The cornea was staphylomatous and he was unable to close the lids over the front of the eye. The epithelium, which is keratinised at the surface, is shown dipping down in the form of numerous finger-like processes into the deeper tissue. Case recorded by Treacher Collins, Trans. Ophth. Soc. of the U. K., X, I 890, 62 .

3. In cases of anterior staphyloma, where the bulging cornea protrudes through the palpebral fissure and the eyelids fail to close over it, so that it is not lubricated with moisture, keratinisation and often hyperkeratosis take place, a dry crust or horn-like structure being formed on its surface.

4. In epithelial new growths on the surface of the globe keratinisation is always present.

5. In the asthenic stage of some general diseases, the conjunctival secretions are diminished and winking is abolished. The patient lies in an apathetic condition with the eyes rolled up and the eyelids only partially closed. The lower part of the cornea is left exposed in the palpebral fissure and the epithelium overlying it becomes dry and keratinised. Ulceration of the cornea in such a condition is not an uncommon sequela. 
6. In marasmic children, and in adults who have become emacited from exhausting diseases, such as dysentery or from want of food in times of famine, a xerotic condition of the cornea and conjunctiva is met with. This is frequently a precursor of a rapidly destructive form of ulceration known as keratomalacia.

A milder form of xerosis of the conjunctiva occurs in association with night blindness and is known as hemeralopic xerosis. It occurs mostly in children in this country, but is met with also in adults in the tropics. Two factors seem necessary for its production; some defect in general nutrition and exposure to bright reflected light.

It is the ocular conjunctiva opposite the palpebral aperture on each side of the cornea which becomes affected. Triangular dry patches are formed where the mucoid secretion ceases, and the cells become keratinised. The surface tension of such patches is raised, and the oily secretion of the Meibomian glands collects on them in the form of a white foam in which the so-called xerosis bacilli (see page 520), are always to be found. These bacilli do not, however, appear to play any part in the causation of the disease but are saprophytic in character. They may be transferred from the maternal vagina to the child's eyes at birth.

Experimentally xerophthalmia has been produced in animals fed on diets deficient in the fat soluble vitamin $\mathrm{A}$, rapid cure taking place when the proper fat diet is restored. It seems, therefore, most probable that the xerophthalmia occurring in children, as above described, is a deficiency degeneration due to the want of vitamin $\mathrm{A}$. This vitamin is contained in the green parts of plants; from cows eating grass it passes into their milk, being soluble in fatty material, and is therefore present in butter but absent in many of its substitutes, like margarine, which are prepared at a high temperature. A diet deficient in fresh milk, butter and green vegetables is likely to favour the development of xerophthalmia. 
As it is the most exposed part of the ocular conjunctiva which is first affected, it seems probable that the desiccating influence of the air plays some part in its production. The night-blindness is also probably due to exposure, being best accounted for by a failure in the reformation of the visual purple which has been bleached by bright light. It is a prolongation of the temporary condition which is experienced normally on passing from a bright light into a dark place. Possibly the fat soluble vitamin is essential for the manufacture and secretion of visual purple, as well as the nutrition of the conjunctival epithelium. A diet containing liver has for generations been a popular remedy for night-blindness of this description in Eastern countries, and has been tried with success under medical supervision in the prisons in India. Cod-liver oil, which has been shown to contain the fat-soluble vitamin $\mathrm{A}$, has also proved very efficacious in the treatment of hemeralopia and xerophthalmia.

(d) Mucoid, Fatty, and Hyaline Changes.-Fatty changes in the epithelium of the cornea and conjunctiva are but seldom met with. In xerosis a few granules are found present on staining with osmic acid or with Sudan III.

The mucoid degeneration of the epithelial cells of the conjunctiva, which to a limited extent is a normal condition, becomes enormously increased in the various hyperemic and inflammatory conditions of that membrane. It is especially marked in diplobacillary conjunctivitis, the discharge in which affection is mainly composed of mucus so formed.

In the tubular epithelial recesses, or false glands, which become formed in the palpebral conjunctiva where papillary formation has taken place, mucoid and hyaline degenerative products frequently accumulate. They form concretions which are seen clinically as little yellow spots on the inner surface of the lids.

Microscopically these concretions are composed of a homogeneous or slightly granular, laminated material. They 
do not contain lithia or give any amyloid staining reactions. They are apparently composed of a hyaline material, the product partly of the degenerated epithelial cells and partly of degenerate leucocytes, which have made their way in through the epithelium. After they have existed for some time calcareous salts may become deposited in them.

\section{LENTICULAR EPIBLASTIC DEGENERATIONS}

Opacity of the lens, or cataract, is due to degenerative changes. A primary inflammation of the lens or phakitis cannot occur, though secondary invasion by inflammatory cells from surrounding parts may take place, when the capsule has been perforated.

Cataracts may be classified as follows according to the region in which the opacity first appears: (a) capsular; (b) subscapsular; (c) equatorial, and $(d)$ nuclear or perinuclear. Such a classification helps to bring out the different ways in which the degenerative changes in the lens may arise.

In capsular cataract the opacity is mainly due to a thickening of the epithelial cells lining the capsule. In subcapsular cataract a degeneration of the capsule cells allows diffusion of unaltered aqueous humour into the cortical lens fibres, which then break up and become opaque. In equatorial cataract the lens fibres formed laterally undergo premature sclerosis, spaces are formed between them in which albuminous globules collect. Nuclear and perinuclear cataracts originate in early life, the degenerative changes which occur involve the whole lens, later new healthy lens fibres are laid on and clear cortical substance is formed.

A cataract commencing in one region will often spread to others, so that in an advanced cataract, it may be impossible to say either clinically ur pathologically, in which of the above divisions it should be included. In some forms of cataract it is still uncertain which is the exact starting-point 
of the affection, and an inclusion of them specifically under one or other of the above headings can only be provisional.

(a) Capsular Cataract.-The hyaline capsule of the lens itself does not become opaque; so-called capsular cataracts are due to thickenings of the cells which line it. Normally these cells form a single layer extending round the anterior capsule to a little behind the equator. Their proliferative activity is to some extent controlled by the intracapsular tension, when it becomes lowered through a wound or from breaking down of some of the lens fibres, an increased mitosis of the cells takes place and several layers instead of a single row are produced. In the later stages of many cataracts the intracapsular tension becomes lowered, capsular cataract is, therefore, frequently found associated with other forms, and is usually present in hypermature senile cataract where the cortex has become liquefied.

The capsule cells may also be stimulated to proliferate by some toxic agent, or having been killed by some toxin, the unaffected cells in the neighbourhood may proliferate to make good the defect.

Most capsular cataracts are secondary cataracts, i.e., they follow on some disease of the surrounding parts. The commonest form of capsular cataract is an anterior polar cataract, which is usually secondary to ulceration of the cornea.

A more widely spread capsular cataract occurs in connection with iritis where, as the result of the inflammation, the iris becomes adherent to the lens capsule.

The changes taking place in the proliferated cells of the capsule, which constitute a capsular cataract, are the same whether it is located at the anterior pole or elsewhere; a description of them, therefore, in connection with anterior polar cataract will suffice.

Anterior polar cataract most frequently occurs as a sequela of ulceration of the cornea in infancy, not necessarily a perforating ulcer. In later life anterior polar cataracts are met with, dating from infancy and giving a history of 
having had ophthalmia neonatorum, but in which no opacity of the cornea or only a very faint nebula is to be found.

Anterior polar cataracts also occur as congenital defects (see page 50) apart from any signs of inflammation; the clinical and histological appearances of these antenatal opacities are the same as those in the postnatal cases.

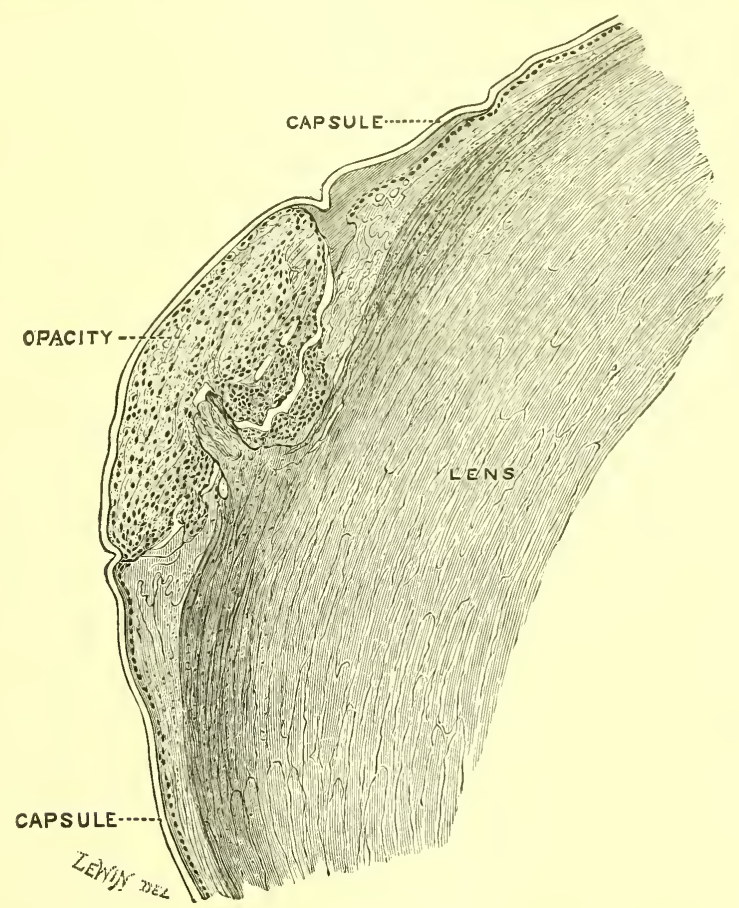

FIG. 265.- Section through an anterior polar cataract of less than six weeks' formation. The changes shown, which are entirely beneath the capsule, consist of proliferation of the capsule cells and degeneration of the cortical lens fibres.

Anterior polar cataracts vary in size and shape, they do not extend over the whole of the pupillary area, and may be less than $\mathrm{I} \mathrm{mm}$. in diameter. Sometimes they form a flat white patch which is not raised above the level of the surrounding capsule, and at others a pyramidal-shaped mass which protrudes forward from the surface of the lens; hence the term "pyramidal cataracts." 
In cases where an anterior polar cataract has existed for several years, a second opacity at some little depth in the lens substance is sometimes seen lying under the one at the anterior pole, and separated from it by clear lens substance.

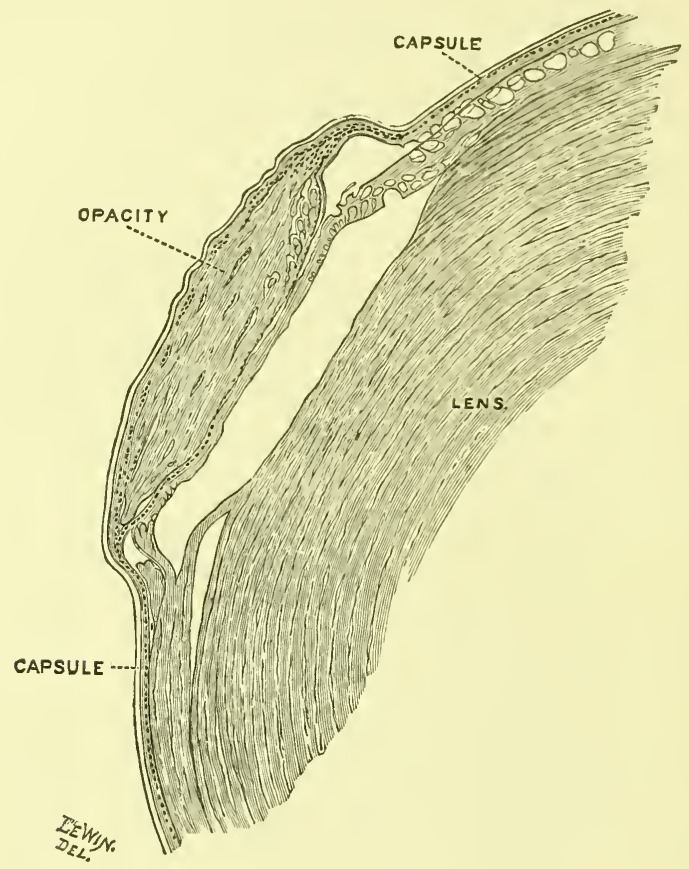

FIG. 266.- Section through an anterior polar cataract of seven months' formation. The mass of capsule cells has become converted into condensed laminated tissue. A layer of cells is shown beneath this new-formed tissue continuous with $t$ hose lining the capsule elsewhere.

Occasionally the separation is incomplete, the two opacities remaining united by a narrow central opaque band so that they present the appearance of a collar stud.

The changes which produce anterior polar opacities are situated within the capsule and are of two descriptions: degenerative changes in the subcapsular cortical lens fibres at the anterior pole, and proliferation of the cells lining the capsule. The degenerate cortical fibres become broken up into irregularly shaped hyaline masses and a depression is formed (Fig. 265). This depression so 
exactly corresponds in shape and extent to the mass formed by the proliferated cells, that there can be no doubt it is due to a change taking place during life and not merely the result of the hardening reagent. The mass of proliferating cells may simply fill up the depression, but more frequently it raises up the lens capsule into a conical projection above the level of the surrounding part.

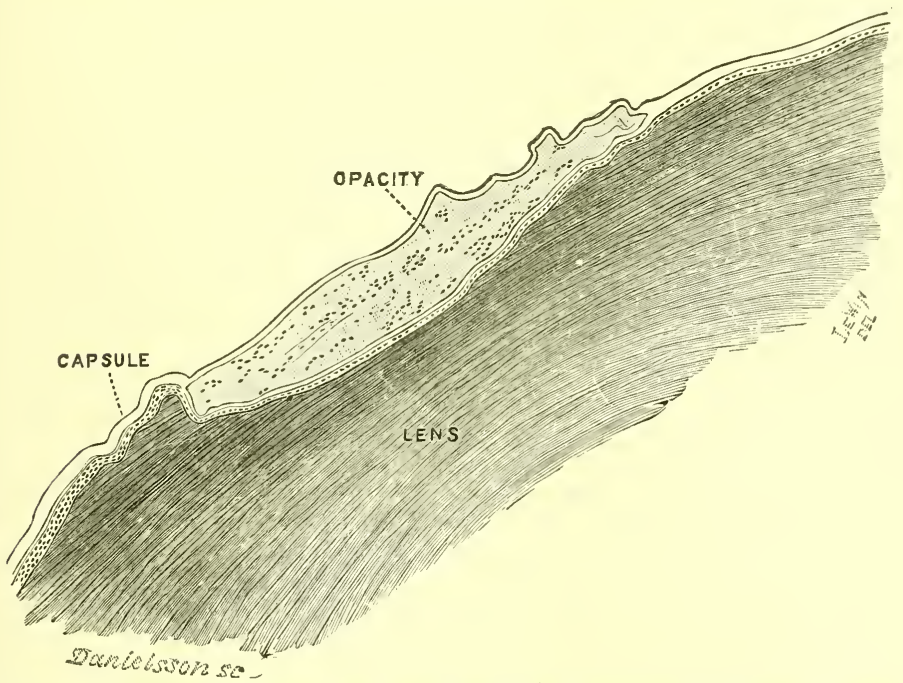

FIG. 267.- Section through an anterior polar cataract of eleven years' formation. A complete layer of hyaline capsule, lined by cells continuous with those lining the capsule elsewhere, is shown behind the opacity, and a hyaline layer in front of it.

In course of time the newly formed capsular cells which are at first polygonal become flattened out, many of them losing their nuclei, so that a condensed laminated mass of tissue is developed, studded here and there with elongated cells which have retained their nuclei. In long standing cases calcareous salts become deposited in the laminated mass.

For a time, the mass formed by the capsule cells which have proliferated and the degenerated cortical fibres, lie in contact. Later on they become separated, by the cells of the capsule at the margins of the affected area multiply- 
ing and insinuating themselves between, so that a complete single row of cells becomes formed separating the two structures (Fig. 266), from which in course of years a new hyaline layer, like that of the capsule, becomes secreted ${ }^{1}$ (Figs. 267 and 268). The laminated mass is then enclosed entirely by hyaline capsule, the normal capsule at its margin

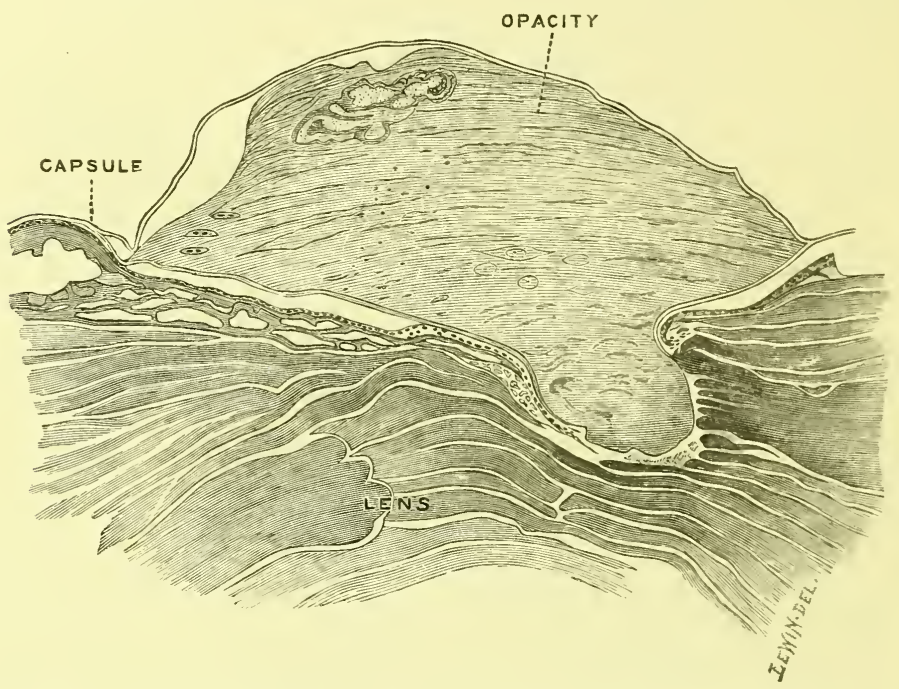

FIG. 268.- Section through an anterior polar cataract of twenty-one years' formation. A hyaline layer of capsule lined by cells is shown behind the opacity and a hyaline layer of equal thickness in front of it.

appearing to divide into two parts, one going in front of it and the other behind (Figs. 267,268 ). This shutting off of an anterior polar opacity by a layer of capsule from the rest of the lens, accounts for the easy way in which it can sometimes be detached in the course of an operation.

Anterior polar cataract usually forms early in life when the lens is comparatively small; as it enlarges new cortical fibres are laid on, some of which extend forwards between the laminated mass formed from the capsule cells and the area of degenerated fibres. It is in this way that two opacities overlying one another, and entirely or partially separate. are produced (Fig. 269).

1 Treacher Collins. Trans. Ophth. Soc. of the U. K., XII, I 892, 89. 
The cause of the proliferation of the capsule cells in anterior polar cataract has not yet been definitely determined. Some writers have attributed it to the diffusion through the capsule of some toxic material, produced in connection with the accompanying ulceration of the cornea. It has been suggested that the toxin may stimulate the cells directly to proliferate, or that by first destroying the

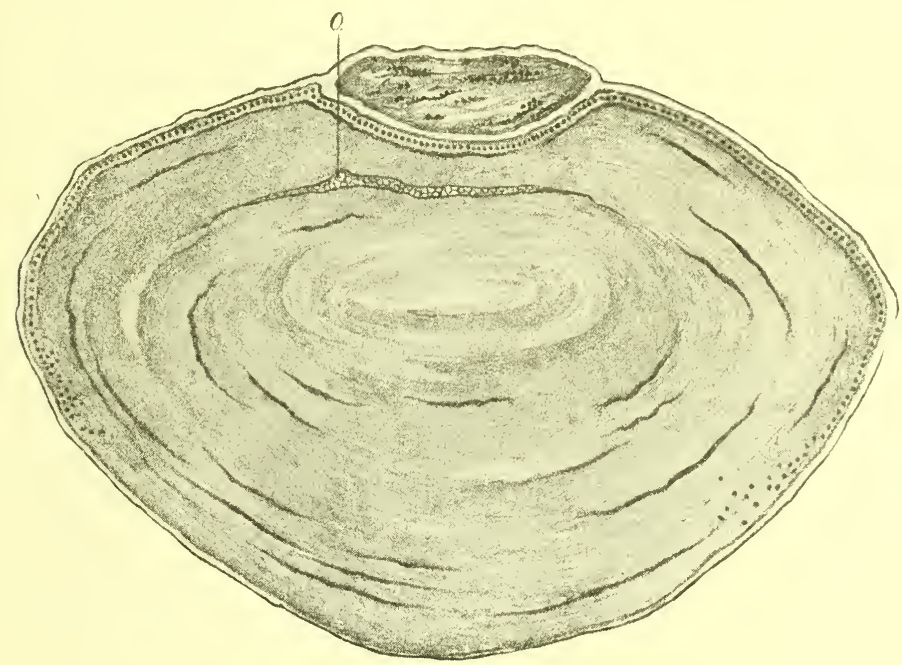

FIG. 269.--Section through a lens with an anterior polar opacity of eleven years' formation, in which there was a second opacity a little deeper in the lens. The opacity formed from proliferation of the capsule cells is shown enclosed between two layers of hyaline capsule. The second opacity due to degeneration of the cortical lens fibres is shown at $O$. New cortical lens fibres have grown in and separated the two opacities.

cells over the affected area, cause those bordering it to multiply in an irregular fashion and occupy the denuded spot. ${ }^{1}$ The difficulties in accepting an explanation based on the presence of a toxin are. first, that it does not account for the congenital anterior polar cataracts, which arise apart from any inflammation, and in cases with perfectly clear corneæ. Secondly, that in cases of nonperforating ulcers of the cornea, in which the inflammation is confined to its anterior layers, it seems unlikely that a

${ }^{1}$ E. Fuchs. Annales d'oculis, CL, I9I3. 
toxin could affect the cell of the anterior lens capsule without exciting any changes in the deep layers of the cornea, or in the endothelium of Descemet's membrane.

Another theory attributes the changes to contact of the lens at its anterior pole with the back of cornea. In an infant's eye the anterior chamber is exceedingly shallow, so that very little swelling of the cornea suffices to bring it and

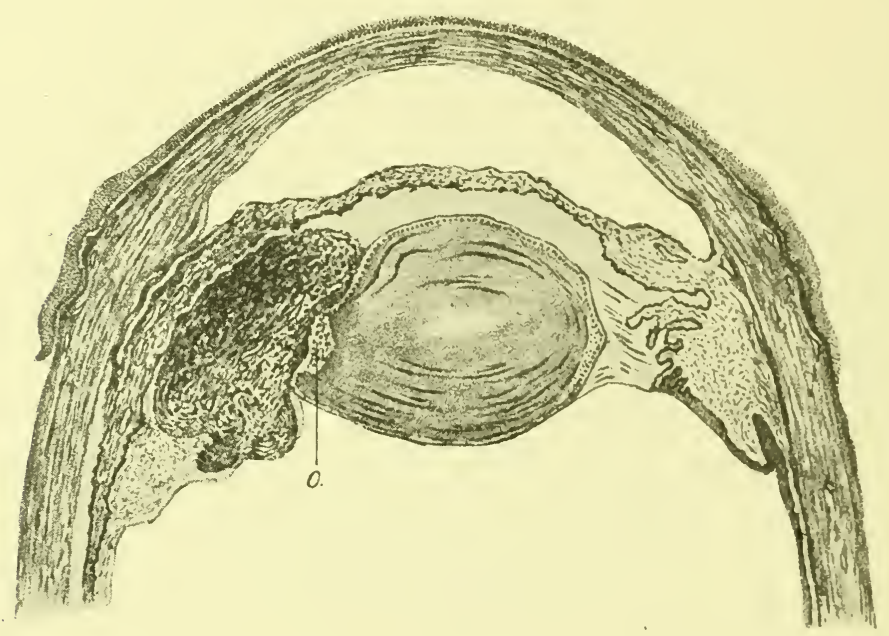

FIG. 270.- Section through the front half of an eye with a melanotic sarcoma of the ciliary body. It has pressed on the side of the lens and caused there a capsular cataract, $O$, similar in appearance to an anterior polar cataract.

the lens in contact in the pupillary area. Where the two come in contact there is obstruction to the passage of nutrient fluid through the capsule, and the lens fibres in that locality degenerate and break down. This breaking down of the lens fibres decreases the intracapsular tension in their vicinity, and so allows of a more rapid proliferation of the capsular cells which forms the main mass of the opacity.

A prolongation of the contact of lens and cornea during fetal life, after the fibro-vascular sheath of the lens had disappeared, would account for congenital anterior polar cataract in accordance with this theory. By it also might 
be explained the occurrence ${ }^{1}$ of capsular cataract at the periphery of the lens where a sarcoma of the ciliary body has come into apposition with it (Fig. 270). It does not, however, seem consistent with this theory that a lens dislocated into the anterior chamber may lie in contact with the cornea for a long time without becoming opaque or showing any capsular changes.

(b) Subcapsular Cataract.-The nutrition of the lens, after the disappearance of its vascular sheath in fetal life, is maintained by endosmosis, through the capsule, of the surrounding fluid. Experiments seem to show that fluid never passes directly from the vitreous humour into the lens. It is from the aqueous that it obtains its nutrient material.

The composition of the lens, and of the fluid surrounding it, differ considerably in the living eye, so that osmosis through its capsule does not take place according to the ordinary law of diffusion. The cells lining the capsule evidently have some selective power, as after their death the ordinary law of diffusion does assert itself, the composition of the lens and aqueous then tending to approximate.

In describing traumatic cataract (see page 383) it has been pointed out that globulin, the albuminous substance of which the lens is mainly composed, is soluble in a weak solution of chloride of sodium such as the aqueous humour. When the lens capsule is perforated and the aqueous gains entrance to the lens fibres they swell, break up, and become dissolved.

The capsular cells check the passage of the sodium chloride into the lens, and so protect it from the disintegrating action of the unaltered aqueous.

When degenerative changes take place in the capsule cells their selective power is destroyed, the unaltered aqueous humour with its chloride of sodium, can then diffuse through the capsule and cause destruction of the lens fibres. These destructive changes commence immediately beneath

${ }^{1}$ Treacher Collins. Trans. Ophth. Soc. of the U. K., XVIII, I 898, I24. 
the capsule, and very frequently, as in traumatic cataracts, the opacity is first noticed at the posterior pole.

The imbibition of fluid causes a condition which may be well described as edema of the lens. The cortical fibres become separated from one another and from the capsule, the spaces between them being filled with coagula so that the

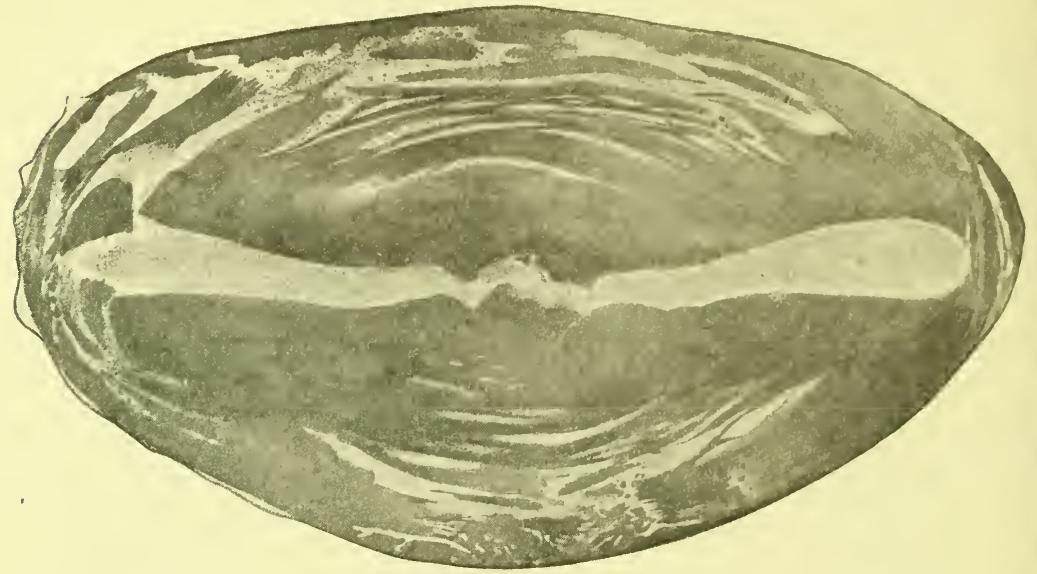

FIG. 27I.- Section through a cataractous lens from an eye with a melanotic sarcoma of the choroid. Note the band of degeneration passing across the nucleus, composed of numerous small globules of coagula. There are spaces between the cortical fibres and some degeneration of the lens fibres bounding them filled with a similar material. Case recorded by Treacher Collins, Trans. Ophth. Soc. of the U. K., X, I890, I 45 .

lens is enlarged (Fig. 272). Subsequently the fibres become broken up and dissolved, and the whole lens becomes opaque. In course of time these lenses tend to shrink, and in rare instances the whole of lens substance may enter into solution and become absorbed from the intact capsule. In specimens which have been examined, where such spontaneous absorption of cataract through the closed capsule has taken place, the capsular epithelium was found to be absent.

In a few cases of secondary cataract a band of irregularly shaped globules has been found extending transversely from side to side across the centre of a sclerosed nucleus (Fig. 27 I). 
Degenerative changes in the capsular epithelium may be brought about in a variety of ways. Friction of the outer surface of the lens capsule, as in the operation for maturation of immature cataract, has been found to produce changes in the capsule cells resulting in the for-

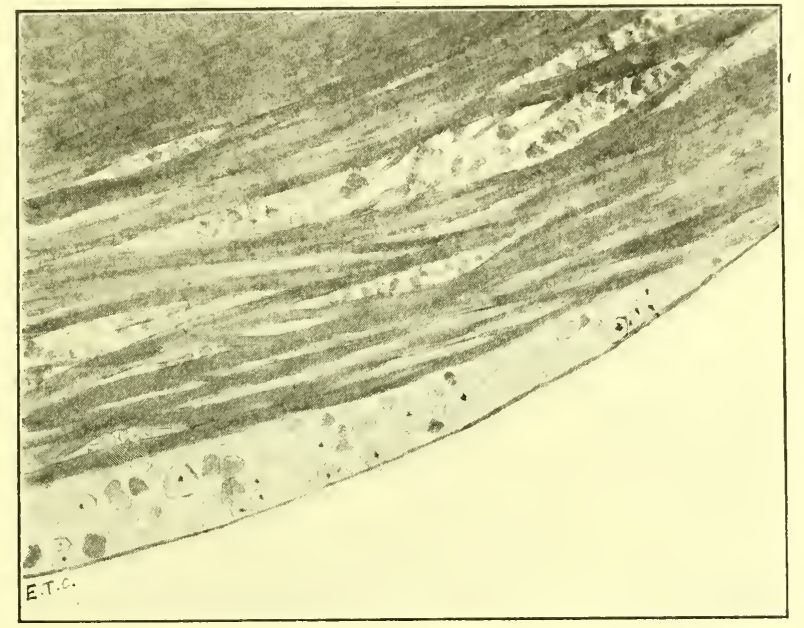

FIG. 272.- Section through the posterior part of a sub-capsular cataract, showing separation of the cortical fibres from the capsule and one another, the spaces being filled with albuminous coagula, "Morganian globules." Between the capsule and the lens fibres there are some vesicular or bladder cells.

mation of vacuoles. Subsequently the cortical subcapsular fibres become acted upon by the aqueous humour, which can then gain access to them, and the cataract becomes complete.

Death of the capsular epithelium, and cataract, together with hyperemia of the ciliary body and iris, have been found in rabbits who have had the sparks of six combined Leyden jars directed against their heads in the supraorbital region. ${ }^{1}$

In the cataracts which have been observed to follow lightning injuries or the accidental exposure to the effects of powerful electric discharges, destruction of the capsular cells from electrolytic action probably occurs.

${ }^{1}$ K. Kiribuchi. Arch. f. Ophth., B. L., I900, I, I. 
Diabetic Cataract.-Cortical subcapsular cataract may arise at any age in patients suffering from diabetes. It usually occurs in both eyes either simultaneously or with an interval of short duration. As a rule the amount of sugar in the urine in those affected with cataract is large, but the patients are not necessarily much debilitated or emaciated.

Some cataracts which are met with in elderly patients with glycosuria, do not begin as subcapsular opacities, and are indistinguishable from the ordinary senile form. The cataracts occurring in association with diabetes in early life are always subcapsular. The lens swells, the opacity progresses rapidly, and the whole of the cortex becomes converted into a milky white material. The nuclei of the cells of the capsule are found, microscopically, to stain unequally or to become replaced by vacuoles.

In a few cases where the opacity in the lens has been of only short duration it has been observed to decrease in amount, or even disappear altogether, as the result of improvement taking place in the patient's general condition.

Experiments have shown that the immersion of the human lens in 5 per cent. solution of sugar will, as the result of dehydration, cause it to become opaque. Diabetic cataract cannot, however, be attributed to such a change because the aqueous and vitreous humours never contain as much as 5 per cent. of sugar. In a patient ${ }^{1}$ who had 8 per cent. sugar in the urine the aqueous was found to contain only 0.5 per cent. Five per cent. of sugar in the aqueous would, moreover, produce opacity of the cornea as well as of the lens.

Small traces of sugar have been discovered in clear lenses in diabetic patients, and diabetic cataracts have been found not to contain any sugar, so the opacity does not seem to be due to any direct action of sugar on the lens fibres.

In patients with diabetes changes have frequently been observed in the pigment epithelial cells on the back of the iris, they become swollen, vacuolated and depigmented.

\footnotetext{
${ }^{1}$ Deutschmann. Archiv. f. Ophth., XXIII, I877, 3, I43.
} 
It has been suggested that some of the nutrient fluid of the lens may be derived from the iris, and that degenerative changes in the pigment epithelium interferes with its formation, so that cataractous changes in the lens ensue.

A more probable explanation of the degenerative changes in the pigment epithelium of the iris and the cells of the lens capsule is, that they are both due to some defective metabolism from a deficiency of insulin. The cataract being produced by the removal of the protective influence which the capsular epithelium affords.

\section{Cataracts Secondary to Disturbance in the Circulation} of the Ciliary Body.- The fluid which nourishes the lens is secreted by the ciliary body. Excision of the ciliary body and iris in a rabbit's eye is followed by opacity of the lens. Cataract is also formed after section of the long posterior ciliary arteries or ligature of the vortex veins, it is, however, of slower formation after ligature of the latter than after section of the former. In the cataracts thus produced the epithelial cells lining the capsule are found degenerate and vacuolated, and the opacity commences in the subcapsular fibres. In association with diseases of the eye in which the circulation of the ciliary body becomes interfered with, secondary cataracts form similar in character to those produced experimentally in the ways above mentioned.

Cyclitis, especially the quiet form, which manifests itself by bleaching of the stroma of the iris and keratitis punctata, is frequently accompanied by opacity of the lens. The changes commence, as in traumatic cataract where the anterior capsule is perforated, at the posterior pole, probably from some separation of the sectors of the lens in that situation; from that region they extend forwards to the anterior subcapsular fibres. Like all cataracts commencing subcapsularly, the lens when extracted comes away very completely.

In absolute glaucoma, as the result of the intraocular pressure, the ciliary processes usually become atrophied; 
the supply of nutrient fluid to the lens becoming thereby deficient and secondary cataract frequently ensues.

Diseases of the choroid affecting its blood-vessels, such as choroiditis, retinitis pigmentosa and high myopia, may involve the long posterior ciliary arteries, and by interfering with the nourishment of the ciliary body gives rise to secondary cataract.

Naphthalin Cataract.-Cataract has been produced in rabbits by the introduction into their circulation of naphthalin. ${ }^{1}$ Fine streaks appear first near the equator of the lens, these coalesce, and in the course of ten days the entire subcapsular cortex becomes opaque. The lens swells quickly, fluid collecting between the capsule and lens fibres; the capsular epithelium shows signs of degeneration. The cortical fibres first disintegrate and later those of the nucleus; finally crystals of phosphate of lime become deposited.

The changes in the eye produced by the ingestion of naphthalin are not limited to the lens, occurring also in the ciliary body, choroid, vitreous, and retina. There is congestion of the uveal tract accompanied by hemorrhages, edema of the retina, and later detachment. A high degree of edema of the ciliary body always precedes the formation of cataract. The aqueous humour contains an abnormal amount of albumin from the first appearance of the cataract, the amount increasing in quantity with the extension of the opacity.

The opacity in the lens is not due to the direct action of the naphthalin on the lens fibres or on the capsular epithelium. Large quantities of it have been introduced into the anterior chamber without causing any alteration in them, and lenses immersed in naphthalin and olive oil do not become opaque. The primary change is probably in the ciliary body which secretes the aqueous humour from which the lens gains its nutrient material.

${ }^{1}$ H. Magnus. Arch. f. Ophth. XXXVI, I890, 4, I50. 
A defect in the nutrition of capsule cells allows of the permeation of aqueous humour into the subcapsular fibres, and the formation of the subcapsular cataract.

Senile Degeneration of Capsular Epithelium.-The cells lining the lens capsule in early life are cubical in shape; as the pressure within it increases with age they become gradually flattened out. They may shrink so much in old age that little more than their nuclei can be seen on the inner surface of the hyaline membrane. It is possible that this physiological shrinking of the capsule cells may pass on into senile degeneration, and that some cases of senile cataract are in this way brought about. The large majority of senile cataracts, however, commence not as subcapsular but as equatorial opacities.

In many eyes, especially though not exclusively in those of advanced years, by means of the slit lamp and corneal microscope an appearance suggestive of shagreen may be seen on the surface of the lens. It is due to a number of small rounded areas arranged in a regular fashion, which reflect more light than the elements around them. The precise site and significance of these rounded particles has not been definitely determined. Some observers think that they are situated in the subcapsular lens fibres, and others that they are in the epithelium of the anterior capsule. Their absence from the posterior capsule, where there is no epithelium, and their presence on fragments of capsule removed by capsulotomy favour the latter view. ${ }^{1}$

(c) Equatorial Cataract.-Experiments on animals, in whicis the effect on the lens has been observed of the introduction of various chemical and staining substances into their circulation, seem to show that fluid enters it chiefly at the equator in the region of the suspensory ligament. It is at the equator of the lens where new fibres are constantly being formed, that the most active changes take place.

The cells lining the capsule, like epithelial cells generally, have the power of proliferating throughout life. As

${ }^{1}$ H. G. A. Gjessing. Klin. Monatsbl. f. Augenheilk., I92o. 
they divide they extend round the capsule until they reach the equatorial zone, there the large supply of nutrient fluid which they receive enables them to grow. Their expansion laterally is prevented by the pressure of surrounding parts, and they lengthen out anteriorly and posteriorly into long flat prismatic bands, which are at first nucleated. Gradually the nuclei in them break down and disappear. The rapidity with which these new lens fibres are produced becomes lessened as age advances, from the increasing intracapsular tension tending to check the proliferative activity of the capsule cells.

The continual laying on of fresh lens fibres causes gradual expansion of the capsule and condensation of its contents. Careful measurements of a large number of lenses at different ages has demonstrated that it continues to grow throughout life. Of a jelly-like consistency in early life it gradually becomes harder and heavier. As the new fibres are laid on laterally the centre of the lens is the part which becomes most compressed and hardest, forming what is termed its nucleus. The hard nucleus increases in size at the expense of the soft cortex with the advance of years.

Microscopically, in the parts of the lens undergoing sclerosis, its fibres are seen to be more closely approximated; the interfibrillar cementing substance, which stains slightly deeper than the fibres, becoming decreased in amount. The fibres themselves become narrower and their margins crenated, or even dentated, the toothed prominence on one fibre fitting into a corresponding depression in its neighbour. This dentation of the fibres is exceedingly well marked in the hard round lenses in fishes eyes.

Senile Cataract. - It has been estimated ${ }^{1}$ that 93 per cent. of senile cataracts originate at the equator. In the large majority of these the changes are first seen in the lower and inner quadrant; they consist of streaks, cones or dots of opacity, which stand out black against the red reflex of the fundus when viewed by reflected light, and appear grey by

${ }^{1}$ H. Magnus. Arch. f. Ophth., XXXVI, I890, 4, 150. 
focal illumination. The nucleus of the lens though not opaque may be of a yellowish colour and give a grey reflex to the pupil. Peripheral opaque striæ frequently remain a long time without undergoing any alteration. When the cataract commences to extend, the peripheral striæ and dots run into one another, and the opacity spreads into the layers around the nucleus. The subcapsular fibres for a

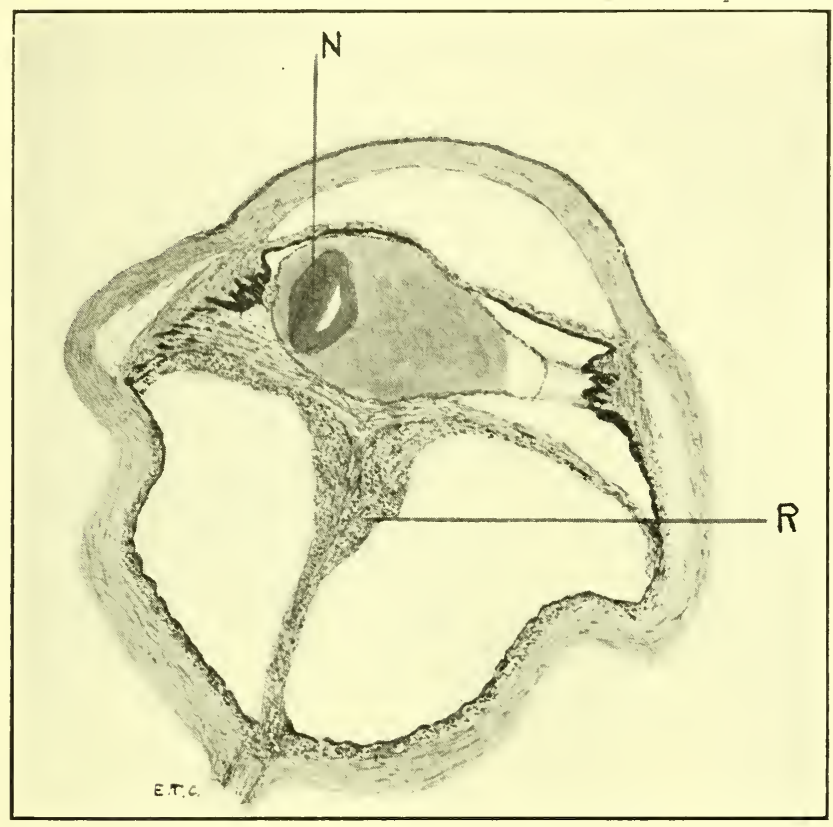

FIG. 273.-Section through a shrunken eyeball, showing puckering of the sclerotic posterior to the insertion of the recti muscles, and complete detachment of the retina $R$ from the position of the ora serrata up to the optic disc. The cortex of the lens has become liquefied; $N$ points to the nucleus lying in it, so-called Morgagnian cataract.

time remain clear, so that the pupillary margin of the iris throws a shadow on the surface of the opacity. When in course of time the subcapsular cortex also becomes involved, the opacity extends right up to the posterior surface of the iris and a shadow from the pupillary margin is no longer formed, the cataract is then said to be "ripe." The fibres 
of the lens may be seen as striations on the surface of the opacity, and sometimes a star-shaped figure is formed where the ends of the fibres come into apposition. Even when a senile cataract has reached this stage of maturity the nucleus on extraction, though it may be amber coloured, is frequently found transparent.

Following on the stage of ripeness comes one of hypermaturity in which the cortex liquefies, being transformed

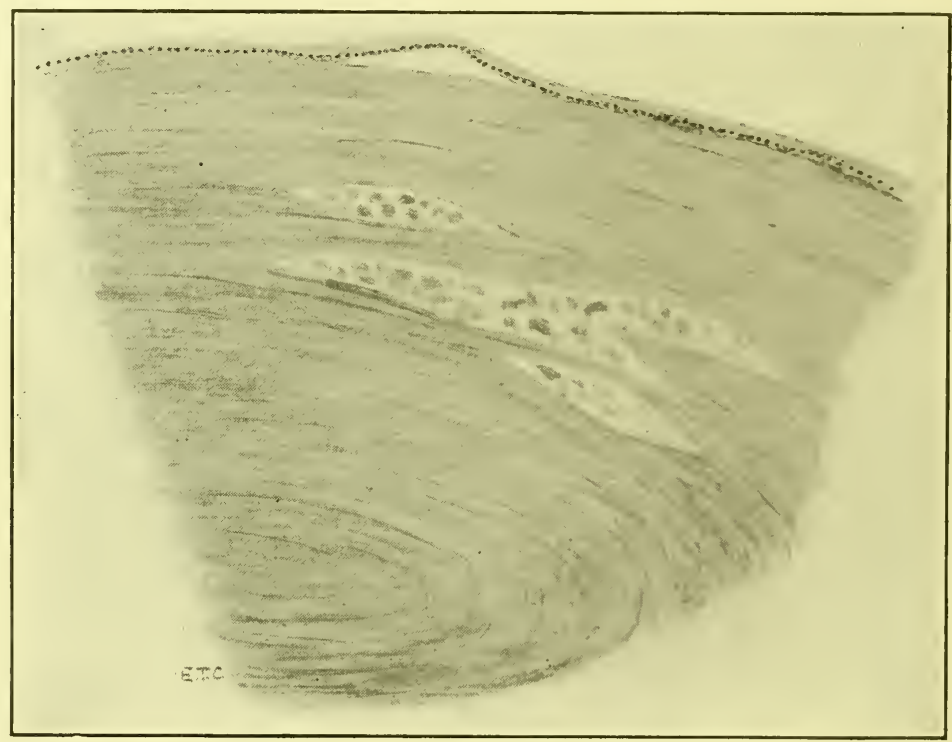

FIG. 274.- Shows the early changes in the lens in senile cataract which give rise to striæ of opacity.

into an opaque milky fluid in which the nucleus sinks (Fig. 273). The surface of the opacity in the pupillary area then loses its radial markings and presents a homogeneous appearance, on the surface of which little bright white specks may sometimes be seen. These are due to calcareous deposits in secondary capsular cataracts, which form as the result of the diminished intracapsular tension.

Sclerosis and shrinking, which normally occur in the nuclear lens fibres, may take place prematurely in some of 
the cortical fibres. As the result of their shrinkage cleft like spaces are formed between them, in which the interfibrillar fluid collects and coagulates into drops or spheroidal bodies, the "Morgagnian globules" (Fig. 274).

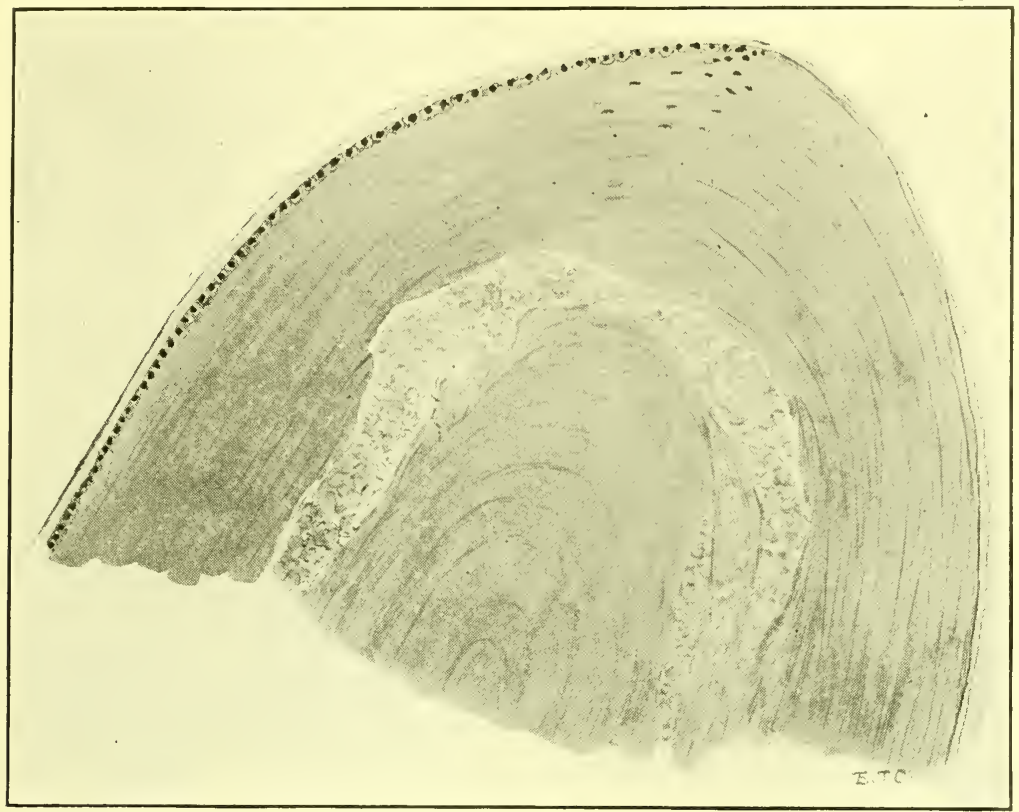

FIG. 275.-Shows spreading changes in senile cataract which give rise to perinuclear opacity.

They have a different refractive index from that of the lens fibres and, therefore, give rise to streaks or patches of opacity situated at the equator. The lens fibres at the margins of these fissures may later undergo a degeneration, minute droplets forming in them giving rise to a granular appearance; these run together and the fibre breaks down. In this way spaces become formed in the cortex of the lens containing a mixture of albuminous Morgagnian globules and drops of fat. As the cataract progresses these spaces open up into one another, and more and more fibres undergo degeneration (Fig. 275.) Ultimately in the hypermature cataract the whole cortex becomes liquefied. 
As the result of the degeneration of the lento-protein, which takes place in cataractous lenses, various crystalline substances may be produced, the commonest of these is cholesterine, tyrosin ${ }^{1}$ and leucin ${ }^{2}$ have also been met with. The mineral constituents of the lens also undergo alteration, ${ }^{3}$ the potash salts being considerably decreased and the calcium salts considerably increased.

When degeneration takes place in the lens fibres in the equatorial region, the capsule cells frequently fail to grow into lens fibres and spread instead round the posterior capsule, in some cases lining it in its entire extent.

When the intracapsular tension has become decreased, capsule cells may become enlarged as they would if they were going to develop into lens fibres, but there being no pressure causing them to become flattened out into fibres, they swell up into large spherical cells, termed vesicular or bladder cells, which have a homogeneous contents and a faintly staining nucleus, the latter may in time disappear (Fig. 272).

Equatorial cataracts, both pre-senile and senile, are usually bilateral, though the extent of the degenerative process is often more advanced in the one eye than the other. They may also be transmitted through several generations, and affect several members of a family in the same generation. It has been shown that they present a tendency to anticipation, i.e., a manifestation of the morbid change at an earlier age in members of each succeeding generation as a whole, or in successively born children of one parentage. ${ }^{4}$

To account for this bi-lateral and hereditary premature sclerosis of the equatorial lens fibres, occurring as it does apart from any inflammation, deficiency or toxæmia, it

${ }^{1}$ Burdon-Cooper. Trans. I7th Internat. Med. Congress. Ophth., 19I3, I 33 .

${ }^{2}$ G. Coats. Trans. Ophth. Soc. of the U. K., XXXII, I9I2, I53.

${ }^{3}$ W. E. Burge. Arch. of Ophth., XXXVIII, 449.

${ }^{4}$ E. Nettleship. Trans. Ophth. Soc. of the U. K., XXIX, 1909, Lxxix. 
seems necessary to assume some inherent defect in their vitality, and to classify the affection as an abiotrophy.

(d) Nuclear or Perinuclear Cataract.-The growth of the nucleus of the lens is the chief cause of the loss of its accommodative power with the advance of years, presbyopia. As the lens becomes harder, it is less capable of altering its shape, and adapting its focus for objects at different distances.

The harder the nucleus the more light there is reflected from its surface and the duller the pupillary reflex. The clear black pupil of youth is due to the soft nucleus reflecting little light; the dull eye of old age, to the hard nucleus causing much reflection. In association with this sclerosing process some pigmentation frequently takes place, the nucleus becoming an amber colour.

The degree of sclerosis of the nucleus and the rapidity of its growth vary in different individuals. Occasionally in elderly people the sclerosis of the nucleus is excessive, and a great increase in its refracting power is produced, contrasting markedly with that of the unsclerosed cortex. Such an eye may be found to be myopic in the centre of the lens and less myopic, emmetropic, or even hypermetropic at the periphery. Looked at by focal illumination it seems to have a greyish yellow nuclear opacity, when examined by reflected light the nucleus may be clear but present a well defined margin, so that it looks like a large oil drop in the centre of the lens. Where, however, it is much tinted it gives rise to an ill defined central haze. The retinal vessels, seen ophthalmoscopically through differently refracting parts of the lens, present a bent and distorted appearance.

When this change takes place in the lenses of elderly people they find, to their surprise and delight, that whereas with advancing years they became more and more dependent on the use of convex glasses for near work, they can once again read with weaker glasses or even abandon their use altogether. To see distinctly in the distance, however, where formerly no glasses were required minus glasses 
would have to be employed. This lenticular myopic degeneration is popularly spoken of as second sight.

Sometimes the sclerosing process becomes so extensive that it involves the whole lens, there being then no differentiation of it into a hard nucleus and softer cortex. In such cases the pigmentation is also excessive, the lens becoming dark brown in colour, so dark in some cases that on reflecting light into the eye no red reflex can be obtained from the fundus, and the patient's sight is greatly impaired. This condition is somewhat inaccurately spoken of as black cataract. It is not strictly speaking a cataract at all, only an advanced stage of senile sclerosis, the whole lens having undergone a change which is usually restricted to the nucleus.

Examined by slit-lamp microscopy ${ }^{1}$ the human lens at birth is found to be slightly yellow. This yellowness gradually increases with age, and is more marked in the nucleus than the cortex. The posterior cortex appears darker than the anterior because it is viewed through the latter.

The lenses of the natives of India are more frequently, and relatively more deeply, pigmented than those of Europeans; this has been attributed to the effects of either light or heat.

The pigmentation always commences in the nucleus of the lens and cannot, therefore, be derived from blood pigment. Compared with which it presents entirely different spectroscopic characteristics. The pigment in the lens is of the nature of melanin, and comparable to the pigment of the hair or that formed pathologically in the basal cells of cuticular epithelium in senile atrophy.

The lens, hair, nails, and enamel of the teeth, structures which are all epiblastic in origin, all yield tyrosin on hydrolysis with weak acids, ${ }^{2}$ and may all either physiologically

${ }_{1}$ A. Vogt Atlas, I92 I.

${ }^{2}$ Burdon Cooper. Trans. I7th Internat. Med. Congress. Ophth., I913, I33. 
or pathologically become pigmented. One of the tests for tyrosin is the production of pigment on its oxidation by the juice or alcoholic extract of a certain species of fungus. In this test all the shades of colour which are observed in the crystalline lens, from light amber to dense black, are produced. It has been suggested that the pathological discolouration of the lens is due to an oxidation of tyrosin, which is formed by hydrolysis of the lens proteid, i.e., a decomposition in which by assimilating a molecule of water it breaks down into one or more simpler substances. As discolouration of the lens substance occurs without any histological change taking place in the lens fibres, the acceptance of this theory necessitates the assumption that it is possible for hydrolysis of the lens protein to take place whilst its fibres remain intact.

The hard sclerosed nucleus of the lens has little tendency to undergo degenerative changes which result in the formation of opacity; it frequently remains clear and unaltered when the whole cortex has become opaque.

As a rule it is only in early life, while the nuclear fibres are still soft, that changes which give rise to opacity occur in them. Even then the perinuclear layers are generally affected to a greater extent than the more central ones.

Zonular or Perinuclear Cataract.-In both perinuclear and nuclear cataracts, when the pupil is dilated, a grey circular opacity is seen with an area of transparent cortex around it. On examination by reflected light the affected area is found to present a duller reflex than the marginal unaffected zone. In a perinuclear cataract the margin of the opaque area is much darker than the centre, whereas in a nuclear cataract the centre is as dark or darker than the edge.

Pathologically no very sharp line can be drawn between perinuclear and nuclear cataracts; the same changes are met with in both, differing only in their locality and degree.

On the surface of the disc-shaped area of opacity dots and streaks of denser opacity are often seen; the latter may 
project beyond the disc into the clear cortex, forming what are called "riders." At times these riders are forked, the limbs of the fork embracing the two surfaces of the affected disc-shaped area.

Microscopically the opacity in these cataracts is found to be due to a number of small globules, the coefficient of the refraction of which differs from that of the surrounding

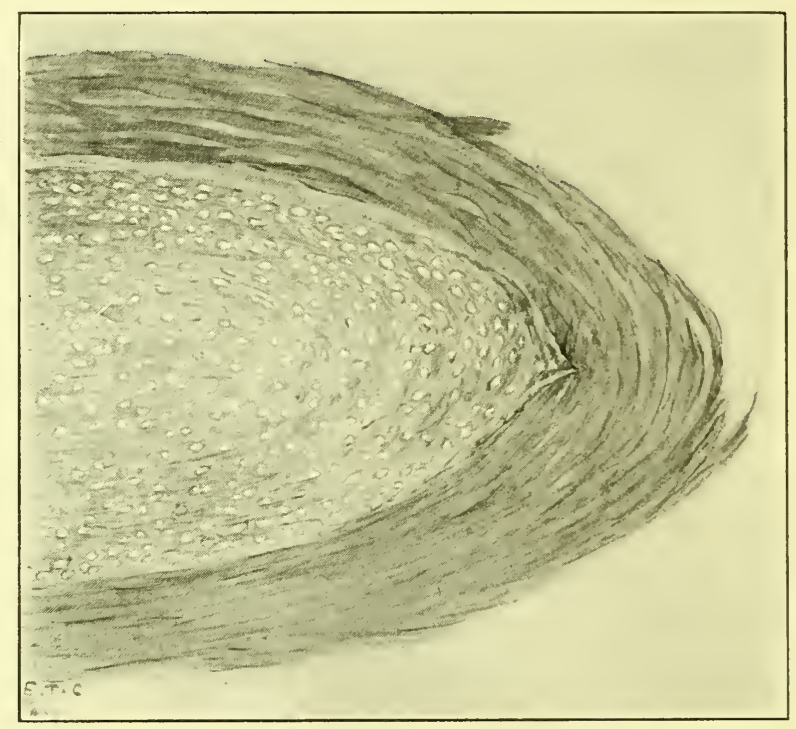

Fig. 276.- Section of a lens which had a zonular cataract, showing a number of small vacuoles scattered throughout the nucleus.

lens fibres (Fig. 276). These globules vary in size from $0.0125 \mathrm{~mm}$. to $0.025 \mathrm{~mm}$. and are mostly round or oval in shape; some writers have described them as vacuoles, because in sections stained by ordinary methods they look like vacant spaces, in others, however, stained in a different way, they are seen to be filled with a hyaline or granular substance. After prolonged immersion in hæmatoxylin solution some globules become deeply stained, whilst others become stained only in the centre and present much the appearance of a nucleated cell. 
The globules lie between the lens fibres, corresponding in size very closely to that of the cells lining the lens capsule, which are estimated to be o.o I $\mathrm{mm}$. in diameter in early life and $0.02 \mathrm{~mm}$. in later life. Though the nature of the globules has not been definitely determined it seems probable that they are either, (a) degenerate lens cells, which from some defect in their nutrition have failed to develop into lens-fibres, or, (b) lens-fibres, which having commenced to develop, through defective nutrition, have subsequently undergone degeneration.

In perinuclear cataract these vacuoles are most numerous in the region of the densest opacity, i.e., around the nucleus, though they may be found distributed throughout the entire nucleus. In nuclear cataracts their distribution in the opaque discoid area is more uniform.

Besides the vacuoles, fissures are met with between the lens fibres, which may or may not contain a granular substance, and run concentrically with the nucleus separating it from the cortex. They are produced probably by contraction of the nucleus and cause the "riders" above referred to.

A third change sometimes met with are spaces larger than the vacuoles, measuring on an average $.02 \mathrm{~mm}$. across, mostly circular with very irregular margins and containing a granular substance which stains deeply with hematoxylin. These correspond to the denser dots seen on the surface of the opaque zone. Some degeneration of the lens fibres apparently occurs in their formation.

There has been considerable discussion as to whether the perinuclear cataracts are formed before birth or after, i.e., whether they are congenital or infantile. There can be little doubt that they are due to some general disturbance of nutrition, as they are bilateral and frequently associated with fits in infancy, rickets, and a defective condition of the enamel of the permanent teeth.

The size of the opaque area, and consequently the size of the clear zone external to it, vary considerably in differ- 
ent cases. Direct measurement of the opaque area in ten cases ${ }^{1}$ by different observers gave an average transverse diameter of $4.5 \mathrm{~mm}$; the largest being $6 \mathrm{~mm}$. and the smallest $3.25 \mathrm{~mm}$. Cases have been seen in which, external to the central complete disc of opacity, there was a second or third incomplete opaque zone.

The transverse diameter of the lens at the ninth month of fetal life has been found to be $5.75 \mathrm{~mm}$., and that of the lens under a year old $7.46 \mathrm{~mm}$. When these measurements are compared with the transverse diameters of the opaque zone in perinuclear cataracts above mentioned, it will be seen that opaque area is sometimes about that of the lens at birth and sometimes very much smaller.

By the continual laying on of lens fibres at the sides the nuclear fibres gradually become more and more tightly compressed, so that under normal conditions, the lens substance present at birth occupies a larger area than it does later in life when fresh cortex has developed.

The rate at which the capsular epithelial cells proliferate and develop into lens fibres is regulated by the intracapsular tension. As life advances, and the intracapsular tension increases, the rate at which new lens fibres are formed decreases.

If during the first year of life some disturbance of nutrition causes the lens fibres to shrink, the intracapsular tension is lowered and an increased number of cortical fibres are formed. These being healthy fibres, clear cortex would be formed surrounding the shrunken opaque area, the diameter of which, from shrinking and compression, would be less than that of the normal lens at birth.

These measurements tend to confirm what has been found clinically, that perinuclear cataracts may develop either in utero or after birth. Cases have been recorded in which infants who have been seen to have clear lenses have subsequently developed perinuclear cataracts. Newly born

${ }^{1}$ Treacher Collins. "Researches into Anatomy and Pathology of the Eye," I 896, 33. . 
infants have also been seen with well developed perinuclear cataracts in both eyes. Cataracts of this type have been produced experimentally in fetal rabbits' eyes, by feeding the mother animal on naphthalin during the second and third months of pregnancy.

A honeycombed appearance of some of the permanent teeth is very commonly met with in those affected with perinuclear cataract; out of twenty-six cases only four showed no abnormality of the teeth. ${ }^{1}$ The condition is best described as a "hypoplasia," due to imperfect calcification; a condition in which the calsopherites have not completely coalesced to form the normal tissue. The teeth most frequently affected are the permanent first molars, incisors and cuspids, i.e., those in which the enamel organ undergoes calcification during the first two years of life. The amount of enamel affected varies considerably in different cases, and also in the different teeth involved in the same case; the transition from the affected area to the normal enamel is always sudden when the disturbing influence has ceased to act, whereas, the onset of the hypoplasia is much less sharply defined. In the perinuclear cataracts there is a similar abrupt transition on the outer surface of the affected zone to normal lens fibres, whilst transition from the most affected zone inwards is generally gradual and ill-defined.

Hypoplastic enamel in the parts of teeth formed before birth is rarely if ever met with. If the affected zone in the lens is small, so as to make it probable that the dystrophic influence which caused the change occurred before birth, there is usually no defect in the enamel of the permanent teeth. Where perinuclear cataract is an hereditary affection, the zone of opacity is generally small, and the enamel of the teeth is unchanged.

Teeth with hypoplasia of the enamel have frequently been described as "rachitic;" perinuclear cataracts were also regarded at one time as due to rickets; the convulsions

${ }^{1}$ Norman Bennett. Trans. Ophth. Soc. of the U. K., XXI, I9Io, 43. 
which are such a frequent accompaniment being attributed to craniotabes. Many cases of perinuclear cataract and of hypoplasia of the enamel of the teeth occur without any manifestations of rickets, and many cases of rickets occur without the formation of any opacities in the lenses or any changes in the enamel of the teeth. It seems, therefore, only possible to regard rickets as a concomitant of these affections and not as a cause, the conditions which predispose to the former also predisposing to the latter.

The convulsions which occur in connection with perinuclear cataract are often met with during the first few months of life, before rickets could have developed sufficiently to have resulted in any abnormal softening of the bones of the skull, it seems impossible, therefore, to attribute them to craniotabes. Moreover they are met with in cases in which no other signs of rickets have been detected.

The convulsions may be accompanied by carpo-pedal spasms and laryngismus stridulus, being similar to what is known as "infantile tetany." Cases have been recorded of tetany occurring in later life in which cataract has developed. Though these cataracts have not presented the same clinical appearances as zonular cataract, they have been found microscopically to show globules, shrinking and degenerative changes of a similar character. In these cases besides the lens changes there has generally been found loss of hair, necrosis of the nails and an affection of the skin.

There is much experimental and clinical evidence ${ }^{1}$ to show that the parathyroid glands are intimately associated with the origin of tetany. Children suffering from infantile tetany quickly respond to the administration of emulsions of ox-parathyroid.

The excision of the parathyroids experimentally in a dog has been followed by the development of cataracts, together with fits and tremors. Cases have been recorded in which, after operations on the thyroid for goitre, tetany developed together with cataracts, in some of these cases there was

${ }^{1}$ Treacher Collins. Trans. Ophth. Soc. of the U. K., XL, I920, 406. 
also an affection of the hair, skin and nails. In myxœedema, an affection attributable solely to thyroid insufficiency, and in which the parathyroids are said to be normal, tetany and cataracts are not met with. It would seem, therefore, that after operations on the thyroid where such symptoms arise, some interference with the parathyroids had probably occurred.

The foregoing observations may be summarised as follows: Tetany is due to parathyroid deficiency. Cataract occurs in association with tetany in adults, and has been produced by removal of the parathyroids. The convulsions which occur in infancy in connection with zonular cataract are due to infantile tetany. It would seem probable, therefore, that the lens changes in such cases are also the result of parathyroid deficiency.

It has been shown by experiments that the parathyroids regulate the metabolism of guanidin in the body, and by doing so probably exercise a controling influence on the tone of the muscles; there is, however, nothing known of guanidin in relation to the crystalline lens.

The parathyroids have also been shown to regulate in some way the calcium metabolism. Experimental aparathyroidea in dogs has been accompanied by a marked diminution of calcium in the blood and brain, and it is possible to keep off for a time the symptoms which accompany it by the administration of calcium.

The three conditions with which perinuclear cataracts are found clinically to be frequently associated, viz. hypoplasia of the permanent teeth, rickets and infantile tetany are all accompanied by disturbed calcium metabolism. In hypoplasia of the teeth there is imperfect calcification of the enamel organ. In rickets there is imperfect calcification of the bones. In infantile tetany, as in dogs with experimental aparathyroidea, it has been shown that there is a deficiency of calcium in both blood and brain.

It seems possible that a deficiency of calcium in the blood might have some effect in the development of the lens fibres, 
as calcium is one of the constituents of the ash of the normal lens. Its amount is exceedingly small but is considerably increased in senile cataracts.

Though in the vast majority of cases of perinuclear cataract the affection is bilateral, rare cases are met with in which the lens in one eye only is involved. The bilateral character of the change suggests a constitutional cause. In the recorded cases of unilateral perinuclear cataract the history suggested that some local cause, such as an injury or an attack of inflammation, gave rise to the opacity.

The experimental production of perinuclear cataracts in young rabbits by feeding the pregnant mother animal on naphthalin, shows that they may be produced by some defect in intra-uterine nutrition. When these cataracts occur at birth in brothers and sisters, both parents being free and no history obtainable of ancestral or collateral cases, they are probably due to defective intra-uterine nutrition, possibly parathyroid deficiency in the mother. The parathyroids, like the thyroids, are normally in a state of heightened activity during pregnancy, and it is not until some months after birth that these glands in an infant attain full functional activity. If, therefore, the secretion of the parathyroid glands is necessary for the development of the tissues of a fetus in utero, and for the first few months of life, it must be obtained from the maternal blood before birth, and from the maternal milk after birth. An infant deprived of it from either of these sources would be expected to manifest the symptoms of aparathyroidia.

In some cases perinuclear cataract has been found to be truly hereditary, not merely familial; it may be transmitted by the father as well as the mother. When transmitted by the father it is necessary to assume the presence of some germinal cause. 


\section{NEURAL EPIBLASTIC DEGENERATIONS}

The degenerations which take place in the tissues of the eye derived from neural epiblast will be described under the following headings: (a) Degenerations of the ganglion cells of the retina; $(b)$ degenerations of the rods and cones of the retina; $(c)$ atrophy of the optic nerve; $(d)$ degeneration of the pigment epithelium of the retina; $(e)$ degeneration of the neuroglia of the optic nerve and retina; $(f)$ degeneration of the vitreous humour; $(g)$ degenerations of the pars ciliaris retinæ and pars iridis retinæ.

(a) Degenerations of the Ganglion Cells of the Retina.The earliest degenerative changes in the ganglion cells of the retina are best demonstrated by Nissl's method of staining (see page 706). This method picks out small granules called Nissl granules, which in the normal condition are scattered throughout the cytoplasm of the cell. Their number and concentration are influenced by exposure to light, which makes them become diffuse and disappear; absence of light favouring their reformation. ${ }^{1}$

In degenerative conditions, whether the result of postmortem changes; anæmia, as in experimental ligature of the carotid artery; or toxic conditions, such as quinine and alcoholic amblyopia, these Nissl granules become smaller and grouped in the peripheral parts of the cell, finally disappearing altogether.

After the disappearance of the Nissl bodies in a degenerating ganglion cell, vacuoles form in its cytoplasm, most of them containing fatty globules, which tend to run together. The nucleus also shows changes, ceasing to stain with the usual nuclear stains and then likewise becoming vacuolated. Finally the cell shrinks, and when completely destroyed leaves a space in the neuroglia where it was situated.

Coagulative necrosis occurring in the ganglion cells ${ }^{2}$ and their axions is the cause of the opacity of the retina which is

${ }^{1}$ Birch-Hirschfeld. Arch. f. Ophth., L., I900, I66.

${ }^{2}$ W. I. Hancock. R. Lond. Ophth. Hosp. Reps., XVII, 1908, 437. 
seen in embolism of the central retinal artery, and quinine amblyopia. It does not extend over the whole retina, but is limited to parts around the macula and optic disc, i.e., to the parts in which the ganglion cells are chiefly located. It is absent at the macula where there are no ganglion cells, and in the peripheral parts where they are very few in number.

As the nerve fibres of the retina degenerate their outlines become obscured; sometimes hyaline material collects around them in irregular clumps; finally they break up and disappear altogether.

Should atrophy occur in a retina, where there is an abnormal development of medullary sheaths around the nerve fibres (i.e., opaque nerve fibres), the medullary substance breaks up and is removed. What was ophthalmoscopically an opaque white area in the retina then becomes transparent, and allows the red reflex of the choroid to be seen through it.

Degeneration of the ganglion cells of the retina is soon followed by changes in the bipolar cells and in the inner nuclear layer, as shown histologically by their diminished staining reaction, their shrinkage, and finally complete disappearance. The internuclear layer may become obliterated, and the two nuclear layers appear to run together. Degeneration of the ganglion cells does not, however, lead to atrophy of the rods and cones, which will remain unchanged when the neurons have entirely disappeared.

Amaurotic Family Idiocy.-This is a disease of the nervous system and retina, the primary change being in the nerve cells. ${ }^{1}$ It is rarely met with in any but Jewish children, and frequently affects several members of the same childship. It manifests itself, when the child is from three to six months old, by a weakness in the muscles of the back and neck. The child is unable to sit up or hold its head erect. In association with this weakness a defect of vision

${ }^{1}$ Kingdon and Russell. Med.-Chir. Trans., LXXX, 1897. 
is soon noticed. Ophthalmoscopic examination reveals a circular white opaque area in the retina around the macula in each eye, while the macula itself appears of an unusually dark red colour, much darker than the cherry-red macula seen in embolism of the central artery. The optic disc though not at first affected, later on becomes pale or even quite white, and the retinal arteries are markedly constricted. The muscular weakness increases and all the cases end fatally from marasmus.

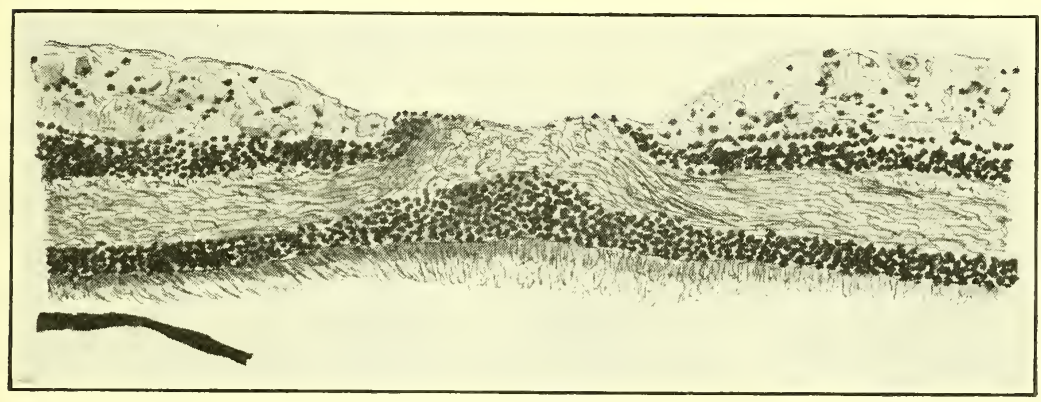

FIG. 277.- Section through the retina at the macula in a case of amaurotic family idiocy. Showing cytological degeneration, and in places complete disappearance of the ganglion cells. Case recorded by Hancock and Coats, Brain, XXXVIII, I9II, 5 I 4 .

There is no evidence in this disease of any inflammatory changes in the parts affected; the essential histological features in it are, progressive loss of the Nissl substance in all the neurons of the body, and the increase of the neuroglia fibril substance to an abnormal degree. Chemical examination of the the brain has shown:1 (I) a decrease of nucleoprotein, which may be associated with the disappearance of the Niss1 substance in the neurons; and (2) the increase of simple protein, which may be correlated with the increase of glial fibres.

The cause of the opacity in the retina is change in its ganglion cells, ${ }^{2}$ similar to that which takes place in the ganglion cells elsewhere; at first they appear somewhat

${ }^{1}$ F. Mott. Archives of Neurology, III, I907, 2 I9.

${ }^{2}$ Holden. Journ. Nerv. and Mental Dis., XXV, I898, 538. 
swollen, then there is a gradual progressive loss of Nissl substance, followed by the formation of vacuoles in the cytoplasm, and finally shrinkage or disappearance of the cell (Fig. 277). The long continuance of the opacity of the retina is due to the long time the degenerative change in the cells goes on, befor ethey finally disintegrate. It is very different from the short duration of the opacity of the retina after embolism of the central artery, which is due to a coagulative necrosis of the ganglion cells, after which they become rapidly absorbed and disappear. The atrophy of the nerve fibres is secondary to the changes in the ganglion cells.

The racial and familial characters of the affection show that it is due to some inborn tendency to neuronic decay, some failure in the inherent specific trophic influence of the nucleus of the nerve cell upon which the vitality of the whole neuron depends, it may therefore be classed as an abiotrophy.

(b) Degeneration of the Rods and Cones of the Retina.Degeneration of the ganglion cells of the retina is not followed by consecutive atrophy of its neuro-epithelium; in eyes blind from embolism of the central artery of the retina the rods and cones, and outer nuclear layer, remain intact. Degeneration of the percipient elements of the retina is always followed by atrophy of its neurons. The outer layers of the retina receive their nourishment from the choroidal blood-vessels, and the inner layers from the retinal vessels.

Shrinking and atrophy of the rods and cones is followed first by similar changes in the outer nuclear layer, and then all the nervous elements in the retina disappear, leaving behind only the supporting network of neuroglial tissue. Into the vacant spaces of this network, pigment epithelial cells migrate, finding their way to the sheaths of the retinal blood vessels, around which they congregate and give rise to branching, irregular, bone corpuscle-shaped patches of pigmentation. 
Degeneration of the rods and cones may be caused by inflammation or disturbances in the circulation of the choroid, or arise as a primary, hereditary, bilateral affection; an abiotrophy; so-called "retinitis pigmentosa."

Degeneration of the retina the result of inflammation is described in connection with choroiditis (see page 459). The disturbances in the circulation of the choroid which may give rise to degeneration of the rods and cones are: lesions severing the short ciliary arteries before they enter the globe, compression of the choroidal vessels by subchoroidal hæmorrhage, and sclerosis of the walls of the vessels narrowing their lumen.

Experiments made on animals, of dividing the posterior ciliary arteries, have definitely proved that the nutrition of the outer layers of the retina depends on the choroidal circulation. When this is cut off, both retina and choroid atrophy and become adherent, pigmentation of the former taking place. The same has been found to occur, both clinically and pathologically in man, after the operation of optico-ciliary neurotomy, and after gunshot wounds of the back of the orbit in which the posterior ciliary arteries have been divided without the eyeball being perforated. The sequelæ of subchoroidal hemorrhages are dealt with in the chapter on injuries (see page 360).

Retinitis Pigmentosa. ${ }^{1}$ - It is most essential to recognise the difference between primary degeneration of the neuroepithelium of the retina, and that which occurs secondary to sclerosis of the choroidal vessels. The ophthalmoscopic appearances of the two affection may be very similar, though the prognosis and possibilities as regards treatment are very different.

That a primary degeneration of the retinal neuroepithelium may take place, independent of any change in the choroidal vessels, has been proved by the pathological

${ }^{1}$ Treacher Collins. Trans. Ophth. Soc. of the U. K., XXXIX, I9I9, 165. 
examination of the eyes from several cases of retinitis pigmentosa by different observers.

The first symptom of which a patient suffering from retinitis pigmentosa complains is a difficulty of seeing in dim lights, "night-blindness."

In the dark-adapted eye the part of the retina which is most sensitive to light is not the fovea, but the area surrounding it. This was first noticed by astronomers, who found that stars of low magnitude can be seen better by indirect than by direct vision. Experiments have since shown that in an eye adapted for darkness the sensibility increases centrifugally with each degree, until at $10^{\circ}$ to $20^{\circ}$ from the fovea the maximum is reached. On the other side of the maximum the sensibility gradually decreases towards the periphery. In typical cases of retinitis pigmentosa the part of the retina in which functional activity first fails, is not the extreme periphery or the central area, but a ring around that from which the papillomacular nerve fibres proceed. The inner border of the ring scotoma which is produced corresponds as a rule to about the $15^{\circ}$ or $10^{\circ}$ circle in perimeter charts. It will be seen, therefore, that in such cases the part of the retina first involved is that which in the dark-adapted eye is the most sensitive to light, this explains why night-blindness is the earliest symptom of the disease. The central area included in the ring scotoma remains unaffected until the outer border of the affected belt has extended to the extreme periphery. The extension of the belt outwards is not always symmetrical, small islands of vision or relatively functional areas, being at times met with scattered throughout the scotoma.

The night-blindness precedes the appearance of pigmentation in the retina by some years. The term "retinitis pigmentosa sine pigmento" usually signifies nothing more than the initial stage of the ordinary disease. The loss of field of vision, and the area of the retina which is 
pigmented, frequently do not correspond, the extent of the former being considerably larger than the latter.

The pigment epithelial cells of the retina normally possess the power of amœboid movement. In response to the stimulus of light they throw out filamentous processes, containing the pigment granules, between the rods and cones; and on removal of the stimulus the processes retract, the pigment granules returning to the body of the cells. It is this capacity for positive phototactic movement in these cells, which accounts for the pigmentation of the retina that takes place when the rods and cones become atrophied. Normally the phototactic movements of these cells is kept in check by their anatomical relation to surrounding structures. The membrana limitans externa checks their movement inwards in the direction from which light is coming. When, however, the rods and cones have atrophied and disappeared, gaps are left in the membrana limitans interna through which they can make their way. When all the nervous elements of the retina have disappeared, and only a network of neuroglia is left, the pigment cells can migrate through its spaces to its innermost layers, and there become grouped in the sheaths around the blood-vessels, giving rise to the branching patches which are seen ophthalmoscopically.

In some forms of degeneration of nervous structures, a disappearance of the nervous elements is accompanied by an overgrowth of the interstitial neuroglia supporting tissue. That such overgrowth is a sequel and not the cause of the atrophy of the nerve elements, as in a sclerosis, is shown by its being a loose network of softer consistency than the structures which have perished.

In the retina, in retinitis pigmentosa, together with the atrophy of its nerve structures, there is an overgrowth of neuroglia and a contraction of the retinal blood-vessels, both of which are consecutive and not primary. It is the overgrowth of neuroglia in the vicinity of the optic disc which gives rise to its peculiar and characteristic waxy 
appearance in advanced cases of the disease. In this overgrown neuroglial tissue on the optic disc hyaline nodules sometimes form. Ophthalmoscopically they are seen as semi-transparent rounded bodies, which may become a protruding conglomerate mass. Their histological characters and pathology are dealt with in connection with the degenerations of the neuroglia (see page 648).

Retinitis pigmentosa is not a congenital affection, the percipient elements of the retina first attain their full development and then degenerate. The tendency to it, however, is innate, as is shown by the large majority of the cases being hereditary; the descent of the disease is usually continuous from parent to child, though occasionally the direct line of descent is interrupted by a healthy generation. Consanguinity of parents, though it cannot be regarded as a cause is frequently met with in connection with the disease, and evidently adds considerably to its tendency to develop. The onset of the symptoms is so insidious that it is often difficult to determine the age at which they commence. Though they generally begin in early life they may sometimes be delayed until quite an advanced age.

Other degenerations, in the internal ear causing deafness, or in the nervous system, may occur, either associated with retinitis pigmentosa or in members of an affected family apart from any eye disease. The deafness varies in degree in different cases, it is always bilateral and when complete it is usually associated with dumbness. A characteristic lisp in speech is present in some cases. The affections of the nervous system may be cerebral or spinal. In order of frequency they are; idiocy, mental deficiency, epilepsy and spinal paralysis.

From the description which has been given of retinitis pigmentosa it will be seen that it is not an inflammatory disease as its name would imply. It is a typical abiotrophy. It is a bilateral hereditary affection in which the rods and cones loose their vitality and degenerate, apart, so far as can be ascertained, from any nutritional deficiency. 
Symmetrical Macular Pigmentary Degeneration.-In the typical cases of retinitis pigmentosa the degeneration begins in a circular area around the macula, slowly extending outwards, and not involving the central vision until the whole of the peripheral vision has been destroyed. Many

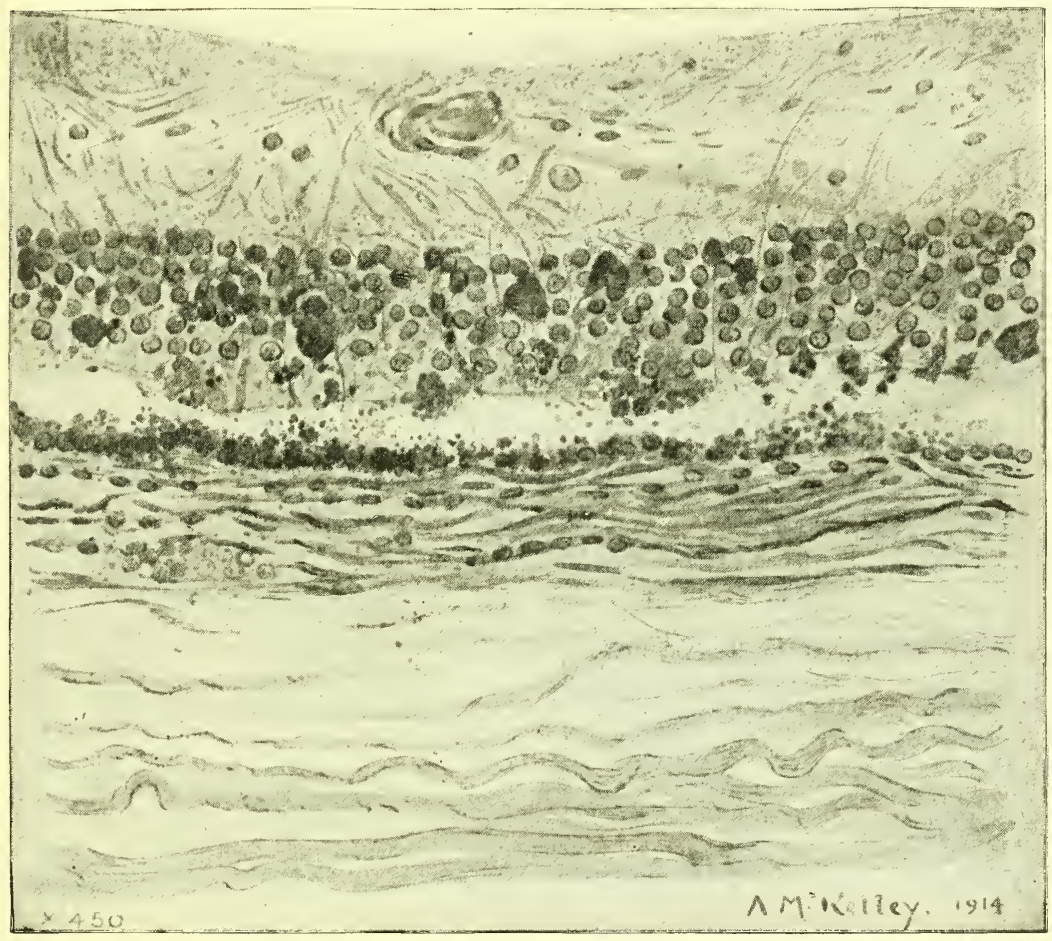

Fig. 278.- Section through retina in a case of symmetrical macular pigmentary degeneration in association with cerebral degeneration. Shows complete disappearance of the rods and cones together with the inner nuclear layer. Migration of the retinal pigment and disappearance of ganglion cells. Case recorded by Batten and Mayou, Proc. of the Roy. Soc. of Med., Ophth. Sec., VIII, I9I5, 70.

atypical cases have been described, some of which have undoubtedly been atrophy of the retina secondary to sclerosis of the choroidal vessels, and not primary degeneration of the rods and cones. The presence of bone corpuscleshaped patches of pigment in the retina is not pathognomonic of primary retinitis pigmentosa. Such patches 
are found in any condition in which the rods and cones atrophy so as to allow the migration of pigment epithelial cells inwards. They are met with in cases of sclerosis of the choroidal vessels, after concussion injuries in which there

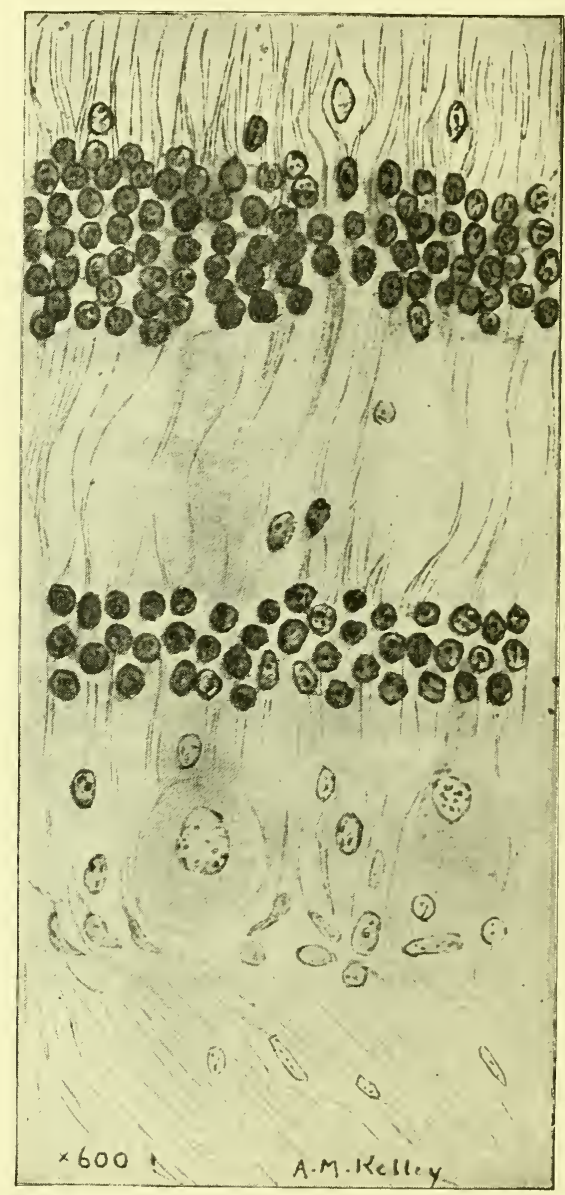

FIG. 279.- Section of retina from the same case depicted in Fig. 278, showing early signs of degeneration in a ganglion cell.

has been extensive subchoroidal hæmorrhage, and in cases of detached retina in which the displaced membrane, after atrophy of its rods and cones, has become restored to its normal position. 
There is, however, one well defined class of primary retinal degeneration, accompanied by pigmentation, which runs a different course from that which has been described in the typical cases.

In these cases the degeneration begins at the macula and failure of acuity of vision is the first symptom noted, its date of onset is, therefore, clearly defined and may be in either the first or second decade of life. The cones being affected at the macula, ${ }^{1}$ and not the rods in the perimacular region, the patients see best in dim lights and complain of day-blindness not night-blindness. There being no blood-vessels in the retina at the macula for the pigment cells to encircle, when they migrate into it, pigmented circular dots instead of bone corpuscle-shaped patches become formed. Generally when the neuro-epithelium at the macula is the first part to degenerate, the peripheral parts do not become involved (Figs. 278 and 279), the central vision having been destroyed no further loss occurs. In exceptional cases, in which further changes take place, the last part of the retina to become affected is usually that which in the typical cases is the first to fail viz., the perimacular zone. The cases which commence with a central failure of vision are frequently, but not always, associated with mental defects, due to degeneration of the ganglion cells in the brain. They often occur in several members of the same childship, but are not handed down, like the typical cases, from one generation to another.

(c) Atrophy of the Optic Nerve.-Atrophy of the optic nerve may be (I) secondary or (2) primary.

I. Secondary atrophy may be again divided into: $(a)$ atrophy secondary to changes in the interior of the eye, and (b) atrophy secondary to changes in the parts around the nerve outside the eye.

(a) Atrophy of the optic nerve secondary to changes in the interior of the eye may be the outcome of neuronic degeneration of the retina, produced either by inflammation,

${ }^{1}$ Batten and Mayou. Proc. Roy. Soc. Med., Ophth. Sec., VIII, I9I5, 70. 
traumatism, insufficient blood supply, toxic substances, increased intra-ocular tension, or abiotrophy.

As the majority of the fibres of the optic nerve are axons of the ganglion cells of the retina, degeneration of the former necessarily entails atrophy of the latter.

It has been shown experimentally in animals, ${ }^{1}$ that a wound of the retina results in the degeneration of a corresponding tract of fibres in the optic nerve.

The changes which take place in the optic nerve from degeneration of the neurons in the retina are well demon-

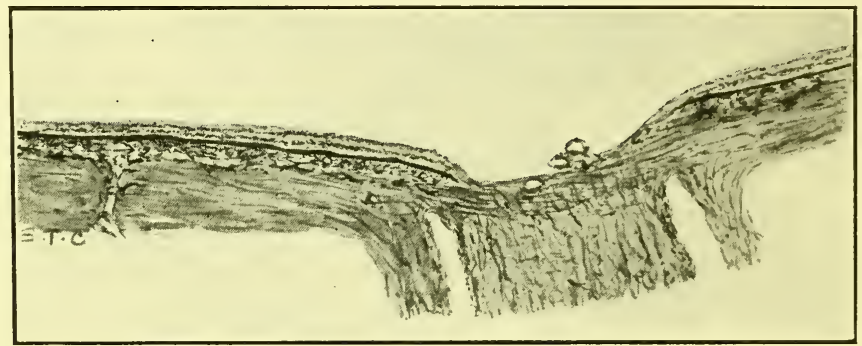

FIG. 280.-Section through the head of the optic nerve in a case of simple optic atrophy. Note the reduction in the size of the nerves, and that a depression has formed on the inner surface of the lamina cribrosa in the position normally occupied by the optic papilla.

strated by Marchi's method of staining (see page 706). As the atrophic process proceeds, the medullary sheaths break up into rows of globules along the whole course of the affected fibres. They are then slowly carried away either in the lymph stream or absorbed by leucocytes. The axis cylinders become beaded, then broken up, and finally disappear. As the nerve fibres become destroyed spaces are left in the supporting connective tissue framework, some proliferation of the neuroglia may take place, but the size of the nerve tends to become considerably reduced (Fig. 280).

Atrophy of the optic papilla secondary to papillitis or papilledema can be distinguished clinically from simple atrophy by the presence of newly formed tissue on the disc,

${ }_{1}^{1}$ Usher and Dean. Trans. Ophth. Soc. of the U. K., XVI, I896, 248. 
which gives it a white or bluish-white appearance, obscures the markings of the lamina cribrosa, and fills in any depression on its surface. The swelling of the disc, moreover, on its subsidence leaves behind some irregularities in its margin and thickenings of the walls of the retinal boodvessels, which are seen as white lines along their course. In simple atrophy the optic disc has sharply defined margins, is pale, slightly excavated, and shows grey markings over a large part of its surface, due to the exposed lamina cribrosa.

(b) Atrophy of the optic nerve, secondary to changes in the parts around the nerve outside the eye, may result from inflammation, traumatism, or pressure.

It might be supposed, in accordance with the Wallerian law, that the optic nerve largely made up of the axons of the retinal ganglion cells, would not degenerate when divided. This however, is not the case, after division, all the fibres in the ocular portion slowly atrophy and the optic disc becomes quite white. The ganglion cells in the retina, moreover, when stained by Nissl's method show chromatolysis. The explanation of this is that the optic nerve is an intercentral and not a peripheral nerve, to which latter the Wallerian law alone applies.

For atrophy secondary to inflammation see retrobulbar neuritis (page 473).

Injury to the optic nerve may be due to a direct wound, or be caused indirectly by a fracture of the base of the skull extending into the optic foramen (page 404).

Pressure on the optic nerve or the chiasma causing atrophy may occur in a variety of ways. It may be due to new growths, orbital, nasal, or intracranial; to organised inflammatory exudates in connection with orbital cellulitis; to deformity in the skull; to hemorrhage into the sheath of the nerve; or the formation of callus after a fracture of the base of the skull.

Tumours of the orbit, which produce optic atrophy from compression of the nerve, may arise in structures around it 
or in the nerve itself; the latter may be extradural or intradural (see page I78). Tumours may sometimes start in the intercranial portion of the nerve.

Solid or cystic tumours may press on the chiasma, starting in either the pituitary body or infundibulum. They frequently produce a characteristic defect of vision, bitemporal hemianopsia, loss of colour vision in the upper part of each temporal field being the first sign. At the chiasma the nerve fibres coming from the nasal half of each retina decussate, those coming from the temporal halves being continued on to the optic tract of the corresponding side without decussation. It is only from pressure on the chiasma at the point of decussation that atrophy of the fibres going to the nasal halves of the two retinæ takes place, and loss of the temporal halves of the two fields occurs.

A tumour at the chiasma which at first causes bitemporal hemianopsia may extend, so as to involve the whole of the nerve or tract on one side, and produce complete blindness of one eye. On still further development both optic nerves may be involved and complete blindness result.

Though bitemporal hemianopsia is a typical symptom of tumour of the pituitary body, other forms of defect of sight may occur from such growths. In twenty-two cases ${ }^{1}$ in which the diagnosis was confirmed postmortem in 23 per cent. the loss of sight was bitemporal, in 23 per cent. unilateral temporal, in 22 per cent. concentric, in 13 per cent. central scotoma, in 9 per cent. homonymous, in 9 per cent. only a sector remaining, and in 4 per cent. irregular.

Tumours in this region, besides the loss of sight, are frequently accompanied by acromegaly, abnormal adiposty, loss of sexual desire, or arrested growth.

In connection with the deformity of the skull known as " "tower skull" or "oxycephaly" atrophy of the optic nerve, simple and postpapillitic, has several times been observed. In some of these skulls a narrowing of the optic foramina has been discovered, to which the atrophy may be attributed.

${ }^{1}$ Quoted by Macnab. Trans. Ophth. Soc. of the U. K., XXIX, I909, I4I. 
In others an alteration in the position of the great wing of the sphenoid pushing forward the dorsum sellæ turcicæ, so that it caused pressure on the optic nerves or chiasma. Other deformities of the skull besides oxycephaly are sometimes accompanied by optic atrophy.

The optic tracts may be the seat of growths or become pressed upon by tumours in the brain. They also become affected by pressure in cases of hydrocephalus.

Lesions in the brain or optic tracts which lead to atrophy of the optic nerves, owing to the decussation of fibres which occurs at the chiasma, affect the vision of both eyes. As such lesions are usually unilateral the symptom to which they give rise is homonymous hemianopsia, i.e., the loss of the whole or part of the temporal field of vision in one eye, and the whole or part of the nasal field of vision in the other.

In complete homonymous hemianopsia the dividing line between the blind and seeing halves of the fields of vision, when mapped out by the perimeter, is found to run in a vertical direction except in the vicinity of the fixation area. It there curves in such a way as to include that region in each of the seeing halves. Presumably fibres from each fovea decussate at the chiasma and extend to both sides of the brain.

The hemiopic pupil reaction, or Wernicke's light test, helps to differentiate lesions which give rise to homonymous hemianopsia situated above the basal or primary optic ganglia from those below. In the latter, light thrown on the blind half of the retina does not proauce such a brisk reaction of the pupil as when thrown upon the seeing half, while in the former the amount of reaction is equal upon whichever half it is thrown.

2. Primary atrophy of the optic nerve includes the atrophy which occurs in connection with disseminated sclerosis, with tabes and general paralysis, and hereditary optic atrophy. 
In the atrophy which occurs in connection with disseminated sclerosis, isolated areas are found in the nerve in which there has been an active proliferation of the connective tissue and glia cells. As these newly formed cells organise and contract they compress the nerve fibres so that their medullary sheaths become broken up and absorbed. The axis-cylinders for a long time remain unchanged in the affected area, in which particular there is a marked difference from what occurs in tabetic atrophy, where the axis-cylinders soon become destroyed. This prolonged resistance of the axis-cylinders, in sclerosis of the nerve, allows of the transmission of visual impulses through the affected part until the late stages of the affection.

Tabetic Atrophy. ${ }^{1}$ - The atrophy of the optic nerve which occurs in connection with tabes and general paralysis manifests itself clinically as a simple atrophy, being unaccompanied by any preceding signs of papillitis. It usually occurs early in the course of the disease, and is always bilateral, though one eye is often involved before the other. The impairment of sight is usually preceded, in some cases for a considerable time, by the pupillary phenomena known as "Argyll Robertson pupil," in which its reaction to light is lost whilst that to accommodation and convergence is maintained. The pupils are also often unusually small "Spinal Miosis." The visual defect generally commences by some loss of dark adaptation, then contraction of the field and defective colour perception, which are followed by impairment of the acuity of central vision. The visual fields show, however, remarkable variability, and in different cases there is a marked disproportion between the loss of visual acuity and the loss of visual field. The frequency with which optic atrophy occurs in connection with tabes has been differently estimated by different observers, some putting it as high as 50 per cent., others as low as 19 per cent.

Just as there is considerable uncertainty in tabes, as to the nature of the pathological process and its starting point in

${ }^{1}$ Paton. Brit. Jour. of Ophth., VI, 1922, 289. 
connection with the spinal cord, so also is there in connection with the optic nerve.

The pathological process in both situations has been attributed to: (i) neuronic degeneration commencing in the ganglion cells, (ii) selective action of the virus on the nerve fibres, (iii) the formation of syphilitic granulation tissue in the connective tissues heaths, and its extention along the lymph channels in the nerve.

In connection with the spinal cord the starting point of the affection has been located in the posterior columns, the posterior root ganglia, and the posterior roots. In connection with the optic nerve it has been located in the ganglion cells of the retina, in the intra-orbital portion of the nerve and in the intracranial portion of the nerve, the chiasma or optic foramen.

There can be little doubt that the optic atrophy of both tabes and general paralysis is due to a local production of toxins by the spirochætes, though so far all attempts to find them in the nerve have failed.

In opposition to the view that the nerve atrophy is secondary to degeneration of the ganglion cells of the retina, it has been pointed out:-(i) that the retinal changes are similar to those met with after experimental section of the nerve; (ii) that they are unlike those which follow degeneration of the ganglion cells from ischæmia, as in embolism of the central artery or in quinine poisoning; (iii) that in several early cases which showed definite changes in the nerve no changes were found in the retina; (iv) that in cases with complete atrophy of the optic nerve a few quite normal ganglion cells have been found still present in the retina.

The presence of some infiltration in the optic nerves in cases of tabes with optic atrophy has been demonstrated in a series of cases, it consisted chiefly of plasma cells in the pial sheath extending into the nerve along its septa, either in the region of the chiasma or in course of the nerve, and 
often especially well marked about the entrance of the central vessels into the nerve.

A suggestion which has been made which would help to reconcile many of the different findings, and also explain the varying ways in which the visual loss occurs, is, that the nerve degenerations and the connective tissue degenerations are coordinate results, due to the presence of the spirochætes in both tissues.

Hereditary Optic Atrophy ${ }^{1}$ (Leber's Disease).-This disease commences usually at the age of twenty and occurs more frequently in men than women. It is transmitted usually through the mother who is unaffected. Direct is less common than collateral inheritance.

The affection comes on in both eyes either rapidly or gradually with failure of sight, due to the formation of a central scotoma, which generally becomes absolute for white and colours. Peripheral vision as a rule is unaffected. After the central scotoma has become absolute the condition usually remains stationary, rarely some sight is regained, still more rarely the case goes on to complete blindness. Ophthalmoscopically no changes are at first seen; in some cases a slight papilledema occurs, the margin of the disc becoming blurred; and later atrophy sets in. In others pallor of the temporal half or of the whole disc slowly develops, without any preceding symptoms of papilledema.

Up to the present no pathological examination has been made of the affected parts in these cases, so that we are still ignorant as to the real nature of the disease.

Two hypotheses have been suggested to explain its manifestations. One regards it as an abiotrophy, due to deficient vital energy of the structures implicated, so that they are unable to maintain their nutrition much beyond full development, and therefore gradually degenerate. ${ }^{2}$ The other attributes it to some temporary disturbance in

${ }^{1}$ S. H. Habershon. Trans. Ophth. Soc. of the U. K., VIII, I888, 190.

${ }^{2}$ Sir William Gowers. Medical Ophthalmoscopy, 4th Ed., I904, 283. 
the pituitary body of moderate degree about puberty. ${ }^{1}$ This hypothesis has received some support from $\mathrm{x}$-ray examinations of the region of the sella turcica, but several negative results from such examinations have also been reported.

(d) Degenerations of the Retinal Pigment Epithelium.In degenerations of the pigment epithelium the cells may either atrophy and disintegrate, or reassume some of their embryonic characteristics, i.e., they may proliferate at an increased rate and form hyaline material. This layer of cells is nourished by the capillaries of the choroid and it degenerates when the capillary layer is from any reason destroyed.

When the retina is detached and the choroidal circulation unchanged, the pigment epithelial cells, though no longer required to fulfill their normal function receive their usual nutrient supply. Under such circumstance they proliferate and become several layers thick. The cells in the innermost layers swell up and become vacuolated, globules of fat form in them, and finally they break down liberating fatty material and granules of pigment into the subretinal fluid. As the outcome of the fatty changes cholesterine crystals may be formed, either in the subretinal fluid or in the masses of cells on the inner surface of the elastic lamina. When the pigment epithelial cells rapidly proliferate they are often less deeply pigmented than usual, and sometimes have only a few scattered granules in them.

In atrophic conditions of the retina or choroid when the two membranes become united, as after division of the posterior ciliary arteries, rupture of the elastic lamina, sclerosis of the choroidal vessels, and in the affection termed "retinitis pigmentosa," pigmentation of the retina takes place.

The pigment epithelial cells when they proliferate, having phototactic movements, are attracted inwards towards the light. The rods and cones having atrophied, gaps are left

${ }^{1}$ J. H. Fisher. Trans. Ophth. Soc. of the U. K., XXXVI, I9I6, 298. 
in the membrana limitans externa, through which the pigment epithelial cells can wander into the substance of the retina; if they undergo degeneration there and rupture, the pigmented granules become dispersed.

The membrane of Bruch or elastic lamina of the choroid when stained with eosine appears a perfectly homogeneous structure. When, however, it is stained with Weigert's elastic tissue stain it is seen to be composed of two layers, an inner which is homogeneous and becomes diffusely blue, and an outer which is finely reticular and takes on the deep blue colour characteristic of elastic tissue. ${ }^{1}$

The difference between the two layers shows best where the membrane is thickest, that is, in the vicinity of the optic disc. At the margin of the papilla they end at different levels, the inner one ceasing with the pigment epithelium, and the outer continuing on for some little distance into the substance of the papilla.

These two layers of the membrane of Bruch have a different origin, the inner homogeneous layer, like the capsule of the lens and Descemet's membrane, which it closely resembles, being formed as a kind of secretion from the pigment epithelium cells which line it, ${ }^{2}$ and the outer reticular elastic fibre layer being continuous with the elastic fibre network of the choroid.

The inner homogeneous part of the membrane of Bruch, though it becomes slightly thickened with the advance of years, is mainly a product of the pigment epithelial cells during embryonic life. Under normal conditions the capacity which the cells have of producing hyaline tissue ceases in the fully developed eye. Under pathological conditions the pigment epithelium reacquires this hyalineproducing capacity, and nodules of it may be formed on the inner surface of Bruch's membrane (Fig. 28I). They occur in connection with various atrophic conditions of the

${ }^{1}$ G. Coats. R. Lond. Ophth. Hosp. Reps., XVI, 1905, I64.

${ }^{2}$ Treacher Collins. "Researches into the Anatomy and Pathology of the Eye," I 896, p. 87. 
choroid, but are sometimes met with as a senile affection when the other parts of the eye are apparently healthy, and sometimes as an hereditary bilateral disease, a form of abiotrophy.

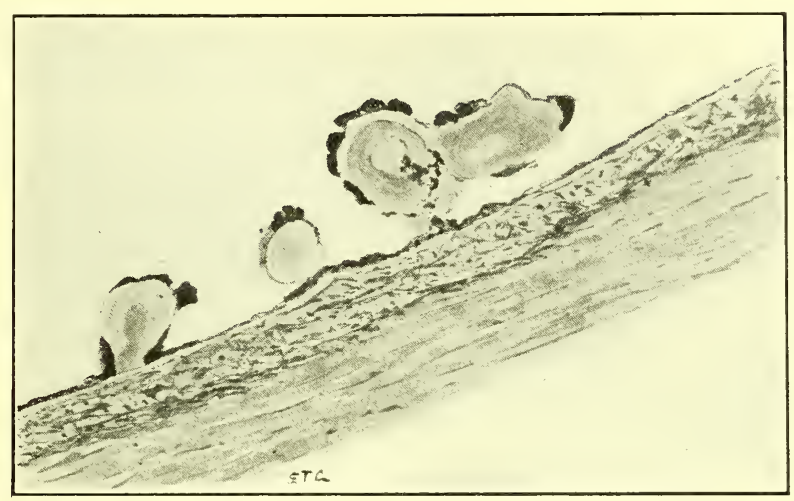

FIG. 28r.-Hyaline formations on the inner surface of the elastic lamina of the choroid.

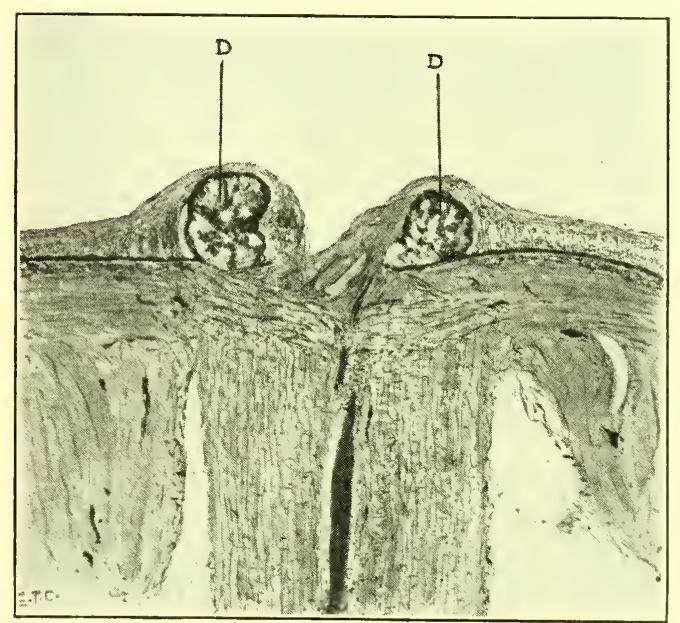

FIG. 282.-Hyaline formations in the head of the optic nerve at the termination of the elastic lamina of the choroid and pigment epithelium.

The senile hyaline or colloid formations are most frequently met with around the macula, and give rise ophthalmoscopically to small oval or circular, pale, yellowish-white 
dots. The condition has been termed central guttate choroiditis, Tay's choroiditis, and also more accurately senile hyaline infiltration of the retina.

In rare cases similar hyaline nodules form in connection with the termination of the elastic lamina at the margin of the optic disc, giving rise to elevations in it (Fig. 282).

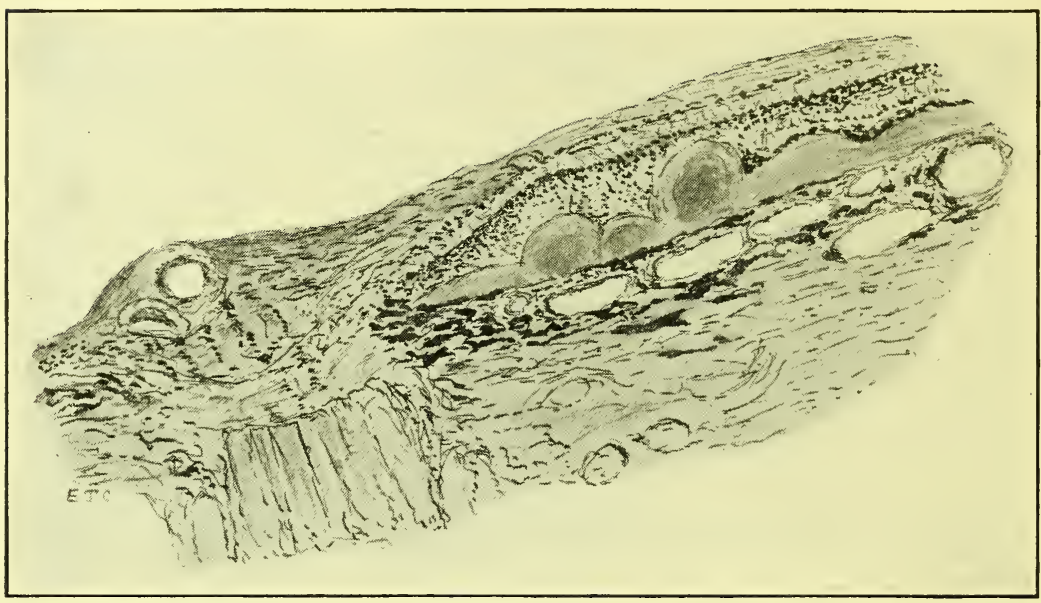

FIG. 283.-Section showing changes in hereditary hyaline infiltration of the retina. Doyne's family or honeycomb choroiditis.

These have to be differentiated from another form of hyaline degeneration met with in connection with the head of the nerve to be referred to later (see page 648). Their situation, always at the margin of the disc, and continuity with the elastic lamina are their chief distinguishing characteristics.

Hereditary hyaline infiltration of the retina, also known as Doyne's ${ }^{1}$ honeycomb family choroiditis, may appear in early adult life, at first it gives rise to no disturbance of vision and its progress is exceedingly slow, so it is frequently impossible to fix the exact time at which it does commence. Light coloured patches form in the neighbourhood of the optic

${ }^{1}$ R. W. Doyne. Trans. Ophth. Soc. of the U. K., XIX, I 899, 7 I. Ibid., $\mathrm{XXX}, 1910,274$. 
disc and macula, sometimes with, and sometimes without, any pigmentary disturbance. In course of time the patches become larger and join up with one another, as they extend in the macular region, so the vision becomes more and more affected. The two eyes are always involved, and the disease having once started steadily and slowly progresses. It is hereditary, affecting several members of the same family in different generations.

Microscopically ${ }^{1}$ a new formation of hyaline substance is found situated between the retina and choroid, the inner surface of which presents nodular elevations with depressions between them (Fig. 283). In places the hyaline material is covered by a single layer of pigment epithelial cells, in others it is devoid of any such covering, whilst here and there in the recesses between the nodules heaped up masses of pigment cells are seen. The only changes to be found in the retina and choroid are such as can be accounted for by the pressure of the hyaline mass, which is evidently of primary formation, and attributable to some hereditary degeneration in the pigment epithelium. It is a condition very similar to the senile degeneration previously described but differs from it in beginning much earlier in life, in the size and steady enlargement of the patches, and in being hereditary. It is best classed as an abiotrophy of the pigment epithelium.

(e) Degenerations of the Neuroglia of the Retina and Optic Nerve.-When the nerve elements of the retina atrophy its supporting connective tissue sometimes increases in amount. These thickened areas of neuroglia may subsequently undergo cystic, fatty or hyaline changes.

In the eyes of old people there is frequently found in the retina, in the neighbourhood of the ora serrata, some atrophy of the nerve elements with secondary hypertrophy of the neuroglia in which small cystic spaces form. The condition is probably the result of sclerosis of the retinal capillaries.

1 Treacher Collins. Ophthalmoscope, IX, I9I3, 537. 
The formation of cystic spaces in the neuroglia of detached retinæ is described on page 288 .

The cells of the neuroglia both in the retina and in the optic nerve sometimes undergo a hyaline or amyloid degeneration, either secondary to nerve degeneration or as a primary affection.

Hyaline Nodules in the Optic Disc.-Hyaline bodies in the form of masses of small rounded, translucent nodules, sometimes protrude forward from the centre of the disc, but may proceed from its margins. Both eyes are usually affected; the vision is unimpaired. It is probably a condition which commences in early life and progresses with extreme slowness. It has several times been found associated with retinitis pigmentosa.

The nodules of hyaline material are found microscopically to be grouped around the central retinal vessels, in front of the lamina cribrosa, and show a faint lamination. Sometimes they contain calcareous granules.

It is clear from their position that they are not the product of the pigment epithelium, or in any way connected with the membrane of Bruch, though they present the same microscopical appearances and give the same chemical reactions as the hyaline nodules met with in connection with it. These latter nodules, as already stated, are due to the perverted action of the pigment epithelial cells of the retina, which are derived from the outer layer of the secondary optic vesicle. The cells which form the neuroglia of the optic nerve have a similar origin, being formed from the pedicle of the secondary optic vesicle, into which the axions of the ganglion cells grow. It seems probable therefore that these cells might also, under perverted condition, produce a hyaline substance, and that the nodules met with in the optic disc are derived from them. It has been already mentioned that in retinitis pigmentosa, an affection in which these hyaline nodules on the disc are sometimes met with, there is an abnormal overgrowth of neuroglia that would be very likely to manifest degenerative changes. 
Hyaline nodules, which must evidently be derived from neuroglial tissue, are found in the lining membrane of cystic protrusions from congenitally microphthalmic eyes (see page 20). This lining membrane is composed of tissue derived from the secondary optic vesicle, and immediately external to it is fibrous tissue continuous with the sclera-no choroid and no elastic lamina or pigment epithelium intervening-so that the only tissue from which the hyaline nodules could have taken origin is that derived from neural epiblast.

Amyloid Bodies in the Optic Nerve and Retina.Amyloid material like hyaline presents microscopically a

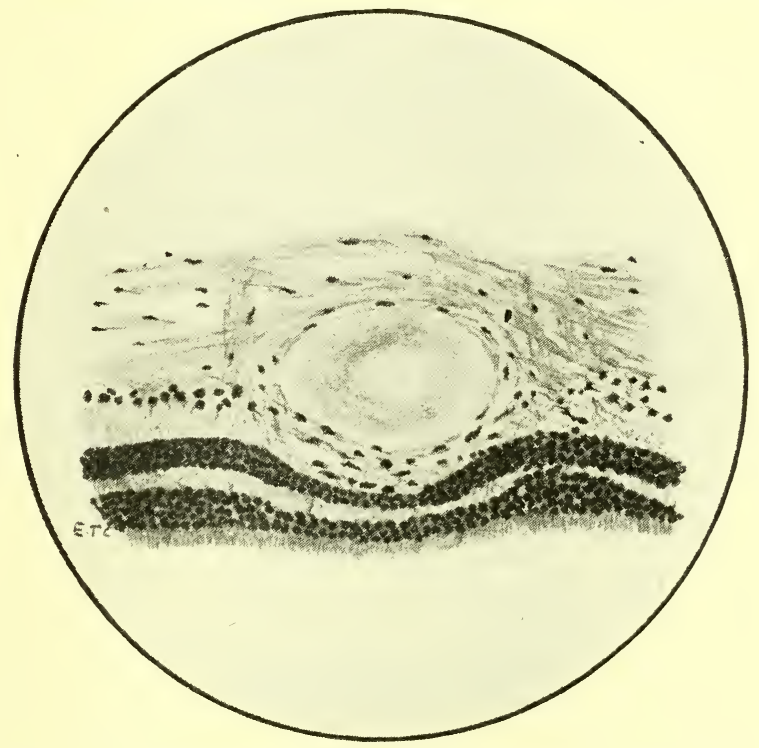

FIG. 284.- Section showing an amyloid body in the retina.

homogeneous appearance; it is, however, distinguished from it by turning a brownish-red colour when stained with iodine, and a purple-red colour with methyl violet. It is often found in association with hyaline material, and also with an homogeneous substance which gives staining reactions that are not quite typical of one or the other. It seems probable that hyaline is derived from the amyloid 
substance, and that the atypically staining material represents an intermediate stage in the transformation.

Corpora amylacea, similar to those found in the central nervous system, are met with in the optic nerve and retina when atrophied, and in elderly people when no other signs of atrophy are present. They consist of varying sized, round or oval bodies, of a homogenous structure, encircled by a well defined capsule which stains a bright red with eosin. They show only very slight tendency to lamination, and always remain discrete, never forming conglomerations (Fig. 284). They lie between the nerve fibres, and in the optic nerve are met with mostly in the intercranial portion or in the chiasma, whilst in the retina they are usually in the nerve fibre layer, rarely in the ganglion cell layer and never external to it. They have been met with in the optic papilla, and in one case hyaline bodies were found in the optic papilla and amyloid bodies in the retina in the same eye. ${ }^{1}$

( $f$ ) Degenerations of the Vitreous Humour.-The vitreous humour is composed of a network of delicate fibres, showing some tendency to arrangement in layers, which radiate from the region of the ciliary body to that of the central canal of Cloquet.

It possesses only a few fixed cells which are situated, widely separated apart, in the external limiting membrane and at its position of attachment to the optic disc.

The vitreous humour may not inaptly be compared to a sponge filled with water, the tissue of the sponge corresponding to its network of fibres and the water in the sponge to the fluid in the network.

It has only a low degree of vitality, and having once reached its full dimensions manifests no powers of increased growth or of repair. The fluid which circulates in it, and from which it receives nourishment, is derived from the cells of the pars plana of the ciliary body, with which its

${ }^{1}$ G. Coats. Trans. Ophth. Soc. of the U. K., XXXII, I9I2, I I9. 
framework retains some delicate connections. When loss of vitreous takes place through a wound, no new frame work of tissue is formed, but fluid of the same consistency as the aqueous humour and derived from the ciliary body, fills the vacant space.

The vitreous humour may undergo degeneration as the result of atrophic changes in the ciliary body, due to inflammatory or hemorrhagic effusions into it, from increase in size of the vitreous chamber, and as a primary affection apart from any other manifest changes in the eyeball.

Changes in the vitreous the result of atrophy of the ciliary body, and of inflammatory and hemorrhagic effusions into it, have already been described (see pages 255 and 469 ).

When the vitreous chamber becomes enlarged, as it does in axial myopia, no new formation of the fibrous network of the vitreous humour taking place, it becomes either more rarefied and fluid in consistency or its hyaloid membrane becomes detached from the retina (see page iा6).

Primary degeneration of the vitreous humour which occurs mostly as a senile condition, causes its fibrous network to become destroyed so that it acquires a fluid instead of a jelly like consistency. In this fluid fragments of the network float about on movements of the eye and can be seen as floating opacities, both by the patient, and by the surgeon ophthalmoscopically. The degree and rapidity of movement of the opacities permits of some opinion being formed as to the amount of liquefaction which has occurred.

A fatty change may sometimes take place in the fibrils resulting in the deposition of crystals of cholesterin, what is termed Synchisis Scintillans being produced. When viewed with the ophthalmoscope these floating crystals reflect light from their flat surfaces, giving rise to the appearance of a shower of gold and silver spangles. It is remarkable how very little disturbance of vision may be associated with this very striking ophthalmoscopic change. 
Detachment of the vitreous humour may be produced in pathological specimens as the result of hardening reagents, but it does also occur during life, either from degeneration due to disturbances of nutrition or from the shrinking of exudates.

Normally the vitreous humour is firmly attached to the optic disc, where during fetal life the central artery passes into it, and to the pars plana of the ciliary body at the ora serrata, from cells connected with which its matrix is derived. Anteriorly its cupped surface, the patellar fossa, is seen by slit-lamp microscopy not to be everywhere in contact with the back of the lens, a relatively "optically empty space" filled with fluid intervening. This corresponds with the region in which the central hyaloid artery, after having divided up into separate branches, spreads out in front of the vitreous humour to form the vascular sheath of the lens.

On the outer surface of the vitreous from the ora serrata to the optic disc stretches a definite layer of hyaline membrane, containing flattened cells widely spaced apart. On the anterior surface of the vitreous, where it covers the posterior surface of the suspensory ligament and the back of the lens, a definite hyaline layer is less easily differentiated. Slit-lamp microscopy reveals behind the retro-lental optically empty space a characteristically folded membrane.

The commonest form of detachment of the vitreous is what is termed "infundibular detachment" (Fig. 29I), in which it becomes separated from the retina everywhere from the optic disc to the ora serrata in tent like fashion. When in addition to detachment in these positions it also becomes torn away from the optic disc it is termed a "globular detachment" (Fig. I90); sometimes in such globular detachments, a circular hole can be seen ophthalmoscopically in the hyaloid membrane, at the position where it was attached to the optic disc. ${ }^{1}$

${ }^{1}$ W. T. Lister. Internat. Cong. of Ophth., Washington, 1922, 60. 
Detachments of the vitreous humour from the suspensory ligament of the lens, that is extending from the ora serrata to the margin of the patellar fossa, are termed "anterolateral detachments" (Fig. 222).

(g) Degeneration of the Pars Ciliaris Retinæ and Pars Iridis Retinæ.-In the ciliary body the hyaline membrane external to its pigment epithelium may, like the elastic lamina of the choroid, show some increase of thickness with the advance of years. A senile hyperplasia also occurs in both the pigmented and unpigmented retinal layers of the ciliary body, the latter may become protruded inward in the form of folds and, as already described, give rise to cysts (see pages 233 and 234).

A peculiar form of degeneration is met with in the pigment epithelium on the back of the iris in cases of diabetes (see page 606).

Degenerative changes of a hyaline character in the dilator muscle fibres of the iris, which, as already stated, are developed from the anterior layer of the secondary optic vesicle on the back of the iris, are the probable cause of the resistance of the pupil to dilatation in elderly people, what is termed senile miosis. This miosis, by the stenopaic effect it produces, has sometimes the effect of enabling old people to read with weaker glasses than would be expected for the correction of their presbyopia.

\section{DEGENERATIONS IN TISSUES DERIVED FROM MESOBLAST}

The degenerations in the tissues of the eye derived from mesoblast may be divided into those of: (a) fibrous tissues; (b) blood vessels; (c) muscles; and (d) inflammatory exudates.

(a) Degenerations in the Fibrous Tissues.-Degenerations in the outer fibrous tissue coat of the eyeball occur more frequently in the avascular cornea than in the vascular sclerotic. 
Degenerations in the cornea may be due to disturbances in its lymph supply, to senility or abiotrophy.

Transverse Calcareous Film of the Cornea.-A transverse calcareous film of the cornea consists of a superficial band of opacity, which extends across it in the line of the palpebral fissure, a little below the centre. Its upper border is usually concave and the lower nearly straight; hence its extremities are broader than the centre. It never quite reaches to the corneal margin on either side, a clear area always intervening. The two extremities of the film are usually denser than the centre, and on magnification small grey and white dots are seen in it. When commencing it consists of two separate opacities, one on each side of the cornea, which gradually extend inward until they unite. Its formation is exceedingly slow and a complete band may take several years to develop. It is met with mostly in shrunken eyes which have had cyclitis, or in blind glaucomatous eyes, but may form in the eyes of elderly people which in all other respects appear healthy.

On pathological examination, ${ }^{1}$ the cornea, in the situation of the film, is found to be thicker antero-posteriorly than elsewhere. This thickening is due to the new formation of laminated, non-vascular, fibrous tissue anterior to Bowman's membrane. Bowman's membrane itself, in the situation of the film, is divided up into short pieces, the gaps between which are filled with fibrous tissue (Fig. 285). Lying on the anterior surface of the membrane, and encrusting it, are calcareous granules which stain deeply with hematoxylin, and evolve bubbles of gas on the application of acids. The epithelium covering the film is either thickened or degenerate.

A new formation of fibrous tissue anterior to Bowman's membrane is also met with in eyes which have had glaucoma or inflammation of the uveal tract, when there has been a formation of vesicles on the cornea. These are due to edema of its anterior layers. The similarity of the condi-

${ }^{1}$ C. H. Usher. R. Lond. Ophth. Hosp. Reps., XIII, I893, p. 508. 
tion pathologically to transverse film of the cornea suggests, that it also may commence as a localised edema.

The lymph spaces which pass antero-posteriorly through Bowman's membrane become distended, and the surface epithelium raised in places, by the accumulation of albumin-

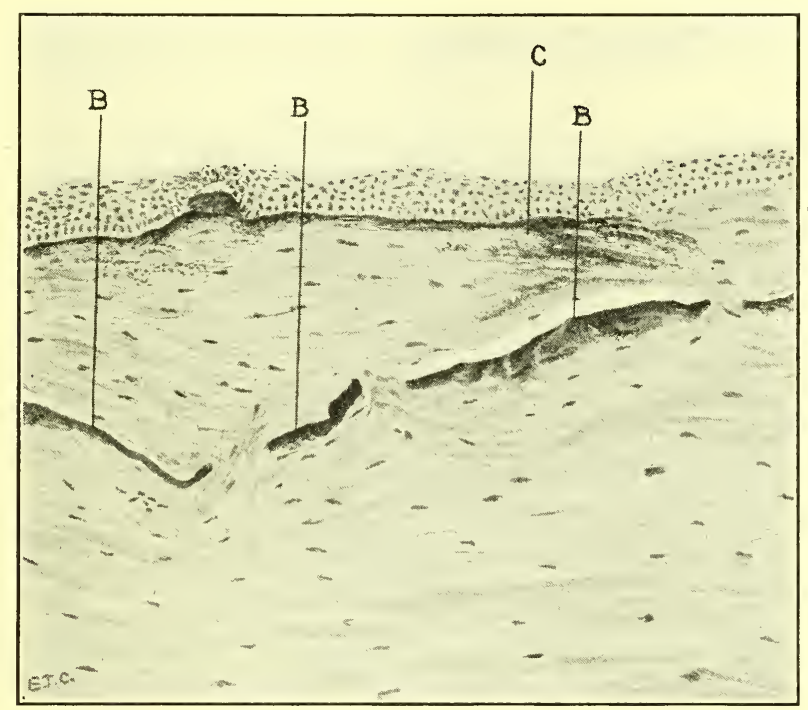

FIG. 285.-Section through a transverse calcareous film of the cornea. $B$, points of pieces of Bowman's membrane encrusted with calcareous granules. Anterior to Bowman's membrane and in the enlarged nerve channels in it is newly formed laminated fibrous tissue. $C$, points to calcareous material in this fibrous tissue immediately beneath the surface epithelium.

ous coagulable fluid beneath it. Cells, formed by proliferation of the corneal corpuscles, then extend into the fibrinous matrix in the dilated lymph channels and beneath the epithelium. These cells develope into fibrous tissue, which lying in the channels in Bowman's membrane split it up into separate pieces. The nutrition of these pieces of Bowman's membrane becoming impaired calcareous salts are formed in connection with them.

A transverse calcareous film forms, as already stated, opposite the palpebral fissure, i.e., in the part of the cornea most exposed to atmospheric influences, and that least 
pressed upon by the lid margins in winking. Though exposure may play some part in its production, it is not the sole cause. The affections of the conjunctiva opposite the palpebral aperture, which are due to exposure, viz., pterygium and pinguecula, are not found associated with transverse calcareous film of the cornea.

It seems highly probable, that a want of the normal amount of pressure of the lid margin over the front of the cornea, is an important factor in its formation. The curved upper margin of the film corresponds to the curved margin of the upper lid. Normally in winking the edges of the lids have a sort of squeezing action on the surface of the cornea, being kept closely in contact with it by the muscle fibres of Riolani. They considerably depress the surface epithelium and flatten out any inequalities, thus tending to prevent any accumulation of fluid between it and Bowman's membrane. The conditions in which this action of the lids is interfered with are those which favour the formation of a transverse film. When the eyeball is shrunken it is recessed away from the lids. When the cornea is anæsthetic, as in glaucoma, the normal afferent stimulus for winking is abolished.

Arcus Senilis.-The opacity which forms in the periphery of the cornea in elderly people, termed "arcus senilis," is probably due to a fatty degeneration of substantia propria of the cornea. It commences in the form of two narrow grey arcs, one at the upper and the other at the lower border of the cornea, these tend to lengthen at their extremities until they become united, and a complete ring of opacity is then produced. The outer margin of the opacity is sharply defined, and is separated from the limbus by a semi-diaphanous zone. The inner margin, on the other hand, shades off gradually into the clear central area of the cornea.

Microscopically the opacity is found to be due to a number of highly refracting globules of various sizes which are situated in Bowman's membrane, in the fibrous lamellæ, 
and in the corneal corpuscles. The sharp outer margin of the opacity corresponds in position to the termination of Bowman's membrane, which does not extend quite to the extreme periphery of the clear cornea. As a large number of the globules in the affected area are situated in Bowman's membrane, the extreme periphery, where it is absent, appears comparatively clear.

In the substantia propria the degeneration is most marked in the anterior layers fading off in the deeper ones, though the tissue immediately adjoining Descement's membrane is often markedly affected. In an antero-posterior section through the cornea the opaque zone is seen to extend deeper toward the perphery than toward the centre. Some globules are occasionaly found in the deep layers of the sclerotic.

There has been much difference of opinion as to the nature of the globules to which the opacity is due, mainly because they are not stained black by osmic acid. With that stain their outlines become sharply defined and they refract light more highly, but they do not turn black. With Sudan III, ${ }^{1}$ however, they stain in every way like fat, and their distribution and arrangement can then be clearly seen. They are dissolved by both ether and chloroform. It seems, therefore, that they must be regarded as a form of fat which does not turn black with osmic acid.

A few cases have been recorded of an unusual form of degeneration of the cornea in which, apart from any inflammation, a grey opacity slowly spreads from the margins inwards, to a greater extent above and below than at the sides, so that only a central oval area of clear cornea remains. $^{2}$ The affection is symmetrical in the two eyes and has been met with in one case in a patient aged $2 \mathrm{I}$. Sometimes, but not always, vessels continuous with those of the conjunctiva at the limbus extend into the affected area. So far nothing is known as to the pathology of the affection.

${ }^{1}$ J. H. Parsons. R. Lond. Ophth. Hosp. Reps., XIII, I893, p. 508.

${ }^{2}$ Treacher Collins. Trans. Ophth. Soc. of the U. K., XXIV, I904, 45 and XXIX, 1909, 225. 
Marginal Atrophy of the Cornea and Marginal Keratectasia. ${ }^{1}$ - This is usually a bilateral condition which occurs in elderly or middle aged persons, and is nearly always associated with an arcus senilis. A groove develops, without any signs of ulceration or inflammation, in the upper peripheral part of the cornea between its margin and the arcus. The outer edge of the groove shelves gradually whilst its inner edge is abrupt and steep; its floor is almost transparent and is traversed by fine blood-vessels which terminate in loops. The furrow tends slowly to increase, and in course of years may extend completely round the cornea. In its later stages the thinned cornea tends to give way and an ectasia is formed, which alters the curvature of the cornea so as to produce an astigmatism against the rule, with corresponding impairment of vision. Slight violence may cause a rupture of the ectasia.

Pathological examination in these cases has shown, that the furrow is due to a loss of the superficial lamellæ of the cornea, which terminate rather abruptly at its central edge, and are replaced by loose tissue like that of conjunctiva. Bowman's membrane ceases some distance from the edge of the furrow; the membrane of Descemet which lines it posteriorly is at first intact, but becomes ruptured when bulging takes place. There is no inflammatory infiltration in the lamellæ left in the floor of the furrow, but minute fatty granules have been found in them, otherwise they show no changes. No cause for the condition, apart from senility and fatty degeneration, has so far been ascertained.

Hyaline Thickenings in Connection with Descemet's Membrane and the Ligamentum Pectinatum.-The structures composed of hyaline in the normal eye are the capsule of the lens, the inner part of Bruch's membrane, and Descemet's membrane. The two former, as already pointed out, are produced by the epithelial cells lining them. Descemet's membrane is developed in the same way from its lining endothelial cells. Just as the epithelial cells

${ }^{1}$ G. Coats. Trans. Ophth. Soc. of the U. K., XXXI, I9II, 5. 
lining the two first-named membranes, under pathological conditions produce abnormal formations of hyaline, so may the endothelial cells lining Descemet's membrane; and the endothelial cells, which are of a similar character, on the anterior surface of the iris.

After wounds of Descemet's membrane the endothelial cells which extend across the gap left by its retracted ends will, in course of time, secrete a new layer of hyaline tissue ${ }^{1}$

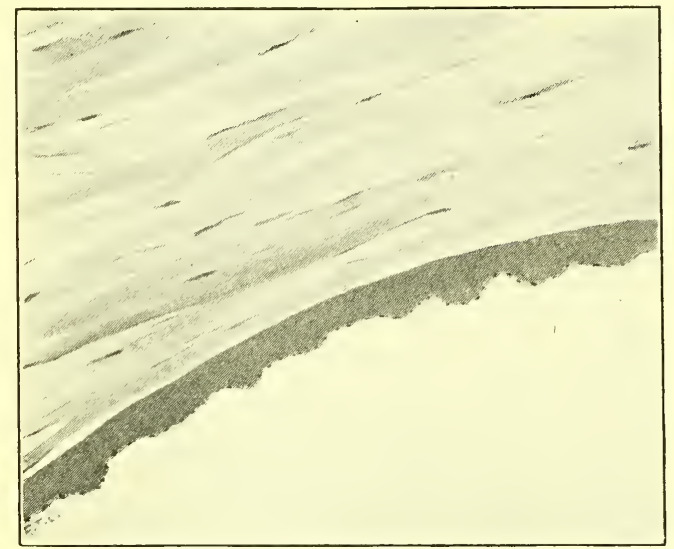

FIG, 286.- Hyaline excrescences on the inner surface of Descemet's membrane.

(see page 369). As a senile change small hemispherical projections of hyaline material sometimes form on the inner surface of Descemet's membrane, usually toward its periphery (Fig. 286). They are comparable to the hyaline nodules which occur on Bruch's membrane. Viewed by slit lamp-microscopy they appear as black, round, pit-like depressions in the olive yellow hexagonal mosaic of the endothelium, and are often confluent.

In cases of glaucoma, especially glaucoma in early life (buphthalmos), where there is a peripheral adhesion of the iris to the cornea, a new formation of a hyaline membrane on the anterior surface of the iris may be produced beneath its layer of endothelial cells. This new membrane generally

${ }_{1}^{1}$ Treacher Collins. R. Lond. Ophth. Hosp. Reps., XIV, I896, 305. 
appears to be continuous with Descemet's membrane round the angle of the anterior chamber. A similar formation on the anterior surface of the iris is also sometimes found in connection with anterior synechiæ. Excrescences may form in these iritic hyaline membranes, like those described in connection with Descemet's membrane.

The fibres of the ligamentum pectinatum are composed of elastic tissue of a similar nature to Descemet's membrane; both stain a deep reddish brown colour after prolonged immersion in acid orcein, whilst the surrounding substantia propria of the cornea only becomes a dull pink. Like Descemet's membrane they also are probably formed as a kind of secretion from the endothelial cells lining them, and as age advances they tend to become thicker. It has been suggested, that this senile sclerosis of the fibres of the ligamentum pectinatum may cause some obstruction to the passage of aqueous between them, and so favour the development of glaucoma.

Nodular and Reticular Opacity of the Cornea.-In the degenerations met with at the margin of the cornea, arcus senilis and marginal atrophy, no hereditary tendency has been noted, and they are probably due to some disturbance of nutrition. There is, however, a degenerative condition of the centre of the cornea which is markedly hereditary, occurring often in several generations of the same family, and which has all the characteristics of an abiotrophy. This degeneration presents itself in two different forms, either as grey nodules of opacity (Fig. 287) or as a network of fine lines (Fig. 288). In an affected family some members may have the one form and some the other, so that they are probably only different manifestations of the same condition. ${ }^{1}$ The appearances which they present clinically are, however, so different that they have been described as two separate affections.

Both conditions are bilateral, start usually about puberty, and progress very slowly without any signs of inflammatory

${ }^{1}$ Doyne and Stephenson. The Ophthalmoscope, III, I905, 2 I 3. 
reaction. In the nodular variety small, round or irregular, grey, discrete opacities form in the superficial central layers of the cornea, the periphery as a rule remaining clear and unaffected. Over the larger opacities, which may reach the diameter of $1 / 4 \mathrm{~mm}$., the epithelium is slightly raised: between them lie many smaller dust like opacities.

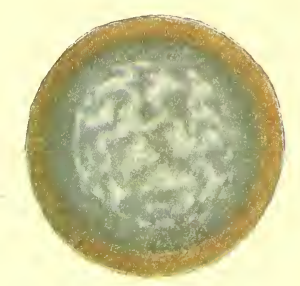

FIG. 287.- Nodular opacity of the cornea in a man aged twenty-six. Case recorded by Treacher Collins, Trans. Ophth. Soc. of the U. K., XXII, I902, I48.

The reticular or lattice-work opacity, like the nodular opacity, is an affection of the central superficial layers of the cornea. It is composed of a network of fine grey lines,

FIG. 288.- Reticular opacity of the cornea in a man aged twenty-four. Case recorded by Treacher Collins, Trans. Ophth. Soc. of the U. K., XIX, I899, 30.

having much the appearance of a cobweb, which may give rise to ridge like elevations of the surface of the cornea. Sometimes small grey dots are also present.

Pathological examination of the nodular variety has shown changes of a degenerative character in the basal 
cells of the epithelium, Bowman's membrane and the superficial layers of the substantia propria. Also the deposition of a substance, often in layers, immediately under the epithelium which gives an acidophile reaction; and a second basophile granular substance in the cornea proper. What the primary condition is in these cases is still uncertain. The arrangement of the opacity in the reticular cases in the form of a network suggests the presence of some anatomical basis. The lines do not represent the tracks left by blood-vessels. In no stage of the disease does the cornea become vascularised, and its peripheral parts always remain clear and free from lines. Any tracks left by blood-vessels would necessarily extend out to the margin of the cornea, to join the vessels from which they were originally derived. The only other anatomical structure in the cornea presenting the appearance of branching lines is its nerve fibres. It has been noticed, in both the nodular and reticular cases, that the sensibility of the cornea to touch is lessened. It may be, therefore, that the primary change in these cases, will be found to be in the central nerve fibres of the cornea or their end-organs, and that the alterations in the fibrous tissue around them is of a secondary nature.

Staphylomata.-The eyeball is a hollow chamber distended with fluid; in a state of health the toughness of its coats is such that they are able to withstand the normal intraocular pressure without stretching or bulging. If the intraocular pressure becomes increased, or the walls of the globe become softened, then some bulging may take place. Often both factors are present. Where the sclerotic bulges it is thinned, and acquires a grey colour from the pigment in the uveal tract showing through. From irregular degrees of bulging the prominence often has a nodular outline, and a fancied resemblance which it may thus possess to a bunch of grapes has led to the condition being termed a staphyloma.

The factors favouring the formation of an ectasia of the walls of the eyeball may be considered under the follow- 
ing headings: (I) age; (2) inflammation; (3) anatomical peculiarities; (4) congenital abnormalities.

I. Age.-The cornea and sclerotic in early life are very expansile structures and gradually toughen as age advances. In glaucoma due to congenital malformation (buphthalmia, see page 86) the walls of the eye, though expansile, are nearly equally resistant in all parts, so that the whole globe becomes enlarged and both cornea and sclerotic are thinned. In late life, high intraocular pressure may exist for a considerable time without any appreciable alteration in the shape of the eyeball.

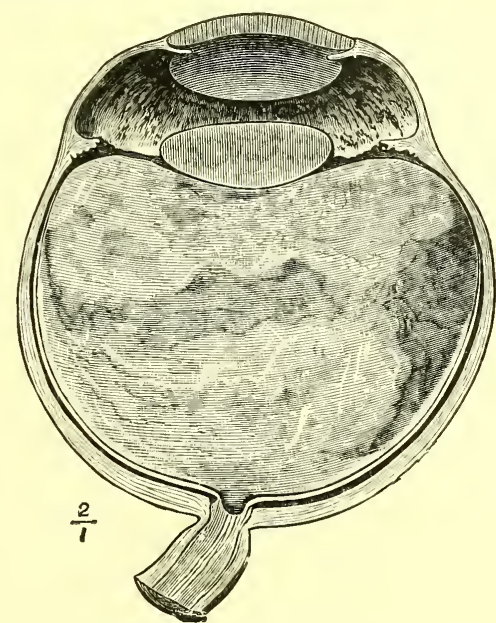

FIG. 289.- Shows an anterio-posterior section of an eye lost from primary glaucoma. There is a staphyloma in front of the ciliary body in the region of the canal of Schlemm (intercalary). The optic disc is deeply cupped. Specimen in the R. Lond. Ophth. Hosp. Museum.

2. Inflammation.-As a result of inflammation a localised softening or thinning of the coats of the eye may take place, so that their resistance becomes lowered, and they stretch and bulge before the normal intraocular tension. Or, they may be sufficiently resistant to withstand normal tension and only stretch, and become thinned. when it is increased.

A staphyloma of the cornea is the outcome of an inflammation of that structure; frequently a perforating ulcer, 


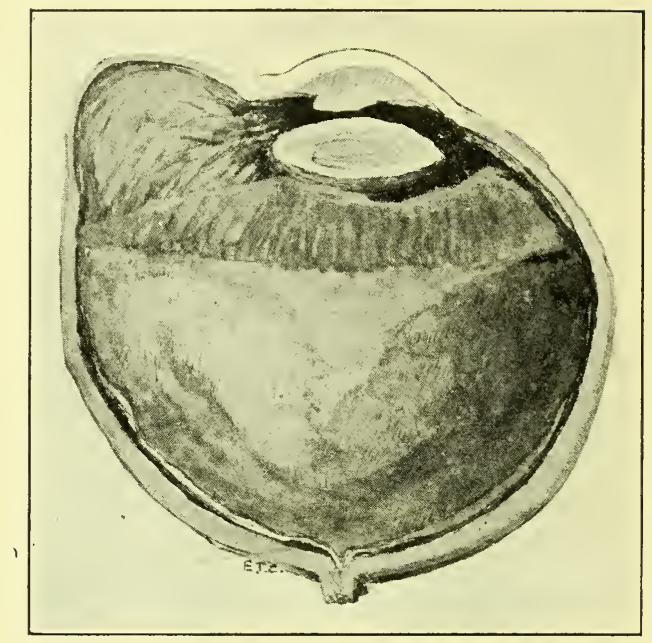

FIG. 290.- Shows an antero-posterior section $\times 2$ of an eye lost from primary glaucoma. On the left side there is a large ciliary staphyloma and considerable atrophy of the ciliary body lining it. Specimen in the R. Lond. Ophth. Hosp. Museum.

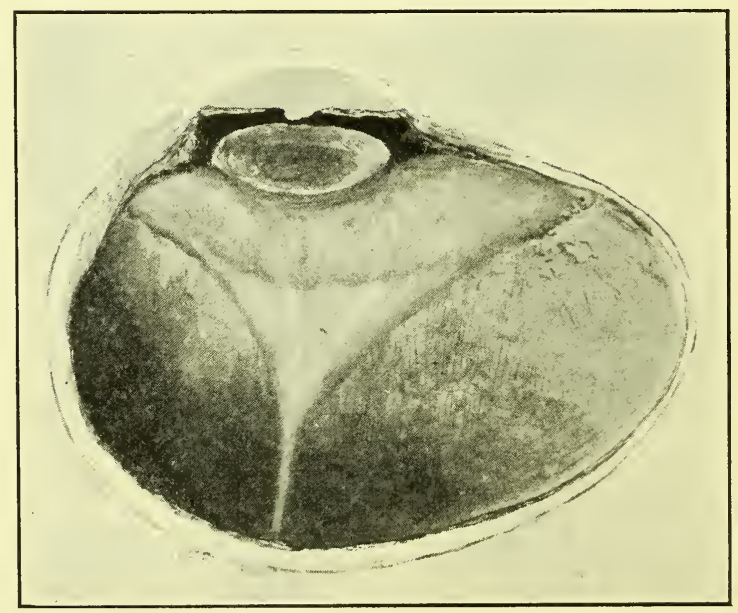

FIG. 29I.-Shows an antero-posterior section $\times 2$ of an eye which went blind from primary glaucoma. On the right-hand side there is a large equatorial staphyloma with considerable thinning of all the coats of the eye. The vitreous humour is shrunken and detached except at the ora serrata and optic disc. Specimen in the R. London Ophth. Hosp. Museum. 
which besides softening the cornea, causes increase of tension from anterior adhesion of the iris (see page 452).

3. Anatomical Peculiarities. - The strength of the fibrous tissue covering of the globe is not equal in all parts. It is weakened at certain positions by the passage through it of blood-vessels and nerves. Ectasias may occur in these positions as the result of increased tension apart from any inflammation.

An intercalary staphyloma is one which occurs in the region of the canal of Schlemm and the anterior ciliary veins, in front of the ciliary body (Fig. 289).

A ciliary staphyloma occurs in the region of the ciliary body where the anterior ciliary arteries perforate (Fig. 290).

An equatorial staphyloma is situated about midway between the corneal margin and the optic disc, where the vortex veins pass out from the eye (Fig. 29I).

A posterior staphyloma is situated at the posterior pole, where the long and short ciliary arteries and the ciliary nerves enter the eye. It is the common cause of axial myopia (see page II2).

Cupping of the optic disc takes place where the optic nerve enters the eye, and where the fibrous tissue coat is considerably reduced in thickness, being only represented by the lamina cribrosa. This is the weakest part of the walls of the globe, and the first to become ectatic as the result of increased tension.

4. Congenital Abnormalities.-In the region of the fetal ocular cleft, as already pointed out (see page 20), some weakness in the walls of the globe may exist; frequently in association with a coloboma of the choroid. Ectasia may then occur as the outcome of normal intraocular tension, even sufficient in some cases to produce a cyst-like protrusion. A bulging in the region of the ocular cleft is frequently associated with a crescent at the lower margin of the optic disc, resembling in every way a myopic crescent. That there is some congenital weakness at the posterior pole of the eye, the nature of which is as yet undetermined, 
which predisposes to the production of axial myopia, seems highly probable.

The view has been advanced that the bulging of the cornea, which results in the production of keratoconus, is occasioned by some developmental weakness near its centre.

Two forms of degeneration occur in the fibrous tissue of the ocular conjunctiva opposite the palpebral fissure under very similar circumstances, these are known as pinguecula and pterygium.

A pinguecula is a raised yellow patch in the ocular conjunctiva, of a triangular shape with the base toward the corneal margin. It occurs most frequently opposite the palpebral aperture, on the inner side of the globe, but is also often present on the outer. It is met with in people who have been exposed to much wind and dust, and is very common in those who have resided in the tropics.

Before its histological characters had been accurately investigated it was supposed, from its yellow colour, to be composed of fat hence the name applied to it. Really it does not contain fat, the yellow colour being due to collections of finely granular hyaline material fused together. ${ }^{1}$ These on high magnification of the nodule can be seen as a number of small yellow spots. They are situated at varying depths in the tissue, some being immediately beneath the epithelium and others in the superficial layers of the . sclerotic. In addition to the hyaline nodules there is a new formation of yellow elastic tissue, the fibres composing which are thick and show signs of degeneration. The blood-vessels in the affected area have thickened walls due to hyaline degeneration. The epithelium overlying the patch is thicker than normal, its superficial cells are flattened, and sometimes contain granules of keratohyaline.

A pterygium is a triangular fold of the conjunctiva which extends on to the cornea. For descriptive purposes it is said to have a head or apex, where it is attached to the cornea, a body or base, where it springs from the conjunctiva

${ }^{1}$ E. Fuchs. Arch.f. Ophth., XXXVII, I89I, II I, I43. 
covering the sclerotic, and a neck situated at the limbus, where a pleat is formed at its upper and lower borders, beneath which a probe can be inserted for a short distance. The arrangement of the blood-vessels in the fold simulates somewhat that seen in the wings of some insects, hence the name by which the affection is known.

A true pterygium arises apart from any corneal ulceration or inflammation, and is always situated opposite the palpebral aperture on either the inner or outer side of the cornea, both eyes being usually affected. A false pterygium is the term applied to a fold of conjunctiva drawn over on to the cornea by the cicatrisation of a marginal ulcer, or a large abrasion, such as that following a lime burn. It may be present at any part of the corneal circumference.

A true pterygium, arises under similar conditions and is situated in the same locality as a pinguecula, by which affection it is frequently preceded. ${ }^{1}$

It is an affection of later life, occurring in those whose occupations expose them to much wind and weather, hence it is more commonly met with in men than in women. It is especially common in dry and dusty districts.

A zone of cornea immediately in front of the head of the pterygium is opaque and grey; into this it tends to spread, advancing slowly further and further inward, ultimately reaching the pupillary area and affecting vision. In rare cases where there are two present in the same eye, on opposite sides of the cornea, they may extend inward until they meet in the centre. If a patient affected with a pterygium be removed from exposure to climatic conditions its progress is sometimes arrested. The apex then flattens down, the opaque zone in front of it disappears, the body becomes less vascular, and it ceases to spread.

A pinguecula frequently precedes the formation of a pterygium, but as the latter develops the pinguecula becomes flattened out and disappears. Where there is a large pterygium the semilunar fold also becomes decreased in

${ }^{1}$ E. Fuchs. Arch. f. Ophth., XXXVIII, I892, 2, I. 
size, or even entirely obliterated. This shows that the condition is more an extension of the conjunctiva on to the cornea rather than a new formation of tissue. The head of a pterygium, unlike the conjunctiva covering the sclerotic, is firmly adherent to the underlying fibrous tissue. On its surface there are several small horizontal folds or puckers. Microscopically these folds are found to be lined by laminated epithelium with globlet cells, and sometimes the appearance of tubular gland-like formations is produced. Occasionally the orifice of a surface depression, or tube-like formation, becomes occluded, and a small cyst lined by epithelium is developed.

The subepithelial tissue in the head of a pterygium resembles the subepithelial tissue of the ocular conjunctiva at the limbus; it is composed of blood-vessels, white fibrous tissue, and yellow elastic fibres. The strands of the fibres appear tightly stretched and not waved. Collections of hyaline substance, like that met with in pingueculæ, may sometimes be found.

Underlying the head of the pterygium, Bowman's membrane becomes broken up by extensions into it of the overlying vascularised subepithelial tissue, which may also penetrate into the anterior layers of the substantia propria. It is this involvement of the substantia propria which causes some opacity to be left after the head of the pterygium has been dissected up.

Prolonged congestion of the ocular conjunctiva is certainly one factor in the causation of a pterygium, but it is not the sole cause. In chronic inflammatory conditions of the conjunctiva there is prolonged congestion, and sometimes a slight extension of the terminal loops of vessels inward, but no development of such a condition as a pterygium. There is probably, in addition to congestion, some softening or degeneration of Bowman's membrane and the anterior layers of the substantia propria, this giving rise to the grey zone around the advancing margin of the pterygium. The degeneration may be a senile change, or the 
result of nutritional disturbance from the exposure to climatic conditions.

(b) Degenerations in the Blood-vessels.-The earliest vascular degeneration which takes place in the eyeball is a physiological process, and occurs during fœtal life. The central artery of the vitreous, which supplies it and the lens with nourishment during their most active period of growth, has usually, together with itslenticular branches, disappeared before birth though remnants of them can be detected by slit-lamp microscopy throughout life. How the degeneration of these vessels is brought about has not yet been definitely worked out. It coincides with the formation of the anterior chamber and the nearly full growth of the lens, but abnormal cases have been recorded in which these vessels have persisted, and continued to carry blood, after the anterior chamber has formed, and after the lens assumed nearly its normal dimensions.

Vascular Sclerosis. ${ }^{1-T h e}$ structure of the intraocular blood-vessels is similar to that of vessels in other parts of the body. The central artery of the retina shows a wellmarked elastic tissue coat (membrane of Henlé) which is lined by endothelium on its inner surface, a middle coat composed of unstriped muscular fibres, and an outer coat, or adventitia, of connective tissues with a few fine elastic fibres in it. The veins consist of similar but thinner coats, except that they have no definite membrane of Henlé, and that the amount of elastic tissue in the adventitia and middle coat is not so great. Within the globe the retinal veins have no elastic tissue in their walls.

Vascular sclerosis affecting the ocular vessels may be either primary or secondary. Primary vascular sclerosis is either a senile degenerative change or the result of some toxic substance circulating in the blood, in connection with either chronic alcoholism, syphilis or renal disease.

Secondary vascular sclerosis occurs as the result of a local inflammation in the eye in which the vessels partici-

${ }^{1}$ Marcus Gunn. Trans. Ophth. Soc. of the U. K., XII, I892, I 24. 
pate, their coats becoming thickened and their lumen narrowed. In some diseases, such as syphilis, either primary or secondary sclerosis may occur. Thus during the secondary stage, when the syphilitic infection is general, it causes a widespread endarteritis followed by primary vascular sclerosis; but when the disease is localised, as in disseminated choroiditis, it causes an intense local secondary vascular sclerosis.

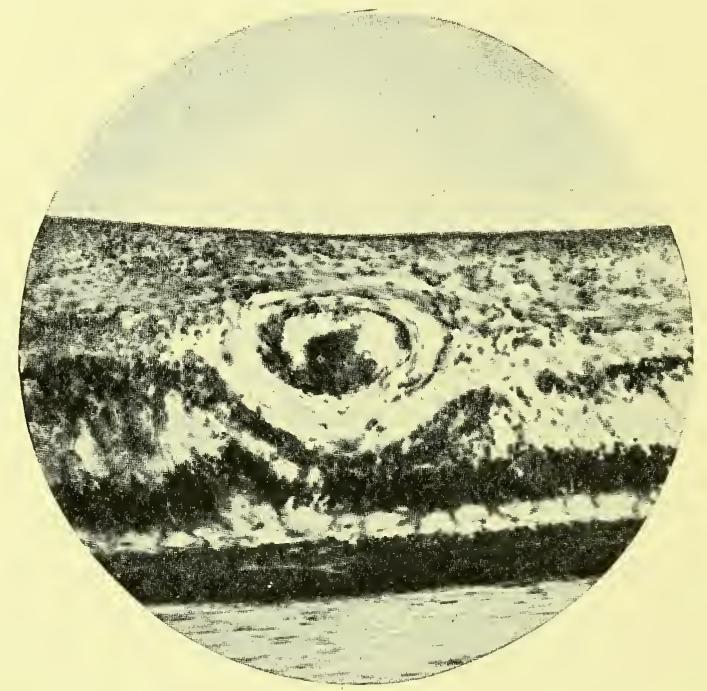

FIG. 292.- Shows a section through the retina, with a sclerosed artery cut transversely. Note the thickening of the vessel's coats.

The effect of vascular sclerosis is to lessen the lumen of the vessel, and so to inhibit the nutrition of the part by diminishing or completely cutting off the blood supply to it. If the constriction of the vessels is general throughout the system the blood pressure is raised, and this, together with the disease in the capillaries, produces changes in the walls of the latter which allows of a transudation of their contents (see page 280). The secondary effects of vascular sclerosis have already been described (see pages $47 \mathrm{I}$ and 476 ).

The actual change found in the larger vessels when sclerosed is an increase of the connective and elastic tissue in their walls (Fig. 292). This is especially marked in the 
intima, which may be either uniformly thickened or have knob-like prominences formed in it, which project into the lumen. The vessel behind the obstruction may become dilated so that miliary aneurisms are produced. This dilatation is especially liable to take place on small thinwalled venous radicles which in some instances may give way, causing intraocular hemorrhage. Partly on account of the stasis produced by the constriction, and partly on account of the irregularity and roughness of the inner surface of the vessel, thrombosis may occur. This may happen in either an artery or a vein.

Sclerosis in the retinal arteries first manifests itself clinically by the bright reflex which appears on their surface, to which the term " copper wire arteries" has been applied; this central light streak on the vessels often has a finely beaded appearance. Thickening of the coat of a retinal artery also produces a loss of its transparency, this is best seen where it crosses a vein, which is not only hidden from view by the artery but also has its course diverted. Instead of passing obliquely under the artery it runs almost transversely and acquires a kinked appearance, one which affords definite evidence of the presence of sclerosis. Proliferation of the lining endothelium of an artery produces irregularities in the size of its lumen, causing it to present a beaded appearance. As an artery's walls become thickened it increases in length and tends to become tortuous, the small vessels in the region of the macula frequently show such tortuosity. When the condition of arterio-sclerosis has existed for some time exudates into the retina occur, and what is termed "arterio-sclerotic retinal degeneration" is produced. Small, white, sharply defined areas of exudation in the retina are formed together with small hemorrhages. The exudate consists mostly of hyaline material situated in the external molecular layer. The arteries may ultimately become converted into cords, the lumen being completely obliterated; sometimes there is an exudation into their perivascular lymph sheaths which gives 
rise to a "pipe-stem" appearance. These changes are usually associated with arterial disease elsewhere, and 50 per cent. of the patients so affected, sooner or later develope cerebral lesions.

Choroidal Vascular Sclerosis.-Both the choroidal arteries and veins may be sclerosed (Fig. 293), but as a rule the

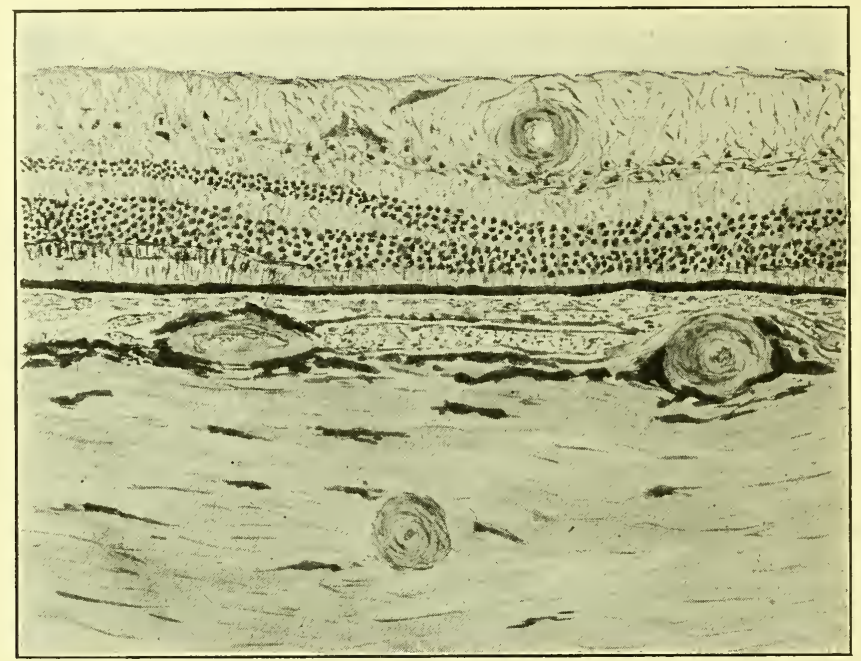

FIG. 293.- Shows a section through the coats of the eye with sclerosed choroidal and retinal vessels cut transversely.

main arteries are chiefly affected, and changes are produced over areas of the choroid supplied by them. The choriocapillaris becomes atrophied, and the larger vessels are seen with the ophthalmoscope as white worm-like bodies in the fundus, occasionally with thin columns of blood within them. The atrophy of the chorio-capillaris has a marked effect on the outer layers of the retina, the pigment cells proliferating and migrating (see page $47 \mathrm{I}$ ). Other secondary degenerative processes follow such as the formation of colloid nodules on the membrane of Bruch (see page 644). In extreme cases the nutrition of the overlying retina is cut off and it loses its function in that locality.

(c) Degenerations in the Ocular Muscles.-As age advances the interstitial connective tissue of the uveal 
tract becomes thickened, and in the eyes of elderly people, that between the bundles of fibres of the ciliary muscle is often considerably increased, so that the muscle fibres themselves become narrowed and degenerate. Weakness of the power of accommodation, due to such muscular changes, cannot be differentiated clinically from that due to hardness of the lens. An unduly early degeneration of this sort might, however, explain the apparent premature onset of presbyopia which is sometimes met with.

Of the extraocular muscles the one in which primary degeneration most frequently occurs is the levator palpebræ superioris, producing a slowly increasing amount of ptosis. It may occur as a senile affection, or as a bilateral, hereditary condition, beginning quite early in life; an abiotrophy of the muscle fibres.

In the hereditary cases the muscular atrophy is sometimes not confined to the levator palpebræ muscle, but involves the recti and oblique muscles also. There is then a slowly progressive ophthalmoplegia externa; as much as thirty or forty years may elapse between the appearance of the first symptom and the complete loss of all power of movement in the eyes. The paralysis comes on without any affection of the nervous system, it seems to be entirely a primary muscular degeneration, and not due to any change in the nerves supplying them or of the nuclei from which those nerves take origin. The condition is not a congenital one, though the tendency to its development is congenital, the patients affected are born with and possess for some years normal movements of the eyes before any loss of power manifests itself.

In one of the cases of ptosis, ${ }^{1}$ from which a piece of the affected muscle was removed, the muscle fibres were found to be much thinner than normal, with marked pigmentary degeneration of their sarcolemma, the connective tissue between them being increased in amount.

${ }^{1}$ E. Fuchs. Archiv. f. Ophth., XXXVI, I890, 234. 
In the facio-scapulo-humeral type of primary muscular degeneration, "Landouzy Déjérine's type," the orbicularis palpebrarum muscle becomes involved together with the other facial muscles, so that the lower eyelid falls away from the eyeball, causing epiphora, and the eyelids cannot be closed.

(d) Degenerations in Inflammatory Exudates. - Inflammatory exudates which do not make their way to the surface and escape, may have blood-vessels extend into them and become organised into fibrous tissue. Such newly formed fibrous tissue tends to contract and obliterate or constrict the blood-vessels supplying it, so that its nutrition becomes affected, and it degenerates into substances more closely allied to inorganic matter, such as hyaline or amyloid material, fat or cholesterine, and calcareous matter or bone.

An exudate composed of a mass of plasma cells may, when the active inflammation subsides, result in the formation of hyaline material from degenerative changes taking place in the cells.

(a) Hyaline Degeneration.-The histological characteristics of the material called hyaline are:

r. Its homogeneous structure and highly refractive power.

2. Its insolubility in strong acids and alkalies.

3. Its capacity of staining; deeply with eosine, acid fuchsin, carmine and picrocarmine; lightly with hematoxylin; and of not being turned brown by iodine.

In old corneal leukomata hyaline may occur in the form of small granules, globules or large irregular shaped masses, the larger formations being produced by a coalescence of the smaller ones. Clinically they give rise to the appearance of yellow spots in the opaque area, and sometimes calcareous granules become deposited in them.

Hyaline degeneration in the conjunctiva occurs as a sequela of trachoma. It has been mentioned in describing that affection how the thickened, smooth, waxy condition 
of the palpebral conjunctiva termed "Stellwag's brawny edema" is due to hyaline degeneration of the plasma cells, which are often present in large numbers. The hyaline degeneration following trachoma is not always limited to the palpebral conjunctiva, in rare instances thickened patches due to it occur in the ocular conjunctiva, and in the cornea when it has been affected by severe pannus.

Amyloid degeneration of the conjunctiva is a rare condition; it usually occurs as a sequela of trachoma, but may arise independently. It has been met with mostly in Eastern Europe. It affects both the palpebral and ocular divisions of the membrane, and also the semilunar folds and caruncle. The whole eyelid becomes much thickened, resulting in ptosis. The conjunctiva presents a yellowish translucent wax-like appearance. It is very friable and non-vascular. Clinically, in the early stages the affection cannot be distinguished from hyaline degeneration; it is of long duration, and in the later stages calcification and ossification of the affected parts may occur.

Fatty Degeneration.-Fatty degeneration is not simply a deposition of fat, but actual change of the albumin into fat. Fat is seen microscopically as highly refractive circular globules which are dissolved by ether and chloroform. When large they stain orange with Sudan III and when small, red. Some forms of fat, but not all, stain black with osmid acid.

Fatty degeneration is a noticeable feature in connection with old inflammatory exudates. In the ossification which takes place in exudates in the uveal tract, a medullary substance containing large globules of fat becomes formed between the trabeculæ of bone.

Cholesterine crystals, which are formed in connection with fatty degeneration, are frequently met with in old hemorrhages and in degenerating inflammatory exudates. Large numbers of them may be found in the subretinal fluid or in the vitreous and aqueous humours where such conditions are present. They are soluble in alcohol, and sections 
of tissue containing them which have been treated with that reagent, show characteristic sharply defined slit-like spaces where they were situated.

Calcareous Degeneration and Bone Formation.-In calcareous degeneration the salts deposited in the tissue are usually the cabonates and phosphates of lime. It generally represents the final stage in a series of degenerative changes, the tissue in which the deposition occurs having very low vitality or being absolutely necrotic.

The pathological hyaline formations already described in connection with the cornea, conjunctiva, choroid and optic disc frequently become calcareous. So also do necrosing inflammatory exudates; breaking down cells of rapidly growing neoplasms; and the laminated tissue formed on the inner surface of the lens capsule in capsular cataracts. If the affected part is non-vascular the deposition takes the form of granular incrustation; where blood-vessels are present osteoblasts form and bone is developed.

The hyaline nodules on the inner surface of the membrane of Bruch are shut off from the blood-vessels of the choroid by that membrane, and usually only become petrified with lime salts. In rare cases, where the membrane of Bruch has been ruptured, they become vascularised and bone is formed in them. When the lens capsule is intact the calcareous changes which are met with in the lens are only of the nature of granular deposition. In rare cases where the capsule has ruptured, and blood-vessels with fibrous tissue has extended into it, a formation of true bone within the capsule has been found.

Ossification of the Choroid.-In the inflammatory exudate which is thrown out in plastic choroiditis, degenerative changes sometimes take place resulting in the formation of bone. The commonest position in which this ossification occurs is immediately around the optic disc; it is also frequently met with in the neighbourhood of the ora serrata. The plate of bone which forms around the optic disc may extend forward, and the ring which forms around the ora 
serrata backward, until the two become united and a complete cup of bone is produced. At the posterior part of such a cup there is always a central hole through which the fibres of the optic nerve pass. The ring of bone at the ora serrata may, besides growing backward, extend inward into an inflammatory cyclitic membrane; the cup of bone will then have a lipped margin.

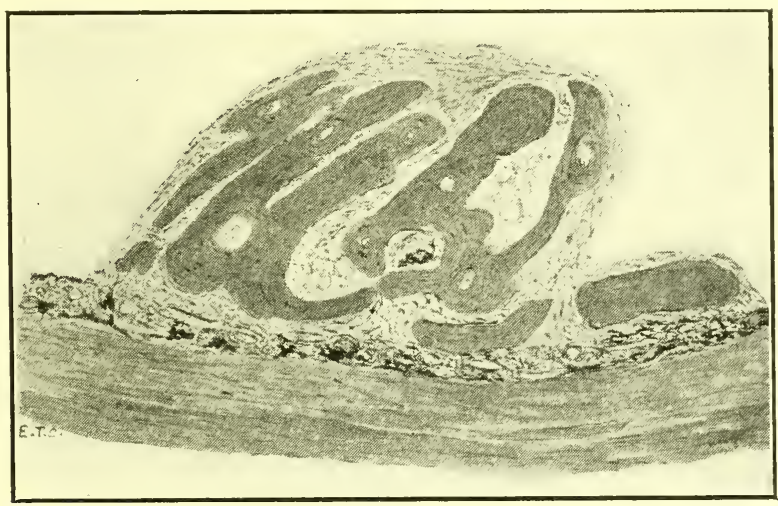

FIG. 294.- Section through a band of bone formed in a mass of organised inflammatory exudate from the choroid. The bone is shown consisting of numerous trabeculæ with medullary fat between them. The eye had been injured by a piece of steel fourteen years previous to removal. Case recorded by Treacher Collins, Ophth. Review, XI, I892, I39.

The development of bone in the choroid is usually a very slow process, and occurs mostly in blind and shrunken eyes. When formed it presents all the characteristics of normal compact bone. It is traversed by blood-vessels lying in Haversian canals, and surrounded by Haversian systems with osteoblasts; sometimes also between its trabeculæ there is medullary fat (Fig. 294).

As the result of plastic inflammation the choroid, in which ossification takes place, is much changed. Large areas of the capillary layer and elastic lamina become destroyed. The outer layers become atrophied and the walls of their blood-vessels sclerosed. The inflammatory exudate is transformed into fibrous tissue. The retina, if not detached, is intimately incorporated with the choroid. 
It is in the fibrous tissue formed from the exudate that the bone develops. Where the elastic lamina is still present this fibrous tissue may be found on either its outer or inner surface. The exudate, from which it forms, accumulates either in the region of the capillary layer or extends through a gap in the elastic lamina to the inner surface. Hence bone formation in connection with the choroid is found sometimes on the outer, and sometimes on the inner surface of Bruch's membrane.

The fibrous tissue, previous to the deposition of the calcium salts in it, assumes a homogeneous appearance resembling hyaline substance. When the salts are deposited, it becomes granular and stains deeply with hematoxylin. The cells in the vicinity of the ossifying area, which are about to become osteoblasts, are seen to assume an angular outline. The process begins on the outer surface of the exudate, concentric layers are gradually formed, and the Haversian systems built up. 


\section{APPENDIX ON LABORATORY METHODS}

In this chapter dealing with laboratory methods it is assumed that the reader has a practical knowledge of the elements of bacteriology and of the various ways of examining the blood, such as are taught in the course of an ordinary medical curriculum. The only methods described are those likely to be required in connection with the work of an ophthalmic hospital or department. They are arranged in the order which is most likely to be of use to the student in the practical examination of a specimen.

\section{Methods of Obtaining Material for Examination}

To make a bacteriological examination the material to be examined should be obtained in an early and progressive stage of the disease, and every care taken to avoid contamination.

In taking material from styes, chalazia, or phlyctenulæ only unopened ones should be selected. The surface of the affected area should be throughly cleansed with antiseptic lotion and then washed with a sterile salt solution. The puncture into the affected tissue should be, made with a sterilised knife, and a platinum loop, which has been sterilised by holding it in the flame of a spirit lamp, passed through the opening. The material adhering to it is then inoculated in two tubes of culture media, one of which should consist of blood-serum.

In making the inoculation, the tube should be held sideways between the base of the thumb and forefinger of the left hand, with the mouth inward toward the palm. The plug of wool, after being passed through the flame of a spirit lamp, is removed with the right hand and held between the bases of the first and second finger of the left hand. The inoculation is made by passing the loop over 
the surface of the medium from below upward. The plug of wool is then passed through the flame and replaced; the tube may be marked with a glass pencil with the name of the patient and the date, and then placed in the incubator in an upright position. If blood-serum cannot be obtained blood agar should be used for taking the primary culture. In the case of the conjunctiva this is readily prepared by pricking a small conjunctival vessel and taking blood and pus together in the loop to smear over the agar medium.

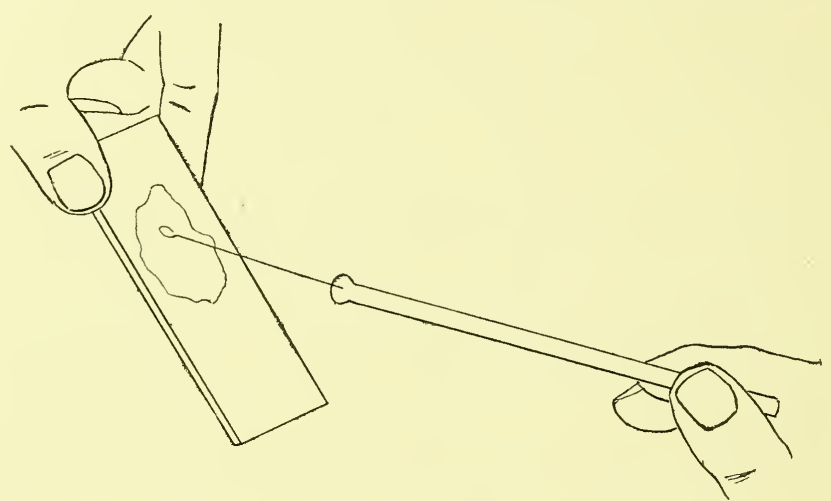

FIG. 295. - Showing the method of making a smear preparation with a platinum loop.

Two smear preparations on microscopic slides should also be made. With the platinum loop the material is smeared as thinly and evenly as possible over the slides, and allowed to dry in the air, no heat being applied. These are subsequently fixed (see Fig. 295), one stained by carbol thionin and the other by Gram's method, and examined with $1 / 12$ oil emersion objective; no cover-glass need be applied if the preparation is not to be kept.

To examine the cytology of the cells of the conjunctiva in cases where there is little or no conjunctival discharge, the conjunctiva is exposed and the platinum loop drawn along the conjunctiva several times. Soon the irritation produced will give rise to mucus, which can be taken up on the loop and a smear preparation made. This method 
is principally of use where it is desired to examine the character of the polymorphonuclear leucocytes, as in cases of suspected spring catarrh. When it is desired to examine the lymphocytes the best method is to rub a cover-glass over the surface of the conjunctiva or express a follicle, squeezing out the material obtained between two coverglasses. To obtain smear preparations from a corneal ulcer, take a sterile discission needle and with the blade lightly scrape the surface of the ulcer, then make smear preparations from the material so obtained.

The examination of the aqueous for organisms is surrounded by many difficulties unless the organism is present

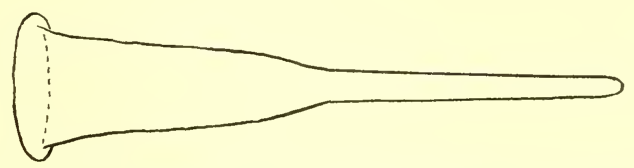

FIG. 296.-Glass tube for centrifugalisation of blood.

in considerable numbers, which is very rarely the case; they cannot be found by an ordinary microscopical examination of smear preparations, but may sometimes be obtained after centrifugalisation of the fluid. For this purpose the fluid is collected in a capillary pipette (see Fig. 296). The capillary end is sealed and it is placed in a centrifugaliser. After centrifugalisation a rubber teat is put on the open end of the tube, and by its means the first drop is squeezed out on to a slide, which is allowed to dry, and is then fixed, stained and examined microscopically.

Cultivations made from the aqueous give more satisfactory results, but the great difficulty is in obtaining the fluid uncontaminated. For this purpose a short fine hollow needle, having a spear point, should be thrust into the anterior chamber at the limbus, the point of entrance being first touched with the cautery so as to ensure its sterilisation. When this latter precaution is neglected the bacillus xerosis is not infrequently found in the cultiva- 
tion, showing that contamination has taken place from the conjunctiva. Aqueous may be obtained for the application of the agglutination test by a simple paracentesis, the fluid being collected as it escapes from the anterior chamber over the surface of the keratome by a pipette.

In removing a piece of conjunctiva for microscopical examination care must be taken not to damage the tissue with forceps. A fine pair of straight iris forceps and sharppointed scissors are the best instruments to use for the purpose. After removal, the piece should be washed in normal saline solution and pinned out on a piece of cork by means of glass pins, which are made by drawing out a piece of capillary glass tubing in the flame of a spirit lamp. The specimen is then placed face downward in the fixing fluid.

In the examination of excised eyes the first thing to decide is what is the particular point of interest about the specimen that is to be investigated; i.e., whether it be bacteriological or histological. If it be bacteriological the eye should be kept as aseptic as possible and examined immediately after excision. After its removal the globe should be cleansed with sterile salt solution and an external examination made; it is then dipped for fifteen seconds in boiling saline solution. This treatment destroys the organisms on the outer surface of the globe.

If the aqueous is to be examined the fluid should be withdrawn from the anterior chamber with a sterile hollowneedle.

If the vitreous, the eye should be divided with a sterile knife, smear preparations and cultivations being taken from it. In cases where the organisms are likely to be very scanty the globe may, after treatment with boiling saline, be placed in a sterile bottle in the incubator for twenty-four or forty-eight hours and then examined; the fluids in the eye acting as a culture medium, the organism will be found to have increased.

If a histological examination is to be made, and it is desired to examine the condition of the retinal blood-vessels, 
as much as possible of the optic nerve should be obtained when the excision is performed.

\section{Postmortem Methods-Injection of the Blood-vessels of the Eye}

Material should be obtained as soon after the death of the patient as possible, before postmortem changes have had time to take place. Such changes in the retina begin to show themselves six hours after death, but they may be inhibited by placing the body in an ice chamber, or injecting undiluted formalin into the orbit immediately on the death of the patient.

After the brain has been taken out the bony roof of the orbit is chiselled through and removed, the ring of bone around the optic foramen being left intact. The periosteum of the orbit is then divided antero-posteriorly, and if the condition of the nerve sheath is to be examined the optic nerve should be ligatured close to the foramen to prevent the loss of fluid therefrom. Having exposed the globe it should be removed by dividing the conjunctiva from the front and the muscles from behind. To prevent the deformity being noticed the oribt may be filled with a piece of liver or an eye from an animal, and the lids stitched together on their posterior surface. In cases in which permission cannot be obtained to remove the whole eye, the posterior half may be taken by isolating the globe posteriorly right up to the limbus and dividing the ora serrata with a sharp knife. Previous injection of the orbit with undiluted formalin renders this easier to perform.

Injection of the blood-vessels of the eye in situ is difficult. It should be carried out as soon as possible after death. Hot carmine-gelatine mass is the best substance to use. It is made as follows:

Pure carmine 4 grm.

Solution of ammonia............... 8 c.c.

Distilled water................. 50 c.c. 
The ammonia is added to the carmine, the water poured on, and the solution filtered.

To this is added, after warming, ro grm. of pure gelatine, which has been allowed to take up as much distilled water as it can. This having completely dissolved, a ro per cent. solution of acetic acid is added drop by drop until the ammonia is quite neutralised; the carmine being precipitated, produces a dull brownish-red colour. The addition of a little salicylic acid prevents decomposition.

The injection should be made through the internal carotid artery after removal of the brain. The artery is ligatured at the base of the skull on the proximal side of the ophthalmic artery.

A hot injection is made by means of a syringe the cannula of which is tied into the distal end of the vessel. It is well to have previously separated the skin of the forehead and eyelids so as to prevent the injection passing into the skin of the face. Before injection is made the tissues should be thoroughly heated by immersion of the head in hot water. The specimen is subsequently hardened in formalin which sets the carmine mass in the tissues.

\section{Methods of Fixation and Hardening}

Smear preparations of cells can be examined in a fresh condition, but not much information can be obtained without first staining them. Wet preparations can be stained with acid methylene blue (see page 70r) but fixation before staining is preferable. The ideal method of fixation is one which will rapidly coagulate the albuminous material of which the cells are composed, without altering their form or their reaction to dyes. There are many fixing agents and the selection must be made according to the points which are to be investigated.

Heat is especially useful for smear preparations when examining for microörganisms. The slide on which the smear preparation has been made is passed rapidly three times through the flame of a spirit lamp or Bunsen burner. 
Blood films or smear preparations of cells may be fixed by holding the film some distance above the flame, thus applying a gentle continuous heat for about thirty seconds. Whole eyes are rapidly fixed by plunging them into hot water and raising it to boiling-point for three minutes. The method is especially useful in the examination of the vitreous, allowing investigation of its consistency, and of exudations in it. Small pieces of growth can rapidly be fixed by this method, and sections be cut by freezing with ethyl chloride, during an operation. The diagnosis of the nature of a tumour can then be made while the patient is still on the operating-table.

Ethyl alcohol is especially useful for small pieces of tissue, such as the conjunctiva, but is of little use for the whole globe, as it will not penetrate the sclerotic readily. Further, it is impossible subsequently to freeze the globe without first removing the alcohol.

Methyl alcohol is one of the constitutents of Leishmann's stain no fixative is, therefore, required for smear preparations stained by his method.

Formalin, of which a 4 per cent. solution in normal saline is the best strength, is extremely useful where the preparation of a macroscopic specimen is desired, since the vitreous is not coagulated by its use and the tissues retain a considerable amount of their normal colour. It is also useful for hardening nerve tissue before transferring it to Müller's fluid. The disadvantages of this method are that it is rather slow in its action and, therefore, does not give good results with regard to the cytology of the cells, and being an acid it interferes with the staining reaction of the cell when aniline dyes are used. It further makes the vitreous swell and causes compression of the retina and choroid.

Müller's fluid consists of

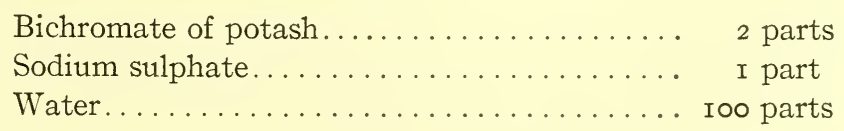


It is especially used for hardening nerve tissue where it is desired to trace or examine the condition of the medullary sheaths. The specimen may or may not have been previously placed in formalin. It should be kept in the Müller's fluid for at least six weeks or two weeks in the incubator at $40^{\circ} \mathrm{C}$., during which time the solution is at first changed every day. The method is also useful in preparing lenses for microscopical examination. The fixation is slow and therefore the condition of the cells from a cytological point of view is not good.

Saturated solution of perchloride of mercury or some mixture containing it, such as Zenker's fluid, which consists of:

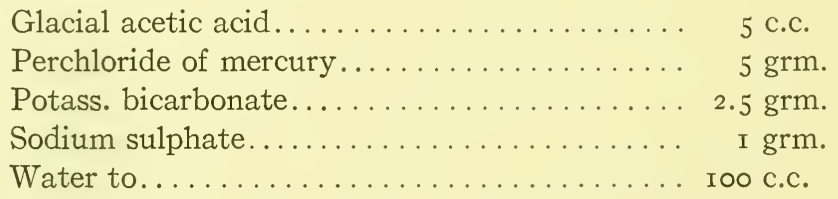

These preparations are extremely valuable for fixing smear preparations which need only be covered with the solution for half a minute; for the rapid fixation of tissues where it is desired to examine the specimen for microorganisms; or where the cytology of the cells is important. Whole eyes should be placed in the solution for twentyfour hours. It has the disadvantage that the vitreous is coagulated by its use and the tissues are rendered white and opaque. After its use the tissues should be thoroughly washed for twelve hours in water. This is best carried out by placing the tissues in a glass jar, inserting a glass funnel and allowing the tap to run into the funnel; this fills the jar and keeps the water circulating without damage to the tissue. A fragment of iodine should be added to the spirit in which the specimen is next immersed to remove all traces of the mercury, otherwise a black precipitate will appear throughout the sections.

Decalcifying Fluid.-After fixation in formalin the specimen is placed for twenty-four to forty-eight hours in sul- 
phurous acid B.P.. This is a very valuable method when the bone in the specimen is not very thick. An alternative solution is Ebner's which consists of:

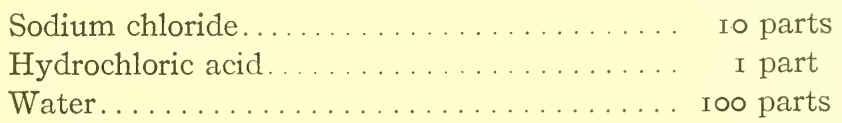

A drop of fresh hydrochloric acid is added daily for two to three weeks until the specimen is soft.

\section{External Examination of an Eyeball after Removal}

Side to Which the Eye Belongs.-This may be determined by the position of the insertion of the oblique muscles which are on the outer side of the globe, that of the superior differing from that of the inferior in being narrower, in the tendinous portion being longer, and in not running so close up to the optic nerve. If, therefore, the eyeball be held with the cornea forward and the surface over which the superior oblique passes upward, the insertion of the oblique muscles will be on the side to which the globe belongs. Assistance may also be gained in many cases in distinguishing the outer from the inner side, by observing the position of the optic nerve, it joining the eyeball to the inner side of the middle line. A silk thread through the superior rectus at the time of the enucleation saves trouble.

Diameters of the Eyeball.-These can be most easily taken with a measure, in which two T-shaped pieces slide on one another. The eyeball is placed between the two crosspieces of the T's, and the diameter read off on the scale. It is usual to measure an eye in three directions, viz., anteroposteriorly, laterally, and vertically. The averages for the normal adult eye in these three directions are anteroposterior $24.3 \mathrm{~mm}$., lateral $23.6 \mathrm{~mm}$., vertical $23.4 \mathrm{~mm}$.

Palpation of the Eyeball. - The tension of the eyeball can be estimated in a similar way to that which is employed during life, by alternating the amount of pressure made by 
the two index-fingers placed on the globe. It should be taken as soon after removal as possible, to be of any value. In cases of intraocular growth an abnormal amount of resistance is sometimes felt, which gives the impression of the presence of some solid substance. Where only a small tumour is present, its position can occasionally be distinguished by this sense of resistance being felt only in one part of the globe, viz., the seat of its attachment. The sensation of a hard substance felt through superficial soft structures is that which is experienced on palpation of an eye in which there has been a deposit of calcareous matter in the choroid. It is found in shrunken or degenerate eyes, and may be either a small nodule or a large shell of bone.

Transillumination.-On holding a healthy eyeball up, with its posterior surface toward the light, a red glow is seen through the pupil. This is obscured when there are any opacities in the media. It may also be entirely or partly absent, or dimmer than usual, in cases of intraocular growth, intraocular hemorrhage, or detachment of the retina. The position of an intraocular growth may occasionally be localised by this red glow, disappearing from view in certain positions of the eyeball and reappearing in others.

Condition of the Cornea.-The normal diameter of the adult cornea is I $2 \mathrm{~mm}$. It may, in certain pathological states, be increased or diminished in size, unduly prominent or flattened out. For purposes of describing the position of any wound, ulcer, or opacity it is convenient to consider the cornea as composed of four quadrants: the upper outer and inner, and the lower outer and inner. In a case of a wound it is always important to observe if it passes beyond the sclero-corneal margin into the so-called ciliary region, which extends backward nearly as far as the insertion of the recti muscles. A pin passed through the tunic of the eye at the insertion of the recti muscles would be found to emerge internally at the ora serrata; the most posterior part of the ciliary muscle ends a little in front of this. 
Condition of the Sclerotic.-It may be altered in shape, being bulged or puckered in one or more directions. So-called squaring of the globe occurs in two opposite conditions; when there is increased tension, from the coats of the eye yielding more in the intervals between the insertions of the recti muscles; and in the diminished tension of a shrinking eyeball, from the pressure caused by the drag of the recti muscles. The sclerotic may be thinned, which is rendered evident by the dark colour of the choroidal pigment showing through. It may be altered in colour, assuming a yellowness where there has been extensive intraocular hemorrhage or becoming pigmented from the invasion of a melanotic growth. It may be wounded or ruptured.

Condition of the Anterior Chamber and Its Contents.The anterior chamber may be entirely obliterated, or its depth may be altered, either uniformly increased or diminished, or of different depths in different parts; as, for example, in the condition known as "iris bombé."

The character of the aqueous may be altered, being simply turbid or containing pus (hypopyon), or blood (hyphema).

Condition of the Iris.-Parts of the iris may have been removed, or it may have acquired altered relations to its surrounding structures. It may be altered in colour, which change is sometimes due to the condition of the aqueous; whether this is so or not can, if thought necessary, be readily determined by tapping the anterior chamber and allowing the aqueous to escape. An oscillating iris is nearly always indicative of an absent or displaced lens.

Condition of the Lens.- The lens may be absent or displaced, sometimes being completely loose and floating about in the vitreous on altering the position of the globe, at other times attached at one part and then only swaying backward and forward. It may have come forward entirely into the anterior chamber, or have been wounded, and some of the 
soft matter, semi-dissolved, rest in front of the iris. It may present various and different amounts of opacities.

\section{Freezing. Division of Eyeball and Macroscopical Exami- nation after Division}

Freezing.-The unopened eye should be wrapped in a piece of protective tissue, and placed in a mixture consisting of one-third salt and two-thirds ice. A coffee tin with holes punched in the bottom is useful for the purpose of holding the mixture. After half an hour the eye may be taken out. It should be divided with a sharp table knife in such a direction as will be most likely to show the peculiar characteristics. A razor thickens too abruptly to make a clean section. After being opened, the eye should be placed in a basin of water and allowed to thaw. Before freezing the eye, it is well to determine the direction the section is to take, and to make an ink-mark on the sclerotic where the knife is first to enter, as after it is frozen there is more difficulty in distinguishing its surfaces from one another. Usually the best section to make is a vertical antero-posterior one, which divides the globe into two lateral halves. A horizontal, or oblique antero-posterior section can also be made, or an equatorial one dividing the globe into anterior and posterior halves. When an antero-posterior section is made, it should pass a little to the outer side of the optic nerve, the whole of which is then left for microscopical examination.

The half eye should then be placed in a museum jar in saline solution (see Fig. 297, p. 692) and carefully examined with a corneal microscope, a method which is of great value for macroscopic specimens. Attention may be directed to the following particulars:

Cornea, as to its thickness and the depth of any ulceration or infiltration there may be in it. Its normal thickness in the centre is $9 \mathrm{~mm}$. and at the margins $\mathrm{I} .2 \mathrm{~mm}$.

Anterior Chamber.-The depth and contents of this can now be more readily appreciated, and also the condition 
of its angle, which may be narrowed by the approximation or adhesion of the root of the iris to the posterior surface of the cornea.

Iris, as to its thickness and its relation to surrounding structures, or any new growth starting from it. In the healthy state the posterior surface of the iris is incontact with the anterior capsule of the lens; in pathological conditions these two structures often become separated and what is called the "posterior chamber" formed.

Lens and Its Capsule.-The measurements of the lens may be taken; the normal diameters at the age of 20 are $3.7 \mathrm{~mm}$. antero-posteriorly, and $8.67 \mathrm{~mm}$. laterally; there is a gradual increase in the size of the lens throughout life, the lateral diameter between 80 and 89 is $9.62 \mathrm{~mm}$.

Vitreous, as to its consistency and the presence of any infiltration or hemorrhages into it. It may have become shrunken and its hyaloid detached from the retina, in which case the fluid between the detached vitreous and retina should be examined.

Retina, as to any hemorrhages, pigmentation, or infiltration within it, or any growth springing from it. In pathological conditions it is often found detached from the choroid; a commencing detachment presents the form of a ruck. An umbrella-shaped detachment is produced when the retina remains adherent only at the optic disc and at the ora serrata, it being collected in the form of a stalk passing through the centre of the globe and expanding anteriorly. In these cases the fluid between the detached retina and choroid requires examination, it may be albuminous and become coagulated by the hardening reagents.

Choroid, as to the presence of any patches of atrophy, pigmentation, or infiltration, any rupture, or any new growth. It may become detached from the sclerotic or be the seat of calcareous deposit.

Optic disc, may be either cupped or swollen. If cupped, attention should be directed to the margin of the cup to see if it shelves gradually or abruptly. 
Optic Nerve.- - If the nerve is to be examined separately from the globe, its outer side should be marked, by making a longitudinal slit with a knife down the dural sheath. If removed from the globe the posterior end should be marked with a piece of silk passed through the dural sheath.

\section{Method of Mounting Macroscopic Specimens}

All tags of tissue are removed from the sclerotic and the divided eye placed in a solution of 4 per cent. formalin and .6 per cent. choride of sodium for twenty-four hours. The specimen is then mounted in the same solution in jars $^{1}$

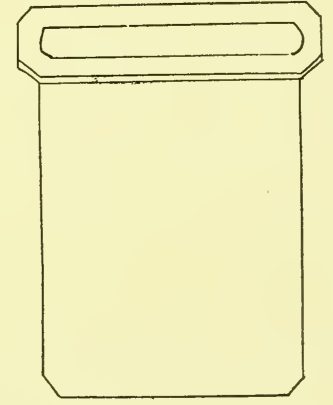

FIG. 297.

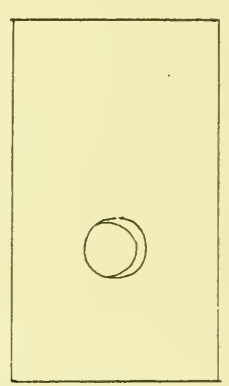

FIG. 298.

Fig. 297.-Museum jar with a polished glass side for mounting macroscopic eye specimens; it is high, broad and deep.

FIG. 298.- Opal glass plate to fit in the above jar to hold the eye in position; it is packed behind with wool. The hole in the plate into which the back of the eye fits measures $3 / 4$ inch in diameter.

specially devised for the purpose (Fig. 297). These are narrow antero-posteriorly and polished on one surface. An opal glass plate, which fits the jar laterally, has a round hole about $3 / 4$ inch in diameter cut in it about one-third of the way up from the botton (Fig. 298). The jar being twothirds filled with the solution, the opal glass plate is inserted, and then the divided eyeball is dropped down in front of the plate so that the cut surface comes against the polished glass side of the jar. The eye is lowered until

1 The jars and plates can be obtained from Messrs. Turner \& Co., I I Foster Lane, London E. C. or Messrs. Angus \& Co., 83 Wigmore St.. London W. 
its convexity rests in the hole of the plate which keeps it in position. The space behind the plate is then packed loosely with wool to keep it in position. Melted pitch is used to cement the cover on. The jars are then labelled with the reference number of the specimens and put in the museum cupboard. For this purpose a modification of the GlobeWernicke bookcases is very useful (Fig. 299). Special shelves are devised so that the shelf above has a piece that

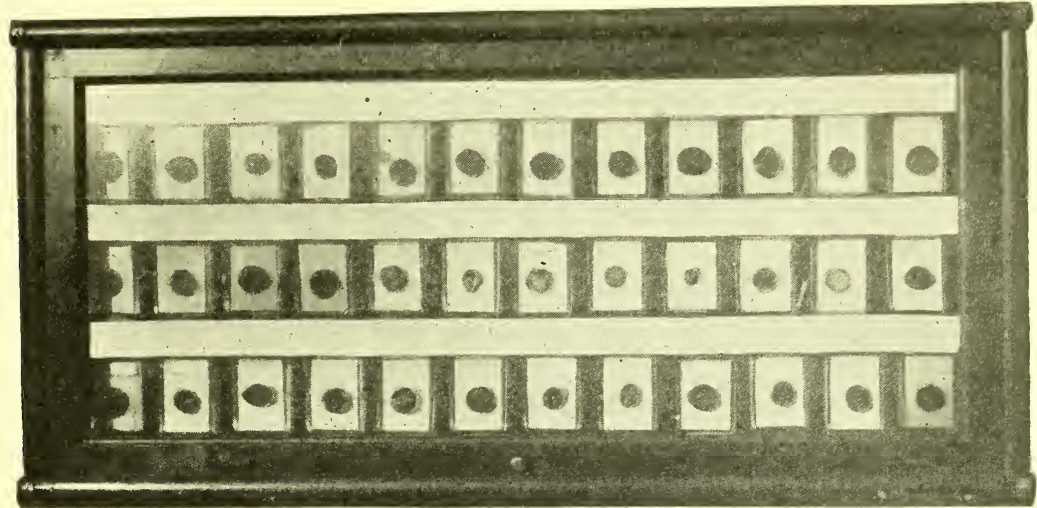

FIG. 299.-Globe Wernicke book case showing the method of arranging macroscopic specimens.

comes down and hides the tops of the jars, on which a label describing the specimen can be placed. The chief advantages of this method are that the specimen is mounted in a few minutes, and can be taken out at any time and microscopically examined.

If it be desired to mount the specimen in glyclrine it should be treated as follows:

I. Place the specimen for three days in the following solution:

Sodæ sulph...................... $40 \mathrm{grm}$.

Mag. sulph....................... $40 \mathrm{grm}$.

Sod. chlor........................ $20 \mathrm{grm}$.

Dissolve in 2000 c.c. of hot water and add I60 c.c. of formalin.

2. Transfer the specimen to 60 per cent. methylated spirit for three minutes in order to bring back its natural colour. 
3. Mount in a jar in the following solution:

Glycerine (pure)................. 60 parts

Distilled water................. 40 parts

Sat. sol. of pot. acetate............. 2 parts

Formalin................... 2 parts

Filter for use.

\section{Embedding and Cutting Microscopical Sections}

Embedding.-The three chief materials used for embedding are celloidin, paraffin and frozen gum. Before embedding a portion of an eyeball for cutting microscopic sections it must first be decided what it is most desirable to show in the specimen. Thus if anatomical conditions are to be investigated celloidin is by far the preferable method to employ, while if microörganisms are to be sought for in the tissues, or fine cytological changes for which aniline dyes have to be used, paraffin should be the method employed. Sometimes it is desirable to strip off the choroid and retina from the sclerotic and embed them separately, as the latter gets unduly tough in the process of hardening so that it becomes difficult to cut it in paraffin. Some reagents, such as Sudan III for staining fat, and methyl-violet when used for amyloid changes, will not act if the specimen is brought in contact with spirit, and in this case the section must be cut with a freezing microtome.

Celloidin (Schering) is sold in chips kept in water. The water should be poured off, the chips dried and dissolved in equal parts of ether and alcohol. Two solutions should be kept; $(a)$ thin celloidin, which is about the consistency of a liqueur and (b) thick celloidin, which is about the consistency of treacle. The specimen to be embedded should be treated as follows:

I. Sixty per cent. of methylated spirit, twenty-four hours.

2. Ninety per cent. alcohol (pure methylated spirit) twenty-four hours.

3. Absolute alcohol twenty-four hours. Instead of using alcohol methylated spirit dehydrated with anhydrous copper sulphate may be employed.

4. Equal parts of ether and alcohol twelve hours. Before placing in the celloidin, if half an eye is being embedded, it is advisable to remove a portion of the sclerotic from the convexity of the specimen with a sharp razor, as by this means not so much celloidin is required, and whilst it is hardening, the eye is less liable to become tilted.

5. Thin celloidin twenty-four hours.

6. Thick celloidin twenty-four hours.

7. Place the specimen in a paper box and fill it quite full with thick celloidin. Arrange the box so as to allow of slow evaporation under 
Cutting.-Apart from good embedding success in getting good sections depends on having a sharp knife. For this reason it is always desirable to cut off the part of the specimen which is not required with an old blade, reserving the use of the sharp blade for the part from which it is desired to obtain good sections. The best form of microtome for paraffin and celloidin is the base sledge (Leitz) (Figs. 300, 30I). For paraffin alone Minot's or the Cambridge rocker are excellent, whilst for celloidin the Jung microtome is good.

In cutting celloidin the knife should be set at an oblique angle to the specimen, and should be kept continuously wet with methylated spirit.

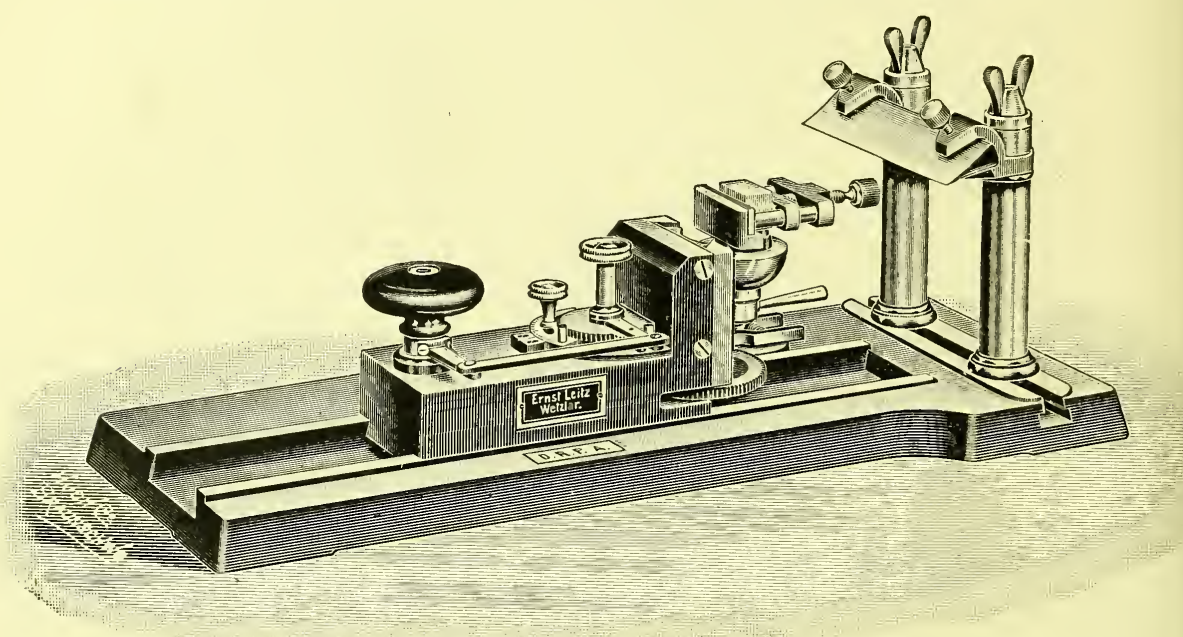

FIG. 30I.-Leitz "base sledge" microtome in use for cutting paraffin sections.

In the base sledge model this is performed by means of a dripper. As the sections are cut they should be placed in 60 per cent. alcohol by means of a camel-hair brush, and if it is desired to mount them in series a long dish or glass pen tray should be used in which to place them in order. In making serial sections by this method it is desirable to mount each one separately, but if it is not required to examine them under the high powers of the microscope, as in tracing the degeneration of nerve fibres, they may be mounted in the following manner:

A large glass plate is smeared with a solution of dextrin; over this is poured a solution of photoxylin in equal parts of ether and alcohol of about the consistency of treacle. When setting, the celloidin sections should be arranged in order on the plate. After it is set the whole is plunged into methylated spirit; the film containing the sections can then be readily stripped from the glass and the sections treated in the ordinary way. 
In cutting paraffin the knife should be set at right angles to the specimen. The sections are removed with a dry camel-hair brush, and floated out on the surface of warm water, which flattens out any folds there may be in them. They are then floated on to microscope slides and blotted on to the surface of the glass with cigarette paper. The slide is next placed to dry in the incubator for twenty-four hours when it will be found that the sections are firmly adherent. If the block is carefully squared ribbons of serial sections may be obtained.

\section{Staining and Mounting}

The following is the method of preparing and staining celloidin sections with logwood and eosin. The section should be transferred from one fluid to another on a large section lifter. All excess of fluid should be drained away on to blotting paper. The celloidin remains permanently in the section; therefore the section should not be put into absolute alcohol or the celloidin will be so softened as to destroy the specimen.

I. From the spiric it is transferred into hematoxylin for five minutes.

2. Acid distilled water I per cent. for one-half hour to take out the excess of stain. No stain should show in the celloidin.

3. Tap water to which a trace of ammonia may be added. The section should remain in this until blue, which usually takes from three to five minutes.

4. Transfer to I per cent. aqueous solution of eosin for about one minute.

5. Methylated spirit 70 per cent., five minutes, to take out the excess of eosin.

6. Methylated spirit, pure, for ten minutes to dehydrate.

7. Carbol-xylol for ten minutes to clear. (Ac. carbolic crystals $i$, xylol iii.)

8. Place flat on a slide and add at once a sufficiency of Canada balsam and a cover-glass. Sections clear gradually during the first twenty-four hours after mounting, or more rapidly if warmed over a spirit lamp.

Care must be taken to keep the hematoxylin, spirit and carbol-xylol covered during the preparation of the sections.

Paraffin sections stuck to the slide are best prepared and stained by passing them through fluids in stoppered glass bottles with necks sufficiently wide to take the slides.

I. Xylol five minutes to remove the paraffin.

2. Absolute alcohol I minute to remove the xylol.

3. Stain.

4. Absolute alcohol-two different bottles for two minutes each to dehydrate. 
5. Xylol three minutes to clear.

6. Mount in Canada balsam.

As the slide is transferred from one bottle to another it should be wiped free from all excess of fluid.

Specimens which are cut by the freezing method may be treated in the same way as celloidin sections but where stains are used, such as Sudan III, which will not stand the alcohol, they are best mounted in glycerine jelly. A fragment of glycerine jelly is placed upon the slide and gently warmed over the Bunsen burner until melted. The section is then placed upon the slide and covered with a cover-glass.

Stains.-The principle on which stains act is either: (I) the dye stains all the tissues equally, and the excess is then washed out from the section, leaving coloured the parts such as the nuclei, which retain the stain more tenaciously. Sometimes, as is the case with hematoxylin, this can be mordanted in the tissue and the rest of the tissue counter-stained with another dye. (2) A chemical is applied to the specimen which will enter into a chemical combination with another substance in the tissue, giving rise to a colouration of the tissue in which it is contained. As, for example, the ferrocyanide reaction with iron; osmic acid and Sudan III for fat; Gram's method; Weigert's method for staining the medullary sheaths of the nerve after the latter has been treated with the salts of chromic acid.

In some instances, such as after using the eosin methylene blue compounds, both factors come into play; e.g., one set of granules in the cytoplasm takes up the eosin (oxyphile or acid granules) whilst other granules take up the methylene blue (basophile). It is obvious that specimens which are to be stained in such a manner should not come in contact with anything but neutral fluids.

The following are some of the stains in general use: ${ }^{1}$

\section{Carbo-thionin}

Thionin..................... I grm.

Solution $(\mathrm{I}-40)$ carbolic acid to Ioo c.c.

Filter immediately before use.

Fixation.-Heat, alcohol, or perchloride.

Use.-For staining organisms in smear preparations, blood films and nerve cells.

Allow the stain to remain on the specimen for five minutes, wash in water, dry and mount.

${ }^{1}$ Grübler's make the best and may be obtained from Messrs. Baird and Tatlock, Hatton Garden, London, E. C. 
The following modification is useful for blood films:

Thionin..................... I. 5 grm.

Absolute alcohol.................. Io. c.c.

Solution of 5 per cent. carbolic acid to roo c.c.

Keep for two weeks, then dilute four times with water before use. Stain for ten minutes.

\section{Borax Methylene Blue}

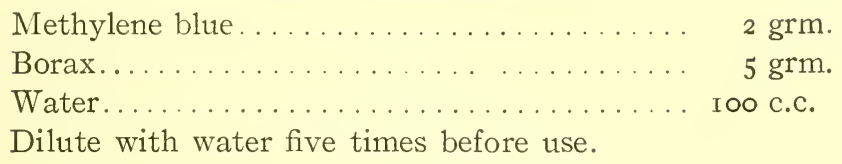

Fixation.-Heat, alcohol, or perchloride.

Use.-It can be employed in place of Lœffler's methylene blue. It is used for the same purposes as the thionin already described.

\section{Carbol-fuchsin}

Fuchsin

I grm.

Solution of carbolic acid $(\mathrm{I}-20) \ldots \ldots \ldots \ldots$ IOo c.c.

Glycerine...................... 50 c.c.

The stain may be diluted with four times its amount of water before use.

Fixation.-Heat, alcohol or perchloride.

Use.-For staining acid-fast bacilli and other organisms; it is especially useful when photographs of organisms are required.

For Staining Tubercle Bacilli.-Heat the stain in the test-tube till it steams. Flood the slide with the stain and allow it to remain on for five minutes. Place the slide in acid sulph. dil. B.P. till the colour is discharged. Wash in tap water and counter-stain with borax methylene blue. Dehydrate and mount.

\section{Carbol-gentian Violet (Gram's stain)}

Saturated alcoholic solution of gentian violet... I part.

Solution of carbolic acid $(\mathrm{I}-20) \ldots \ldots \ldots \ldots \ldots 9$ parts.

Filter before use.

The stain should be made immediately before use; when mixed, the solution will not keep more than one week.

Fixation.-Heat, alcohol or perchloride.

\section{Gram's Iodine Solution}

Iodine.

I part.

Potassium iodide

2 parts.

Water. 300 parts. 
Flood the slide with carbol-gentian violet and allow it to remain on for three minutes. Rinse in water, pour on Gram's iodine solution and allow it to remain on for one-half minute. Decolourise in absolute alcohol or aniline oil and xylol (aniline oil, 2 parts; xylol, I part). Counter-stain with safranine one-half minute, wash, dry and mount.

\section{Safranine}

Saturated aqueous solution of safranine...... I part.

Water........................ Io parts.

Use.-To counter-stain for microörganisms staining by Gram's method, either in the tissues or in smear preparations. It can also be used for staining the keratohyaline granules in the epithelium. In the latter case the decolourisation should not be so thorough as in the former.

Gram's Method of Staining Hair, Scales, Etc.-Take a suspected hair or hairs and cut off all but $1 / 4$ inch from its root. Wash in ether. Stain with carbol-gentian violet fifteen minutes. Place the hairs on a microscope slide and blot with cigarette paper. Cover with Gram's iodine for ten minutes. Decolourise with hydrochlorate of aniline (aniline oil I dram, strong hydrochloric acid 3 minims) frequently changing the solution. Cover with aniline oil one hour. Xylol, balsam.

\section{Weigert's Fibrin Stain}

Fixation.-Formalin, perhcloride, or alcohol.

Embedding.-Paraffin or freezing.

Pass the section into water. Stain with carbol-gentian violet five minutes. Drain off the stain, pour on Weigert's iodine and let it remain for one-half minute.

\section{Weigert's Iodine}

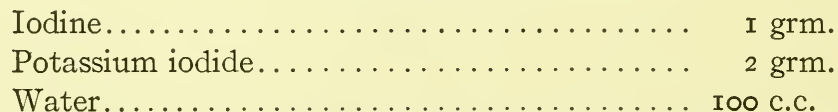

Drain quite dry and differentiate with aniline oil and xylol until no more blue colour comes away. Xylol, balsam.

Weigert's Elastic Tissue Stain.-This stain is best bought ready prepared for use, but it can be prepared as follows: Place in a porcelain dish and warm 200 c.c. of water containing resorcin 2 per cent. and fuch$\sin$ I per cent. After the mixture has been heated add 25 c.c. of ferricchloride solution (German pharmacopœia). Heat and stir for five minutes. Cool and filter, keeping the residue dry. Put filtrate and residue in a dish with 200 c.c. of 94 per cent. alcohol and boil, stir and lift out the 
filter-paper. Cool, filter and make up to 200 c.c. with alcohol 94 per cent. then add 4 c.c. of pure hydrochloric acid.

The sections are best cut in paraffine. The section is placed in the stain which should be kept covered. The stain is allowed to act for five minutes. Decolourise with I per cent. acid alcohol. The sections can subsequently be counter-stained with safranine.

\section{Sudan III}

Fixation.-Formalin or Müller.

Embedding.-Freezing.

Stain.-Sudan III, saturated solution in 96 per cent. alcohol. Filter and add two-thirds of its bulk of 50 per cent. spirit. Filter again before use.

Stain frozen sections with Sudan III, for three minutes. Wash in 30 per cent. methylated spirit. Counter-stain with borax methylene blue. Mount in glycerine jelly.

\section{Acid Methylene Blue}

Borax methylene blue......... 2 parts (see above).

Glacial acetic acid............. I part.

Water.................. 7 parts.

Filter before use,

Use.-For examining wet cytological preparations. It has the advantage of dissolving any red blood corpuscles that may be present, and so allows a better examination of the other cells.

\section{Methylene Blue (Neissler)}

Methylene blue.

I grm.

Alcohol 96 per cent................ 20 c.c.

Glacial acetic acid.............. 50 c.c.

Water.......................... 950 c.c.

\section{Bismark Brown}

Bismarck brown................... $5 \mathrm{grm}$.

Water...................... I000 c.c.

Use.-To stain Klebs-Loeffler and xerosis bacilli to show polar granules. A twenty-four hour culture of the organism on blood serum is made. Films are made and stained with methylene blue for one-half minute. Wash in tap water and treat with bismarck brown for one-half minute. Wash, dry and mount. The polar bodies appear at each end of the bacillus as dark brown dots, the body of the bacillus being stained blue. 


\section{Leishmann's Stain (eosin methylene blue compound)}

The stain should be freshly made and can be bought in tabloids from Burroughs, Wellcome \& Co. These are dissolved in pure methyl alcohol. No fixation is required as the stain is made up with alcohol. Flood the film with the stain and allow it to remain on for two minutes. Then add a few drops of distilled water and allow it to act for another two minutes. Wash in distilled water and dry in the air.

Use.-Can only be used satisfactorily for smear preparations. The basic radicle (methylene blue) picks out the nuclei, the organisms and the basophile granules, whilst the acid radicle (eosin) picks out the acid oxyphile or eosinophile granules. It is especially useful for blood films.

Leishmann's Method of Staining the Spirocheta Pallida.--Smear preparations are made from the lesions by scraping its surface. Dry in air and fix for five minutes in methyl alcohol. Dry. Smear over the surface of the film a little fresh blood serum and allow it to dry. Flood the slide with Leishmann's stain diluted with an equal part of distilled water and allow it to act for one hour; then dry and mount.

Giemsa's Method of Staining the Spirocheta Pallida and So-called Trachoma Bodies.-The stain is prepared by taking ı c.c. of tap water and adding to it I drop of a solution of $\mathrm{I}$ to Iooo solution of potassium carbonate and Io minims of Giemsa's stain (eosin azur). Place in a test-tube and mix by gently inverting the tube once or twice. Do not shake it violently. Very thin smear preparations should be made on clean slides and fixed by a gentle heat. Pour on the stain and heat the slide over a flame until it steams. Allow the stain to remain on for fifteen seconds and then pour it off repeating the same process four times. At the last application it should be allowed to act for one minute. Wash in tap water, dry and mount.

Method of Demonstrating the Spirocheta Pallida on a Dark Background.-This is the easiest way of demonstrating the protozoon in smear preparations.

Take a drop of a solution of ordinary Indian ink and place on a slide. Add to this the scraping from the suspected lesion. Mix thoroughly and make a smear by drawing another slide along the surface. Allow to dry and examine under oil immersion one-twelfth objective. The cells and spirocheta appear white on a dark background.

Silver Method of Staining the Spirochæta Pallida in the Tissues. Fixation.-Formalin, alcohol.

Place the pieces of the tissues suspended by a thread in an amber bottle and cover with the following solution:

I.5 per cent. nitrate of silver.......... 90 c.c. Pyridin...................... Io c.c. 
for three hours in the cold and three hours in the incubator at $45^{\circ} \mathrm{C}$.

Remove the tissue and place for twelve hours in the following developing solution:

4 per cent. pyrogallol................. 90 c.c.

Acetone... . . . . . . . . . . . . . . . . . . . . Io c.c.

Add I 5 c.c. of pyridin to every 85 c.c. of the mixture.

Embed in paraffin and counter-stain.

The solution should be freshly prepared.

Care must be taken to distinguish connective-tissue fibrillæ from the spirocheta pallida.

The method is not so satisfactory as the previous methods which should be applied wherever possible.

To demonstrate capsules of bacteria (McConkey's method).

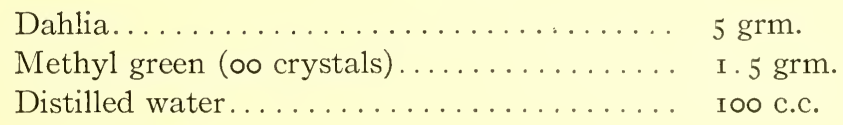

Rub these together in a mortar and add saturated alcoholic solution of carbol-fuchsin, ro c.c., followed by 9 o c.c. of distilled water.

Filter and allow to stand two weeks before use. It should be kept in an amber glass bottle.

Method.-Fix the film by heat. Flood the slide with the stain and allow it to remain on for seven minutes. Wash with distilled water, dry and mount.

To Demonstrate Flagella of Bacteria.-Examine a wet preparation for motility by making a hanging drop preparation.

To Make a Hanging Drop Preparation.-Take a slide and with a wooden match make a ring of vaseline on the slide. Take a perfectly clean cover-glass and with the platinum loop place a single drop of water on the surface. A loopful of a twenty-four hour cultivation of the organism is then mixed with the water on the cover-glass. With forceps the cover-glass is inverted on to the ring of vaseline so that the drop hangs downward in the cell so formed. Examine under one-twelfth objective. When finished with the preparation should be thrown into a bowl of lysol which should be always kept on the bench for used slides and cover-glasses.

Bacteria exhibit three kinds of movement: $(a)$ molecular or Brownian movement, (b) streaming, due to currents in the fluids, and $(c)$ true motility, due to flagellae. This is best identified by watching a single organism and comparing its movements with others. 


\section{Staining Flagellæ. (Pitfield Method Modified)}

Make a smear preparation of a twelve hour culture after emulsifying it with water in a watch glass. Care should be taken not to damage the organisms in making the smear. When dry it should be fixed by gentle heat.

\section{Mordant}

Tannic acid to per cent. aqueous solution....... Io c.c. Perchloride of mercury saturated aqueous solution 5 c.c. Alum saturated aqueous solution.......... 5 c.c. Carbol-fuchsin................. 5 c.c.

Mix well and a precipitate forms. This should always be removed by centrifugalisation immediately before use. The mordant is poured over the film and held high up over a Bunsen burner till it steams. Allow it to remain in contact with the films for two minutes, wash and dry. Pour on the following stain freshly prepared for each occasion:

Saturated aqueous solution of alum......... 25 c.c.

Saturated alcoholic solution of gentian violet.... 5 c.c.

Gently heat the slide above a Bunsen burner till steaming and allow the stain to remain in contact for three minutes. Wash in water, dry and mount.

Flagellæ may be polar or diffuse.

A single one at one pole is termed monotrichous (B. pyocyanus).

A single one at each pole is termed amphitrichous.

A bunch at one pole is termed lophotrichous.

Diffuse is peritrichous (B. typhosus; B. coli).

\section{Pappenheim's Stain}

Methyl green................ 2 knife points.

Pyronine................. 4 knife points.

Distilled water............. 1/2 large test-tube.

Test the stain by dropping on to filter paper. Good rings of individual colour should be produced. The stain does not keep good for more than about two weeks.

Fixation.-Alcohol or perchloride.

Embedding.-Paraffin.

Stain the sections for five minutes and differentiate with resorcin (two knife points) in absolute alcohol (one-half test-tube). This usually takes about five seconds. Dehydrate rapidly and clear in xylol. 
Use.-The stain is useful to demonstrate the gonococcus and bacillus of Morax-Axenfeld. It is also the best stain for examining the cytology of cells, more especially lymphocytes and plasma cells and cells of the central nervous system.

\section{Eosin Aqueous}

Eosin I part.

Water........................ roo parts.

The stain is useful for staining the cytoplasm and acid granules (eosinophiles) in the cells. When used for the latter purpose a very diluted solution (I-rooo) should be employed, the specimen being allowed to remain in it one hour or more and the excess of stain being removed by 50 per cent. methylated spirit.

\section{Van Gieson}

Sections cut in celloidin or paraffin are overstained in hematoxylin. After washing placed for two minutes in a mixture consisting of a saturated aqueous solution of picric acid to which a few drops of acidfuchsin have been added. The intensity of the red colouration can be lessened by putting the sections into picric acid first. By this stain the epithelium and endothelium become a brownish yellow whereas the connective tissue is a bright red. Iron hematoxylin picks out the nuclei better than ordinary hematoxylin.

\section{Iron Hematoxylin}

Hematoxylin $\mathrm{I}$ per cent............... 7 parts.

Liquor ferri sesquichlorati 4 per cent......... 5 parts.

\section{Ehrlich's Hematoxylin}

I. Hematoxylin.................. 2 grm.

Absolute alcohol................... 6o c.c.

2. Equal parts of glycerine and distilled water .. 60 c.c. Glacial acetic acid.................. 3 c.c. Add alum to saturation.

Mix I and 2 together and ripen for at least three weeks in the dark. The stain should be filtered before use.

\section{Method of Staining the Medullary Nerve Sheaths Kultschutzky's Hematoxylin (acid)}

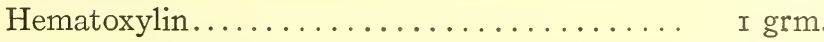

Glacial acetic acid................. 2 c.c.

Water.......................... roo c.c. 
This stain is preferable to Weigert's as it keeps well.

\section{Decolouriser (Weigert's Method)}

Sodium biborate................ 4 grm.

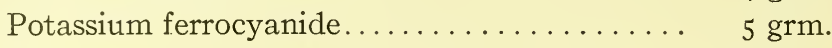

Water....................... 200 c.c.

The tissue which has been hardened in Müller's fluid is cut in celloidin and then treated as follows:

I. Copper acetate one-half saturated solution one-half hour.

2. Rinse in spirit and water.

3. Place in Kultschutzky's hematoxylin for twelve to twenty-four hours in the incubator, taking care to cover the vessel in which it is contained.

4. Wash in spirit and water.

5. Place in the decolouriser and allow it to remain until the colour is removed from everything but the nerve fibres. Wash in distilled water for five minutes and then place in tap water for several hours. Counterstain, dehydrate and mount.

\section{Method of Staining Early Fatty Degenerations in the Medullary Sheaths of Nerve Tissue}

The tissue should not be more than I cm. in thickness. It should be hardened in Müller's fluid from two to three weeks. It is then placed in Busch's fluid.

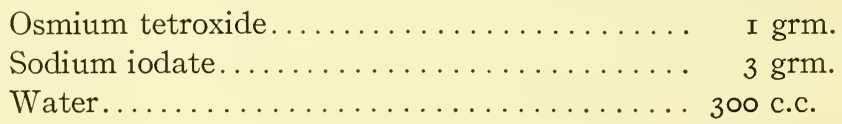

The specimen should be placed in a vessel on glass wool and covered with a solution for 6 to 7 days, a drop or two of fresh osmic acid being added daily. It is then washed for twenty-four hours in water. Dehydrate in alcohol and embed.

\section{Nissl's Method of Staining Nerve Cells}

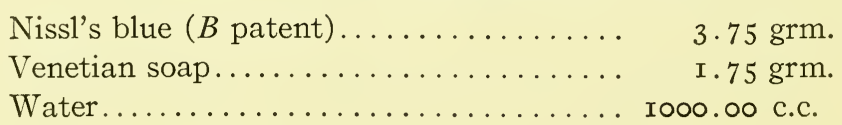

Stain the section for five minutes in then cold. Then warm till the steam rises. Decolourise in aniline alcohol (aniline I part, absolute alcohol 9 parts); xylol, balsam. 


\section{Mallory's Axis-cylinder Stain}

Stain the sections for twenty minutes to one hour in sunlight in the following mixture:

Phosphomolybdate, ro per cent......... Iо. c.c.

Hematoxylin.................. I. 75 grm.

Wash in 50 per cent. alcohol five to twenty minutes. Counter-stain. Axis-cylinders and glia stained dark blue.

\section{Ehrlich's "intravitam" Methylene Blue Method of Staining Axis- cylinders of Nerves}

Ehrlich intravitam methylene blue........ 4 grm.

Saline solution 0.6 per cent........... Ioo c.c.

Inject one-half hour before death. For fresh tissue immerse for one hour in 0.2 per cent. solution in the incubator. Then place in solution of bipicrate of ammonia, Io per cent., for two hours and transfer to equal parts of the same solution and glycerine for six to twelve hours. Tease out tissue and mount in glycerine jelly.

\section{Golgi's Method of Staining Nerve Fibres, Ganglion Cells and Neuroglia}

The fresh retina, after removal from the globe, is cut into strips dipped into thin celloidin and rolled round a piece of pith. Portions of the optic nerve are cut into thin slices, then placed into Formalin 4 per cent. . . . I part and a solution of bichromate of potash 3 per cent. . . . 4 parts for three days, after which they are transferred to a I per cent. nitrate of silver solution for another three days. Sections should be made at once. They can be held together if necessary either by melting the surface of a block of paraffin and implanting the specimen, or by embedding rapidly in celloidin. The sections should be thick and be examined as made. They should be mounted on a slide with a thick layer of Canada Balsam and without a cover-glass.

\section{Method of Staining Karyokinetic Figures in the Nucleus}

I. Fix in Zenker's solution.

2. Removing the sublimate with iodine.

3. Mordant in $\mathrm{I} / 2$ per cent. of ferric alum.

4. Rinse in water.

5. Stain for 12 hours in iron hematoxylin.

9. Rinse in water.

7. Decolourise in ferric alum solution.

8. Counter-stain if desired. 


\section{Method of Demonstrating Hyaline Changes}

Hyaline is insoluble in strong acids and alkalies. It is stained by carmine and less so by hematoxylin. It does not give the iodine reaction, which distinguishes it from amyloid degeneration.

\section{Methyl Violet for Demonstrating Amyloid Changes}

The section should be made by freezing and no alcohol must be applied to the specimen. The section is then placed in I per cent. solution of iodine. Wash in dilute sulphuric acid till blue. Then place in methyl violet for five minutes; wash in dilute potassium acetate until a pink colour is obtained.

Mount direct in glycerine jelly.

\section{Method of Removing Pigment from Cells}

Bleaching Fluid (Permanganate and Oxalic Acid Method).-Immerse the section in one-half saturate solution of potassium permanganate for fifteen minutes; rinse and transfer to ro per cent. solution of oxalic acid until decolourised; wash for one hour and then stain with logwood and eosin.

\section{Method of Demonstrating the Cement Material between Epithelial and Endothelial Cells}

I. Put fresh specimen in watch-glass in $1 / 4$ per cent. nitrate of silver, and expose to the bright sunlight for ten minutes.

2. Transfer into distilled water for quarter of an hour with one drop of 2 per cent. acetic acid in the water.

3. Wash in water.

The section can then be hardened and cut in the usual way if desired.

\section{Method of Demonstrating Cholesterin Crystals}

A few of the crystals are removed in the exudation, placed on a microscope slide and a cover glass applied. They appear as thin transparent rhombic plates, often notched at the angles. If 30 per cent. sulphuric acid is allowed to run under the cover glass it turns the crystals first orange, then rose-red, and finally dissolves them. Lugol's iodine stains the crystals dark brown, and the addition of sulphuric acid changes their colour to bright blue. They are soluble in ether and alcohol, so that when sections are cut in celloidin, only the spaces from which the crystals have been dissolved are seen in the section. 


\section{Method of Demonstrating Calcareous and Bony Changes}

In calcareous changes there is no alteration of the structures of the part beyond the deposit of calcium in the tissues, which gives a gritty feeling with a needle. It is dissolved in a 5 per cent. solution of hydrochloric acid, giving off carbonic acid gas. In true bony changes the specimen must first be decalcified (see page 686), and then sections cut in the ordinary way.

\section{Method of Demonstrating the Staining of the Eye with Iron and Copper}

Siderosis Bulbi, results from a piece of iron being embedded in the eye. It is probably due to the iron being dissolved by carbonic acid and converted into the bicarbonate of the suboxide of iron, for which certain tissues seem to have a selective affinity. Another form of siderosis occurs sometimes as the result of recurrent hemorrhages into the eye, the iron being derived from the hemoglobin in the blood.

Berlin Blue Method.-(I) Place sections in 2 per cent. aqueous solution of ferro-cyanide of potassium for a few minutes.

2. Transfer to a I per cent. solution of hydrochloric acid.

3. Wash.

4. Examine in water, alcohol or glycerine.

The tissue containing iron is stained bright blue; this is usually most marked in the epithelium of the ciliary processes, the retina and the epithelium under the lens capsule.

A freshly prepared solution of Sulphide of Ammonium will turn the iron containing tissue dark green.

Copper in the tissues is turned brown by the Berlin blue method.

\section{DEMONSTRATION OF THE MICROSCOPICAL STRUCTURE OF VARIOUS PARTS OF THE EYE}

Cornea.-The shape of the epithelial cells of the cornea is best demonstrated by macerating in a ro per cent. salt solution to which a little alcohol has been added. After two days the upper layers can be removed, and on the third day the deeper layers can be detached by rubbing the surface with a spatula. The cells can be examined in water or glycerine after staining.

The cement material between the epithelial cells can be demonstrated by the silver method (see page 708).

Lamellæ of the substantia propria can be resolved by placing small pieces of cornea for 24 hours in a solution of picric acid, they should be then teased and mounted in the ordinary way. They may be counterstained if desired.

Corneal Cells. If the cornea of a frog be carefully laid flat on a warm stage, the movements of the corneal cells can be watched. 
Fixed Corneal Corpuscles are best demonstrated by the gold chloride method.

I. The cornea of a frog or other small animal, immediately after death, is placed in freshly filtered lemon juice for 5 minutes.

2. Washed in distilled water.

3. Placed in a I per cent. gold chloride solution in the dark for I $_{5}$ minutes.

4. Transferred by a glass spatula into distilled water and washed.

5. Placed in water containing a few drops of acetic acid and exposed to the sunlight for two or three days.

6. When the corpuscles are tinted a dull red it can be removed and placed in 70 per cent. alcohol in the dark for 24 hours.

The lamellæ may be removed from the posterior surface by a knife and a needle, and either mounted or embedded in celloidin.

The Lymphatic system can be demonstrated either by the silver method, by the injection into the cornea of Methylene Blue, or by Leber's method, as follows:

I. Place a fresh cornea in a I per cent. solution of ferric sulphate for 2 minutes.

2. Remove the epithelium, and again place the tissue in the same solution for 5 minutes.

3. Wash in distilled water.

4. Place in a I per cent. solution of ferrocyanide of potassium, when the specimen becomes blue. Again wash and examine in glycerine, or harden with alcohol and make sections. The lymph spaces appear colourless on a blue field.

Corneal Nerves. Cohnheim's Method. A solution of gold chloride I per cent. . . . 8 parts. Formic acid . . . 2 parts is boiled up in a test tube three times and then placed in a watch-glass to cool. Fresh pieces of cornea are placed in it and left in the dark for about half an hour; then washed in distilled water and exposed to the daylight in Formic acid ro parts and distilled water 40 parts until the pieces become a dark violet colour. This usually takes two or three days. They are then placed in 70 per cent. alcohol in the dark for 24 hours and are transferred to 90 per cent. alcohol, also in the dark, for several days. Steel instruments should not be used for handling the specimens. Tangenital sections are then made. Ehrlich's intra-vitam Methylene Blue method can also be used.

The Elastic Fibres can be demonstrated by Weigert's method (see page 700).

The endothelium of Descemet's Membrane can be stripped off after maceration and the cells mounted. The silver method can be used for the demonstration of the cement material between the cells. 
The Uveal Tract.-The layers of the iris are best demonstrated in meridional sections. Carmine is the best stain to use as the hematoxylin makes the nuclei nearly the same colour as the pigment cells. The surface layer of the endothelium can be demonstrated as follows: a portion of iris, removed by iridectomy from a fresh specimen, is shaken up in a test tube with salt solution so as to remove the pigment; it is then stained by the silver method, dehydrated, mounted on a slide with the anterior surface uppermost. The posterior layers of the iris together with the dilatator pupillæ can be studied in albino subjects; or in bleached sections.

Nerves, can be demonstrated best in an albino's eye enucleated immediately after death, the modified Ehrlich intravitam methylene blue method of staining being employed (see page 707). The specimen should be put in an incubator for half an hour after being injected. Teased preparations should then be made.

Pigment Cells, can be demonstrated by removal of a small piece of hardened choroid and mounting it unstained on the flat. The hexagonal pigment cells, and the pigment cells of the stroma, are seen at different levels by focussing with the microscope.

Choroidal Vessels, the venæ vorticosæ are exposed in a hardened eye by incising the sclera about its equator, and then dissecting it off, forwards as far as the ora serrata, and backwards to the optic nerve. The fibres of the supra-choroidal layer should not be torn away but divided by scissors. The other layers of the choroid can be examined either by a transverse section, or by laying a piece of it out on a microscope slide and focussing the various layers, as already described.

Lens.-Zenker's fluid is the best medium for hardening the lens. In order to get good sections, specimens should be first embedded in celloidin, then the eye divided so as to open the lens capsule. The block is then re-embedded after being passed through the alcohol and ether mixture Portions of the lens capsule removed, by forceps during the operation of cataract extraction, can be stretched out on a microscope slide, stained, and examined, on the flat.

The capsule is very elastic, and when torn curls up. It is very resistant to all staining reagents. The epithelial cells lining the capsule when examined in a fresh condition are bright and granular, with large nuclei and one or two nucleoli. They are mostly hexagonal in shape, towards the equator they become slender and elongated passing into the nucleated lens fibres. The transition zone becomes very indistinct in old age, and the epithelial cells elsewhere also show signs of degeneration, sometimes over a considerable area, the protoplasm swelling up and becoming granular. After wounds of the lens mitosis is seen in the cells after the fourth day. 
The lens fibres consist of hexagonal ribbon-like bands, those at the periphery being broader and thicker than those in the centre. They are held together by a cement material which can be demonstrated by the nitrate of silver method. The lines of suture on the anterior and posterior surface of the lens can be demonstrated by the same method. Their different arrangement at various depths in the lens can be examined by peeling off the layers from without inwards.

The zonule, which has already been described (see page 70), can be best demonstrated in a hardened globe, by dividing it along the equator and removing the vitreous, cornea, sclera and iris. The specimen can then be examined with a corneal microscope both from the front and the back.

Vitreous.-The best method of fixing the vitreous humor is by boiling. The eye is put into hot water and gently brought to the boil. This coagulates the fluid of the vitreous without rendering it very opaque, and the eye can be then treated in the ordinary way.

Retina.-The retina is a transparent membrane, attached in the eye only at the ora serrata and optic disc. During life it is purplish red in colour; the colouring matter being in the outer segments of the rods. It is bleached at once by exposure to daylight. The visual purple is best seen in fishes which have a white tapetum. The macula is yellow in colour due to a pigment which is diffused through all its layers. The fovea is recognised as a bright depression devoid of blood-vessels and surrounded by a very yellow ring. For sections of the retina; the eye is best hardened in Zenker's fluid.

The hexagonal cells of the pigment epithelium can be seen by stretching out a piece of the choroid and examining it on the flat. The cells are cemented together by neurokeratin. Single cells can be examined either at the edge of the specimen or by teasing it out. They consist of an outer zone containing the nucleus and an inner one with projecting processes. These processes are short in the human retina, but long in birds and cold blood animals. The length of the processes, and the position of the pigment in the cells, vary with exposure to light. In the dark the processes shorten and the pigment recedes into the outer nuclear zone. In the light the processes lengthen inwards and the pigment streams forwards into them.

Rods and cones are prolongations through the external limiting membrane of the outer nuclear layer. Weigert's stain for the medullary sheaths, with slight decolourisation, stains their inner segments.

The individual cells in the retina can be demonstrated by means of osmic acid. A fresh retina is placed in a I per cent. solution of osmic acid for 24 hours, and macerated in distilled water for two days, the water being frequently changed; small portions can then be removed and placed on a slide in glycerine and examined under a microscope. 
To demonstrate the external limiting membrane transverse sections of the retina should be made, and the specimens placed in tincture of iodine I part, and serum (e.g. pericardial fluid) roo parts; or Golgi's method of staining should be employed. The membrane is formed by the apposition of the outer ends of the sustentacular fibres of Müller, and is perforated by apertures corresponding to the rods and cones. Those for the cones being larger than those for the rods.

The cells in the outer nuclear layer are the nuclei of the rods and cones. They are best demonstrated by hardening in Zenker's fluid and staining with safranin. The chromatin within the cells often presents a ribbed appearance.

The outer molecular layer presents a granular appearance in sections; the granules are really transverse sections of fibrils which form the reticulum of synapses. The endings of the outer nuclear layer and the arborisation of the bipolar cells of the inner nuclear layer, are best demonstrated by Golgi's method.

The inner nuclear layer from without inwards has three sets of cells. First, the horizontal cells which stain well with hematoxylin, then the bipolar cells which are spindle-shaped and which connect by their synapses the cells of the outer nuclear layer with the ganglion cell layer, and thirdly, flat branched cells known as "spongioblasts."

The inner molecular layer is similar to the outer molecular layer and consists of a plexus of anastomosing nerve fibrills.

The ganglion cell layer can be demonstrated by Golgi's method, by the intravitam methylene blue process, or by Pappenheim's stain. In most parts of the retina they form a single layer, but in the macular region there are several layers. Between the ganglion cells pass the fibres of Müller which are processes of neuroglial cells.

The best means of studying the nerve fibre layer is by the intravitam Methylene blue method. The axis-cylinder are fine fibrillæ held together by a small amount of interfibrillar substance.

The membrane limitans interna is formed by the bases of Müller's fibres.

Optic Nerve.-For hardening the optic nerve Müller's fluid is the best for general purposes. Zenker's fluid should be used when microörganisms have to be demonstrated. Recent degenerative changes in the nerve are best shown by means of Busch fluid. The neuroglia is best stained by Golgi or Weigert's method. Transverse sections of the nerve usually afford the best method of detecting changes in it, its sheaths and blood-vessels. The nerve is covered from without inwards by dural, arachnoidal and pial sheaths. The pial sheath sends septa into the nerve forming the framework which carry the nutrient blood-vessels. Between these septa, supporting the bundles of nerve fibres, there is a fine meshwork of neuroglia. The axis-cylinders are enclosed in thin 
myelin sheaths in which varicosites are frequently seen. The medullary sheaths pass as far forward as the lamina cribrosa where they cease. The lamina cribrosa itself is mainly made up of yellow elastic tissues, it stains with Weigert's elastic stain, and with acid orcein.

Diseases affecting the central retinal artery or vein are best studied in transverse serial sections. The area in the neighbourhood of the lamina cribrosa being the most important, the papilla should be removed from the globe with the nerve attached.

\section{EXAMINATION OF BLOOD AND VARIOUS FLUIDS OF THE EYE}

The following methods applicable to the examination of blood and various fluids of the eye are given, but for the details of the clinical examination of the blood a larger work on clinical pathology should be consulted.

Cleaning Slides and Cover-glasses for Making Smear Preparations.The slides should be cleansed by boiling in 5 per cent. lysol and kept subsequently in the same solution.

Cover-glasses are cleaned by immersing in nitric acid for an hour, washing them and placing for three hours in 5 per cent. solution of ammonia. Subsequently they should be kept in absolute alcohol.

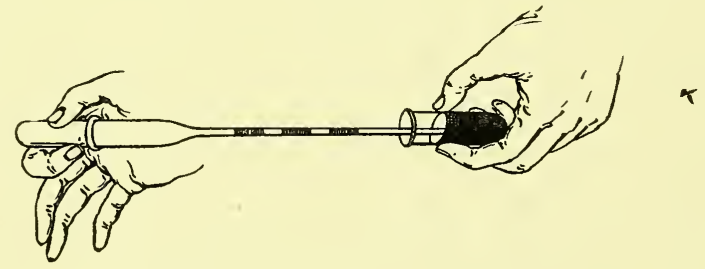

FIG. 302.- Showing the method of drawing up equal volumes of fluid into a capillary pipette. The clear spaces in the tube represent air bubbles.

The best method of wiping slides and cover-glasses is to use an old piece of clean linen. It is most important in preparing cover-glasses for blood films that no trace of grease from the fingers should be left on them.

Pipettes (Fig. 302) are useful for the collection of aqueous from the anterior chamber, and also for mixing minute quantities of fluid in equal proportions. They are made in the following way:

A piece of glass tubing 6 inches long, about the thickness of a lead pencil, is heated in the flame of a blowpipe till thoroughly soft. It is then removed from the flame and drawn out until of sufficient length. The capillary tube thus formed should be about the thickness of a knitting needle. It is then allowed to cool and broken in two. The 
capillary tube so formed is heated near its end in a small flame and again drawn out so that it has a pointed end. When the pipette is required for use a rubber teat is placed on the larger end, and the fine tip of the capillary end is broken off. The fluid is sucked up into the tube by means of the teat. The end may be again sealed leaving the fluid in the capillary part of the tube. When it is required to mix equal parts of two fluids the capillary tube is marked, and the first fluid drawn up into the tube to the mark. An air bubble is then allowed to enter, and the other fluid, with which it is required to mix the first, is drawn up into the tube up to the same mark. They are then blown out on to a slide and mixed.

To Make a Sterile Glass Needle.--Take a piece of capillary glass tubing, such as the thin end of a pipette, and draw it out in the flame of a spirit lamp or wax match; two sterile needles are thus obtained.

Wright's blood capsules (Fig. 303) are the best for taking specimens of blood. They can be bought of R. B. Turner, I I Foster Lane E. C. or made in the laboratory. They are used as follows:

Method of Collecting Blood for Wassermann Reaction, Opsonic Index, Etc.-Let the patient hang his arm downward or swing it for a

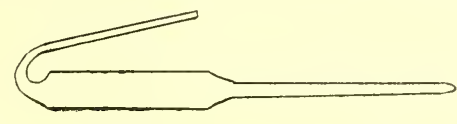

FIG. 303.-Wright's blood capsule.

time. Place a piece of rubber tubing round the finger so as to stop the venous return. After cleansing the skin with alcohol prick the finger on the back above the nail. Hold the blood tube with the curve downward, and apply the open curved end to the drop of blood, when it will be found that the blood will run into the tube. When the tube is about half full, still holding the loop downward, the other end is heated in the spirit lamp and the capillary end finally sealed. As the air contracts in the lower end of the tube the blood will be sucked up into the body of the tube. The curved end of the tube can then be hooked over the side of a vessel. The capsules should be carefully marked so as to recognise the case from which the blood is taken. In children, or where a larger amount of blood is required, the ear is preferable to the finger. It should be rubbed well before making the puncture, which should be performed with a glass needle, the blood subsequently being squeezed out.

As the blood clots in the tube the serum separates. This can be removed by notching the upper part of the body of the tube with a glass knife or file and breaking off the curved end in a cloth. The serum is removed with a pipette as already described. 
When large quantities of blood are desired, as for the examination of it for microörganisms in sympathetic ophthalmia and other metastatic infections of the eye, the following method should be employed: the skin of the arm in the antecubical fossa is thoroughly cleansed with antiseptics. A firm bandage is then placed around the arm sufficiently tight to obstruct venous return. With a sharp sterile hypodermic needle having an all glass syringe attached, capable of holding at least 20 c.c., a puncture of one of the largest superficial veins is made, the point of the needle being directed away from the patient's body so that it faces the blood flow. The piston of the syringe is then gently withdrawn

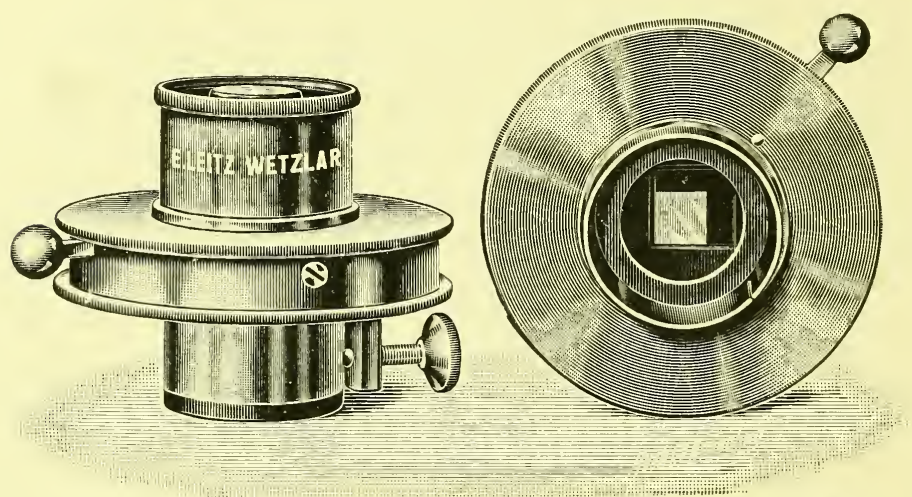

FIG. 304.-Ehrlich eye-piece which divides the field into squares.

till sufficient blood is obtained. The contents of the syringe should be expelled into at least two culture tubes, broth and agar being best for the purpose. If the former is used the blood clot at first retains the organisms, but in the course of a few days in the incubator they will spread out into the surrounding medium. The bandage should be removed from the arm immediately the syringe is withdrawn, otherwise the blood will be extravasated into the tissue.

To Make Blood Films.-Prick the finger and wipe away the first drop of blood. When enough has again accumulated take two coverglasses, one in each hand, and hold them by their opposite corners with the finger and thumb; touch the drop of blood with the surface of one of them; now place the other on it and a thin film will be found between them. Do not squeeze the cover-glasses together. Slide one gently and evenly off the other and allow them to dry in the air. They may subsequently be fixed by heat, alcohol, or perchloride of mercury.

Method of Preparation of Vaccines (see page 425). - The following are the steps: 
I. Determine the organism causing the disease.

2. Make a forty-eight hour culture on solid medium from the patient suffering from the disease, taking care to select a lesion that is likely to be free from contamination with other organisms.

3. Add to the tube a small quantity of sterile normal saline solution and shake gently, so that the colonies are washed off from the medium.

4. Pour off the fluid and dilute with sterile normal saline solution until the fluid is slightly opalescent.

5. Standardise the solution by estimation of the number of organisms. This is best performed by mixing equal parts of the emulsion with diluted blood, and counting the number of corpuscles and organisms in several fields of the microscope. This is performed as follows:

(a) Prick the finger and wipe away the first drop of blood. When the second drop forms draw it up to a mark on a capillary tube.

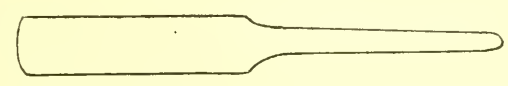

FIG. 305.-Sealed glass capsules for vaccines, serum, etc.

(b) Allow a small air bubble to enter, and then draw up four times the quantity of $1 / 2$ per cent. sodium citrate in normal saline. (Tabloids are prepared of this mixture by Burroughs, Wellcome \& Co., as the solution does not keep.)

(c) Blow the whole out on to a slide and mix thoroughly. There is then a mixture which contains a million red corpuscles to the cubic millimetre of fluid.

(d) Draw up into another tube equal parts of this mixture and of the fluid containing the organism. Mix thoroughly on a slide.

(e) Make a smear preparation of the mixture, stain with carbo-thionin, and count the organisms and corpuscles in ten fields of the microscope. This is much facilitated by the use of Ehrlich's eye piece which divides the field into squares (Fig. 304).

$(f)$ Having estimated the proportion between the red blood corpuscles and the organisms it is easy to calculate the number of organisms to the cubic millimetre; thus if there are 500 red blood corpuscles and so organisms the proportion will be as I to 5. In a cubic millimetre of diluted blood there are Iooo red blood corpuscles, therefore in a cubic millimetre of the fluid there is one-fifth of that amount, namely 200 organisms.

6. Bottle off the fluid containing the organisms into sterile capsules (Fig. 305) and heat them in hot water at $60^{\circ} \mathrm{C}$. for an hour, taking care that the water covers the capsules.

7. Test for sterility. Open a capsule, make a cultivation from the contents and place in the incubator. If sterile it is ready for use. 
Table Showing the Decimal System Equivalent in English Measure

$$
\begin{aligned}
\text { Ioo c.c. } & =35.25 \text { fluid oz. } \\
\text { I litre } & =3.5 \text { fluid oz. } \\
\text { I gràm } & =\text { I } 5.43 \text { grains. } \\
28.4 \text { c.c. } & =\text { I fluid oz. } \\
3.5 \text { c.c. } & =\text { I fluid dram. } \\
.064 \text { gram } & =\text { I grain. }
\end{aligned}
$$

To convert c.c. per litre into minims per oz. (approximately) divide by 2 ; e.g., I 2 c.c. per litre $\mathrm{I} 2 \div 2=6 \mathrm{~mm}$. per oz.

To convert gram per litre into grains per oz. multiply by $7 / 16$ which gives grains per oz.

To convert degrees centigrade to fahrenheit multiply by $9 / 5$ and add 32. 


\section{INDEX}

The figures in italics indicate the chief reference.

Abiotrophy, 587, 632, 642

Achromatopsia, congenital, 39

Acne vulgaris, 494

Actinomycosis, $55^{\circ}$

Adenoma eyelids, I68

lacrymal, I69

Agglutination, 4I8

Agglutinin, 41.5, 419

Albinism, I I, 80, I 23

Alexin, 4.15, 4I7

Amaurotic family idioicy, 626

Amblyopia alcoholic, 273

congenital, 37,39

diabetic, $27 \mathrm{I}$

exanopsia, 1.56

puerperal, 27 I

quinine, 274,626

tobacco, 272

toxic, $27 \mathrm{c}$

uremic, $27 \mathrm{I}$

Ambobceptor, 4I7, 42 I

Anaphylaxis, 428, 585

Anemia, 263

Aneurysm-arterio-venous, 408

Angeoid streaks, 256

Aniridia, 8o, 96 pseudo, 468

Ankyloblepharon congenital, I30, I 44

Ankylostomiasis, 567

Anophthalmia, I0, I6

Anterior chamber, cyst, 226

traumatic, 37 I

examination, 689

filaria in, 566

Antibodies, 415

to lens, 3

Antigen, 4I5

Antitoxin, 4I5
Aphakia congenital, 42, 43

Aqueous, diminished secretion, 333

examination, 68I

formation of, 280, 284, 293, 336, $46 I$

increased excretion, 334

Arcus juvenils, $8 \mathrm{I}$

senilis, 656

Arlt's streak, 573

Artery centralis retinæ, 24I, 669 abnormalities, 60,63

obstruction of, $243,267,625$

ciliary obstruction, 248

cilio-retinal, 60, 63, 241, 243

hyaloid, 57

persistent, 45,60

sheath of, 60

ophthalmic embolism, 268

Aspergillus, 55 I

Astigmatism, 80, $9 I$

Atrophy epithelial, $5^{89}$

Autolysins, 42 I

Bacilli, 5I I

Bacillus aerogenes, 548

coli, $5^{25}$

diphtheriæe, 5 I6, 701

Ducrey, 547

Friedlander, 524

glanders, 546

influenzæ, 5I 5

Koch-Weeks, 512

leprosy, 544

Morax-Axenfeld, $52 \mathrm{I}$

of Müller, 5I.5

of Petit, 524

pyocyaneus, 549

subtilis, 547

tuberculosis, 526, 699 
Bacillus typhosus, 525

xerosis, 520

Zur Nedden, 547

Bacteria, 490

Bacteriological examination, 679

Bacteriolysin, 4I 5, 4I 7

Basophiles, 433

Beriberi, 269

Birth injuries, 365

Bjerrum's sign, 322

Bleaching, 708

Blepharitis, 479, 494

Blood supply of eye, 24I

vessels, degeneration of, 669

injection of, 683

Bone formation, 676

Botryomycosis, $55^{2}$

Bruch, membrane of, 9, 644

Bupthalmia, 79, 86, 305, 3 I I , 659

Burns, 393

Calcium deficiency, 623

Calmette's reaction, 530

Carcinoma glandular of skin, I60, I67

lacrymal, I6o, I69

of meibomian glands, I60, 168

melanotic, I60, I72, I85

metastatic, $22 \mathrm{I}$

squamous, I 58, I $60, I 6 I$

epibulbar, $I 62$

Carmine solution, 683

Cataract, 595

"after," 385

axial fusiform, 43, 52

black, 6 I 6

calcium deficiency, 623

capsular, 595

concussion, 355

congenital miosis in, $4 \mathrm{I}$

coralliform, 43,53

diabetic, 606

disc shaped, 43, 49, $5^{2}$

Doyne's, 54

equatorial, 609

glass blowers, 393,395

glaucomatous, 3 I 6

inherited, 2, 624
Cataract in irido cyclitis, 464

lamellar, 6i 7

Morgagnian, 6I 2

congenital, 43, $5 I$

naphthalin, 608

nuclear, 6 I 5

congenital, 43,44

parathyroid insufficiency, 4, 622

perinuclear, 6I 5, 6I7

polar, anterior, 49, 386, 45I, 596

punctate congenital, 43,55

pyramidal, 597

secondary, 607

senile, 609, 6I0

shrunken fibrous tissue, 43,47

subcapsular, 603

traumatic, 384, 603

triradiate, 43,53

$\mathrm{x}$-rays, 402

zonular, 6I 7

Cavernous sinus thrombosis, 262

Celloidin, 694

Cerebro-macular degeneration, 635

Chalazion, 494

Chancre, 556

Chemotaxis, 424

Chloroma, 266

Cholesterine crystals, 675,708

Chorioplaques, 437

Choroid, angioma of, 2 I 6

blood supply, I07, 242

carcinoma metastatic, 22 I

circulation impeded, 25I

colloid degeneration, 644

coloboma of, $2 \mathrm{I}, 80,105$ macular, I06, I08

detachment of, $280,332,336$

development of, 9, 79

embolism, 267

in glaucoma, $3 \mathbf{I} 5$

hemorrhages, 252, 256

membrane of Bruch, 9, 644

muscle tissue, 80, IIO

neurofibromatosis, 2 I I

ossification, $469,675,676$

rupture of, 357

sarcoma of, 190

causing glaucoma, 302 
Choroid, vascular sclerosis, 25I, 672 vessels examination, 7 I I

Choroideremia, I06

Choroiditis, 459 central guttate, 645

Doyne's "honeycomb," 646

gummatous, 562

sympathetic, 584

syphilitic, 557,562

Tay's, 645

tuberculous, 539

Ciliary body, adenoma of, I 82

coloboma, 66

cyst, $235,337,653$

inflammatory, 233

detachment, 280, 336

development, 9

epithelial hyperplasia, I 82

in glaucoma, $3 \mathrm{I} 4$

gumma of, 562

injuries of pars plana, 349

prolapse, 379

sarcoma of, I 88

muscle changes in myopia and hypermetropia, I I 7

development of, 79

in hypermetropia, 122

rupture of, 346

Cleft-fetal ocular, 7, 20, 65, 70, 665

Cocci, 49 I

Colloid bodies, 644

Colour blindness congenital, 5, I I, 35 , 38

Complement, 4I 7

deviation of, 42 I

Concussion, 344

Conjunctiva, 438

amyloid degeneration,

$$
675,708
$$

calcification, 675

concretions, 594

cyst lymphatic, 238

traumatic, 226

cysticercus of, 565

edema, 292

epithelium of, 438

examination, 682

false glands of, 44I
Conjunctiva, foreign bodies in, 387

hyaline degeneration, 674

keratinisation of, $44^{2}$

lymphagioma, 220

lymphangiectasis, 220

lymphoma of, 205

neurofibromatosis, 209

organisms of, 486

ossification, 675

pinguecula, 59I, 666

pterygium, 666

sub, epithelium of, 443

staining of, 395

wounds of, 380

xerosis, $40 \mathrm{I}, 443,59 \mathrm{I}$

Conjunctivitis, 440

angular, 522, 594

b. coli, 525

chronic, 446

diphtheritic, 5I9

follicular, 446

Friedlander, 524

glanders, 546

gonococcal, 506

insectivorous, 568

influenzal, 5I 5

Koch-Weeks, 5I4

leprosy, 545

meningococcal, 5 I I

Morax-Axenfeld, 522, 594

Parinaud's, 495

pneumococcal, 502

streptococcal, 499

trachoma, 570, 702

tuberculous, 532

Corectopia, 80, IO4

Cornea, abrasion of, 366

abscess of, 448

annular infiltration, 455

arcus senilis, 656

astigmatism, $80,9 I$

atrophy marginal, $65^{8}$

blood staining of, 254

burns, 393

calcareous film, 654

conical, 80,89

corpuscles, 450

cyst traumatic, 226 
Cornea, development of substantia propria, 77

diameters of, 93

edema of, $288,590,654$

epithelium of, 167

examination of, 688,709

foreign bodies in, 387

inflammation (vide keratitis).

in glaucoma, 3Io, 654

herpes zoster, 29I

hyaline bodies of, 658

megalo, 79,89

micro, 79,89

nodular opacity, 660

opacities and vascularity congenital, 79,80

pannus of, 573

pterygium, 667

recurrent bulla, 29 I

repair of, 449

reticular opacity, 660

ring abscess, 455

infiltration of, 449

rupture of, 340

siderosis, 39I

staphylococcal infection of, 496

staphyloma, 663

ulcer internal, 449 (vide keratitis).

Mooren's, 456

serpigenous, 449, 503

wounds of, 365

xerosis, 592

Corpus nigrum, 28

Couching, 355

Cryptophthalmia, I03, I 40

Cutting sections, 696

Cyclitis, 46I

and cataract, 607

gummatous, 562

sympathetic, 584

syphilitic, 562

tuberculous, 538

Cyclodialysis, 326, 332

Cyclopia, I o, I I

Cycloplegia traumatic, 345

Cystic eyeball congenital, I0, 18

Cysticercus, 565
Cystoid Cicatrix, 328, 373

Cysts, 225

dermoid, I29, I35

Cytology, 680

Cytolysin, 3, 4I7, 4I9

Dacryoadenitis, 477

Dacryocystitis, 478

Day blindness congenital, I0, 35

Decalcification, 686

Degenerations, 586

amyloid, 675, 708

calcareous, 676

of cells, 437,586

cerebro-macular, 635

combined, subacute, 265

fatty, 594, 657, 675

hyaline, 594, 674, 708

Landouzy-Déjérine's, 674

mesoblastic tissue, 653

mucoid, 594

neural, 625

neuroglial, 647

optic nerve (vide optic nerve atrophy).

pars ciliaris retinæ, 653

iridis, 653

retina, 628,643

toxic, 587

Demicyclops, I29, I 33

Dermoid, cyst, I 29, I 35

growth, I30, I4I

implantation, 392

Descemet's membrane, development of, 78

Desensitisation, 43 I

Desquamation, 590

Development of eye, 7

Discoria, I04

Disseminated sclerosis, 640

Distichiasis, I30, I46

Doyne's honeycomb, chorioditis, 646

Eclipse blindness, 396

Ectropion, cicatricial, 403

xerosis in, 592

Edema, 280, 590

Effusion serous, 280 
Elliot's sign, 323

Embedding, 694

Embolism of arteria centralis retinæ, 243

Encephalocele, orbito-meningo, I29, I 34

Endothelial cells, 436

Endothelioma, conjunctival, 200 interfasicular, 197

Enophthalmia traumatic, 406, 408

Enophthalmos, congenita1, I 30, I49 in goitre, $15 \mathrm{I}$

Entozoa, 564

Entropion, cicatricial, 403

Eosinophilis, 433 in Spring catarrh, 580

Epibulbar sarcoma, I60, I70, I99

Epicanthus, I29, I33

Epiphora, 403

Episcleritis, 457

Epithelial cells, 437 plaques, I60, 166

Epithelioid cells, 437

Epithelioma, I58, I60, I62 neuro of retina, $160, I 7 I$ peri-bulbar, I 63 peri-corneal, I63

Epithelium atrophy of, 589

Erythropsia, 397

Examination, external, 687

Exophthalmic goitre, 26I

Exophthalmos congenital, I 29, I 34 pulsating, 26I

Eyelid, atrophy senile, 589

burns of, 404

coloboma of, I 29, I 30

contusion of, 402

development of, I28

edema of, 293

pediculosis, 568

scabies, 568

wounds, 402

Face, development of, I 26

Fat orbital hernia of, 130, 136

Favus, $55^{2}$

Fibroma-neuro, 160, I66, 207

Field in glaucoma, $32 \mathrm{I}$
Filaria, 566

bancrofti, 567

loa, 567

oculi, 567

Filtration scars, 328,370

Fixation, 684

Foreign bodies, intra-ocular, 388 orbital, 408

Formol saline, 685

Fovea, 37, I 55, 242

albinotic, 123

cherry red, 244

Freezing, 69o, 695

Fungi, 549

Gas poisoning, 394

Giant cells, 436

Gland lacrymal, 1ymphoma of, 206 of Henlé, 44 I

Glaucoma, 299

aniridia in, 98

blood staining of the cornea in, 254

cataract in, 608

caused by intra-ocular growths, I 92,302

cysts, 228

hypermetropia, I 22, 307

thrombosis, 246

choroidal hemorrhage, 256

congenital, 79, 86

corneal degeneration, 654

haloes, 310

post-operative, 375,377

primary, 307

secondary, 299, 454, 462

Glioma of retina, I6o, $I 7 I$

pseudo, 45, 65, 460, 469, 504, 5 I I

Gliomatosis of optic nerve, $172, I 78$

Gonococcus, 505

Guanidin in cataract, 623

Hemangioma, I60, 214

Hemianopsia, bitempora1, 638

- homonymous, 639

Hemolysins, 420, 492

Hemolysis, 4I 5

Hemophilia, 269 
Hemorrhage, cholesterin in, 675 after glaucoma operations, $33^{2}$ into optic nerve, 26I retinal, 259

in anæmia, 263

subhyaloid, 255, 257

traumatic, 364

Henlé's gland cyst of, 23I

Herbert's sclerotomy, 330

Herpes Zoster, 29I

Heterolysins, 420

Heterophoria, I $30,{ }_{52}$

Hole at optic disc, Io

Hordeolum externum, 494

Horns, I60, I66, 592

Hyaline thickening of Descemet's membrane, 658

Hyaloid detachment of, 288

Hydatid, 565

Hyperkeratosis, 588, 590

Hypermetropia, 80, I I9, I 2 I

Hypersusceptibility, 428

Hypertony concussion, 363

Hyphemia, 252

Hyphomycetes, 549

Hypopyon, 447, 468

Hypotony, 333, 35I

concussion, 363

Idiosyncrasies, 432

Immunity, 4I 4

Implantation cyst, 225

Infection, dissemination of, 48 I

ectogenous, 483

endogenous, 484

methods of, 480

of wounds, 485

Inflammation, $4 \mathrm{IO}$

blood changes in, $4 \mathrm{I} 3$

histology, 4I 2

intra-uterine, 437

Injuries, 339

Insectivora, 568

Intra-ocular foreign bodies, 388

Iridectomy, 331, 375

choroidal hemorrhage, 256

dialysis, 325

glaucoma, 324
Irido cyclitis, $46 I, 484,496$

blowfly, 570

plastic, 464

post operative, 376

serous, 463

suppurative, 468

sympathetic, 465

trypanosomiasis, 564

Iridodialysis, 345

Iridodonesis, 354

Iris, absence of, 80,96

blood supply, 242

bombé, 467

coloboma of, 66, 80, 99, IOI

cyst, edematous, 233, 286

inflammatory, 232

traumatic, 228

cysticercus, 565

cysts, 239

development of, $9,78,98$

dilator, $\mathbf{I} 83$

ectropion of uveal pigment, Io, 27,96

examination, 689

in glaucoma, $3 \mathbf{I} 2$

gumma, 562

hemorrhages, $25^{2}$

heterochromia, I 24

hyaline membrane, 659

hyperplasia, 80

hypoplasia, 80

injuries, 343

new vessels in thrombosis, 246

polycoria, 80

prolapse, 373

pseudo-coloboma of, 80, IOI

retroflexion, 354

rupture of pigment epithelium 345

sphincter, 345

sarcoma of, 188

siderosis, 39 I

sphincter, 183

synechia anterior, congenital, 79 , $8 I$

synnechia anterior, 372

tissue formation on anterior surface, 95 
Iris, wounds of, 383

Iritis, 466

amæbic, 564

gonococcal, 5 IO

gummatous, 562

sympathetic, 584

syphilitic, 556, 562

tuberculous, 534

Ischemia, 264

Isoagglutination, 420

Isolysins, 420

Jaw winking, I30, I47

Keratectasia, 455 marginal, $65^{8}$

Keratinisation, 588, 590

Keratitis, 446 aspergillus, 55I atheromatous, 589

b. of Ducrey, 547

botryomycosis, $55^{2}$

bullous, 367

coli, 525

dendritic, 29I

filamentary, 29I, 367

Friedlander, 524

gonococcal, 509

interstitial, 455, 559

Koch-Weeks, 5I 5

lagophthalmic, 592

leprosy, 545

meningococcal, 5 I I

Morax-Axenfeld, 523

nodular, 659

pneumococcal, 503

profunda, 454

punctata, $436,463,536,446$

punctate superficial, 29I

reticular, 659

sclerosing, $45^{8}$

sequestrating cicatricial, 589

streptococcal, 499

striate, $335,370,454$

syphilitic, 559

trypanosomiasis, 564

tuberculous, 533

vesicular, 289
Keratocele, 45 I

Keratoconus, 8o, 89

Keratomalacia, 499

Koeppe's sign, 314

Krause's gland, cyst of, 23 I

Laboratory methods, 679

Lacrymal canal, absence of, I 29, I 32 canaliculi defective, I 30

concretions, 549

ducts inflammation, 478

fistula, I 30

gland, cyst of, 229

inflammation of, 477

lymphoma, 206

sarcoma of, 206

mixed mesoblastic tumour of, 207

obstruction congenital, I30, I39, 478

syphilitic, $55^{8}$

passages abnormalities, $I 38$

development of, 127

sac, fistula of, $I 39,478$

inflammation of, 478,487

tubercle of, 544

Lagophthalmia, 592

congenital, I30, I44

Lagrange's sclerectomy, 330

Lamina cribosa rupture of, 36I

iridis pupillaris, 78

supra choroidal, cyst of, 239

Landouzy Déjérine degeneration, 674

Leber's atrophy, 642

Lens, absence of, 42,43

adhesion to ciliary processes, 60 , 77

antibodies, 3

bladder cells, 6I 4

capsule adhesions, $374,45 \mathrm{I}$

congenital, defect of, 44,48

coloboma, 6o, 72

degeneration myopic, 6 I 6

degenerations, 595 (see cataract).

development, 7, 4I, 53

dislocation pseudo, 254

traumatic, 343,352 
Lens, displacement forwards, 336

ectopia, 6o, $7 I$

examination, 689, $7 I I$

fibro-vascular sheath, $42,57,78$, 98

glaucoma, 3 I 6

growth, I 59, 620

nucleus, defective development of, 50

displacement of, 43,52

opaque membrane behind, 43 , 45

pigmentation, 616

sclerosis, 6ro, 6I 5

siderosis, 39I

suspensory ligament, coloboma of, 66,70

sutures, 53

synechia, congenital, 79, $8 I$

tubercle, 543

tyrosin in, 616

vesicular cells, 6I 4

wounds of, 383

zones of, 54

Lenticonus anterior, 60,75

posterior, $43,45,47,5^{2}$

Leontiasis ossea, 2 I 2

Leucocidin, 493

Leucocytes large mononuculear, 434

polymorphonuclear, 433

Leucocytic endolysins, 426

Leucoma, 450

adherens, $45^{2}$

Leukemia, 266

Lid, cyst Meibomian, 230

cysts, 230

hemiangioma, 2 I 5

neurofibromatosis, 209

notches, I30, I38

Ligament, suspensory development, 59

Ligamentum pectinatum, development of, 78

defect of development of, 87 , 98

endothelioma of, 198

rupture of, 346

Lightning injuries, 400, 605
Lime burns, 394

Lipemia retinalis, $25 \mathrm{I}$

Loa Loa, 567 .

Lupus, 533

Lymph circulation, 277

Lymphangiectasis, 220

Lymphangioma, I60, 219

Lymphocyte, large, 434 small, 434

Lymphoma, I60, 205

Lympho-sarcoma, I60, 205

Macula, 449

hole, 361

star figure, $25^{\circ}$

Magnesium producing cyclopia, I 5

Malaria, 563

Mast cells, 433

Megalo, cornea, 79, 89

Meibomian cyst, 230, 494

Melanoma, I60, I72, I82

Melanosis, 8o, I24

Membrane, persistent, 80, 93 pupillary, 78 synechia of, $79,8 I$

Mendelism, 5

Meningitis, 475

Meningoccus, 5 IO

Metastases, 22 I

Mickulic's disease, 206

Microblepharon, I30, I44

Microcoria, 80, IO 4

Microcornea, 79, 89

Microphthalmia, I0, 20, 44, 67

Miosis congenital, I I, 40 senile, 653 tabetic, 640

Moles, I60, I70, I6I, 200

Moll's gland, cyst of, 230

Morganian globules, $6 \mathrm{I} 3$

Mounting sections, 697 specimens, 692

Mucocele, 478, 504

Müller's fluid, 685

Muscæ volitantes, 60, 68

Muscle, degeneration, 673 development of, I 29

Müller's, I5I 
Muscle, obliques, I 52 orbicularis development, I48 recti, I5I insertions of, I 53 retractor bulbi, I5 I

Mydriasis traumatic, 345

Myiasis, 568

Myoma, I60, 213

Myopia, axial, 80, III, 65I

in cataract, 616

causation of, I 20

in glaucoma, 316

hemorrhages, 256

in hypotony, 336

index, 73

malignant, I I 9

vitreous in, I I 6

Naphthalene, effect on development, 2

Nebula, 449

Nemathelminthes, 567

Neoplasms, ${ }_{5} 8$

Nerve fibres, absence of, 10, 29 opaque, I, Io, 3I, 626

Neuritis, 473

interstitial, 474

pseudo, 10, 34, I 22

retrobulbar, 269, 473

Neuro-fibroma, I69, 207

fibromatosis, 160, 207

Neuroma plexiform, 208

Neutrophilis, 433

Nevi, I60, I7o, I6I, 200

Night blindness, 270, 397, 593, 630

congenital, I0, 35

Nissl granule's, 625

Nystagmus, 35, 37, 398

in albinism, I 23

miner's, 399

Onyx, 448

Opaque nerve fibres, I, IO, 3 I

Ophthalmia, electrica, 398

gonorroheal, 438

neonatorum, 506

nodosa, 387

sympathetic, 582

Oposonin, 424
Optic chiasma, I 55

pressure, 638

disc, atrophy, 475

waxy, $63 \mathrm{I}$

crescent congenital, Io 8

distraction, I I 5

myopic, I09, II 3

supertraction, I I 5

cupping, $3 \mathrm{I} 6$

congenital, 3 I

physiological, I0, 34

cysts on, 63

hole in, Io, 33

hyaline nodules, 632,648

serous effusion into, 287

foramen, 404

nerve, amyloid bodies, 649

atrophy, 475, 635

disseminated sclerosis, 640

hereditary, 642

Leber's, 642

primary, 639

secondary, 635

tabetic, 640

coloboma, I0, 20, 26, 64

development, 7,8

endothelioma of, 204

evulsion of, $36 \mathrm{I}$

examination, 7 I 3

fibroma of, 2 I 2

in glaucoma, 3 I 6

gliomatosis of, $172, I 78$

gumma, 562

hemorrhage into, 26I

hole in, Io

tubercle of, 543

neuritis, 473

retrobulbar, 473

Orbit, cysts of, 239

edema, 292

endothelioma of, 204

foreign bodies in, 408

fractures of, 404

gumma, 563

hemangioma, 2 I 5

inflammation of, 480

inflammatory pseudo tumour of, 202 
Orbit, injuries of, 404

lymphangioma, $22 I$

lympho-sarcoma, 224

maggots in, 569

myoma of, 213

neurofibromatosis, 210

osteoma of, 2 I 2

sarcoma of, $20 \mathrm{I}$

thyroid carcinoma of, 224

wounds of, 406

Orbital cellulitis, 480,504

Osteoma, I60, 212

Oxycephaly, $I 34,638$

Palsies, ocular progressive, 673 traumatic, 407

Pannus, 573

Panophthalmitis, 45I, 468, 504

Papilledema, 287, 338, 475 traumatic, 405

Papilloma, I60, I65

Paraffin, 695

Parathyroids and cataract, 622

Paresis, ocular congenital, I30, I48

Pediculosis of lids, 568

Pencillum glaucum, 532

Peridacryocystitis, 478

Perineuritis, 475

Perithelioma, 196

Phagocytosis, 423

Phlyctenule, 444

Phthisis bulbi, 469

Pinguecula, 591, 666

Pituitary growths, 638

in Leber's atrophy, 642

Plasma cell, 434

Plica semilunaris, I30, I43

Pneumococcus, 499

Polycoria, 80, 103

Postmortem methods, 683

Precipitin, 4I5, 4I9

Presbyopia, 6I 5, 653, 672

Protein therapy, 427

Pterygium, 666 pseudo, 393, 667

Ptosis, atrophic, 673 congenital, I30, I46 traumatic, 403
Pupil, Argyll Robertson, 640

corectopia, 80, IO 4

discoria, 80, I04

hemiopic reaction, 639

microcoria, 80, IO4

miosis (vide miosis).

occluded, 466

polycoria, 80, I03

secluded, 466

Purpura, 268

Pyemia, 484

Retina, amaurotic family idiocy, 627 amyloid bodies, 649

anemia, 263

angioma, 218

arteria centralis development, 56

arterio-sclerosis, $67 \mathrm{I}$

artery obstruction, $243,267,625$

at birth, 8

cells pigmented, $63 \mathrm{I}$

coloboma, Iо, 20

commotio, $35^{8}$

cones, 39

congenital pigmentation, I0, 27

cysts, 237,288

cyst congenital, 22

degeneration, cerebro-macular, 635

ganglion cells, 625

macular, 633

neuroglial, 647

pars, ciliaris, 653

iridis, 653

pigment epithelium, 631, 643

pigmentary, 629

rods, and cones, 628

detachment, 233, 235, 247, 250, $282,338,343,349,377$, $565,570,643$

malignant, I9I

development, 7

edema, 470

erythræmia, 265

examination of, 712

in albinism, 123

fovea, 37 (see also fovea). 
Retina, glaucomatous, 316

glioma of, I60, $I 7 I$

hemophilia, 269

hemorrhages, 252, 257

filarial, 567

malarial, 563

hereditary hyaline infiltration, 646

hyaline bodies, 649

leukemia, 266

lipemia, 25I

macular degeneration, 633

hole, 361

star figure, $25^{\circ}$

nerve fibres, 319

pars, ciliaris adenoma of, I60, $172, I 8 I$

pigmentation inflammatory, 459

traumatic, 360

purpura, 268

rupture, 358

siderosis, 39I

vascular sclerosis, 669

venous thrombosis, 245, 266, 302

vessels, exudation from, 287

pulsation, 299, $3{ }^{18}$

watered silk, I0, I9, I22

Retinitis, $47^{\circ}$

albuminuric, 248, 282

arterio-sclerotic, $67 I$

circinata, 260

diabetic, 25 I

massive, 247,260

pigmentosa, 25I, 629, 643, 648

proliferans, 248, 256, 257, 472

punctata albescens, I0, 35

purulent, 473

septica, 268, 473

staphylococcal, 497

syphilitic, 558, 669

tuberculous, 543

Ring worm, 553

Roenne's step, 322

Rosettes, 176

Sæmisch section, 449

Sarcoma, I86
Sarcoma, choroid, r9o

epibulbar, 160, 170, I99

intra-ocular, I60, 187

leuco, 194

lympho, I60, 224

in melanosis, 125

orbital, I60, 201

ring, 190, 197

Schlemm's canal, 297

Sclerophthalmia, 8I

Scleritis, 457

annular, $45^{8}$

deep, $45^{8}$

Sclerotic, 457,689

blue, 80 , I Io

development, 79

foreign bodies, 387

in glaucoma, 308

rupture of, 340

staphyloma, 662

wounds of, $38 \mathrm{I}$

Sclerotomy anterior, 378

Scurvy, 270

Seidel's sign, 322

Sensitisation, 429

Side chain theory, 4 I 6

Siderosis bulbi, 39I, 709

Silver nitrate, 443

Skin reactions, 43I

Slit lamp, cataract senile, 609 concussion, 357

conical cornea, 90

hyaline thickenings of Descemet's membrane, 659

hyaloid artery remnants, 57

Koeppe's sign, 314

lens at birth, 616

sutures, 53

zones, 54

membrane pupillary persistent, 93

vitreous, $58,65^{2}$

Snow blindness, 398

Sparganum, Mansoni, 567

Spasmus nutans, 399

Spirochæta, 553, 702

Spring catarrh, 579

Staining, 697 
Staphylococcus, 491,585

Staphyloma, 310, 374, 662

anterior, 452

congenital anterior, 79, 82, 84, 142

partial, 453

total, 453

verum, I 12

xerosis of, 592

Stelwag's brawny edema, 573,578 , 674

Strabismus, 37

concomitant, I30, 154

Streptococcus, 497

Streptothrix, 549

Stye, 494

Supra-choroiditis, 46I

Symblepharon, 394, 403

Sympathetic ophthalmitis, 344,465 , 485,582

Synchisis scintillans, 65 I

Synechiæ congenital anterior, 79, 81 posterior, 466

Synophthalmia, Io, II

Syphilis, 553

Tabes, 640

Tapeworms, 564

Tay's choroiditis, 645

Teeth, lamellar cataract, 62 I

Teleplasms, I43

Tenon's capsule, 479 effusion into, 293

Tenonitis, 479

Tension, intraocular, 293

Teratoma, I 43

Thelaziasis, 567

Thrombosis central retinal vein, 245

Thyroid, metastatic orbital tumours, 224

Tissue culture, I 59

Toxic, degenerations, 587

Toxins, 418

Trachoma, 570, 674, 702

Trephining, 330

Treponema pallidum (see spirochæta pallida).

Trichiasis, congenital, I 30, I44
Trypanosomiasis, 564

Tuberculin, 529

Tuberculous, ocular lesions, 53 I

Typhoid fever, 525

Tyrosin in lens, $6 \mathrm{I} 6$

U1cer rodent, I60, 167

Uveal tract, 458

blood supply, 242

carcinoma, secondary, 22 I

examination, 7 I I

Uveitis leprosy, 546

meningococcal, 5I I

non-superative, 459

staphylococcal, 496

suppurative, $45^{8}$

sympathetic, 584

syphilitic, 556

tuberculous, 534

Vaccines, 425, 716

Vaquez's disease, 265

Vascular sclerosis, 251, 669

choroidal, 672

retinal, 67 I

Vein, central retinal obstruction of, $245,266,302$

vortex obstruction of, 248,309

Vernal catarrh, 579

Vesiculisation, 590

Vessels, rupture of, 252

Virulence, 427

Vitamins, 269, 593

Vitreous, 650

atypical development of 45,60 , 64

cholesterine crystals, 675

coloboma of, 60,68

cysticercus, 565

degeneration, $65^{\circ}$

detachment of, I I 7, 256, 652

development, 7,56

examination, 682, 712

hemorrhages, $255,266,350$

hyaloid membrane, 652

myopia, I I6

opacities, 65 I

prolapse, 343,376 
Vitreous, slit lamp, $5^{8}$

synchisis scintillans, 651,675

Von Pirquet's test, 43I, 530

Von Reckinghausen's disease, 208

Wassermann test, 42I, 7I 5

Wernicke's test, 639

Wound infection, 485
Xerosis, 40I, 443, 591

hemeralopic, 593

$\mathrm{X}$-ray injuries, 4 o I

Zeiss gland, cyst of, 230

Zenker's fluid, 686

Zinn circle of, 24I, 256 





